\title{
Direct measurement of the Higgs boson mass, natural width, and cross section times branching ratio to four leptons using a per-event lineshape in the Higgs to $\mathrm{ZZ}$ to four lepton decay channel with the ATLAS detector
}

\author{
Graham Cree \\ A thesis submitted to the \\ Faculty of Graduate and Postdoctoral Affairs \\ in partial fulfillment of the requirements for the degree of
}

Doctor of Philosophy, Particle Physics

\author{
Department of Physics \\ Carleton University \\ Ottawa-Carleton Institute for Physics \\ Ottawa, Ontario \\ April, 2017
}

(c) Graham Cree, 2017 


\section{Abstract}

The discovery of the Higgs boson by the ATLAS and CMS Collaborations in 2012 remains the crowning achievement of the Large Hadron Collider (LHC) physics programme. Five years since its discovery, Run 2 at the LHC is underway and producing more data than ever before, allowing measurements of the Higgs boson beyond the reach of Run 1. Precise measurement of the Higgs boson's properties help guide particle physicists in understanding the Standard Model, and what lays beyond.

This thesis presents a measurement of the Higgs boson mass, natural width, and cross section times branching ratio in the $H \rightarrow Z Z^{(*)} \rightarrow 4 \ell$ decay channel using the full $2015+2016$ combined dataset from Run 2 at the LHC, totalling $36.1 \mathrm{fb}^{-1}$ of $p$ - $p$ collisions at centre-of-mass energy $\sqrt{s}=13 \mathrm{TeV}$. The analysis is performed using a technique developed by the author, called the per-event response method. The technique is designed to produce a more precise, accurate, and model-independent measurement of the Higgs boson properties grounded directly on the performance of the ATLAS detector.

Using this technique, the Higgs boson mass was measured to be $m_{H}=124.61_{-0.44}^{+0.44}$ (stat.) ${ }_{-0.06}^{+0.06}$ (syst.) GeV. The total Higgs boson production cross section times branching ratio to four electrons or muons in $p$ - $p$ collisions at $\sqrt{s}=13 \mathrm{TeV}$ was measured to be $\sigma_{H} \times \mathrm{BR}(H \rightarrow 4 \ell)=8.55_{-1.15}^{+1.23}$ (stat. ${ }_{-0.52}^{+0.63}$ (syst.) fb. An upper limit was set on the natural width of the Higgs as $\Gamma_{H}<3.65$ (stat.) $\oplus 0.19$ (syst.) $\mathrm{GeV}$ at $95 \%$ confidence. These measurements are consistent with previous measurements and with the Standard Model. This result is the first ever measurement of Higgs 
boson mass using ATLAS at $\sqrt{s}=13 \mathrm{TeV}$, exceeding the precision of any $H \rightarrow 4 \ell$ $m_{H}$ measurement made to this point.

Also presented are the author's contributions to the precision assembly of the small-strip Thin Gap Chambers to be installed in ATLAS in 2019, and to the ATLAS reconstruction software, both essential to continued detector operation and the many physics analyses yet to come from ATLAS. 


\section{Acknowledgments}

As the other students graduated and the office evolved, it rarely occurred to me that my turn would come as well. For having gotten this far, I owe several people my sincere gratitude:

My supervisor, Thomas Koffas, for his guidance, advice, and loyal advocacy on my behalf.

To Christos Anastopoulos, William Leight, Ioannis Nomidis, for an abundance of useful discussions, and for tolerating my pickiness over shared analysis code.

To the other members of the Carleton student office, David Di Valentino, Jim Lacey, Tom McCarthy, Rick Ueno, Stephen Weber, Kate Whalen, for categorically rejecting the miserable grad student trope, and helping me do the same. The last five years have not been without challenges, but for the most part I was just having fun.

To my parents, for a lifetime (mine) of love and support (theirs), without which I would certainly not be here. To my grandmother, who sometimes hears news from the LHC before I do, and to the rest of my family, whose support has been invaluable.

Finally, to Emily, who has been an unwavering ally and my ultimate confidante through this whole affair. I would be neither where I am, nor who I am, without you.

I would also like to acknowledge the support of the Natural Sciences and Engineering Research Council of Canada. 


\section{Table of Contents}

Abstract

Acknowledgments $\quad$ iv

Table of Contents $\quad$ v

List of Tables $\quad$ X

List of Figures $\quad$ xii

List of Abbreviations $\quad$ xix

1 Introduction 1

2 The Standard Model 5

2.1 Particle Content . . . . . . . . . . . . . . . . . 6

2.1.1 Composite particles . . . . . . . . . . . . . . 8

2.2 Interactions . . . . . . . . . . . . . . . . . . . . 10

2.2.1 Strong interaction . . . . . . . . . . . . . . 11

2.2.2 Electroweak interaction . . . . . . . . . . . . . . . . 12

2.2.3 Z boson decay . . . . . . . . . . . . . . . . . 12

2.3 The Higgs boson . . . . . . . . . . . . . . . . . . . . . . . 15

2.3.1 The Higgs mechanism . . . . . . . . . . . . . . . . . 15

2.3.2 Higgs production and decay . . . . . . . . . . . . . . . . . 20 
2.3.3 The $H \rightarrow Z Z^{(*)} \rightarrow 4 \ell$ channel . . . . . . . . . . . . 28

2.4 Beyond the Standard Model . . . . . . . . . . . . . . . . . . . . . 29

3 ATLAS and the LHC 31

3.1 The $\mathrm{LHC} \ldots \ldots \ldots \ldots \ldots \ldots$

3.1 .1 From canister to collision . . . . . . . . . . . . . . . . 32

3.1 .2 Collisions . . . . . . . . . . . . . . . . 34

3.2 The ATLAS Experiment . . . . . . . . . . . . . . . 37

3.2 .1 The ATLAS coordinate system . . . . . . . . . 38

3.2 .2 Inner Detector . . . . . . . . . . . . . . . . . . . 42

3.2 .3 Calorimeters . . . . . . . . . . . . . . . . . . . 45

3.2 .4 Muon Spectrometer . . . . . . . . . . . . . . . . . . . . 48

3.3 New Small Wheel . . . . . . . . . . . . . . . . . . . . . 50

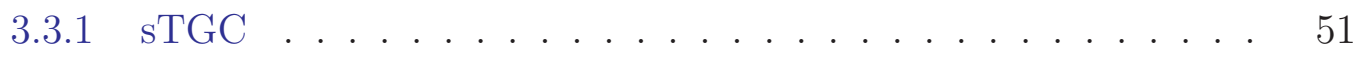

4 Reconstruction $\quad 63$

4.1 Data and Simulation . . . . . . . . . . . . . . 63

4.2 Kinematic formulae . . . . . . . . . . . . . . . 66

4.3 Trigger . . . . . . . . . . . . . . . . . . 70

4.4 Electrons and photons . . . . . . . . . . . . . . 72

4.4 .1 Gaussian Sum Filter . . . . . . . . . . . . . . . 73

4.5 Muons . . . . . . . . . . . . . . . . . . . . . . . . . . 82

4.5.1 Silicon Associated Forward Muons _. . . . . . . . . . 84

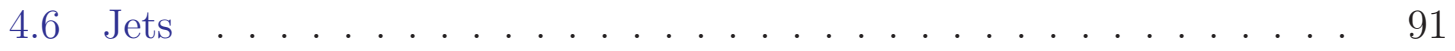

4.7 Missing transverse energy $\ldots \ldots \ldots \ldots \ldots$

4.8 Reconstruction summary . . . . . . . . . . . . . . . . 93

$5 \quad H \rightarrow Z Z^{(*)} \rightarrow 4 \ell$ Event Selection $\quad 94$

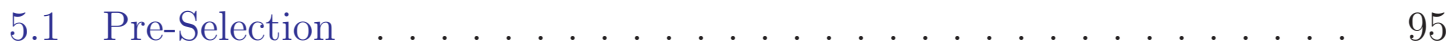


5.2 Event-level selection . . . . . . . . . . . . . . . . . . . . 95

5.3 Lepton selection . . . . . . . . . . . . . . . . . . . . . 98

5.3 .1 Electron selection . . . . . . . . . . . . . . . 98

5.3 .2 Muon selection . . . . . . . . . . . . . . . . . . . . . . . 99

5.4 Jet and photon selection . . . . . . . . . . . . . . 100

5.5 Overlap removal . . . . . . . . . . . . . . . . . . . . . . . . 100

$5.6 \quad 4 \ell$ selection $\ldots \ldots \ldots \ldots$

5.7 Additional Criteria . . . . . . . . . . . . . . . . . 103

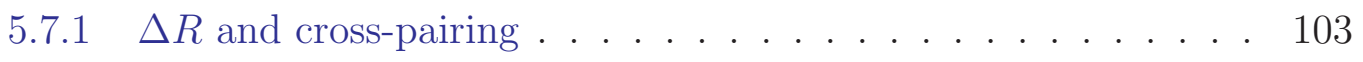

5.7 .2 Isolation . . . . . . . . . . . . . . . . . 103

$5.7 .3 d_{0}$ significance and vertexing $\ldots \ldots \ldots \ldots \ldots$

$5.8 \quad$ FSR recovery . . . . . . . . . . . . . . . . . . . . . . . . . . . 104

5.9 Z Mass Constraint . . . . . . . . . . . . . . . . . . . . 105

5.10 Invariant mass resolution . . . . . . . . . . . . . . . . . . 109

5.11 Analysis categories . . . . . . . . . . . . . . . . 114

6 Background Estimation and Modelling $\quad 115$

6.1 Standard Model $Z Z^{*} \ldots \ldots \ldots \ldots$

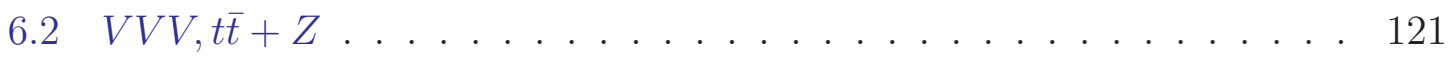

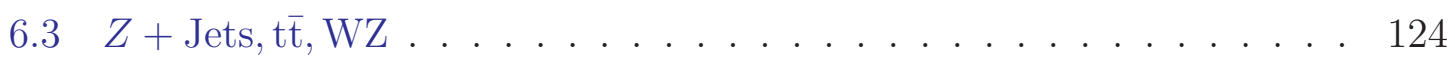

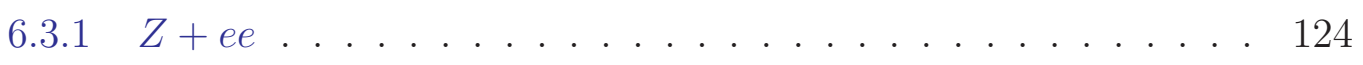

$6.3 .2 Z+\mu \mu \ldots \ldots \ldots \ldots \ldots \ldots \ldots \ldots \ldots \ldots \ldots \ldots$

$6.3 .3 Z$ + Jets, $\mathrm{t} \overline{\mathrm{t}}, \mathrm{WZ}$ PDF $\ldots \ldots \ldots \ldots \ldots \ldots$

6.4 Backgrounds summary . . . . . . . . . . . . . . . . . 135

7 Per-Event Response Signal Model 136

7.1 Signal Normalization . . . . . . . . . . . . . . . . . 137

7.2 Template Method . . . . . . . . . . . . . . . . . . . . . 138 
7.2.1 Signal template smoothing . . . . . . . . . . . . . . . . 140

7.2 .2 Signal template morphing . . . . . . . . . . . . . . . 140

7.3 Per-Event Response method . . . . . . . . . . . . . . . 146

7.3.1 Per-Event Response PDF . . . . . . . . . . . . . . 147

7.3.2 Describing Single Lepton Energy Response . . . . . . . . . . 150

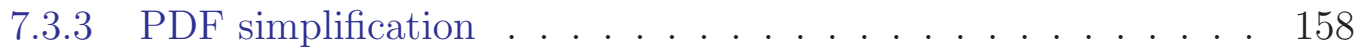

7.3.4 Gaussian sum parameter transformation . . . . . . . . . 162

7.3.5 Response after Z Mass Constraint . . . . . . . . . . . . . 163

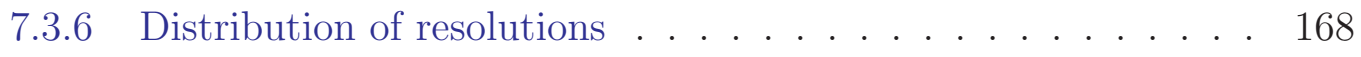

7.4 Choosing a signal model . . . . . . . . . . . . . . . . . 173

8 Systematic Uncertainties $\quad 175$

8.1 Parameterization of systematic uncertainties . . . . . . . . 176

8.2 Sources of systematic uncertainty $\ldots \ldots \ldots \ldots \ldots \ldots$

8.2 .1 Theory systematics . . . . . . . . . . . . . . . . 178

8.2 .2 Experimental systematics _. . . . . . . . . . . . 180

8.2 .3 Analysis Systematics . . . . . . . . . . . . . . . . 182

8.3 Systematic affecting event yields . . . . . . . . . . . . . . . . . 182

8.4 Systematics affecting scale and resolution . . . . . . . . . . . 189

9 Statistics and Property Measurement 197

9.1 The method of maximum likelihood . . . . . . . . . . . . . 198

9.2 Tests using an effective dataset . . . . . . . . . . . . . 204

9.2.1 Tests of expected resolution _ . . . . . . . . . . . . 205

9.2 .2 Tests of model closure . . . . . . . . . . . . . . . . . 210

9.3 Effects of systematics . . . . . . . . . . . . . . . . . 213

9.4 Tests using pseudo-experiments . . . . . . . . . . . . 218

9.4.1 Fit results in pseudo-experiments . . . . . . . . . . . . 219 
9.4 .2 Toy-by-toy comparison . . . . . . . . . . . . . . . . 227

9.5 Validation using the $Z \rightarrow 4 \ell$ resonance $\ldots \ldots \ldots \ldots \ldots$

9.5.1 Per-event response and the $Z \rightarrow 4 \ell$ lineshape . . . . . . . 232

9.5.2 Property measurement of the $Z \rightarrow 4 \ell$ resonance . . . . . . 236

9.6 Results with data . . . . . . . . . . . . . . . . . . . . . . . . . . . . . . 241

10 Conclusion 253

$\begin{array}{ll}\text { Appendix A Backgrounds supplementary plots } & 256\end{array}$

Appendix B Extra Discussion of the Signal Model 260

B.1 Stability of Template interpolation f . . . . . . . . . . 260

B.2 On the definition of $x_{i} \ldots \ldots \ldots \ldots \ldots \ldots \ldots$

B.3 Response model fits . . . . . . . . . . . . . . . . . . . 263

B.4 Response model parameters . . . . . . . . . . . . . . . . . 271

B.5 Model Independence of the PER signal model . . . . . . . . . . 276

$\begin{array}{ll}\text { Appendix C Statistics and Measurement Supplement } & 278\end{array}$

C.1 Data distributions of $m_{4 \ell}^{\mathrm{fsr}} \ldots \ldots \ldots \ldots \ldots \ldots \ldots \ldots$

C.2 Post-fit PDF . . . . . . . . . . . . . . . . . . . . . . 281

C.3 More results from pseudo-experiments . . . . . . . . . . . . 284

C.4 $Z \rightarrow 4 \ell$ analysis using $m_{4 \ell}^{\mathrm{fsr}} \ldots \ldots \ldots \ldots \ldots \ldots \ldots$

C.5 Response distribution of data events . . . . . . . . . 300

$\begin{array}{ll}\text { List of References } & 309\end{array}$ 


\section{List of Tables}

1 Standard Model Fermions . . . . . . . . . . . . . . . . 6

2 Standard Model Bosons . . . . . . . . . . . . . . . . . 7

3 SM Higgs production fractions . . . . . . . . . . . . . . 24

4 Branching Ratio $\mathrm{BR}\left(H \rightarrow Z Z^{*}\right) \ldots \ldots \ldots \ldots \ldots \ldots$

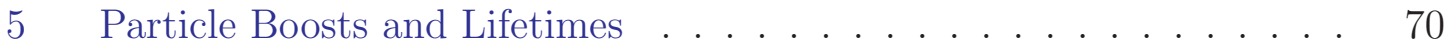

6 Trigger Rates . . . . . . . . . . . . . . . . . . 71

7 Muon Reconstruction Resolution by Type . . . . . . . . . . . 90

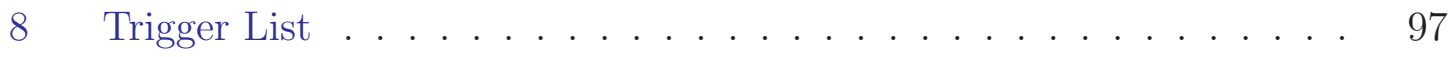

9 Invariant mass resolution . . . . . . . . . . . . . . 110

$10 Z+e e$ Background Estimate . . . . . . . . . . . . . . . 132

11 Backgrounds summary table . . . . . . . . . . . . . 135

12 Expected signal yield . . . . . . . . . . . . . . . . . . . 138

13 Unconstrained PER transformation parameters . . . . . . . . 163

14 Constrained PER transformation parameters . . . . . . . . . 167

15 Dominant systematics for signal yield . . . . . . . . . . . 188

16 Dominant systematics for signal scale . . . . . . . . . . . . . . 193

17 Dominant systematics for signal resolution . . . . . . . . . . . 193

18 Effective dataset results . . . . . . . . . . . . . . . . . 209

19 MC statistical fluctuation of $m_{H} \ldots \ldots \ldots \ldots \ldots$

20 Residual bias of $m_{H} \quad \ldots \ldots \ldots \ldots \ldots \ldots \ldots$

21 Systematics summary on $m_{H}, \sigma_{H \rightarrow 4 \ell}, \Gamma_{H} \ldots \ldots \ldots \ldots \ldots$ 
22 Summary table of toys fitting $m_{H} \ldots \ldots \ldots$. . . . . . . . 221

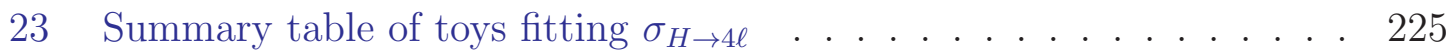

24 Summary table of toys fitting $\Gamma_{H} \ldots \ldots \ldots$. . . . . . . . 226

25 Improvement from PER . . . . . . . . . . . . . . . . . 231

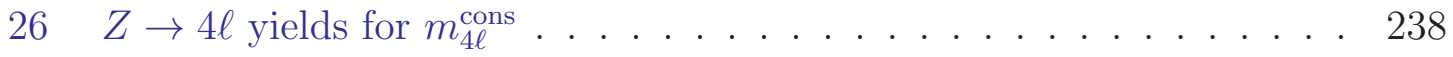

$27 Z \rightarrow 4 \ell$ constrained fit results . . . . . . . . . . . . . . . . . 240

28 Event yields in data . . . . . . . . . . . . . . . . . . . . . 242

29 Fit results in data . . . . . . . . . . . . . . . . . . 249

30 Lepton response model example fits . . . . . . . . . . . . . . 263

31 Lepton response model parameters table . . . . . . . . . . . . . . 271

$32 Z \rightarrow 4 \ell$ yields for $m_{4 \ell}^{\mathrm{fsr}} \ldots \ldots \ldots \ldots \ldots \ldots$

$33 Z \rightarrow 4 \ell$ unconstrained fit results . . . . . . . . . . . . . . . 299

34 Per-event PDFs figures summary . . . . . . . . . . . . . . . 300 


\section{List of Figures}

1 Proton Substructure . . . . . . . . . . . . . . . . . . . 9

2 Strong interaction diagrams . . . . . . . . . . . . . . . 11

3 Electroweak interaction diagrams . . . . . . . . . . . . 13

4 Z boson lineshape . . . . . . . . . . . . . . . . . . . . . 14

$5 \quad$ Higgs Potential . . . . . . . . . . . . . . . 16

6 Higgs interaction diagrams . . . . . . . . . . . . . . . . . 21

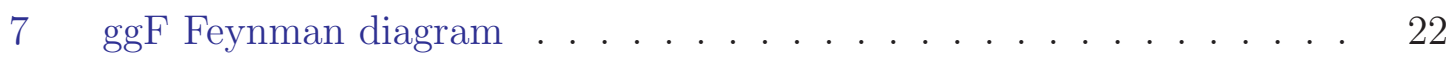

8 VBF Feynman diagram . . . . . . . . . . . . . . . 23

9 VH Feynman diagram $\ldots \ldots \ldots \ldots \ldots$

10 ttH Feynman diagram . . . . . . . . . . . . . . . . . . 24

11 Higgs production cross section versus $m_{H} \ldots \ldots \ldots \ldots \ldots$

12 Higgs branching ratio versus $m_{H} \ldots \ldots \ldots \ldots \ldots$

13 Higgs lineshape . . . . . . . . . . . . . . . . . . . 27

$14 H \rightarrow Z Z^{(*)} \rightarrow 4 \ell$ Feynman diagram . . . . . . . . . . 28

15 LHC Accelerator Chain . . . . . . . . . . . . . . . . . 35

16 LHC Collision Event . . . . . . . . . . . . . . . . . 37

17 ATLAS Detector $\ldots \ldots \ldots \ldots \ldots \ldots \ldots \ldots$

18 ATLAS Coordinate System _. . . . . . . . . . . . . . 39

19 Pseudorapidity Conversion . . . . . . . . . . . . . . . . . . 41

20 ATLAS Inner Detector . . . . . . . . . . . . . . . . 43

21 ATLAS Inner Detector in $r-|z| \ldots \ldots \ldots \ldots \ldots$ 
22 ATLAS Calorimeter System . . . . . . . . . . . . . . 46

23 ATLAS Muon Spectrometer . . . . . . . . . . . . . . . . 49

24 ATLAS Muon Spectrometer in $r-|z| \ldots \ldots \ldots \ldots$

25 NSW Triggering . . . . . . . . . . . . . . . . . 51

26 sTGC and NSW assembly . . . . . . . . . . . . . 53

27 sTGC Alignment Mechanism . . . . . . . . . . . . . . . 55

28 sTGC Alignment Microscope . . . . . . . . . . . . . . 56

29 sTGC Alignment Algorithm . . . . . . . . . . . . . . . 57

30 Alignment Measurement Calibration . . . . . . . . . 58

31 Alignment Measurement Orientation . . . . . . . . . . 58

32 Alignment Microscope Orientation _. . . . . . . . . . . . . . 59

33 Alignment Measurement Example . . . . . . . . . . . . . . . 60

34 sTGC Alignment Measurement Locations . . . . . . . . . . . . . . 62

35 Gaussian sum approximation of Bethe-Heitler . . . . . . . . . . 74

36 Gaussian Sum Filter propagation . . . . . . . . . . 75

$37(q / p)$ pull for different $N_{r e d} \ldots \ldots \ldots \ldots \ldots \ldots \ldots$

38 Tuning of GSF parameter $N_{\text {red }} \ldots \ldots \ldots \ldots \ldots \ldots$

39 Auto-vectorization . . . . . . . . . . . . . . . 80

40 GSF CPU Improvements . . . . . . . . . . . . . . . . 81

41 Pixel and SCT hits by muon type . . . . . . . . . . . 85

42 Muon Types . . . . . . . . . . . . . . . . . . . . 87

43 Muon Kinematics by Type . . . . . . . . . . . . . . . . 88

44 Muon Resolution by Type . . . . . . . . . . . . . . . . . . . . . 89

45 Muon Resolution vs Hits . . . . . . . . . . . . . . . . . . . . . 90

46 Particle Reconstruction in ATLAS . . . . . . . . . . . . . . 93

47 Impact Parameters $d_{0}, z_{0} \ldots \ldots \ldots \ldots \ldots \ldots \ldots$

48 Di-lepton invariant mass cuts . . . . . . . . . . . . . . 103 
$m_{12}$ distributions . . . . . . . . . . . . . . . . . . 108

$50 m_{4 \ell}$ resolution .......................... 110

$51 m_{4 \ell}$ resolution with FSR . . . . . . . . . . . . . 111

$52 m_{4 \ell}$ resolution with $\mathrm{FSR}+\mathrm{ZMC} \ldots \ldots \ldots . \ldots . \ldots . \ldots 112$

53 Inclusive $m_{4 \ell}$ resolution . . . . . . . . . . . . . . . . . . . . 113

$54 q \bar{q} \rightarrow Z Z^{(*)}$ Feynman diagrams . . . . . . . . . . . . . . . . 116

$55 g g \rightarrow Z Z^{(*)}$ Feynman diagram . . . . . . . . . . . . . 117

$56 q \bar{q} \rightarrow Z Z^{(*)} \mathrm{PDF}$ of $m_{4 \ell}^{\mathrm{cons}} \ldots \ldots \ldots \ldots \ldots \ldots$

$57 g g \rightarrow Z Z^{(*)} \mathrm{PDF}$ of $m_{4 \ell}^{\text {cons }} \ldots \ldots \ldots \ldots \ldots \ldots$

58 Resonant $q \bar{q} \rightarrow Z Z^{(*)}$ Feynman diagram . . . . . . . . . . . . . . . . 121

$59 \quad V V V$ Feynman diagrams . . . . . . . . . . . . . . . . . . . . . . . 122

$60 t \bar{t}+Z$ Feynman diagrams . . . . . . . . . . . . . . . . . . . . . 122

$61 V V V, t \bar{t}+Z \mathrm{PDF}$ of $m_{4 \ell}^{\mathrm{cons}} \ldots \ldots \ldots \ldots \ldots \ldots \ldots$

$62 n_{\text {InnerPix }}$ templates from the $Z+X$ CR . . . . . . . . . . . 127

63 Data/MC comparison of $Z+X$ CR . . . . . . . . . . . 128

$64 \mathrm{X}$ electron efficiency vs. $p_{T} \ldots \ldots \ldots \ldots$. . . . . . . . . 129

65 Fit on data of the $3 \ell+X \mathrm{CR} \ldots \ldots . \ldots 131$

$66 \quad Z+\mu \mu \mathrm{CRs} \ldots \ldots \ldots \ldots \ldots \ldots \ldots \ldots$

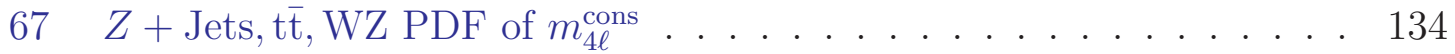

68 Signal Yield Parameterization . . . . . . . . . . . . . . . . 138

69 Template smoothing $4 \mu \ldots \ldots \ldots \ldots$. . . . . . . . . 141

70 Template smoothing 4 . . . . . . . . . . . . . . . . . 142

71 Template smoothing $2 \mu 2 e \ldots \ldots \ldots$. . . . . . . . . . 143

72 Template smoothing $2 e 2 \mu \ldots \ldots \ldots \ldots$. . . . . . . . . 144

73 Template morphing . . . . . . . . . . . . . . . . 145

74 Unconstrained muon response fits $(1 / 2) \ldots \ldots \ldots$. . . . . . 153

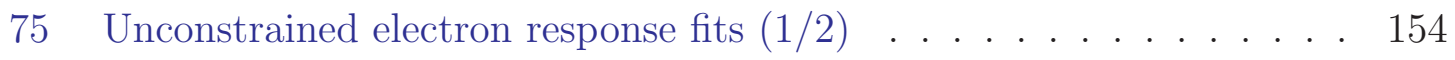


$76 \quad \chi^{2} / n$ distribution of unconstrained lepton response model . . . . . . . 155

77 Lepton response model $\sigma_{1} \ldots \ldots$. . . . . . . . . . . . 156

78 Comparison with CP Resolutions . . . . . . . . . . . . . . . 157

79 Gaussian sum collapsing . . . . . . . . . . . . . . . . 160

80 Unconstrained transformation parameters ggF $125 \mathrm{GeV}$. . . . . . . . 164

81 Unconstrained transformation parameters ggF $124 \mathrm{GeV}$. . . . . . . 165

82 Unconstrained transformation parameters ggF $126 \mathrm{GeV}$. . . . . . 165

83 Unconstrained transformation parameters VBF $125 \mathrm{GeV}$. . . . . . . 165

84 Constrained transformation parameters ggF $125 \mathrm{GeV}$. . . . . . . . . 169

85 Constrained transformation parameters ggF $124 \mathrm{GeV}$. . . . . . . . . 170

86 Constrained transformation parameters ggF $126 \mathrm{GeV}$. . . . . . . . . 170

87 Constrained transformation parameters VBF $125 \mathrm{GeV}$. . . . . . . . . 170

88 Response PDF examples . . . . . . . . . . . . . . . . . . 171

89 Distribution of $\sigma_{\text {eff }} \ldots \ldots \ldots \ldots$. . . . . . . . . . . . . . 172

90 FlexibleInterpVar used for systematics . . . . . . . . . . . . . . . 177

91 Theory systematics . . . . . . . . . . . . . . . . . . . . 184

92 Normalization systematics for $H \rightarrow 4 \ell$ events . . . . . . . . . . . . . . 185

93 Normalization systematics for $q \bar{q} \rightarrow Z Z^{(*)}$ events . . . . . . . . . . 186

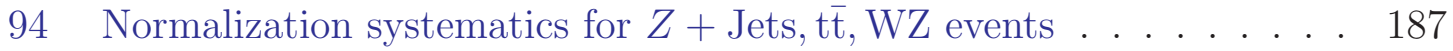

95 Signal systematic morphing . . . . . . . . . . . . . . . 187

96 Scale systematics for $H \rightarrow 4 \ell$ events . . . . . . . . . . . . . . 191

97 Resolution systematics for $H \rightarrow 4 \ell$ events . . . . . . . . . . . . . . . 192

98 Shape systematics for $q \bar{q} \rightarrow Z Z^{(*)} \ldots \ldots \ldots$. . . . . . . 196

99 Scans of $m_{H}$ in effective dataset . . . . . . . . . . . . . . . 206

100 Scans of $\sigma_{H \rightarrow 4 \ell}$ in effective dataset . . . . . . . . . . . . . 207

101 Scans of $\Gamma_{H}$ in effective dataset . . . . . . . . . . . . . 208

102 Systematic pulls on $m_{H} \quad \ldots \ldots$. . . . . . . . . . . . . . . . . . . . . 214 
103 Systematic pulls on $\sigma_{H \rightarrow 4 \ell} \ldots \ldots \ldots \ldots \ldots \ldots$

104 Systematic pulls on $\Gamma_{H} \ldots \ldots \ldots \ldots \ldots \ldots$

105 Toy fit results $m_{H} \ldots \ldots \ldots \ldots \ldots \ldots \ldots \ldots \ldots \ldots \ldots$

106 Toy fit results $\sigma_{H \rightarrow 4 \ell} \ldots \ldots \ldots \ldots \ldots \ldots \ldots \ldots \ldots$

107 Toy fit results $\Gamma_{H} \ldots \ldots \ldots \ldots \ldots \ldots$. . . . . . . . . . . 224

108 Toys 2D correlation . . . . . . . . . . . . . . . . . . . . . . 229

109 Toys expected difference . . . . . . . . . . . . . . . . . 230

110 Toys one-to-one accuracy . . . . . . . . . . . . . . . . . . 230

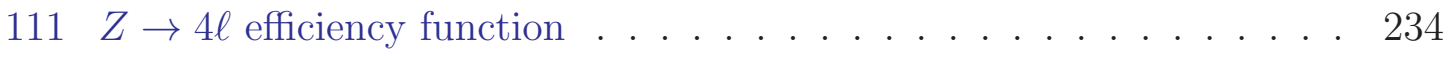

$112 Z \rightarrow 4 \ell$ PER model on simulated data . . . . . . . . . 235

$113 Z \rightarrow 4 \ell$ likelihood scans on effective dataset using $m_{4 \ell}^{\text {cons }} \ldots \ldots \ldots 237$

$114 Z \rightarrow 4 \ell$ likelihood scans on data using $m_{4 \ell}^{\text {cons }} \ldots \ldots \ldots \ldots \ldots$

$115 Z \rightarrow 4 \ell$ constrained PER model post-fit . . . . . . . . . . . . 239

116 Data distribution of $m_{4 \ell}^{\text {cons }} \ldots \ldots \ldots \ldots \ldots \ldots$

$117 m_{4 \ell}^{\text {cons }}$ vs $\sigma_{\text {eff }}$ in data . . . . . . . . . . . . . . 245

118 Scans of $m_{H}$ in data . . . . . . . . . . . . . . . 246

119 Scans of $\sigma_{H \rightarrow 4 \ell}$ in data . . . . . . . . . . . . . . . 247

120 Scans of $\Gamma_{H}$ in data . . . . . . . . . . . . . . . 248

$121 q \bar{q} \rightarrow Z Z^{(*)} \mathrm{PDF}$ of $m_{4 \ell}^{\mathrm{fsr}} \ldots \ldots \ldots \ldots \ldots \ldots \ldots \ldots$

$122 g g \rightarrow Z Z^{(*)} \mathrm{PDF}$ of $m_{4 \ell}^{\text {cons }} \ldots \ldots \ldots \ldots \ldots \ldots \ldots \ldots$

$123 V V V, t \bar{t}+Z \mathrm{PDF}$ of $m_{4 \ell}^{\mathrm{fsr}} \ldots \ldots \ldots \ldots \ldots \ldots \ldots \ldots$

124 Template interpolation check . . . . . . . . . . . . . . . 261

125 Unconstrained muon response fits $(2 / 2) \ldots \ldots \ldots$

126 Unconstrained electron response fits $(2 / 2) \ldots \ldots \ldots \ldots$

127 Constrained muon response fits $(1 / 2) \ldots \ldots \ldots \ldots$

128 Constrained muon response fits $(2 / 2) \ldots \ldots \ldots \ldots$

129 Constrained electron response fits $(1 / 2) \ldots \ldots \ldots$ 


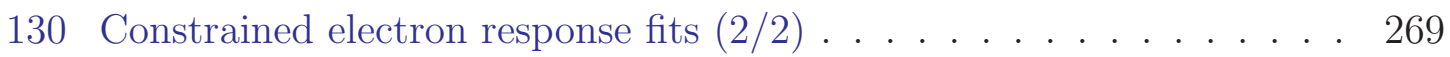

$131 \chi^{2} / n$ distribution of constrained lepton response model . . . . . . . . 270

132 Lepton response model unconstrained $\sigma_{1} \ldots \ldots$. . . . . . . . . . 272

133 Lepton response model unconstrained $\sigma_{2} \ldots \ldots$. . . . . . . . . . . . 272

134 Lepton response model unconstrained $\sigma_{3}$. . . . . . . . . . . . . . . 272

135 Lepton response model unconstrained $\mu_{1}$. . . . . . . . . . . . . . 272

136 Lepton response model unconstrained $\mu_{2}$. . . . . . . . . . . . . . . 273

137 Lepton response model unconstrained $\mu_{3} \ldots \ldots$. . . . . . . . . . 273

138 Lepton response model unconstrained $w_{1} \ldots \ldots$. . . . . . . . . . 273

139 Lepton response model unconstrained $w_{2} \ldots \ldots$. . . . . . . . . . 273

140 Lepton response model constrained $\sigma_{1}$. . . . . . . . . . . . . . . . . 274

141 Lepton response model constrained $\sigma_{2} \ldots \ldots$. . . . . . . . . . . 274

142 Lepton response model constrained $\sigma_{3} \ldots \ldots$. . . . . . . . . . . 274

143 Lepton response model constrained $\mu_{1}$. . . . . . . . . . . . . . . 275

144 Lepton response model constrained $\mu_{2} \ldots \ldots$. . . . . . . . . . 275

145 Lepton response model constrained $\mu_{3}$. . . . . . . . . . . . . 275

146 Lepton response model constrained $w_{1}$. . . . . . . . . . . . . . . . . 275

147 Lepton response model constrained $w_{2}$. . . . . . . . . . . . . . 276

148 Kinematic Model Independence of PER . . . . . . . . . . . . . . 277

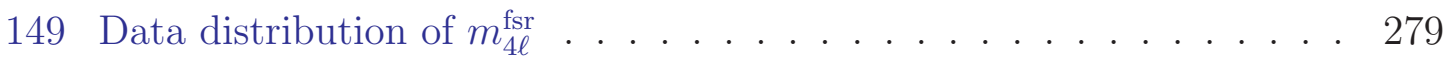

$150 m_{4 \ell}^{\mathrm{fsr}}$ vs $\sigma_{\mathrm{eff}}$ in data . . . . . . . . . . . . . . . . . . . . 280

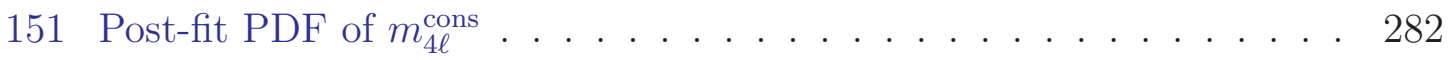

152 Post-fit PDF of $m_{4 \ell}^{\mathrm{fsr}} \quad \ldots \ldots \ldots \ldots$. . . . . . . . . . . . 283

153 Toy fit results $m_{H}$ for constrained PER model . . . . . . . . . . . . . 284

154 Toy fit results $m_{H}$ for Template model . . . . . . . . . . . . . . 285

155 Toy fit results $m_{H}$ for unconstrained PER model . . . . . . . . . . . . 285

156 Toy fit results $\sigma_{H \rightarrow 4 \ell}$ for constrained PER model . . . . . . . . . . . . 286 
157 Toy fit results $\sigma_{H \rightarrow 4 \ell}$ for Template model . . . . . . . . . . . . . 286

158 Toy fit results $\sigma_{H \rightarrow 4 \ell}$ for unconstrained PER model . . . . . . . . . . 287

159 Toy fit results $\Gamma_{H}$ for constrained PER model . . . . . . . . . . . . . 287

160 Toy fit results $\Gamma_{H}$ for unconstrained PER model . . . . . . . . . . . . 288

161 Toys 2D Correlation of $\hat{m}_{H}$ by category . . . . . . . . . . . . . . . . 289

162 Toys 2D Correlation of $\hat{\sigma}_{H \rightarrow 4 \ell}$ by category . . . . . . . . . . . . 290

163 Toys Expected Difference of $\hat{m}_{H}$ by category . . . . . . . . . . . . . . 291

164 Toys Expected Difference of $\hat{\sigma}_{H \rightarrow 4 \ell}$ by category . . . . . . . . . . . . 292

165 Toys One-to-one accuracy fitting $m_{H}$ by category . . . . . . . . . . 293

166 Toys One-to-one accuracy fitting $\sigma_{H \rightarrow 4 \ell}$ by category . . . . . . . . . . 294

$167 Z \rightarrow 4 \ell$ likelihood scans on effective dataset using $m_{4 \ell}^{\mathrm{fsr}} \quad \ldots . \ldots 296$

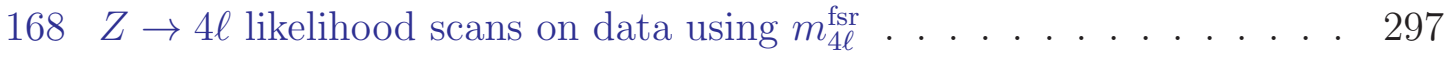

$169 Z \rightarrow 4 \ell$ unconstrained PER model post-fit . . . . . . . . . . . 298

170 Per-event signal PDF of $4 \mu$ events $(1 / 2) \ldots \ldots$. . . . . . . . . . 301

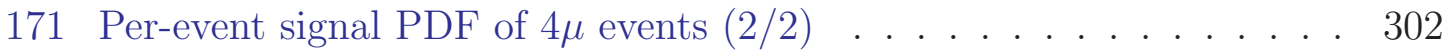

172 Per-event signal PDF of $4 e$ events $(1 / 2) \ldots \ldots$. . . . . . . . . . 303

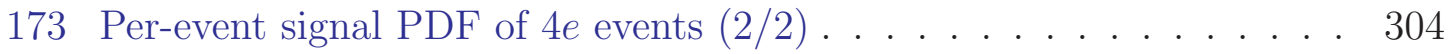

174 Per-event signal PDF of $2 \mu 2 e$ events $(1 / 2) \ldots \ldots$. . . . . . . . . 305

175 Per-event signal PDF of $2 \mu 2 e$ events $(2 / 2) \ldots \ldots$. . . . . . . . 306

176 Per-event signal PDF of $2 e 2 \mu$ events $(1 / 2) \ldots \ldots$. . . . . . . . . 307

177 Per-event signal PDF of $2 e 2 \mu$ events $(2 / 2) \ldots \ldots$. . . . . . . . . . 308 


\section{List of Abbreviations}

\begin{tabular}{|c|c|c|}
\hline Abbreviation & Meaning & First introduced \\
\hline ALICE & A Large Ion Collider Experiment & 3.1 \\
\hline ATLAS & A Toroidal LHC ApparatuS & 3.2 \\
\hline $\mathrm{BH}$ & Bethe-Heitler & 4.4 .1 \\
\hline $\mathrm{BR}$ & branching ratio & 2.3.2.2 \\
\hline BW & Relativistic Breit-Wigner & 2.2 .3 \\
\hline CB & Crystal Ball & 7.3 \\
\hline $\mathrm{CT}$ & Calo-tagged (muon) & 4.5 \\
\hline $\mathrm{CB}$ & Combined (muon) & 4.5 \\
\hline $\mathrm{CDF}$ & cumulative distribution function & 4.4 .1 \\
\hline CL & confidence level & 9.1 \\
\hline $\mathrm{CP}$ & Combined Performance & 7.3 .2 \\
\hline CR & Control Region & 6.3 .1 \\
\hline CERN & European Organization for Nuclear Research & 3.1 \\
\hline CMS & Compact Muon Solenoid & 3.1 \\
\hline
\end{tabular}




\begin{tabular}{|c|c|c|}
\hline c.o.m. & centre of momentum & 3.2 .1 .1 \\
\hline $\mathrm{CSC}$ & cathode strip chamber & 3.2 .4 \\
\hline $\mathrm{DM}$ & Dark Matter & 2.4 \\
\hline DQ & Data Quality & 5.1 \\
\hline ECAL & Electromagnetic Calorimeter & 3.2.3.1 \\
\hline $\mathbf{E W}$ & Electroweak & 2.2 .2 \\
\hline EWSB & Electroweak Symmetry Breaking & 2.3 .1 \\
\hline FCal & Forward Calorimeter & 3.2 .3 .2 \\
\hline FIV & FlexibleInterpVar & 8 \\
\hline FSR & Final State Radiation & 5.8 \\
\hline $\operatorname{ggF}$ & gluon gluon fusion & 2.3.2.1 \\
\hline GRL & Good Runs List & 5.1 \\
\hline HCAL & Hadronic Calorimeter & 3.2 .3 .2 \\
\hline HEC & Hadronic End-Cap Calorimeter & 3.2 .3 .2 \\
\hline $\mathrm{HF}$ & Heavy Flavour & 6.3 .2 \\
\hline HLT & High-Level Trigger & 4.3 \\
\hline HS & Hard Scatter & 3.1 .2 \\
\hline IBL & Insertable B-Layer & 3.2 .2 .1 \\
\hline ID & Inner Detector & 3.2 .2 \\
\hline
\end{tabular}


L1 Level 1 Trigger

LAr Liquid Argon

3.2.3.1

LHC

Large Hadron Collider

3.1

$\mathrm{LHCb}$

LHC-beauty

3.1

LO

leading order

2.2

$\mathrm{MC}$

Monte-Carlo

MDT

monitored drift tube

3.2 .4

ME

Extrapolated (muon)

MM

MicroMegas

NLL

negative log likelihood

9.1

NLO

next-to-leading order

NNLO

next-to-next-to-leading order

N3LO

$(\text { next-to })^{3}$ leading order

2.2

MPI

multiple-parton-interaction

NP

Nuisance Parameter

8.2

NSW

New Small Wheel

3.3

$p-p$

proton-proton

$p-\bar{p}$

proton-antiproton

$\mathrm{Pb}-\mathrm{Pb}$

lead-lead

3.1 
PDF probability density function

$\begin{array}{lll}\text { PER } & \text { Per-Event Response }\end{array}$

$\begin{array}{lll}\text { POI } & \text { paramer of interest }\end{array}$

PS $\quad$ Proton Synchrotron 3.1 .1

PSB $\quad$ Proton Synchrotron Booster 3.1 .1

$\begin{array}{lll}\text { PU Pileup } & 3.1 .2\end{array}$

QCD $\quad$ Quantum Chromodynamics 2.2.1

$\begin{array}{lll}\mathbf{R F} & \text { radio-frequency } & 3.1 .1\end{array}$

$\begin{array}{lll}\text { RPC } & \text { resistive plate chamber } & 3.2 .4\end{array}$

SiAF $\quad$ silicon-associated forward (muon) $\quad 4.5$

SCT $\quad$ Semiconductor Tracker $\quad 3.2 .2 .3$

SFOS same flavour opposite sign $\quad 5.6$

$\begin{array}{lll}\text { SM } & \text { Standard Model }\end{array}$

SPS $\quad$ Super Proton Synchrotron $\quad 3.1 .1$

ST Segment-tagged (muon) $\quad 4.5$

$\begin{array}{lll}\text { sTGC } & \text { small-strip thin gap chamber } & 3.3\end{array}$

$\begin{array}{lll}\text { TGC } & \text { thin gap chamber } & 3.2 .4\end{array}$

TRT Transition Radiation Tracker 3.2.2.4

$\begin{array}{lll}\text { ttH } & \text { top fusion } & 2.3 .2 .1\end{array}$ 
UL

upper limit

9.1

VBF

vector boson fusion

2.3.2.1

VH

associated production

2.3.2.1

ZMC

Z Mass Constraint

5.9 


\section{Chapter 1}

\section{Introduction}

The Higgs Boson, predicted in 1964 [1], proved elusive to searches for nearly half a century. In the wake of its eventual discovery in 2012 [2][3], it was often heralded as the last missing piece of the Standard Model. Since the discovery, focus has shifted to the measurement of its properties. Some properties are free parameters of nature for which our theory gives no prediction. Others can be tested against theory to help determine whether the observed particle is in fact the Higgs boson predicted by the Standard Model, or some manifestation of an underlying theory.

The main objective of this thesis is to present measurements of the mass, natural width, and cross section times branching ratio to four electrons or muons of the Higgs boson using a technique developed by the author. The mass, $m_{H}$, is a free parameter of nature, while the other two measurements, $\sigma_{H} \times \mathrm{BR}(H \rightarrow 4 \ell)$ and $\Gamma_{H}$, are predicted by the Standard Model, once the mass is known. The measurements are made using the Higgs' decay to two Z bosons which subsequently decay to pairs of electrons or muons, called the $H \rightarrow Z Z^{(*)} \rightarrow 4 \ell$ channel. Other hardware and software contributions to the ATLAS Experiment from the author are presented as well.

Chapter 2 presents the Standard Model of particle physics - our modern description of the nature of particles and their interactions. A large focus lies on the Higgs 
boson and its role in the Standard Model.

Chapter 3 presents the impressive hardware used to produce and detect Higgs bosons - the Large Hadron Collider and the ATLAS Detector, respectively. Also presented is an upgrade project for the future of the ATLAS Detector, and an approach used to assure precision alignment of its components developed by the author (in Section 3.3.1.1).

Chapter 4 moves on to the software level, showing how various particles are reconstructed in the ATLAS Detector. Emphasis is placed on the reconstruction of the electrons and muons used this analysis, in particular the author's own contributions are highlighted inline in Section 4.4.1 and Section 4.5.1.

Chapter 5 begins the presentation of the $H \rightarrow Z Z^{(*)} \rightarrow 4 \ell$ analysis with the event selection, showing how candidate events are selected out of the enormous numbers of collisions in the detector. The described selection was implemented by the author, but developed and executed by other members of the HZZ working group within ATLAS.

Chapter 6 presents the various techniques used to estimate the different sources of background surviving the event selection. Particular emphasis is placed on the $Z+e e$ background estimation (Section 6.3.1) developed in part by the author.

Chapter 7 introduces the method used to measure the Higgs boson mass, natural width, and cross section times branching ratio to four electrons or muons, the socalled per-event response method. The chapter focuses on the signal model: how uncertainties at particle-level reconstruction are propagated upwards to the fourlepton candidate level, and combined with the theoretically motivated lineshape to produce a final expected signal distribution for each event observed. Also presented is an alternate method used as a cross-check, called the template method. The full contents of this chapter are the work of the author.

Chapter 8 presents the various sources of systematic uncertainty considered in 
the analysis. The implementation and impact of each uncertainty on the measurements presented in this thesis is the sole work of the author, although the sources of uncertainty combine the work of many other ATLAS collaborators.

Chapter 9 completes the presentation of the per-event response measurement method, incorporating expected yields and descriptions of all sources of signal and background. The chapter includes a description of the statistical methods used, and several validation steps of the measurement procedure. The chapter closes by presenting the final measurements of the Higgs boson mass, natural width, and cross section times branching ratio to four electrons or muons. All results in this chapter are the work of the author, though comparisons are made with results by others, and will be noted as such.

Chapter 10 concludes this thesis with a summary of the results and outlook.

Several appendices supplement the main body of work. Appendix A supplements Chapter 6 by describing the distribution of background events used for a cross-check. Appendix B supplements Chapter 7 with supporting plots and descriptions of the per-event response and template methods. Appendix C supports Chapter 9 with additional plots and checks.

Various publications have emerged in relation to the work of this thesis. Elements of the author's work on the muon reconstruction software entered into [4], though the primary project described in Section 4.5.1 did not (it was completed after this publication). The $H \rightarrow 4 \ell$ event selection and background estimation techniques were described as they are used in this thesis in [5]; both evolve over time as new techniques are developed, and so previous iterations with some differences can be found in [6], and [7]. The per-event response technique (in an earlier version) was also described in [7] where it was used to set a direct limit on the Higgs width, and as a cross-check on the Higgs mass measurement. The mass measurement was combined with CMS in [8]. The author also presented searches for a high-mass Higgs boson with ATLAS 
in [9], which includes the $H \rightarrow Z Z^{(*)} \rightarrow 4 \ell$ channel, along with other di-boson decay channels.

Note that the LHC and ATLAS do not run continuously - they operate for multiyear periods of data taking, split by multi-year periods of shut down for upgrades and maintenance. The two such periods to date are called Run 1 (2011-2013), and Run 2 (2015-present). Though the two different runs have differences right from the hardware level to the final analysis, all descriptions and data in this thesis correspond to Run 2, unless specifically noted. The measurements presented in this thesis were performed on the to-date ATLAS Run 2 dataset, $36.1 \mathrm{fb}^{-1}$ of $p$ - $p$ collisions at $\sqrt{s}=13 \mathrm{TeV}$ 


\section{Chapter 2}

\section{The Standard Model}

Particle physics is the study of fundamental particles and their interactions. Through this lens we gain an understanding about the nature of the universe, from its very origins to the interactions we make use of every day.

Our description of what is fundamental has evolved over time. Historically indivisible particles have been revealed to be composite - made up of something more fundamental and governed by a new interaction heretofore unseen. Our modern description of the fundamental particles and their interactions is called the Standard Model of Particle Physics (SM).

This chapter will describe the content of the SM. Section 2.1 discusses the particle content, while Section 2.2 describes how these particles interact. The main focus of this thesis is a measurement of the Higgs boson, and so Section 2.3 is dedicated to

discuss its role in the SM, how they will be produced and observed at the LHC, and some of its properties. Section 2.4 explores some of the weak points of the SM, and why measuring the properties of the Higgs boson can be useful in resolving them.

The complete mathematical formulation of the SM is beyond the scope of this thesis, and is used only where it is essential for understanding the content. 


\subsection{Particle Content}

The fundamental particles of SM are divided into fermions, and bosons. Fermions may be thought of as matter particles, and bosons as force-carriers. The fermion content of the SM is summarized in Table 1, and the boson content in Table 2.

Table 1: Fermion content of the SM, based on [10]. The top mass is updated using a recent measurement [11]. Note that the corresponding anti-particles for each of the listed particles has an equal spin and mass, but opposite charge. The uncertainties on the quark masses are much larger than those of the leptons.

\begin{tabular}{cccccc}
\hline & symbol & common name & spin & charge & mass $[\mathrm{MeV}]$ \\
\hline & $\mathrm{u}$ & quarks & & & \\
\hline \multirow{2}{*}{ up-type quarks } & $\mathrm{c}$ & up & $1 / 2$ & $+2 / 3$ & 3 \\
& $\mathrm{t}$ & charm & $1 / 2$ & $+2 / 3$ & 1200 \\
& $\mathrm{~d}$ & dop & $1 / 2$ & $+2 / 3$ & 172,440 \\
\hline \multirow{2}{*}{ down-type quarks } & $\mathrm{s}$ & strange & $1 / 2$ & $-1 / 3$ & 7 \\
& $\mathrm{~b}$ & bottom & $1 / 2$ & $-1 / 3$ & 4,300 \\
\hline \multirow{5}{*}{ charged leptons } & $e^{-}$ & electron & $1 / 2$ & -1 & 0.510999 \\
& $\mu^{-}$ & muon & $1 / 2$ & -1 & 105.659 \\
& $\tau^{-}$ & tau & $1 / 2$ & -1 & 1776.99 \\
\hline & $\nu_{e}$ & electron neutrino & $1 / 2$ & 0 & $<10^{-6}$ \\
& $\nu_{\mu}$ & muon neutrino & $1 / 2$ & 0 & $<10^{-6}$ \\
& $\nu_{\tau}$ & tau neutrino & $1 / 2$ & 0 & $<10^{-6}$ \\
\hline \multirow{2}{*}{ neutrinos } & & & &
\end{tabular}

Fermions are spin-1/2 particles. They are divided in leptons and quarks based on their interactions. The leptons come in pairs of a negatively charged lepton $\left(\ell^{-}\right)$and its corresponding neutrino $\left(\nu_{\ell}\right)$. There are three such generations, ordered by increasing mass $m_{\ell}$ (while neutrinos definitively have mass [12][13], we do not know their mass hierarchy, and their masses are not included in the SM). The three generations of 
Table 2: Boson content of the SM, based on [10]. The Higgs boson properties are the latest ATLAS+CMS combination [8].

\begin{tabular}{ccccc}
\hline symbol & common name & spin & mass $[\mathrm{GeV}]$ & interacts with \\
\hline$H$ & Higgs boson & 0 & 125.09 & $H, W^{ \pm}, Z, \ell, q$ \\
\hline$g$ & gluon & 1 & 0 & $g, q$ \\
\hline$W^{ \pm}$ & W boson & 1 & 80.42 & $H, W^{ \pm}, Z, \gamma, \ell, \nu_{\ell}, q$ \\
\hline$Z$ & Z boson & 1 & 91.19 & $H, W^{ \pm}, \ell, \nu_{\ell}, q$ \\
\hline$\gamma$ & photon & 1 & 0 & $W^{ \pm}, \ell, q$ \\
\hline
\end{tabular}

leptons are called the electron, muon, and tau $(\ell=e, \nu, \tau)$. Quarks also come in three generations of "up-type" (charge $+2 / 3$ ) and "down-type" (charge $-1 / 3)$ quarks. The three generations of quarks are the up and down $(q=u, d)$, the charm and strange $(c, s)$, and the top and bottom $(t, b)$. For each of these 12 particles, there is a corresponding anti-particle. The only one with a special name is the anti-electron, called the positron. The others are simply denoted as $\ell^{+}, \overline{\nu_{\ell}}, \bar{q}$ according to their type. In some contexts the particle-anti-particle distinction is either implied or unimportant. For example in the decay $Z \rightarrow \mu^{+} \mu^{-}$, including the charge designation is redundant so it is often omitted.

In the language of the SM, fermions are separated into left(L) and right-handed(R) chirality. The left-handed fermions are described mathematically as doublets, and the right-handed fermions are described as singlets. There are no right-handed neutrinos. For the first generation fermions, this looks like

$$
\begin{aligned}
& E_{L}=\left(\begin{array}{c}
\nu_{e} \\
e^{-}
\end{array}\right)_{L}, e_{R}, \\
& Q_{L}=\left(\begin{array}{l}
u \\
d
\end{array}\right)_{L}, u_{R}, d_{R},
\end{aligned}
$$


and the second and third generation can be written by the obvious substitution of $e \rightarrow \nu, \tau, \quad u \rightarrow c, t$, and $d \rightarrow s, b$. This structure will return later in Section 2.3.1.

The bosons are much fewer in number than fermions. The only scalar boson (spin-0) is the Higgs boson which couples to all particles with mass. The vector bosons (spin-1) are the gluon which mediates the strong force between quarks, the photon which mediates the electromagnetic force between charged particles, and the $W^{ \pm}$and $Z$ bosons which mediate the weak force.

\subsubsection{Composite particles}

Gluons and quarks share a property called colour charge. Colour charge is to the strong force as electric charge is to the electromagnetic force: it decides with whom gluons will interact; since quarks are the only fermions that carry colour charge, they are the only fermions that gluons interact with.

A very important property is colour confinement, which imposes the requirement that stable particles be colour-neutral. The result is that a bare quark, which carries one unit of colour (call it $r e d=r$ ), cannot exist in isolation. It can either be paired with an anti-quark (carrying anti-red $=\bar{r}$ ), or grouped with two other quarks (carrying $b l u e=b$, and green $=g$ colour charges), such that the overall particle is colour-neutral. These objects are held together by exchange of gluons, which themselves are colourneutral, carrying for example $r \bar{r}$ colour. Collectively these composite particles are called hadrons, while two-quark hadrons are called mesons, and three-quark hadrons are called baryons. The only quark which does not form hadrons is the top; because its lifetime is so short it has no time to hadronize before it decays.

The most familiar baryon is the proton. A proton is composed of two up quarks and a down quark $(u u d)$, held together by a constant exchange of gluons. In fact, these quarks represent only what we call valence quarks in the proton: much like valence electrons determine the electromagnetic interactions of atoms, valence quarks 
determine the electroweak interactions of hadrons. "Underneath" the valence quarks is a sea of other $q \bar{q}$ pairs. A visualization of the substructure of a proton is shown in Figure 1.

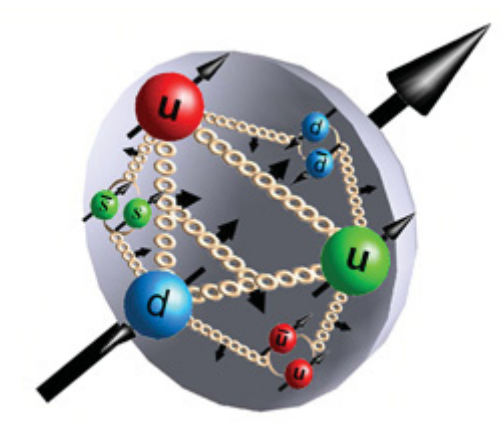

Figure 1: Substructure of the proton, taken from [14].

The other familiar baryon is the neutron $(u d d)$, though many other baryons states are produced at the LHC. A myriad of mesons are also produced in collisions at the LHC. Some survive long enough to be observed directly (e.g. the most common is $\left.\pi^{ \pm}=u \bar{d}, \bar{u} d\right)$, while others decay very quickly and are observed indirectly by their decay products. A sub-class of mesons worth distinguishing are "B mesons", a term used to mean any meson with a valence bottom quark. While all mesons produce jets within ATLAS' calorimeters (the calorimeters are discussed further in Section 3.2.3, while jet reconstruction is discussed further in Section 4.6), the topology jets from B mesons differ from those of the lighter mesons.

The quarks and gluons that make up a hadron are collectively called partons. The momentum fraction of the proton that is carried by each parton is distributed according to their parton distribution function, a probability density function $\left(\mathrm{PDF}^{1}\right)$ on the range $[0,1]$. Parton density functions are empirically determined, but as an approximate figure, a proton's momentum is divided roughly into 6 equal pieces to its three constituent quarks and three gluons. The important implication for the LHC

\footnotetext{
${ }^{1}$ Much of the literature will use 'PDF' to mean parton distribution function, but as this thesis is more often concerned with probability density functions, the acronym is defined accordingly.
} 
is that when protons collide at $6.5 \mathrm{TeV}$, in reality it is partons with approximately $\approx 1 \mathrm{TeV}$ energies which collide.

\subsection{Interactions}

It is the interactions between these particles which allow us to observe and understand them $^{2}$. Together the Strong and Electroweak interactions can describe virtually every interaction occurring inside ATLAS. The formal language of the SM describes these interactions using symmetry groups. Loosely speaking, the SM must be invariant under certain symmetry transformations, and this requirement precipitates the interactions we observe. Theories beyond the SM, discussed more in Section 2.4, try to impose new symmetry groups on the SM, and make predictions of processes that will be observed, if the postulated theory is true. The detailed mathematical structure of the SM is beyond the scope of this thesis, so this section aims for a mostly qualitative understanding: which particles interact with each other, and how these interactions can be used to describe processes observed in ATLAS.

Particle interactions can handily be illustrated using Feynman diagrams. These diagrams can be used to show the structure of any process, but are even more useful as calculation tools for evaluating the cross section of the process. For the purposes of this thesis, these calculations are left to phenomenologists, and the diagrams are used only graphically. In general any diagram which can be written down (using the basic pieces discussed in the following sections) does occur, but the rate of the interaction must be calculated, and could be negligible. The diagrams can also be rotated, flipped, or twisted in any orientation, and still be valid. The usual convention has time flowing from left-to-right each diagram. The greater the number of vertices

\footnotetext{
${ }^{2}$ This statement holds true from a practical standpoint, that the particles must interact in some way in our particle colliders and detectors for them to be recorded, but it also holds true from an anthropic standpoint, since particles must be able to interact to form atoms and chemistry which allows the evolution of (eventually) intelligent life which can go on to build particle colliders and ask these questions.
} 
in the diagram, the smaller its cross section. The form of a diagram with the least number of vertices is called the leading order (LO) diagram for a process, and gives a useful visual of the initial and final-state particles. For precise predictions, evaluating higher order diagrams is necessary. As more vertices are added, these are called next-to-leading order (NLO), next-to-next-to-leading order (NNLO), and even nextto-next-to-next-to-leading order (NNNLO, or just N3LO). All the diagrams shown in this thesis are given at LO, though higher-order calculations are always used, or corrected for, in the simulated data. This is discussed further in Section 4.1.

\subsubsection{Strong interaction}

The Strong interaction is described by the theory of Quantum Chromodynamics (QCD) [15]. It is mediated by gluons, which interact (or "couple to") colour charge. In practice this means they interact only with quarks and other gluons. In the mathematical formulation of the SM, the strong force is described by an $\mathrm{SU}(3)_{C}$ symmetry group, where $C$ is the colour-charge, and the the gluons are actually an octet of 8 particles. Most of the LHC collisions begin with QCD, since the colliding particles are composed of quarks and gluons. The basic Feynman diagram pieces for QCD are shown in Figure 2. Any combination of these pieces can be "snapped" together to create a more complex process.
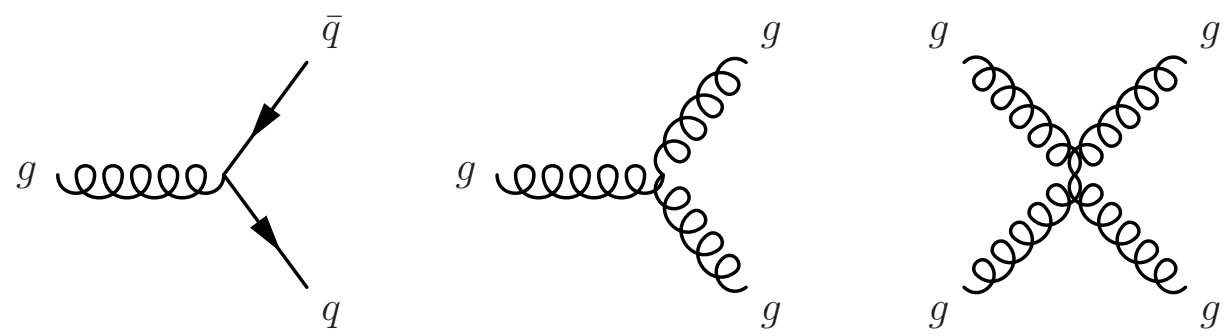

Figure 2: Feynman diagram components for strong interactions. 


\subsubsection{Electroweak interaction}

The electroweak (EW) interaction is the unification of the electromagnetic and weak forces under one theory [15]. At low energy scales, far below $m_{W}$, they can be described separately, but at higher energies they are combined into the single EW force, mediated by photons and the vector bosons, $W^{ \pm}, Z$. The symmetry governing these interactions is $S U(2)_{L} \times U(1)_{Y}$, where $L$ denotes left-handed chirality, and $Y$ is the weak hyper-charge (analogous to the electric charge). A truly self-consistent picture of the EW interactions is impossible without the Higgs mechanism which will be discussed in Section 2.3.1, so for now its content must be taken at face value. Photons couple to electric charge, so interact only with charged particles. The $W^{ \pm}, Z$ bosons couple to all left-handed fermions. When a $W^{ \pm}$interacts with a fermion, it always changes the particle's species (flavour) and charge, whereas neither the $\gamma$ nor $Z$ do so. The basic Feynman diagram pieces for EW interactions are shown in Figure 3.

These diagrams, together with Figure 2 are all that is needed to understand the source of the main backgrounds to the analysis in this thesis, and will return in Chapter 6. They are also essential in understanding the signal process under study in this thesis, $H \rightarrow Z Z^{(*)} \rightarrow 4 \ell$.

\subsubsection{Z boson decay}

Of special importance to this thesis is the $\mathrm{Z}$ boson. The $\mathrm{Z}$ boson is an inherently unstable particle, with a large natural width $\Gamma_{Z}$. This width parameter determines, in part, the shape of the production cross section of the $\mathrm{Z}$ boson, and therefore the distribution of Z boson masses. Although the "Z mass" is a single value, $m_{Z}=91.1876 \mathrm{GeV}$ [16], individual $\mathrm{Z}$ bosons come with a range of masses - call them $m_{\ell \ell}$ for the di-lepton pair they may decay into. They are distributed, as for any unstable particle, according to 

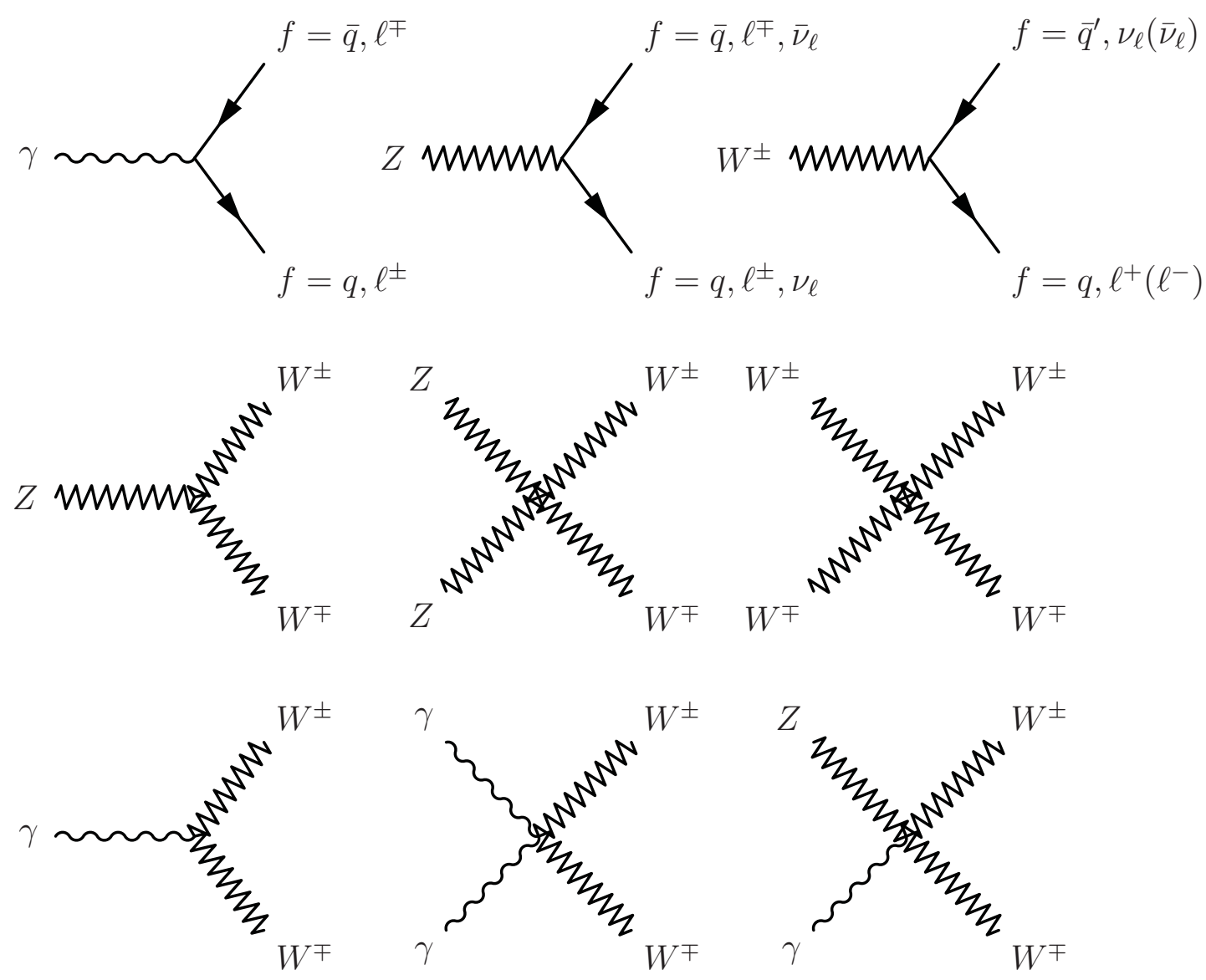

Figure 3: Feynman diagram components for electroweak interactions.

a Relativistic Breit-Wigner (BW) distribution[15]:

$$
B W\left(m_{\ell \ell} ; m_{Z}, \Gamma_{Z}\right)=\frac{k}{\left(m_{\ell \ell}^{2}-m_{Z}^{2}\right)^{2}+m_{Z}^{2} \Gamma_{Z}^{2}}
$$

where $k$ is a normalization constant depending on $m_{Z}$ and $\Gamma_{Z}$. This distribution is called the Z boson's lineshape. It is shown in Figure 4 . The width, $\Gamma_{Z}$, is the full width at half-maximum of the distribution.

At the LHC where the colliding quarks and gluons have a range of momentum, the production of the $\mathrm{Z}$ boson cannot be finely controlled, and so $\mathrm{Z}$ bosons are produced according to this lineshape. At an $e^{+}-e^{-}$collider, where the colliding particles' energy is finely controlled, the collider operator is in control of where on the Z lineshape the 


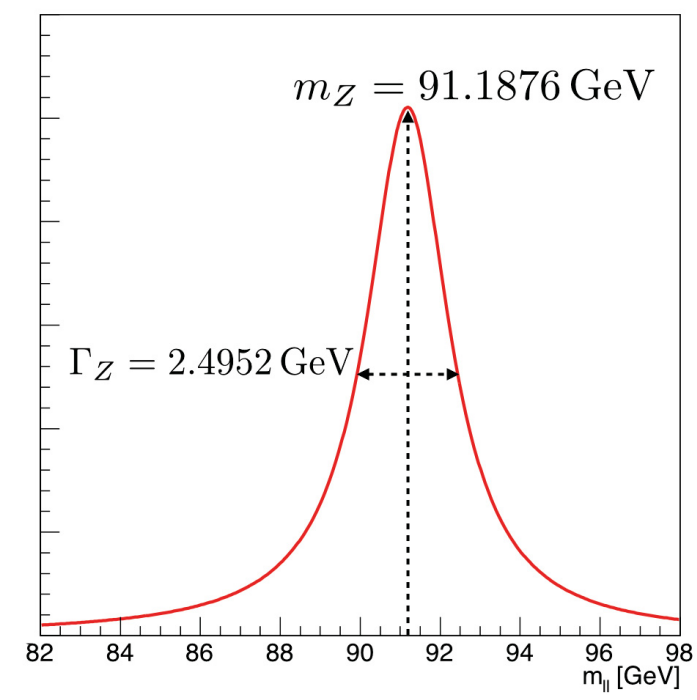

Figure 4: Relativistic Breit-Wigner lineshape for Z boson decays.

bosons are being produced. When the collider is tuned to the peak of the distribution, Z bosons are being produced "on-shell" (and at a higher rate), whereas away from the peak of the distribution, they are being produced "off-shell" (and at a lower rate). The same applies at the LHC, except that both on-shell and off-shell Z bosons will be produced simultaneously, distributed according to (2).

The decays $Z \rightarrow e^{+} e^{-}$and $Z \rightarrow \mu^{+} \mu^{-}$form the latter half of the $H \rightarrow Z Z^{(*)} \rightarrow 4 \ell$ signal analyzed in this thesis (only $\ell=e, \mu$ are considered, $\tau$ 's are not part of the signal, by definition). They proceed exactly as seen in the top-center diagram of Figure 3. The branching ratio for these two decays [16] are

$$
\begin{aligned}
& \operatorname{BR}\left(Z \rightarrow \mu^{+} \mu^{-}\right)=3.363 \pm 0.007 \% \\
& \operatorname{BR}\left(Z \rightarrow e^{+} e^{-}\right)=3.363 \pm 0.004 \%
\end{aligned}
$$

The signal process studied in this thesis contains two $Z$ bosons, which must both decay via one of these channels to be part of the signal. 


\subsection{The Higgs boson}

The structure of the SM requires that its interactions be invariant under transformations of $\mathrm{SU}(3)_{C} \times \mathrm{SU}(2)_{L} \times \mathrm{U}(1)_{Y}$ (combining the Strong and EW symmetry groups). The only way to achieve this is to require that all the fermions and bosons in the theory be massless, which stands in clear contention with experimental evidence. The reconciliation of this problem came in 1964 [1][17][18], with the introduction the (later-called) Higgs mechanism, which could explain the masses of the fermions and bosons by spontaneously breaking the $\mathrm{SU}(2) \times \mathrm{U}(1)$ symmetry in the theory. This mechanism is explained in Section 2.3.1. An important consequence of this mechanism is the prediction of another massive boson, the Higgs boson, whose production, decay, and use in this thesis are described in Section 2.3.2 and Section 2.3.3.

\subsubsection{The Higgs mechanism}

The Higgs mechanism works by introducing into the SM a doublet of complex scalar fields, called the Higgs doublet [15],

$$
\Phi=\left(\begin{array}{c}
\phi^{+} \\
\phi^{-}
\end{array}\right)
$$

which transforms under $S U(2)_{L}$. By introducing this doublet, the SM Lagrangian, which encodes all the particles and interactions in the theory, gets an additional contribution from

$$
\mathcal{L}_{S M} \supset\left|D^{\mu} \Phi\right|^{2}-V(\Phi)
$$

where $D^{\mu}$ is the covariant derivative, used to represent the interactions of the doublet with other particles (technically, fields), and the $V(\Phi)$ is the Higgs potential, which 
can be parameterized as

$$
V(\Phi)=\mu^{2} \Phi^{\dagger} \Phi+\lambda\left(\Phi^{\dagger} \Phi\right)^{2}
$$

To guard against vacuum instability, we require $\lambda>0$ (otherwise the potential falls to $-\infty$ for large values of $\left.\Phi^{\dagger} \Phi\right)$. If $\mu^{2}<0$, the potential takes on the form shown in Figure 5.

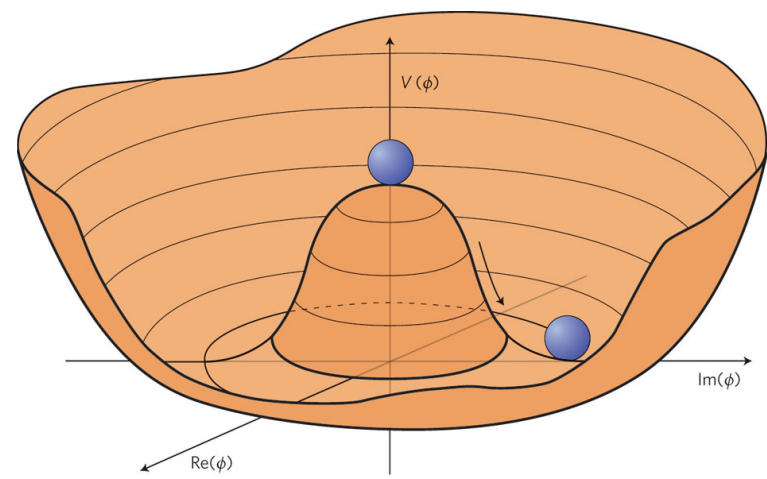

Figure 5: Shape of Higgs potential function leading to Spontaneous Electroweak Symmetry Breaking. Taken from [19].

This potential now has a non-zero minimum located in a ring of radius $\Phi^{\dagger} \Phi=v / \sqrt{2}$, where $v=\sqrt{-\mu^{2} / \lambda}$. This value $v$ is called the vacuum expectation value, equal to $246 \mathrm{GeV}$. As the vacuum falls down to this non-zero minimum, the $\mathrm{SU}(2)_{L} \times \mathrm{U}(1)_{Y}$ symmetry describing the EW interactions is spontaneously broken. This process is called Electroweak Symmetry Breaking (EWSB).

Using a clever transformation to simplify (4), $\Phi$ can be expressed using a new field, $H$, which is a perturbation about the minimum of $V(\Phi)$,

$$
\Phi=\frac{1}{\sqrt{2}}\left(\begin{array}{c}
0 \\
v+H
\end{array}\right)
$$


Using this expression, (6) becomes

$$
\begin{aligned}
V(\Phi) & =\frac{-\lambda v^{2}}{2}(v+H)^{2}+\frac{\lambda}{4}(v+H)^{4} \\
& =\frac{\lambda}{4} H^{4}+\lambda v H^{3}+\lambda v^{2} H^{2}+\text { constant } .
\end{aligned}
$$

The covariant derivative will include a term $\frac{1}{2}\left(\partial_{\mu} h\right)^{2}$, such that the terms in the Lagrangian involving directly and only the $H$ field become

$$
\mathcal{L}_{S M} \supset \frac{1}{2}\left(\partial_{\mu} H\right)^{2}-\lambda v^{2} H^{2}-\lambda v H^{3}-\frac{\lambda}{4} H^{4}
$$

Here we recognize the Lagrangian of a self-interacting scalar, the SM Higgs boson. The term $\frac{1}{2}\left(\partial_{\mu} H\right)^{2}$ is the kinetic term, the $-\lambda v^{2} H^{2}$ represents a mass term for the $H$, where $\frac{1}{2} m_{H}^{2}=\lambda v^{2}$, and so $m_{H}=\sqrt{2 \lambda} v=\sqrt{-2 \mu^{2}}$. The final two terms, $-\lambda v H^{3}$ and $-\frac{\lambda}{4} H^{4}$ are three-Higgs and four-Higgs self-interaction terms.

To see why the Higgs mechanism is so important to the SM, we turn to the covariant derivative, $D^{\mu}$, which will show how the Higgs doublet's interactions with the EW sector are affected by EWSB. The derivative has the form

$$
\left|D_{\mu} \Phi\right|^{2}=\left|\left(\partial_{\mu}+\frac{i g_{2} \tau_{a} W_{\mu}^{a}}{2}+\frac{i g_{1} B_{\mu}}{2}\right) \Phi\right|^{2} .
$$

In this expression, the $W_{\mu}^{a}=W_{\mu}^{1,2,3}$ are the massless fields associated to the $\mathrm{SU}(2)_{L}$ symmetry, and the $B_{\mu}$ is the massless field associated to $\mathrm{U}(1)_{Y}$. The $\tau$ are Pauli matrices, and $g_{1}\left(g_{2}\right)$ is the electromagnetic (weak) coupling constant. Inserting (7) and expanding the expression gives

$$
\left|D_{\mu} \Phi\right|^{2}=\frac{1}{2}\left(\partial_{\mu} H\right)^{2}+\frac{1}{8} g_{2}^{2}(v+H)^{2}\left|W_{\mu}^{1}+i W_{\mu}^{2}\right|^{2}+\frac{1}{8}(v+H)^{2}\left|g_{2} W_{\mu}^{3}-g_{1} B_{\mu}\right|^{2},
$$

where the first term was already extracted and used in (9). This expression can be 
simplified by a linear transformation to four new fields defined by,

$$
\begin{aligned}
W_{\mu}^{ \pm} & =\frac{1}{\sqrt{2}}\left(W_{\mu}^{1} \mp i W_{\mu}^{2}\right), \\
Z_{\mu} & =\cos \left(\theta_{W}\right) W_{\mu}^{3}-\sin \left(\theta_{W}\right) B_{\mu}, \\
A_{\mu} & =\sin \left(\theta_{W}\right) W_{\mu}^{3}+\cos \left(\theta_{W}\right) B_{\mu},
\end{aligned}
$$

where $\tan \theta_{W}=g_{1} / g_{2}$ defines the weak mixing angle, $\theta_{W}$. Using this transformation, several terms in (11) can be identified as

$$
\left|D_{\mu} \Phi\right|^{2} \supset \frac{g_{2} v^{2}}{4} W_{\mu}^{+} W^{-\mu}+\frac{g_{2} v^{2}}{8 \cos ^{2}\left(\theta_{W}\right)} Z_{\mu} Z^{\mu}+0 \cdot A_{\mu} A^{\mu}
$$

As before, terms quadratic in a field represent masses of particles, from which we can find the masses of these newly defined fields,

$$
\begin{aligned}
M_{W^{ \pm}} & =\frac{1}{2} g_{2} v, \\
M_{Z} & =\frac{1}{2} \frac{g_{2} v}{\cos \left(\theta_{W}\right)}, \\
M_{A} & =0 .
\end{aligned}
$$

These fields are recognized (if the naming convention wasn't a giveaway already) as the physical $W^{ \pm}, Z$ weak vector bosons, and the massless photon. The weak vector bosons acquired their mass through the introduction of the Higgs doublet, which breaks the $\mathrm{SU}(2)_{L} \times \mathrm{U}(1)_{Y} \mathrm{EW}$ symmetry. The remaining symmetry, only on the massless $A$ field, is called $\mathrm{U}(1)_{E M}$, and is the more familiar symmetry group of the electromagnetic interaction. As a by-product of this symmetry breaking, a massive $H$ field was introduced, which we recognize as the Higgs boson. This is the essence of the Higgs mechanism.

A similar mechanism works to generate the fermion mass terms. By introducing a coupling parameter for each fermion, the interaction with the Higgs doublet can be 
written as (for, e.g., the electron)

$$
\mathcal{L}_{S M} \supset-\lambda_{e} \bar{E}_{L} \Phi e_{R}
$$

where $\lambda_{e}$ is a coupling parameter, and $\bar{E}_{L}, e_{R}$ were introduced in (1). By expanding $\Phi$ about the minimum using (7), we get a term

$$
\mathcal{L}_{S M} \supset-\frac{1}{\sqrt{2}} \lambda_{e} v \bar{e}_{L} e_{R}
$$

which can be interpreted as a mass term for the electron,

$$
m_{e}=\frac{1}{\sqrt{2}} \lambda_{e} v
$$

Note that since there is no right-handed neutrino, $\nu_{R}$, neutrinos do not acquire a mass in this way. The SM is unable to explain the non-zero (but very small) neutrino masses observed experimentally.

For quarks the situation is a bit more complex as a linear transformation must be done to translate to the mass eigenstates. The final product however, appears similar to that of the fermions, where mass terms appear in the Lagrangian giving masses to the quarks,

$$
m_{u}=\frac{1}{\sqrt{2}} \lambda_{u} v, \quad m_{d}=\frac{1}{\sqrt{2}} \lambda_{d} v .
$$

Also important for anyone wishing to observe a Higgs boson is to see how it interacts with particles, beyond just giving them mass. To see how the Higgs field couples with these new massive vectors bosons, we collect the terms in (11) with factors of $H$ and $H^{2}$, leaving the following in the Lagrangian,

$$
\mathcal{L}_{S M} \supset\left[m_{W}^{2} W_{\mu}^{+} W^{-\mu}+\frac{1}{2} m_{Z}^{2} Z_{\mu} Z^{\mu}\right]\left(\frac{2 H}{v}+\frac{H^{2}}{v^{2}}\right) .
$$


This expression suggests interactions of the type $H V V, H H V V$, (where $V=W, Z$ ) with couplings

$$
g_{H V V}=-2 i \frac{m_{V}^{2}}{v}, \quad g_{H H V V}=-2 i \frac{m_{V}^{2}}{v^{2}}
$$

Similarly, the Lagrangian will contain terms for the fermions of the form

$$
\mathcal{L}_{S M} \supset-m_{f} \bar{f} f \frac{h}{v}
$$

which suggestion fermion couplings

$$
g_{H f f}=i \frac{m_{f}}{v}
$$

In all cases, the Higgs bosons' couplings are proportional to the mass of the particles with which it is interacting. The consequence is that the Higgs preferentially interacts with the heaviest particles in the SM: $t$ quarks, $Z, W^{ \pm}$, and $b$ quarks. This coupling behaviour determines the ways in which Higgs bosons are produced and decay, discussed in the following section.

\subsubsection{Higgs production and decay}

The Higgs boson couples to any massive particles, which in the SM means it couples to all particles except the gluon and photon. The Feynman diagrams for Higgs interaction are shown in Figure 6. Only the top two diagrams contribute meaningfully to this thesis. With these two diagrams, and the preceding diagrams of the QCD and EW interactions, all of the Higgs production and decay modes considered in this thesis can be described.

In the bottom row, the three-Higgs and four-Higgs diagram are called the Higgs self-interaction diagrams, and the associated couplings are called the trilinear Higgs coupling and quartic Higgs coupling, respectively. Measuring the strength of the 
trilinear Higgs coupling is one of the goals of the final stage of the LHC programme, called the High-Luminosity LHC (HL-LHC). Recent studies[20] project that it will be just in reach with $3000 \mathrm{fb}^{-1}$ of data at $\sqrt{s}=14 \mathrm{TeV}$, which the HL-LHC anticipates to collect by the end of its life. The quartic Higgs coupling is far out of reach of the LHC, and may only be accessible in a future collider. Measuring these couplings is valuable because it is equivalent to a measurement of each term in the Higgs Lagrangian, (9). Measurement of these terms would fully determine the shape of the Higgs potential, (6) (and shown in Figure 5), which has consequences as far reaching as the long-term stability of the universe.
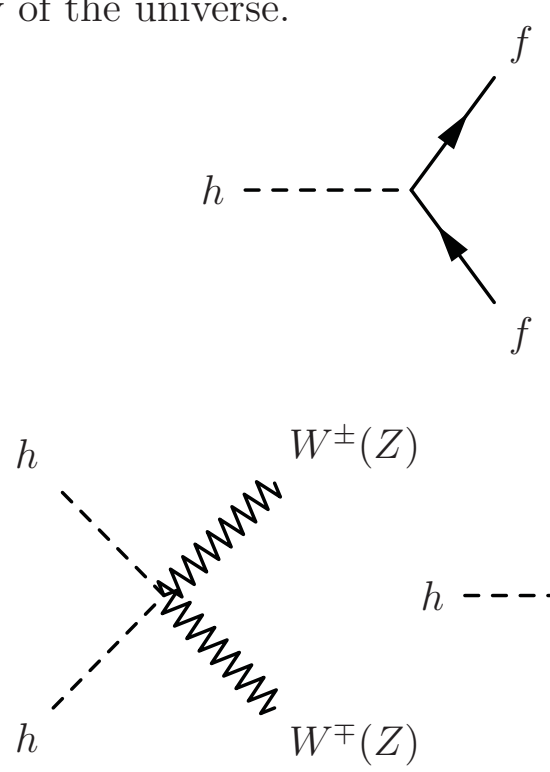
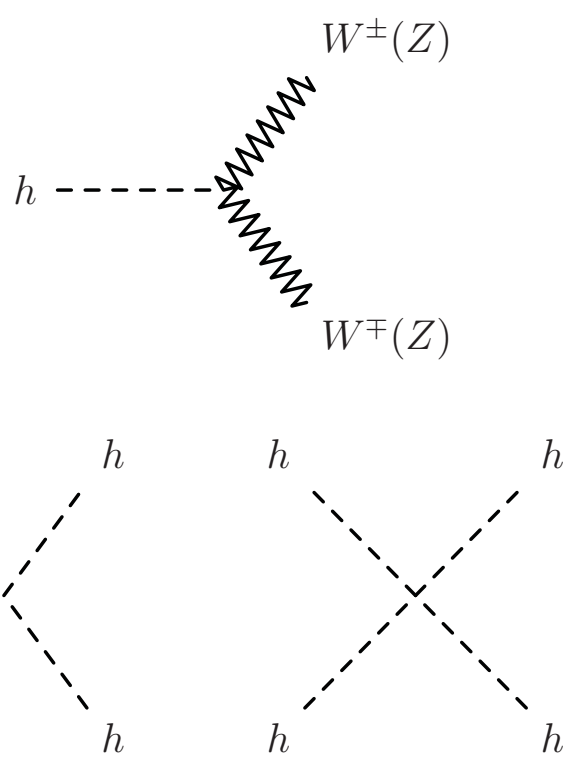

Figure 6: Feynman diagram components for Higgs interactions. Only the upper diagrams contribute at rates relevant to this thesis.

\subsubsection{Production}

The mode of production of Higgs bosons depends on the collider at which they are produced, as well as its energy. At the LHC, the dominant production modes are necessarily either gluon or quark-initiated, whereas at an $e^{+}-e^{-}$collider they would be different. The Higgs bosons analyzed in this thesis are produced through four production modes: 
ggF The dominant production mode by far is gluon gluon fusion (ggF), wherein two gluons fuse via a loop of top quarks to produce a Higgs. The extremely high mass of the top quark is what makes this production mode so dominant, as its coupling to the Higgs is far higher than any other particle. A contribution from a similar loop of bottom quarks is included in this production cross section, but it represents a very small fraction of ggF production, scaling like $\left(m_{b} / m_{t}\right)^{2}$ compared to the top loop. Other quarks technically enter into the loop as well, with even smaller contributions. The leading-order Feynman diagram for ggF production is shown in Figure 7.

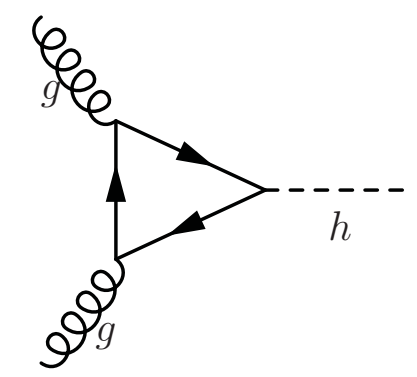

Figure 7: Feynman diagram for ggF Higgs production.

VBF The second most important production mode is vector boson fusion (VBF), approximately an order of magnitude down from ggF. In VBF production, a pair of vector bosons (either $Z Z$ or $W^{+} W^{-}$) radiate from quarks in the two colliding beams. These bosons fuse to produce the Higgs boson. The signature which distinguishes $\mathrm{VBF}$ from $\mathrm{ggF}$ is the pair of quarks appearing in the final state (observed as jets, to be explained in Section 4.6). The leading-order Feynman diagram for VBF production is shown in Figure 8. 


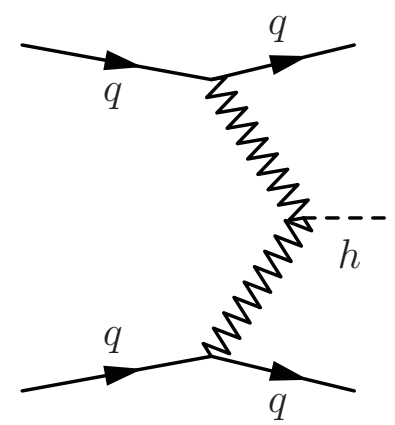

Figure 8: Feynman diagram for VBF Higgs production.

VH The third leading Higgs production mode is associated production (VH), in which a Higgs boson is produced in association with a vector boson. In this mode, an incoming quark-anti-quark pair fuse to produce a $W^{ \pm}$or $Z$ boson, which then radiates a Higgs boson. The leading-order Feynman diagram for VH production at the LHC is shown in Figure 9.

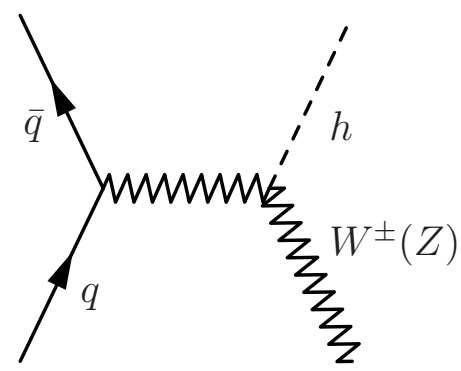

Figure 9: Feynman diagram for VH Higgs production.

$\mathbf{t t H}, \mathbf{b b H}$ The rarest Higgs production mode mentioned in this thesis is top fusion, or bottom fusion (ttH, or bbH). In this process, a Higgs boson is produced in association with a $t \bar{t}$ or $b \bar{b}$ pair. The ttH process is enhanced over bbH by the Higgs' strong coupling to top quarks due to their high mass, but suppressed by the enormous energy required to produce two tops (and a Higgs) in a single collision, as compared to that required to produce two bottoms (and a Higgs). Coincidentally, at $\sqrt{s}=13 \mathrm{TeV}$, these two competing factors balance out, and ttH and bbH have nearly identical cross sections. The leading diagram for both 
processes resembles that for ggF, but the quarks no longer form a loop, instead they survive as final state particles. It is shown in Figure 10.

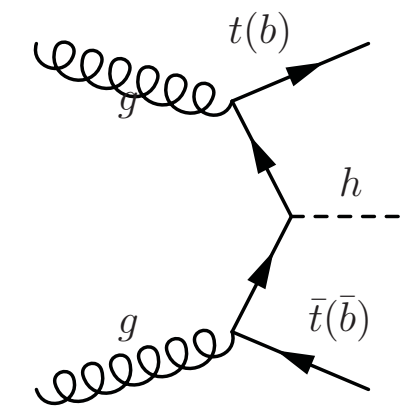

Figure 10: Feynman diagram for ttH (bbH) Higgs production.

Figure 11 shows the cross section for each production mode, as a function of the Higgs boson mass. In this diagram, ggF is labelled as $p p \rightarrow H, \mathrm{VBF}$ is labelled as $p p \rightarrow q q H$, and $\mathrm{VH}$ is split into $p p \rightarrow W H$ and $p p \rightarrow Z H$. Table 3 summarizes the cross sections and fraction of each production mode expected by the SM for a $m_{H}=125 \mathrm{GeV}$ Higgs in $\sqrt{s}=13 \mathrm{TeV}$ collisions at the LHC.

Table 3: Production cross sections for a $m_{H}=125 \mathrm{GeV}$ Higgs boson in $\sqrt{s}=13 \mathrm{TeV}$ collisions. Values taken from [21], with uncertainties symmetrized and summed in quadrature, where necessary.

\begin{tabular}{ccc}
\hline production mode & cross section $[\mathrm{pb}]$ & fraction [\%] \\
\hline ggF & $48.58 \pm 2.45$ & 87.3 \\
$\mathrm{VBF}$ & $3.78 \pm 0.08$ & 6.8 \\
$\mathrm{VH}$ & $2.26 \pm 0.04$ & 4.1 \\
$\mathrm{ttH}$ & $0.51 \pm 0.04$ & 0.9 \\
$\mathrm{bbH}$ & $0.49 \pm 0.11$ & 0.9 \\
\hline
\end{tabular}




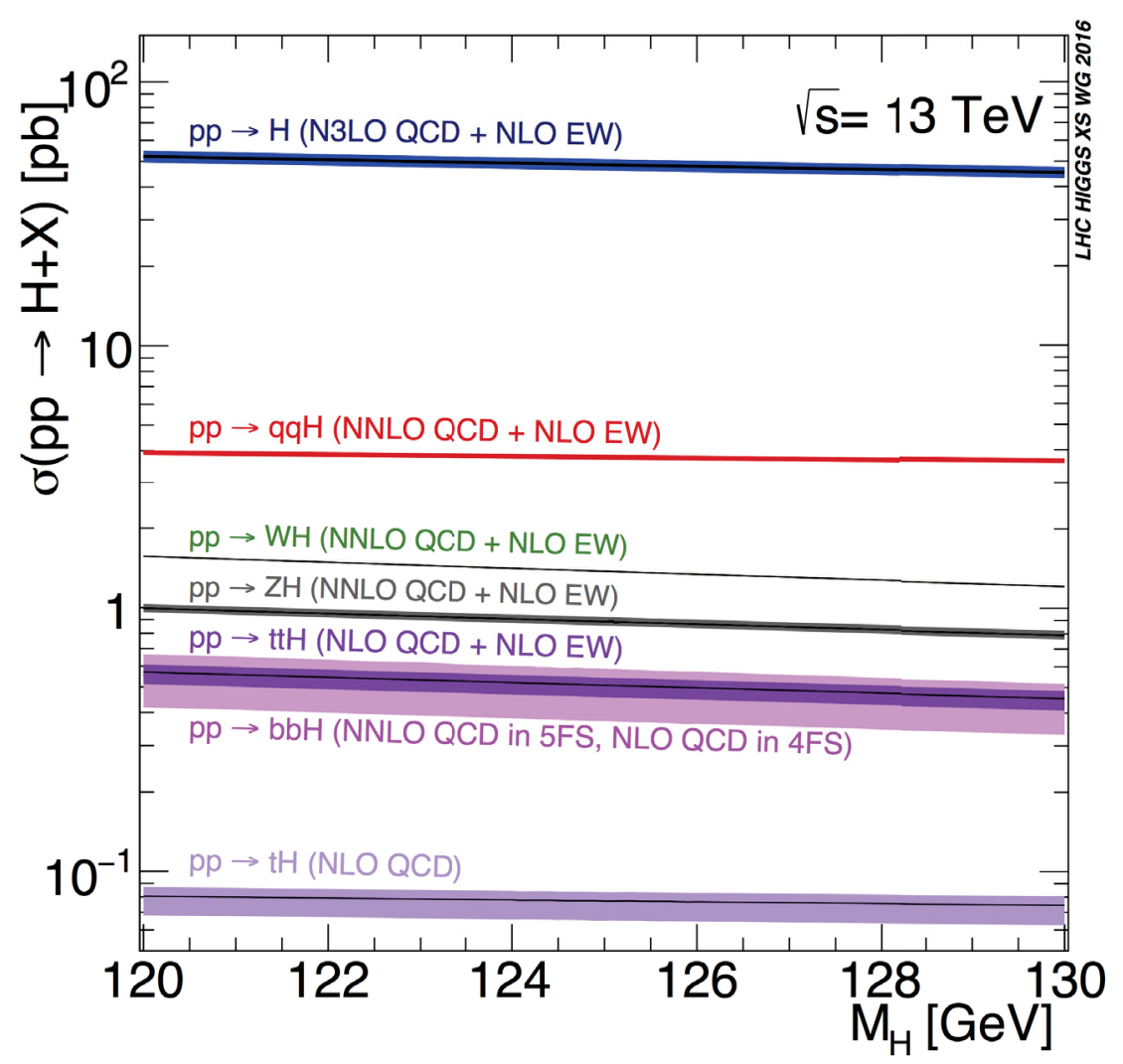

Figure 11: Plot of the SM predicted Higgs cross section for different production modes at the LHC at $\sqrt{s}=13 \mathrm{TeV}$ as a function of the Higgs mass. Taken from [21]

\subsubsection{Decay}

Like many massive particles, the Higgs boson is inherently unstable. The SM predicts the lifetime of a $125 \mathrm{GeV}$ Higgs to be approximately $10^{-22} \mathrm{~s}$. The lifetime is related to the total width of the Higgs boson by $\tau_{H}=1 / \Gamma_{H}$, where $\Gamma_{H}$ is the Higgs total width, or natural width. The decays of the Higgs boson occur by several modes, in proportion to their branching ratios $(\mathrm{BR})$. The Higgs BR is predicted by the SM as a function of the Higgs mass, $m_{H}$, and is independent of the collider at which it is produced. An unstable particle decays preferentially to the particles it is most strongly coupled (assuming enough kinematic phase space to allow conservation of energy and momentum). For the Higgs, this means it prefers decays to heavy particles, though these often subsequently decay to light particles. 
The BR of the SM Higgs boson as a function of its mass is shown in Figure 12. There is no decay to $t \bar{t}$, because the Higgs is lighter than the top quark. The decays to massless $g g, \gamma \gamma$, and (partly massless) $Z \gamma$ occur via a top loop (like the ggF production process, Figure 7, in reverse). The decays to $W, Z$ boson pairs depend most strongly on $m_{H}$ because the combined mass of the decay products exceed the mass of the Higgs. For this reason, one of the bosons must be produced off-shell, so is denoted as (e.g.) $Z^{*}$. The higher the $m_{H}$, the closer to on-shell the $Z^{*}$ can be, so the $\mathrm{BR}\left(H \rightarrow Z Z^{*}\right)$ grows very quickly: by over $20 \%$ between $124-126 \mathrm{GeV}$. By including the branching ratios for the subsequent $\mathrm{Z}$ decay, the total branching ratio to $4 \ell$ final states can be computed. The values of the branching ratios $\mathrm{BR}\left(H \rightarrow Z Z^{*}\right)$ and $\operatorname{BR}\left(H \rightarrow Z Z^{(*)} \rightarrow 4 \ell\right)$ are given in Table 4 for a few $m_{H}$ points.

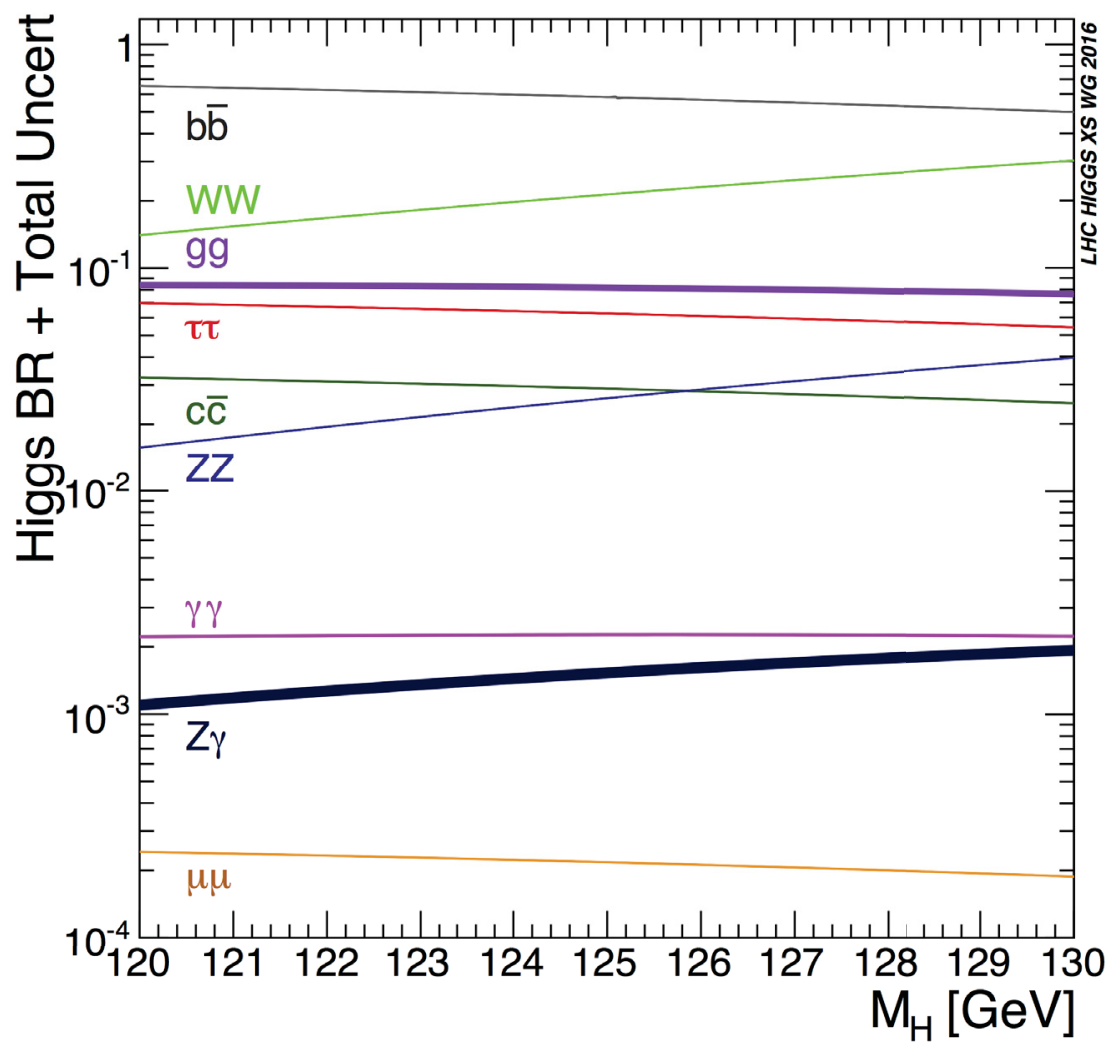

Figure 12: Plot of the SM predicted Higgs branching ratio as a function of the Higgs mass. Taken from [21]. 
Table 4: Branching ratios $\mathrm{BR}\left(H \rightarrow Z Z^{*}\right)$, and $\mathrm{BR}(H z z f l)$ for different masses $m_{H}$. Values taken from [21]. The final column implicitly includes the middle column - they should not be multiplied.

\begin{tabular}{ccc}
\hline$m_{H}[\mathrm{GeV}]$ & $\mathrm{BR}\left(H \rightarrow Z Z^{*}\right)[\%]$ & $\mathrm{BR}\left(H \rightarrow Z Z^{(*)} \rightarrow 4 \ell\right)[\%]$ \\
\hline 124.0 & $2.38 \pm 0.04$ & $(1.13 \pm 0.03) \times 10^{-2}$ \\
124.5 & $2.50 \pm 0.04$ & $(1.19 \pm 0.03) \times 10^{-2}$ \\
125.0 & $2.62 \pm 0.04$ & $(1.24 \pm 0.03) \times 10^{-2}$ \\
125.5 & $2.74 \pm 0.04$ & $(1.30 \pm 0.03) \times 10^{-2}$ \\
126.0 & $2.87 \pm 0.04$ & $(1.36 \pm 0.03) \times 10^{-2}$ \\
\hline
\end{tabular}

Like the $\mathrm{Z}$ boson, the production cross section of the Higgs, and thus its invariant mass distribution, follows a BW. The lineshape for a SM $m_{H}=125 \mathrm{GeV}$ Higgs is shown in Figure 13. Note that the SM Higgs width is nearly three orders of magnitude smaller than that of the $\mathrm{Z}$ boson. For $m_{H}=125 \mathrm{GeV}$, the width predicted by the SM is just $4.1 \mathrm{MeV}$. This lineshape will return in an important way in Chapter 7.

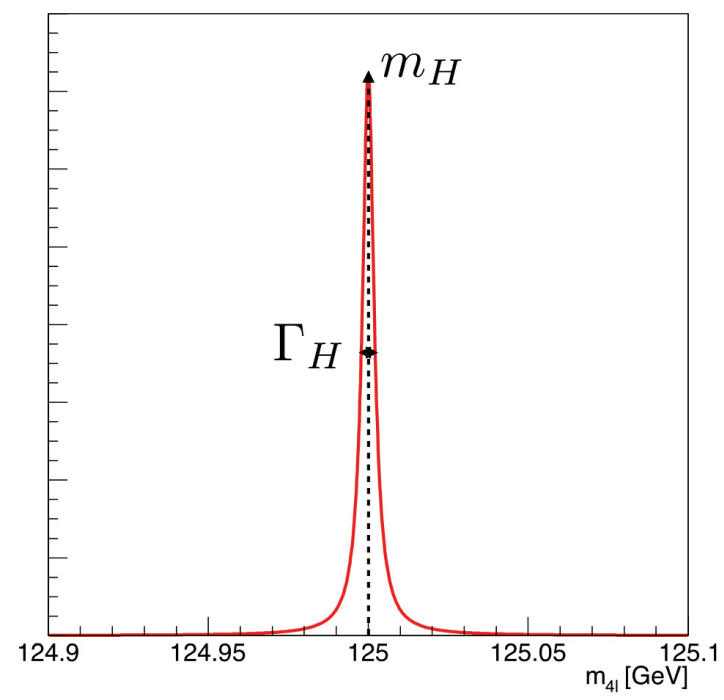

Figure 13: Relativistic Breit-Wigner lineshape for Higgs boson decays, drawn for a $m_{H}=125 \mathrm{GeV}$ Higgs. 


\subsubsection{The $H \rightarrow Z Z^{(*)} \rightarrow 4 \ell$ channel}

This thesis presents measurements of the Higgs boson through its decay to four leptons: the $H \rightarrow Z Z^{(*)} \rightarrow 4 \ell$ decay channel. By combining the diagrams in the preceding sections, the dominant way this process happens at the LHC is shown in Figure 14.

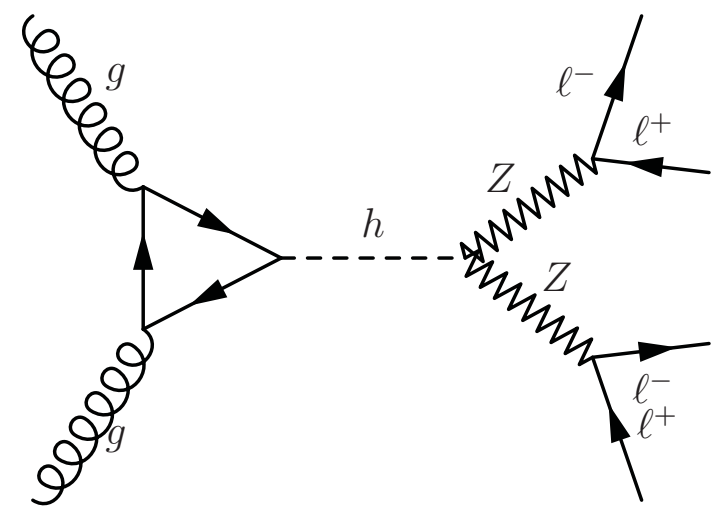

Figure 14: Feynman diagram for production and decay of $H \rightarrow Z Z^{(*)} \rightarrow 4 \ell$.

This decay channel is a very powerful tool for measuring the Higgs' properties. The primary reason is that the final state is composed entirely of muons and electrons, which are both precisely reconstructed with very high efficiency. Secondly, the channel has a very high signal-to-background ratio (after the event selection described in Chapter 5, it is $\mathcal{O}(1))$. For this reason, it was called the golden channel for Higgs discovery, and was one of the two channels essential to the discovery of the Higgs boson in 2012. In this thesis, it is used to make direct measurements of the Higgs mass, $m_{H}$, width, $\Gamma_{H}$, and total cross section times branching ratio, defined as

$$
\sigma_{H \rightarrow 4 \ell} \equiv \sigma_{p-p \rightarrow H} \times \mathrm{BR}\left(H \rightarrow Z Z^{(*)} \rightarrow 4 \ell\right)
$$

where $\sigma_{p-p \rightarrow H}$ is the total cross section at $\sqrt{s}=13 \mathrm{TeV}$ combining all production modes, and $\ell=e, \mu$. In the SM, $m_{H}$ is a free parameter left to measure, while $\Gamma_{H}$ and $\sigma_{H \rightarrow 4 \ell}$ can be calculated once it is known. The SM predictions, when $m_{H}=125 \mathrm{GeV}$, 
are

$$
\begin{aligned}
m_{H} & =125.00 \mathrm{GeV}, \\
\Gamma_{H} & =4.09 \pm 0.03 \mathrm{MeV}, \\
\sigma_{H \rightarrow 4 \ell} & =6.90 \pm 0.31 \mathrm{fb} .
\end{aligned}
$$

Measuring these parameters is the primary goal of this thesis. Although the SM offers no prediction of $m_{H}$, it has an innate value as a fundamental parameter of the theory. Additionally, precise knowledge of $m_{H}$ improves the precision of other SM predictions, among them $\Gamma_{H}$ and $\sigma_{H \rightarrow 4 \ell}$. More importantly, precise $m_{H}$ knowledge improves the predictive precision on the more rare Higgs production processes, which allow one to disentangle the Higgs' couplings to individual particles. The best existing measurement of $m_{H}$ comes from an ATLAS+CMS combination of the Run 1 LHC data in the $H \rightarrow Z Z^{(*)} \rightarrow 4 \ell$ and $H \rightarrow \gamma \gamma$ channels [8], where the mass was measured to be $m_{H}=125.09 \pm 0.24 \mathrm{GeV}$. Although the measurement in this thesis cannot surpass the precision of this combined measurement, it exceeds the precision of any $H \rightarrow 4 \ell$ mass measurement conducted so far. A review of the other existing measurements of $m_{H}, \sigma_{H \rightarrow 4 \ell}$, and $\Gamma_{H}$ will accompany the results of the measurements in Chapter 9 for ease of comparison.

\subsection{Beyond the Standard Model}

While the SM succeeds is describing nearly all observed phenomena with incredible precision, it is still an incomplete theory. Beyond Standard Model (BSM) physics refers to the phenomena observed in nature but not explained by the SM[22], and also to extensions of the SM attempting to explain these phenomena. Most BSM models will in some way affect the Higgs sector, which makes precision Higgs physics a powerful probe for testing them.

One of the most important questions for future experiments is the nature of Dark 
Matter (DM) - a class of particles making up $84 \%$ the matter content of our universe yet wholly unknown to the SM. The existence of Dark Matter is supported by an abundance of evidence from astrophysical and cosmological measurements [23], but has never been observed in a laboratory. One thing we do know is that some of it (and maybe most of it) is composed of non-SM particles, which we call Weakly Interacting Massive Particles (WIMPS). To explain the observed phenomena, WIMPS must have mass. The only known way particles can acquire mass is through the Higgs mechanism, which would mean that the WIMPS, even if they don't interact with any other SM particles, will interact with the Higgs boson. If this were the case, it is possible that the Higgs boson could decay to DM particles. Searches by ATLAS [24] have not detected any such decays, but even if the Higgs does not decay to DM particles, for example because they are too heavy for a $125 \mathrm{GeV}$ Higgs to decay into, their coupling would still affect the production cross sections for the Higgs, and thus $\sigma_{H \rightarrow 4 \ell}$.

Many BSM theories postulate an extended Higgs sector [25] to help resolve discrepancies in the SM. In these models, the observed Higgs near $125 \mathrm{GeV}$ is the lightest of several Higgs bosons. These heavier counterparts have been searched for, including one search by the author [26], though no direct evidence of a heavy Higgs has been found. Direct detection is however not the only way an extended Higgs sector can reveal itself. The existence of a heavy Higgs also alters the couplings of the $125 \mathrm{GeV}$ Higgs such that the cross sections, thus $\sigma_{H \rightarrow 4 \ell}$, and the total width, $\Gamma_{H}$, are modified compared to the SM. As a result, precision measurement of these properties is itself an indirect test of a whole class of BSM theories.

The above examples are not meant to be complete, but rather to illustrate how precision measurement of an SM particle can be a powerful tool for finding clues, and guiding future searches and measurements, of BSM physics. 


\section{Chapter 3}

\section{ATLAS and the LHC}

Though production of Higgs bosons can occur spontaneously in nature, the rate for this occurrence is prohibitively low for any observation[27]. For this reason, experimental searches and studies of the Higgs boson use high-energy particle colliders to actively produce Higgs bosons, while detectors observe the outcome of these collisions. By happenstance the Higgs boson has a mass near $125 \mathrm{GeV}$, and the collider currently best suited to its production is the Large Hadron Collider. Had the mass been lower, another collider such as the Tevatron or Large Electron-Positron collider would have preempted the LHC in the discovery and initial property measurement of the Higgs boson.

Section 3.1 will describe the Large Hadron Collider, used to produce the Higgs bosons under study. The products of these collisions are recorded by the ATLAS Detector, described in Section 3.2, and it is these data analyzed throughout the thesis. Section 3.3 will describe plans for a future hardware upgrade of the ATLAS Detector, with the author's particular involvement described in Section 3.3.1.1.

\subsection{The LHC}

The Large Hadron Collider (LHC) [28] is the largest particle collider in the world. It is a circular collider with a circumference of approximately $27 \mathrm{~km}$, located $100 \mathrm{~m}$ 
underground straddling the border between Switzerland and France, operated by the European Organization for Nuclear Research (CERN, an acronym based on its French name).

The primary use of the LHC is as a proton-proton $(p-p)$ collider. Two adjacent beam pipes contain beams of protons circulating in opposite directions around the ring. The beams are clumped in 2808 bunches, each bunch containing approximately $10^{11}$ protons, each proton with an energy of $6.5 \mathrm{TeV}$. At this enormous energy, protons circle the LHC 11, 000 times per second, travelling at 99.9999990\% the speed of light. The opposing beams cross at four collision points along the ring. During each so-called "bunch crossing", separated by intervals of $25 \mathrm{~ns}$ during normal operation, some of the protons in the bunches may collide, while the remainder of the bunch continues to circulate the LHC. The products of these collisions are observed by detectors at each of the four collision points: ATLAS, CMS, ALICE, and LHCb.

ATLAS (A Toroidal LHC Apparatus) [29] and CMS (Compact Muon Solenoid) [30] are both general-purpose detectors with similar physics goals, but distinct designs. ALICE (A Large Ion Collider Experiment) [31] is a more specialized detector designed to study quark-gluon plasma using the LHC's other mode of operation: collision of lead ions $\left({ }^{208} \mathrm{~Pb}^{82+}\right)$, called $\mathrm{Pb}-\mathrm{Pb}$ collisions. LHCb (LHCbeauty) [32] is also a specialized detector, focusing on the study of bottom quarks and their interactions. The relative locations of the four detectors on the LHC ring is shown in Figure 15.

\subsubsection{From canister to collision}

Protons in the LHC begin their journey [33] in a small can of hydrogen gas. An electric field strips the electrons from the hydrogen, leaving only the nuclei which are bare protons. These protons pass into Linac2, a linear accelerator which uses Radio-Frequency Quadrupoles (RFQs) to focus the beam of protons and accelerate 
them to $50 \mathrm{MeV}$.

Linac2 is the only linear accelerator in the chain, before the protons pass into circular accelerators. The advantage of circular accelerators over linear accelerators is that the particles pass through the system many times, allowing the machine to give many small "kicks", adding more energy with each orbit. All circular accelerators share the same main components: radio-frequency $(\mathrm{RF})$ cavities are used to accelerate the particles, electromagnetic dipoles are used to bend the particle trajectories along the ring, while higher-order multipoles (quadrupole, sextupoles, octopoles) are used to shape and focus the beam. In order for the particles to be accelerated as they orbit, the dipole field strength, $B$, must ramp in sync (thus they are called "synchrotrons") with the particles momenta, $p$, such that the bending radius given by

$$
\rho=\frac{p c}{B}
$$

remains constant, and equal to the physical radius of a circular accelerator. Here $c$ is the speed of light, and we assume particles with charge equal to one proton (or electron). The field strength must also compensate the incomplete coverage of the circumference ring by the dipole magnets (because of practical limitations), so must be proportionately stronger than for full coverage. As a result of the dynamic field strength of a synchroton, all particles circulating must have the same momentum, so adding more particles during the acceleration is impossible. Typically the accelerator is filled at a lower starting energy, then all particles are accelerated to the target energy, and finally they are all extracted (or collided) to empty the accelerator and start over.

In the second stage of the accelerator chain, the protons enter the Proton Synchrotron Booster (PSB), a set of four stacked synchrotrons. The proton beam is split into these four synchrotrons, and then RF cavity harmonics with the orbiting 
beams are used to split these beams into bunches. The beams are merged (but not de-bunched) during extraction.

The third stage of the accelerator chain is the Proton Synchrotron (PS). The PS further splits the PSB bunches, and accelerates the protons up to $25 \mathrm{GeV}$.

The fourth stage of the accelerator chain is the Super Proton Synchrotron (SPS) which once operated as a proton-antiproton $(p-\bar{p})$ collider, providing collisions for the Nobel-prize-winning [34] discovery of the W [35] and Z [36] bosons in 1984. Several injection cycles from the PS are required to fill the SPS with the full bunch structure of the LHC. Once full, the SPS accelerates the protons up to $450 \mathrm{GeV}$.

The final stage of the chain is the LHC itself. Bunches are extracted from the SPS at two points, leading to the two separate beam pipes in the LHC: one circulating clockwise and the other anticlockwise. Once the $\mathrm{LHC}$ is filled at $450 \mathrm{GeV}$, the particles are accelerated over the course of about 20 minutes to reach their maximum energy of $6.5 \mathrm{TeV}$ per proton, when they are finally ready to be collided. The full accelerator chain is illustrated in Figure 15.

\subsubsection{Collisions}

A critical parameter in collider physics is the luminosity of the collisions, roughly speaking the amount of data collected. The amount of data being collected per unit time is the instantaneous luminosity, denoted by $\mathcal{L}$, whereas the total amount of data collected (in 2015 for example) is called the integrated luminosity (or commonly as in this thesis, just luminosity), written in shorthand as

$$
\int \mathcal{L}=\int \mathcal{L}(t) d t
$$

The importance of the luminosity becomes clear when we consider the measurement of (or search for) some process, $p p \rightarrow X$. If we observe some number of events, 


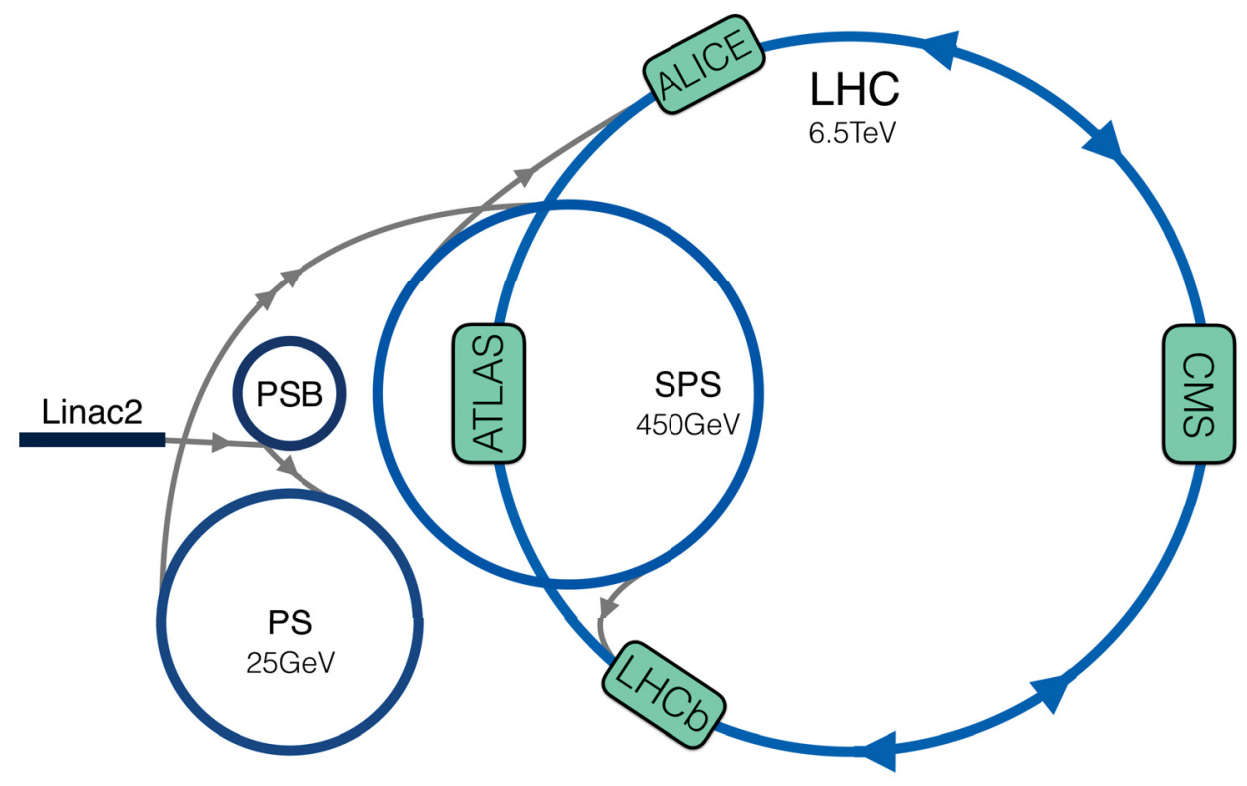

Figure 15: Illustration of the LHC accelerator chain and the four detectors, based on [33]. Energies are per-proton. Sizes are not to scale.

$N_{p p \rightarrow X}$, we may wish to compare to a predicted cross section, $\sigma_{p p \rightarrow X}$, from theory. To do this we use the integrated luminosity as follows:

$$
\sigma_{p p \rightarrow X}=\frac{N_{p p \rightarrow X}}{\epsilon \int \mathcal{L}}
$$

Where the quotient $\epsilon$ is introduced to represent an efficiency: what fraction of the produced events were expected to be observed. In Section 7.1, the $\sigma_{H \rightarrow 4 \ell}$ parameter being measured in this thesis is parameterized in a similar way, with some additional caveats.

The instantaneous luminosity can be calculated directly from beam parameters [37]. For head-on beams, it is expressed as

$$
\mathcal{L}=\frac{n_{b} n_{1} n_{2} f_{r}}{2 \pi \Sigma_{x} \Sigma_{y}}
$$

where $n_{b}$ is the number of bunches per beam, $n_{i}$ is the number of protons in 
beam $i, f_{r}$ is the revolution frequency of the beams, $\Sigma_{\mu}=\sqrt{\sigma_{1 \mu}^{2}+\sigma_{2 \mu}^{2}}$ for $\mu=x, y$ represents the $\mathrm{x}-\mathrm{y}$ decomposition of the beam profiles. In reality the beams of the LHC cross at a small angle in order to control the point of collision, and in practice the equation must be modified slightly to take this into account. From (28) it is clear that $\mathcal{L}$ has units of $\mathrm{s}^{-1} \mathrm{~m}^{-2}$, and thus $\int \mathcal{L}$ has units of $\mathrm{m}^{-2}$. The units of inverse area are commonly expressed in $\mathrm{pb}^{-1}$ or $\mathrm{fb}^{-1}$, which provides a convenient scale to work in (e.g. this thesis presents an analysis of $36.1 \mathrm{fb}^{-1}$ of data).

To understand the collisions themselves it must noted that, as introduced in Section 2.1, protons are not fundamental particles, they are made up of partons. It is the partons which interact directly, not the protons themselves ${ }^{1}$. The two partons whose interaction makes up the bulk of the momentum transfer form what is called the hard-scatter (HS) interaction (located at the hard-scatter vertex). It is in these high-energy interactions where we search for Higgs bosons.

While the hard-scatter interaction is the most interesting for physics analyses, it is not the only interaction during a collision event. The remainder of the two protons form what is called the underlying event (UE) [39]. These leftover partons undergo multiple-parton-interaction (MPI), a collective interaction involving soft scatter and hadronization. The MPI process produces showers of particles, referred to as hadronic jets. UE is also taken to include beam-beam remnants, where other protons in the beam scatter elastically, producing additional jets near to the beam axis. Figure 16 illustrates a collision event with these components indicated.

A further source of activity in the detector is what we call pileup (PU). Pileup

\footnotetext{
${ }^{1}$ This is only a partial truth for two reasons:. At low momenta, such that the de Broglie wavelength, $\lambda=h / p$ (where $h$ is Planck's constant, and $p$ is the particle momentum), is much larger than the effective radius of the proton, the substructure is irrelevant to the interactions, and we can consider direct proton-proton interactions. At high momenta, maybe 10 or $10^{10}$ times more than the LHC, perhaps it will be revealed that quarks themselves are not fundamental, and the same arguments can be made for them. The more complete truth is that the substructure of protons is essential to describing interactions at the LHC, whereas the [unknown, possibly non-existent] substructure of quarks and gluons is, so far, not [38].
} 


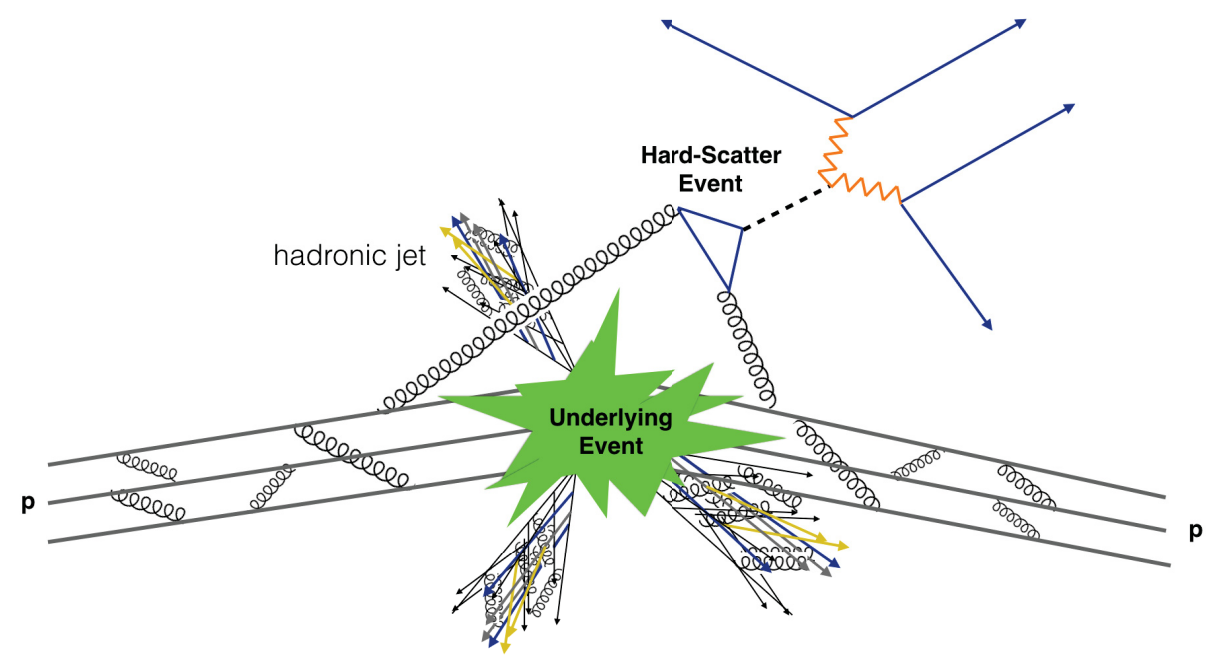

Figure 16: Diagram showing distinction between underlying event and hard-scatter. The HS event in this case is a $H \rightarrow Z Z^{(*)} \rightarrow 4 \ell$ event. Diagram inspired by [40].

occurs in two forms: in-time pileup, and out-of-time pileup. In-time pileup refers to interactions from other protons within the same bunch crossing as the hard-scatter event. Out-of-time pileup refers to interactions from preceding or subsequent bunch crossings. In both cases, the products of the less interesting collisions are recorded by the detector indistinguishably from the hard-scatter, obfuscating the signal of interest. Pileup is sensitive to the instantaneous luminosity given in (28). The LHC beam parameters are tuned to maintain a level of pileup ATLAS and the other detectors can cope with, while still delivering the maximum amount of data.

\subsection{The ATLAS Experiment}

The ATLAS Experiment (or just "ATLAS") is the largest detector on the LHC ring [29]. It is approximately cylindrical in shape, standing $25 \mathrm{~m}$ tall (and wide), and $45 \mathrm{~m}$ long. The detector weighs nearly 7000 tons, and is located along the LHC beam line, $100 \mathrm{~m}$ underground. The LHC beams collide at exactly (or nearly) the geometric center of the detector, and the debris flies outward in all directions through 
the detector from there.

The function of ATLAS is to record the particles that fly out of the collisions: what species of particle, what was its charge, momentum, where did it originate, etc. Knowing this information allows the study of the physical processes at hand. To this end, ATLAS is composed of many subdetectors specialized for different types of measurements. These subdetectors are illustrated in Figure 17 and will be described in detail in the following sections.

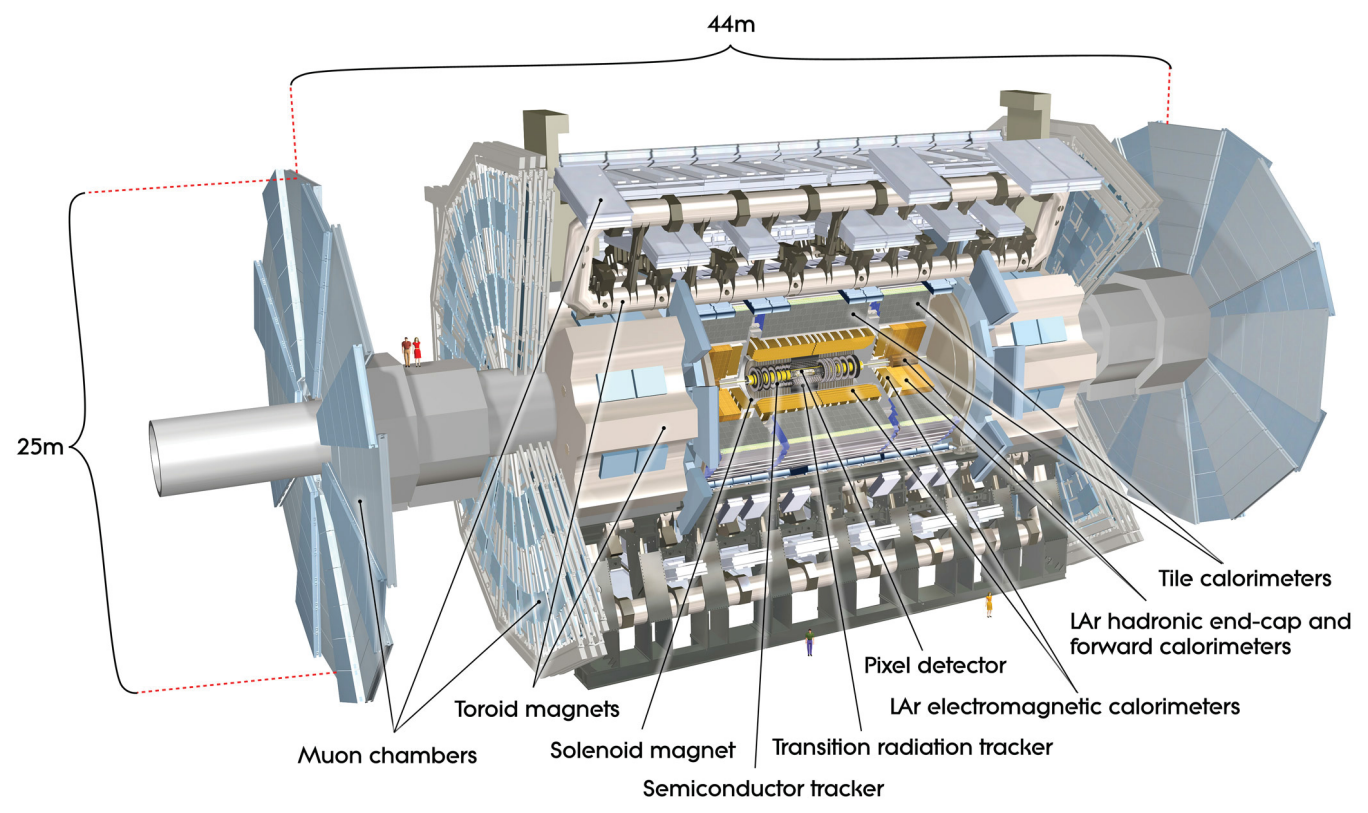

Figure 17: Diagram of the ATLAS Detector with various components illustrated. Image taken from [41].

\subsubsection{The ATLAS coordinate system}

In order to describe the detector, we must first define a coordinate system. The ATLAS detector uses a mix of Cartesian, cylindrical, and spherical coordinates. Depending on the application, one system of coordinates may lend itself more readily than another. All coordinate systems share the same origin, the geometric center of 
ATLAS, defined by $x=y=z=r=0$.

The Cartesian coordinate system, $(x, y, x)$, used in ATLAS is defined as follows. The $x$ axis points toward the center of the LHC. The $y$ axis points up toward the sky (but is off vertical by $0.704^{\circ}$, because the LHC is not completely level). the $z$ axis points along the beam line in the anti-clockwise direction. The Cartesian coordinate system is illustrated in Figure 18a.

The cylindrical coordinate system, $(r, z, \phi)$, shares the same $z$ coordinate as the Cartesian system. The transverse plane $(x-y)$ is transformed into a radial distance $r$, and an azimuthal coordinate $\phi$ on the range $(-\pi, \pi]$, with $\phi=0$ on the positive $x$-axis, and positive (negative) $\phi$ representing the top (bottom) half of the detector. The cylindrical coordinate system is illustrated in Figure 18b.

The spherical coordinate system introduces the polar angle $\theta$, on the range $[0, \pi]$, defined as the angle from the positive $z$-axis. While normally a spherical coordinate system would include a radial coordinate, this has little practical use in ATLAS. The spherical coordinate system is illustrated in Figure 18c.

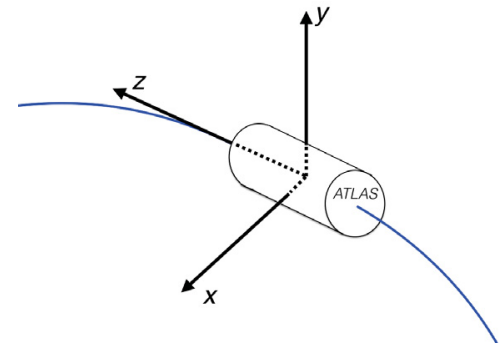

(a) Cartesian

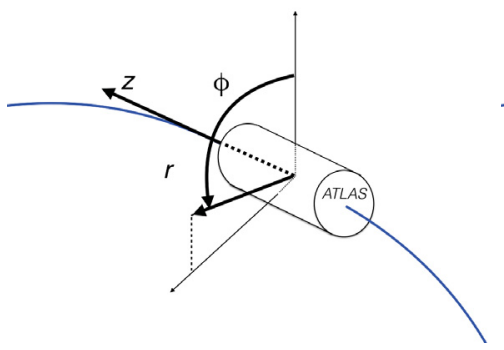

(b) Cylindrical

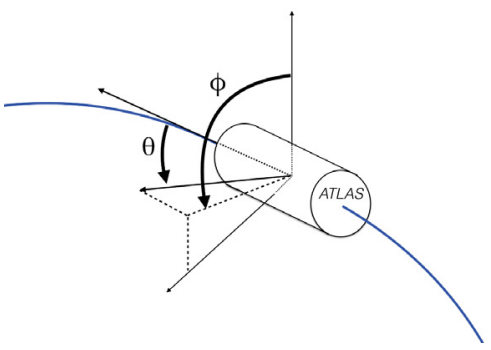

(c) Spherical

Figure 18: Illustration of the three coordinate systems used in ATLAS.

\subsubsection{Rapidity and pseudorapidity}

Before moving on, consider the $p-p$ collisions at the LHC. Both protons participating in the collision have the same momentum, $6.5 \mathrm{TeV}$, but the actual colliding partons have only a random fraction of this momentum, distributed according to their PDFs. 
This means that the center-of-momentum (c.o.m.) of the collision is generally not the rest frame of the ATLAS detector: the two have a large relative velocity along the $z$-axis.

How can we compare multiple data from one detector when each process occurred in a different c.o.m. frame? The straightforward idea would be to boost the data recorded in the ATLAS frame to the HS frame. Unfortunately this is generally not possible. To do so would require reconstructing the momentum of all particles in the detector, and selecting correctly only those which came from the HS vertex in order to balance the momenta and identify the HS frame. This is ruled out both by the production of neutrinos in many physical processes, and by the presence of products from UE and PU processes (especially near to the $z$-axis).

The solution to this problem is the introduction of rapidity [42], defined by

$$
y=\frac{1}{2} \ln \left(\frac{E+p_{z} c}{E-p_{z} c}\right) .
$$

For a relativistic coordinate transformation (called a "boost") at speed $v=\beta c$ along the $z$-axis, the rapidity transforms as

$$
y^{\prime}=y-\tanh ^{-1} \beta,
$$

which leads to a valuable property. Consider two particles measured in the ATLAS detector to be separated in rapidity by $\Delta y=y_{1}-y_{2}$ in the ATLAS frame. What was their rapidity separation in their c.o.m. frame, which as discussed above is boosted along the $z$-axis?

$$
\Delta y^{\prime}=y_{1}^{\prime}-y_{2}^{\prime}=\left(y_{1}-\tanh ^{-1} \beta\right)-\left(y_{2}-\tanh ^{-1} \beta\right)=y_{1}-y_{2}=\Delta y .
$$

Ergo, separations in rapidity are invariant under boosts along the $z$-axis. This very helpful property allows the comparison of rapidity separations from many different 


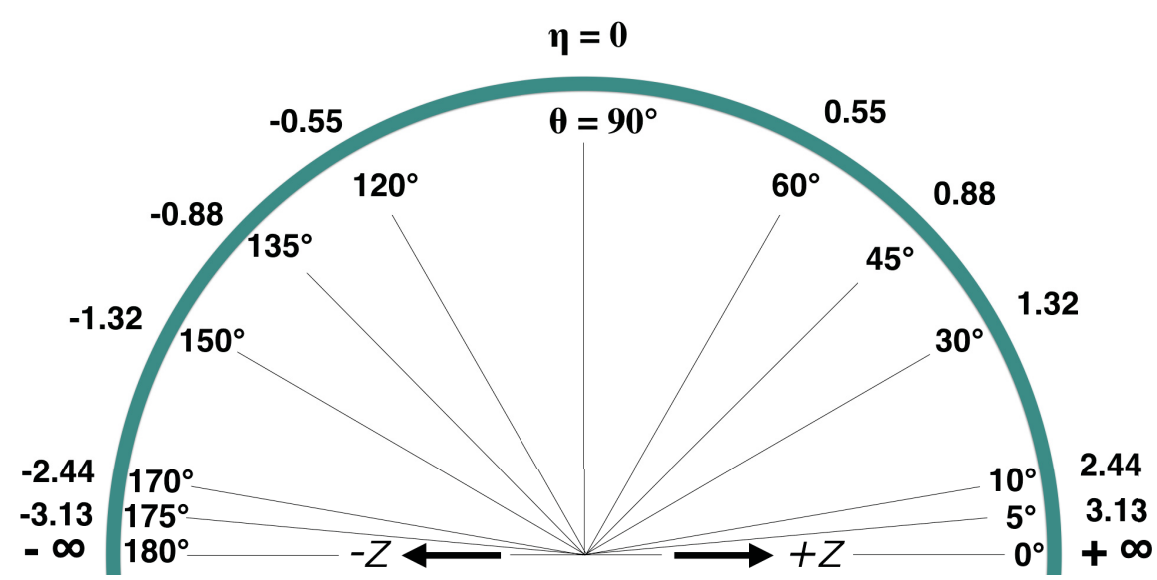

Figure 19: Diagram showing conversion between polar angle $\theta$ and pseudorapidity $\eta$.

HS frames, as the separation in each HS frame is identical to what we can measure in the ATLAS frame.

In practice we can rarely measure both the momentum (or its component $p_{z}$ ) and the energy of a particle independently, and so it is hard to calculate the rapidity from (29). However if we examine the behaviour for highly relativistic particles, where $E, p \gg m$, we can reduce (29) to a simpler expression for a variable called pseudorapidity, defined by

$$
\eta=-\ln \left(\tan \left(\frac{\theta}{2}\right)\right)
$$

All particles directly observed (e.g. electrons, muons) in ATLAS will satisfy this condition. Instead of describing directions by $(\theta, \phi)$, they are described by $(\eta, \phi)$. Figure 19 offers a fast visual conversion between $\theta$ and $\eta$. Heavier particles, such as $h, Z, W$ bosons, must be described by the full rapidity, $y$, and Figure 19 cannot be used.

The pseudorapidity has another more qualitative advantage over polar angle, which is that particle production in a collider is roughly flat in $\eta$. This is useful 
information in detector design as it allows predictions for relative particle fluxes between different components of the detector - important to consider when designing for radiation hardness, granularity, detector live-time, etc. As a consequence, in the following sections many of the detector components are described by their location in $\eta$. Low (high) $|\eta|$ regions are also called forward (central) or end-cap (barrel), though the specific boundary in $\eta$ varies by context.

\subsubsection{Inner Detector}

The ATLAS Inner Detector (ID) is the closest sub-detector to the collision point, and the first that particles encounter. It occupies the space $|r|<1.15 \mathrm{~m},|z|<3.5 \mathrm{~m}$ with pseudorapidity coverage up to $|\eta|=2.5$. The ID is composed of four main subsystems; from the beampipe outwards they are: the Insertable B-Layer (IBL), the Pixel Detector, the Semiconductor Tracker (SCT), and Transition Radiation Tracker (TRT). The entire ID is enclosed within a superconducting solenoid with inner radius $r=1.2 \mathrm{~m}$ and magnetic field $2 \mathrm{~T}$ oriented along the $z$-axis. Due to the orientation of this field, charged particle tracks in the ID bend in the $r$ - $\phi$ plane. As a result, momentum measurement in the ID depends more heavily on the precision in this plane, and not as much along the $z$-axis.

A 3D Render of these components is shown in Figure 20, excluding the solenoid. It should be noted that, in reality, additional detection planes would cover either end very closely and obscure any view of this sort - these are stripped away in the diagram. A cross section in $r-\phi$ is shown in Figure 21, including the specific envelopes of each ID component, excluding the IBL. 


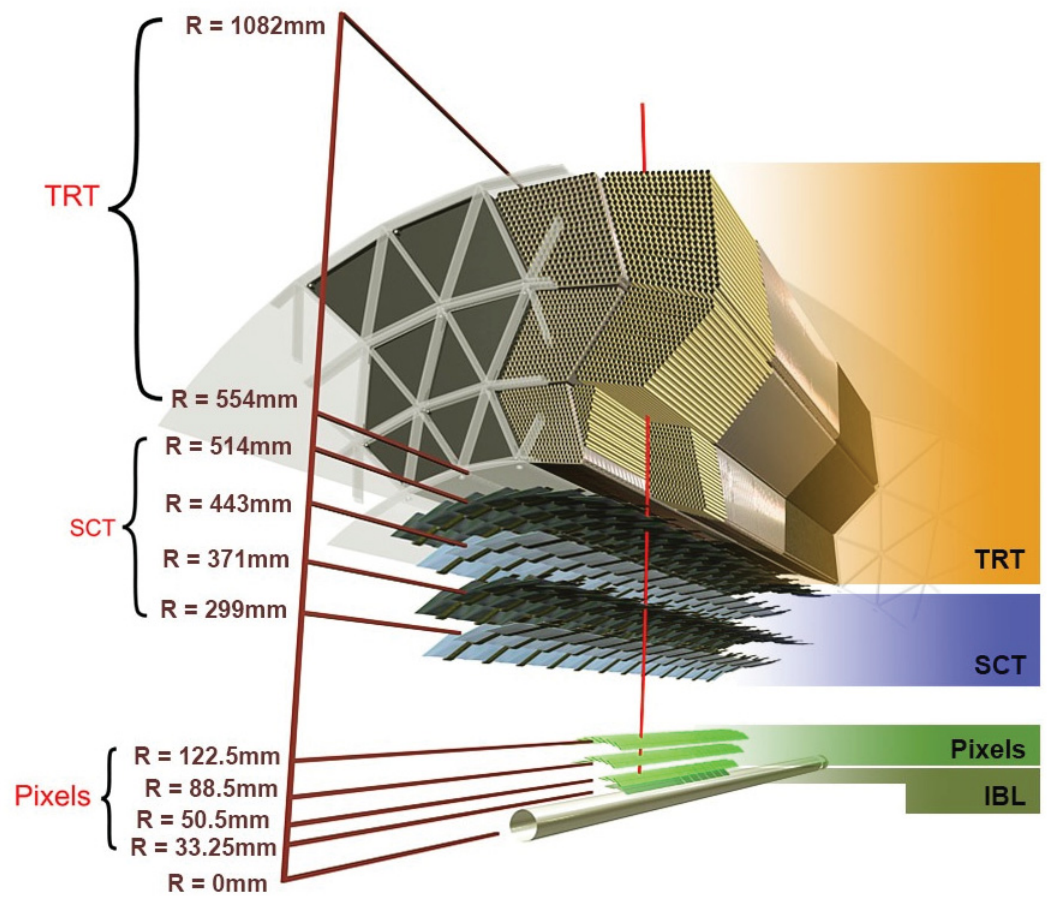

Figure 20: Render of the barrel components (those within $|z|=750 \mathrm{~mm}$ ) of the ID as used in Run 2 at the LHC. Taken from [43].
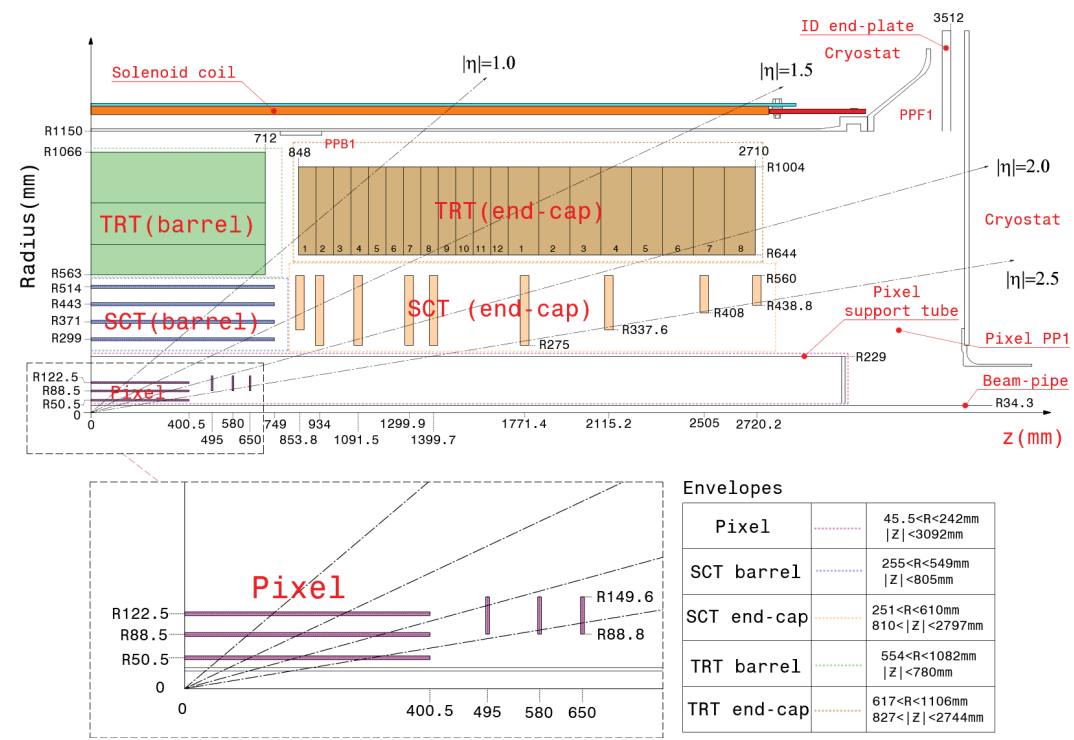

Figure 21: Diagram showing all components of the ID projected in the $r-z$ plane. The detector is symmetric in $z$, so shows only the positive $z$-axis. This diagram is taken from [44], and as such it describes the ID as it was in Run 1. The only change for Run 2 was the addition of the IBL between the beam pipe and the innermost Pixel layer. 


\subsubsection{IBL}

The IBL [45] is a layer of semiconductor pixel detectors immediately surrounding the beam pipe. It is the first active layer that particles produced in a collision will encounter. It is called the "B-Layer" because often B mesons will cross it before they decay, creating a distinct signature in the detector (a single track turning into 2 or more particles), and thus allowing them to be identified. In contrast, heavier unstable particles like Z bosons have smaller $E / m$, and so aren't as boosted, and thus decay much sooner, before reaching or even significantly approaching the BLayer. While this function can be useful for someone interested in B mesons, in the case of this thesis it is used to reject background leptons from B decays. Furthermore the additional tracking layer slightly increases the lever arm used to measure track bending radius (discussed in Chapter 4), and so improves momentum resolution, in particular for electrons.

\subsubsection{Pixel Detector}

The Pixel Detector uses a similar technology to the IBL: layers of semi-conducting pixel detectors surround the beam pipe, which provide three-dimensional hit measurements along charged particle trajectories. Three concentric barrels surround the beampipe, while three coaxial disks cover the forward $(2.0<|\eta|<2.5)$ regions. Coverage between regions is overlapping, and so any particles produced with $|\eta|<2.5$ will cross at minimum three layers. Because of its proximity to the interaction point and high granularity, the Pixel Detector drives the precision of reconstruction of collision vertices.

\subsubsection{SCT}

The SCT uses layers of silicon microstrip detectors as its detection mechanism. In the barrel, the strips run nearly along the $z$ direction, providing measurements in the $r$ - $\phi$ 
plane. Each SCT layer indicated in Figure 21 is composed of 2 sets of strips, which are both at a small (and different) angle from the $z$-axis, providing a less precise measurement in the $z$ coordinate as well.

\subsubsection{TRT}

The TRT uses a technology called straw detectors. The TRT barrel (endcap) is composed of 50,000 $(320,000)$ individual straws oriented along the $z(r)$ direction. The straws have a $4 \mathrm{~mm}$ diameter, and are filled with a gas mixture of $\mathrm{Xe}_{\mathrm{e}} \mathrm{CO}_{2}$, and $\mathrm{O}_{2}$. As charged particles traverse the straws, they ionize the gas and this ionization is read out on a sense wire through the center of each tube. Between the straws is a radiator which induces passing electrons to emit transition radiation. This design feature gives electrons a unique signature in the TRT as compared to other charged particles, and is used as a quality criteria for identifying good electrons.

\subsubsection{Calorimeters}

The second sub-detector encountered by particles (and often the last) is the calorimeter. Located immediately outside the solenoid, it extends out to $r=4.25 \mathrm{~m}$, and provides the most forward coverage in ATLAS, out to $|\eta|=4.9$. It is designed to fully absorb electrons, photons, and hadrons, while allowing muons (and of course neutrinos) to pass through. Like in any calorimeter, the absorption happens by a dense material inducing the particle(s) to shower, and then measuring the total energy deposited in a shower. The ATLAS calorimeters are composed of two subsystems: the Electromagnetic Calorimeter, and the Hadronic Calorimeter. They are depicted in Figure 22. 


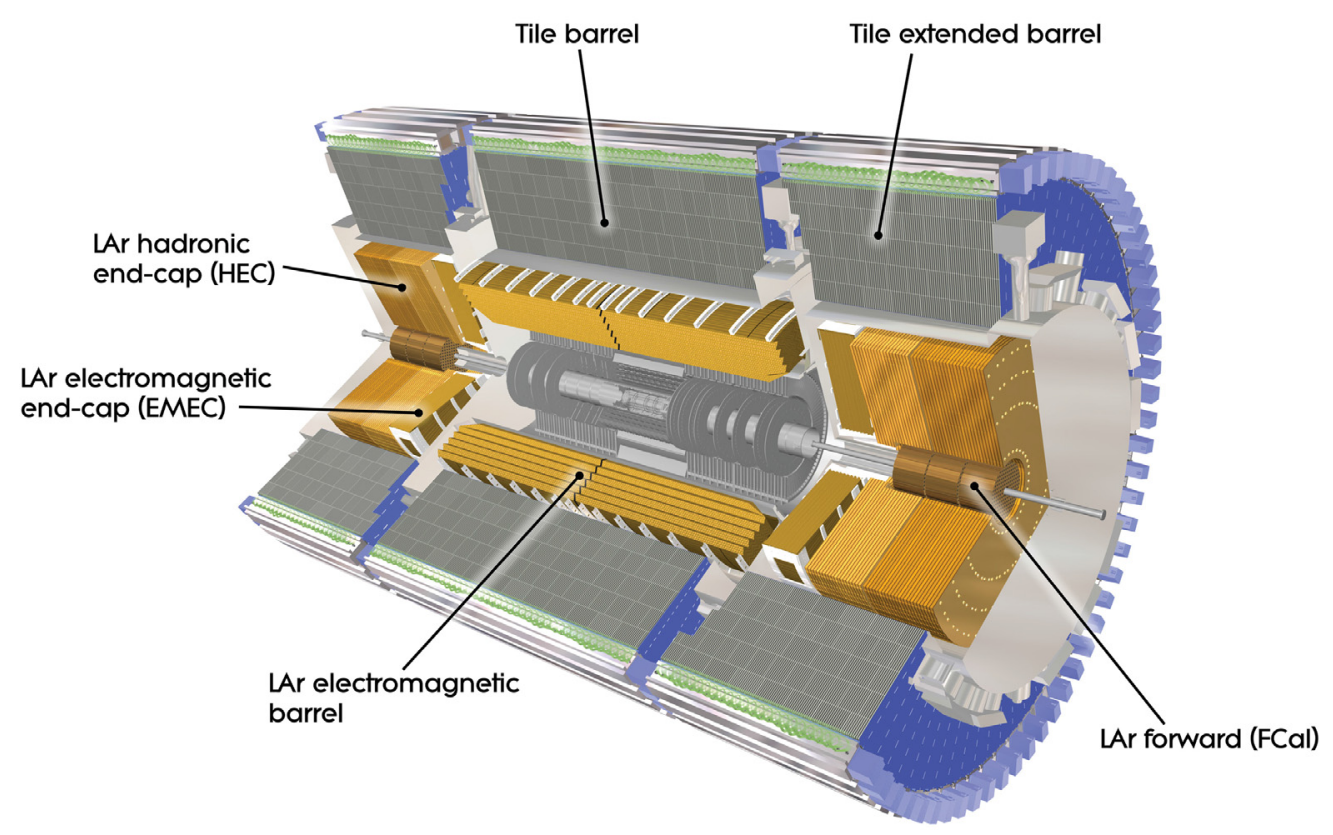

Figure 22: Render of the ATLAS Calorimeter systems, taken from [46]

\subsubsection{Electromagnetic Calorimeter}

The Electromagnetic Calorimeter (ECAL) is designed to absorb the showers of photons and electrons. The ability of a calorimeter to absorb EM showers can be characterized by its depth in radiation lengths, $X_{0}$. An $X_{0}$ is the length over which a high-energy electron loses an $e$-fold of its energy to bremsstrahlung radiation, and 7/9 the length that pair-produced photon intensity falls by an $e$-fold. The ECAL has a depth of $22 X_{0}$ in the barrel, and $24 X_{0}$ in the endcap. Both barrel and end-cap components are lead-liquid argon (LAr) calorimeters, which use alternating layers of lead as the absorbing material, and LAr as the detection medium to measure the showers. The geometry of the calorimeter is a nested accordion, which provides total symmetry in $\phi$ with no gaps in coverage. 


\subsubsection{Hadronic Calorimeter}

Hadronic jets have a much deeper profile than electromagnetic showers, so as a result require a thicker calorimeter. The characteristic length for hadronic showers is the Nuclear Interaction Length, $\lambda_{I}$, the mean distance travelled before a hadron undergoes an inelastic collision, equivalently the distance over which particle intensity falls by an $e$-fold. The Hadronic Calorimeter (HCAL) has a depth of $9.7 \lambda_{I}$ in the barrel, and $10 \lambda_{I}$ in the most forward region. The HCAL is divided into three main subsystems: the Tile Calorimeter, the Hadronic End-Cap Calorimeter (HEC), and the LAr Forward Calorimeter (FCal) [29].

The Tile Calorimeter covers the outermost extent of the calorimeter system in the $r$ direction, from $r=2.28 \mathrm{~m}$ to $4.25 \mathrm{~m}$ across its full length to $|z|=6.0 \mathrm{~m}$ (equivalently up to $|\eta|=1.7)$. It is a sampling calorimeter, using scintillating tiles as the detection medium and steel as the absorber.

The HEC bridges the space between the Tile Calorimeter and the FCAL, providing coverage in both endcaps from $1.5<|\eta|<3.2$. Like the ECAL, the HEC uses LAr as the detection medium, but instead uses thick copper plates as the absorber.

The FCal is the most forward main component of ATLAS, located from $3.1<|\eta|<4.9$ (note the overlap between the Tile Calorimeter, HEC, and FCal is deliberate to ensure gap-free coverage). Unlike the other hadronic calorimeters, the FCal is not overlapped by the ECAL, and so it is also responsible for absorbing EM showers. The FCal also uses LAr as the detection medium, and uses copper (first layer - for EM showers) and tungsten (subsequent layers - for hadronic jets) as the absorber. Because of the enormous particle flux at these values of pseudorapidity, the FCal uses a matrix of parallel tubes filled with the LAr to achieve a high granularity and prevent ion buildup that would otherwise occur in larger gas volumes. 


\subsubsection{Muon Spectrometer}

The outermost set of detectors in ATLAS comprise the Muon Spectrometer (MS) [47].

The MS is a tracking detector immersed in an extensive magnetic field produced by a series of toroid coils. The combined barrel and endcap toroids produce a complex magnetic field oriented roughly in the $\hat{\phi}$ direction, thus causing particle trajectories to bend in the $r$ - $z$ plane. The MS is primarily designed to measure muons, though some hadronic punch-through, where a jet is too energetic to be stopped by the calorimeters, does occur. The MS occupies the space beyond the calorimeter system, out to $r=11 \mathrm{~m}$ and $|z|=22 \mathrm{~m}$. Most of that physical volume is empty however - the MS has a very long lever arm for measuring bending radius.

The MS is composed of four detector technologies. Monitored drift tubes (MDTs) and cathode strip chambers (CSCs) provide precision measurements in the bending plane, while resistive plate chambers (RPCs) and thin gap chambers (TGCs) are used for fast triggering (see more in Section 4.3) and measurement in the orthogonal coordinate.

The geometry of the MS is designed to provide at least three measurements of any track passing through it. Three concentric barrels at increasing radii surround the calorimeters. They are called respectively Inner, Middle, and Outer stations, and each contains overlapping chambers, called Small and Large according to their size in $\phi$, to provide unbroken coverage up to $|\eta|<1.05$. In Figure 24, which shows only the location of the Large chambers, they are labelled as BIL (meaning Barrel-InnerLarge), BML, BOL. Each station is composed of several layers of MDTs, while the Middle (Outer) station also contains two (one) layers of RPCs for triggering. In the forward region, three coaxial disks, called Inner, Middle, Outer, also composed of Small and Large components, provide coverage up to $|\eta|<2.7$. Precision measurement is made by MDTs at all three stations, except for the Inner station and the region $2.0<|\eta|<2.7$, where the measurement is made by CSCs. Three layers of 
TGCs in the Middle station provide triggering, while the TGCs in the Inner station (also called the 'Small Wheel') are used only as a track measurement (but not triggering). In Figure 24, these chambers are labelled as EIL (ie Endcap-Inner-Large), EML, EOL. Though the combined Barrel and Endcap chambers provide near-complete coverage, some additional chambers are necessary to provide coverage in certain gaps caused by structural, service, or instrumentation requirements.

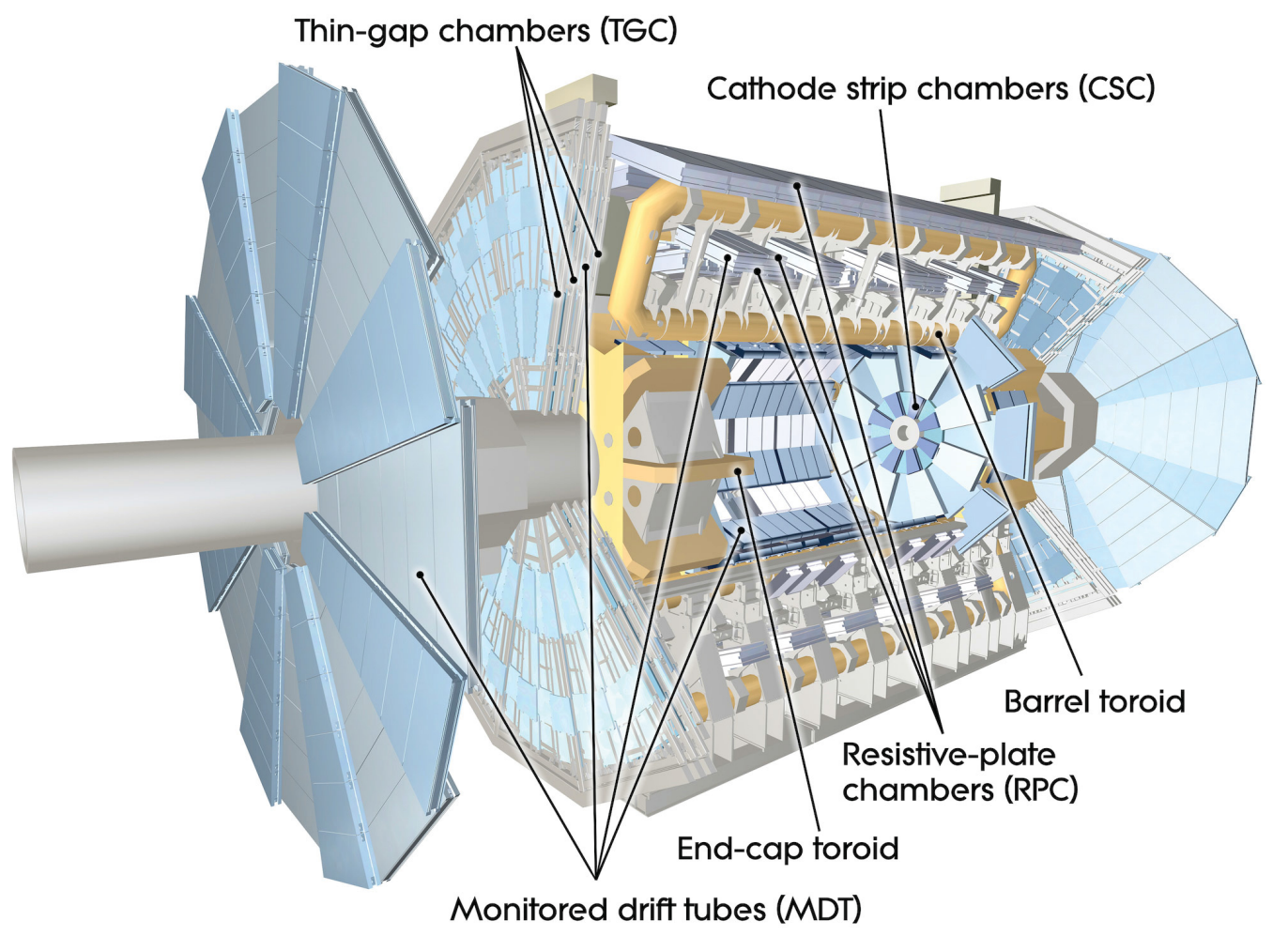

Figure 23: Render of ATLAS Muon Spectrometer showing various detector components. The disk closest to the front standing on its own is one of the Outer Wheels $\left(\mathrm{EO}^{*}\right)$, while the equal-sized disk tightly capping the rest of the detector is the Large Wheel $\left(\mathrm{EM}^{*}\right)$. Taken from [48] 


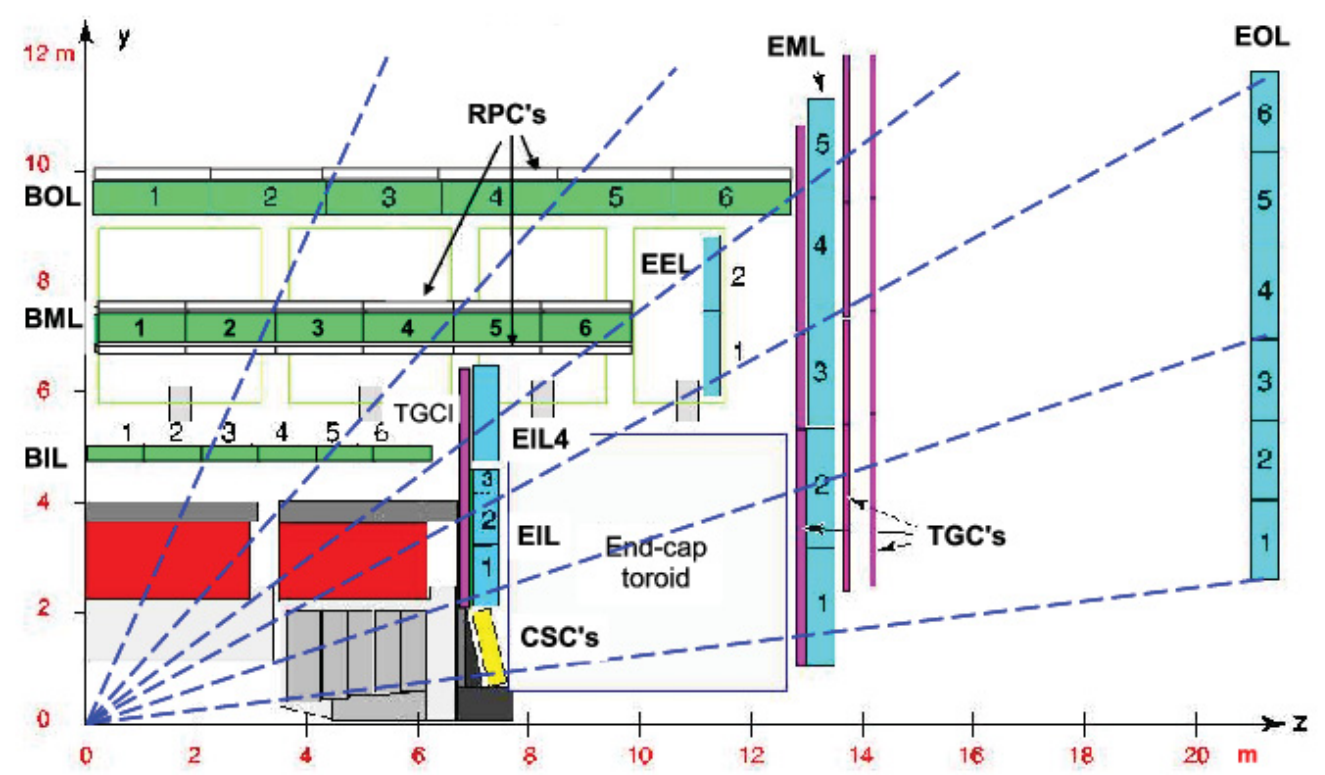

Figure 24: Diagram of MS in $r-|z|$ plane, taken from [49]. Note that for each set of chambers labelled here ending in an "L" (for Large), there is a complementary "S" (for Small) chamber, at a slightly increased $r(z)$ in the barrel (endcap). The Small and Large chambers have partial overlap, as can be seen in Figure 23, to provide nearly hermetic coverage for $|\eta|<2.7$.

\subsection{New Small Wheel}

Over the course of the LHC run program, the instantaneous luminosity of collisions is expected to exceed the design value of $1 \times 10^{34} \mathrm{~cm}^{-2} \mathrm{~s}^{-2}$ by a factor of five [50]. The resultant increase in particle flux through the detector presents two key challenges for ATLAS: a degradation in tracking performance as a result of the increased activity in the detector, as well as an unsustainable Level-1 trigger rate (thus requiring the trigger thresholds to be raised). These problems are more present in the end-cap region region $(|\eta|>1.0)$ of the MS which receives the largest particle flux.

Resolving these problems is critical for an effective physics program. Precision momentum measurement in the MS, as well as maintaining a high-efficiency trigger, particularly with low momentum threshold, is critical for any analyses using finalstate muons. The solution undertaken by ATLAS is to replace the existing Small 
Wheel in ATLAS, with the (aptly named) New Small Wheel (NSW).

The NSW (actually two wheels - one for each end-cap) is composed of MicroMegas (MM) and small-strip thin gap chambers (sTGC). The MM will provide precision tracking, while the sTGCs provide fast triggering. The detectors are grouped into multi-layers 4 planes thick, and ordered as sTGC-MM-MM-sTGC. This configuration adds space between the two sTGC multi-layers, which improves angular resolution in triggering. The remainder of this section will discuss the sTGCs, as Carleton University is actively involved in their construction. The MM are certainly deserving of equal consideration, but are beyond the scope of this thesis. The location of the NSW, and an illustration of its importance in triggering is shown in Figure 25.

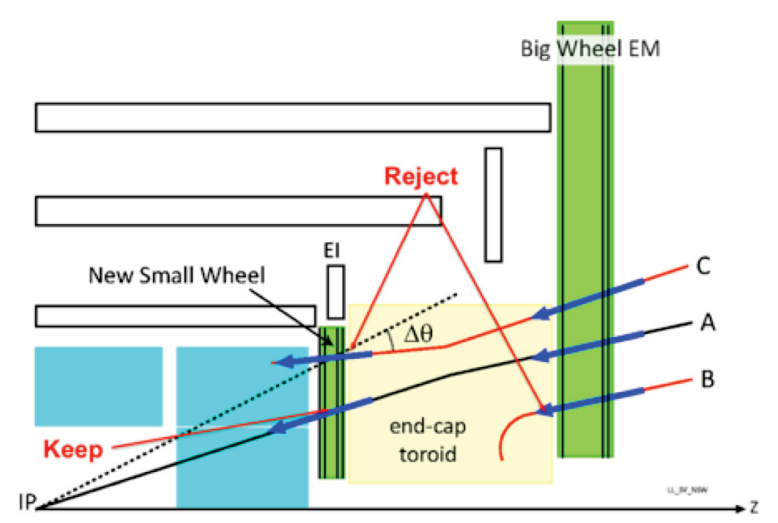

Figure 25: Diagram showing location of the NSW inside one quadrant of the detector. The importance of the NSW for triggering is also depicted: Using the Big Wheel alone, three tracks are observed, A, B, and C. When combining the information from the NSW, tracks B and C can be rejected because they did not originate from near the collision point.

\subsection{1 sTGC}

The sTGC detection mechanism is the same as that of a normal TGC. A plane of parallel wires is held at high voltage inside a thin gas volume, which is sandwiched between two cathodes made up of a resistive graphite epoxy backed on one side by 
large rectangular copper pads, and the other by copper strips orthogonal to the wires. this design is pictured in Figure 26a. When a charged particle passes through the plane of the detector, it ionizes the gas. This ionization deposits charge on the wires, strips, and pads, allowing a measurement of the particle's trajectory in the $x-y$ plane of the detector.

The ATLAS environment is highly demanding, where sTGCs can expect a particle flux of $20 \mathrm{kHz} / \mathrm{cm}^{2} \mathrm{~s}$. The wires are held at $2900 \mathrm{~V}$ to ensure a small drift time, accommodating these high rates. The graphite coating in the existing Small Wheel TGCs is too resistive to allow such operation. Furthermore, the strip spacing in the current TGC is inadequate to provide sufficient granularity and angular resolution for the high luminosity environment of Run 3 and beyond at the LHC. The sTGC uses a strip spacing of $3.2 \mathrm{~mm}$, which will eventually provide a hit resolution of $100 \mu \mathrm{m}$ in the $r$ coordinate (recall the bending plane of the toroids is the $r-z$ ). This high resolution measurement will be used for triggering as well as a complementary measurement to the MM in the offline reconstruction.

Each gas volume is called a gap, or singlet. During assembly, two singlets are paired into a doublet, and then two doublets into a quadruplet. An existing doublet built at Carleton is pictured in Figure 26b. All quadruplets are trapezoidal in shape, with the strips oriented along the parallel sides. Several size variants of the quadruplets are needed, so that together with the MM detectors, they can be assembled into wedges (Figure 26c), and these wedges into wheels (Figure 26d).

The operating principle of the sTGCs as a fast trigger detector is quite simple. Inside one quadruplet, a 3-of- 4 coincidence in the pads is used to identify tracks pointing approximately toward the interaction point. The pads are used to select nearby strips so only a small number of strips need to be read out. These strips provide a track measurement in the bending plane to be used for triggering. If the trigger fires, then all pads, strips, and wires can be read out for optimum track 


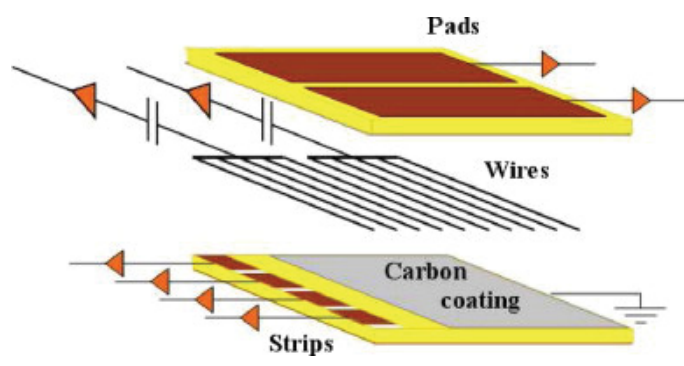

(a) singlet design

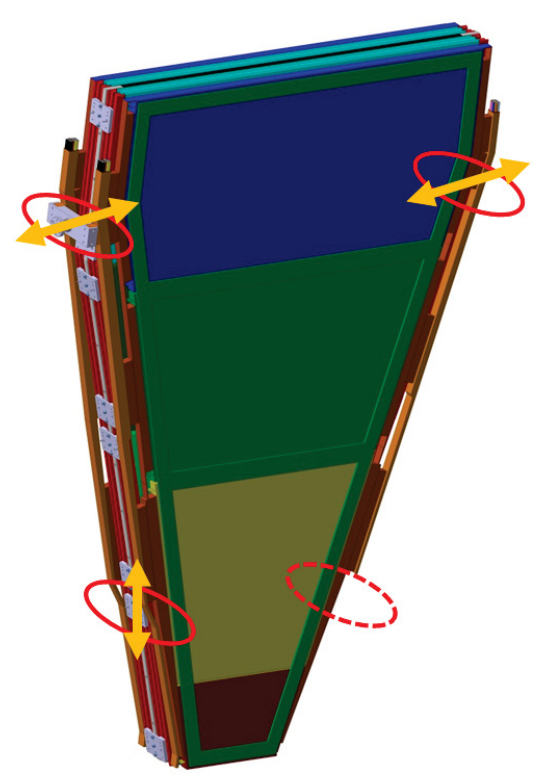

(c) wedge design

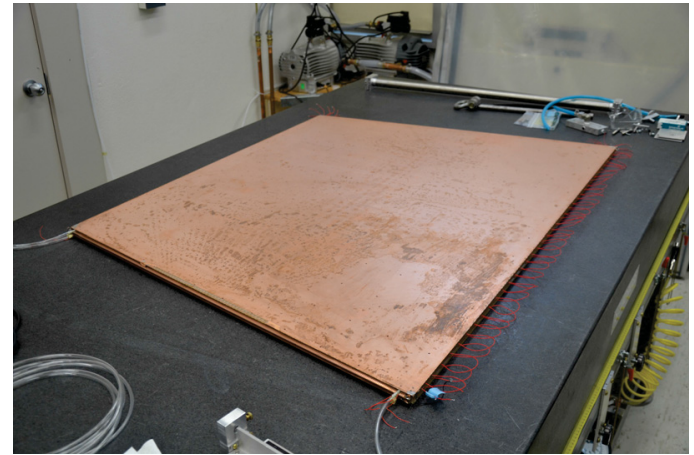

(b) doublet

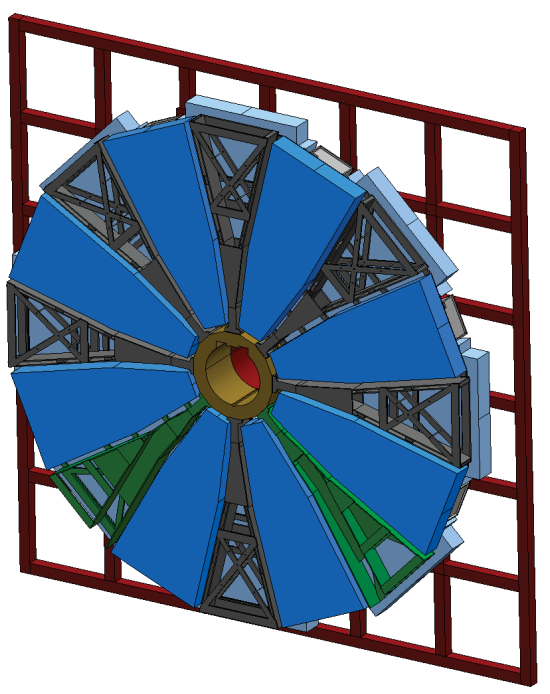

(d) wheel design

Figure 26: Different stages of assembly of sTGC detectors into the final NSW. Figures 26a, 26b, 26d are taken from [50], while Figure 26b is a photo of an actual doublet assembled at Carleton. 
resolution in the offline reconstruction. The nature of the algorithms to combine this information into coordinates for tracking is still under development.

\subsubsection{1 sTGC alignment}

An important aspect of sTGC assembly is alignment. High precision measurement within a detector component is worthless if you do not know where that component is. To do this, a optical locater device is placed on the exposed side of each quadruplet, which locates the quadruplet with respect to the ATLAS global coordinate system. Still unknown however are the relative locations of each singlet, and thus their strips, within the quadruplet.

The relative position of each singlet within the quadruplet is fixed (literally - by glue) at assembly time, in the assembly lab at Carleton. Any movement after this point would be the result of catastrophic failure (components breaking or warping due to mishandling) that would result in a chamber being discarded. Measurement of the alignment within a quad serves a dual purpose: it can be used for quality assurance and control (QA/QC) during assembling, and it can be used in offline reconstruction to correct the location of hits on a gap-by-gap basis. When used for QA/QC, the design specification requires that strips be aligned to their nominal location within $100 \mu \mathrm{m}$. Any misalignment larger than this requires special note and may result in a quadruplet being rejected.

The process of assembling boards with $100 \mu \mathrm{m}$ alignment across dimensions larger than $1 \mathrm{~m}$ is non-trivial. During gluing of a doublet (quadruplet), one gap (doublet) is held in alignment with the other using an alignment jig. The jig is screwed into a flat granite table, assuring that its two precision pins sit vertically, as shown in Figure 27a. Brass inserts built into one of the angled sides on each strip board, shown in Figure 27b, are then pulled flush against the vertical pins, holding the boards in an aligned position during gluing. This whole system is held flat against the granite 
table under a plastic cover with light vacuum of 0.95 atm to maintain planarity over the large area of the detector.

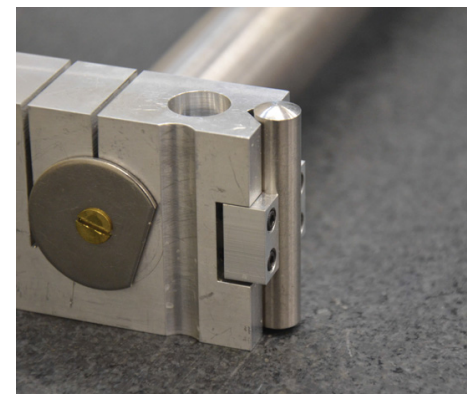

(a) Alignment jig

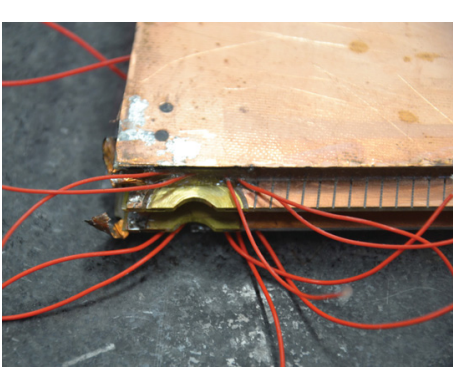

(b) Brass inserts

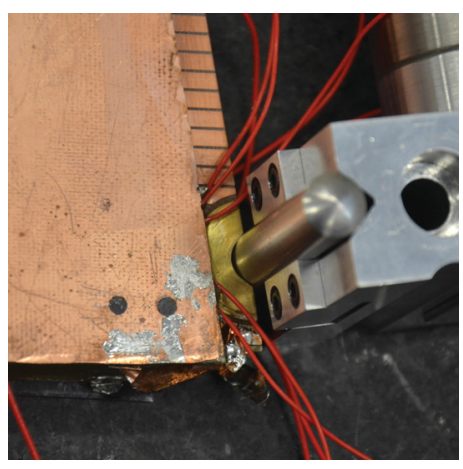

(c) Jig against inserts

Figure 27: During gluing of a doublet (quadruplet) sTGC singlets (doublets) are aligned using built-in brass inserts held firmly against the vertical pins of the alignment jig. Photos taken from Carleton assembly lab.

By design this alignment system should hold the singlets tightly to their nominal respective alignment, but there are a number of reasons the system may fail to do so

- The alignment jig pins may be slightly out of parallel. Because of the roughly 2:1 proportions of the sTGCs, and the position of the alignment features on the short side of the boards, a small misalignment at the location of the pins could be twice as large on the opposite side.

- Any small mote of dust between the pin and the insert may hold the board out of alignment, despite appearing flush to the pin. This is mitigated by checking for electrical continuity between the pin and the insert.

- The alignment may drift while glue is curing, a process which takes several hours. Because the entire assembly is hidden under a plastic bag (used to assure planarity), it is not possible to visually monitor the alignment during this time.

These factors all lead to a need to measure the relative alignment of the gaps 
within doublets and quadruplets, which the author developed. The method can be used while glue is wet and a problematic alignment can be addressed, or after glue is dry as a method of quality control.

A microscope assembly, pictured in Figure 28 was designed to sit on the granite table facing the exposed ends of the strips in the doublets or quadruplets (the other side is obscured by the alignment jig). A $45^{\circ}$ reflecting prism allows a view very low to the surface of the table - an important feature since the bottom set of strips in a doublet is less than $5 \mathrm{~mm}$ from the surface of the table. This microscope assembly is used to record an image of the two sets of strips to measure. An example of such an image is Figure 33a.

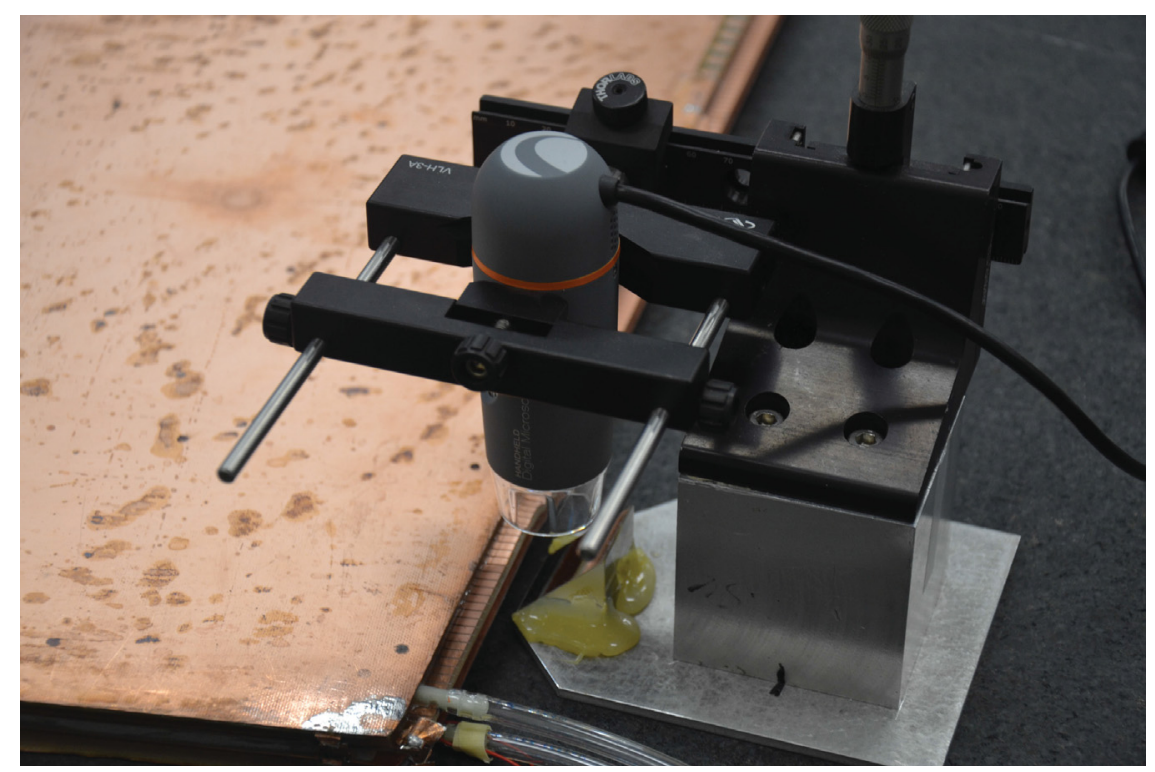

Figure 28: Microscope assembly used for measuring alignment of sTGC doublets and quadruplets.

This image is then opened using the alignment code written by the author. The user (assembly technician) needs only to mark off the corners of the strips in the image, separately for the top gap and the bottom gap. Typically an image can contain several $(4,5,6, \ldots)$ pairs of strips. Once this user input is complete, the alignment algorithm takes over to compute the alignment. 
An idealization of the alignment algorithm for a small number of strips is shown in Figure 29. A description of the algorithm is as follows:

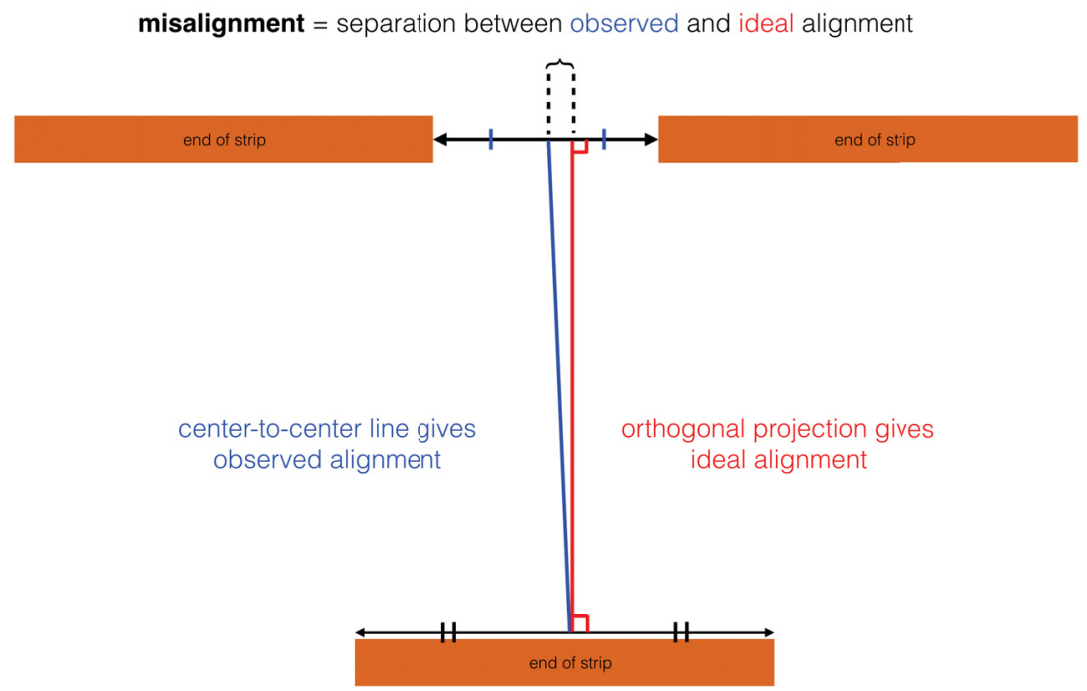

Figure 29: Idealization of algorithm for sTGC alignment measurement. The same algorithm is applied on several pairs of adjacent strips, and the measured misalignments are averaged. Sizes not to scale.

1. Find the image scale: The algorithm makes no assumption about the scale of the image, or the perspective of the camera relative to the strips. The scale is calibrated off the input image and user input (marked strip edges), using the known strip pitch of $3.2 \mathrm{~mm}$. By finding the number of strips selected (based on the number of marked points), a conversion is made between pixel coordinates and real coordinates. This process is depicted in Figure 30.

2. Compute the strip-centers and centers-between-strips: Any pair of adjacent gaps in a doublet or quadruplet have their strips alternatively aligned, that is the strip-centers in one are designed to align with the centers-between-strips in the other (and vice-versa). Collectively these are just called the "centers", and so henceforth this detail of alternatively aligned gaps is implied. 


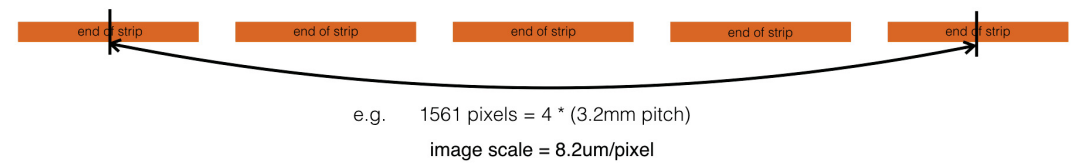

Figure 30: Diagram showing calibration of pixel scale in each image. This process is performed separately and automatically on every image, the numbers printed here serve only as an example.

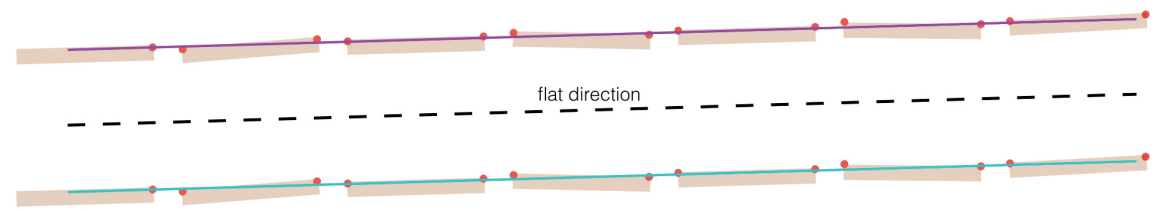

Figure 31: Diagram showing calibration of flat direction in each image. This process is performed separately and automatically on every image. Minor perspective effects, where the two rows of strips are not parallel, are mitigated by averaging the top and bottom slopes.

3. Compute the "flat" direction: A linear $\chi^{2}$ fit is performed separately on the top set of centers and the bottom set of centers. Then the slopes of these two vectors is averaged to find the flat direction. This process is depicted in Figure 31.

4. Compute the perpendiculars: For each center in the bottom gap, a line intersecting this point and perpendicular to the flat direction is computed.

5. Compute the misalignments: Find the distance between each perpendicular and its corresponding center in the top gap, in the flat direction.

6. Compute the final misalignment: A final value and its error is computed by averaging the values from the previous step and finding their standard deviation, respectively.

A reasonable question that may arise, because of the trapezoidal shape of the 


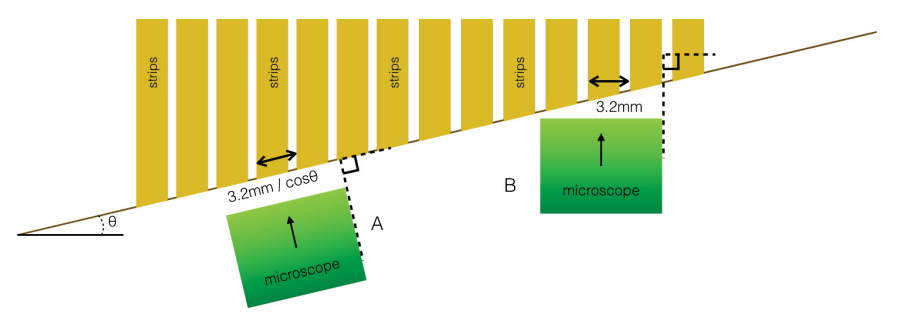

Figure 32: The two possible ways to orient the microscope while measuring misalignment. As explained in the text, configuration B is not possible because of the shallow depth of field of the microscope. Configuration A is the one used throughout the text here.

sTGC chambers, is whether the microscope should be aligned to the cathode board face, or look directly down the strips. These two configurations are depicted in Figure 32, labelled as A and B, respectively. In fact, the configuration B is ruled out because of the shallow depth of field of the microscope: in this configuration it is impossible to have more than one strip (or even one complete strip) in focus simultaneously. This leaves as the only option to face the edge of the cathode board, configuration A in the diagram. In this case, a further consideration is whether the self-calibration of the pixel scale is correct, given the observed strip pitch will be affected by the trapezoid angle $\theta$, such that when facing the cathode board edge, $p^{\prime}=p / \cos \theta$ (where $p$ represents the true strip pitch, and $p^{\prime}$ represents the observed pitch). Fortunately, any misalignments of the boards perpendicular to the strip direction will also be scaled by this same amount, so when computing the misalignment the self-calibration nulls the effect of this term. The corollary is that the alignment algorithm is robust to any facing angle, it does not require the microscope to be precisely oriented to produce accurate alignment measurements.

The actual code itself produces a visualization of its work-flow, an example of which is shown in Figure 33b; the final alignment measured is printed on the image. The measurement uncertainty arises from uniformity in strip etching, microscope focus, image resolution, and user error in marking strip corners. Each of these sources 
affects the value of the individual misalignments measured (step 5), and so the standard deviation of these values captures all sources of uncertainty together. The error as computed from the the standard deviation is confirmed by repeated measurement of the same set of strips producing values all within this error. The overall precision of this technique is around $25 \mu \mathrm{m}$, significantly better than the design specification for the sTGCs of $100 \mu \mathrm{m}$.

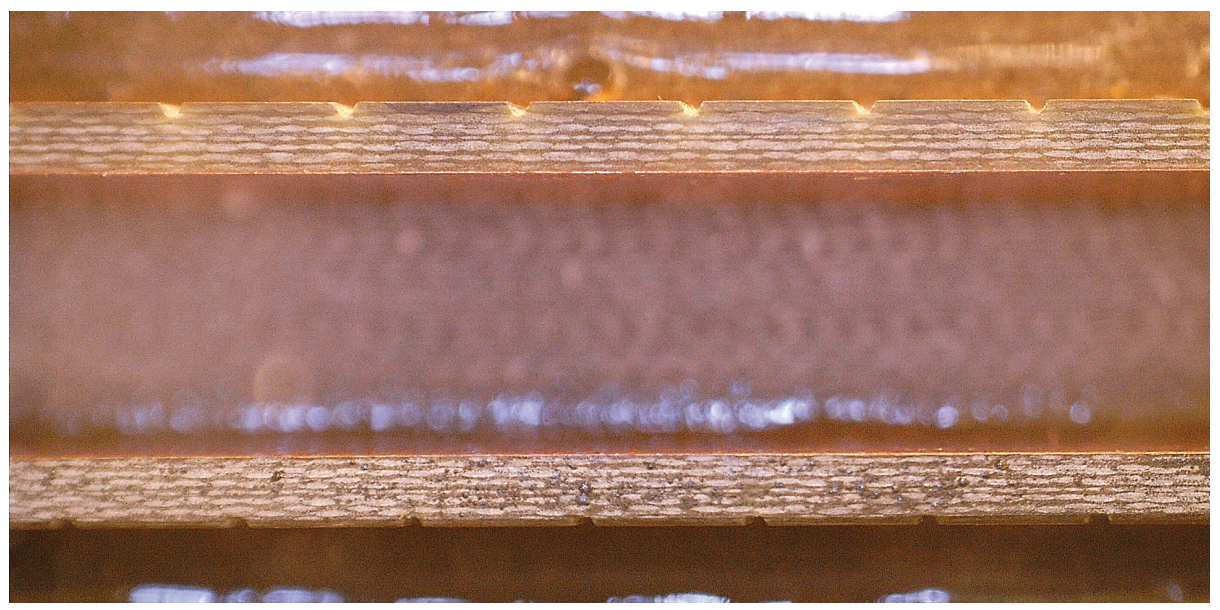

(a) Image from microscope

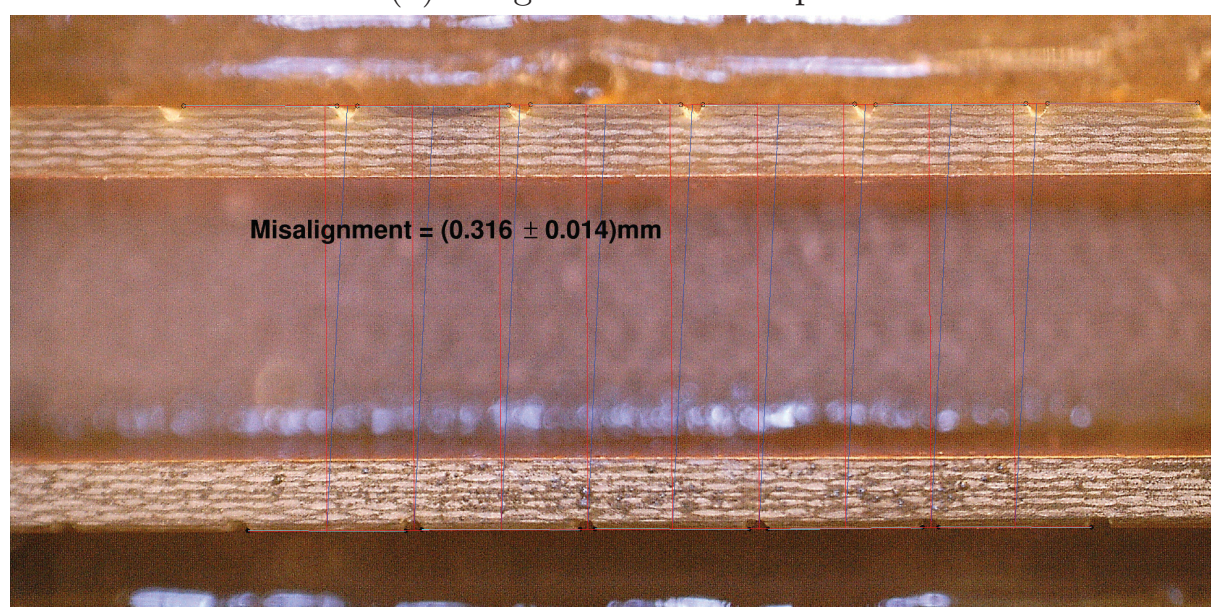

(b) Image after processing

Figure 33: Example of image from an early doublet built at Carleton showing significant misalignment. Shown at the image directly from the microscope (a), and the image after processing (b). Both images crop off useless portions at the top and bottom. 
In order to describe the overall alignment of one quadruplet, multiple measurements are needed. Measuring the gap-to-gap misalignment near each of the four corners is sufficient to give a complete picture of the relative alignment of these two gaps, under the assumption that the relative alignments can be interpolated between these measurements. In the quadruplet, the relative alignment of each gap should be with respect to one single reference gap which will eventually have the precision optical alignment device to tie it into the ATLAS global coordinate system. Call these final misalignments $a_{12}^{i}, a_{13}^{i}, a_{14}^{i}$, where $a_{x y}^{i}$ represents the alignment of gap $y$ with respect to gap $x$, as measured at point $i$.

Because of geometrical constraints, the alignment microscope is only able to capture a field of view approximately $25 \mathrm{~mm}$ tall, limiting the measurement to adjacent strip boards inside a quadruplet. This necessitates calculating the desired misalignments from those that can actually be measured. Figure 34 indicates which values are calculated and measured, as well as the locations of the measurements. The final misalignments are calculated (except $a_{12}^{i}$, which requires no calculation) as follows

$$
\begin{aligned}
& a_{12}^{i}=a_{12}^{i}, \\
& a_{13}^{i}=a_{12}^{i}+a_{23}^{i}, \\
& a_{14}^{i}=a_{12}^{i}+a_{23}^{i}+a_{34}^{i} .
\end{aligned}
$$

Assuring and measuring the alignment of sTGCs at assembly time is one of many details to be understood during their construction and testing. It is a small detail, but it can have resounding effects. Eventually when the NSW are installed in ATLAS, precision alignment (or misalignment that can be corrected for) assures precision hit resolution. Hit resolution aids in efficient triggering and reconstruction of muons, discussed in the next chapter. Precision reconstruction in turn improves resolution of measurements such as the Higgs boson mass in final states containing muons. Though 


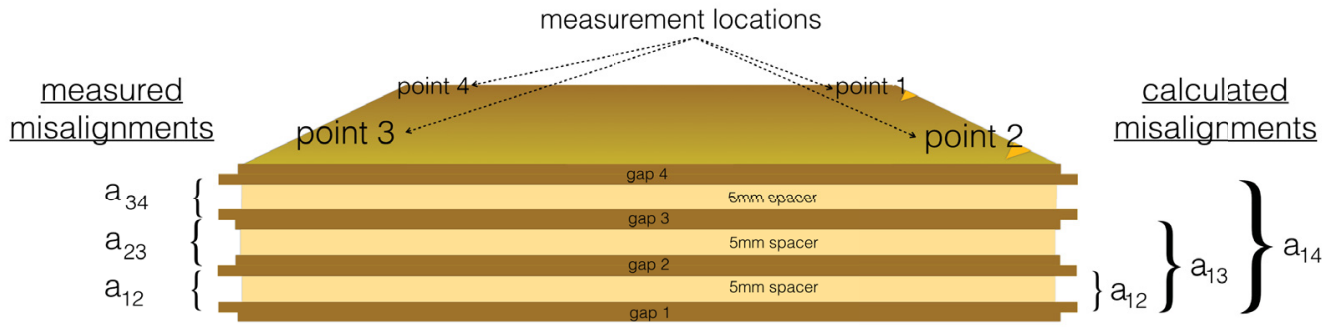

Figure 34: Diagram indicating the four corners used for alignment measurements. At each corner, three measurements are made as indicated, and the remaining relative alignments are calculated from them.

these particular alignment measurements in the sTGCs have no bearing on the analyses discussed in the remaining chapters of this thesis, they will have a consequence for analyses in 2019 and beyond. 


\section{Chapter 4}

\section{Reconstruction}

Data from the ATLAS detector arrives in the form of hits in tracking detectors and energy deposits in various calorimeter cells. The interpretation of this information into useful "physics objects" to be used in analyses is called reconstruction, and is the subject of this chapter.

This chapter begins by differentiating simulated data and real data in Section 4.1, as simulated samples are used throughout the proceeding chapters. Next, Section 4.2 delves into the reconstruction, explaining important quantities we measure, and how they can be used to understand what happened in a collision. The sections that follow then describe the specific algorithms used to reconstruct the physics objects used in this thesis. More emphasis is placed on those prominent later in this thesis, and in particular to the author's own contributions in Section 4.4.1 and Section 4.5.1.

\subsection{Data and Simulation}

Simulated data are broadly used in ATLAS as a tool to design components of the detector, develop software, and prepare physics analyses [51]. Using simulated data permits many things not possible in real data ${ }^{1}$. The subset of these which are useful

\footnotetext{
${ }^{1}$ The phrase "real data" is used in this section for emphasis, though most of this thesis will just use "data".
} 
to this thesis are :

- Rare processes, such as Higgs events, can be simulated with far more statistics than can be collected in real data. Simulating these processes means we know what to look for in real data. The simulated dataset of a specific process is called a simulated sample.

- Processes can be simulated with different parameters of nature, e.g. values of $m_{H}$, which can be in turn used to measure the parameters in real data.

- In simulated data, the true particle kinematics are known, but not in real data. With this information in hand, we can compare reconstruction algorithms which attempt to measure these kinematics.

All simulated samples used in this thesis are generated by the Monte Carlo (MC) method [52]. Different generators can be used to simulate different physical processes. The plethora of generated particles, with their respective kinematics, are then passed through the digital model of the ATLAS detector using the GEANT4 framework [53] to simulate their interaction with the detector. The detector response is then digitized, and the regular ATLAS reconstruction algorithms can be ran on these simulated data [51]. Regardless of how many events are simulated, the generated events can be re-weighted to the SM cross sections (or some other cross section, if a non-SM process is being simulated) and some integrated luminosity, so that the large number of simulated events will have a total weight equal to the number of events expected from that process in real data (e.g. 1,000,000 simulated events may be given a total weight of 8.53). Each of the simulated samples are further re-weighted in various ways to correct the simulations to better reflect real data, since neither the generators nor the model of ATLAS are perfect.

The details of the MC generators - why one is used for some samples but not others for instance - is beyond the scope of this thesis. They are included below for 
completeness only, and the reader is invited to consult the references inline.

The $H \rightarrow Z Z^{(*)} \rightarrow 4 \ell$ signal samples are predominantly generated using the POWHEG MC event generator. POWHEG calculates ggH [54] and VBFH [55] production at NNLO, and a separate weighting is applied to make the Higgs $p_{T}$ spectrum match the latest theoretical calculation as prescribed by [56]. This generator is interfaced to PYTHIA8 [57] for simulation of showering and particle hadronization, and to EVTGEN [58] for the the simulation of B-hadron decays. Events produced by VH production are generated using PYTHIA8, and ttH and bbH production are generated using MadGraph5_aMC@NLO [59], interfaced to Herwig++ [60] and PYTHIA8, respectively. The cross section for ggF is calculated to N3LO QCD + NLO EW. For VBF and $\mathrm{VH}$ it is calculated to NNLO QCD + NLO EW. For ttH it is calculated to NLO QCD + NLO EW. For bbH it is calculated to NNLO or NLO, under different flavour schemes. The values of the cross sections used for $m_{H}=125 \mathrm{GeV}$ were reported in Section 2.3.2, and sourced from [21].

Using the above generators, 500,000 ggF events, 300,000 VBF events, and 200,000 $\mathrm{ttH}$ and bbH events, where $H \rightarrow 4 \ell(=e, \mu)$ were generated at $m_{H}$ values of 120,122,124,125,126,128,130 GeV. For VH, 200,000 ZH and WH events (each), where $H \rightarrow 4 \ell(=e, \mu, \tau)$ were generated at $m_{H}$ values of $124,125,126 \mathrm{GeV}$.

The sources of background entering this thesis are described later in Chapter 6 . The dominant source is from $q \bar{q} \rightarrow Z Z^{(*)}$, for which samples are generated using POWHEG interfaced to PYTHIA8 and EVTGEN as with the signal. For this background, $10,000,000$ events with $4 \ell=e, \mu$ were generated in the mass window $100-150 \mathrm{GeV}$, and 4,000,000 (not statistically independent) events were generated with no mass window cut. Background samples from $g g \rightarrow Z Z^{(*)}$ are generated in the same way, with 1,000,000 total events generated.

Backgrounds from tri-boson production - ZZZ, WZZ, and WWZ - are generated using SHERPA [61]. For ZZZ, 70,000 events with at least 4 real leptons were generated 
for each. For WZZ, and WWZ, 50,000 and 60,000 events were generated, respectively. For background from $t \bar{t} Z$, SHERPA was used to generate 1,500,000 events (this sample was used by other analysis groups - only a small fraction of these events decay via $t \bar{t} \rightarrow W b W b(W W \rightarrow \ell \nu \ell \nu)$ in order to be relevant).

The background from Z+Jets is generated using SHERPA. Many samples were generated separately for $\mathrm{Z}+1,2,3$,4Jets, filtered into $\mathrm{Z}+\mathrm{b}$-jets, $\mathrm{Z}+\mathrm{c}$-jets, and $\mathrm{Z}+$ light jets according to the true origin of the quark-initiated jets. Alternative samples used for cross checks were generated using POWHEG and MADGRAPH, both interfaced to PYTHIA8 and EVTGEN. As Z+Jets samples are of broad use within ATLAS, huge numbers (10s of millions) of events were generated overall, though the vast majority do not enter the analysis in this thesis. Events from $t \bar{t}$ were generated using POWHEG with PYTHIA6 [62] for parton shower and hadronization, with PHOTOS [63] for QED processes, and with TAUOLA [64] for simulation of $\tau$ decays. Finally, WZ background events were generated using POWHEG interfaced to PYTHIA8 and EVTGEN.

A few other simulated samples are also used only in the current chapter for testing certain aspects of the reconstruction, and will be mentioned as needed.

\subsection{Kinematic formulae}

When a particle is produced in a collision (in data or simulation), it has some real kinematics, energy and momentum, that we call the truth, and that we attempt to measure through reconstruction. How well the reco matches the truth is the ultimate measure of how well reconstruction is performing, and is something that can be quantified using simulated data, where the truth is known. The difference between reco and truth comes back to play a major role in Chapter 7. In this section, the formulae apply equally to either reco or truth quantities.

We can describe any particle's kinematics by its four-momentum, 


$$
\begin{aligned}
p & =(E, \vec{p}), \\
& =\left(E, p_{x}, p_{y}, p_{z}\right),
\end{aligned}
$$

where $E$ is the energy, and $\vec{p}$ is the classical three-momentum. A particle's mass can be calculated from the four-momentum²:

$$
m^{2}=p \cdot p=p^{2}=E^{2}-|\vec{p}|^{2} .
$$

Note the minus sign appearing in this expression, but not, for example, in the classical momentum dot product: $\vec{p} \cdot \vec{p}=p_{x}^{2}+p_{y}^{2}+p_{z}^{2}$. The value of the four-momentum always depends on the reference frame of the observer, and this minus sign appears as a consequence of the transformation properties under changes of reference frame, called Lorentz boosts. To change to a frame moving with $\operatorname{speed}^{3} \beta$, which corresponds to a Lorentz boost factor $\gamma=1 / \sqrt{1-\beta^{2}}$, the four-momentum transforms as

$$
\begin{aligned}
& E \rightarrow \gamma\left(E+\beta p_{z}\right), \\
& p_{x} \rightarrow p_{x}, \\
& p_{y} \rightarrow p_{y}, \\
& p_{z} \rightarrow \gamma\left(p_{z}+\beta E\right),
\end{aligned}
$$

In contrast, the dot products of four-momenta, in any combination, do not depend on reference frame; for this reason they are called Lorentz Invariants, and the particle mass $(m)$ is more precisely called the invariant mass, also the rest mass because it is always equivalent to the value in the rest frame. The corollary is that even though collisions do not occur in the rest frame of ATLAS, for reasons explained in Section 3.2.1.1, the invariant mass of any particle will be the same as if it did.

Conservation of energy and momentum dictate that four-momentum must be

\footnotetext{
${ }^{2}$ Note that this is the famous Einstein mass-energy equivalence relationship $E=m c^{2}$, except here expressed in a moving frame where $\vec{p} \neq 0$, and using a system of units where $c=1$

${ }^{3}$ Note that speed ranges from 0 to 1 , with a value 1 meaning the speed of light
} 
conserved through time. The useful consequence for ATLAS is that when an unstable particle decays and we measure only its decay products, we can access the kinematics of the initial particle without ever directly observing it.

For example, in a $Z \rightarrow \mu \mu$ decay,

$$
\begin{aligned}
p_{Z} & =p_{\mu_{1} \mu_{2}}=p_{\mu_{1}}+p_{\mu_{2}}, \\
m_{\mu_{1} \mu_{2}}^{2} & =\left(p_{\mu_{1}}+p_{\mu_{2}}\right)^{2}, \\
& =p_{\mu_{1}}^{2}+p_{\mu_{2}}^{2}+2 p_{\mu_{1}} \cdot p_{\mu_{2}}, \\
& =m_{\mu_{1}}^{2}+m_{\mu_{2}}^{2}+2\left(E_{\mu_{1}} E_{\mu_{2}}-\vec{p}_{\mu_{1}} \cdot \vec{p}_{\mu_{2}}\right) .
\end{aligned}
$$

At the high energies at the LHC, most final state particles have a relatively small invariant mass, e.g. $m_{\mu} \ll E_{\mu}$, and so the terms $m_{\mu}^{2}$ can be neglected. If we are neglecting $m_{\mu}$, then (35) tells us that $|\vec{p}|=E$, and so we find

$$
m_{\mu_{1} \mu_{2}}^{2}=2 E_{\mu_{1}} E_{\mu_{2}}\left(1-\cos \theta_{12}\right),
$$

where $\theta_{12}$ is the relative angle between the two muons. These mathematics extend easily to a more complex decay, such as $H \rightarrow Z Z^{(*)} \rightarrow 4 \ell$, which will be exploited in Chapter 7 .

Returning to the four-momenta of individual final state particles, we can establish a few other useful formulae. The transverse momentum of a particle, defined by $p_{T}=\sqrt{p_{x}^{2}+p_{y}^{2}}$ is a useful quantity because HS events generally produce particles transverse to the beam. Additionally, the $p_{T}$ is also invariant under boosts along the $z$-axis, so it is a useful quantity in which to compare experimental observation with theory (for example, in a differential cross section measurement like [65]). Similarly the transverse energy is defined by $E_{T}=\sqrt{p_{T}^{2}-m^{2}}$. 
A more intuitive way to express a particle's four-momentum is in cylindrical coordinates:

$$
p=(E, y, \phi, m)
$$

where $y$ is the rapidity defined in Section 3.2.1.1. For light particles (including electrons and muons) using the pseudo-rapidity, $\eta$, is usually more convenient. Some expressions can be used to relate the quantities presented so far:

$$
E=E_{T} \cosh y, \quad p_{z}=E_{T} \sinh y, \quad p_{x}=p_{T} \cos \phi, \quad p_{y}=p_{T} \sin \phi,
$$

or, when $m \ll E$, the expressions become $\left(p_{x}, p_{y}\right.$ are unchanged)

$$
E=|\vec{p}|=p_{T} \cosh \eta, \quad p_{z}=p_{T} \sinh \eta .
$$

As explained in Section 3.2.1.1, separations in $y$ are invariant under boosts along the beamline. Using this, we can define a boost-invariant angular separation between two final state particles as

$$
\Delta R=\sqrt{\Delta \phi^{2}+\Delta \eta^{2}} .
$$

Finally, some useful relations involving the speed $\beta$, the Lorentz boost factor $\gamma$, and the lifetime $\tau$, of particles are

$$
\gamma=E / m, \quad \beta=|\vec{p}| / E=\sqrt{1-1 / \gamma^{2}}, \quad \tau=\gamma \cdot \tau_{\text {rest }} .
$$

These expressions are useful in understanding the behaviour of particles: how fast are they travelling? will they decay before we observe them? will they pass through the whole detector before decaying? These questions can be answered quickly for a few example particles, shown in Table 5. Note that these simple expressions can be used to derive the canonical description of the LHC as "protons colliding at $99.9999988 \%$ 
light speed".

Table 5: A few example particles, with their Lorentz boost $(\gamma)$ and speed $(\beta)$ calculated. Also calculated is the lifetime in the ATLAS detector frame, and the travel distance before decay, $c \beta \tau$. All particles use masses and decay widths from [16], except the Higgs boson for which a SM Higgs of $m_{H}=125 \mathrm{GeV}$ is used.

\begin{tabular}{lcccc}
\hline Particle and momentum & $\gamma$ & $\beta$ & $\tau$ & $c \beta \tau$ \\
\hline $50 \mathrm{GeV}$ muon & 473 & 0.9999978 & $1 \mathrm{~ms}$ & $312 \mathrm{~km}$ \\
$20 \mathrm{GeV} \mathrm{B} B^{+}$ & 3.91 & 0.967 & $6 \mathrm{ps}$ & $1.93 \mathrm{~mm}$ \\
$20 \mathrm{GeV} Z$ boson & 1.02 & 0.214 & $3 \times 10^{-7}$ as & $79.9 \mathrm{~nm}$ \\
$20 \mathrm{GeV}$ Higgs boson & 1.01 & 0.158 & $2 \times 10^{-4}$ as & $45.6 \mu \mathrm{m}$ \\
$6.5 \mathrm{TeV}$ proton & 6600 & 0.999999988 & N.A. & N.A. \\
\hline
\end{tabular}

Though the above kinematic description is most useful for physics analyses, these are not always the quantities directly measured during reconstruction. A general outline to keep in mind is that calorimeters directly measure energy $E$, and coarse directional information $\theta, \phi$, while tracking detectors directly measure the parameters than define the particle trajectory: $q / p$ (charge-to-momentum ratio), $\theta, \phi, d_{0}$ (transverse impact parameter), $z_{0}$ (longitudinal impact parameter). These measured quantities are used to derive the four-momenta discussed above. The detector signature is also used to identify the species of particle, and so its known mass (e.g. for electrons $m_{e}=511 \mathrm{keV}$ ) can be used to construct the four-momentum.

\subsection{Trigger}

The first stage of ATLAS reconstruction is the Trigger. The Trigger decides which events to keep for further analysis, and which events to discard. Because of the enormously high event rate at the LHC, it is impossible to keep all events. Events which are kept are said to fire the trigger. Triggering happens online, in real-time 
as collisions happen, while the remainder of the reconstruction happens offline, and normally happens in the following days as data is transferred to the various computing sites which comprise the Worldwide LHC Computing Grid (WLCG) [66].

The trigger proceeds in two stages, called Level-1 (L1), and high-level trigger (HLT). The L1 stage is entirely implemented in hardware; it uses relatively simple vetoes to reject a huge number if uninteresting events. The HLT is implemented in software, and uses complex computations to identify the best remaining events. The approximate rate $^{4}$ of events passing each trigger under nominal running conditions is shown in Table 6.

Table 6: Expected average trigger rates for Run 2, from [67].

\begin{tabular}{cc}
\hline Level & rate \\
\hline no trigger & $1.6 \mathrm{GHz}$ \\
L1 & $100 \mathrm{kHz}$ \\
HLT & $1 \mathrm{kHz}$ \\
\hline
\end{tabular}

The software running at the trigger level exists at a balance point between providing efficient trigger (capturing all the events with exciting physics), and fast triggering (being fast enough to process all events it receives). At the HLT level, the reconstruction algorithm is only slightly pared down from the offline reconstruction as it is described in the following sections.

This triggering procedure causes a certain bias in the data: triggers introduce specific search criteria, and so the data which passes the trigger already has a certain degree of model-dependence. To protect against this, ATLAS also records and analyses so-called minimum-bias events [68] - events triggered entirely at random with no requirements so as to be completely model-independent.

\footnotetext{
${ }^{4}$ Through the course of a collision run at the LHC, the two beams are depleted (protons are collided or lost by other means), and so the trigger rate slowly falls over time. If the rate falls a certain threshold, it becomes more worthwhile to dump the remaining beams and refill, rather than continue colliding at a low rate.
} 


\subsection{Electrons and photons}

Electrons and photons are described together because their reconstruction [69][70] is very closely tied.

The reconstruction starts by building clusters out of energy deposits in the ECAL. Second, ID tracks are constructed using a combination of pattern-recognition tools, and performing a helical fit of the hits on each identified track, with the bending direction of the helix determining the track's charge. The fit assumes a charged pion hypothesis as a baseline for energy loss. As the fit extrapolates the particle trajectory layer-by-layer through the ID, it allows a Gaussian uncertainty on the track parameters, which is re-estimated at each step. This technique is called a Kalman filter.

The clusters in the ECAL are matched, if possible, with ID tracks. Electrons and photons are identified in the following ways

- The cluster matches no tracks: the particle is identified as an "unconverted photon", meaning it was produced in the collision event as a photon, and remained so until it reached the ECAL.

- The cluster matches a pair of oppositely-charged tracks: the particle is identified as a "converted photon", meaning it was produced in the collision event as a photon, but converted to an $e^{+} e^{-}$pair before (or while) it crossed the ID. A converted photon can also be identified by a single track if it has no hits in the innermost Pixel layers.

- The cluster matches a single track: this particle is identified as an electron (or positron). 


\subsubsection{Gaussian Sum Filter}

As electrons traverse the material of the ID, they radiate energy (in the form of photons) by bremsstrahlung (translated as "braking radiation", often simply called brem). The fraction of energy remaining, $z=E_{f} / E_{i}$, after traversing a thickness of material $t=x / X_{0}$ (normalized to radiation lengths) follows a Bethe-Heitler $(\mathrm{BH})$ distribution [71],

$$
p_{B H}(z)=\frac{(-\ln (z))^{\frac{t}{\ln 2}-1}}{\Gamma\left(\frac{t}{\ln 2}\right)}, \quad 0<z<1,
$$

with some higher order corrections ${ }^{5}$.

Because the BH distribution is highly non-Gaussian, the approximation used by the Kalman Filter can fail to account for the extreme tails of energy loss. When the electron radiates a large fraction of its energy into a brem photon (an uncommon, but not entirely rare occurrence), its trajectory will exhibit a strong kink. Because the Kalman Filter uses a Gaussian description of the stochastic energy loss processes of electrons, it fails to account for these uncommon-but-not-entirely-rare events, and so electrons can be lost or else poorly reconstructed (e.g. bad momentum estimate).

To mitigate these problems, electrons are re-fit with a dedicated procedure called the Gaussian Sum Filter (GSF) [72][73]. The GSF approximates the BH as a weighted sum of Gaussian distributions,

$$
p_{G S F}(z)=\sum_{i=0}^{N_{G}} w_{i} G\left(z ; \mu_{i}, \sigma_{i}\right)
$$

The particular sum of Gaussians used was optimized in [73] by minimizing the cumulative distribution function (CDF) distance, defined by

$$
D_{C D F}=\int_{-\infty}^{+\infty}\left|p_{B H}(z)-p_{G S F}(z)\right| d z
$$

\footnotetext{
${ }^{5}$ At the energies we are concerned with, $7 \mathrm{GeV}$ and above, the most noteworthy contribution is from multiple scattering
} 
Figure 35 compares the six-Gaussian sum $p_{G S F}(z)$ with $p_{B H}(z)$ optimized by this metric.

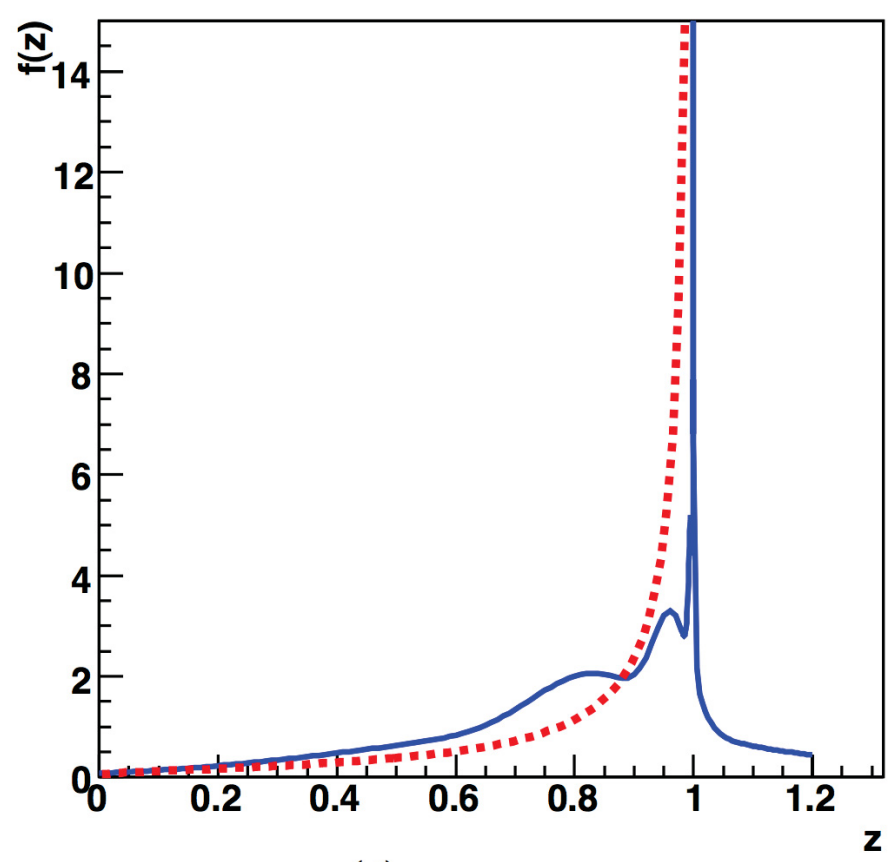

Figure 35: Comparison of Gaussian sum approximation (solid, blue) with $N_{G}=6$ Gaussians to the Bethe-Heitler distribution (dashed, red), shown for $t=0.02$. Taken from [73], Figure 5.2.

With this Gaussian-sum approximation in hand, the track fitting can proceed as many weighted Kalman Filters in parallel. At each step, the track parameters' uncertainties are represented by a sum of multi-dimensional Gaussians. The dimension is equivalent to the number of track parameters, in this case five ${ }^{6}$. As the track is extrapolated to the next measurement, it passes through some material which causes an increase in the uncertainties according to the $\mathrm{BH}$ distribution. By describing this distribution as a sum of $N_{G}$ Gaussians, the GSF allows the complex distribution of uncertainties to be propagated through material. At each propagation step, the number of Gaussians needed to describe the uncertainties increases by a factor $N_{G}$, as each initial Gaussian uncertainty is propagated through the Gaussian sum describing

\footnotetext{
${ }^{6} \mathrm{As}$ mentioned at the top of Section 4.2 they are $q / p, \theta, \phi, d_{0}, z_{0}$
} 
the $\mathrm{BH}$ distribution. As the propagation reaches each measurement, the track parameters can be updated and the uncertainties re-estimated. This process is illustrated in Figure 36.

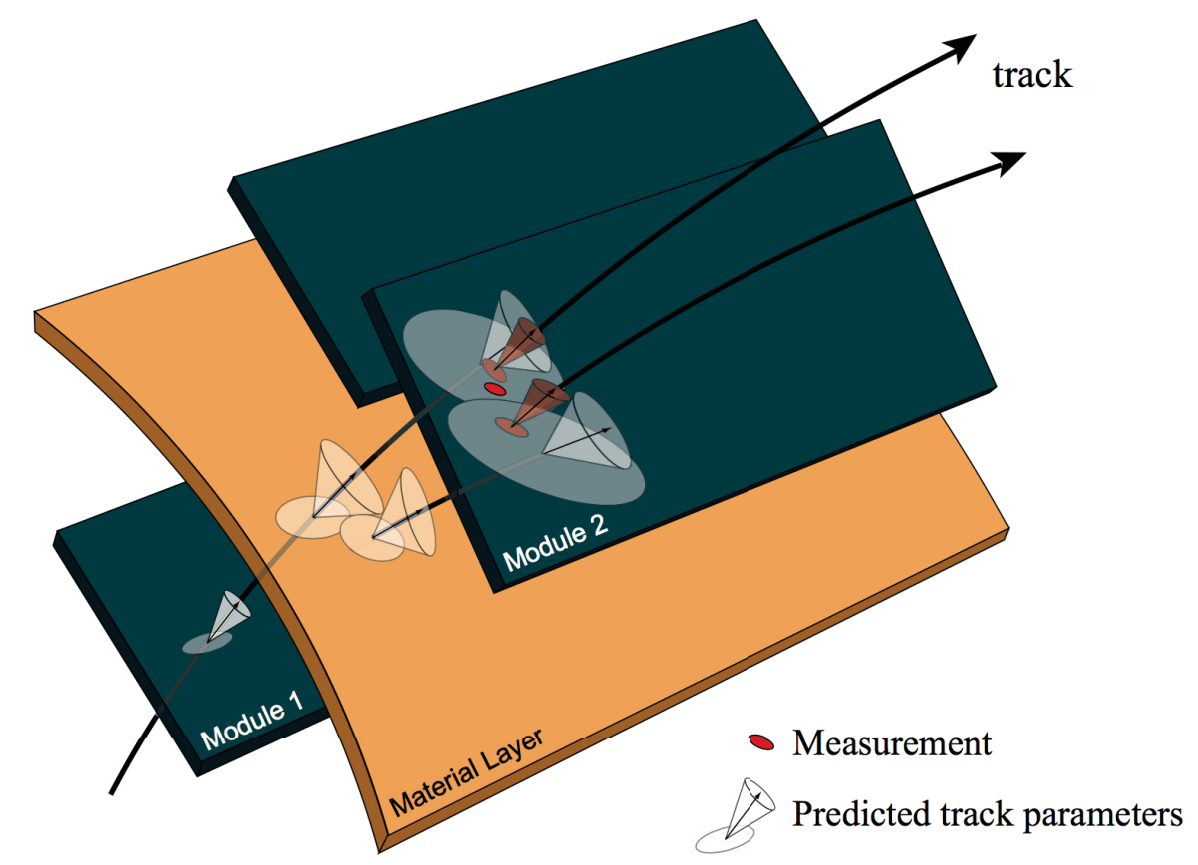

Figure 36: Illustration of the GSF error propagation for a simplified example with $N_{G}=2$ Gaussians. The initial track parameters, on Module 1, have their initial uncertainties described by a single Gaussian. As the track is propagated through the Material Layer, the sum of Gaussians describing the energy loss is propagated through the track parameter uncertainties, which are thereafter described by a sum of two weighted Gaussians. When the track reaches the measurement on Module 2, the same process is repeated for each of these two Gaussians, leaving four Gaussians. As a final step (not shown), the parameters would be re-estimated according the the actual measurement on this Module. Taken from [71], Figure 4.4

The astute observer will see a potential danger with this approach: the number of Gaussians required explodes exponentially, as at step $k$, the number of Gaussians would be $\left(N_{G}\right)^{k}$. To prevent this catastrophe, after each propagation step the GSF reduces the number of Gaussians back to a manageable number. This process is a necessary approximation: the full details of the uncertainties are most accurately encoded in some huge number of Gaussians, but if most of that same information 
can be encoded in a smaller number of Gaussians (call it the "Number of Reduced Components", or $N_{r e d}$ ) such that the uncertainties are still accurate, then the same result can be achieved in a more computationally feasible way. To understand how this reduction is done, we must establish some notation, which is adapted from [73].

The distribution of errors, $\boldsymbol{\epsilon}$, may be described in generic form as a sum of $n$ multi-dimensional Gaussians:

$$
p(\boldsymbol{\epsilon})=\sum_{i=1}^{n} w_{i} G\left(\boldsymbol{\epsilon} ; \boldsymbol{\mu}_{i}, \boldsymbol{V}_{i}\right)
$$

where $\boldsymbol{\mu}_{i}$, and $\boldsymbol{V}_{i}$ are the mean and covariance matrix of the $d$-dimensional Gaussian $i$, defined by

$$
G(\boldsymbol{\epsilon} ; \boldsymbol{\mu}, \boldsymbol{V})=\frac{1}{(2 \pi)^{d / 2} \sqrt{\operatorname{det}(\boldsymbol{V})}} \exp \left(-\frac{1}{2}(\boldsymbol{\epsilon}-\boldsymbol{\mu})^{T} \boldsymbol{V}^{-1}(\boldsymbol{\epsilon}-\boldsymbol{\mu})\right) .
$$

If the sum is too large, that is if $n>N_{\text {red }}$, then the goal is to reduce it to a manageable number. To do this, we identify "similar" Gaussians: if the information from two Gaussians is redundant or nearly redundant, it is obvious those should be the first to go. In practice, a scalar distance is computed between each pair of Gaussians in the sum, called the Kullback-Leibler distance [73][74]. For Gaussians $i=a, b$, this distance is

$$
D_{\mathrm{KL}}(a, b)=\operatorname{tr}\left[\left(\boldsymbol{V}_{a}-\boldsymbol{V}_{b}\right)\left(\boldsymbol{V}_{b}^{-1}-\boldsymbol{V}_{a}^{-1}\right)\right]+\left(\left(\boldsymbol{\mu}_{a}-\boldsymbol{\mu}_{b}\right)^{T}\left(\boldsymbol{V}_{a}^{-1}+\boldsymbol{V}_{b}^{-1}\right)\left(\boldsymbol{\mu}_{a}-\boldsymbol{\mu}_{b}\right)\right) .
$$

When the pair of Gaussians with the smallest $D_{\mathrm{KL}}$ are found in the sum of (47), 
they are replaced by a single Gaussian with parameters

$$
\begin{aligned}
w & =w_{a}+w_{b}, \\
\boldsymbol{\mu} & =\frac{1}{w_{a}+w_{b}}\left(w_{a} \boldsymbol{\mu}_{a}+w_{b} \boldsymbol{\mu}_{b}\right), \\
\boldsymbol{V} & =\frac{1}{w_{a}+w_{b}}\left(w_{a} \boldsymbol{V}_{a}+w_{b} \boldsymbol{V}_{b}\right)+\frac{w_{a} w_{b}}{\left(w_{a}+w_{b}\right)^{2}}\left(\boldsymbol{\mu}_{a}-\boldsymbol{\mu}_{b}\right)\left(\boldsymbol{\mu}_{a}-\boldsymbol{\mu}_{b}\right)^{T} .
\end{aligned}
$$

This merged Gaussian preserves the total weight, the mean (1st moment), and the covariance (2nd moment) of their combined distribution. The terms involved are mostly obvious (e.g. the combined mean is the weighted sum of the individual means), except for the final term of $\boldsymbol{V}$, which is the contribution to the combined variance introduced by the difference between means of the two Gaussians. Once this combined Gaussian replaces Gaussians $a, b$ in the sum, new $D_{K L}$ are computed, and the process repeats iteratively until $n \leq N_{\text {red }}$.

\section{Tuning of $N_{\text {red }}$}

One study undertaken by the author was to optimize the configuration in the GSF of the parameter $N_{\text {red }}$. Too small a value results in poor description of the energy loss and thus poor momentum resolution, while too large a value results in a significant CPU time penalty. The initial value of $N_{\text {red }}=12$ was established in 2006 [73], and this study sought to verify this value was still optimal, considering the volume of changes to other aspects of the ID track fitting code since that time.

To evaluate the quality of track reconstruction, a sample of 10,000 simulated electrons with $E_{T}=25 \mathrm{GeV}$ was used. These electrons were reconstructed by the GSF under different configurations of $N_{\text {red }}$. The relative residual distributions, defined by

$$
1-\frac{(q / p)_{\text {reco }}}{(q / p)_{\text {truth }}},
$$

are plotted for different $N_{\text {red }}$ in Figure 37. These distributions are used to evaluate 
the performance according to three metrics:

- The $\sigma$ of a Gaussian fit to the core of the distribution

- The Full-Width-at-Half-Max (FWHM) of the distribution

- The smallest width to contain $50 \%$ of the integral of the distribution

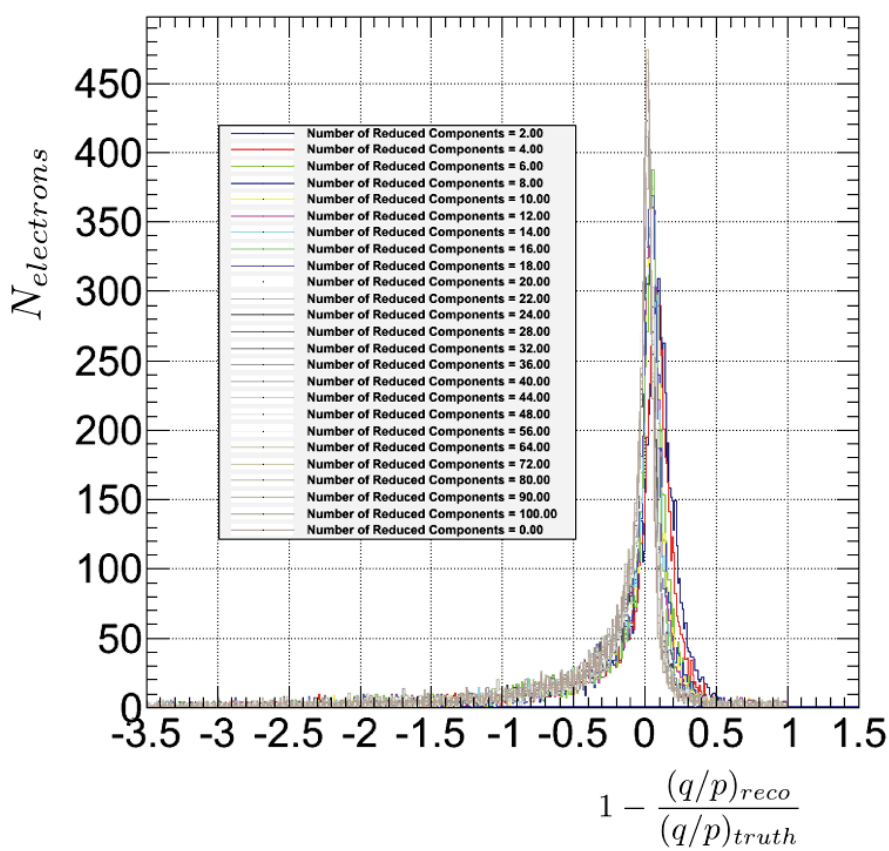

Figure 37: Distribution of $(q / p)$ pulls for a sample of 10,000 electrons with $E_{T}=25 \mathrm{GeV}$ reconstructed with the GSF under different configurations of the parameter $N_{\text {red }}$. The author learned how to produce much nicer plots since this study.

Figure 38 shows the evaluation time of the GSF algorithm, and the performance as a function of $N_{\text {red }}$, in steps of 2,5 , or 10 as $N_{\text {red }}$ increases. The evaluation time behaves approximately linearly with $N_{\text {red }}$, and will be discussed in a subsequent section. The resolution, as quantified by the three metrics above, exhibits a plateau of diminishing gains for higher $N_{\text {red }}$. The "smallest width" metric appears to be the most robust, providing a mostly smooth dependence as one expects, and the most distinct representation of the plateau. From this study, the value of $N_{\text {red }}=12$ was 
confirmed to be optimal, and is the current value used by the GSF algorithm for all ATLAS electron reconstruction.
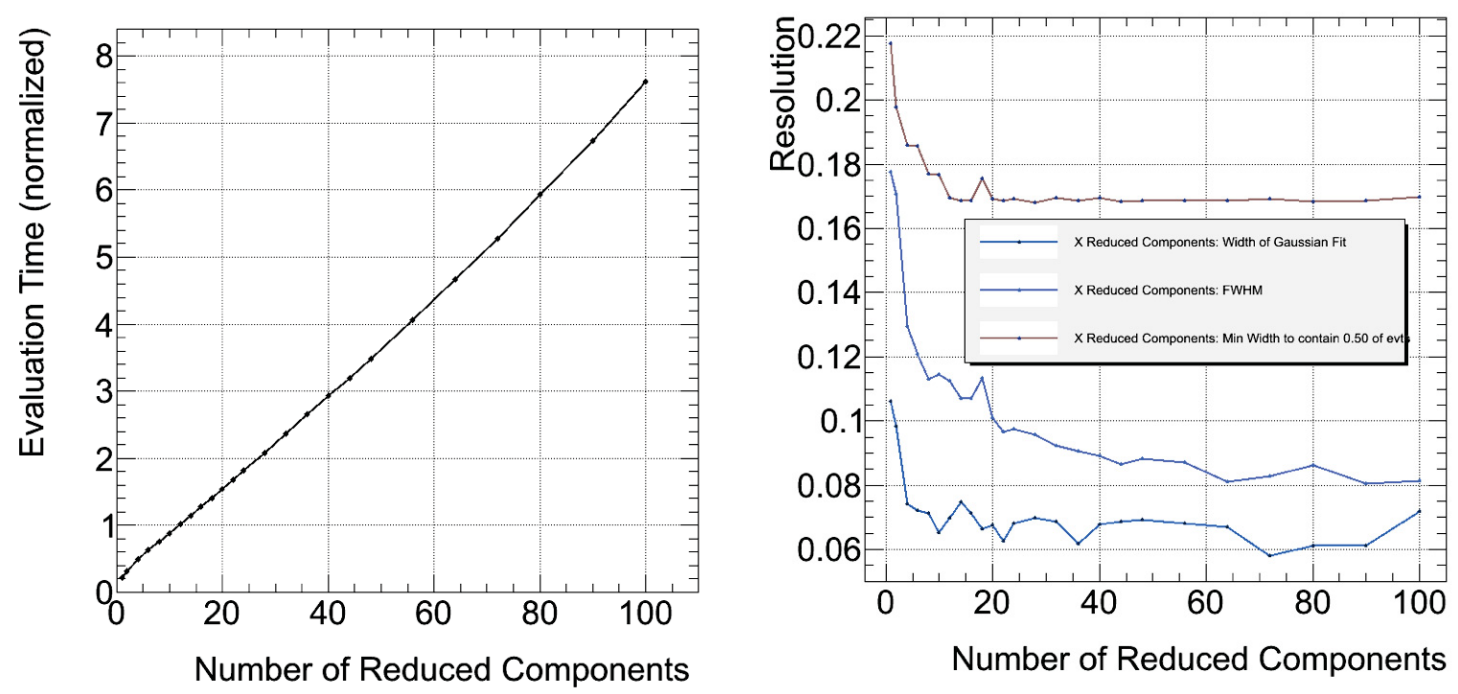

Figure 38: Tuning of GSF parameter $N_{\text {red }}$. Left shows the evaluation time of the fitter normalized to the $N_{r e d}=12$ time. Right shows three metrics of performance as a function of $N_{r e d}$, evaluated on the histograms of Figure 37. All show a consistent picture of diminishing returns from an increase of $N_{\text {red }}$ after approximately $N_{\text {red }}=12$.

\section{CPU optimizations}

Another study by the author sought to improve CPU performance of the GSF. While CPU gains themselves don't directly improve physics analysis, gaining (e.g.) $0.1 \mathrm{~ms}$ in one part of the code can leave "free time" to perform more expensive calculations to potentially improve resolution in another part.

The strategy used was to implement auto-vectorizable [75] code where possible within the GSF algorithm. Auto-vectorization is a compiler directive which allows a loop to be split onto multiple CPU cores and so allow iterations to be done in parallel in cases where one iteration of the loop does not affect another. A simple code example where this could occur is in array multiplication (this example taken 
from $[75])$ :

$$
\begin{aligned}
& \text { for }(\text { int } \quad \mathrm{i}=0 ; \mathrm{i}<12 ;+\mathrm{i})\{ \\
& \mathrm{A}[\mathrm{i}]=\mathrm{B}[\mathrm{i}] * \mathrm{C}[\mathrm{i}]
\end{aligned}
$$

Here it is clear that each iteration $i$ has no effect on the execution of some other iteration $j \neq i$. In this case the iterations can be split up onto $N_{C P U} \mathrm{CPU}$ cores and executed in parallel. Theoretically this can result in gains by a factor of as much as $N_{C P U}$ for such sections of code. This optimization is schematically illustrated for the above loop in Figure 39.

\section{$\underline{\text { no auto-vectorization }}$}

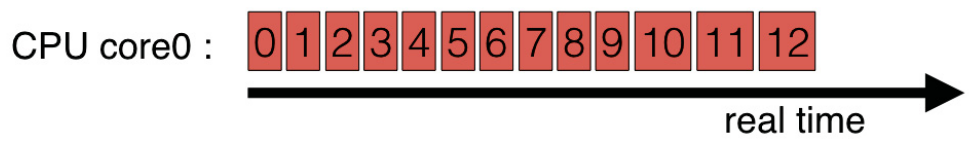

with auto-vectorization

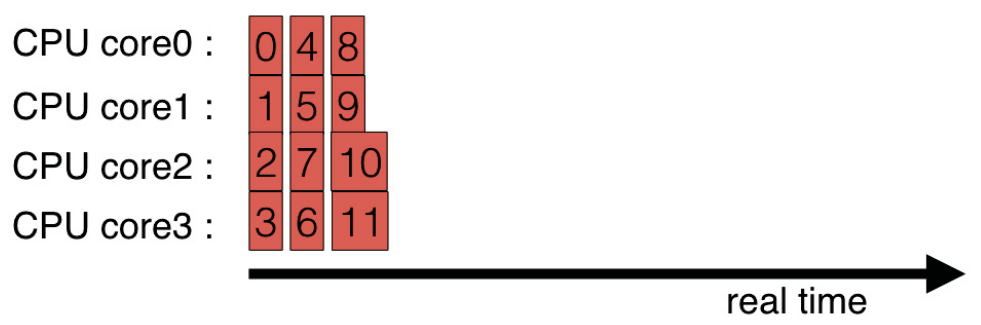

Figure 39: Diagram showing theoretical example of auto-vectorization on a loop of size 12, and a CPU with 4 cores. The numbers in boxes represent the index of the loop.

From this example one can imagine potential opportunities for the use of autovectorization in the GSF code, which relies heavily on matrix manipulation, vector addition and subtraction, and in general numerical computation. The author implemented auto-vectorizable code into some such cases, and here are presented the results in terms of CPU performance. Figure 40 shows the same CPU scaling with $N_{\text {red }}$ as 
seen in Figure 38, this time comparing before and after the code improvements. Note that since both lines, before and after, are normalized with respect to themselves, only the scaling can be compared, and not the absolute CPU consumption.

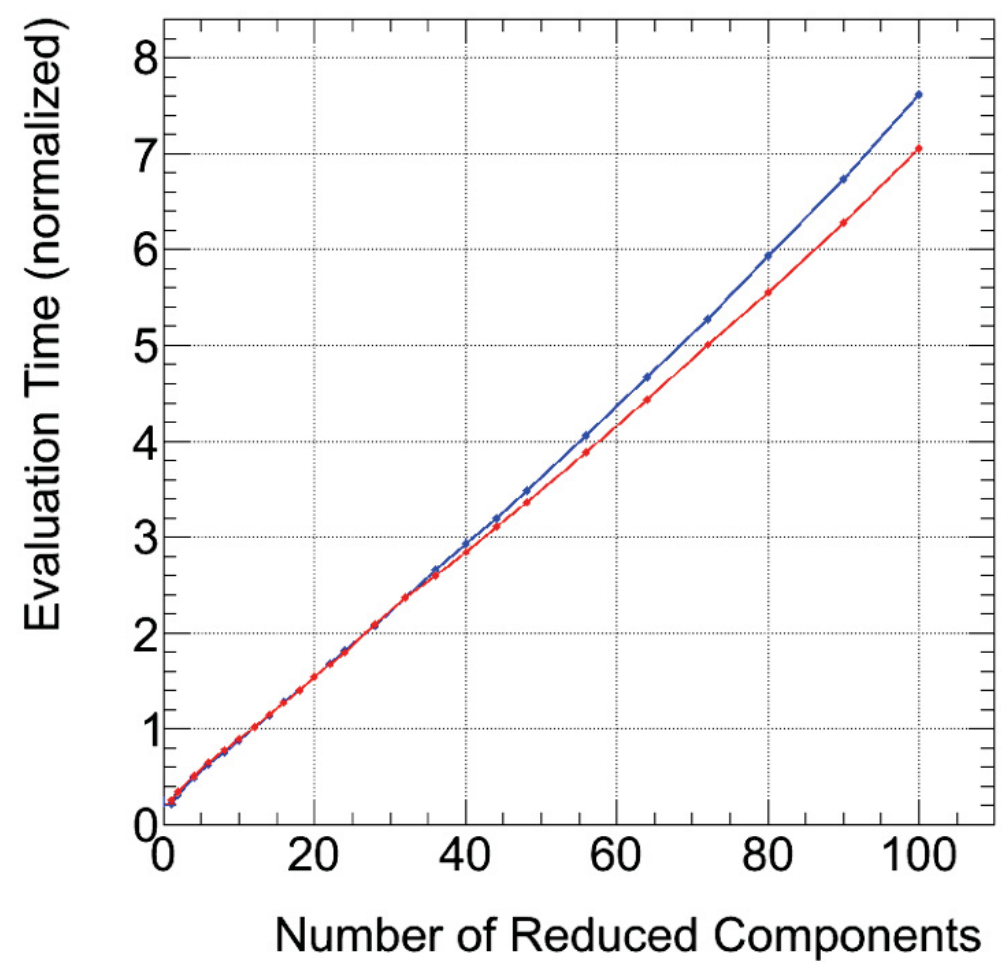

Figure 40: Plot showing improved scaling of CPU time with $N_{\text {red }}$ brought by autovectorization of GSF code. The blue line is prior to changes, and red line is after changes by the author. The vertical scale is normalized to $N_{\text {red }}=12$ separately for both lines.

We can compare the CPU performance prior to changes (labelled $A$ ), and after the changes (labelled $B$ ) by fitting with a simple 2 nd order polynomial ${ }^{7}$. The results of these two fits are

$$
\begin{aligned}
& \left(\frac{t}{t_{12}}\right)_{A}=0.111\left(\frac{N_{\text {red }}}{12}\right)^{2}+0.576\left(\frac{N_{\text {red }}}{12}\right)+0.221 \\
& \left(\frac{t}{t_{12}}\right)_{B}=0.089\left(\frac{N_{\text {red }}}{12}\right)^{2}+0.617\left(\frac{N_{\text {red }}}{12}\right)+0.212
\end{aligned}
$$

\footnotetext{
${ }^{7}$ for this fit I exclude the points below $N_{\text {red }}=12$, because the appeal of ever lowering this parameter is very small
} 
where $t_{12}$ is the CPU time for $N_{\text {red }}=12$, separately for the two different code configurations. From this result it is clear the scaling with $N_{\text {red }}$ is rendered more linear by the implementation of auto-vectorizable code. These improvements have made the penalty paid to increase $N_{\text {red }}$ more affordable, should a replication of the preceding study find a higher value is more optimal to improve reconstruction resolution.

\subsection{Muons}

In contrast with the preceding objects which are all fully contained by the ATLAS detector, muons have much higher penetrating power (i.e. they deposit very little energy on average per unit length), and so they escape the detector entirely. Fortunately, as they do so, they deposit small amounts of energy through each detector they pass (ID, Calorimeters, then MS), which allows their trajectory to be reconstructed. Since they are charged particles, and most of the detector is immersed in strong magnetic fields from either the Solenoid or the Toroid Magnets, the bending of their trajectory allows measurement of their momentum.

Muon reconstruction initially proceeds separately in the ID and the MS [4]. In the ID, tracks are reconstructed exactly as for electrons, except the GSF re-fit is not used. ID tracks are labelled as muon-associated if they are matched to a calorimeter energy deposit consistent with a muon (low ionization, no shower), and if they satisfy the following requirements:

$$
\begin{aligned}
& d_{0} \leq 50 \mathrm{~mm}, \\
& N_{p i x} \geq 1, \\
& N_{S C T} \geq 3, \\
& N_{S i} \geq 4,
\end{aligned}
$$

where $N_{p i x(S C T)}$ is the number of hits in the Pixel (SCT) detector, and $N_{S i}$ is the combined silicon hits (BLayer, Pixel, SCT). 
In the MS, a separate algorithm designed around its large physical size is needed. First, in each MDT chamber and its associated TGC or RPC trigger chamber, a pattern-recognition algorithm, the Hough Transform [76], is used to identify sets of hits pointing in straight lines. These "track segments" are fit with a straight line, and required to point approximately toward the interaction region.

Full MS tracks are built by running a matching algorithm on the reconstructed track segments. When a possible match of segments is found, all the hits in the constituent segments are refit as one (curved) track. Tracks must have at least two segments, with a few geometrically-motivated exceptions. If the tracks contain hits found also on other tracks, they are removed from the track with the worse fit $\chi^{2}$. If a track passes close to hits which were not initially associated to one of its segments, and so were not included in the track fit, these hits are recovered. If the hit content of a track changes, it is refit. The collection of tracks produced by this algorithm are called MS tracks.

Muon objects to be used in physics analyses are finally built from ID tracks, calorimeter information, and MS tracks. Depending on the available information, these muons are classed into five types as follows, ordered roughly by performance (efficiency, purity, and momentum resolution).

- Combined (CB) Muons: built from a combined fit of matching ID and MS tracks. Tracks are matched using an outside-in pairing first, followed by insideout pairing. Because ID tracks vastly outnumber MS tracks, the former accounts for most of the $\mathrm{CB}$ muons reconstructed. The hits from both tracks are refit together to create the combined track.

- Segment-tagged (ST) Muons: in some cases an ID track can only be paired with MS track segments in a particular MDT or CSC chamber, rather than full tracks. Since these tracks have lower performance than CB tracks, they are marked as a separate type. 
- Silicon Associated Forward (SiAF) Muons: discussed separately in Section 4.5.1.

- Extrapolated (ME) Muons: In the region $2.5<|\eta|<2.7$, there is coverage by the MS but not the ID. In this region, MS tracks are refit with a loose constraint to have originated from the interaction point.

- Calorimeter-tagged (CT) Muons: In the region $|\eta|<0.1$, the MS has very poor coverage due to service and instrumentation constraints (service and cabling for the ID and Calorimeters runs through this region). In this region muons are identified based only on the muon-associated ID track.

\subsubsection{Silicon Associated Forward Muons}

Silicon Associated Forward Muons are described in their own section as the author was responsible for implementing their combined refit into the reconstruction algorithm, and for the performance studies presented in this section.

On close examination of the inset of Figure 21, one can see that there is some ID coverage above $|\eta|>2.5$ coming from the Pixel detectors. SiAF Muons attempt to take advantage of this coverage to improve muon performance in the narrow band around $|\eta|=2.5$. For a real muon which passed only through these edges of Pixel detectors and then out into the MS, inclusion of these tracklets for ID-MS track matching (always as a second choice behind full ID tracks) can provide a more com-

plete reconstruction, and therefore more precise momentum measurement, than the MS track alone.

In the rest of the ID, ie $|\eta|<2.5$, tracks are required to meet the criteria of (53) to ensure a high level of purity. In the forward region, a separate set of ID "tracklets" can be reconstructed, almost exclusively from Pixel hits. For these tracklets, the SCT hit requirements of (53) are lifted, and the $N_{S i}$ requirement is loosened to $N_{S i} \geq 3$. By comparison, Figure 41 shows the ID hit distributions for CB and SiAF muons 
(ME muons are not included because by definition have no ID hits) in a simulated sample. The difference in Pixel hit distribution is accounted for by requirements on $N_{S i}$ : while CB muons are allowed fewer Pixel hits in practice, because they are able to make up for it in SCT hits, SiAF muon have usually no (or few) SCT hits to make up the difference. The particular shape of the $N_{S C T}$ distribution for SiAF muons (e.g. the lack of $N_{S C T}=1$ muons) is a product of the detector geometry in the endcap region. The combined Pixel and SCT hits are also shown.
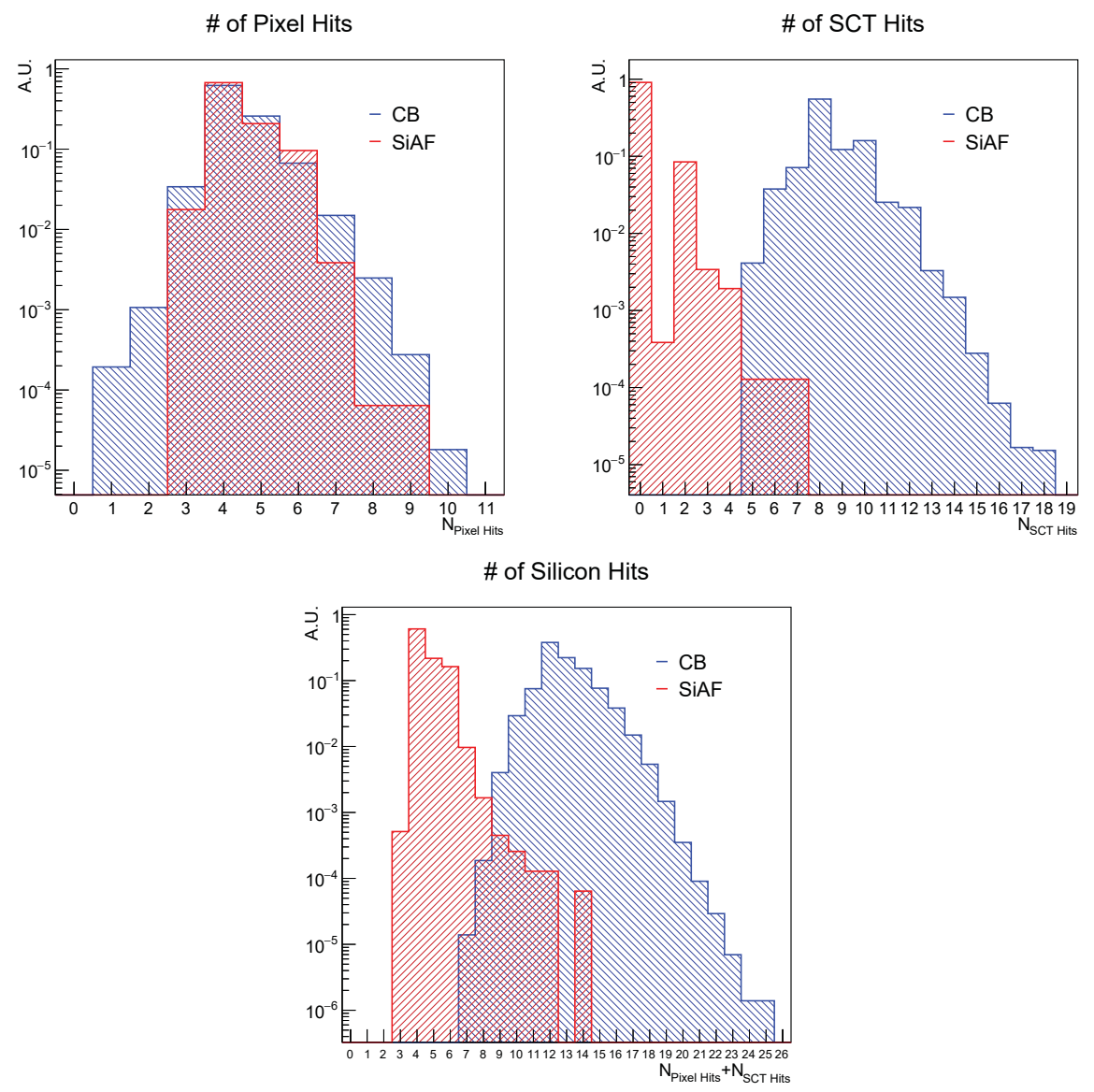

Figure 41: Number of hits in Pixel (left) and SCT (center), and combined Pixel+SCT (right) systems for the ID track or tracklet of CB and SiAF muons. Number of hits includes hits plus dead sensors the track passed through. Certain loose quality cuts matching those of the $H \rightarrow 4 \ell$ event selection, discussed later in Section 5.3.2, are applied. Distributions are normalized for comparison.

For the SiAF muons, the matching of ID tracklets to MS tracks is done in the same 
way as for $\mathrm{CB}$ muons $^{8}$. First the remaining unmatched MS tracks are extrapolated inwards to find a matching ID tracklet. As a secondary approach, inside-out matching is done where the ID tracklets are extrapolated outwards to be matched to MS tracks. The possible matches are compared using a combined fit $\chi^{2}$, and the combination producing the smallest $\chi^{2}$ is taken, unless no good match is found, in which case the MS track is left unpaired, and an ME muon is created. The net effect of the inclusion of this algorithm is a migration of some otherwise-ME muons to be SiAF muons.

To quantify the benefit of SiAF muons, we can look at simulated data containing $Z \rightarrow \mu \mu$ decays. Figure 42 shows the breakdown of muon types for muons reconstructed in these events ${ }^{9}$. Note that the rate of SiAF muons $(2 \%)$ is approximately equal to that of ME muons, indicating that roughly half of the otherwise-ME muons were able to be reconstructed as SiAF muons. The ST and CT muons are reconstructed quite independently of this discussion, so are not included in proceeding plots.

It can be useful to examine directly the reconstructed kinematic distributions for the various muon types. Figure 43 shows a comparison of some kinematic distributions of $\mathrm{CB}$, SiAF, and ME muons reconstructed in the simulated $Z \rightarrow \mu \mu$ events. In particular the $\eta$ distribution gives the most physical insight as to where these muons arise: values of $|\eta|$ near 2.5 skim the edge of the ID, exactly where one expects SiAF muons. At higher values of $|\eta|$, approaching 2.7, the rate for SiAF muons falls while that of ME muons rises ${ }^{10}$.

We also expect improved reconstruction performance arising from the use of ID

\footnotetext{
${ }^{8}$ but with lower priority: if an MS track can be paired to create a CB muon, it will take precedence over a SiAF pairing of the same MS track

${ }^{9}$ note that these muons already have certain quality criteria applied. The same selection is used for the $H \rightarrow 4 \ell$ event selection, and discussed in Section 5.3.2

${ }^{10}$ This plot extends to $|\eta|=3$, even though muons used in analysis are required to have $|\eta|<2.7$
} 
muon types in $Z \rightarrow \mu \mu$

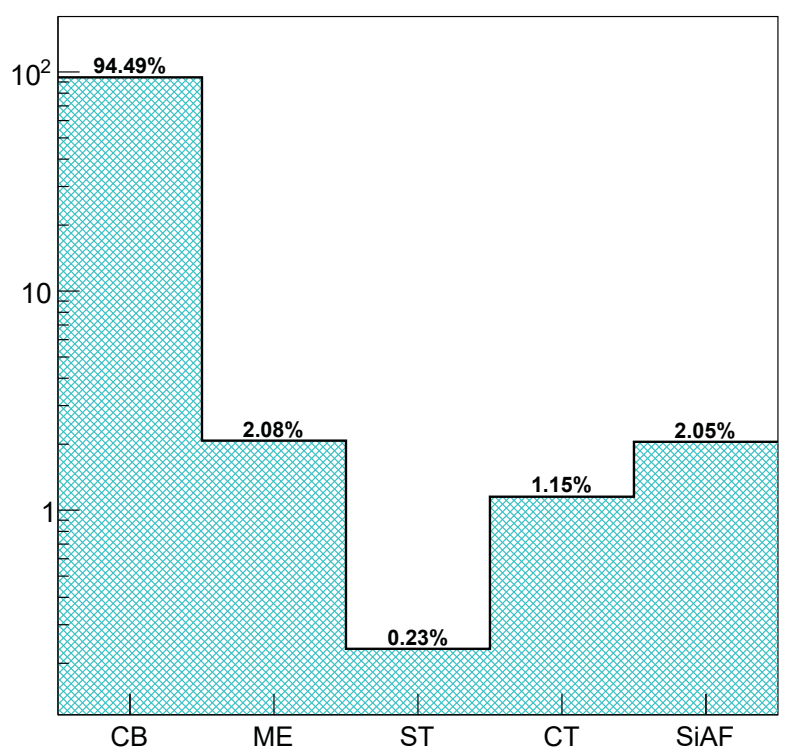

Figure 42: Composition of reconstructed muons from simulated $Z \rightarrow \mu \mu$ events by muon type.

tracklets in a combined fit. Using simulated data permits the comparison of reconstructed kinematics to the truth kinematics, which we use to measure this benefit as explained in Section 4.2. Figure 44 shows the reconstruction performance for several kinematic variables - a narrower distribution indicates reconstructed values are closer (on average) to their truth values, and so reconstruction is performing better. For a balanced comparison, these plots require $|\eta|>2.4$, an approximate pseudorapidity region $^{11}$ where all three muon types are reconstructed. This cut creates an apparent bias in the $\eta$ estimation for $\mathrm{CB}$ muons which should be ignored (i.e. do not use this shift to draw conclusions about the muon types). From these figures we see the expected result: CB muons are always the best, but SiAF muons demonstrate a marked improvement over ME muons. To quantify what we can see by inspection, Table 7 compares the standard deviation of each of these distributions. SiAF muons

\footnotetext{
${ }^{11}$ More central pseudorapidities, for instance $|\eta|<2.4$, have less pileup, and less material for muons to traverse (thus less scattering), making reconstruction "easier", and giving an unfair advantage to CB muons.
} 

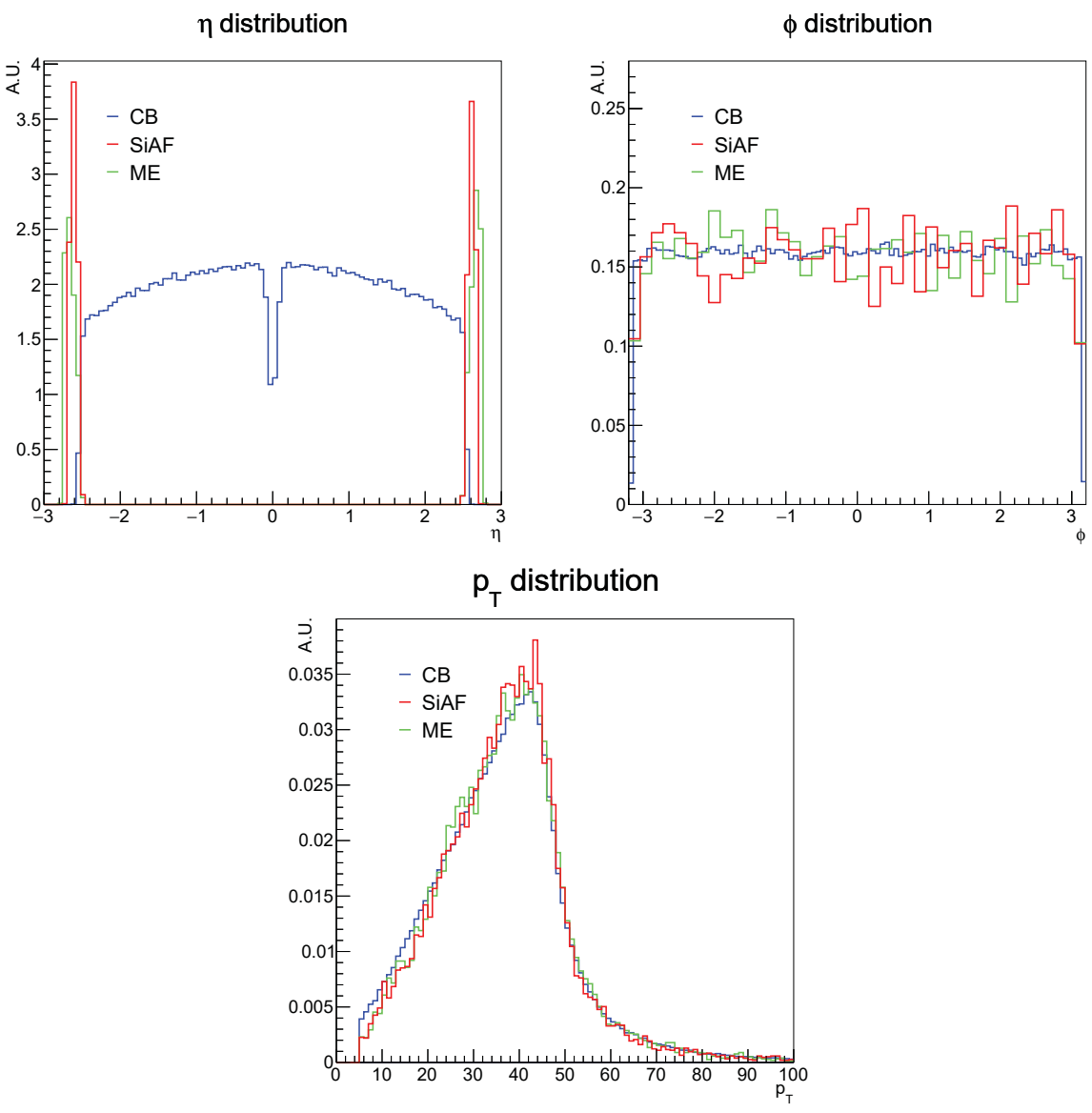

Figure 43: Kinematic distributions for muons reconstructed in simulated $Z \rightarrow \mu \mu$ events, separated by muon type. Note that the vertical scale and bin size of each histogram is arbitrary - the histograms are independently scaled and binned for convenient comparison. 

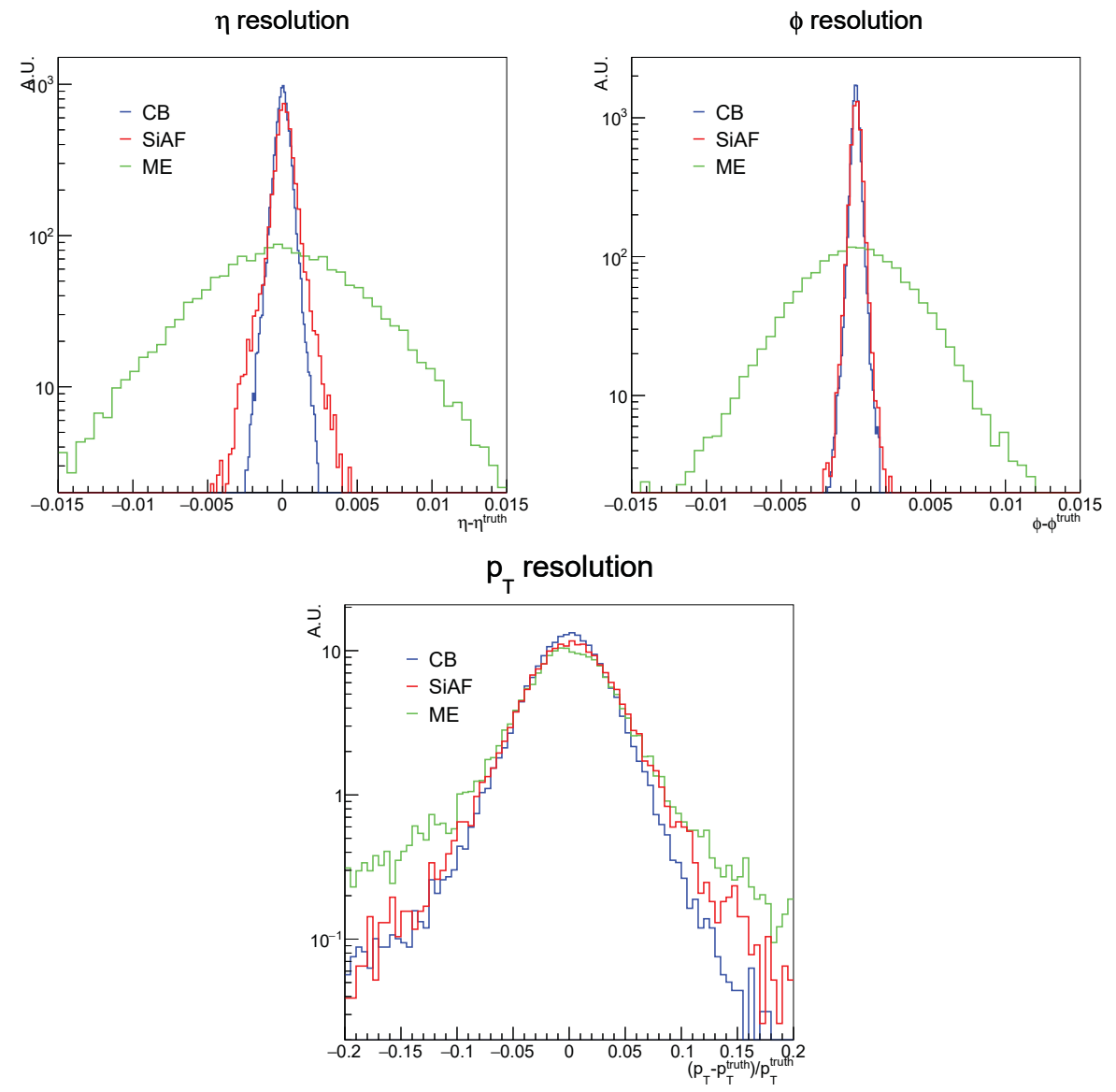

Figure 44: Resolution distributions for muons reconstructed in simulated $Z \rightarrow \mu \mu$ events, separated by muon type, plotted for $|\eta|>2.4$. Note that the vertical scale and bin size of each histogram is arbitrary - they are normalized and binned for convenient comparison.

are found to improve angular resolutions as compared to ME muons by a factor of 4.5 or better, and momentum resolution by a factor of 1.2 .

This observed improvement in resolution arises because of the addition of Silicon hits to the final muon track. Delving deeper, we can compute the above resolutions as a function of the number of silicon hits on the ID track (compared to Table 7 which shows inclusive numbers). Figure 45 shows exactly this result. ME muons are excluded from these plots as they have no ID hits. It should be noted that CB muons will in general also have TRT hits, which brings some benefit to resolution, 
Table 7: Performance of reconstruction for different muon types in kinematic variables $\eta, \phi, p_{T}$, for $|\eta|>2.4$. The table values are the sample standard deviations (denoted as $\sigma$ ) of the distributions shown in Figure 44, such that smaller numbers indicate better resolution. Note that while $\eta$ and $\phi$ are shown as absolute resolutions (e.g. $x-x^{\text {truth }}$ ), the $p_{T}$ is shown as a relative resolution $\left(\left(p_{T}-p_{T}^{\text {truth }}\right) / p_{T}^{\text {truth }}\right)$. Statistical uncertainties are an order of magnitude below the final presented digit, though systematic uncertainties were not evaluated.

\begin{tabular}{cccc}
\hline muon type & $\sigma_{\eta}\left(\times 10^{-3}\right)$ & $\sigma_{\phi}\left(\times 10^{-3}\right)$ & $\sigma_{p_{T}}$ \\
\hline $\mathrm{CB}$ & 0.56 & 0.32 & $3.7 \%$ \\
$\mathrm{SiAF}$ & 1.1 & 0.71 & $4.3 \%$ \\
$\mathrm{ME}$ & 5.1 & 3.9 & $5.3 \%$ \\
\hline
\end{tabular}

but especially helps in rejection of electron tracks in the ID faking muons.

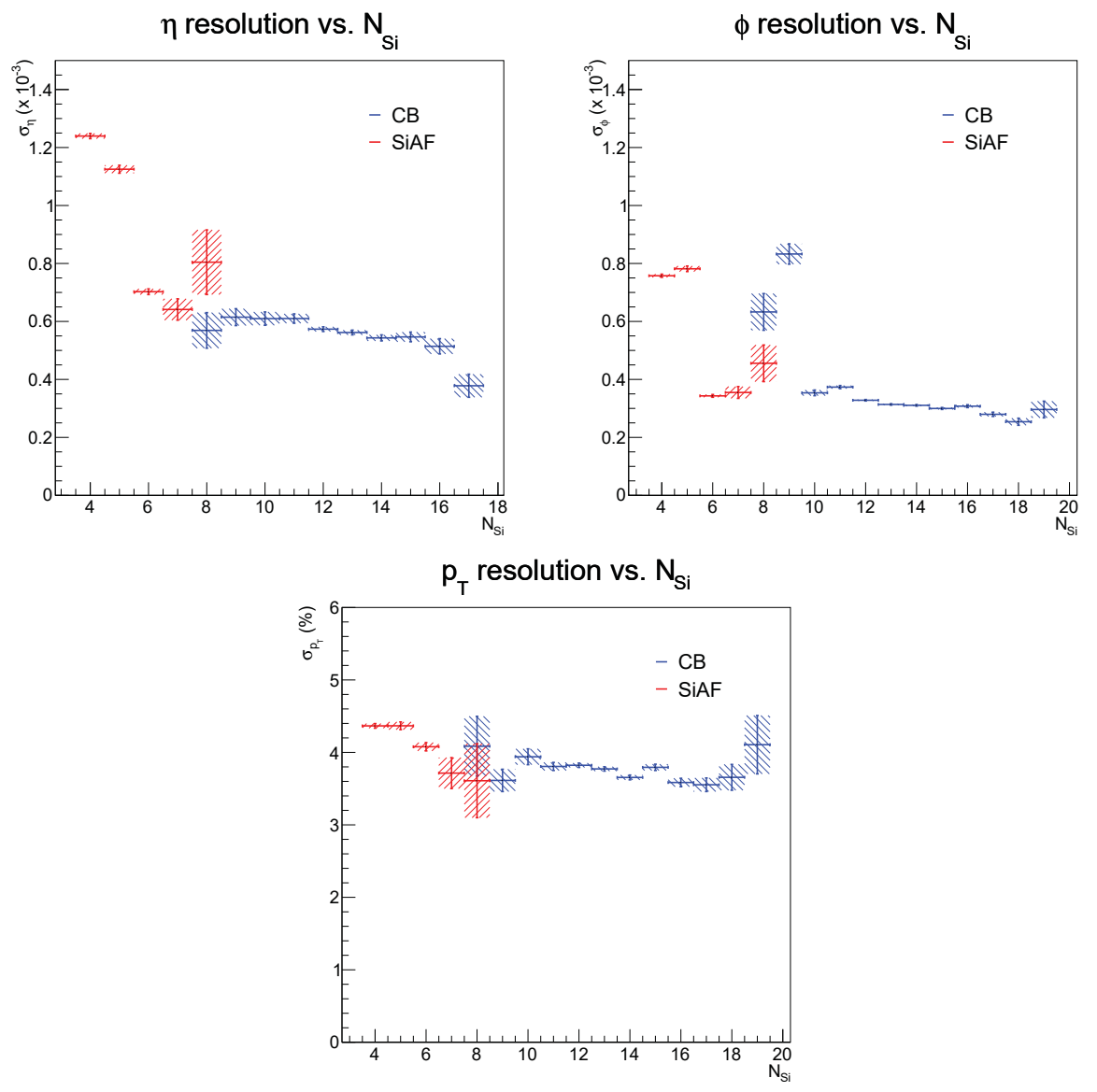

Figure 45: Resolution values for different muon types as a function of $N_{S i}$, for $|\eta|>2.4$. The value plotted is the standard deviation of the histograms shown in Figure 44, split into bins of $N_{S i}$. 
From these studies we can draw some conclusions. SiAF muons represent a distinct upgrade from ME muons they would otherwise be. This improvement arises from the addition of silicon hits to the track, in particular because they are so close to the interaction point. The more silicon hits the muon uses, the better its resolution will be, which can be seen from Figure 45. In some cases, SiAF muons can be nearly as well reconstructed as $\mathrm{CB}$ muons. Of course, the resolution of reconstructed quantities is not the only factor that should establish the quality of a certain algorithm. Also important is the purity: the fraction of reconstructed muons which were actually muons and not some other source of background. Because they lack TRT hits and have fewer silicon hits, it is expected that SiAF muons will have lower purity than $\mathrm{CB}$ muons, though it is left for future work to determine.

\subsection{Jets}

In contrast to photons, electrons, or muons, which are all both SM particles and a "physics object", jets are a purely reconstruction-based concept. They are built from energy deposits in the HCal. Jets are the manifestation of a variety of strongly interacting final-state particles. Because of the hadronic nature of $p-p$ collisions, many jets are produced from quarks and gluons directly, and also from baryons and mesons produced in collisions.

Jets are reconstructed using the anti- $k_{T}$ algorithm with a cone size of $R=0.4$ [77]. The algorithm iteratively groups nearby calorimeter clusters into jets, starting with the clusters with highest $p_{T}$ and working downwards. The cone size, roughly speaking, defines the distance in which to search for nearby clusters, as defined in (42).

Jets are used only tangentially in the analysis in this thesis where their overlap with muons and electrons is concerned, described in Section 5.5. 


\subsection{Missing transverse energy}

Missing Transverse Energy (MET, also denoted $E_{T}$ ) is not directly reconstructed so much as it is calculated. When neutrinos are produced in collision, for example from a $W \rightarrow \ell \nu$ decay, they stream freely through the detector (and in all likelihood several light-years of space) without interacting. The only window by which we observe neutrinos produced in the detector is by looking for the energy we didn't see - what they carried away from the collision.

As a consequence of conservation of momentum, the total momentum of all incoming particles in a collision is equal, unequivocally, to the total momentum of all outgoing particles from the collision. As mentioned in Section 3.2.1.1, the longitudinal momentum of partons participating in a collision is unknown, because the protons' very precisely known momentum is shared at random according to parton distribution functions. Only the momentum vector in the transverse $(r-\phi)$ plane is known, and it is zero. When summing the transverse momentum of all recorded particles (measured as described in the previous sections), any remaining imbalance called Missing Transverse Momentum, whose magnitude is the MET.

$$
\begin{aligned}
& \not{p}_{T}=-\sum_{\text {particles }} \vec{p}_{T} \\
& \not E_{T}=\left|\vec{p}_{T}\right|
\end{aligned}
$$

This description of MET is a simplified version, as MET only enters tangentially to the analysis presented in this thesis. For a study which relies more heavily on MET, additional subtleties, such as when to use track-based or calo-based MET, must be considered and detailed. 


\subsection{Reconstruction summary}

Figure 46 provides a summary of how different types of long-lived particles are reconstructed within ATLAS. Note that the distinction "long-lived" is important here, because short-lived particles (whether they are occasionally short-lived, or always short-lived) will have drastically different signatures: photons can convert to a $e^{+} e^{-}$ pair, the neutral hadron $\pi_{0}$ always decays immediately to a $\gamma \gamma$ pair, among a plethora of other examples.

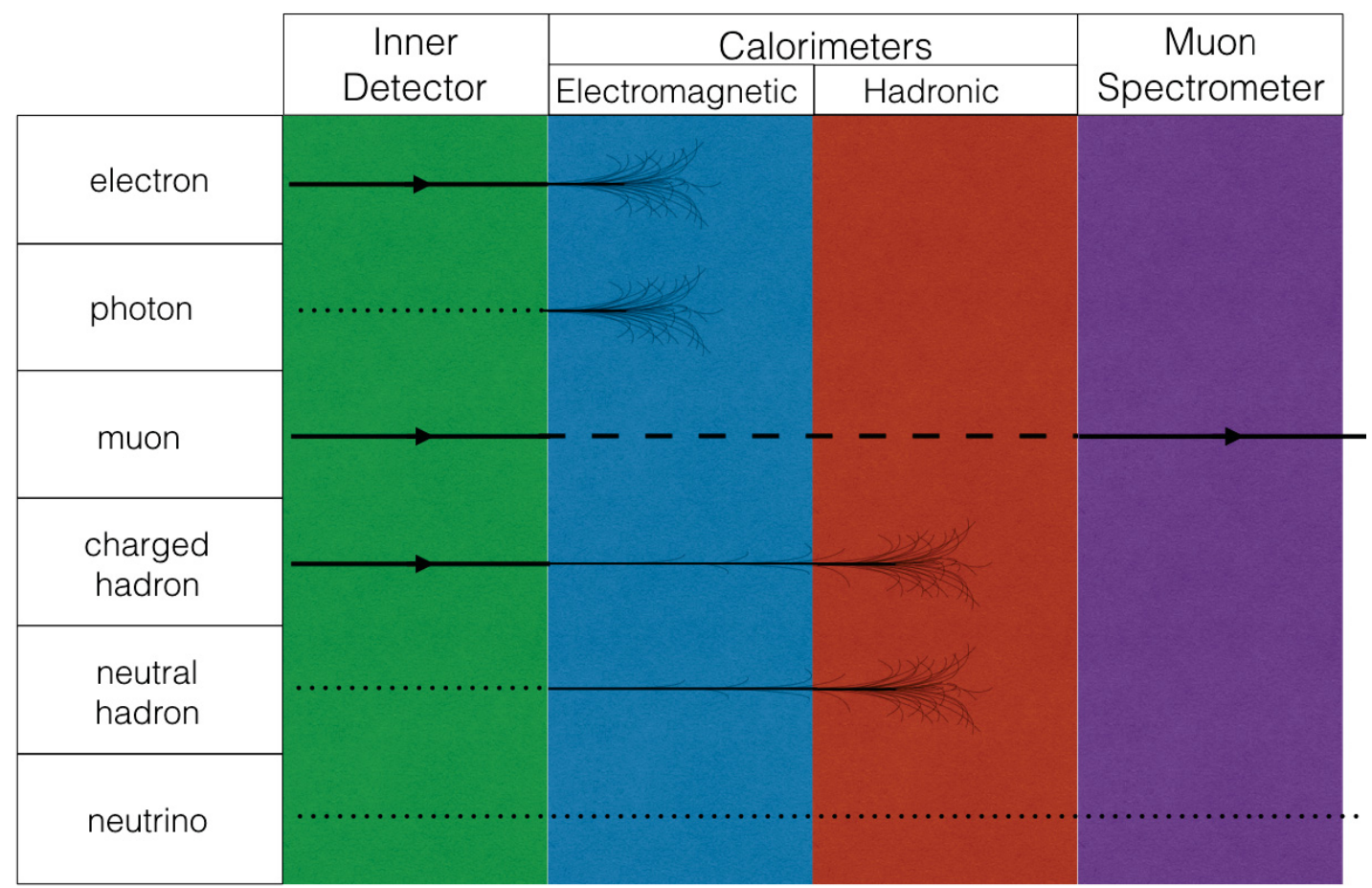

Figure 46: Diagram showing how different types of particles are reconstructed in ATLAS. Solid lines represent tracks, dashed lines represent small energy deposits, and dotted lines represent a complete absence of any signature. Electromagnetic and hadronic showering are indicated schematically to show in which component of the detector most of the energy is deposited. Sizes of any kind are not to scale. 


\section{Chapter 5}

\section{$H \rightarrow Z Z^{(*)} \rightarrow 4 \ell$ Event Selection}

Among the billions of collision events recorded by ATLAS, a very small minority contain $H \rightarrow Z Z^{(*)} \rightarrow 4 \ell$ decays. This chapter describes how these events are identified for further analysis. While the full event selection described was implemented by the author, the development of the selection criteria and execution of the event selection was performed by ATLAS collaborators.

Events are selected using a series of requirements, or cuts. The cuts are designed around a trade-off between signal efficiency and background rejection: any cut in general could be modified to accept more signal at the cost of also accepting more background (called looser), or be adjusted to reject more background at the cost of also rejecting more signal (called tighter). Another pragmatic consideration affecting the event selection criteria is to only make cuts on variables that are well-described by $\mathrm{MC}$, and only in a phase space which the ATLAS detector performance is wellunderstood, both factors which take higher priority over optimizing signal significance. The events surviving the event selection described in this chapter are called candidate events, and are used in the statistical analyses described in Chapter 7, 8, and 9.

Before any data are looked at, the analysis (which includes the event selection) is developed from end to end using MC simulated samples. This is done to prevent potential bias that could arise from modifying the analysis strategy after looking at 
the $\operatorname{data}^{1}[78]$.

\subsection{Pre-Selection}

A pre-selection of events is designed to remove data which are unsuitable to be used for analysis. One reason is a detector component may malfunction or fail during a data collection run (e.g. because of a high voltage trip). An offline component can create an effective "hole" in the detector from which particles can escape during collisions, and so it is safest to discard events during which this happens. Since ATLAS data is divided into runs - periods of LHC collisions usually lasting between hours to days - and further into lumi-blocks - 1 minute blocks of data - the first cut in the event selection is the Good Runs List (GRL), a list of runs and lumi-blocks that have been approved by the Data Quality (DQ) group for use in physics analyses. Malfunctioning detector components can often be rebooted by shifters in the ATLAS control room, so only a minimal number of lumi-blocks are lost. Further removal of events flagged for detector problems is done by checking for error states recorded in the SCT, the LAr, Tile, or for data corruption.

\subsection{Event-level selection}

The second set of cuts are designed to remove events which show no promise of having $H \rightarrow Z Z^{(*)} \rightarrow 4 \ell$ decays based on event-level quantities.

The first is to require that events have a primary vertex with at least 2 tracks. The primary vertex is defined as the vertex with the highest $\sum p_{T}^{2}$ in the event. This definition is designed to identify hard scattering collision events, i.e. events most

\footnotetext{
${ }^{1} \mathrm{~A}$ worst-case scenario would be to choose analysis cuts which "cut around the data": a dangerous bias is introduced if one decides to make a cut of $p_{T}>4.9 \mathrm{GeV}$ instead of $p_{T}>5.0 \mathrm{GeV}$, just because it accepts several new data events (or vice-versa to remove these events). The cut at $4.9 \mathrm{GeV}$ would have been acceptable in general only if it was decided upon before looking at the data.
} 
likely to contain interesting physics such as a Higgs boson.

Events are then required to have passed one of the triggers listed in Table 8. The requirement to have passed any one (or more) of these triggers is nearly $100 \%$ efficient for signal events. Note that the triggers presented here are those used in the final period of data taking in 2016 - earlier run periods use slightly different configurations according to their availability at the time. 
Table 8: Trigger list for the $H \rightarrow Z Z^{(*)} \rightarrow 4 \ell$ event selection. Rows are grouped by trigger type, as labelled.

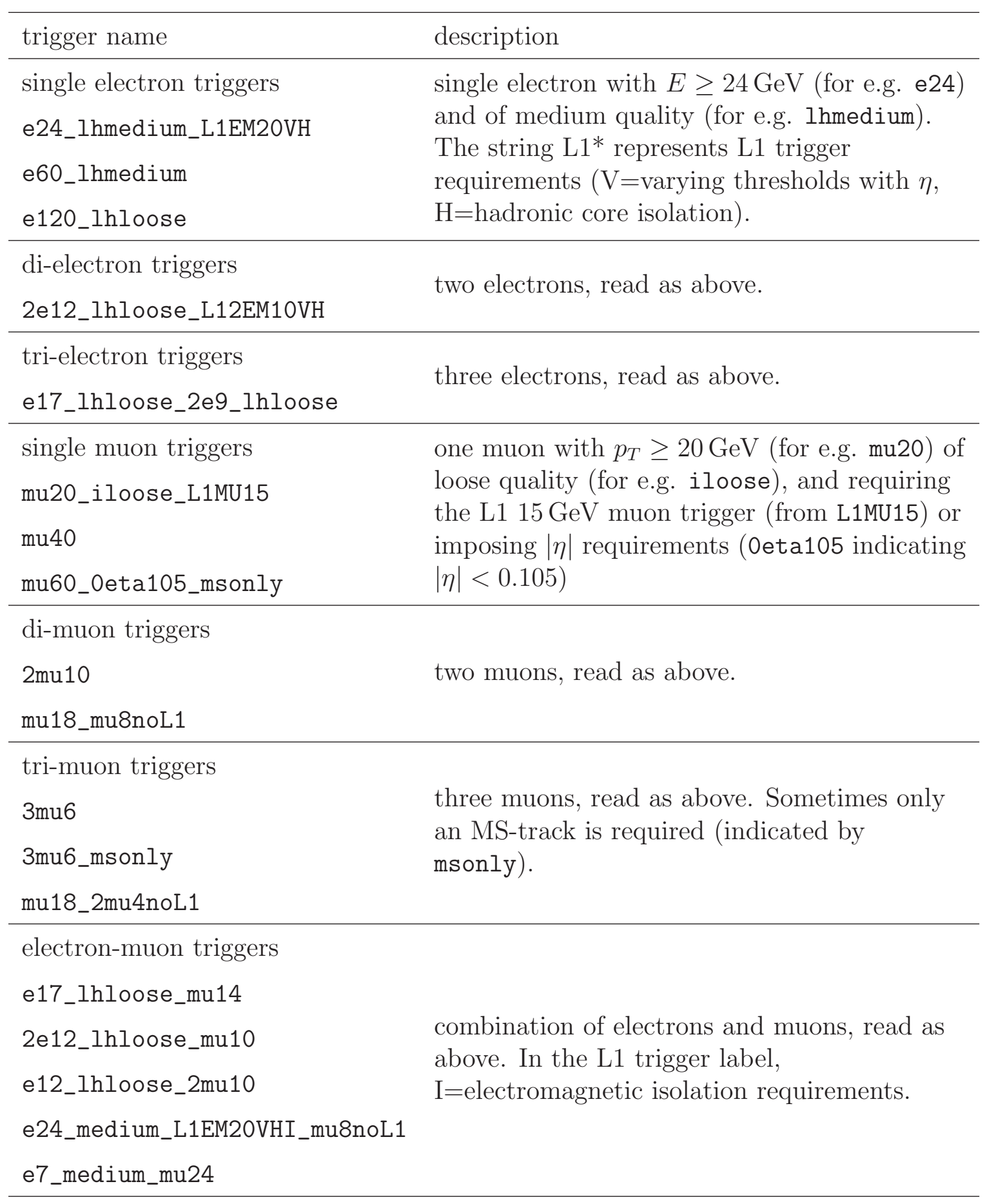




\section{$5.3 \quad$ Lepton selection}

For mass measurement in the $H \rightarrow Z Z^{(*)} \rightarrow 4 \ell$ channel, the lepton selection is critical: the invariant mass of the four leptons, $m_{4 \ell}$, will be used as the observable sensitive ultimately sensitive to $m_{H}, \Gamma_{H}$, and $\sigma_{H \rightarrow 4 \ell}$. This section describes the criteria for leptons to be used in the remainder of the event selection and analysis.

Note that "lepton" here and in the following refers only to electrons and muons. Neither taus nor neutrinos are included in this analysis as both result in MET and so require significantly different analysis strategies. They are also insensitive to the Higgs mass for the same reason.

\subsubsection{Electron selection}

In order to have precise four-momentum reconstruction, electrons need a good ID track. This is achieved by requiring a hit on the inner-most tracking layer, the BLayer, as well as a minimum number of hits in the Pixel and SCT sub-detectors.

True electrons are distinguished from fake electrons using a multivariate likelihood discriminant (the predecessor method used during $8 \mathrm{TeV}$ collisions is described in [79]). The discriminant is built from a set of variables with separating power between real electrons and fake electrons both reconstructed by the algorithms described in Section 4.4. It includes variables related to energy leakage into the hadronic calorimeter, fraction of energy deposited in the back layer of the EM calorimeter, variables related to shower width, and track-cluster matching. PDFs are built using the distributions of these variables in simulation for true electrons and fakes. These PDFs are combined in a likelihood to compute a single score for each electron. A cut is placed on this score, tuned on MC, so that together with the ID cuts a $95 \%$ efficiency for true electrons is achieved - this working point is called Loose, because it achieves high efficiency at a sacrifice of some purity. Section 6.3.1 provides a detailed 
measurement of the contamination from fake electrons passing this event selection.

Only electrons with $p_{T}>7 \mathrm{GeV}$ and $|\eta|<2.47$ (where ID tracking is available) are used.

\subsubsection{Muon selection}

Where they are available, CB muons are preferred for their high resolution and purity; they are found roughly in $0.1<|\eta|<2.5$. CT and ST muons are kept in the region $|\eta|<0.1$ where no (or partial) MS coverage is available. SiAF and ME muons are kept in the region $2.5<|\eta|<2.7$ where there is no (or trailing) ID coverage; of these, SiAF muons are preferred

A working point for muons, similarly called Loose, is built by placing cuts on ID and MS hits, specific to each muon type (one cannot ask ID hits of an SA track, nor ask MS hits of a CT track, for example). The goal of these requirements is to reject fake muons from pion and kaon decays. A measurement of the compatibility between ID and MS tracks, called $q / p$ significance and calculated using the ID and MS track momenta and resolutions, also enters into the working point. These selections are described, excepting SiAF muons, in [4].

$\mathrm{CB}, \mathrm{ST}, \mathrm{SiAF}$, and ME muons are used for $p_{T}>5 \mathrm{GeV}$, while CT muons are used for $p_{T}>15 \mathrm{GeV}$.

Muons are required to have $d_{0}<1 \mathrm{~mm}$, and $\left|z_{0} \sin (\theta)\right|<0.5 \mathrm{~mm}$, except for SA muons. These cuts are designed to reject muons which did not originate at the primary vertex, possibly originating from cosmics, displayed vertices, or pileup vertices. The definitions of $d_{0}$ and $z_{0}$ are shown in Figure 47. 


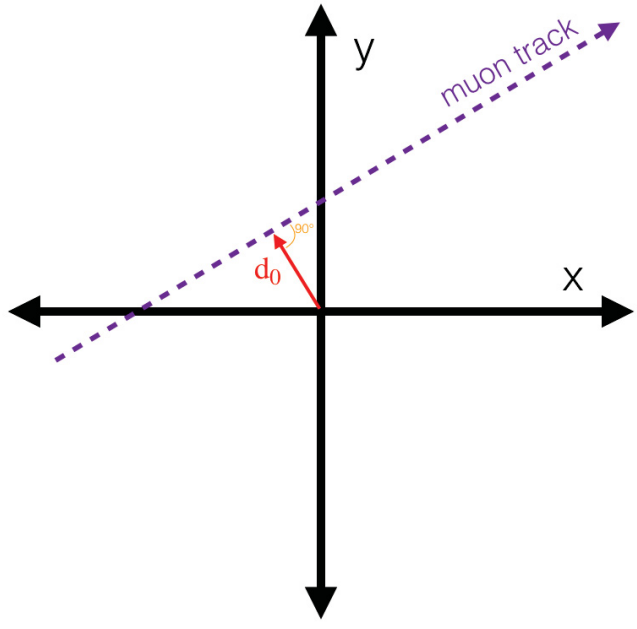

(a) d0

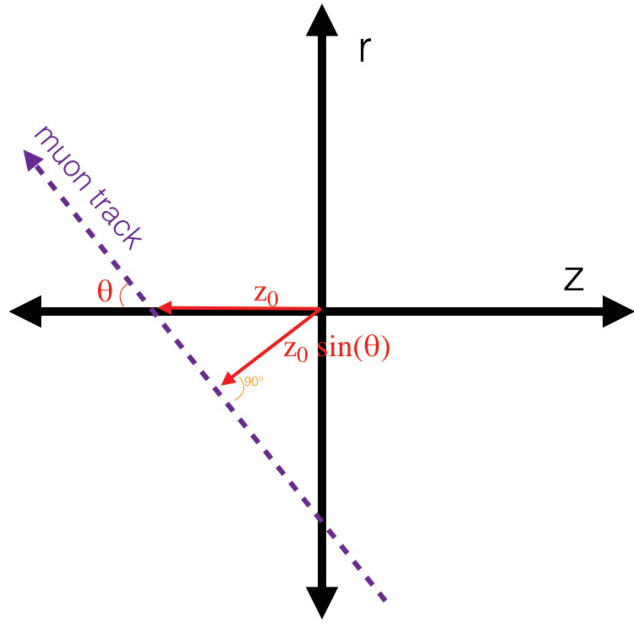

(b) z0

Figure 47: Impact Parameter definitions. $d_{0}$ is the distance from the track to the z-axis at its point of closest approach (perigee). $z_{0}$ is the $\mathrm{z}$-coordinate of the perigee, defined with respect to the primary vertex.

\subsection{Jet and photon selection}

Jets are required to pass a quality selection called Bad Loose in ATLAS [80]. Central, low-momentum jets (defined by $p_{T}<60 \mathrm{GeV}$ and inside $|\eta|<2.4$ ) are required to pass a jet-vertex tagger cut designed to eliminate contamination from pileup. Jets passing these requirements, as needed, are kept for $p_{T}>30 \mathrm{GeV}$ and $|\eta|<4.5$.

Photons are required to pass basic selection criteria based on shower properties as measured in the EMC.

\subsection{Overlap removal}

The reconstruction algorithms for each physics object run independently of other objects that have been reconstructed, leaving the possibility that two selected objects may share tracks or clusters in the detector. For example, the ATLAS reconstruction algorithm for electrons thinks a particular ID track belongs to an electron, and the reconstruction algorithm for muons thinks it belongs to a muon - both potentially 
reasonable conclusions, but at least one is incorrect. These cases are removed by the following cuts:

- For two electrons which share ID tracks, the electron with the highest track $p_{T}$ is kept.

- For two electrons with nearby clusters, the electron with the highest cluster $E_{T}$ is kept. "Nearby" is taken to mean $|\Delta \eta|<0.075$ and $|\Delta \phi|<0.125$.

- When an electron shares an ID track with a CT muon, only the electron is kept.

- When an electron shares an ID track with any other muon, the muon is kept.

- When a jet overlaps with an electron within $\Delta R<0.2$, the jet is removed.

\section{$5.64 \ell$ selection}

The goal of the event selection is to identify and re-assemble the $H \rightarrow Z Z^{(*)} \rightarrow 4 \ell$ decay in the detector from the leptons passing the previously described selection. To do this, the following requirements are imposed in order.

Events are required to have either four muons, four electrons, or two electrons and two muons.

Next, all possible pairs of same flavour (electron-electron, or muon-muon), opposite sign (positive-negative charge) leptons are identified, called SFOS. These are

the candidate $Z$ boson decays. Events are required to have at least two independent SFOS pairs.

The SFOS pairs are themselves paired up to form all possible quadruplets, and the event selection proceeds by applying cuts to these quadruplets. If an event contains no quadruplet passing the selection, the event itself is cut. 
The quadruplets must pass a kinematic cut which requires the leptons, ordered by $p_{T}$, to pass

$$
\begin{aligned}
& p_{T_{1}}>20 \mathrm{GeV}, \\
& p_{T_{2}}>15 \mathrm{GeV}, \\
& p_{T_{3}}>10 \mathrm{GeV} .
\end{aligned}
$$

Quadruplets are also required to contain one (or more) of the leptons which caused the trigger to fire for this event.

Since the decay of the Higgs proceeds via two $\mathrm{Z}$ bosons, knowledge of this intermediate step can be used in the event selection. For $m_{H}$ near $125 \mathrm{GeV}$, one of the $Z \mathrm{~s}$ can be on-shell. In the quadruplets reaching this stage of the event selection, the primary di-lepton pair, called Z1, is identified as the one with an invariant mass, $m_{Z 1}$, closest to $m_{Z}^{P D G}=91.1876 \mathrm{GeV}$. Any quadruplets not containing this Z1 are removed. Among the remaining quadruplets, the one with a secondary di-lepton pair, called Z2, closest to $m_{Z}^{P D G}$ is kept, while all others are discarded.

Note that this definition of Z1 and Z2 is what distinguishes the two channels called $2 e 2 \mu$ and $2 \mu 2 e$ - they are named according to which leptons form the primary and secondary di-lepton pairs (in that order). The channels $4 e$ and $4 \mu$ use the same definitions of Z1 and Z2, though their naming introduce less grounds for confusion. The above cuts are applied on each channel separately, so at this stage of the cuts there remains at most one quadruplet per event and per channel.

Cuts are imposed on the di-lepton masses, $m_{Z 1}$ (also called $m_{12}$ ), and $m_{Z 2}$ (also called $m_{34}$ ) as shown in Figure 48. Note that the $m_{Z 2}$ cut depends on the four-lepton invariant mass, $m_{4 \ell}$, because with increasing $m_{4 \ell}, m_{Z 2}$ is increasingly able to be onshell, so a stricter cut can be imposed. For $m_{H}$ near $125 \mathrm{GeV}, m_{Z 2}$ from a signal event can never be on-shell. 

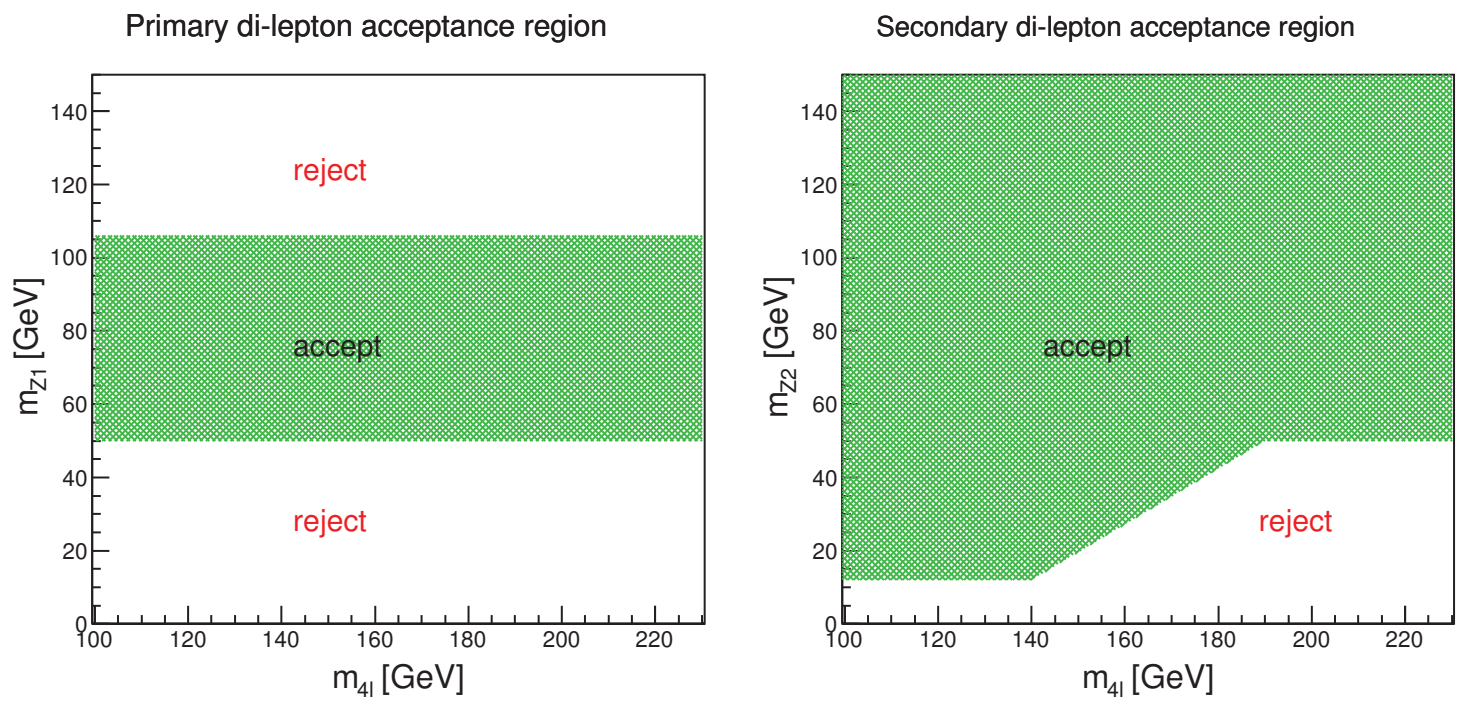

Figure 48: Cuts imposed on the di-lepton masses $m_{Z 1}$ and $m_{Z 2}$. Note that both sets of cuts extend flatly off the plotted range in $m_{4 \ell}$, and that the $m_{Z 2}$ acceptance region is only a lower bound (it extends indefinitely off the plotted vertical range).

\subsection{Additional Criteria}

\subsection{1 $\Delta R$ and cross-pairing}

To reject boosted B-meson decays, quadruplets are required to have for all sameflavour leptons a separation of $\Delta R_{\ell_{i} \ell_{j}}>0.10$, and for all different-flavour leptons a separation of $\Delta R_{\ell_{i} \ell_{j}}>0.20$. Additional possible contamination from B-meson decays is rejected by cutting events where any cross-paired (one lepton from the Z1, the other from the Z2) SFOS di-lepton pair has $m_{\ell \ell}<5 \mathrm{GeV}$.

\subsubsection{Isolation}

To ensure high momentum resolution, and to reject backgrounds primarily from $t \bar{t}$ events, the leptons are required to be isolated from nearby particles in the detector. This isolation requirement is imposed in two steps: track isolation, and calo isolation, which are applied to all four leptons in the quadruplet. 
Track isolation is a measure of nearby tracks to that of the lepton. The $p_{T}$ of tracks within $\Delta R<0.30$ of the lepton is summed (excluding the lepton itself), and expressed as a ratio over the lepton $p_{T}$. A cut is applied requiring $p_{T}^{\text {varcone30 }} / p_{T}^{\text {lepton }}<0.15$ for both electrons and muons. In plainer language, no more than $15 \%$ of the lepton's $p_{T}$ should be found in the $p_{T}$ of other tracks within $\Delta R<0.30$ of the lepton.

Calo isolation applies a similar cut on the $E_{T}$ of clusters within $\Delta R<0.20$ of the lepton cluster. For electrons (muons) the cut is $\left.E_{T}^{\text {topocone20 }} / E_{T}^{\text {lepton }}<0.20(0.30)\right)$. This is read in a similar way: no more than $20 \%$ (30\%) of an electron's (muon's) $E_{T}$ should be found in the $E_{T}$ of other clusters within $\Delta R<0.20$ of the lepton.

\subsection{3 $d_{0}$ significance and vertexing}

A cut is applied to reject displaced vertices by requiring $d_{0} / \sigma_{d_{0}}<3$ for muons and $d_{0} / \sigma_{d_{0}}<5$ for electrons, where $d_{0}$ is defined as in Figure $47 \mathrm{a}$, and $\sigma_{d_{0}}$ is the resolution on that quantity from the track reconstruction.

In addition to this, a simultaneous fit of the four leptons' ID tracks ${ }^{2}$ is performed with a constraint to have come from a common vertex. A cut of $\chi^{2}<5$ is applied to the fit result.

\subsection{FSR recovery}

In a small percentage of events, one of the final-state leptons can emit final-state radiation (FSR), which carries away energy, leading to a loss of $m_{4 \ell}$ resolution. An attempt is made to recover this energy by identifying candidate FSR photons to be re-added to the quadruplet. At most one such photon will be recovered.

Collinear $(\Delta R<0.15)$ photons are recovered only to muons coming from Z1. Electrons can also emit collinear radiation (bremsstrahlung), but this is typically

\footnotetext{
${ }^{2}$ When a quadruplet contains an ME muon, only three ID tracks are used.
} 
caught in the same EM cluster as the electron, and so there is no need to recover it at this stage ${ }^{3}$. A collinear photon is accepted only if alters $m_{Z 1}$ from within $66-89 \mathrm{GeV}$ to $66-100 \mathrm{GeV}$. Where multiple photons exist, the one with the highest $E_{T}$ is used.

When no collinear FSR can be recovered, an attempt is made to recover far FSR $(\Delta R>0.15$ for all leptons in the quadruplet). This is done for both flavours of lepton from both the selected Z1 and Z2. A photon can be recovered only if $m_{4 \ell}<190 \mathrm{GeV}$, and if the recovery alters the di-lepton mass from within $m_{\ell \ell}<81 \mathrm{GeV}$ to $m_{\ell \ell}<100 \mathrm{GeV}$. Where multiple photons exist, the one with the highest $E_{T}$ is used. When separate candidate FSR photons meet these requirements both for Z1 and Z2, the photon which alters the di-lepton mass by the smallest amount is taken.

The invariant mass after the FSR recovery procedure (even if no FSR is recovered) is called $m_{4 \ell}^{\mathrm{fsr}}$, which has a slightly improved resolution compared to $m_{4 \ell}^{\mathrm{reco}}$, to be demonstrated in Section 5.10. This gain in $m_{4 \ell}$ resolution translates directly to the final $m_{H}$ resolution.

\subsection{Z Mass Constraint}

Knowledge of the decay chain $H \rightarrow Z Z^{(*)} \rightarrow 4 \ell$ can be used to gain additional $m_{4 \ell}$ resolution for the final state. For the same reason that the true invariant mass distribution of the Higgs' decay products will follow a Relativistic Breit-Wigner (with to-be-measured parameters $m_{H}, \Gamma_{H}$ ), so too does the decay of any on-shell $Z$ boson in the decay chain. Unlike the Higgs, the parameters of the $\mathrm{Z}$ boson lineshape are very well-known: LEP measured [81] the mass $m_{Z}=91.1875 \mathrm{GeV}$, and the width $\Gamma_{Z}=2.4952 \mathrm{GeV}$ with uncertainty in the final two digits ${ }^{4}$. This known lineshape

\footnotetext{
${ }^{3}$ The electron reconstruction described in Section 4.4 would already have included co-linear FSR inside the cluster.

${ }^{4}$ This uncertainty could in principle be included in the statistical procedure to follow, but because it is so much smaller than individual lepton measurement uncertainties in ATLAS, it would have a
} 
can be used to constrain the measurement uncertainties for leptons coming from an on-shell Z decay, a procedure call the Z Mass Constraint (ZMC) [82].

The mass of the $Z$ boson to di-leptons (called $\ell_{1}, \ell_{2}$ with four-momenta $p_{1}, p_{2}$ ) before detector resolution is taken into account is called the true mass, and can be expressed as

$$
\begin{aligned}
\left(m_{12}^{\text {true }}\right)^{2} & =\left(p_{1}^{\text {true }}+p_{2}^{\text {true }}\right)^{2}, \\
& =p_{1}^{\text {true }}{ }^{2}+p_{2}^{\text {true }}{ }^{2}+2 p_{1}^{\text {true }} \cdot p_{2}^{\text {true }}, \\
& =2 E_{1}^{\text {true }} E_{2}^{\text {true }}\left(1-\cos \theta_{12}^{\text {true }}\right),
\end{aligned}
$$

where $\theta_{12}$ is the relative angle between the two leptons. The final equality applies only in the high energy environment of the LHC, where the invariant mass of an individual electron or muon, $p_{i}^{2}=m_{i}^{2}$, is small enough to neglect, leaving $E_{i} \approx\left|\vec{p}_{i}\right|$. The quantity $m_{12}^{\text {true }}$ is distributed as a BW distribution with parameters $m_{Z}, \Gamma_{Z}$, introduced in (2).

When reconstruction effects - a combination of many stochastic processes - are taken into account, the reco-level $m_{12}$ no longer follows its true distribution. Each of the lepton energies, $E_{i}^{\text {true }}$ are measured to be $E_{i}^{\text {reco }}$ with some resolution $\sigma_{E_{i}}$ (the same is true of the lepton direction, and thus $\theta_{12}$, but this effect is far smaller), which effectively smears out the distribution of $m_{12}^{\text {true }}$ into that of $m_{12}^{\text {reco }}$ (also called $m_{Z 1}$ ). The goal of the ZMC is to use our knowledge of the $P\left(m_{12}^{\text {true }}\right)$ distribution to remove or reduce the effect of measurement uncertainties on the leptons. To do this, we write down a likelihood expression for $m_{12}^{\text {true }}$ (a function of $E_{i}^{\text {true }}$ ) given the observed mass $m_{12}^{\text {reco }}$ (a function of $E_{i}^{\text {reco }}$ ) and resolution $\sigma_{m}$ (a function of $E_{i}^{\text {reco }}, \sigma_{E_{i}}$ ) as vanishingly small effect. 


$$
\begin{aligned}
P\left(m_{12}^{\text {true }} \mid m_{12}^{\text {reco }}, \sigma_{m}\right) & =\frac{P\left(m_{12}^{\text {reco }} \mid m_{12}^{\text {true }}, \sigma_{m}\right) P\left(m_{12}^{\text {true }}\right)}{P\left(m_{12}^{\text {reco }}\right)} \\
& \propto P\left(m_{12}^{\text {reco }} \mid m_{12}^{\text {true }}, \sigma_{m}\right) P\left(m_{12}^{\text {true }}\right) \\
& \propto N\left(m_{12}^{\text {reco }} ; m_{12}^{\text {true }}, \sigma_{m}\right) \mathrm{BW}\left(\mathrm{m}_{12}^{\text {true }} ; \mathrm{m}_{\mathrm{Z}}, \Gamma_{\mathrm{Z}}\right)
\end{aligned}
$$

where the first equality uses Bayes' Theorem [83], and the final proportionality assumes a Gaussian resolution distribution, and substitutes the actual BW distribution for $m_{12}^{\text {true }}$.

By maximizing this likelihood, the constrained Z1 mass is derived:

$$
m_{12}^{\text {cons }} \equiv \underset{m_{12}^{\text {true }}}{\operatorname{argmax}} P\left(m_{12}^{\text {true }} \mid m_{12}^{\text {reco }}, \sigma_{m}\right) .
$$

This mass should be interpreted as the "most likely true mass for this event, given reconstructed quantities", and should not be interpreted as equivalent to $m_{12}^{\text {true }}$. A noteworthy consequence is that $P\left(m_{12}^{\text {cons }}\right) \neq P\left(m_{12}^{\text {true }}\right)$, which can be seen by looking at the distributions of these quantities in simulated data, shown in Figure 49. Note that these distributions are presented in a chronological order: an event occurs (or is generated) with a di-lepton mass $m_{12}^{\text {true }}$, which then passes through the detector and is reconstructed with mass $m_{12}^{\text {reco }}$, then has FSR recovered to become $m_{12}^{\text {fsr }}$, and finally the $\mathrm{ZMC}$ is applied to give $m_{12}^{\text {cons }}$.

Note that in practice it is the lepton energies which are adjusted by this process, such that the invariant mass calculation remains consistent:

$$
\left(m_{12}^{\text {cons }}\right)^{2}=\left(p_{1}^{\text {cons }}+p_{2}^{\text {cons }}\right)^{2}
$$




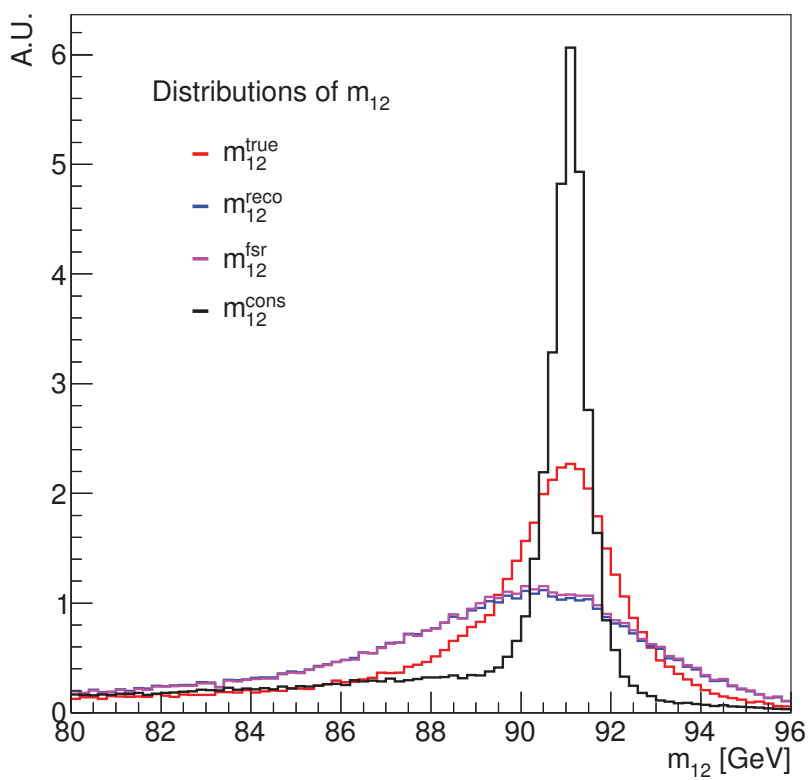

Figure 49: Distributions of $m_{12}^{\text {true }}, m_{12}^{\text {reco }}, m_{12}^{\text {fsr }}$ and $m_{12}^{\text {cons }}$ on simulated signal sample inclusive of production modes with $m_{H}=125 \mathrm{GeV}$.

These same constrained leptons ${ }^{5}$ can then be used to calculate a constrained fourlepton invariant mass

$$
\left(m_{4 \ell}^{\text {cons }}\right)^{2}=\left(p_{1}^{\text {cons }}+p_{2}^{\text {cons }}+p_{3}^{\text {cons }}+p_{4}^{\text {cons }}\right)^{2}
$$

which is used as the ultimate mass discriminant in this thesis. Note that the the momenta $p_{3,4}^{\text {cons }}$ are unchanged from $p_{3,4}$, but the notation is kept consistent with leptons 1,2 to mean after the $Z M C$, even if no change was made in this case.

\footnotetext{
${ }^{5}$ The superscript "cons" should be interpreted to mean the leptons after the ZMC procedure, whether or not a lepton was affected by the constraint. For events around $125 \mathrm{GeV}$, the constraint only makes sense for the leptons from Z1, as only one Z boson can be on shell. At higher masses, approximately above $180 \mathrm{GeV}$, the constraint is applied to the $\mathrm{Z} 1$ and $\mathrm{Z} 2$ pairs, as they could have both come from on-shell $\mathrm{Z}$ boson decays.
} 


\subsection{Invariant mass resolution}

Simulated signal samples are used to verify the effects of the FSR recovery, and the ZMC on the final $m_{4 \ell}$ resolution. To do this, samples with $m_{H}=125 \mathrm{GeV}$ of all SM Higgs production modes are combined and weighted according to their respective cross sections. The $m_{4 \ell}$ distributions in each decay channel for events passing the full selection are compared in the three cases: The $m_{4 \ell}^{\text {reco }}$ distributions with no corrections are shown in Figure 50, the $m_{4 \ell}^{\mathrm{fsr}}$ distributions with FSR recovery are shown in Figure 51, and the $m_{4 \ell}^{\text {cons }}$ distributions with FSR recovery + ZMC are shown in Figure 52. The inclusive distributions (all decay channels merged) are shown for the three cases in Figure 53.

In each plot, the core of the invariant mass distribution ${ }^{6}$ is fit with a Gaussian. Because the samples used have a very narrow width $\left(\Gamma_{H}=4.1 \mathrm{MeV}\right)$, the spread of events is determined almost entirely by detector resolution, and so this Gaussian can be used to quantify the detector resolution. As Chapter 7 will expound greatly on, a simple Gaussian is insufficient to describe the entire resolution, though for now it serves as an adequate first-order approximation. The detailed results of these fits are found in Table 9, and by this method we conclude the FSR recovery brings on average a $1.4 \%$ benefit to the $m_{4 \ell}$ resolution, and the ZMC brings an additional $19 \%$ benefit, as compared by the Gaussian-fit $\sigma$ of the inclusive distribution in each case.

\footnotetext{
${ }^{6}$ The core is defined somewhat arbitrarily by the mean and standard deviation (s.d.) of the distribution to be the range [mean - s.d. , mean + s.d.]
} 


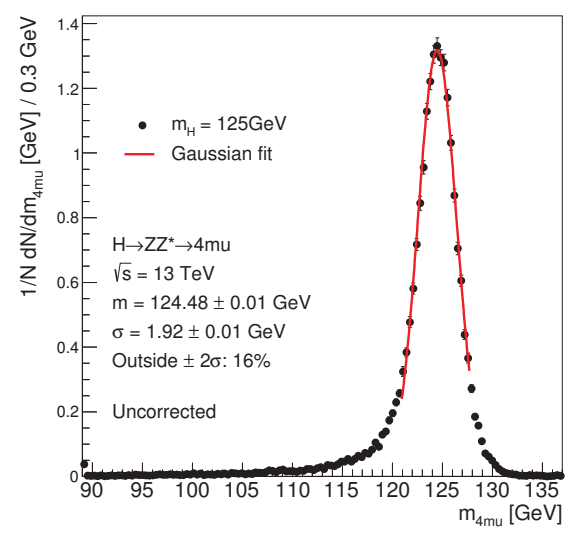

(a) $4 \mu$

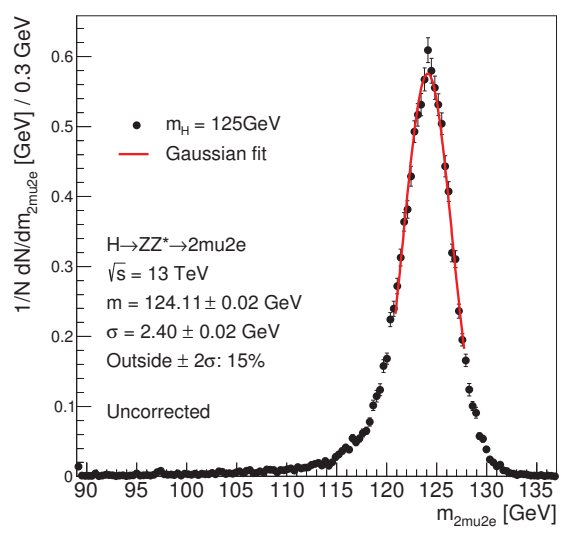

(c) $2 \mu 2 e$

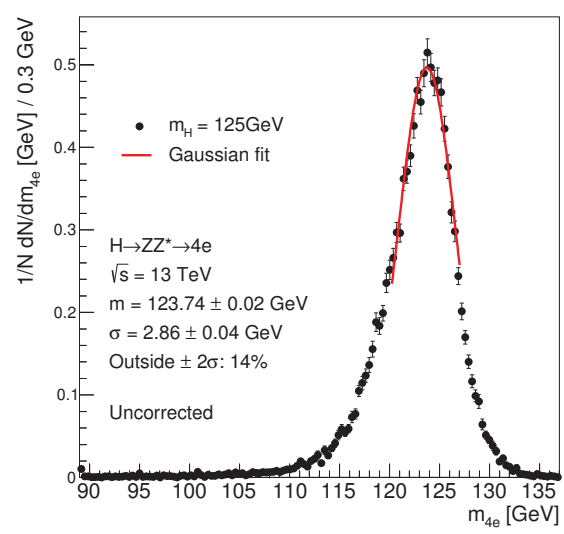

(b) $4 e$

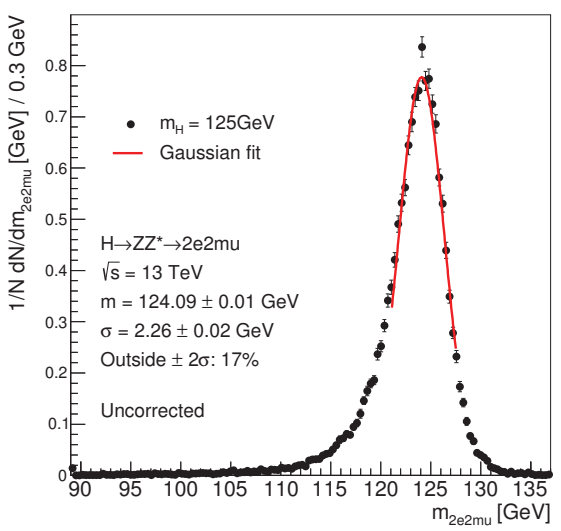

(d) $2 e 2 \mu$

Figure 50: Four-lepton invariant mass distribution and resolution from simulated $m_{H}=125 \mathrm{GeV}$ signal in the four decay channels with no corrections.

Table 9: Summary table of four-lepton invariant mass resolution. Shown are the Gaussian-fit parameters, $\mu$ and $\sigma$, on the invariant mass distribution (i) uncorrected, (ii) with FSR recovery, and (iii) with FSR recovery and Z Mass Constraint applied, as determined on MC simulation at $m_{H}=125 \mathrm{GeV}$.

\begin{tabular}{ccccccc}
\hline & \multicolumn{2}{c}{ Uncorrected } & \multicolumn{2}{c}{ With FSR } & \multicolumn{2}{c}{ With FSR and ZMC } \\
channel & $\mu[\mathrm{GeV}]$ & $\sigma[\mathrm{GeV}]$ & $\mu[\mathrm{GeV}]$ & $\sigma[\mathrm{GeV}]$ & $\mu[\mathrm{GeV}]$ & $\sigma[\mathrm{GeV}]$ \\
\hline $4 \mu$ & 124.48 & 1.92 & 124.55 & 1.88 & 124.80 & 1.55 \\
$4 e$ & 123.74 & 2.86 & 123.78 & 2.82 & 124.46 & 2.29 \\
$2 \mu 2 e$ & 124.11 & 2.40 & 124.20 & 2.37 & 124.40 & 2.17 \\
$2 e 2 \mu$ & 124.09 & 2.26 & 124.11 & 2.24 & 124.78 & 1.65 \\
\hline $4 \ell$ & 124.22 & 2.21 & 124.29 & 2.18 & 124.70 & 1.77 \\
\hline
\end{tabular}




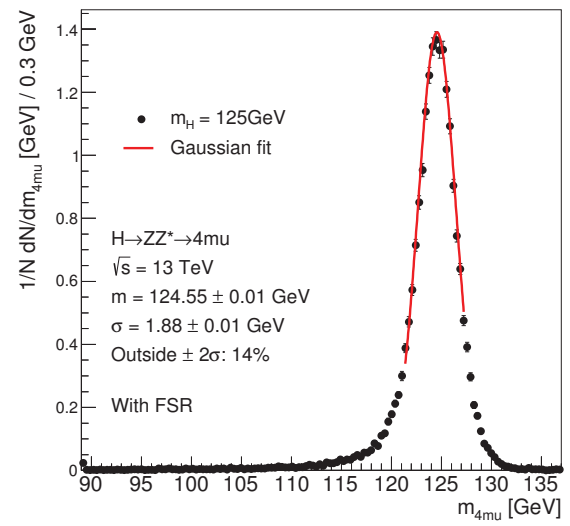

(a) $4 \mu$

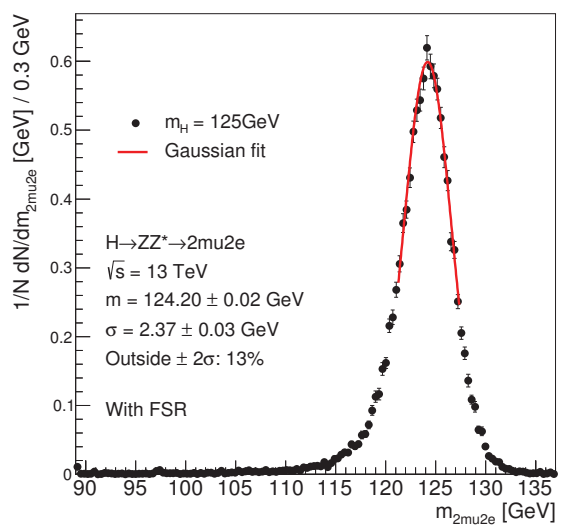

(c) $2 \mu 2 e$

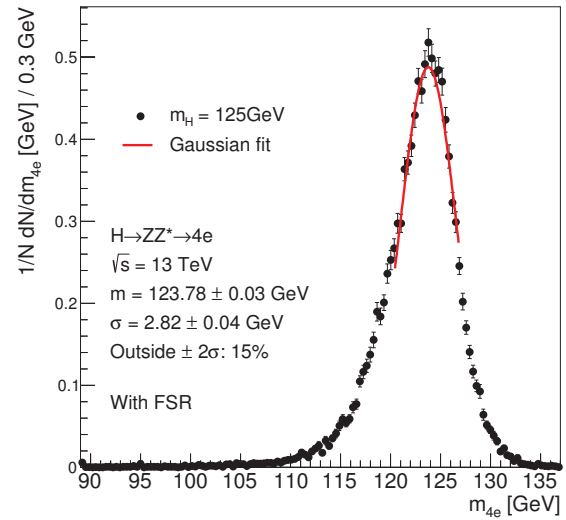

(b) $4 e$

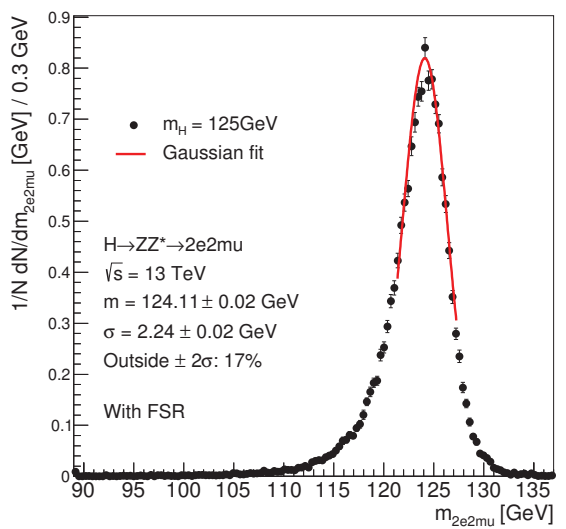

(d) $2 e 2 \mu$

Figure 51: Four-lepton invariant mass distribution and resolution from simulated $m_{H}=125 \mathrm{GeV}$ signal in the four decay channels with FSR recovery. 


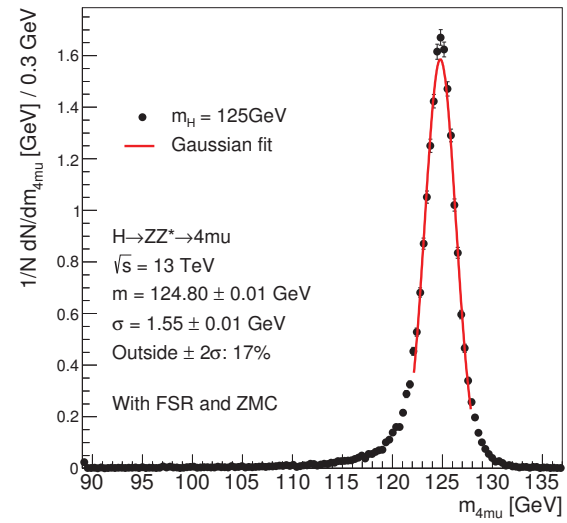

(a) $4 \mu$

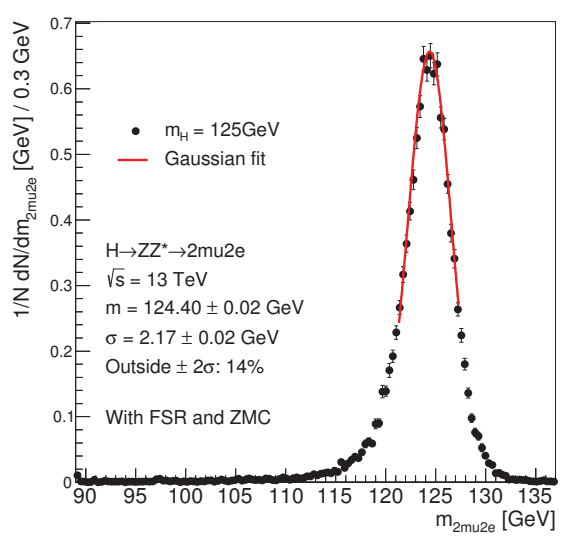

(c) $2 \mu 2 e$

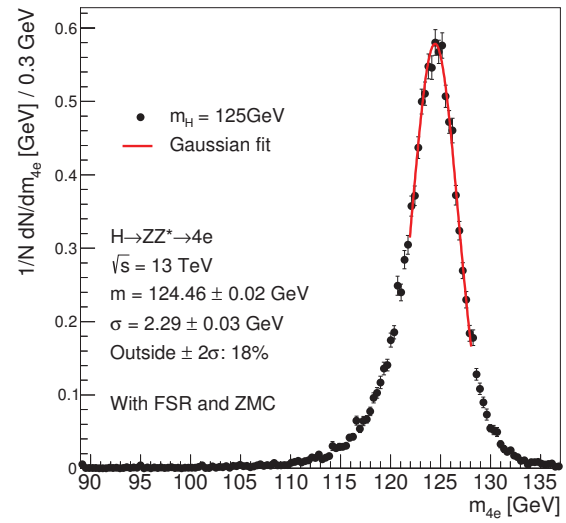

(b) $4 e$

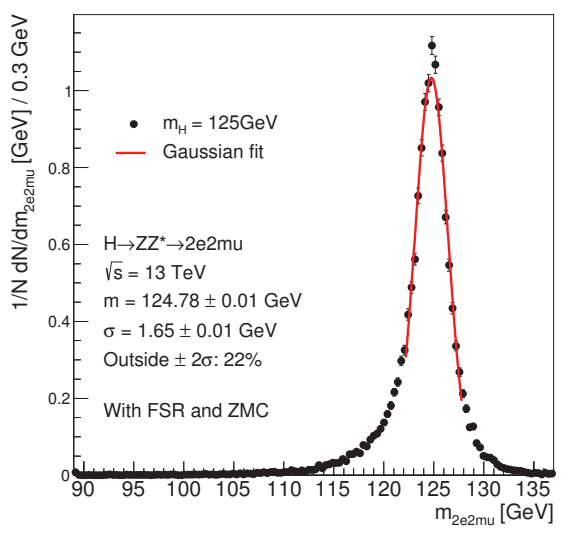

(d) $2 e 2 \mu$

Figure 52: Four-lepton invariant mass distribution and resolution from simulated $m_{H}=125 \mathrm{GeV}$ signal in the four decay channels with FSR recovery and $\mathrm{Z}$ Mass Constraint. 


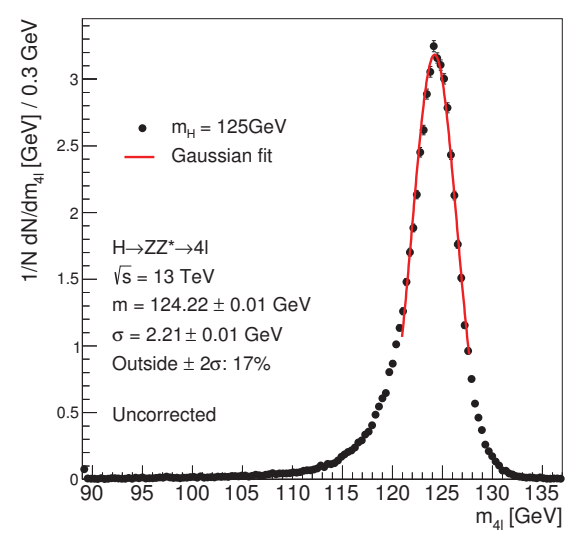

(a) Uncorrected

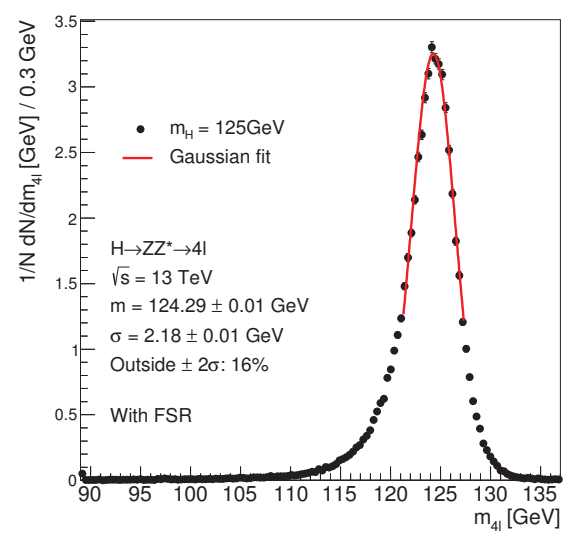

(b) FSR

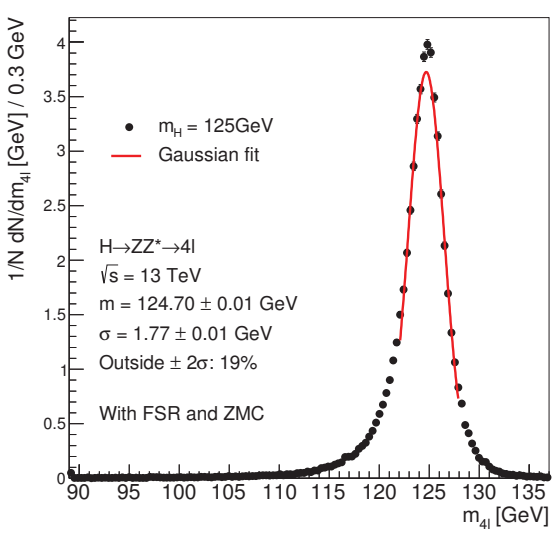

(c) $\mathrm{FSR}+\mathrm{ZMC}$

Figure 53: Four-lepton invariant mass distribution and resolution from simulated $m_{H}=125 \mathrm{GeV}$ signal inclusive of all decay channels, shown without corrections, with FSR recovery, and with FSR recovery + Z Mass Constraint 


\subsection{Analysis categories}

In the following chapters, models for the signal and backgrounds will be described. To enhance sensitivity, the analysis is divided into four categories according to the decay channel: $4 \mu, 4 e, 2 \mu 2 e, 2 e 2 \mu$, where the mixed channels are defined as described in Section 5.6. Included in the definition of each category is a mass window surrounding the Higgs boson mass: $110 \mathrm{GeV} \leq m_{4 \ell} \leq 135 \mathrm{GeV}$. The analysis will be executed using both $m_{4 \ell}^{\mathrm{fsr}}$ and $m_{4 \ell}^{\text {cons }}$, and will use the same mass window in both cases. The mass window is chosen to capture any reasonable signal distribution (very conservatively, say $122 \mathrm{GeV} \leq m_{H} \leq 128 \mathrm{GeV}$ ) with high efficiency. The use of a mass window is purely pragmatic: using an arbitrarily large window puts a greater onus on the background modelling to be described in Chapter 6, without gaining much in return.

This is a good opportunity to distinguish between the two terms channel, and category, which at first may appear interchangeable. The channel is a physics-based concept, defined according to the decay mode of the two $Z$ bosons in the decay chain. In reconstructed events this can't be known with perfect accuracy, but it is labelled according to the best of our knowledge. The category, however, is a statistical concept - nearly all analyses in ATLAS are separated into categories to increase sensitivity of the measurement. This improvement in sensitivity comes when categories are defined so as to separate classes of events with different resolutions, or different signal over background ratios. In this thesis, the analysis categories are defined according to the decay channel, because the decay channel is a reasonably good separator of event resolution as seen in Section 5.10. This concept is further explored in Chapter 7. 


\section{Chapter 6}

\section{Background Estimation and Modelling}

The event selection described in Chapter 5 is designed to identify $H \rightarrow Z Z^{(*)} \rightarrow 4 \ell$ events in ATLAS data. Any events which pass this selection, but did not come from Higgs boson decays, constitute the backgrounds to this analysis. Understanding and describing the backgrounds is essential to measuring the properties of the Higgs signal events.

This chapter is divided into four sections. Section 6.1 describes the backgrounds from SM (non-Higgs) $Z Z^{*}$ production, called irreducible background because the four-lepton final state that gets reconstructed is nearly indistinguishable from signal events. Section 6.2 describes backgrounds from other SM processes producing four (or more) leptons - because of additional final state particles, or different topologies, these are more easily distinguished from signal. Section 6.3 describes backgrounds arising from other particles which fake electrons or muons in the detector; these backgrounds are called reducible, because generally they can be reduced by tighter quality cuts, primarily those in Section 5.3 and Section 5.7. Finally Section 6.4 summarizes the expected yields of each background.

For each background, two aspects are described:

Yield How many events from each source we expect to observe in the data, also called the normalization. 
PDF The Probability Density Function (PDF); the expected distribution of $m_{4 \ell}^{\mathrm{fsr}}$ or $m_{4 \ell}^{\text {cons }}$, defined in Section 5.8 and Section 5.9, respectively, for each source.

These two concepts are modelled independently, but in general they can be linked by other variables (e.g. a systematic uncertainty may affect the yield in an $m_{4 \ell^{-}}$ dependent way, so would be included in both the yield and the PDF). In all cases, the yield and the PDF will depend on the analysis category, as defined in Section 5.11.

\subsection{Standard Model $Z Z^{*}$}

The irreducible background from $\mathrm{SM} Z Z^{*}$ is produced by two main modes: $q \bar{q} \rightarrow Z Z^{(*)}$ (nicknamed qqZZ), and $g g \rightarrow Z Z^{(*)}$ (nicknamed ggZZ), where both Z bosons decay to di-lepton pairs. The leading Feynman diagrams for qqZZ and ggZZ are found in Figure 54 and Figure 55, respectively. Of the two, the qqZZ is dominant by more than an order of magnitude, because of the structure of the interaction. Both productions modes are non-resonant inside the mass window, so form continuums in $m_{4 \ell}$.

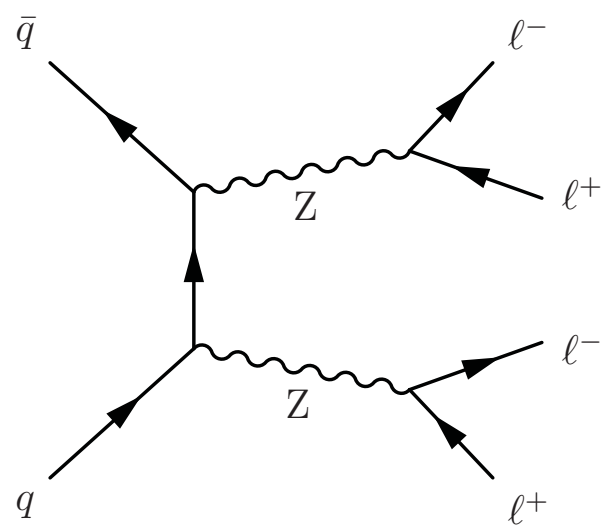

Figure 54: Feynman diagram for $q \bar{q} \rightarrow Z Z^{(*)}$ background.

The yield for qqZZ is estimated using the $100-150 \mathrm{GeV}$ filtered MC simulated 


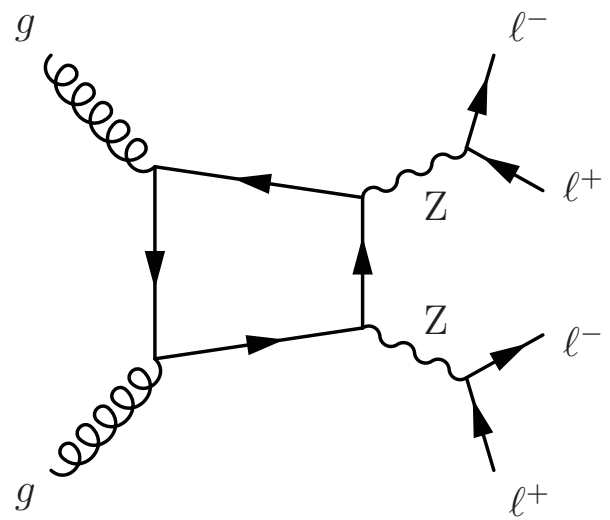

Figure 55: Feynman diagram for $g g \rightarrow Z Z^{(*)}$ background. The intermediate particle (the "box"), not labelled on the diagram, is a quark.

sample, based on the number of events passing the full selection into each category, with the sample weighted according to the SM cross section. The yield for ggZZ is estimated in the same way, using both the $2 \ell 2 \ell$ and $4 \ell g g \rightarrow Z Z^{(*)} \mathrm{MC}$ simulated samples.

The PDFs for qqZZ and ggZZ are built using the same samples as used for the yields. In both cases they are constructed using kernel smoothing (by the class RooKeysPdf [84]) of the simulated data. This smoothing algorithm uses the unbinned data as input, and creates a PDF by summing a Gaussian centered on each datum, with widths dynamically adjusted according to the local event density, and a smoothing parameter $\rho$ (where higher $\rho$ indicates more smooth, and lower $\rho$ indicates less smooth). The algorithm is designed to capture fine structural details in the population distribution where many input data are available, without over-smoothing regions with fewer input data. Furthermore the algorithm includes an option of mirroring the data across the boundaries of the input dataset range ${ }^{1}$. When the input distribution is non-zero at the boundary of the smoothing range, this option helps regulate the output PDF to prevent it from falling to zero ${ }^{2}$.

\footnotetext{
${ }^{1} \mathrm{An}$ as example, in the case that the smoothing range ends at $140 \mathrm{GeV}$, the input data are mirrored such that the resulting PDF from $135 \rightarrow 140$ and from $145 \rightarrow 140$ are identical.

${ }^{2}$ Without input data on the other side of the boundary, events within the smoothing range "spill out" of the boundaries, but no events outside "spill in" to compensate.
} 
The qqZZ background uses $\rho=3.4$, with mirroring enabled. The smoothing range is set to $105 \mathrm{GeV} \leq m_{4 \ell} \leq 140 \mathrm{GeV}$ (a $5 \mathrm{GeV}$ extension on either side of the fit range) to ensure good behaviour near the boundary of the fit range. The qqZZ smoothing results for the constrained mass, $m_{4 \ell}^{\text {cons }}$, are found in Figure 56. The ggZZ uses $\rho=3.0$, and otherwise the same configuration as with qqZZ. The ggZZ smoothing results for the constrained mass are found in Figure 57. The corresponding plots for the unconstrained mass, $m_{4 \ell}^{\mathrm{fsr}}$, are found in Appendix A, as they are not substantially different.

At lower masses, outside of the fit window for the $m_{H}$ analysis, $q \bar{q} \rightarrow Z Z^{(*)}$ can be resonant at the $\mathrm{Z}$ mass through the diagram shown in Figure 58 (the same diagram contributes inside the mass window, but to a smaller extent). This diagram enters the event selection when both bosons decay to di-lepton pairs. This diagram forms a peak in the $m_{4 \ell}$ spectrum around $m_{4 \ell}=m_{Z}$, and will be exploited later in Section 9.5. 


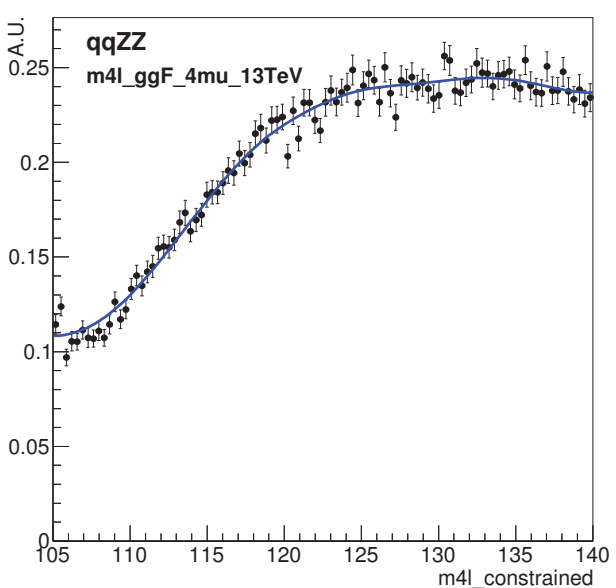

(a) $4 \mu$

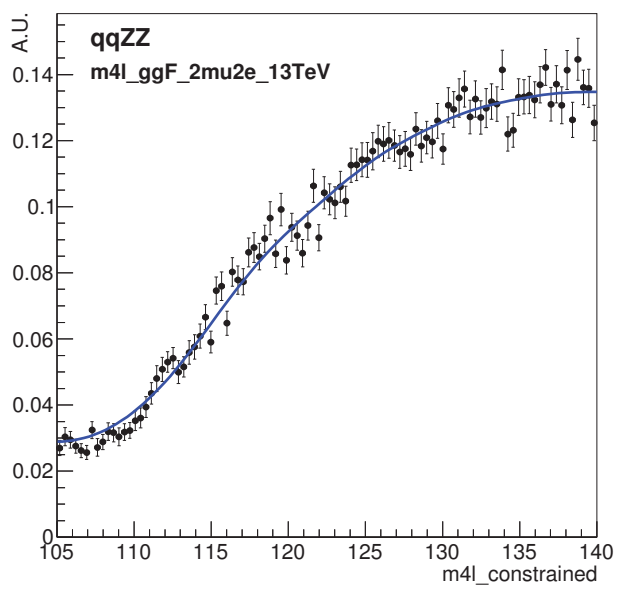

(c) $2 m u 2 e$

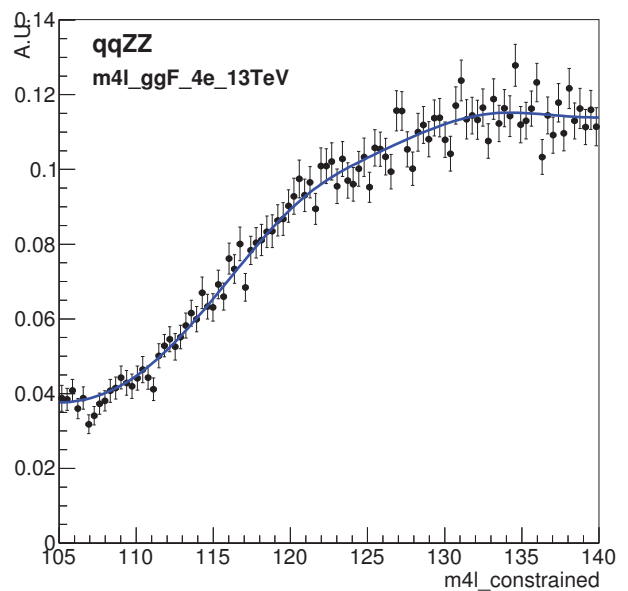

(b) $4 e$

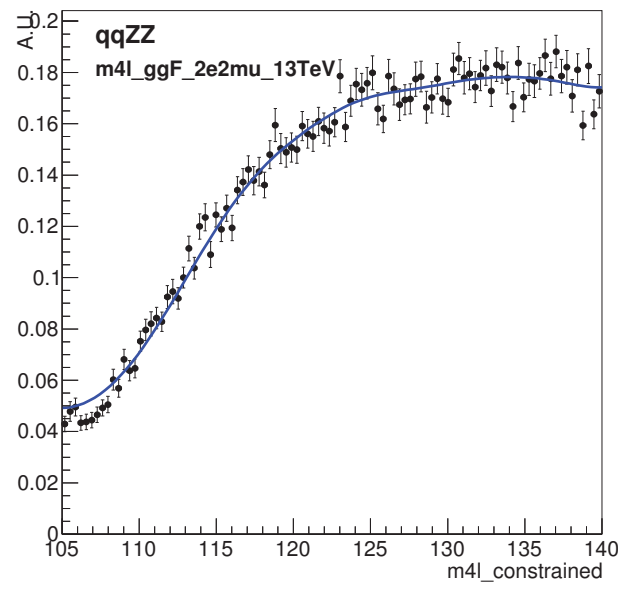

(d) $2 e 2 m u$

Figure 56: Smoothed distribution of $m_{4 \ell}^{\text {cons }}$ from simulated $q \bar{q} \rightarrow Z Z^{(*)}$ events. The black points represent the simulation, the solid blue line is the kernel smoothing result used as the PDF. 


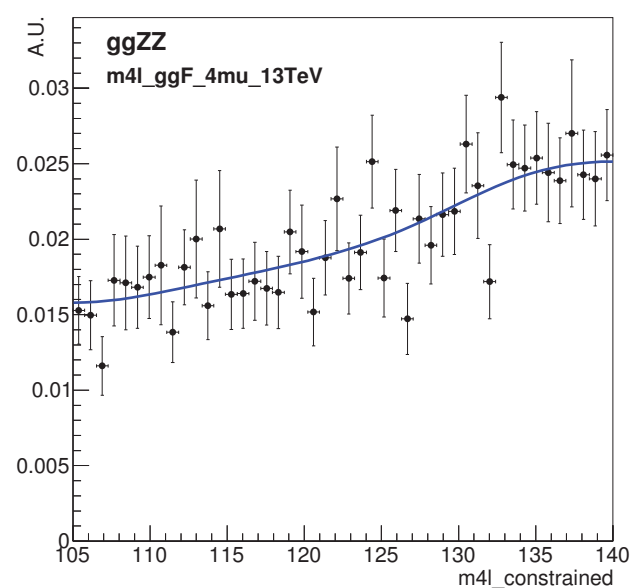

(a) $4 \mu$

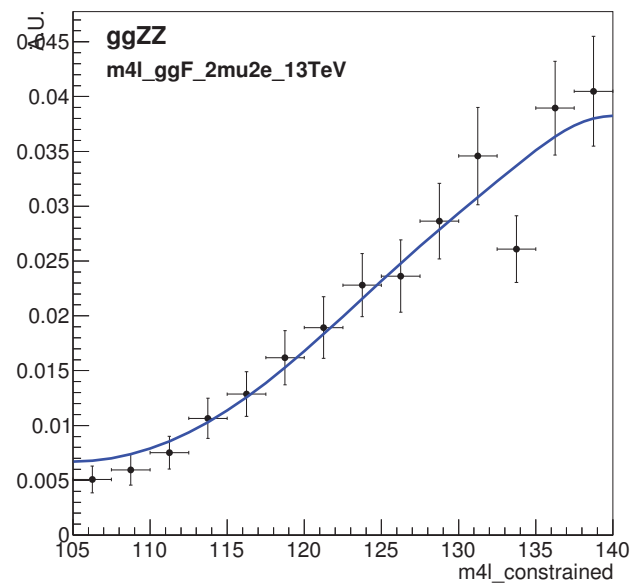

(c) $2 m u 2 e$

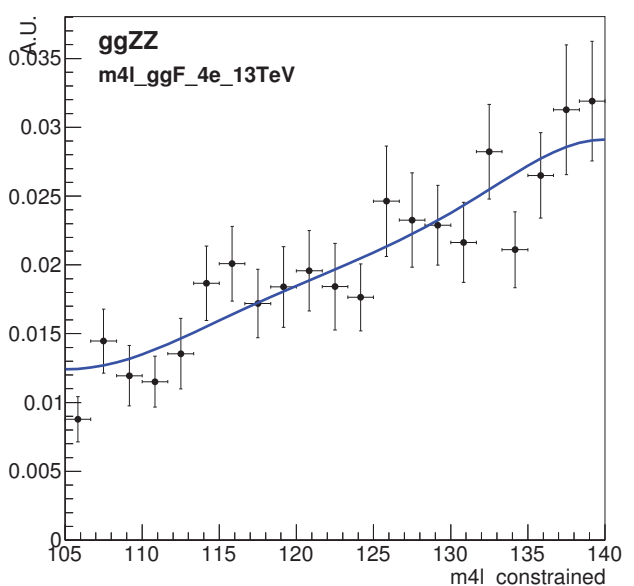

(b) $4 e$

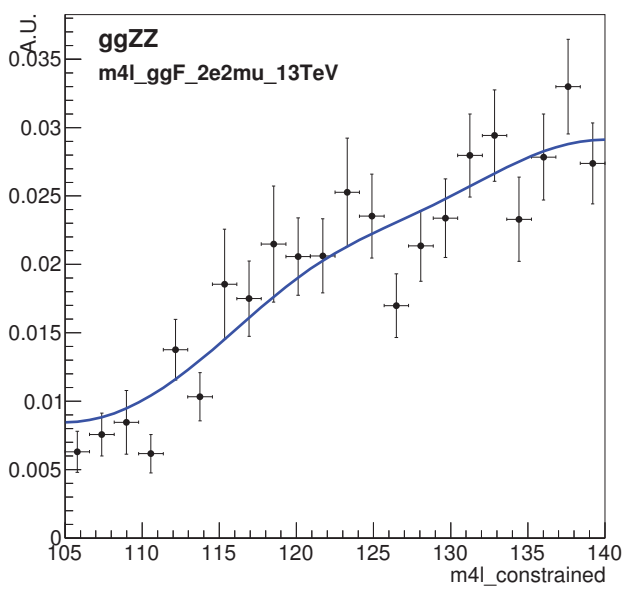

(d) $2 e 2 m u$

Figure 57: Smoothed distribution of $m_{4 \ell}^{\text {cons }}$ from simulated $g g \rightarrow Z Z^{(*)}$ events. The black points represent the simulation, the solid blue line is the kernel smoothing result used as the PDF. 


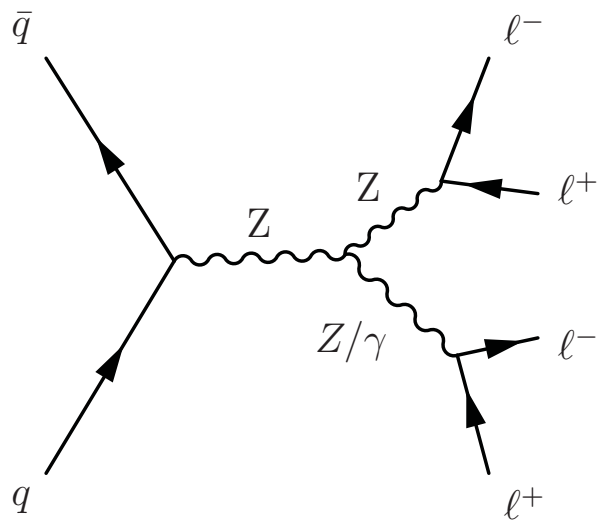

Figure 58: Feynman diagram for resonant $q \bar{q} \rightarrow Z Z^{(*)}$ background. This diagram is most important near $m_{4 \ell}=m_{Z}$ where it forms a resonance.

\section{$6.2 \quad V V V, t \bar{t}+Z$}

The background nicknamed VVV has two main components. The first is tri-boson production, $V V V$ (meaning $Z Z Z, Z Z W, Z W W$ ). When some combination of the vector bosons decay leptonically, these events can enter the event selection. Examples of the leading diagrams for each are shown in Figure 59. The diagrams shown here are meant to be instructive but not complete - a plethora of diagrams with the bosons and leptons swapped into different configurations also contribute.

The second background grouped under the nickname VVV is $t \bar{t}+Z$. The leading diagrams for this process are shown in Figure 60.

The yield from both these processes is estimated together using MC simulated samples for each, weighted according to their SM cross sections. The PDF is built using kernel smoothing of the sample samples, with $\rho=2.8$, mirroring enabled, and the smoothing range extended $5 \mathrm{GeV}$ beyond the fit range, as was done for the SM $Z Z^{*}$ backgrounds. The results when smoothing $m_{4 \ell}^{\text {cons }}$ are shown in Figure 61, while $m_{4 \ell}^{\mathrm{fsr}}$ are found in Appendix A. 

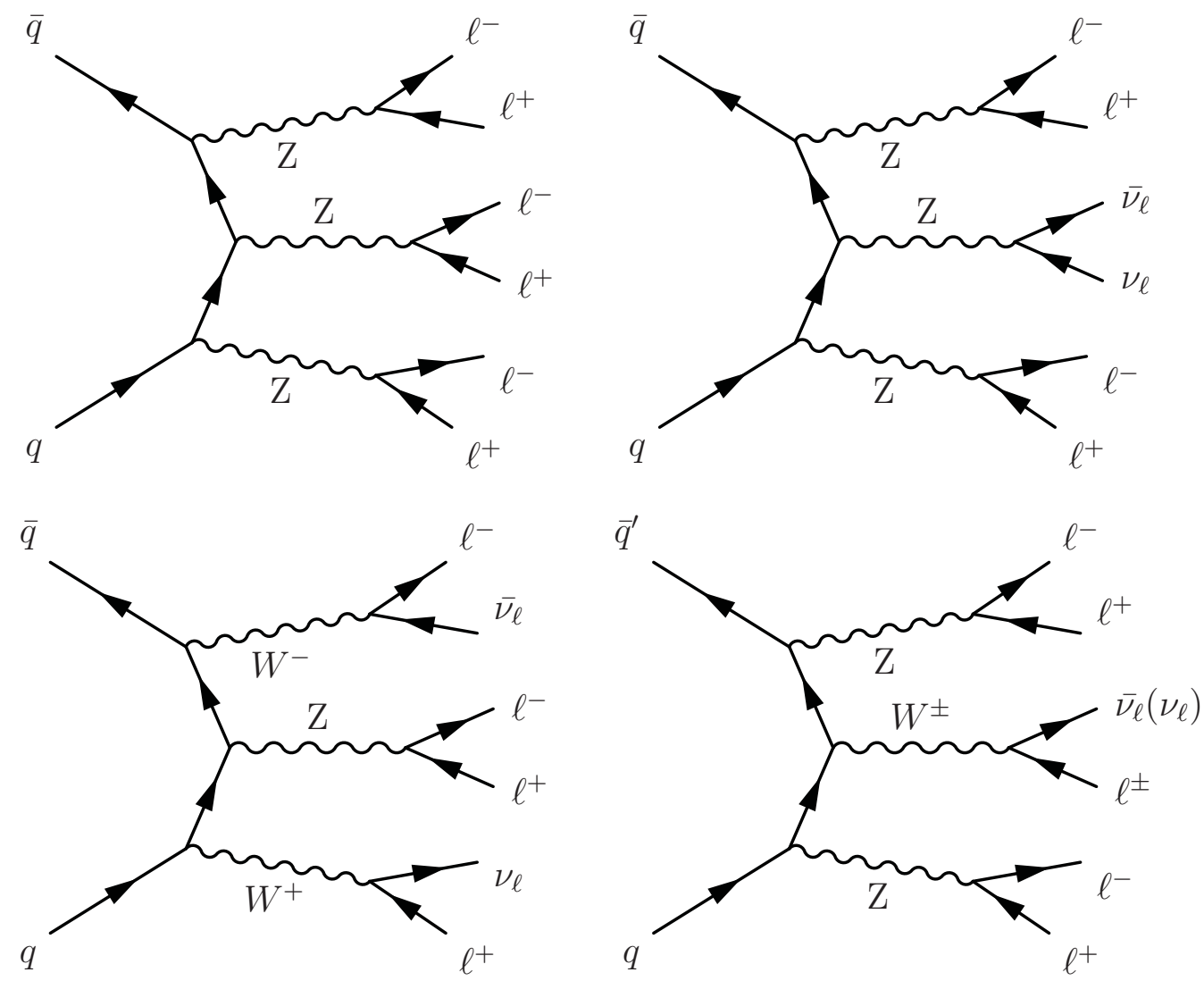

Figure 59: Feynman diagrams for tri-boson background.
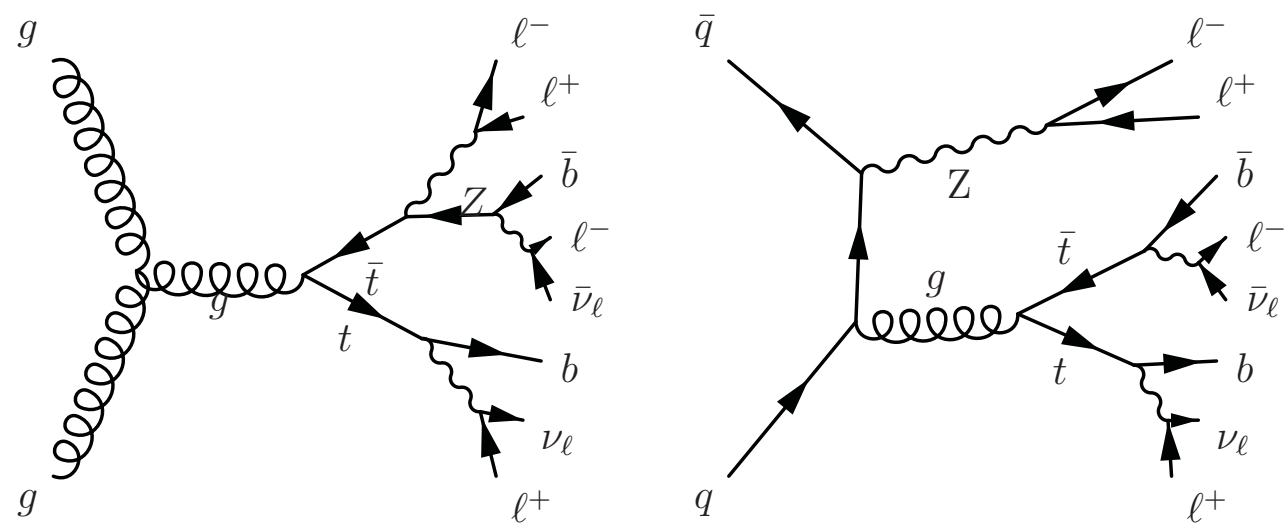

Figure 60: Feynman diagram for $t \bar{t}+Z$ background. For both diagrams, the top pair decays as $t \rightarrow W^{+} b, \quad \bar{t} \rightarrow W^{-} b$ (and then $W \rightarrow \ell \nu$ ), but it is left unlabelled to reduce clutter. In the analysis, this process is grouped together with $V V V$ under the same label. 


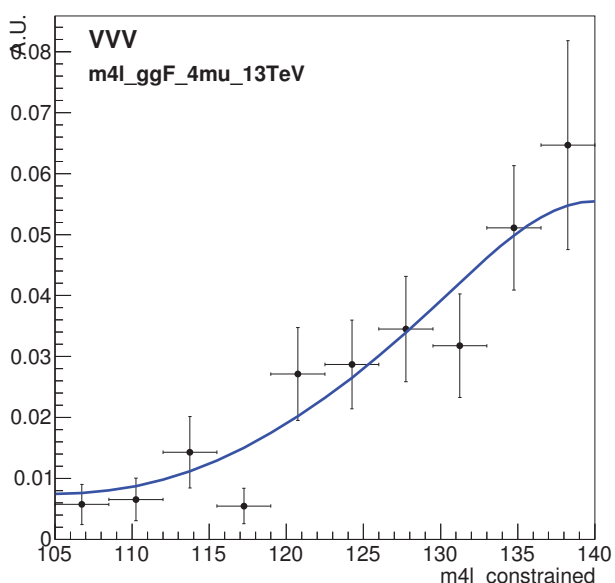

(a) $4 \mu$

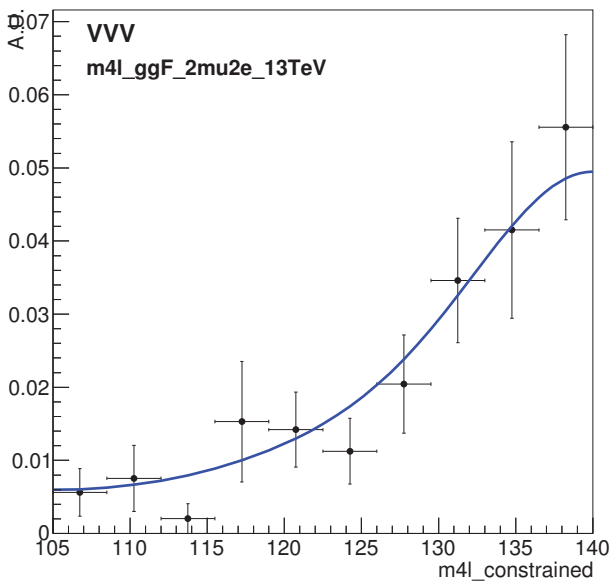

(c) $2 m u 2 e$

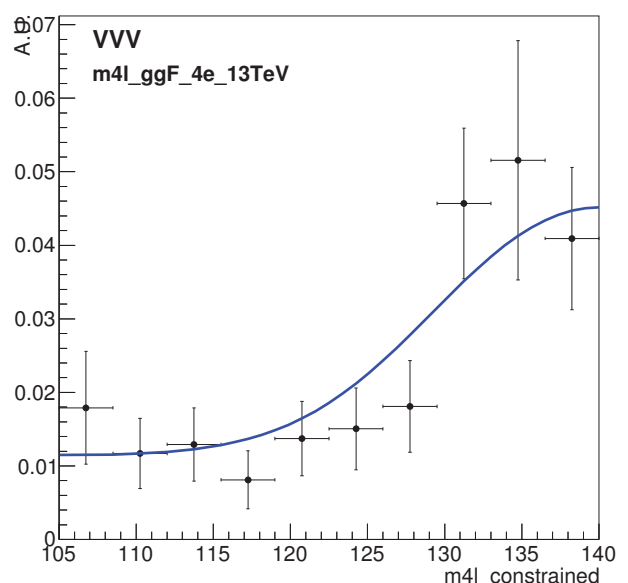

(b) $4 e$

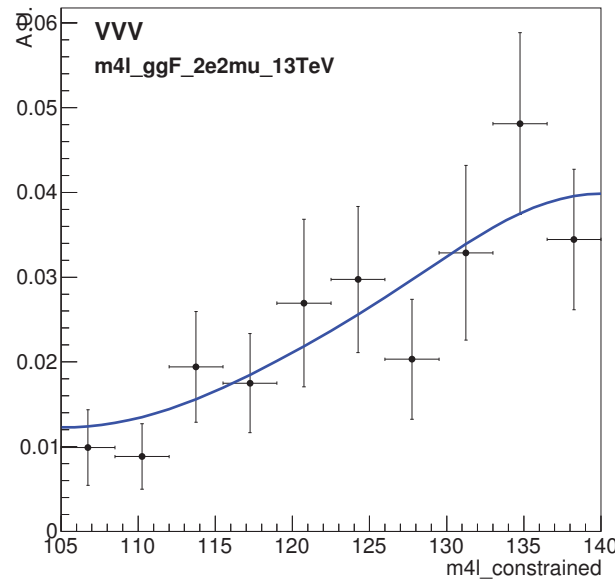

(d) $2 e 2 m u$

Figure 61: Smoothed distribution of $m_{4 \ell}^{\text {cons }}$ from simulated $V V V, t \bar{t}+Z$ events. The black points represent the simulation, the solid blue line is the kernel smoothing result used as the PDF. 


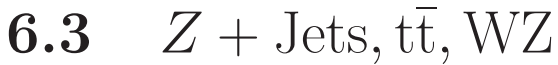

The background $\mathrm{Z}+$ Jets, $t \bar{t}$ and $W Z$ is collectively nicknamed $\mathrm{Z}+$ Jets, for its dominant component. It is the most challenging background entering the analysis, because it is composed of other particles faking prompt electrons and muons ${ }^{3}$. These processes are often difficult to simulate, and quite rare, such that the MC simulation-based approach used for the other backgrounds can not be used. For these reasons, Z+Jets is the only background in this thesis whose yield is estimated from data. Because the estimation of fake electrons differs from that of fake muons, the two are treated separately: Section 6.3.1 describes the so-called $Z+e e$ yield estimation for events containing fake electrons (entering the $4 e$ and $2 \mu 2 e$ categories), and Section 6.3.2 describes the same for fake muons called $Z+\mu \mu$ (entering the $4 \mu$ and $2 e 2 \mu$ categories). More detail is presented for the $Z+e e$ estimation than for $Z+\mu \mu$, as the author was directly involved in the former, but not the latter. The strategy and statistical tools for the $Z+e e$ estimation were developed by the author in collaboration with Ioannis Nomidis and William Leight, while the final execution (estimates, plots), were performed by Ioannis Nomidis. Section 6.3.3 describes how the PDF for the Z+Jets backgrounds is built. Note that the yield estimation described in these sections is inclusive of $m_{4 \ell}^{\text {cons }}$; the final $\mathrm{Z}+$ Jets yield used in the analysis is derived by integrating the inclusive yield, distributed according to the PDF, from $110 \mathrm{GeV}-135 \mathrm{GeV}$.

\subsection{1 $Z+e e$}

The electron background estimation is separated by the source of the fake electrons. While the final statistical analysis is unconcerned with the source of the fake electrons (only their number matters), understanding the composition of the background provides more confidence in its estimation, and is important for constructing the $\mathrm{Z}+\mathrm{J}$ Jets

\footnotetext{
${ }^{3}$ This includes non-prompt, but real-electrons and muons, which did not originate from the HS event.
} 
PDF. The different sources of fake electrons are labelled as follows, ordered from most-to-least abundant:

$\boldsymbol{f} \quad$ Light jets. These are initiated by 1 st or 2 nd generation quarks, or gluons, where in certain cases the calorimeter shower profile can be reconstructed as an electron.

$\gamma \quad$ Events where a photon converts via $\gamma \rightarrow e^{+} e^{-}$. In cases where the tracks are not separately reconstructed, and the EM showers overlap, these can be mistakingly reconstructed as a single electron or positron.

$\boldsymbol{q} \quad$ Heavy quarks, when they decay semi-leptonically, can produce real electrons that enter the event selection. These are still grouped together with the fake electrons because they are produced non-promptly (not an immediate product of the HS event) . Unlike the other two sources, the $q$ background is estimated from MC.

In $\mathrm{MC}$ simulation, the true origin of any electron (real or fake) is known from its truth information, but in data the composition must be estimated ${ }^{4}$. To estimate the composition, a variable called $n_{\text {InnerPix }}$ is used. It is defined as the number of pixel hits on the inner-most (closest to the beam-line) active pixel layer. In some cases the physically inner-most pixel modules are non-functioning, and the second layer is used. This variable is a powerful discriminant between $f$ and $\gamma$, because each occupy mostly different bins of the $n_{\text {InnerPix }}$ distribution.

To estimate the background in the Signal Region (SR) (i.e. the background which passes the full event selection), several Control Regions (CR) are used. Conceptually, the procedure begins "farther" from the signal region, and moves "closer" until an estimate in the $\mathrm{SR}$ is derived.

\footnotetext{
${ }^{4}$ The effective composition of individual electrons could be estimated too, but this is already done by the electron selection likelihood in Section 5.3.1. The working point used in that section deliberately allows some fake electrons to pass, as the necessary sacrifice to have high signal efficiency.
} 


\subsubsection{1 $Z+X$ Control Region}

First, a $\mathrm{CR}$ called $Z+X$ is built in simulation and data. The selection for this $\mathrm{CR}$ proceeds similarly to the regular event selection, except,

- Only the single-electron and di-electron triggers of Table 8 are used, to mitigate selection bias on additional leptons in the event.

- Only a single di-lepton pair, called Z1, is required. It has all the same selection requirements as imposed in the main selection, including those of Section 5.7 modified for only two leptons. The only alteration is that a mass window cut of $76 \mathrm{GeV}<m_{Z 1}<106 \mathrm{GeV}$ is applied to be more confident a real $Z$ boson was selected for the Z1.

- In addition to the Z1 pair an additional electron, called an $X$-electron, is required. This electron is not required to pass the likelihood criteria, or the ID hit requirements of Section 5.3.1. The only track quality cuts imposed are on the total number of pixel and SCT hits, and the $d_{0}$ significance cut (described in Section 5.7.3) . The isolation cuts of Section 5.7.2 are also removed for this electron.

The events passing the $Z+X$ selection in MC simulated are used to build templates of the $n_{\text {InnerPix }}$ distribution for $\gamma$ and $f$ fake electrons. These expected distributions from simulation are shown in Figure 62.

In practice, the variable $n_{\text {InnerPix }}$ is not perfectly modelled by simulation. To account for this, a data/MC comparison in the $Z+X \mathrm{CR}$ is used to correct the template for $f$. Since $f$ is the leading contributor (by far) to these events, only the $f$ template is corrected by this procedure. Figure 63 shows the comparison of data and $\mathrm{MC}$ used to derive the correction. Included in this plot is the contribution from real electrons (combining non-prompt from $q$, and prompt electrons) from simulation. 


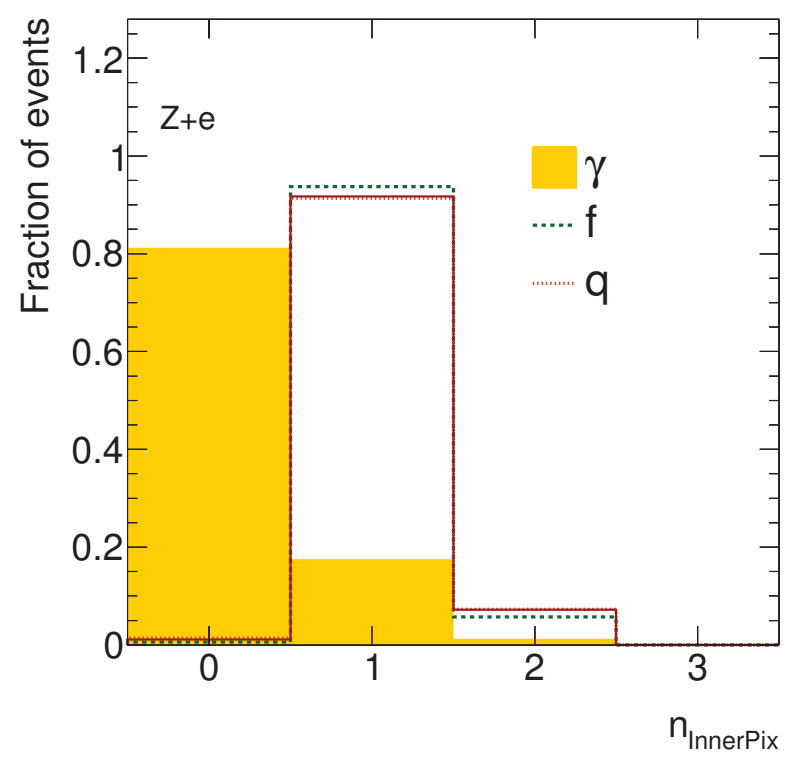

Figure 62: Templates of the $n_{\text {InnerPix }}$ distribution for $\gamma$ and $f$-classified fake electrons built using the $Z+X \mathrm{CR}$ in simulation. The template for real electrons (from $\left.Z Z^{*}\right)$ follows the exact same shape as for $q$. This plot was provided by Ioannis Nomidis.

The truth information is used to classify the electrons in simulation.

Next, the $Z+X \mathrm{CR}$ is divided into the $f$-enriched, and $\gamma$-enriched $Z+X$ CRs by requiring $n_{\text {InnerPix }}=0$ and $n_{\text {InnerPix }} \geq 1$, respectively. After these selections, the purity of the $X$-electrons, defined here as $N_{\gamma} / N_{X}$ in the $\gamma$-enriched CR, and $N_{f} / N_{X}$ in the $f$-enriched CR, is measured in simulation as $90 \%$ and $98 \%$ in the two CRs, respectively, indicating they are highly pure samples.

The data events in these enriched-CRs are used to determine the efficiency of the $X$-selection (defined, somewhat paradoxically, as the efficiency of the cuts that were not imposed on the $X$-electron) in data for $f$ and $\gamma$. Like the template distributions, the yield must also be corrected for data/MC discrepancies. A scale-factor is defined for each species as:

$$
N_{f(\gamma)}^{\text {data }}=s_{f(\gamma)} N_{f(\gamma)}^{\mathrm{MC}}, \quad N_{f(\gamma)}^{\text {data,pass X }}=s_{f(\gamma)}^{\text {pass } \mathrm{X}} N_{f(\gamma)}^{\text {MC,pass X }},
$$




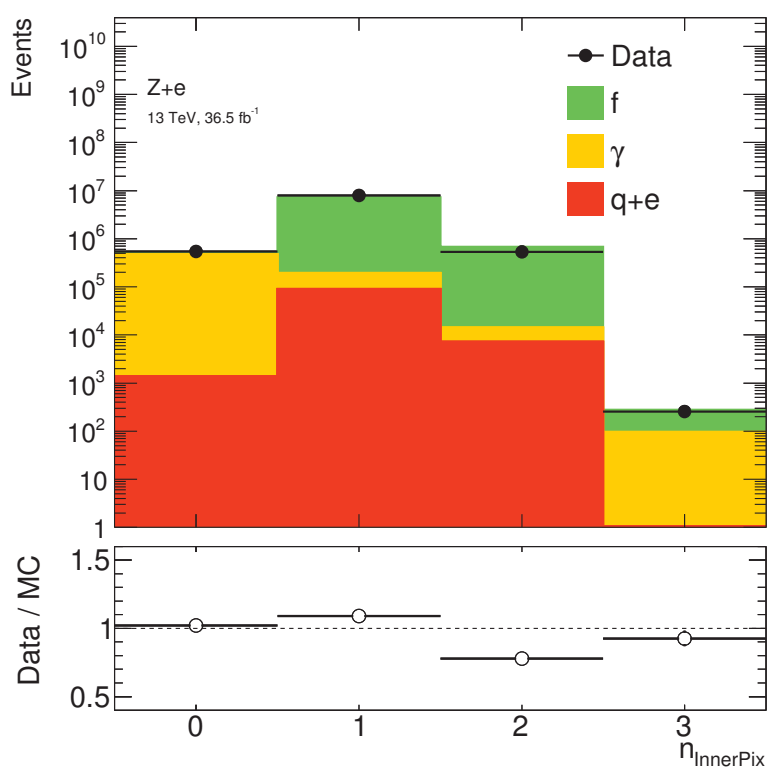

Figure 63: Comparison of data and MC simulation of events passing the $Z+X \mathrm{CR}$ selection. The distribution is simulation is separated by the origin of the $X$ electron as determined by the MC truth information. The difference between the data and simulation is used to correct the $n_{\text {InnerPix }}$ template for $f$ electrons. This plot was provided by Ioannis Nomidis.

where $N_{f(\gamma)}^{\mathrm{data}(\mathrm{MC})}$ is the yield in data (MC) of $\gamma(f)$ electrons, $s_{f(\gamma)}$ denotes the data/MC scale factor to be determined for source $f(\gamma)$, and "pass X" denotes electrons which pass the $X$-selection (like those in the SR). Of course, the yield of a given species of background can't be determined exactly in data - it must be measured. This is done through an iterative procedure using the expected yields in MC simulation of each species of background. The two-step iteration starts by determining $s_{\gamma}$ from the $\gamma$-enriched $Z+X$ CR by subtracting from data the expected yields of $q+e$ and $f$,

$$
s_{\gamma}=\frac{N^{\text {data }}-N_{q+e}^{\mathrm{MC}}-N_{f}^{\mathrm{MC}}}{N_{\gamma}^{\mathrm{MC}}},
$$

and then using this value of $s_{\gamma}$, determines $s_{f}$ from the $f$-enriched $Z+X \mathrm{CR}$,

$$
s_{f}=\frac{N^{\text {data }}-N_{q+e}^{\mathrm{MC}}-s_{\gamma} N_{\gamma}^{\mathrm{MC}}}{N_{f}^{\mathrm{MC}}},
$$


and then re-evaluates $s_{\gamma}$, by using the updated $s_{f}$,

$$
s_{\gamma}=\frac{N^{\text {data }}-N_{q+e}^{\mathrm{MC}}-s_{f} N_{f}^{\mathrm{MC}}}{N_{\gamma}^{\mathrm{MC}}} .
$$

This process is repeated three times (terminating after the third calculation of $s_{\gamma}$ ), which is sufficient for the estimate to stabilize to fluctuations far below the systematic uncertainties assigned to the final estimate. The same iterative procedure is used to calculate $s_{f(\gamma)}^{\text {pass X }}$ from events passing the $X$-selection.

Finally the efficiency for the $X$-selection can be calculated as

$$
\epsilon_{f(\gamma)}=\frac{N_{f(\gamma)}^{\text {data,pass X }}}{N_{f(\gamma)}^{\text {data }}}=\frac{s_{f(\gamma)}^{\text {pass X }} N_{f(\gamma)}^{\mathrm{MC}, \text { pass X }}}{s_{f(\gamma)} N_{f(\gamma)}^{\mathrm{MC}}}
$$

In fact, this efficiency is calculated in three separate $p_{T}$ bins, to account for the varying $X$-selection efficiency as a function of $p_{T}$. These efficiencies are shown in Figure 64 for two different MC generators. The resulting efficiencies, averaged over $p_{T}$, will be recorded in Table 10, though the following section is necessary to understand the remainder of the table.

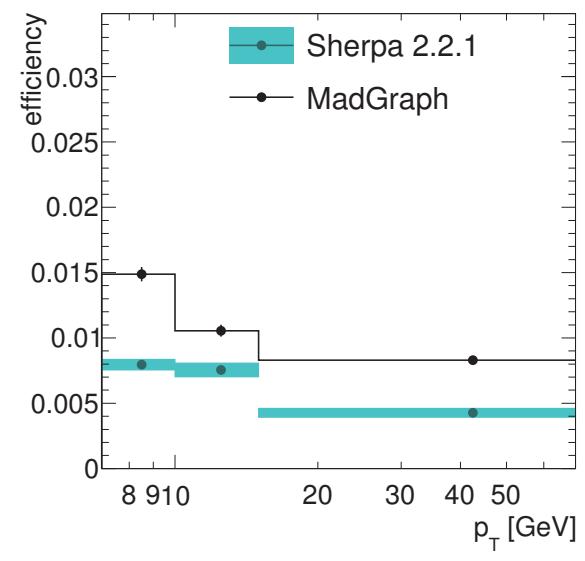

(a) $\gamma$

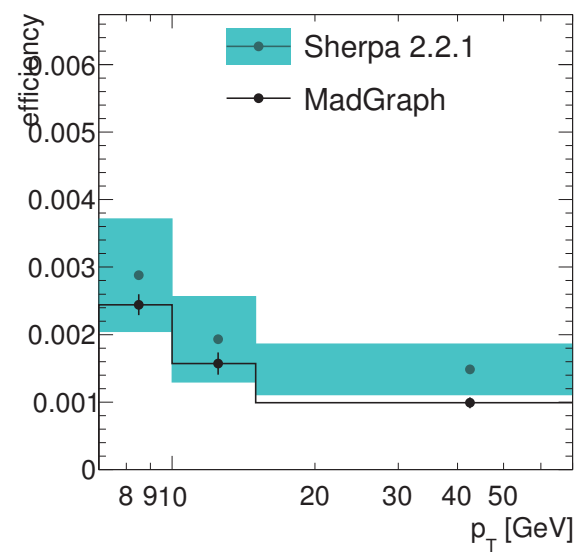

(b) $f$

Figure 64: Final X selection efficiencies as a function of $p_{T}$ of $\gamma$ and $f$ fakes, produced using two different generators of the MC simulated samples. These plots were provided by Ioannis Nomidis. 


\subsubsection{2 $3 \ell+X$ Control Region and Extrapolation to the Signal Region}

A second $\mathrm{CR}$, called $3 \ell+X$, is built in simulation and data. This CR is more closely related to the SR selection described in Chapter 5, having all the same selection, except:

- The lowest- $E_{T}$ electron of the Z2 pair has the exact same relaxed requirements as in the $Z+X$ control region. This electron is the $X$ in $3 \ell+X$.

- For the Z2 pair, only same-sign $\left(e^{ \pm} e^{ \pm}\right)$pairs are considered. This requirement is designed to reject most of the contribution from $Z Z^{*}$, though even after this cut about $5 \%$ of the events in the $\mathrm{CR}$ are from $Z Z^{*}$. These surviving events occur when a fake lepton accompanies the $Z Z^{*}$ event, and replaces one of the true leptons in the selection.

- To consider fakes occurring in any potential Z2 candidate, multiple quadruplets which share the same Z1 are allowed to pass the selection.

The key assumption of this background estimation technique is that the $X$ in $3 \ell+X$ and the $X$ in $Z+X$ share the properties. This was tested by comparing the $n_{\text {InnerPix }}$ distributions of $X=f, \gamma, q$, respectively, for the $Z+X$ selection and $3 \ell+X$ selection using MC simulated samples, where the PDFs of $n_{\text {InnerPix }}$ were found to agree.

The data distribution of $n_{\text {InnerPix }}$ in the $3 \ell+X$ CR was fit for the composition of $N_{f}, N_{\gamma}$ using the data-corrected templates built using the $Z+X \mathrm{CR}$. This fit is shown in Figure 65. After the fit, the expected yields of $Z Z^{*}$ and $q$ as determined from the $3 \ell+X \mathrm{CR}$ in simulation are subtracted from the fit results. The remainder is the yield of $f, \gamma$ in the $3 \ell+X$ CR.

The final step is to extrapolate these yields to the SR using the efficiencies derived from the $Z+X \mathrm{CR}$. To account for the $p_{T}$-dependence of the efficiencies, the tool 


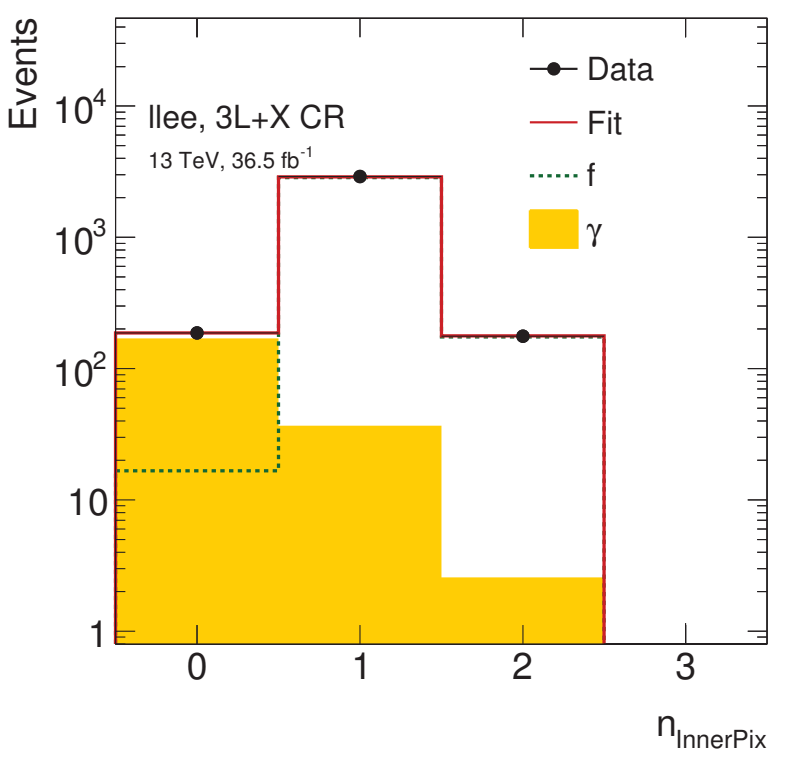

Figure 65: Fit on the $n_{\text {InnerPix }}$ distribution in the $3 \ell+X$ CR in data. The fit templates are built from the $Z+X \mathrm{CR}$.

sPlot [85] is used to unfold the decomposed $n_{\text {InnerPix }}$ distributions into decomposed $p_{T}$ distributions, according to actual 2D distribution in data of $n_{\text {InnerPix }}$ and $p_{T}$ in the $3 \ell+X \mathrm{CR}$. This tool works by fitting the $n_{\text {InnerPix }}$ distribution for the relative contributions from each source, and then projecting this fit result across the observed $2 \mathrm{D}$ data distribution of $n_{\text {InnerPix }}$ and any other variable (in this case $p_{T}$ ). This decomposed $p_{T}$ spectrum is multiplied by the $p_{T}$-dependent efficiencies to produce the final estimate for each background in the SR. Table 10 shows the yield estimates fit in the $3 \ell+X \mathrm{CR}$, and extrapolated to the SR. The efficiencies quoted in the table are averaged over $p_{T}$.

\subsection{2 $Z+\mu \mu$}

Much like the electron background, the background from muons arises from multiple sources. The dominant source is from Z bosons accompanied by heavy flavour hadrons (e.g. B-mesons) which decay semi-leptonically, called Z+HF. Also contributing are 
Table 10: Summary table for reducible electron background estimate. The final yields in the SR are quoted with statistical uncertainty propagated from the fit $3 \ell+X$ fit, and systematic uncertainty from the efficiency estimate. For the background from heavy flavour, the uncertainty is not separated.

\begin{tabular}{cccccc}
\hline type & data fit & $Z Z^{*}+q$ & $N_{i}^{3 \ell+X}$ & efficiency [\%] & $N_{i}^{S R}$ \\
\hline$f$ & $3075 \pm 56$ & $280 \pm 6$ & $2795 \pm 56$ & $0.22 \pm 0.01$ & $5.68 \pm 0.36 \pm 1.19$ \\
$\gamma$ & $208 \pm 17$ & $19.4 \pm 0.5$ & $179 \pm 17$ & $0.72 \pm 0.03$ & $1.34 \pm 0.35 \pm 0.27$ \\
$q$ & & estimated from simulation & $5.7 \pm 1.7$ \\
\hline
\end{tabular}

muons coming from light jets via $\pi^{ \pm} / K^{ \pm} \rightarrow X+\mu^{ \pm}$, as well as $t \bar{t}$, and $W Z$. The $W Z$ contribution is estimated from simulation, while the others are estimated from data.

The estimation of $Z+\mu \mu$ proceeds as described in [5], with the dataset and all expectations from simulation updated to $36.1 \mathrm{fb}^{-1}$. The measurement defines several CRs each designed to be enriched in the different source of background. The selection for each CR is similar to the nominal event selection, with a few cuts inverted/relaxed in order to enhance the background contribution. The full details of the selections are omitted to put more emphasis on the cuts which do the most to enrich each background.

- Inverted d0: By inverting the $d_{0}$ significance cut, this $\mathrm{CR}$ in enriched in heavy flavour decays which tend to have large impact parameters (since the heavy flavour hadron has non-negligible $\beta c \tau$ before it decays)

- $\boldsymbol{e} \boldsymbol{\mu}+\boldsymbol{\mu} \boldsymbol{\mu}$ : By requiring that the Z1 is formed from an $e^{ \pm} \mu^{\mp}$ di-lepton pair, this $\mathrm{CR}$ is enriched in $t \bar{t}$, while the contribution from $Z$ decays is highly suppressed.

- Inverted Isolation: By inverting isolation on the sub-leading muon of the Z2 pair, this CR is enriched in light jets.

- Same-sign: In this CR, the Z2 is required to have same-sign leptons $\left(\ell^{ \pm} \ell^{ \pm}\right)$. All of the background sources enter into this CR. 
In each $\mathrm{CR}$, the expected distribution of each source is modelled using MC simulation. Using this model, the data in each CR enter a simultaneous fit to determine the yield of the different backgrounds in each CR. As an illustration, the fits from a preliminary result [5] using $14.8 \mathrm{fb}^{-1}$ are shown in Figure 66. Finally these yields are extrapolated back to the signal region using transfer factors for the inverted/modified cuts, determined using simulation.

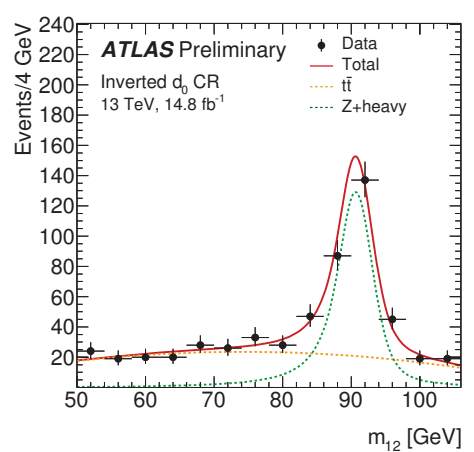

(a) Inverted $d_{0}$

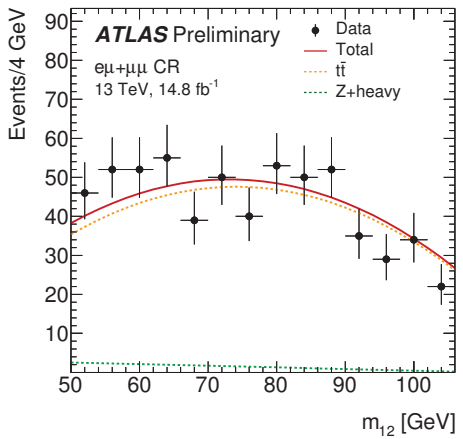

(b) $e \mu+\mu \mu$

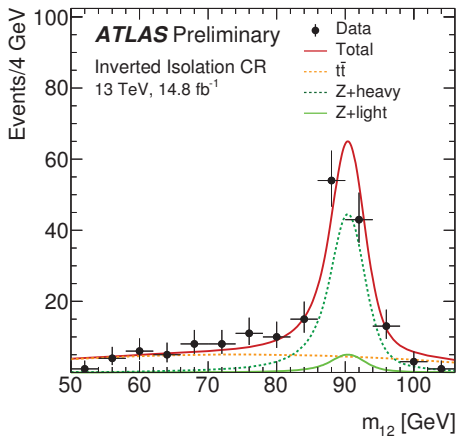

(c) Inverted Isolation

Figure 66: Distributions in the different CRs used in the simultaneous fit to estimate $Z+\mu \mu$ background composition, taken from [5]. These plots are shown for $14.8 \mathrm{fb}^{-1}$, while the estimations used in this thesis (performed by ATLAS collaborators) use the full $36.1 \mathrm{fb}^{-1}$.

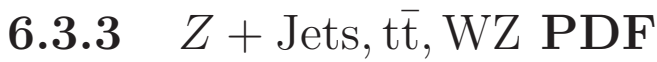

Similar to the yield estimation, the PDF of the Z+Jets background is split between a $Z+e e$ shape (used for the $4 e$ and $2 \mu 2 e$ categories), and a $Z+\mu \mu$ shape (used for the $4 \mu$ and $2 e 2 \mu$ categories).

For the $Z+\mu \mu \mathrm{PDF}$, the $m_{4 \ell}^{\text {cons }}$ distribution of simulated $\mathrm{Z}+\mathrm{HF}$ events passing the nominal event selection is kernel smoothed to build a PDF. The same is done separately for $t \bar{t}$. Next, these two distributions are summed according to their relative expected yields ${ }^{5}$ from the data-driven estimates, to build the Z+Jets PDF. The

\footnotetext{
${ }^{5}$ By definition, the PDF is always normalized to unity.
} 
relative contribution of each is varied in accordance with the uncertainty of the yield estimate to create systematic variations of the shape. The backgrounds from light flavour, and $W Z$ are small enough that they are assumed to be absorbed within these systematic variations.

For the $Z+e e$ PDF, a partly data-driven approach is taken. In the same way that sPlot was used in the fit on $n_{\text {InnerPix }}$ in the $3 \ell+X$ CR to decompose the distribution of the $X$-lepton $p_{T}$, it can also be used to decompose the distribution of $m_{4 \ell}^{\text {cons }}$ (or any other variable) in the CR. This distribution is then multiplied by the $p_{T}$-dependent efficiencies from the $Z+X \mathrm{CR}$ to extract the $m_{4 \ell}^{\text {cons }}$ distribution of $f$ and $\gamma$ backgrounds in the SR. The shape for $q$ is taken from kernel smoothing of MC simulation. Finally, these three distributions are added together according to their relative expected yields to create the PDF for $Z+e e$. As done for the $Z+\mu \mu$, the yields are varied within their uncertainties to create systematically varied shapes to be used as an uncertainty.

The final shapes for Z+Jets, together with their systematic uncertainties are shown in Figure 67.

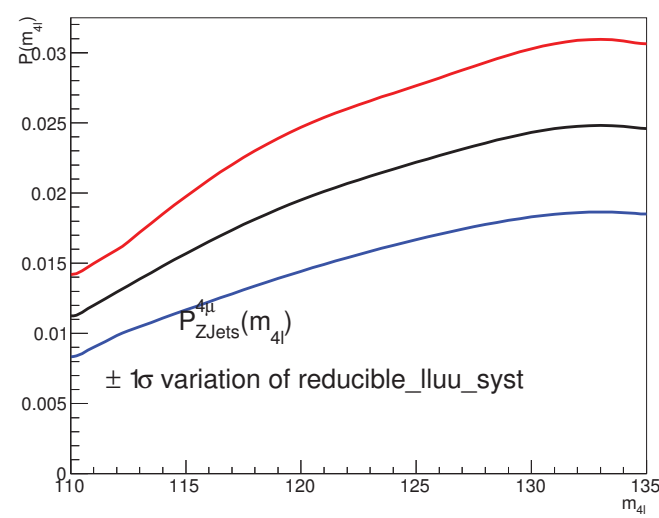

(a) $4 \mu$ and $2 e 2 \mu$

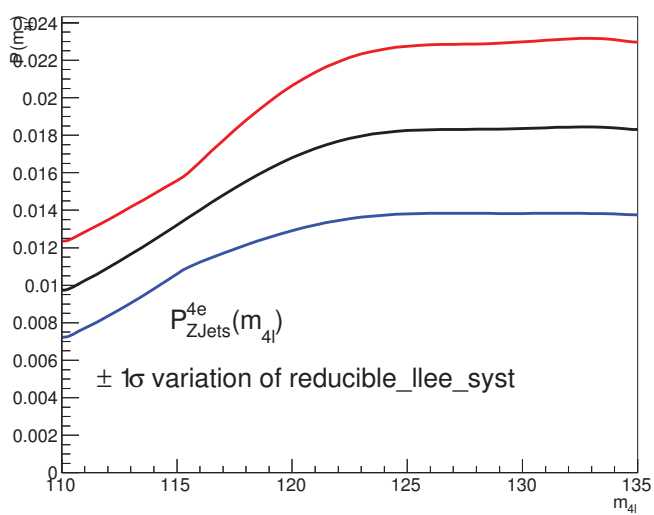

(b) $4 e$ and $2 \mu 2 e$

Figure 67: PDF used to describe distribution of $m_{4 \ell}^{\text {cons }}$ for $Z+$ Jets, têt, WZ backgrounds (black line). The red and blue lines represent systematic uncertainties both on the reducible background yield and shape (they are not normalized PDFs). 


\subsection{Backgrounds summary}

The final results of the background yield estimates for the full $36.1 \mathrm{fb}^{-1}$ dataset is found in Table 11. The categories are defined as in Section 5.11. Note that the source of uncertainties on these values (except the Z+Jets) has yet to be explained in this thesis; this will be covered in Chapter 8, together with all other systematic uncertainties. The central values are estimated as described above. The Z+Jets yield in this table is smaller than in Table 10, because the latter was inclusive of $m_{4 \ell}$, and here the analysis mass window of $110-135 \mathrm{GeV}$ is applied on $m_{4 \ell}^{\text {cons }}$. The yields using a window in $m_{4 \ell}^{\mathrm{fsr}}$ are nearly identical, as the ZMC has a minimal effect on the background distributions.

Table 11: Number of expected background events in each fit category for the full dataset. All sources of systematic uncertainty are included.

\begin{tabular}{ccccc}
\hline Category & $q \bar{q} \rightarrow Z Z^{(*)}$ & $g g \rightarrow Z Z^{(*)}$ & $V V V, t \bar{t}+Z$ & $Z+$ Jets, $\mathrm{t} \overline{\mathrm{t}}, \mathrm{WZ}$ \\
\hline $4 \mu$ & $15.18 \pm 1.49$ & $0.65 \pm 0.39$ & $0.18 \pm 0.01$ & $1.56 \pm 0.40$ \\
$4 e$ & $6.39 \pm 0.74$ & $0.29 \pm 0.18$ & $0.13 \pm 0.01$ & $1.97 \pm 0.47$ \\
$2 \mu 2 e$ & $6.83 \pm 0.77$ & $0.20 \pm 0.12$ & $0.11 \pm 0.01$ & $2.05 \pm 0.47$ \\
$2 e 2 \mu$ & $10.59 \pm 1.02$ & $0.31 \pm 0.19$ & $0.18 \pm 0.01$ & $1.56 \pm 0.40$ \\
\hline combined & $39.00 \pm 2.10$ & $1.45 \pm 0.49$ & $0.60 \pm 0.03$ & $7.14 \pm 0.87$ \\
\hline
\end{tabular}




\section{Chapter 7}

\section{Per-Event Response Signal Model}

This chapter describes the signal model used for the mass measurement. Rather, it presents two signal models: the template method in Section 7.2 and the per-event response method in Section 7.3. The per-event response method is one of the primary contributions of this thesis - it is presented second for pedagogical and chronological reasons: the template method, in various manifestations, has been used to measure particle masses since the 1980's; it is a relatively straightforward technique, and a good introduction to the goals of a signal model. The per-event response model is a more ambitious strategy, which, well-executed, can deliver a more robust and precise measurement. The overarching goal of both models is to describe the expected distribution of $m_{4 \ell}$ from $H \rightarrow Z Z^{(*)} \rightarrow 4 \ell$ events as a function of $m_{H}$ and $\Gamma_{H}$, which will allow both to be measured in data from the events passing the selection of Chapter 5. The template method does not include $\Gamma_{H}$ dependence, and is forced to assume the SM value.

Before this, the chapter begins on much more humble footing. Section 7.1 describes how the expected number of signal events is modelled as a function of $m_{H}$, common to both methods. Also common to both methods are the background distributions described in Chapter 6, and the category definitions as described in Section 5.11. 


\subsection{Signal Normalization}

The expected number of signal events in each category can be parameterized as

$$
\begin{aligned}
N_{\mathrm{H}, \mathrm{c}}^{\text {exp. }} & =\sigma_{p-p \rightarrow H} \times \mathrm{BR}(H \rightarrow 4 \ell) \times L \times A_{c}^{4 \ell}\left(m_{H}\right) \times\left(\frac{4 \mathrm{BR}(H \rightarrow c)}{\mathrm{BR}(H \rightarrow 4 \ell)}\right), \\
& =\sigma_{H \rightarrow 4 \ell} \times L \times A_{c}^{4 \ell}\left(m_{H}\right) \times\left(\frac{4 \mathrm{BR}(H \rightarrow c)}{\mathrm{BR}(H \rightarrow 4 \ell)}\right),
\end{aligned}
$$

where $c$ is the category $(4 \mu, 4 e, 2 \mu 2 e, 2 e 2 \mu), \sigma_{H \rightarrow 4 \ell}$ is the total Higgs production cross section times branching ratio to four electrons or muons as it was defined in Section 2.3.3, and $L$ is the integrated luminosity, $36.1 \mathrm{fb}^{-1}$. The factor $A_{c}^{4 \ell}\left(m_{H}\right)$, is the detector acceptance times efficiency into category $c$ as a function of $m_{H}$. For every $1 \mathrm{fb}$ of $\sigma_{H \rightarrow 4 \ell}$ and $1 \mathrm{fb}^{-1}$ of data, this is how many events are expected to be reconstructed in category $c$. It is parameterized using a second order polynomial, fit on the number of events passing the event selection into each category in the MC simulated samples, combining production modes, at different mass points from $m_{H}=120-130 \mathrm{GeV}$. This parameterization is shown in Figure 68. The final factor is an $\mathcal{O}(1)$ constant used to give the correct share of the $4 \ell$ branching ratio to each of the sub-channels. The branching ratios are normalized by

$$
\mathrm{BR}(H \rightarrow 4 \ell)=\sum_{c} \mathrm{BR}(H \rightarrow c)
$$

but they are not identical. The $H \rightarrow$ eeee, $\mu \mu \mu \mu$ channels have about $10 \%$ more total width than the $H \rightarrow 2 e 2 \mu, 2 \mu 2 e$ channels.

The signal yields expected from the SM, with detector acceptances time efficiency as parameterized by (68), are given for a few mass points in Table 12. The sources of uncertainty on these values are discussed later in Chapter 8. 
Table 12: Expected number of $H \rightarrow 4 \ell$ signal events, $N_{\mathrm{H}, \mathrm{c}}^{\text {exp. }}$, in $36.1 \mathrm{fb}^{-1}$ of data in each category for select values of $m_{H}$.

\begin{tabular}{cccc}
\hline category & $m_{H}=123 \mathrm{GeV}$ & $m_{H}=125 \mathrm{GeV}$ & $m_{H}=127 \mathrm{GeV}$ \\
\hline $4 \mu$ & $19.84 \pm 1.29$ & $20.86 \pm 1.35$ & $21.80 \pm 1.42$ \\
$4 e$ & $10.45 \pm 0.86$ & $11.12 \pm 0.92$ & $11.70 \pm 0.97$ \\
$2 \mu 2 e$ & $10.47 \pm 0.81$ & $11.31 \pm 0.87$ & $12.06 \pm 0.93$ \\
$2 e 2 \mu$ & $13.98 \pm 0.84$ & $14.79 \pm 0.89$ & $15.48 \pm 0.93$ \\
\hline combined & $54.74 \pm 3.52$ & $58.08 \pm 3.73$ & $61.04 \pm 3.92$ \\
\hline
\end{tabular}

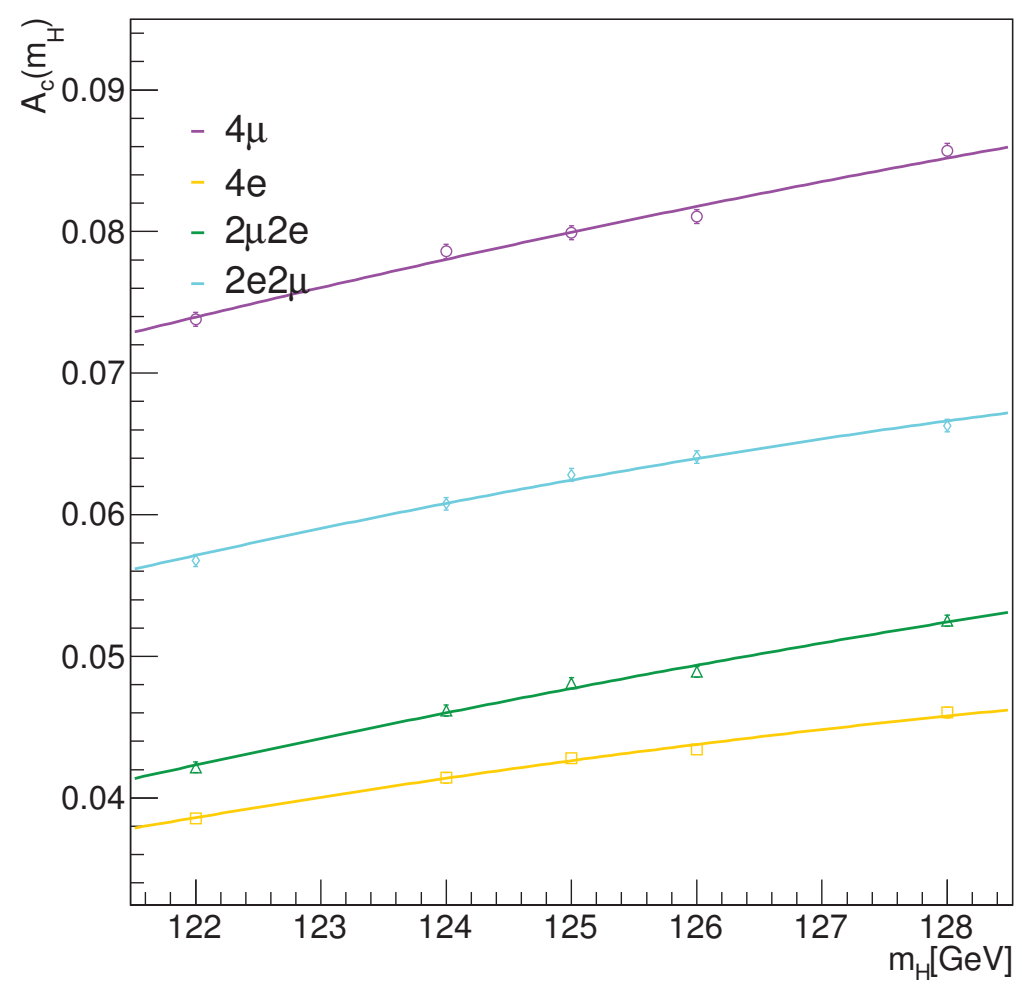

Figure 68: Parameterization of expected signal yield (per unit of cross section times branching ratio) as a function of $m_{H}$. The points are derived using simulated signal samples, and fit with a 2 nd order polynomial.

\subsection{Template Method}

The template method serves as a benchmark against which the per-event response method can be tested by using a well-established method for measuring the mass of a heavy particles experiment. It was used in the first $\mathrm{W}$ boson mass measurement in 
1987 [86] up to ATLAS' measurement of the Higgs boson mass from the Run 1 LHC data in 2013 [7]. Conceptually the method is straightforward:

1. Produce simulated data with a range of mass hypotheses.

2. Choose an observable whose distribution is sensitive to the hypothesis mass, and build a template for that distribution at each mass point.

3. Look at the observed distribution in data, and see which hypothesized mass point best matches; this is the best fit value.

In practice, these steps include more nuance and flexibility. One possibility is to use multiple observables (as in [7], among other examples), and correspondingly multidimensional template PDFs; this adds the complication of accounting for correlations between the observables in the templates. Another point of flexibility is whether the fit will be binned (using $\chi^{2}$ or binned likelihood fit), or unbinned (using an unbinned likelihood fit). If the fit will be unbinned, then one must decide how the templates at each mass point will be built: the most basic method would be a histogram from the simulated data [87], but a more physically motivated approach ${ }^{1}$ is to create a smooth distribution using numerical methods, or an analytical parameterization ([7] provides examples of both in the signal model for the $H \rightarrow 4 \ell$ channel, and $H \rightarrow \gamma \gamma$ channel, respectively). Finally, one must decide how to perform the final fit: in modern implementations, the templates are usually interpolated between simulated mass points to create a continuous parameterization, but discrete parameterizations together with some basic assumptions can also be functional ${ }^{2}$.

The template method as presented in this thesis uses a 1D unbinned fit on observable $m_{4 \ell}^{\text {cons }}$, using templates built from smoothed MC and interpolated using morphing to create a continuous parameterization.

\footnotetext{
${ }^{1}$ Unless infinite MC statistics are available, then a histogram must have finite bins and the template will be "boxy", which is not a physical PDF.

${ }^{2}$ One example, [87], assumes these discrete simulated mass points which form the likelihood curve will be parabolic, and so fits the minimum based on this assumption.
} 


\subsubsection{Signal template smoothing}

Template PDFs of $m_{4 \ell}^{\text {cons }}$, called $P\left(m_{4 \ell}^{\text {cons }}\right)$, are built for each simulated mass point available from $m_{H}=120-130 \mathrm{GeV}$, separately for each analysis category. The templates are built by kernel smoothing the MC simulated data using the KEYS algorithm, described previously in Section 6.1, using $\rho=2.2$. This value was chosen to produce a smooth, unimodal distribution, without any evidence of the result "chasing" the statistical fluctuations in the input data. Results of the smoothing in the $4 \mu$ category are shown in Figure 69, for the $4 e$ category in Figure 70, for the $2 \mu 2 e$ category in Figure 71, and for $2 e 2 \mu$ in Figure 72 , for each of the available simulated mass points.

\subsubsection{Signal template morphing}

The templates at each mass point are combined together to form a continuous parameterization in $m_{H}$ using the class RooParamKeysPdf [88]. This class assumes that for some shift values $\Delta$,

$$
\begin{array}{r}
P\left(m_{4 \ell} \mid m_{H}+\Delta\right) \approx P\left(m_{4 \ell}-\Delta \mid m_{H}\right), \\
\text { e.g. } \quad P\left(m_{4 \ell} \mid 125.2\right) \approx P\left(m_{4 \ell}-0.2 \mid 125\right) .
\end{array}
$$

In this way, the signal templates at the simulated mass points can be interpolated to mass points values that were not simulated by what amounts to a small horizontal 

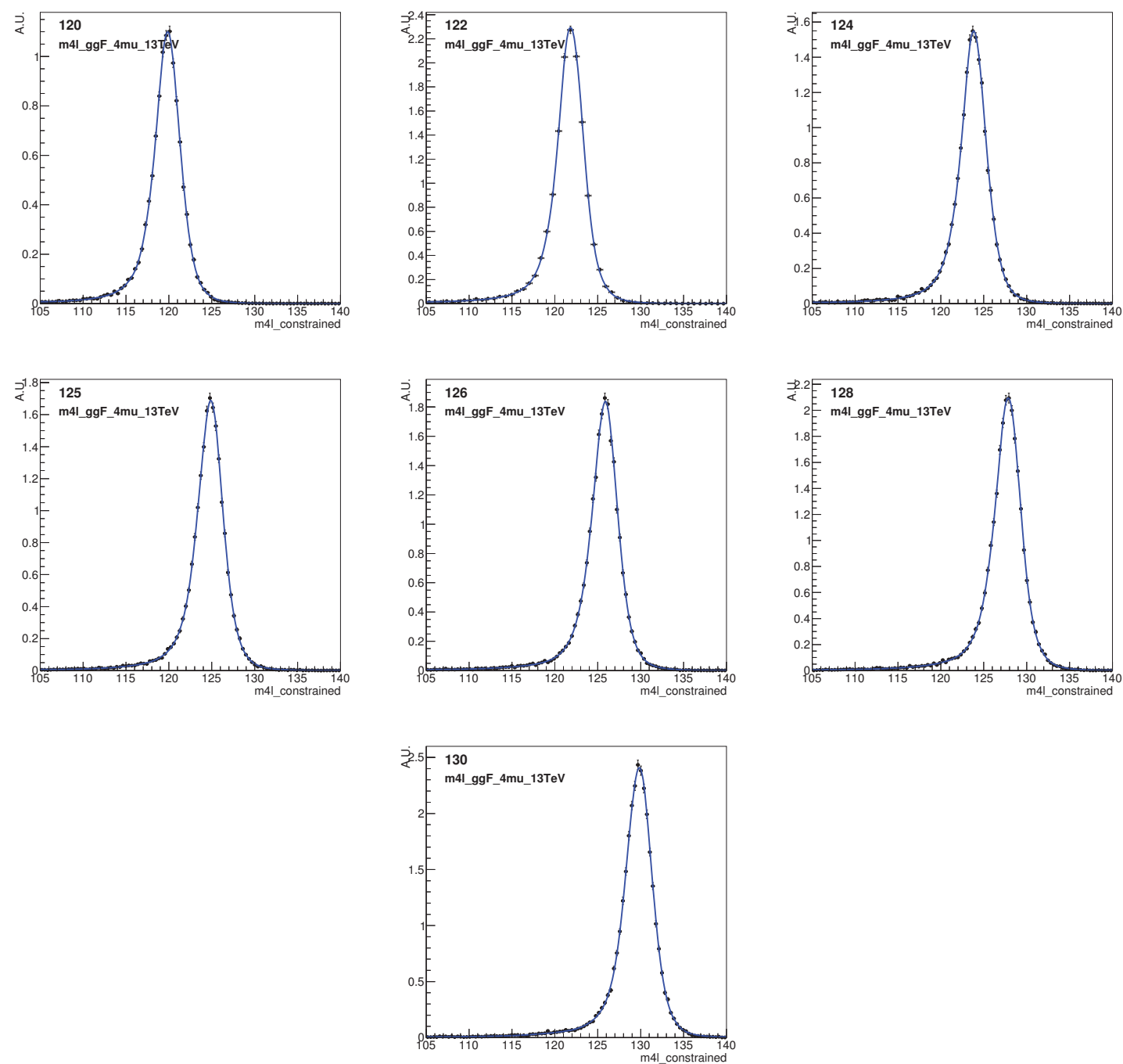

Figure 69: Smoothing result for template signal model. The $m_{4 \ell}^{\text {cons }}$ distribution in category $4 \mu$ is shown for $\mathrm{MC}$ simulated mass points in order $m_{H}=$ $120,122,124,125,126,128,130 \mathrm{GeV}$.

shift. In practice, a sum of several nearby templates with masses $m_{H}^{i}$ used,

$$
\begin{aligned}
P_{\text {morph }}\left(m_{4 \ell} \mid m_{H}\right) & =\sum_{m_{H}^{i}} w_{m_{H}^{i}} P_{m_{H}^{i}}\left(m_{4 \ell}^{\text {cons }}-\left(m_{H}^{i}-m_{H}\right)\right), \\
\text { where } & \sum_{\text {template i }} w_{m_{H}^{i}}=1,
\end{aligned}
$$



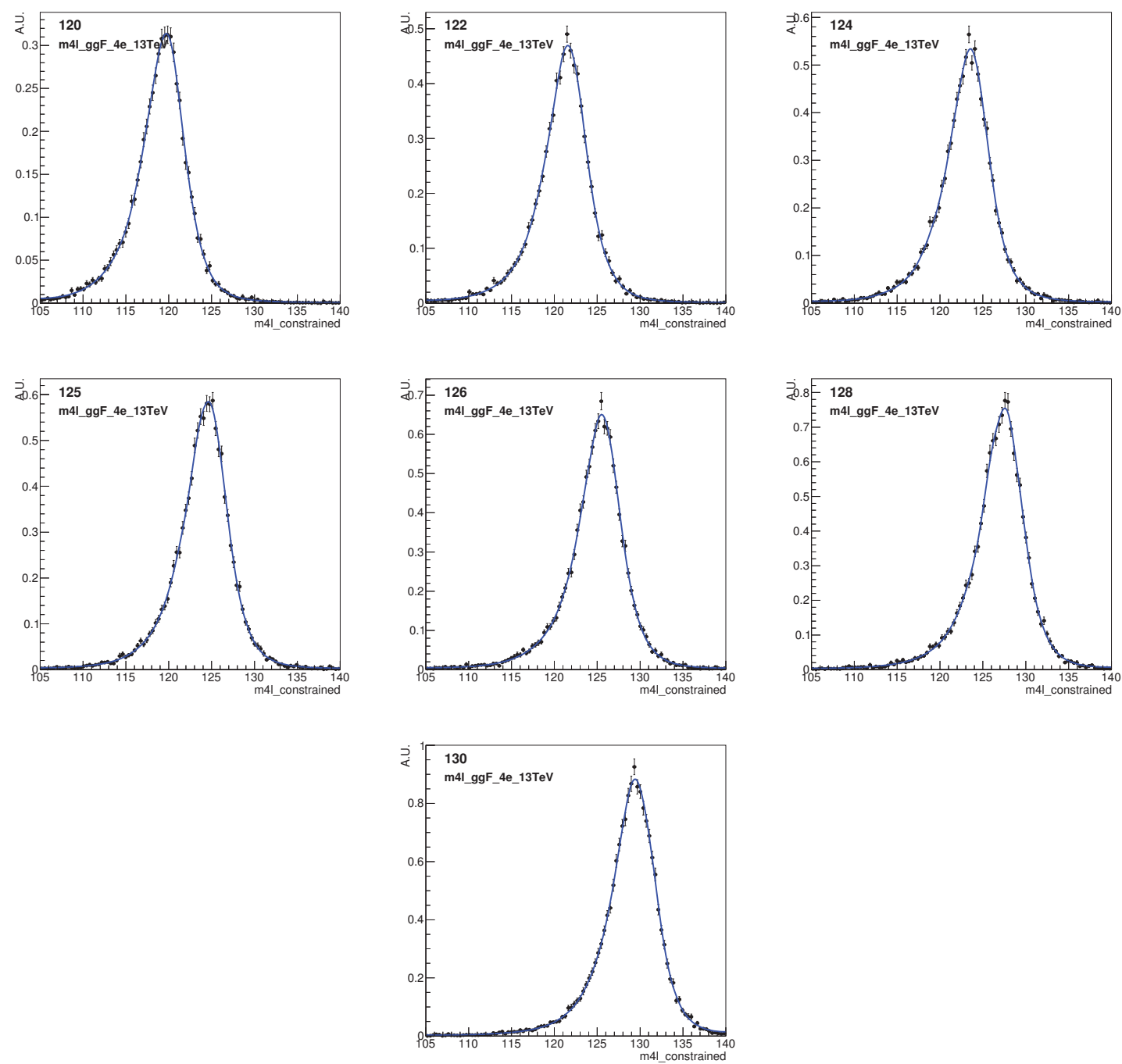

Figure 70: Smoothing result for template signal model. The $m_{4 \ell}^{\text {cons }}$ distribution in category $4 e$ is shown for $\mathrm{MC}$ simulated mass points in order $m_{H}=$ $120,122,124,125,126,128,130 \mathrm{GeV}$.

where the weights are determined according to the distance between the template mass value and the hypothesis value, such that

$$
\begin{array}{r}
w_{m_{H}^{i}} \text { is largest when } \quad\left(\mathrm{m}_{\mathrm{H}}^{\mathrm{i}}-\mathrm{m}_{\mathrm{H}}\right) \rightarrow 0, \\
w_{m_{H}^{i}} \rightarrow 0 \text { when } \quad\left|\mathrm{m}_{\mathrm{H}}^{\mathrm{i}}-\mathrm{m}_{\mathrm{H}}\right| \text { is large. }
\end{array}
$$



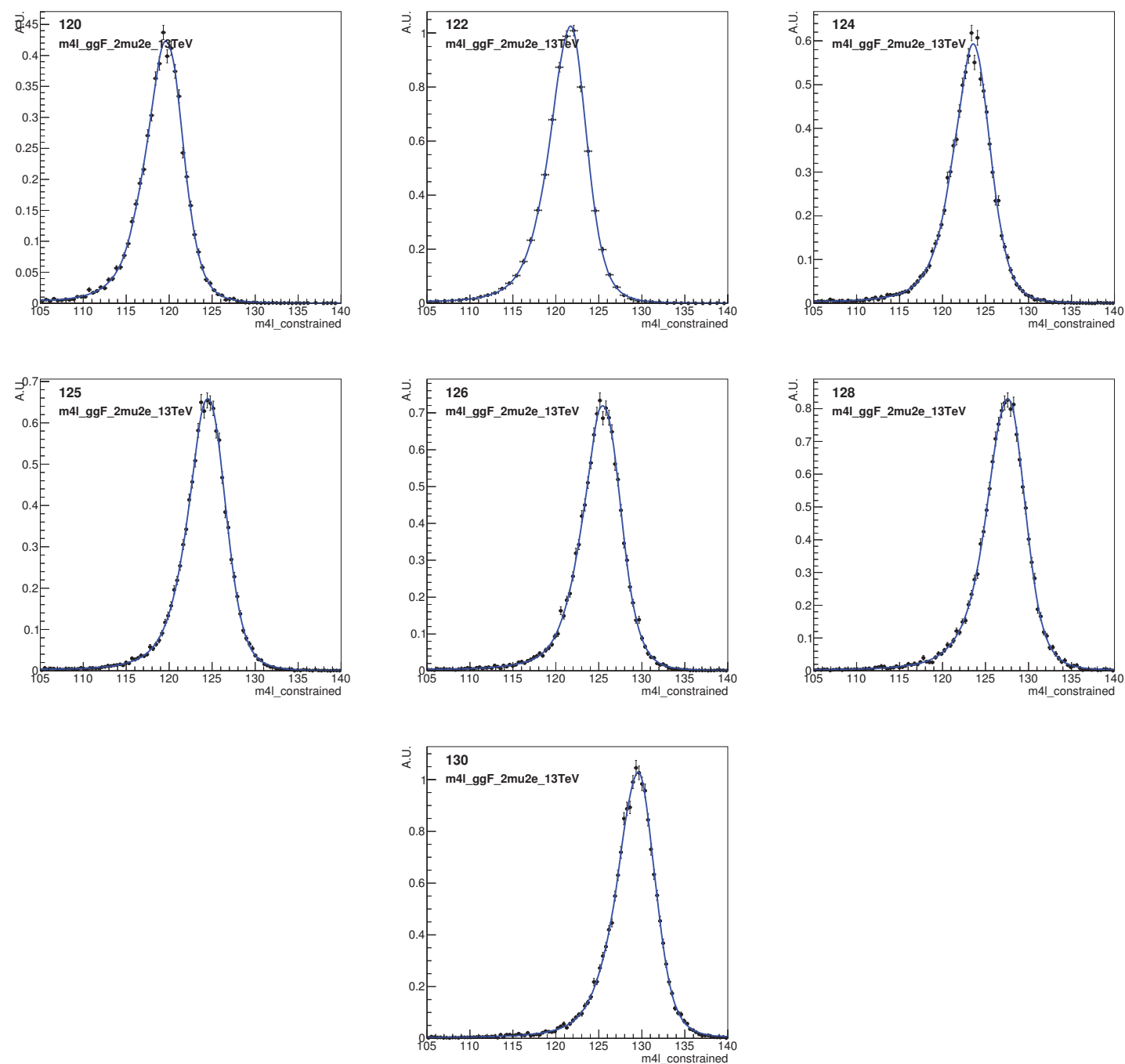

Figure 71: Smoothing result for template signal model. The $m_{4 \ell}^{\text {cons }}$ distribution in category $2 \mu 2 e$ is shown for $\mathrm{MC}$ simulated mass points in order $m_{H}=$ $120,122,124,125,126,128,130 \mathrm{GeV}$.

This process prevents discontinuities in morphing across the template values, and produces a smooth dependence in $m_{H}$. The result of this morphing is shown in Figure 73 for each of the analysis categories, in steps of $0.5 \mathrm{GeV}$ from $120-130 \mathrm{GeV}$, demonstrating the smooth interpolation of the morphing. One consequence of the above weighting strategy is that although several of the input mass points are included in this plot (e.g. $\left.m_{H}=125 \mathrm{GeV}\right)$, the exact templates themselves are not: the input templates which surround $125 \mathrm{GeV}$ are used to improve the continuity of the shape 

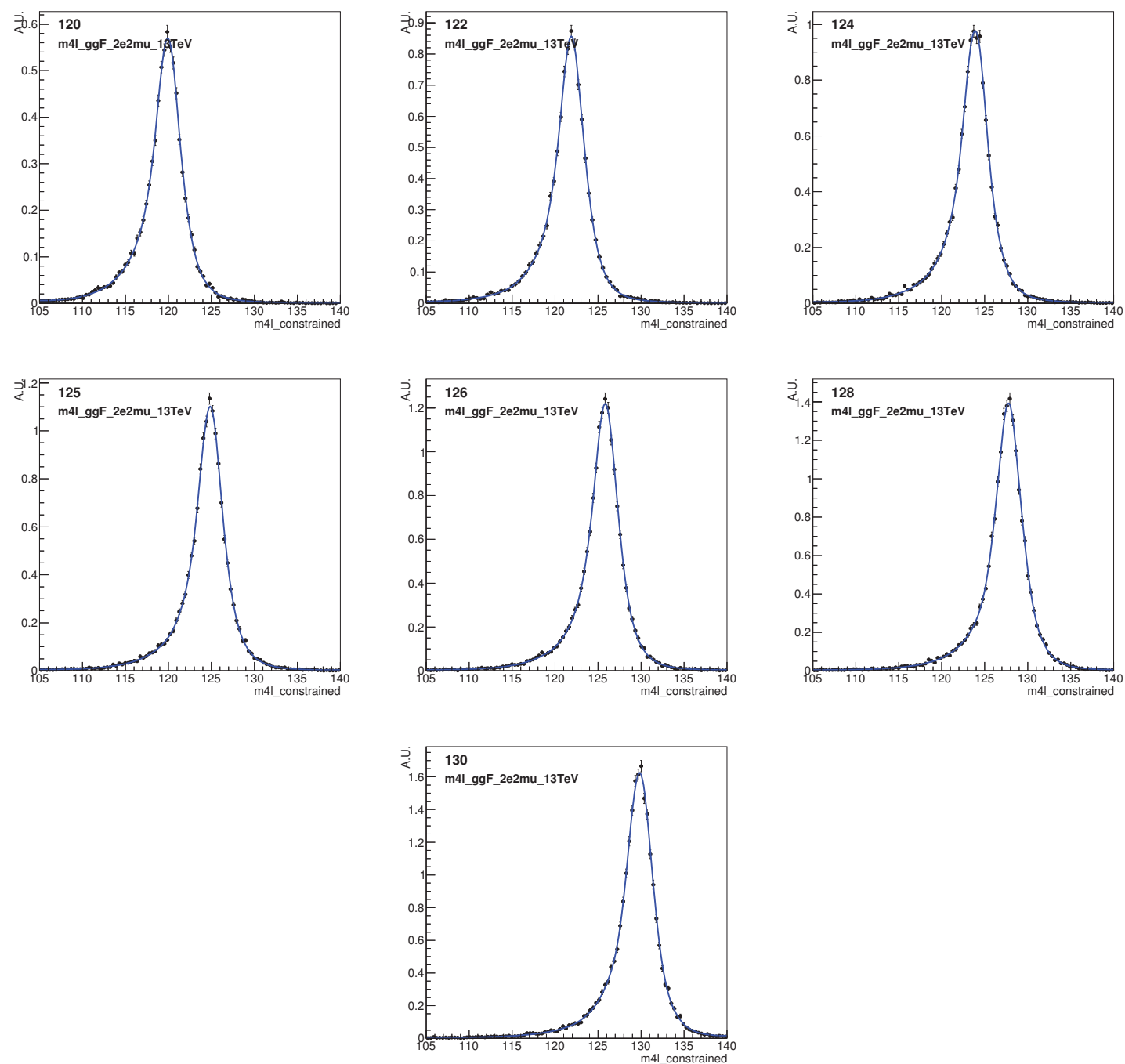

Figure 72: Smoothing result for template signal model. The $m_{4 \ell}^{\text {cons }}$ distribution in category $2 e 2 \mu$ is shown for $\mathrm{MC}$ simulated mass points in order $m_{H}=$ $120,122,124,125,126,128,130 \mathrm{GeV}$.

at this mass point, modelling it more reliably than the $125 \mathrm{GeV}$ template alone. In this specific example, the weights take on the values as follows,

$$
\begin{aligned}
P_{\text {morph }}\left(m_{4 \ell} \mid 125\right) & =\ldots+w_{124} P_{124}\left(m_{4 \ell}-1\right)+w_{125} P_{125}\left(m_{4 \ell}\right)+w_{126} P_{126}\left(m_{4 \ell}+1\right)+\ldots \\
& =\ldots+0.17 P_{124}\left(m_{4 \ell}-1\right)+0.64 P_{125}\left(m_{4 \ell}\right)+0.17 P_{126}\left(m_{4 \ell}+1\right)+\ldots
\end{aligned}
$$

where the even more distant templates carry the remainder of the weight. This morphing is stable even when individual mass samples are removed, a fact which is 
demonstrated in Appendix B.1.

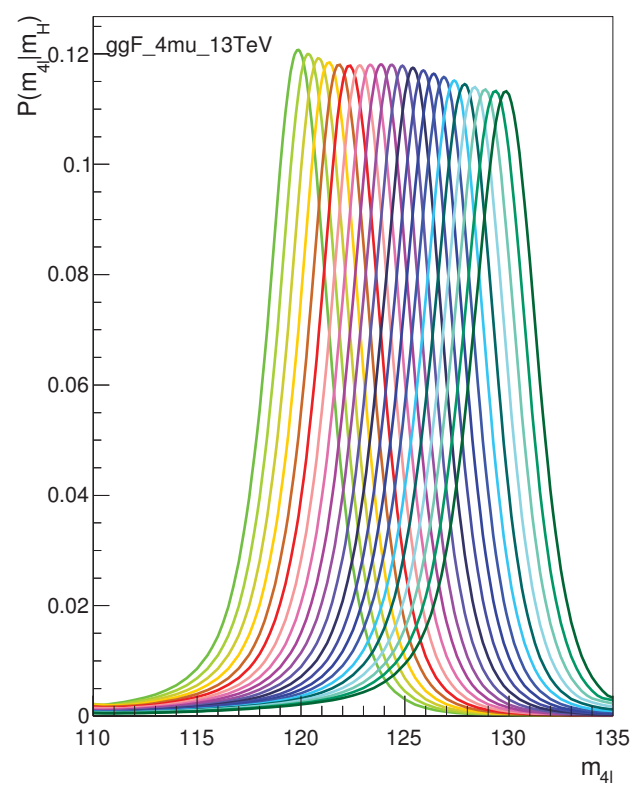

(a) $4 \mu$

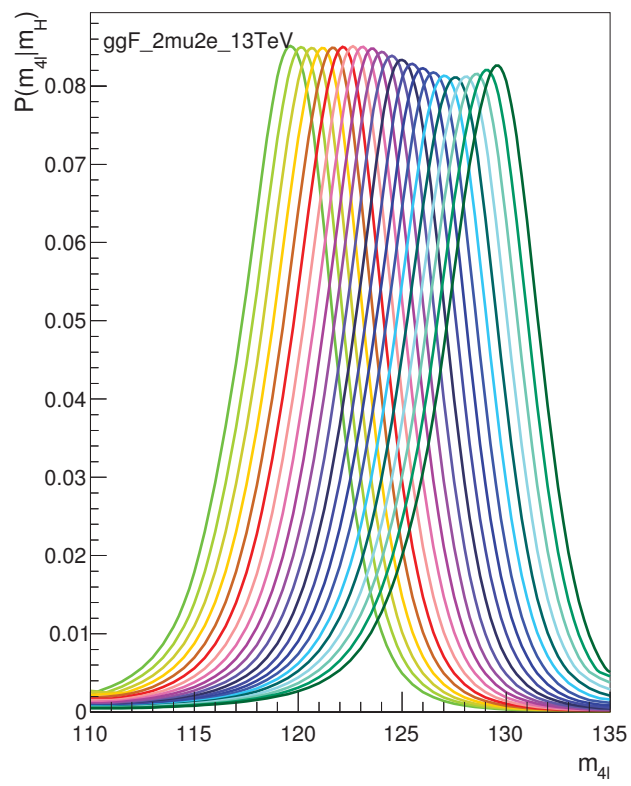

(c) $2 \mu 2 e$

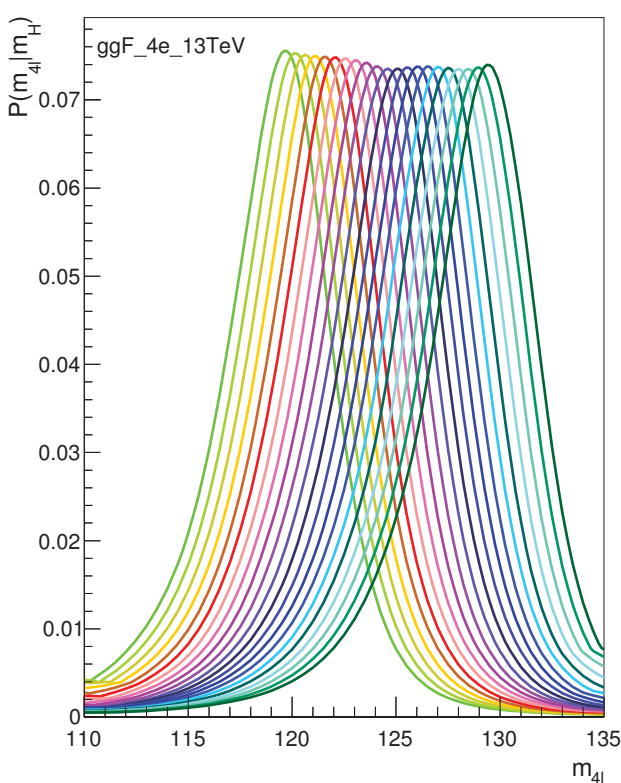

(b) $4 e$

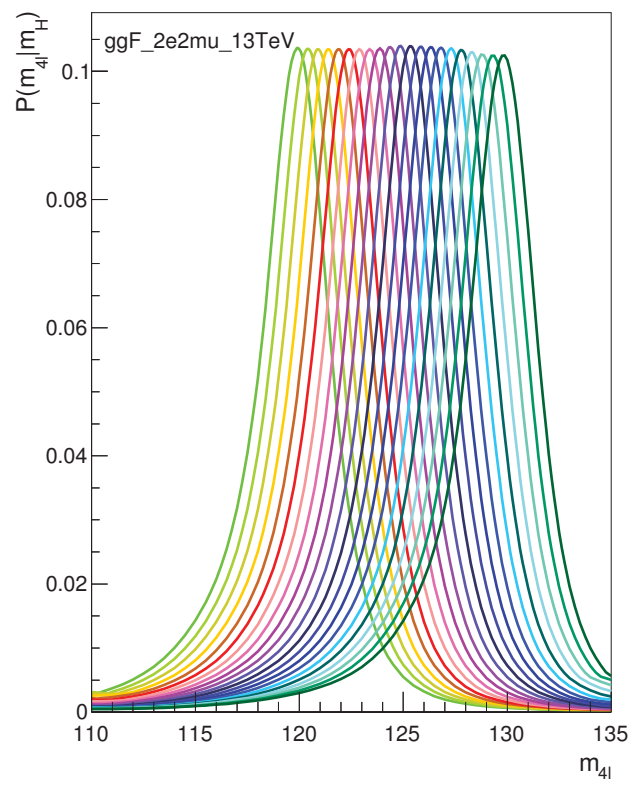

(d) $2 e 2 \mu$

Figure 73: Demonstration of template morphing using RooParamKeysPdf in the four categories. The PDF $P\left(m_{4 \ell}^{\text {cons }} \mid m_{H}\right)$ is drawn in $0.5 \mathrm{GeV}$ steps from $120 \mathrm{GeV}$ to $130 \mathrm{GeV}$. 


\subsection{Per-Event Response method}

The per-event response (PER) method, and measurements performed with it, are the principal contribution presented in this thesis. It is a novel application of a Gaussianmixture approach to describe the detector response (in this case, the $m_{4 \ell}$ response) of each event. It is inspired by other uses in ATLAS, such as the Gaussian Sum Filter for electron tracking described in Section 4.4.1, where a Gaussian mixture is used to describe the Bethe-Heitler energy loss distribution. In the PER method, a Gaussian mixture is used to describe the energy response distribution of the four leptons in each selected event. These energy response distributions are propagated to calculate the $m_{4 \ell}$ response for each event, which is consequently also described by a Gaussian mixture. By convolving this predicted $m_{4 \ell}$ response distribution - unique to each event - with the common truth distribution for signal events with unknown parameters $m_{H}, \Gamma_{H}$ the PER signal PDF is constructed.

The method bears similarities to a per-event error fit, so it is worthwhile to distinguish them. The principle in both cases is that every event has a unique detector resolution: some are better-measured, some are worse-measured than others. It is natural that these better-measured events should have more importance in the fit than worse-measured events. This is accomplished in practice for a $H \rightarrow 4 \ell$ analysis by having the PDF of $m_{4 \ell}$ depend explicitly on the measurement resolution. Where the two methods differ is that while per-event errors assumes the general form of the PDF [89] as a Gaussian, Crystal Ball (CB) [90] function, CB+Gaussian, two-sided$\mathrm{CB}$, etc., and includes a per-event resolution analytically in the width parameter, the PER method does not make such an assumption. Instead it predicts the entire PDF from first principles, based on the lepton kinematics in the event. The benefits of the PER method are most pronounced in channels with low statistics, or highly variable resolution between events, both of which are satisfied in the $H \rightarrow 4 \ell$ channel. The PER method also more neatly disentangles the detector component of the $m_{4 \ell}$ 
distribution (the response) from the physics component (the truth distribution). Both these methods differ from the template method, where the PDF is a fixed shape for all events in a given category, created by implicitly averaging the resolution of all events in that category when building each template. As a result, the template method is unable to given better-measured events within each category more importance in the fit than the poorly-measured events, whereas the PER method does exactly that.

It should be noted that in the earlier stages of development of the PER method, it was used as a cross-check of the ATLAS Run 1 Higgs mass measurement [7], and as a direct measurement of the Higgs boson natural width. The development and execution of the method at this time was done by the author in close collaboration with others from CERN, Harvard University, and Max Planck Institute for Physics. The method at this stage was also presented in detail in [91]. As it is presented in this thesis, the method is executed at every stage by the author, and makes several improvements over the aforementioned version through the mathematical formulation, the treatment of FSR photons, the single muon response modelling, the use of tuning parameters to improve the model (to be explained in Section 7.3.4), and the treatment of systematics, as well as technical logistics throughout.

\subsubsection{Per-Event Response PDF}

In the PER model, the PDF for signal events is uniquely determined for each event based on the lepton kinematics, and the hypothesized Higgs parameters, taking the form $P\left(m_{4 \ell}^{\mathrm{fsr}} \mid m_{H}, \Gamma_{H}\right.$, kinematics $)$.

To reach this expression, note that any given lepton $i$ has a true energy $E_{i}^{\text {true }}$, and an energy after reconstruction and FSR recovery ${ }^{3} E_{i}^{\mathrm{fsr}} \equiv E_{i}^{\mathrm{reco}}+E^{\mathrm{FSR}}$. The true energy used here is the born energy [92] - the lepton's energy at the Z boson decay vertex and, importantly, before any FSR. Using these quantities, we can define the

\footnotetext{
${ }^{3}$ The FSR photon recovered in Section 5.8 is matched to a particular lepton using the $\Delta R$ separation
} 
lepton energy response as

$$
x_{i}=\frac{E_{i}^{\mathrm{fsr}}-E_{i}^{\mathrm{true}}}{E_{i}^{\mathrm{fsr}}},
$$

where $x_{i}$ is distributed according to some general distribution $P\left(x_{i}\right)$ that will be described in Section 7.3.2. Some additional remarks about the definition of $x_{i}$ are found in Appendix B.2.

Next, examine the four-lepton invariant mass at the born level:

$$
\begin{aligned}
\left(m_{4 \ell}^{\text {true }}\right)^{2} & =\left(p_{4 \ell}^{\text {true }}\right)^{2}, \\
& =\left(\sum_{i=1, . ., N_{\mathrm{part}}} p_{i}^{\text {true }}\right)^{2}, \\
& =\sum_{i}\left(p_{i}^{\text {true }}\right)^{2}+\sum_{i, j \neq i} p_{i}^{\text {true }} \cdot p_{j}^{\text {true }},
\end{aligned}
$$

where $N_{\text {part }}=4$ for the four-lepton system ${ }^{4}$. Since the individual particles are muons and electrons, $\left(p_{i}^{\text {true }}\right)^{2}=m_{e}^{2}, m_{\mu}^{2}$ can be neglected compared to the much larger terms. Continuing,

$$
\begin{aligned}
\left(m_{4 \ell}^{\text {true }}\right)^{2} & =2 \sum_{i, j>i} p_{i}^{\text {true }} \cdot p_{j}^{\text {true }} \\
& =2 \sum_{i, j>i} E_{i}^{\text {true }} E_{j}^{\text {true }}\left(1-c_{i, j}\right)
\end{aligned}
$$

where $c_{i, j}$ is the cosine of the relative angle between the leptons, assumed to be exactly measured in reconstruction ${ }^{5}$, such that $c_{i, j} \equiv c_{i, j}^{\mathrm{fsr}}=c_{i, j}^{\text {true }}$. Next rearrange (75) to get

\footnotetext{
${ }^{4}$ Except for the unimportant labelling of $m_{4 \ell}$, the formulation is completely general to any number of particles

${ }^{5}$ This is an imperfect but low-impact assumption: angular uncertainties contribute a much smaller effect to multi-lepton invariant masses than the uncertainties on individual lepton energies/momenta.
} 
$E_{i}^{\text {true }}=E_{i}^{\text {fsr }}\left(x_{i}-1\right)$, which when substituted into $(77)$ gives

$$
\begin{aligned}
\left(m_{4 \ell}^{\text {true }}\right)^{2} & =2 \sum_{i, j>i} E_{i}^{\mathrm{fsr}}\left(x_{i}-1\right) E_{j}^{\mathrm{fsr}}\left(x_{j}-1\right)\left(1-c_{i, j}\right), \\
& =2 \sum_{i, j>i} E_{i}^{\mathrm{fsr}} E_{j}^{\mathrm{fsr}}\left(1-x_{i}-x_{j}+x_{i} x_{j}\right)\left(1-c_{i, j}\right), \\
& =2 \sum_{i, j>i} E_{i}^{\mathrm{fsr}} E_{j}^{\mathrm{fsr}}\left(1-c_{i, j}\right)-2 \sum_{i, j>i} E_{i}^{\mathrm{fsr}} E_{j}^{\mathrm{fsr}}\left(x_{i}+x_{j}\right)\left(1-c_{i, j}\right), \\
\left(m_{4 \ell}^{\mathrm{fsr}}\right)^{2}-\left(m_{4 \ell}^{\mathrm{true}}\right)^{2} & =2 \sum_{i, j>i} E_{i}^{\mathrm{fsr}} E_{j}^{\mathrm{fsr}}\left(1-c_{i, j}\right)\left(x_{i}+x_{j}\right), \\
& =\sum_{i}\left(\sum_{j \neq i}\left(m_{i j}^{\mathrm{fsr}}\right)^{2}\right) x_{i},
\end{aligned}
$$

where in the third line we have dropped the term with a factor $x_{i} x_{j}$ which is negligible, and in the final line $m_{i j}$ is the di-lepton mass of leptons $i$ and $j$ together. Finally if we divide both sides by $\left(m_{4 \ell}^{\mathrm{fsr}}+m_{4 \ell}^{\mathrm{true}}\right) \approx 2 m_{4 \ell}^{\mathrm{fsr}}$ then $^{6}$ we reach a neat expression

$$
m_{4 \ell}^{\mathrm{fsr}}-m_{4 \ell}^{\mathrm{true}}=\frac{1}{2 m_{4 \ell}^{\mathrm{fsr}}} \sum_{i}\left(\sum_{j \neq i}\left(m_{i j}^{\mathrm{fsr}}\right)^{2}\right) x_{i} .
$$

If we consider $\left(m_{4 \ell}^{\mathrm{fsr}}-m_{4 \ell}^{\mathrm{true}}\right)$ to be a random variable, we are very interested in its PDF, which can be calculated using the properties of random variables to be

$$
P\left(m_{4 \ell}^{\mathrm{fsr}}-m_{4 \ell}^{\mathrm{true}}\right)=\frac{1}{2 m_{4 \ell}^{\mathrm{fsr}}} \prod_{i}\left(\sum_{j \neq i}\left(m_{i j}^{\mathrm{fsr}}\right)^{2}\right) P\left(x_{i}\right),
$$

where $\prod$ here represents repeated convolution, and not repeated multiplication. This is the response distribution of any particular event, if the $P\left(x_{i}\right)$ are known.

Considering the invariant mass again as a random variable, for each event the

\footnotetext{
${ }^{6}$ To account for the difference between reconstruction and true, this factor is not exactly 2 , but is determined on an event-by-event basis based on the mean of the predicted distribution $P\left(\left(m_{4 \ell}^{\mathrm{fsr}}\right)^{2}-\left(m_{4 \ell}^{\mathrm{true}}\right)^{2}\right)$.
} 
trivial expression $m_{4 \ell}^{\mathrm{fsr}}=\left(m_{4 \ell}^{\mathrm{fsr}}-m_{4 \ell}^{\mathrm{true}}\right)+m_{4 \ell}^{\text {true }}$ leads to the expression

$$
P\left(m_{4 \ell}^{\mathrm{fsr}}\right)=P\left(m_{4 \ell}^{\mathrm{fsr}}-m_{4 \ell}^{\mathrm{true}}\right) * P\left(m_{4 \ell}^{\mathrm{true}}\right)
$$

By substituting (80), we have the expression

$$
P\left(m_{4 \ell}^{\mathrm{fsr}} \mid \text { kinematics }\right)=\left[\frac{1}{2 \mathrm{~m}_{4 \ell}^{\mathrm{fsr}}} \prod_{\mathrm{i}}\left(\sum_{\mathrm{j} \neq \mathrm{i}}\left(\mathrm{m}_{\mathrm{ij}}^{\mathrm{fsr}}\right)^{2}\right) \mathrm{P}\left(\mathrm{x}_{\mathrm{i}}\right)\right] * \mathrm{P}\left(\mathrm{m}_{4 \ell}^{\text {true }}\right)
$$

This derivation shows that if the lepton energy responses are accurately known, no matter the form of their distributions, then the correct PDF for any given event can be calculated exactly, for any given signal hypothesis of $P\left(m_{4 \ell}^{\text {true }}\right)$ and its corresponding parameters. In this thesis, the parameters are $m_{H}$ and $\Gamma_{H}$, as will be seen shortly. The following section describes how we model $P\left(x_{i}\right)$ as a Gaussian-mixture for any given lepton kinematics.

\subsubsection{Describing Single Lepton Energy Response}

The PDF of each lepton's energy response, $P\left(x_{i}\right)$, is described using a weighted sum of three Gaussian distributions. The number of Gaussians is chosen as the smallest number sufficient to describe the energy response $x_{i}$ in any kinematic region. Note that using Gaussians is not obligatory - any parameterization of $P\left(x_{i}\right)$ which accurately describes the distribution would do fine. The choice of a Gaussian mixture for the PDF is convenient because

- The Gaussians can usually (but not necessarily) be associated with particular effects: a Gaussian resolution core, and radiative/bremsstrahlung tails.

- The convolutions necessitated by (80) are always analytical.

- The response distribution of (80) will also be a sum of weighted Gaussians. 
- The response distribution can be simplified in an efficient way - a procedure to be described in Section 7.3.3.

- The propagation of systematic uncertainties is more easily understood, and technically implemented.

The response PDF for each lepton is then parameterized using 8 free parameters:

$$
P\left(x_{i}\right)=\sum_{g=1,2,3} w_{g} G\left(x_{i} ; \mu_{g}, \sigma_{g}\right)
$$

where the weights are normalized $\sum_{g=1,2,3} w_{g}=1$ (reducing the free parameters from 9 to 8$)$. These Gaussian parameters $\left(w_{g}, \mu_{g}, \sigma_{g}\right)$ describing the lepton energy response in general depend on the species and kinematics of the lepton.

To construct a model of the lepton energy response, all available signal and $q \bar{q} \rightarrow Z Z^{(*)} \mathrm{MC}$ simulated samples are used. Only leptons from events passing the full selection and entering the $m_{4 \ell}$ fit range are used. These leptons are separated by species (muon, or electron), and divided into bins of (Z1 or Z2) $\mathrm{x}$ ( $p_{T}$ bins) $\mathrm{x}$ $\left(|\eta|\right.$ bins). The $p_{T}$ and $|\eta|$ bins are chosen based on available MC statistics (finer granularity would always be better than coarser), expected resolution behaviour as quantified by ATLAS Combined Performance (CP) groups using Data and MC comparisons [79][4] (rapidly varying resolution requires more bins, slowly varying resolution requires fewer), and detector geometry (transitions in detector layout as a 
function of $|\eta|$ normally define bin boundaries). The bins used are as follows:

- muons

- Z bins: 1,2

$-p_{T}$ bin boundaries $(\mathrm{GeV}): 5 . \quad$, 6.5, $7.5,8.5,9.5,10.5,11.6,12.7,13.8$, 15.0, 16.2, 17.4, 18.6, 19.8, 21., 22.3, 24 , 26 , 28.1, 30., 31.5, 33, 34.5, 36, $38,40,42,44,46,49,52,56,60,64,68,72,77,82,87,92,98,104,110$, $118,126,134,144,154,166,178,180,200,225,250,400,1000$

- $|\eta|$ bin boundaries: 0., 0.1, 0.22, 0.34, 0.46, 0.58, 0.70, 0.82, 0.94, 1.06, $1.18,1.30,1.42,1.54,1.68,1.80,1.92,2.04,2.19,2.34,2.50,2.70$

- electrons

- Z bins: 1,2

- $p_{T}$ bin boundaries $(\mathrm{GeV}):$ 7., 8.6 , 10.0 , 11.0 , 12.2 , 13.4 , 14.6 , 15.8, 17, $18.4,19.8,21.2,22.6,24,25.6,27.2,28.8,30.6,32.3,34.2,36,38.2$ , 40.4 , 42.6 , 44.8 , 47, 49.4 , 51.8, 55, 58, 61, 65, 69, 74, 79, 85, 91, 97, $103,110,118,126,135,144,154,164,176,188,200,225,250,1000$

- $|\eta|$ bin boundaries: $0 ., 0.1,0.15,0.2,0.4,0.6,0.8,1.00,1.20,1.37,1.52$, $1.6,1.75,1.90,2.0,2.1,2.3,2.4,2.5$

As a result, the muons are subdivided into $2 \times 55 \times 21=2310$ bins. The electrons are subdivided into $2 \times 51 \times 18=1836$ bins. In each of these bins the distribution of $x_{i}$ as defined in (75) is fit using an unbinned maximum likelihood fit $^{7}$ with the PDF of (83). The parameters are stored in a database for later retrieval. It's not practical to include the 4146 fits (in fact, double this number, as Section 7.3.5 will explain) in this text, but a few samples bins are included in Figure 74 for muons, and in Figure 75 

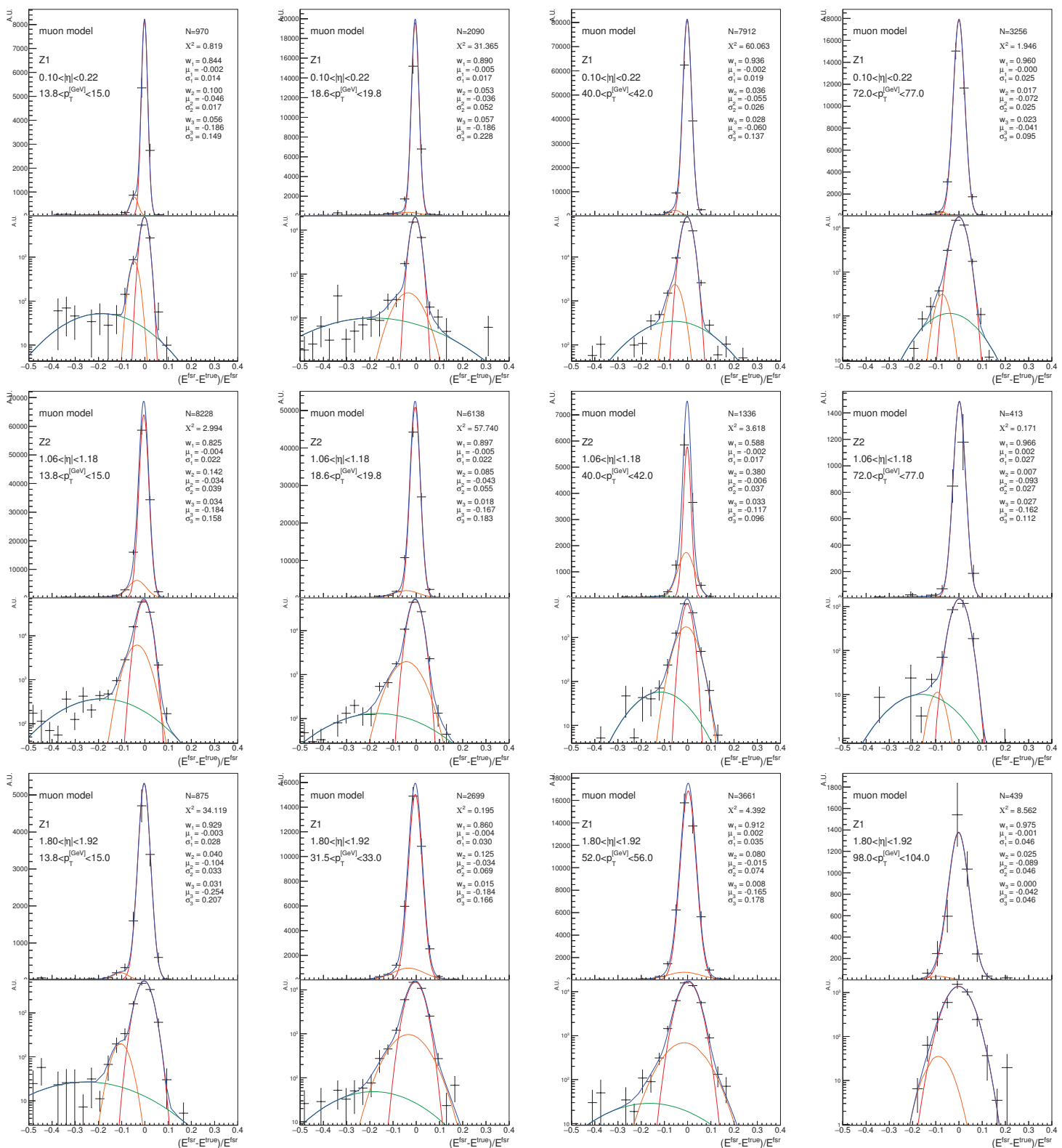

Figure 74: Example fits of unconstrained muon energy response $(1 / 2$ - see Appendix B.3 for more). The distributions are built from MC simulation (black points) fit using a sum of three Gaussians (blue curve). Each plot shows the same fit in linear and log scale. The kinematic bins and the fit parameters are printed on each plot.

for electrons. Additional examples are included in Appendix B.3.

\footnotetext{
${ }^{7}$ The fit is re-executed as many as 30 times with randomly varying seeds in order to escape local minima and find the best fit possible in this bin
} 

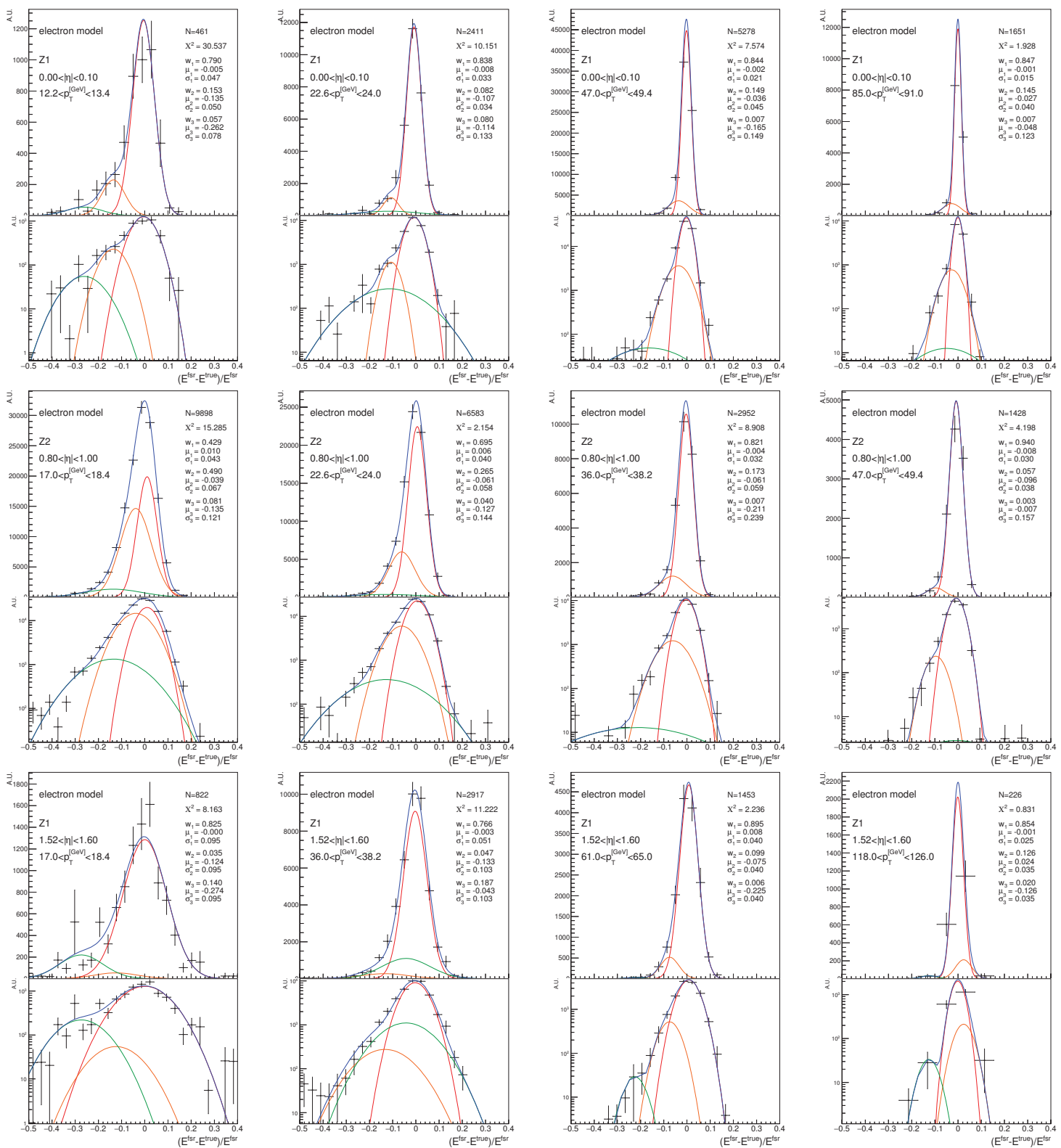

Figure 75: Example fits of unconstrained electron energy response (1/2 - see Appendix B.3 for more). The distributions are built from MC simulation (black points) fit using a sum of three Gaussians (blue curve). Each plot shows the same fit in linear and log scale. The kinematic bins and the fit parameters are printed on each plot.

Note that the response distribution for muons is nearly Gaussian (meaning $w_{1}$ is large), and the additional Gaussians are only needed to account for small nonGaussian behaviour in the tails, whereas the electrons have more significant asymmetries which requires larger $w_{2}, w_{3}$ to describe. To gain an overall picture of the 
performance of the fits, the distribution of $\chi^{2} / n$, defined as in [93], from each of the fits is plotted in Figure 76. There is no analytical prediction against which to measure this distribution, because it combines the $\chi^{2} / n$ values from many fits with different values of $n^{8}$, so it can only be examined qualitatively. Lower values indicate a closer agreement of model and data, and a value of 1 is expected from statistical fluctuation alone, on average.

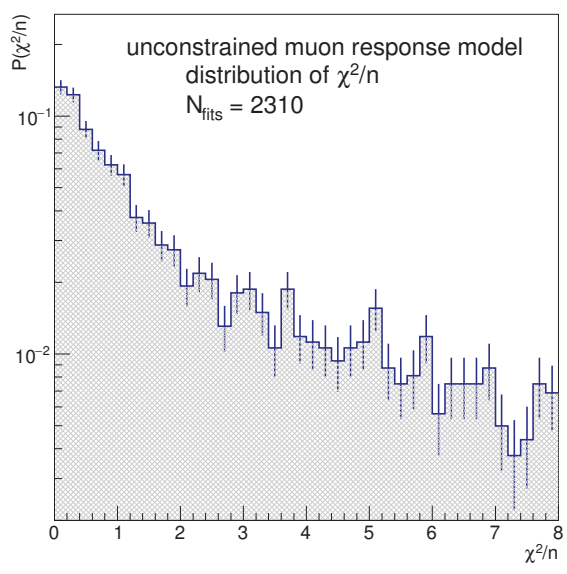

(a) muons

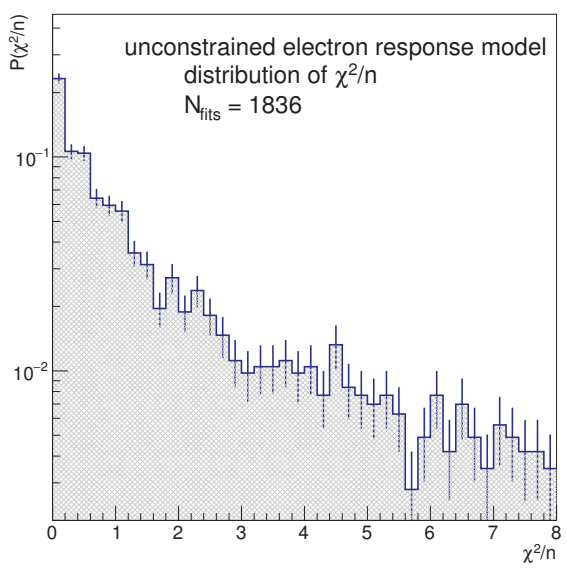

(b) electrons

Figure 76: Distribution of $\chi^{2} / n$ from unconstrained lepton response model fits.

It can be interesting to examine trends in the model, in particular by looking at $\sigma_{1}$, the width of the leading Gaussian, or most simply thought of as the scalar energy resolution of the lepton. The dependence of $\sigma_{1}$ on $p_{T}$ is $\operatorname{plotted}^{9}$ in all $\mathrm{Z}$ and $|\eta|$ bins in Figure 77. These plots exhibit the expected behaviour for both species of lepton: for electrons, calorimeter energy resolution is proportional to $\sqrt{E}[94]$, so the fractional resolution falls as $1 / \sqrt{E}$, which is nicely replicated in the plot. For muons, since the momentum is measured according to the bending of the muon track, the fractional resolution grows with $E$ as the track becomes more straight. Some

\footnotetext{
${ }^{8}$ The $\chi^{2}$ is only defined for non-empty bins, so the number of bins used to calculate it can vary from fit to fit

${ }^{9}$ Note that these plots don't include the full $p_{T}$ range covered by the model; they include the range where most $H \rightarrow 4 \ell$ leptons exist, and where the $p_{T}$ dependence is most interesting.
} 
deviations from this behaviour exist because the leading (sub-leading and sub-subleading) Gaussian(s) is (are) not forced to represent the core (tails) of the resolution distribution - they are free to find the best representation of $P\left(x_{i}\right)$ given the MC simulated distribution in that bin. The behaviour of the other parameters of the Gaussian sum is presented in Appendix B.4, as they are less instructive than $\sigma_{1}$.

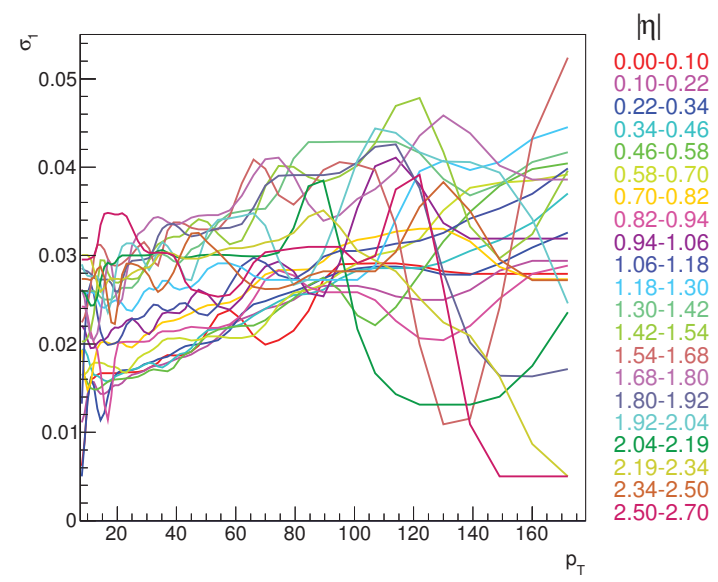

(a) muon Z1

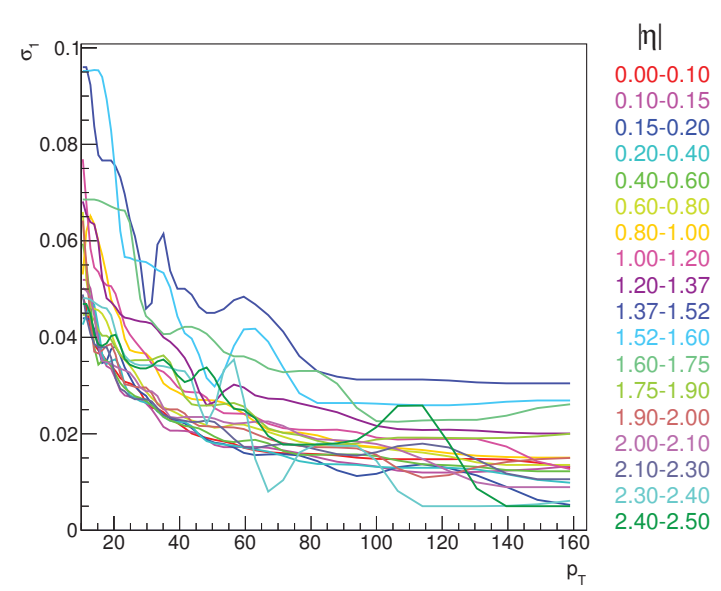

(c) electron Z1

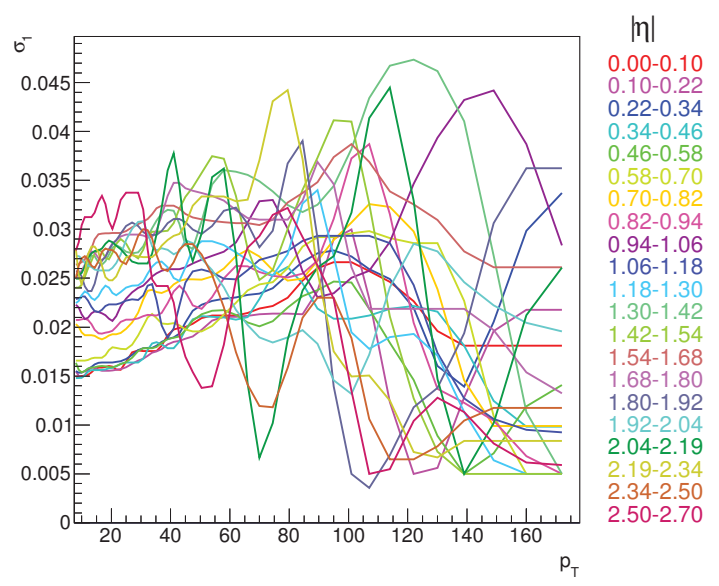

(b) muon Z2

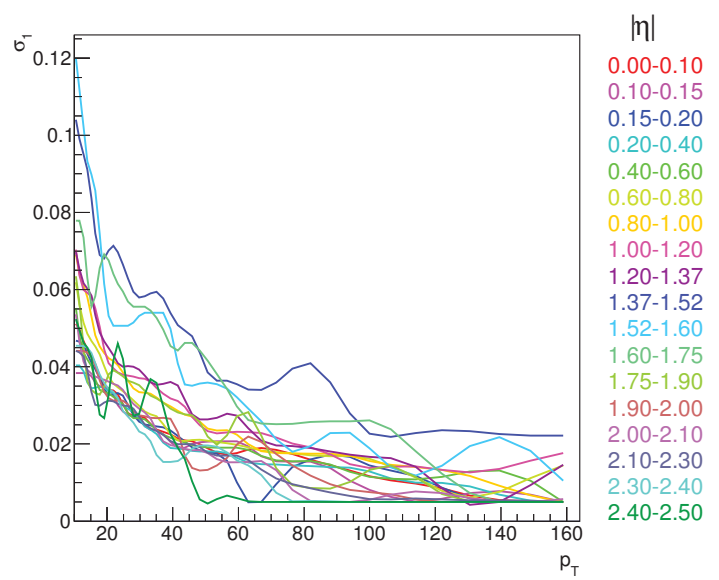

(d) electron Z2

Figure 77: Behaviour of parameter $\sigma_{1}$ in lepton response model as a function of $p_{T}$ in the $|\eta|$ bins for muons and electrons, split by Z1 and Z2. The parameters take on discrete values, but a piece-wise linear interpolation is drawn to emphasize the trend.

As further validation, the lepton resolution as described by the PER model was compared with that from the ATLAS CP groups. Note that this can never be a 
perfect apples-to-apples comparison: the PER model is by definition a response, not a resolution. To make a comparison, the PER model was reduced to a single number by using only the leading-Gaussian width. Then the two models of resolution were compared by examining the ratio of $\sigma_{1} / \sigma_{\mathrm{CP}}$ for many leptons from simulated signal events . This comparison is shown in Figure 78 (note: on the plot $\sigma_{1}$ is labelled as $\left.\sigma_{\text {PERg1 }}\right)$. As expected, the two methods do not agree perfectly, but they do show indications of providing complimentary descriptions of the lepton resolution: At higher values of $p_{T}$, where the electron response becomes more Gaussian-like, the two resolution models converge. For muons the same convergence exists, but there is more $|\eta|$-dependence, so it is less distinct.

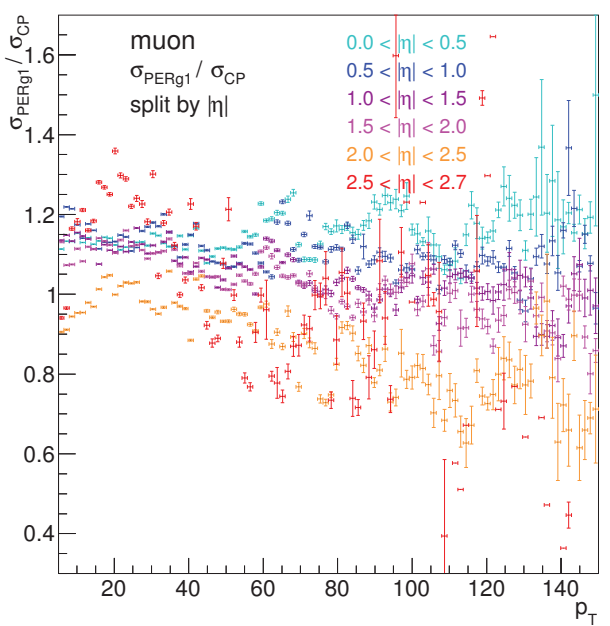

(a) muons

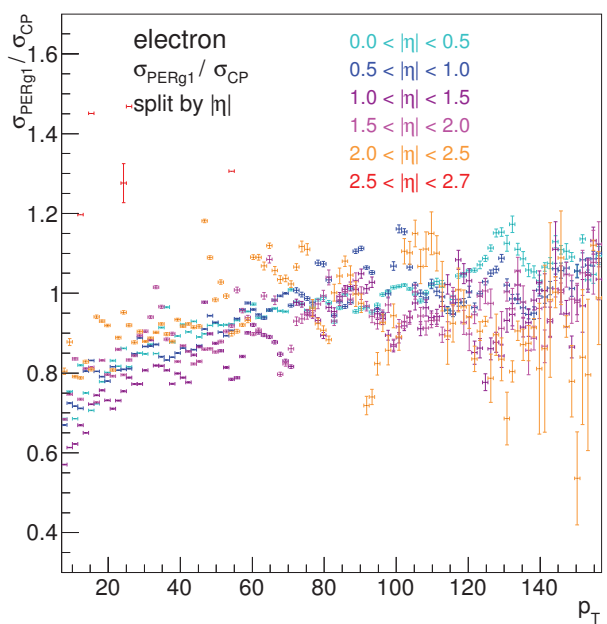

(b) electrons

Figure 78: Comparison of lepton energy resolution as modelled in PER method, with the resolution as modelled by $\mathrm{CP}$ groups. The markers show the average ratio of the PER leading-Gaussian resolution (labelled $\sigma_{\text {PERg1 }}$ ), with the resolution as parameterized by the ATLAS CP group (labelled $\sigma_{\mathrm{CP}}$ ) as a function of $p_{T}$, in slices of $|\eta|$. The vertical errors bars represent the standard deviation of the same ratio, for all leptons in the same bin. The leptons come from ggF and VBF Higgs signal simulated samples at various mass points. 


\subsubsection{PDF simplification}

As the lepton response $x_{i}$ is dimensionless by definition, it is important to see how the factors of mass found in (82) enter the expression to create a $m_{4 \ell}$ response. Using the properties of random variables we can see that

$$
\begin{aligned}
\text { if } \mathrm{P}\left(\mathrm{x}_{\mathrm{i}}\right) & =\sum_{g} w_{g} G\left(x_{i} ; \mu_{g}, \sigma_{g}\right), \\
\text { then } \mathrm{P}\left(\mathrm{Ax}_{\mathrm{i}}\right) & =\sum_{g} w_{g} G\left(A x_{i} ; A \mu_{g}, A \sigma_{g}\right),
\end{aligned}
$$

where A can be any scalar number, but is often the factor $\left(\sum_{j \neq i}\left(m_{i j}^{\mathrm{fsr}}\right)^{2}\right)$ from (79). In this expression now the parameters of the Gaussians carry the same dimensions as $A$ (e.g. mass). We can also see what happens when these distributions are convolved, as instructed by (80):

$$
\begin{aligned}
P\left(A x_{i}+B x_{j}\right) & =\left[\sum_{g} w_{g}^{i} G\left(A x_{i} ; A \mu_{g}^{i}, A \sigma_{g}^{i}\right)\right] *\left[\sum_{g^{\prime}} w_{g^{\prime}}^{j} G\left(B x_{j} ; B \mu_{g^{\prime}}^{j}, B \sigma_{g^{\prime}}^{j}\right)\right] \\
& =\sum_{g, g^{\prime}} w_{g}^{i} w_{g^{\prime}}^{j}\left[G\left(A x_{i} ; A \mu_{g}^{i}, A \sigma_{g}^{i}\right) * G\left(B x_{j} ; B \mu_{g^{\prime}}^{j}, B \sigma_{g^{\prime}}^{j}\right)\right], \\
& =\sum_{g, g^{\prime}} w_{g}^{i} w_{g^{\prime}}^{j} G\left(A x_{i}+B x_{j} ; A \mu_{g}^{i}+B \mu_{g^{\prime}}^{j}, \sqrt{\left(A \sigma_{g}^{i}\right)^{2}+\left(B \sigma_{g^{\prime}}^{j}\right)^{2}}\right) .
\end{aligned}
$$

From this expression we see that when the input lepton energy response model is a Gaussian mixture, so too will be the final invariant mass response.

A consequence of (85) is that when 3 weighted Gaussians are used to describe $P\left(x_{i}\right)$, then a sum of $3^{N_{\text {part }}}=81$ weighted Gaussians will be needed to describe the $m_{4 \ell}$ response. This number is evidently quite unwieldy and must be reduced. The reduction follows the same procedure used in electron reconstruction using a Gaussian Sum Filter [72]. The 81 Gaussians are compared using the Kullback-Leibler 
distance [73] defined for a pair of Gaussians (call them $a, b$ ) by

$$
D_{\mathrm{KL}}=\frac{\left(\sigma_{a}^{2}-\sigma_{b}^{2}\right)^{2}+\left(\mu_{a}-\mu_{b}\right)^{2}\left(\sigma_{a}^{2}+\sigma_{b}^{2}\right)}{\sigma_{a}^{2} \sigma_{b}^{2}},
$$

and the closest pair (smallest $D_{\mathrm{KL}}$ ) is identified. This pair is replaced in the sum by a single Gaussian with parameters

$$
\begin{aligned}
w^{\prime} & =w_{a}+w_{b}, \\
\mu^{\prime} & =\frac{w_{a} \mu_{a}+w_{b} \mu_{b}}{w_{a}+w_{b}}, \\
\sigma^{\prime 2} & =\frac{w_{a} \sigma_{a}^{2}+w_{b} \sigma_{b}^{2}}{w_{a}+w_{b}}+\frac{w_{a} w_{b}}{\left(w_{a}+w_{b}\right)^{2}}\left(\mu_{a}-\mu_{b}\right)^{2} .
\end{aligned}
$$

The algorithm is repeated until the number of remaining Gaussians reaches the target, $N_{G}=4$. This procedure by design preserves the 1st moment (mean) and 2nd moment (variance) of the original distribution. Higher order moments are not preserved, but by retaining $N_{G}=4$ Gaussians, most of the unique shape of any individual event's $m_{4 \ell}$ response is preserved. An example of this procedure for a single MC event is shown in Figure 79, where the Gaussian sum representing the mass response for this event is shown at various stages of the collapse. In practice the procedure stops at $N_{G}=4$, though here it is shown down to $N_{G}=1$.

Returning to (82), the PER signal PDF can be written down as the convolution of this Gaussian mixture (describing the response), with the true distribution for any given signal hypothesis,

$$
P\left(m_{4 \ell}^{\mathrm{fsr}} \mid \text { kinematics }\right)=\left[\sum_{g=1}^{N_{G}} w_{g} G\left(m_{4 \ell}^{\mathrm{fsr}}-m_{4 \ell}^{\mathrm{true}} ; \mu_{g} ; \sigma_{g}\right)\right] * P\left(m_{4 \ell}^{\mathrm{true}}\right),
$$

where the weighted sum in square brackets and all the Gaussians parameters incorporate the invariant mass factors implicitly (the same ones explicitly shown in (85)). 


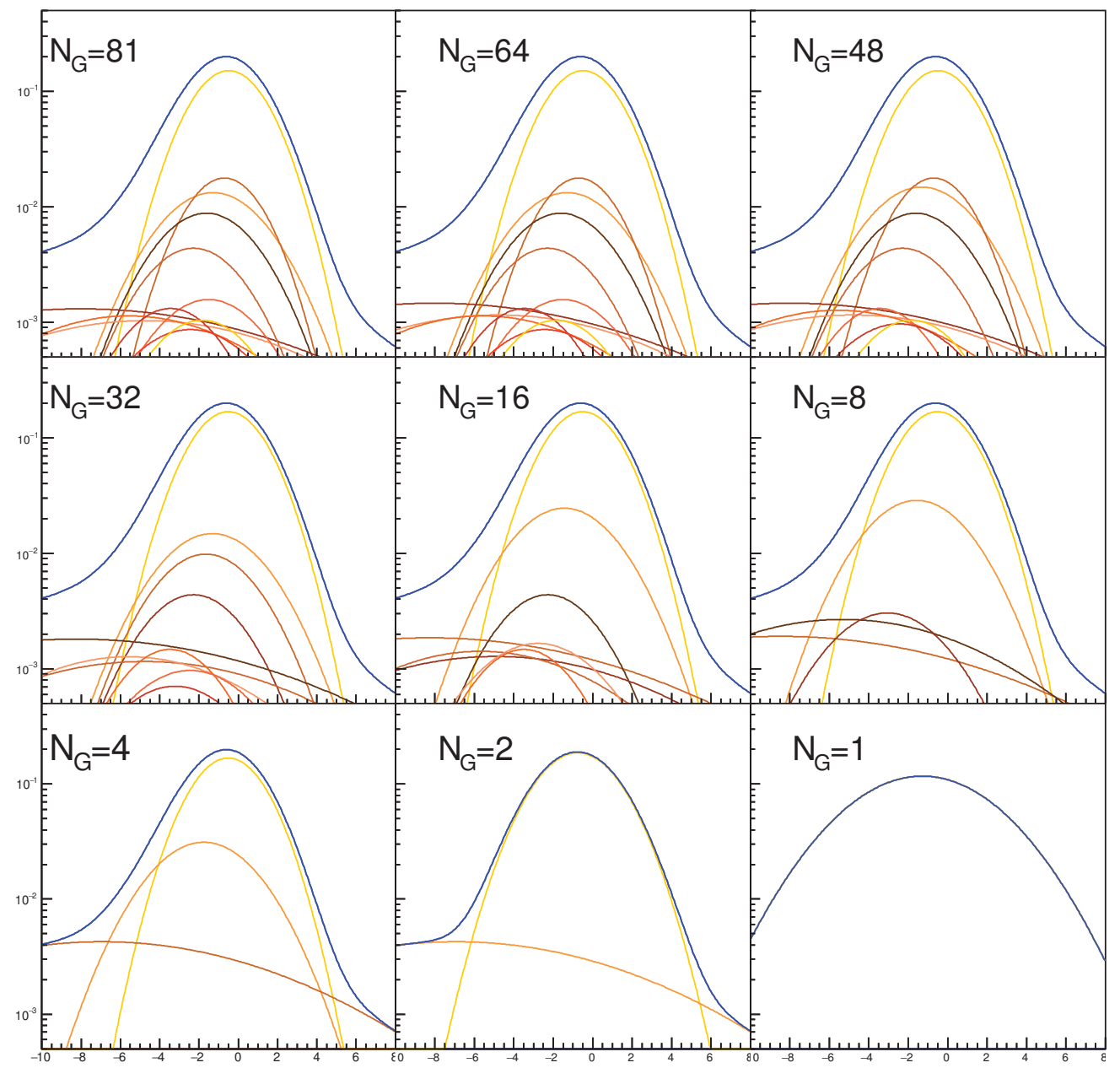

Figure 79: Demonstration of collapsing procedure on an example MC signal event. The initial 81-Gaussian sum is shown at various stages of collapse down to a single Gaussian. The $\mathrm{x}$-axis is $m_{4 \ell}^{\mathrm{fsr}}-m_{4 \ell}^{\text {true }}$ in units of $\mathrm{GeV}$, left unlabelled to minimize clutter. Some Gaussians have weights and sigmas such that they are below the plotted y-axis range.

The signal hypothesis used in this thesis is of a Higgs boson with mass $m_{H}$, and natural width $\Gamma_{H}$. Its true mass will be distributed, as described in Section 2.3.2.2, by a BW distribution,

$$
P\left(m_{4 \ell}^{\text {true }}\right)=\mathrm{BW}_{\epsilon}\left(m_{4 \ell}^{\text {true }} ; m_{H}, \Gamma_{H}\right)
$$

where the $\mathrm{BW}_{\epsilon}$ indicates the $\mathrm{BW}$ is corrected by an efficiency function $\epsilon\left(m_{4 \ell}^{\text {true }}\right)$ to 
account for non-uniform detector efficiency across the width of true lineshape when $\Gamma_{H}$ is large. For small widths, as in the SM, the efficiency is sufficiently flat that this factor has no effect (even up to $\Gamma_{H}=100 \Gamma_{H}^{\mathrm{SM}}$, the effect is very small). Conveniently, this factor is exactly equivalent to the acceptance polynomial function of Section 7.1,

$$
\begin{aligned}
\mathrm{BW}_{\epsilon}\left(\mathrm{m}_{4 \ell}^{\text {true }} ; \mathrm{m}_{\mathrm{H}}, \Gamma_{\mathrm{H}}\right) & =\epsilon\left(m_{4 \ell}^{\text {true }}\right) \mathrm{BW}\left(\mathrm{m}_{4 \ell}^{\text {true }} ; \mathrm{m}_{\mathrm{H}}, \Gamma_{\mathrm{H}}\right) \\
& =A_{c}\left(m_{4 \ell}^{\text {true }}\right) \mathrm{BW}\left(\mathrm{m}_{4 \ell}^{\text {true }} ; \mathrm{m}_{\mathrm{H}}, \Gamma_{\mathrm{H}}\right) .
\end{aligned}
$$

The description of $P\left(m_{4 \ell}^{\text {true }}\right)$ as a BW distribution assumes by definition that we select the correct four leptons in an $H \rightarrow 4 \ell$ signal event. For ggF and VBF production, events passing the event selection and the mass window used in this analysis have the correct four leptons $99.97 \%$ of the time. For VH and ttH production modes with additional leptons, the correct four leptons are selected in $97.59 \%$ of the events in the same mass window (most events with a wrong lepton selected end up outside the mass window). When these production modes are weighted by their SM cross section the non-resonant contribution is extremely small.

The PER signal PDF as it is used in this measurement can be written down by combining (88) and (89),

$P\left(m_{4 \ell}^{\mathrm{fsr}} \mid m_{H}, \Gamma_{H}\right.$, kinematics $)=\left[\sum_{g=1}^{N_{G}} w_{g} G\left(m_{4 \ell}^{\mathrm{fsr}}-m_{4 \ell}^{\mathrm{true}} ; \mu_{g} ; \sigma_{g}\right)\right] * \mathrm{BW}{ }_{\epsilon}\left(m_{4 \ell}^{\mathrm{true}} ; m_{H}, \Gamma_{H}\right)$.

This final expression contains one single observable $\left(m_{4 \ell}^{\mathrm{fsr}}\right)$, several conditional observables (kinematics, which are transformed into $w_{g}, \mu_{g}, \sigma_{g}$, combined as $\vec{g}$ ) whose distribution is not specified in advance but must be observed in data, and two parameters of interest $\left(m_{H}, \Gamma_{H}\right)$ that can be fit. 


\subsubsection{Gaussian sum parameter transformation}

While the method described above very closely predicts the lineshape of each event, the final result can be improved by tuning on the $m_{4 \ell}$ distribution from signal MC. This transformation accounts for effects introduced by the choice of binning in the lepton response model, for MC statistics availability in each of these bins and their effect on the Gaussian parameters, and for any small shape detail lost during the collapsing procedure. This transformation uses one tuning parameter for each parameter of the Gaussian sum in (91), which are transformed as follows:

$$
\begin{aligned}
w_{i} & \rightarrow n_{i} w_{i}, \\
\sigma_{i} & \rightarrow r_{i} \sigma_{i}, \\
\mu_{i} & \rightarrow \mu_{i}+s_{i}+\left(1 .-r_{i}\right)\left(\mu_{i}-\mu_{1}\right),
\end{aligned}
$$

where $r$ denotes a modification on the resolution, $s$ denotes a modification on the scale, $n$ denotes a modification on the relative contributions of each of the Gaussians. Note that the $r_{i}$ are used both to scale the resolution of the Gaussians, and to shift them closer together (reducing the RMS of their combined distribution). The parameters are fit simultaneously for all events in $m_{H}=125 \mathrm{GeV} g g F \mathrm{MC}$ sample, separately for each category, and recorded in Table 13. No improvement was found for the $2 e 2 \mu$ category, and so no transformation was applied. Note that values of $s_{i}=0, r_{i}=1, n_{i}=1$ indicate no effect; the transformation overall has a very small (but none-the-less valuable) effect.

Figure 80 shows the effect of this transformation on the $m_{H}=125 \mathrm{GeV} \operatorname{ggF} \mathrm{MC}$ sample (the same one used to derive the parameters). The transformation yields a distinct improvement over the uncorrected case. Values of $\chi^{2} / n$ are printed on each plot for comparison. For a robust fit result, these parameters must be independent of $m_{H}$ and of the Higgs production mode - by definition they exist only to correct minor 
Table 13: Transformation parameters used to account for slight discrepancies in the invariant mass response, as used in the unconstrained PER model. The parameters are derived by a simultaneous fit on simulated ggF event with $m_{H}=125 \mathrm{GeV}$.

\begin{tabular}{ccccc}
\hline parameter & $4 \mu$ & $4 e$ & $2 \mu 2 e$ & $2 e 2 \mu$ \\
\hline$r_{1}$ & 0.963 & 0.997 & 0.948 & 1 \\
$r_{2}$ & 0.953 & 0.984 & 1.001 & 1 \\
$r_{3}$ & 0.994 & 0.933 & 1.051 & 1 \\
$r_{4}$ & 1.110 & 1.995 & 1.154 & 1 \\
$s_{1}$ & 0.060 & 0.148 & 0.101 & 0 \\
$s_{2}$ & -0.252 & 0.043 & -0.040 & 0 \\
$s_{3}$ & -1.214 & -0.611 & -0.836 & 0 \\
$s_{4}$ & -1.140 & -2.997 & -2.967 & 0 \\
$n_{1}$ & 0.998 & 0.983 & 1.012 & 1 \\
$n_{2}$ & 1.001 & 1.000 & 0.981 & 1 \\
$n_{3}$ & 1.026 & 1.117 & 0.988 & 1 \\
$n_{4}$ & 1.155 & 1.177 & 0.794 & 1 \\
\hline
\end{tabular}

imperfections in the response model, something wholly detector-based. To verify this, the same set of transformation parameters are tested on the $m_{H}=124 \mathrm{GeV}$ and $m_{H}=126 \mathrm{GeV}$ ggF MC samples, shown in Figure 81 and Figure 82 respectively, as well as the $m_{H}=125 \mathrm{GeV}$ VBF MC sample in Figure 83. In each case, the same set of transformation parameters perform very well at improving model agreement.

\subsubsection{Response after Z Mass Constraint}

As demonstrated in Section 5.10, the introduction of the Z Mass Constraint reduces the resolution of four-lepton invariant mass by about 14\%, and consequently makes $m_{4 \ell}^{\text {cons }}$ more sensitive to $m_{H}$ than $m_{4 \ell}^{\mathrm{fsr}}$ by an equal amount. Inclusion of this benefit 


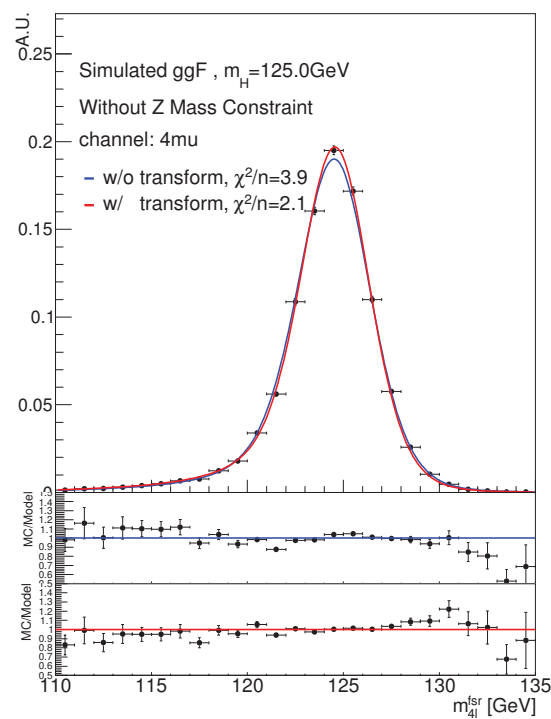

(a) $4 \mu$

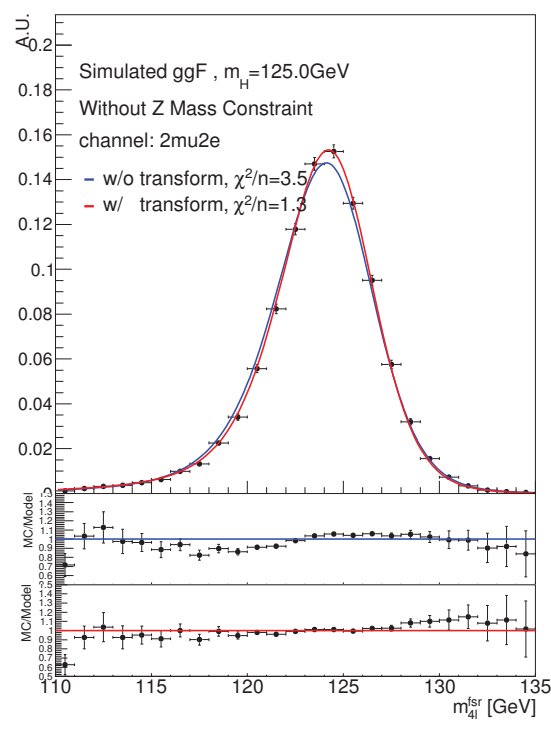

(c) $2 \mu 2 e$

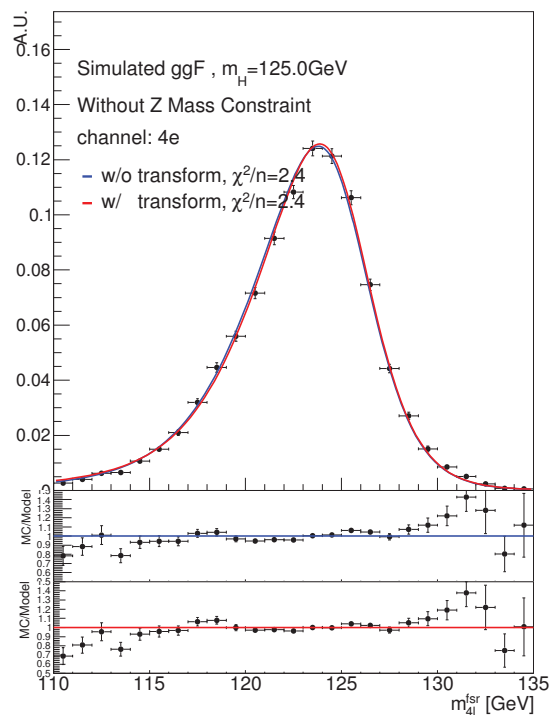

(b) $4 e$

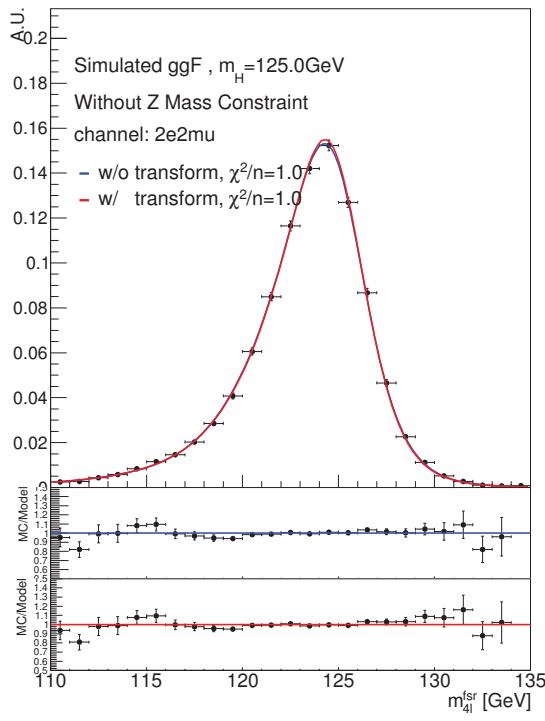

(d) $2 e 2 \mu$

Figure 80: Demonstration of effect of transformation of Gaussian parameters on the unconstrained PER model on the $m_{H}=125 \mathrm{GeV}$ ggF MC sample, separated by category. The transformation parameters, defined in (92), are derived by a simultaneous fit on this sample. The black points show the MC simulation, the blue line shows the prediction from the untransformed per-event response model, and the red line shows the prediction from the transformed per-event response model. At the bottom the ratio of the MC simulation over each prediction is shown. 


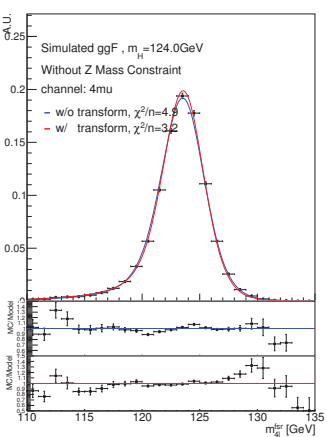

(a) $4 \mu$

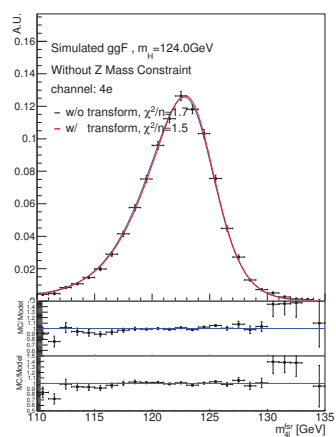

(b) $4 e$

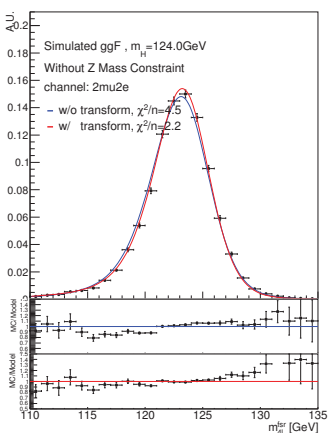

(c) $2 \mu 2 e$

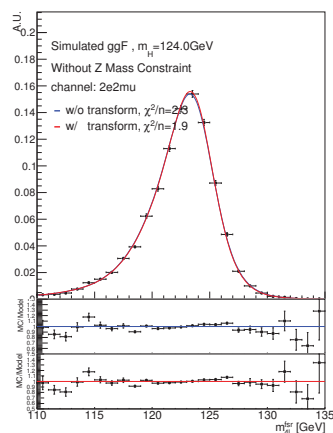

(d) $2 e 2 \mu$

Figure 81: Demonstration of effect of transformation of Gaussian parameters on the unconstrained PER model on the $m_{H}=124 \mathrm{GeV}$ ggF MC sample.

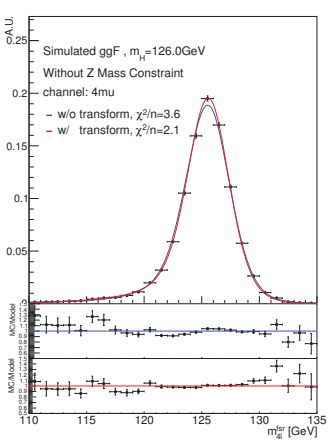

(a) $4 \mu$

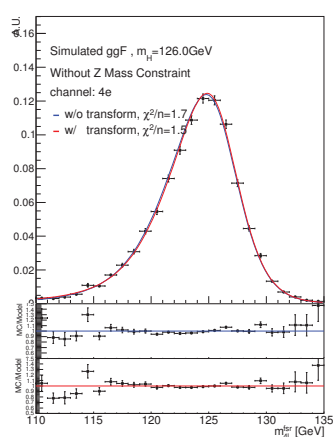

(b) $4 e$

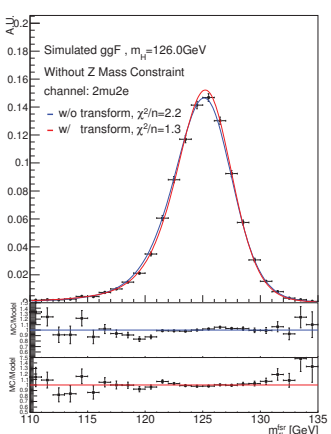

(c) $2 \mu 2 e$

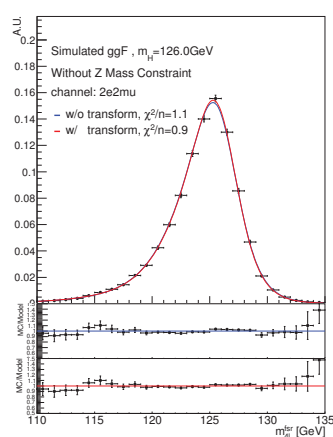

(d) $2 e 2 \mu$

Figure 82: Demonstration of effect of transformation of Gaussian parameters on the unconstrained PER model on the $m_{H}=126 \mathrm{GeV}$ ggF MC sample.

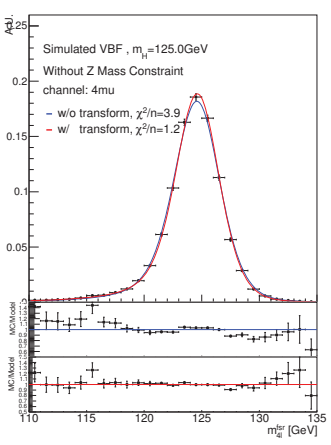

(a) $4 \mu$

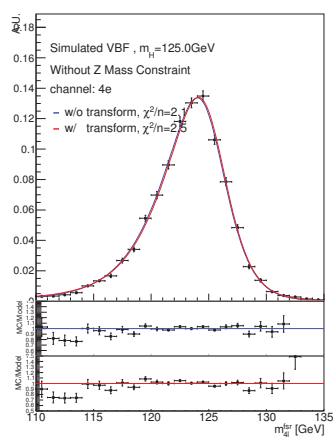

(b) $4 e$

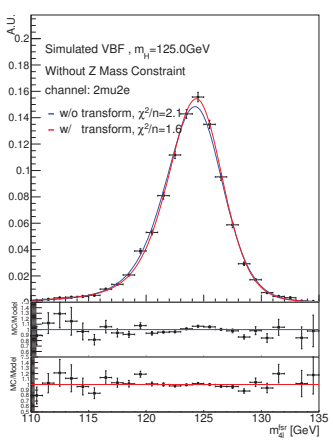

(c) $2 \mu 2 e$

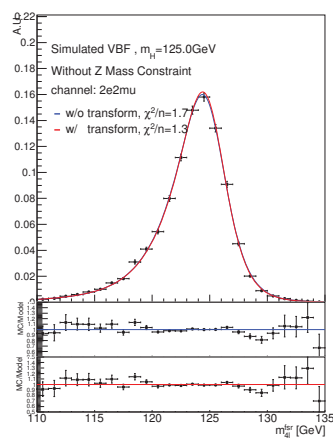

(d) $2 e 2 \mu$

Figure 83: Demonstration of effect of transformation of Gaussian parameters on the unconstrained PER model on the $m_{H}=125 \mathrm{GeV}$ VBF MC sample. 
is evidently worthwhile for a precision measurement of $m_{H}$.

If the constrained four-momenta are considered as an alternative estimate of the lepton kinematics ${ }^{10}$, the model described in Section 7.3.2, and Section 7.3.3 can be modified to describe these new responses simply by substituting $E_{i}^{\mathrm{fsr}} \rightarrow E_{i}^{\mathrm{cons}}, m_{4 \ell}^{\mathrm{fsr}} \rightarrow$ $m_{4 \ell}^{\text {cons }}$. With this substitution, the mathematics which predict the detector response of $m_{4 \ell}^{\text {cons }}$ are unchanged as compared to $m_{4 \ell}^{\mathrm{fsr}}$. The fits of the lepton response (those shown in Figures 74,75) are altered by this new definition, though visually they are not substantially different; for this reason they are recorded in Appendix B.3. Both versions of the response model serve valuable purposes: the unconstrained PER is used as a proof-of-principle and cross check, and the constrained PER is used in the final $m_{H}$ measurement.

An additional caveat when using the ZMC is that the constraint introduces a relationship between the two leptons which form the Z1 pair. As shown in Section 5.9, the most likely values $m_{Z 1}$ on an event-by-event basis form a distribution significantly narrower than the $\mathrm{Z}$ lineshape. As a result, the energies $E_{1}^{\text {cons }}, E_{2}^{\text {cons }}$ are correlated in order to make a di-lepton invariant mass very close to $m_{Z}^{\mathrm{PDG}}=91.19 \mathrm{GeV}$. This introduces a potential pitfall in the error propagation of the PER method, particularly (80), which assumes the lepton energy responses are uncorrelated; using this relationship with correlated random variables is incorrect ${ }^{11}$. To account for this effect in the PER method, the transformation parameters in Section 7.3.4 are re-interpreted to not only account for small discrepancies in the model, but also to absorb the effect of the relationship between $x_{1}, x_{2}$ in the error propagation. The approach is to say that while the co-dependent uncertainties can't be propagated analytically, their effect can be observed numerically in simulation, and incorporated parametrically In

\footnotetext{
${ }^{10}$ Some revolutionary calibration procedure which improves the invariant mass resolution by $18 \%$ would be treated the same way.

${ }^{11}$ An obvious solution may appear to be to introduce a correlation $\rho_{12}$ between $x_{1}$ and $x_{2}$, and use simple correlated Gaussian error propagation: $\sigma^{\prime 2}=\sqrt{\sigma_{1}^{2}+\sigma_{2}^{2}-2 \rho \sigma_{1} \sigma_{2}}$ in (85), but the correlation is not a simple one, and this expression is inadequate to describe the relationship between $x_{1}$ and $x_{2}$.
} 
this way we can still benefit from the increased mass resolution of the ZMC, together with the PER method. As in the unconstrained case, the transformation parameters are derived by a simultaneous fit on simulated ggF event with $m_{H}=125 \mathrm{GeV}$. The parameters are given in Table 14.

Table 14: Transformation parameters used to account for the effect of the ZMC on the invariant mass response, as used in the constrained PER model. The parameters are derived by a simultaneous fit on simulated $g g F$ event with $m_{H}=125 \mathrm{GeV}$.

\begin{tabular}{ccccc}
\hline parameter & $4 \mu$ & $4 e$ & $2 \mu 2 e$ & $2 e 2 \mu$ \\
\hline$r_{1}$ & 0.850 & 0.917 & 0.896 & 0.826 \\
$r_{2}$ & 0.949 & 0.978 & 0.955 & 1.023 \\
$r_{3}$ & 0.956 & 0.942 & 0.952 & 0.964 \\
$r_{4}$ & 1.011 & 1.998 & 1.078 & 1.070 \\
$s_{1}$ & 0.079 & 0.185 & 0.115 & -0.010 \\
$s_{2}$ & -0.081 & 0.059 & 0.068 & 0.131 \\
$s_{3}$ & -0.815 & -1.021 & -0.223 & -0.453 \\
$s_{4}$ & -0.592 & -2.990 & -0.795 & -1.974 \\
$n_{1}$ & 0.906 & 0.996 & 1.039 & 1.017 \\
$n_{2}$ & 1.047 & 1.041 & 1.071 & 0.964 \\
$n_{3}$ & 1.051 & 1.016 & 1.299 & 1.023 \\
$n_{4}$ & 1.013 & 0.896 & 1.105 & 1.201 \\
\hline
\end{tabular}

Note in particular the parameter $r_{1}$, which transforms the core width of the resolution distribution ${ }^{12}$. The values for each category (e.g. a $15 \%$ reduction in $4 \mu$ ) are consistent with those found by fitting the core of the distributions alone, summarized in Table 9. Results of this transformation on the $m_{H}=125 \mathrm{GeV} \operatorname{ggF} \mathrm{MC}$ sample can be seen in Figure 84. The same transformation parameters applied to the $m_{H}=124 \mathrm{GeV} \mathrm{ggF}, m_{H}=126 \mathrm{GeV}$ ggF, and $m_{H}=125 \mathrm{GeV}$ VBF MC samples can be seen in Figure 85, Figure 86, and Figure 87, respectively, justifying that these

\footnotetext{
${ }^{12}$ The Gaussian-parameter indices are ordered by largest weight. Index 1 corresponds to the largest weight, making up the core of the resolution distribution, while index 4 corresponds to the far far tails.
} 
parameters correct only the response model, independent of $m_{H}$ or the production mode.

\subsubsection{Distribution of resolutions}

While the macro properties of the PER model shown in the preceding sections provide a good measure of its overall performance through agreement with simulation, it can also be interesting to examine individual events. Figure 88 shows the PDF predicted by the constrained PER model for a few events randomly chosen from ggF simulation. The distributions are plotted assuming $m_{H}=125 \mathrm{GeV}$, with $\Gamma_{H}=4.4 \mathrm{MeV}$.

Included on the distributions is an effective resolution [95], $\sigma_{\text {eff }}$, defined as half the smallest width that contains $68.3 \%$ of the distribution; this is a simple metric that enables comparison of the generally complex resolution distributions. For a Gaussian distribution, it is equivalent to the Gaussian $\sigma$. This quantity can also be used to visualize the spread of resolutions within each category; this is plotted in Figure 89.

The distributions of $\sigma_{\text {eff }}$ exhibit some expected features: The $4 \mu$ effective resolution has significantly less variance than the categories containing electrons. This is not surprising when Figure 77 is considered: energy resolution varies much more rapidly for electrons than for muons, leading to larger variance in the final $m_{4 \ell}$ resolution (both with and without constraint). The mean of each histogram, printed on the same plots, represents the average resolution in each category. Events with $\sigma_{\text {eff }}$ smaller than this value are better-than-average events, and those with larger $\sigma_{\text {eff }}$ are worse-than-average; consequently, these events become either more or less important in the final $m_{H}$ fit, respectively. 


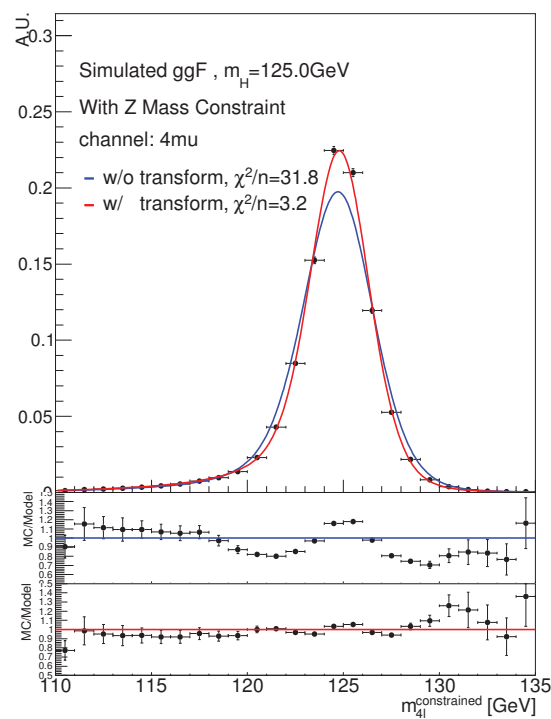

(a) $4 \mu$

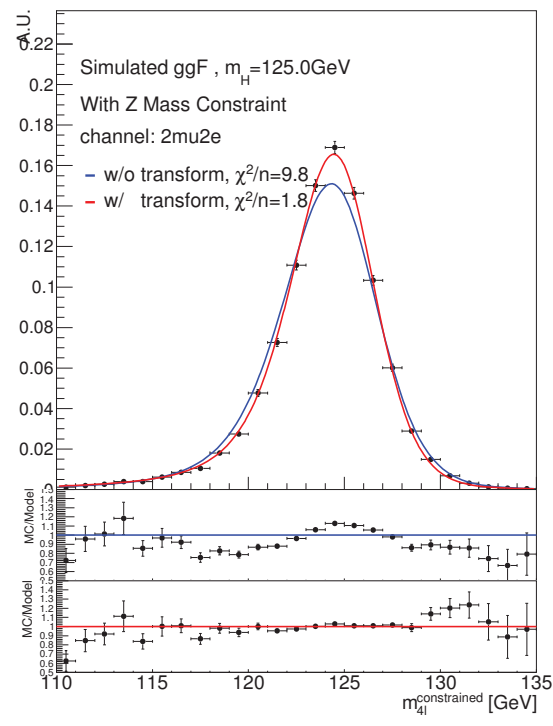

(c) $2 \mu 2 e$

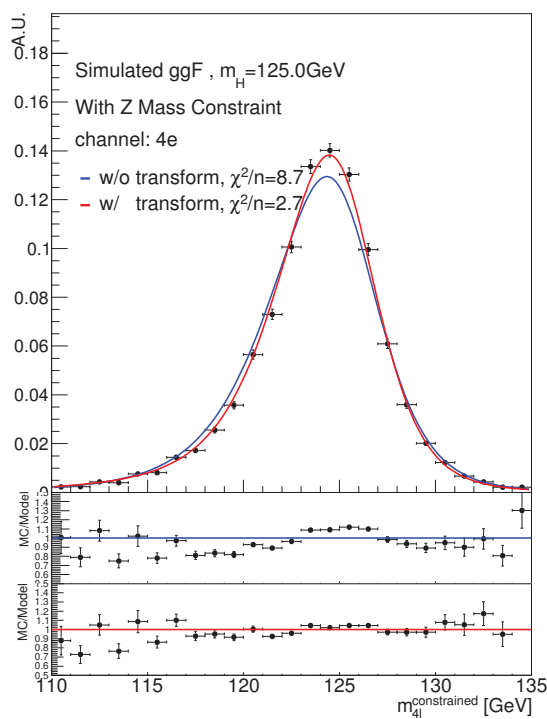

(b) $4 e$

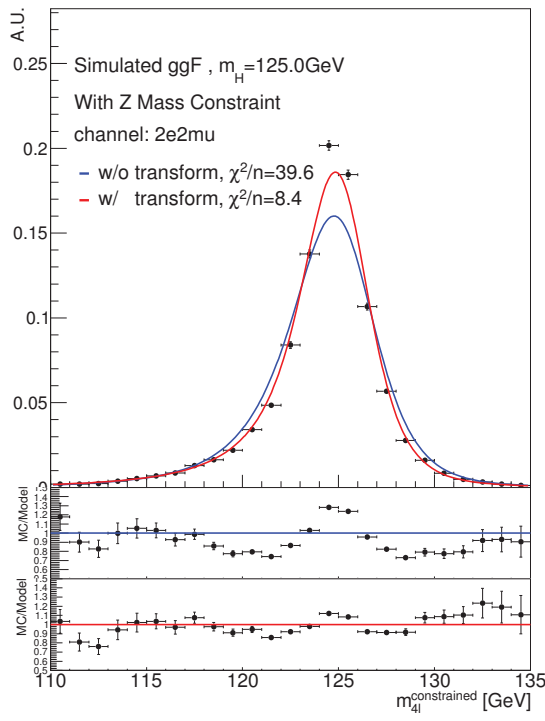

(d) $2 e 2 \mu$

Figure 84: Demonstration of effect of transformation of Gaussian parameters on the constrained PER model on the $m_{H}=125 \mathrm{GeV}$ ggF MC sample, separated by category. The transformation parameters, defined in (92), are derived by a simultaneous fit on this sample. The black points show the MC simulation, the blue line shows the prediction from the untransformed per-event response model, and the red line shows the prediction from the transformed per-event response model. At the bottom the ratio of the MC simulation over each prediction is shown. 


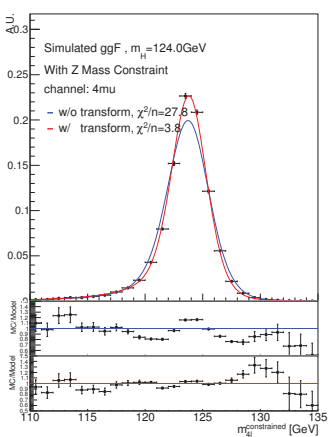

(a) $4 \mu$

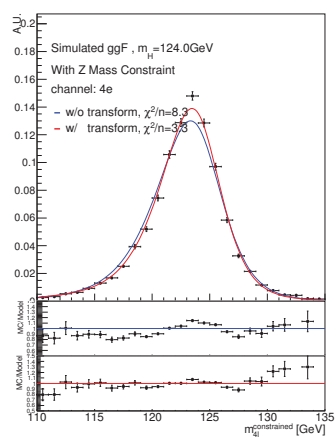

(b) $4 e$

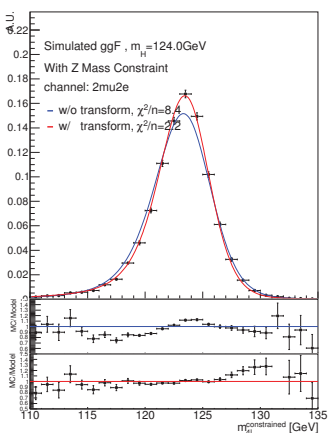

(c) $2 \mu 2 e$

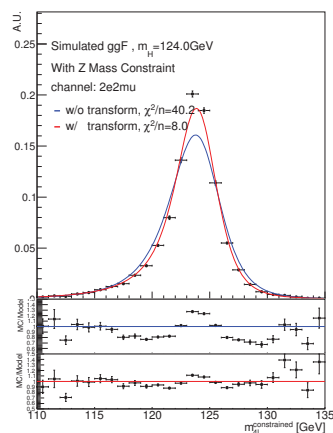

(d) $2 e 2 \mu$

Figure 85: Demonstration of effect of transformation of Gaussian parameters on the constrained PER model on the $m_{H}=124 \mathrm{GeV}$ ggF MC sample.

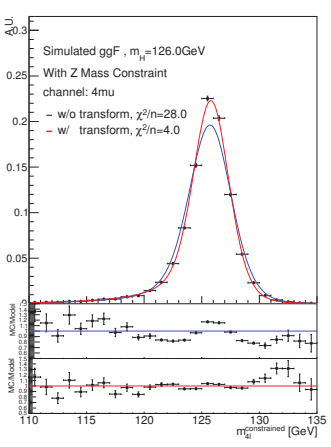

(a) $4 \mu$

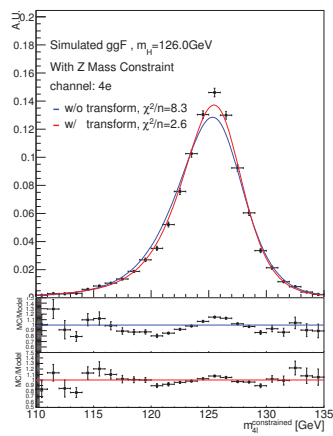

(b) $4 e$

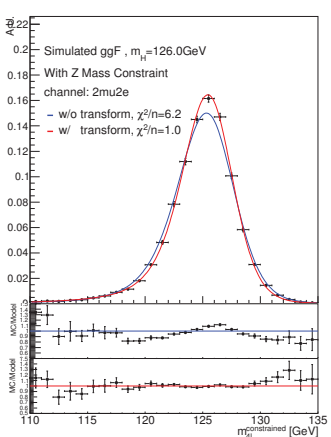

(c) $2 \mu 2 e$

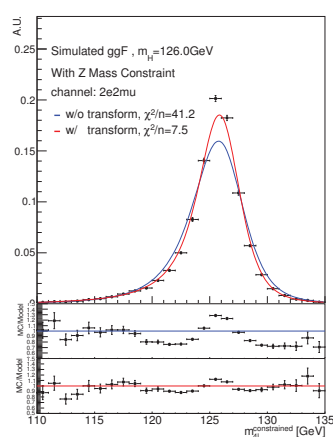

(d) $2 e 2 \mu$

Figure 86: Demonstration of effect of transformation of Gaussian parameters on the constrained PER model on the $m_{H}=126 \mathrm{GeV}$ ggF MC sample.

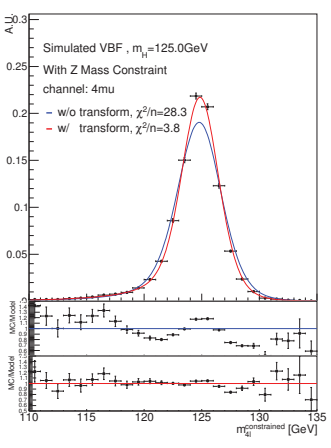

(a) $4 \mu$

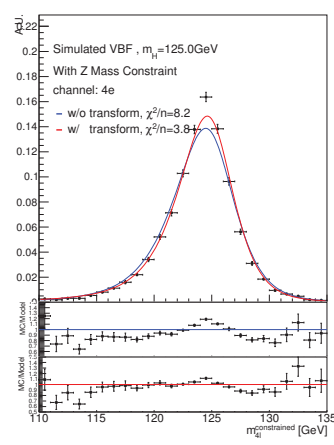

(b) $4 e$

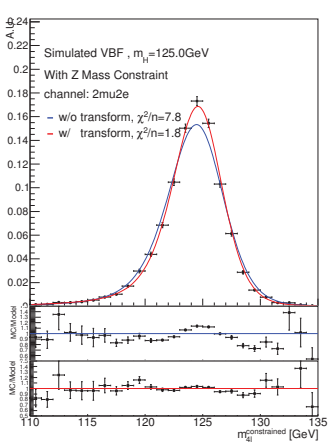

(c) $2 \mu 2 e$

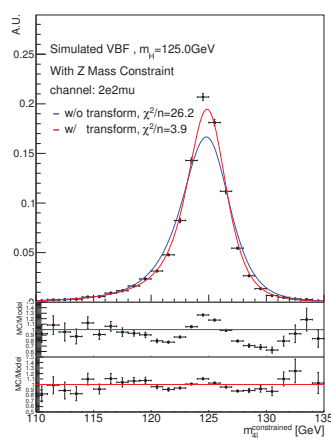

(d) $2 e 2 \mu$

Figure 87: Demonstration of effect of transformation of Gaussian parameters on the constrained PER model on the $m_{H}=125 \mathrm{GeV}$ VBF MC sample. 

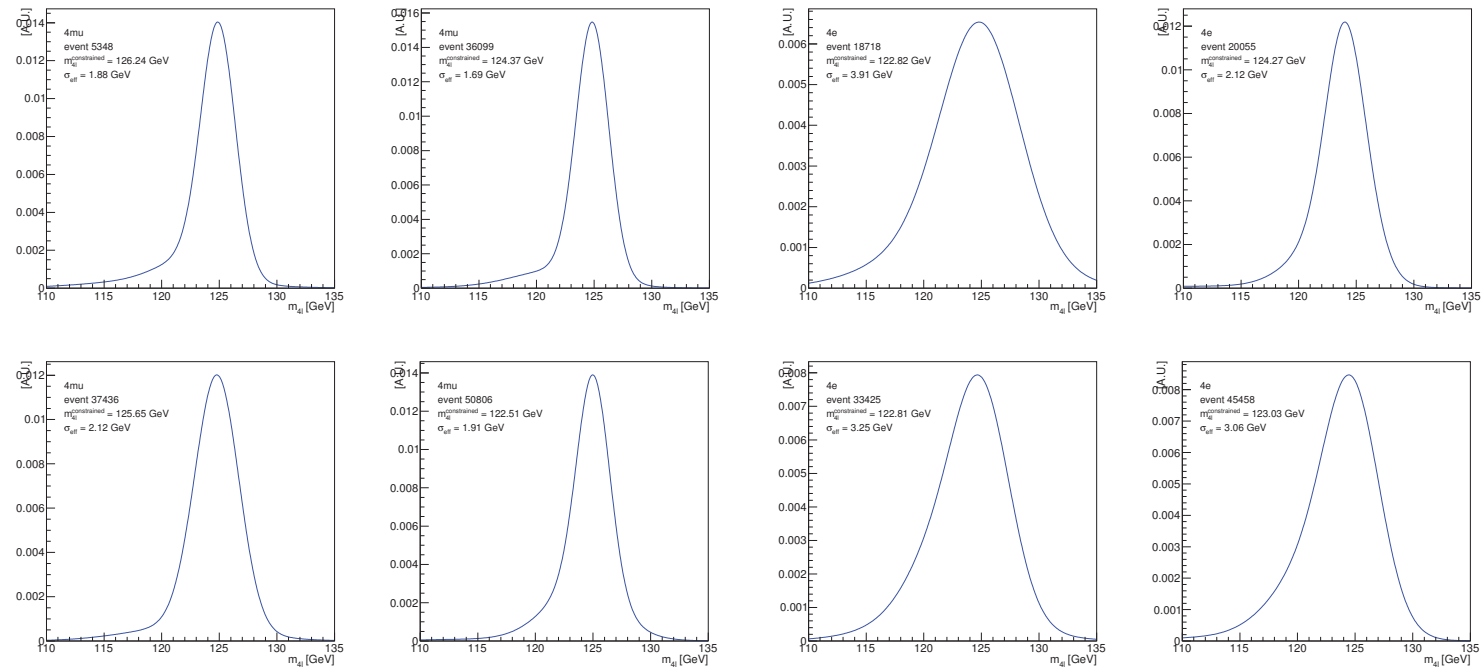

(a) $4 \mu$

(b) $4 e$
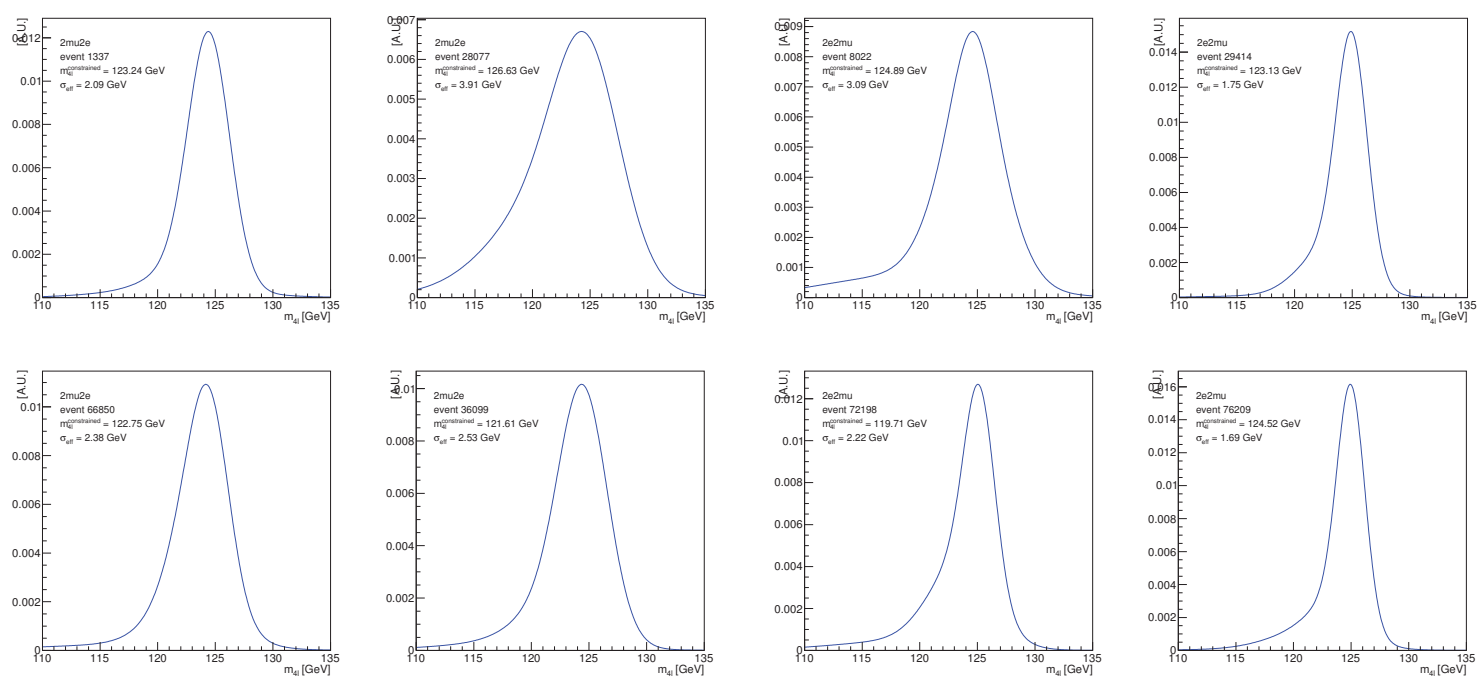

(c) $2 \mu 2 e$
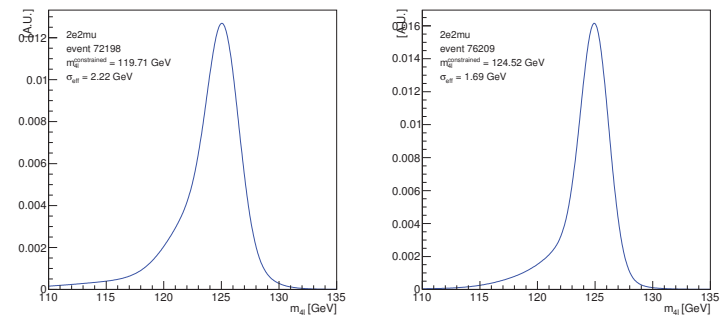

(d) $2 e 2 \mu$

Figure 88: Predicted $P\left(m_{4 \ell}^{\text {cons }}\right)$ from the constrained PER model for a sample of simulated ggF events in each category. Printed on the plots are the $m_{4 \ell}^{\text {cons }}$ from each event, and the effective resolution, $\sigma_{\text {eff }}$. 


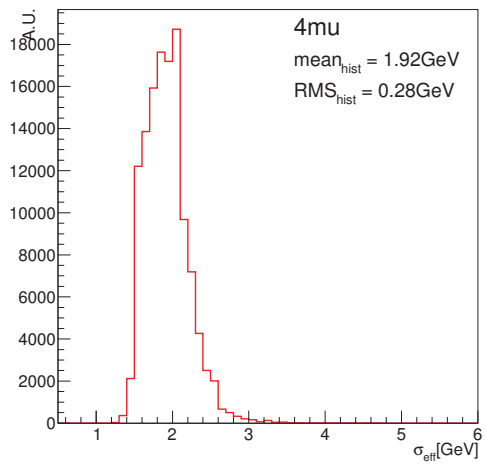

(a) $4 \mu$

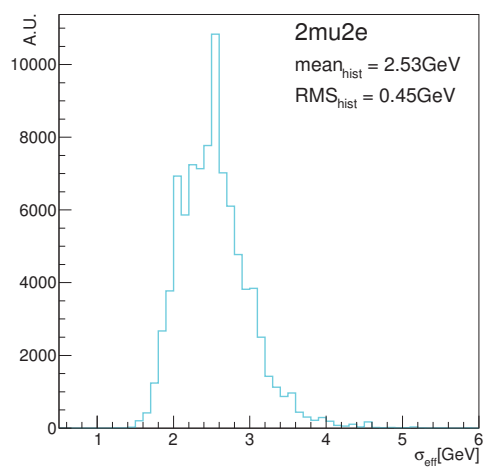

(c) $2 \mu 2 e$

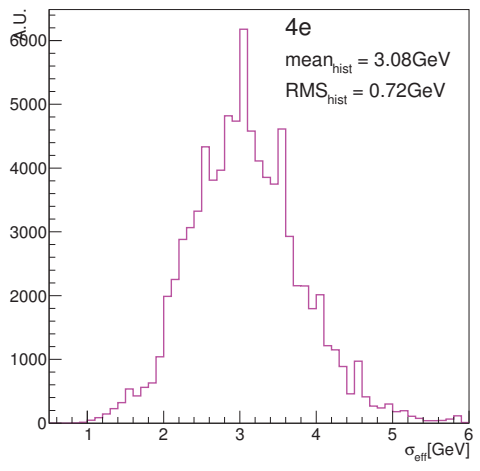

(b) $4 e$

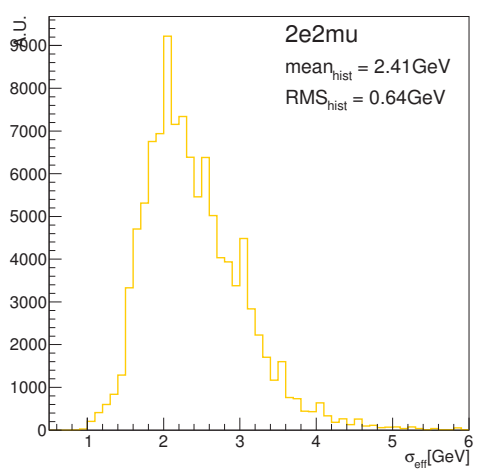

(d) $2 e 2 \mu$

Figure 89: Distribution of $\sigma_{\text {eff }}$ in the constrained PER model from simulated ggF events in each category. Better-measured events have lower values of $\sigma_{\text {eff }}$, and more importance in the fit. Printed on the plots are the mean and standard deviation of the distributions. 


\subsection{Choosing a signal model}

Both signal models presented in this chapter - the per-event response method, and the template method - are valid techniques for measuring the Higgs boson mass, $m_{H}$. It is also quite clear that the PER method is significantly more complex, requires more ingredients, and proportionately more validation. Perhaps less obvious is that the CPU consumption of the PER method is drastically higher than for the template; a consequence of each event carrying its own signal PDF, each of which requires a numerical convolution ${ }^{13}$. Naturally this begs the question: why use the PER method? Or phrased another way, what are the advantages of the PER method which outweigh these potential drawbacks?

There are several advantages to the PER method which clearly justify the complexity of the method, and the CPU requirements:

- The PER provides a first-principles derivation, starting from the lepton kinematics and propagating uncertainties upwards to the $m_{4 \ell}$ lineshape. This method makes a clear delineation between detector and physics, something impossible through template-style methods.

- Only the PER method is capable of fitting the Higgs width, $\Gamma_{H}$, since the truth lineshape is included analytically within the PDF.

- Only the PER method is capable of incorporating systematics uncertainties on the detector resolution into the model. Chapter 8 will present the treatment of systematic uncertainties, and elaborate on this point.

\footnotetext{
${ }^{13}$ This point is not to be understated: along with the non-inclusion of the ZMC, the CPU consumption was a limiting factor in the implementation of a PER method during Run 1 . This drawback has been radically improved by the implementation presented in this thesis: where the the Run 1 implementation requires two numerical convolutions per-event ( resolution $* \mathrm{FSR} *$ truth ), the implementation in this thesis requires only one ( (resolution + FSR $) *$ truth $)$. This change, among others, has drastically reduced CPU consideration as a limiting factor from the method, though it is still important.
} 
- The PER method is as model-independent as possible. While the PER and template methods both use the same simulated samples as input, the template method by definition assumes the kinematic distributions of the sample to be correct (so, implicitly, the SM is correct), while the PER does not. Should the model used in the simulation be incorrect, because either the SM is flawed, or new higher-order corrections alter the model, then the lineshape predicted by the template method will be incorrect, and so the $m_{H}$ value it measures will be subject to change. In contrast, since the PER method predicts the PDF of the observed events based only on the observed lepton kinematics and the ATLAS detector, the $m_{H}$ value measured is not subject to change based on a different model hypothesis. The only model assumption embedded in the PER is the very safe claim that the true distribution of $H \rightarrow 4 \ell$ events follows a Breit-Wigner lineshape, which is a basic property of any unstable particle (see Section 2.3.2.2). A demonstration of this model independence is found in Appendix B.5.

- The statistical power of each event in determining $m_{H}$ is more closely related to its measurement uncertainty when using the PER method. As Section 7.3.6 demonstrates, the resolution of $4 \ell$ events reconstructed in ATLAS can vary quite significantly. While the template method separates events by category to control this effect, it is clear that within each category there are still large differences. Because the template method effectively assumes the average resolution within each category, better-than-average events are wasted (in a statistical sense), and worse-than-average events are given too much impact. In the PER method, each event has a statistical impact proportionate to its resolution.

It is these advantages which make the PER method the most powerful tool available for measurement of the Higgs boson resonance properties. A clear demonstration of the power of the method is found in Chapter 9. 


\section{Chapter 8}

\section{Systematic Uncertainties}

Every measurement has an uncertainty composed of statistical and systematic components. The statistical component decreases as more data are added, giving better precision. The systematic component however is an inherent limitation in the measurement strategy, and (mostly) doesn't decrease as more data are added. Systematic uncertainties arise from many sources: the theory inputs used to generate the MC simulation, the lepton energy/momentum calibrations, the scale factors used to correct the simulation to match the data, the available MC statistics ${ }^{1}$, as well as the measurement implementation, such as the choice of signal and background models. These uncertainties are the subject of this chapter.

Section 8.1 begins by describing how systematics uncertainties are parameterized in the statistical model. Section 8.2 then describes all the different sources of systematic uncertainty entering the analysis. The following sections then divide the systematics based on where their effect is felt: Section 8.3 describes uncertainties affecting the yield of the signal or backgrounds, while Section 8.4 describes those affecting the energy scale or resolution. Depending on whether the measurement is of $m_{H}, \sigma_{H \rightarrow 4 \ell}$, or $\Gamma_{H}$, these systematics alter the expected resolution of the measurement, the central value of the measurement, or both.

\footnotetext{
${ }^{1}$ This is an example of a "statistical systematic" - systematic uncertainties which could be improved by increased data or MC statistics, although their effect on the measurement is indirect.
} 
The strategies used to estimate the systematics which follow were developed by a large number of individuals, both within and without the ATLAS $H \rightarrow 4 \ell$ analysis group. The systematics are then made available to analyzers like the author through tools and documentation. Some systematics - e.g. the theory systematics - can be used directly in the statistical analysis, while others need a further step of calculation. In the latter case, it may be that ATLAS detector performance groups have provided systematic variations of the (e.g.) energy of an individual lepton. For any such systematic which impacts the event selection ${ }^{2}$, the full selection was repeated with the systematically varied quantities set to their nominal and $\pm 1 \sigma$ values. The event selection was run on all the MC simulated signal and background samples to evaluate the impact of the systematic uncertainty associated to each sample. The author's direct involvement appears at the final step: evaluating the impact on the $m_{4 \ell}$ distribution of candidate events, and thus the impact on $m_{H}, \sigma_{H \rightarrow 4 \ell}$, and $\Gamma_{H}$ measurements.

\subsection{Parameterization of systematic uncertainties}

The fluctuation of each systematic uncertainty is described using a nuisance parameter (NP), and the set of NPs is denoted as $\boldsymbol{\theta}$. These NPs effectively normalize the effect of every systematic to the same scale, so the effect of two systematics, e.g. A with a $\pm 1 \%$ effect and $B$ with a $\pm 2 \%$ effect, are both mapped to the same NP value: $\theta_{A(B)}= \pm 1$. For the same reason, this definition normalizes asymmetric systematics, so that the effect of $C,{ }_{-4 \%}^{+3 \%}$, is also mapped to $\theta_{C}= \pm 1$. This definition lets the full set of NPs, $\boldsymbol{\theta}$, be constrained by symmetric Normal distributions to their nominal values, and allows the fluctuations of each to be more easily interpreted and compared. Two systematics which fit to the same value are being "pulled" equally hard by the data,

\footnotetext{
${ }^{2}$ In this example, if an energy scale uncertainty variation moves a lepton across a $p_{T}$ cut, the entire selection algorithm for this event will be altered.
} 
although they may have wildly different effects. How the nuisance parameters and their constraints enter the statistical model used for Higgs property measurement will be shown in Chapter 9.

In practice, the systematics are all implemented using the class FlexibleInterpVar (FIV). This class takes as input a nominal and $\pm 1 \sigma$ value of a systematic $A$, and produces a continuous function $f\left(\theta_{A}\right)$ to represent the effect of the systematic. Inside (outside) the given range of $\pm 1 \sigma$, the class interpolates (extrapolates) piece-wise-linearly to determine the effect of the systematic. Figure 90 shows an example of two functions built in this way. Better still, the class accepts as input the nominal and $\pm 1 \sigma$ values of multiple systematics simultaneously, and so builds a function $f(\boldsymbol{\theta})$ incorporating the effect of many systematics in an efficient manner.

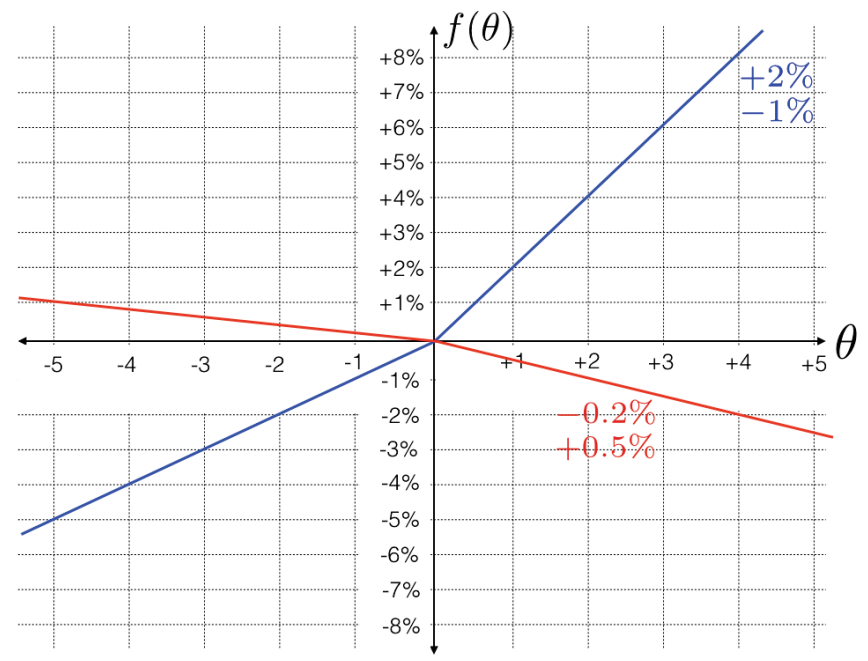

Figure 90: Example of the continuous function created by FlexibleInterpVar to model the effect of a given systematic which is specified at its nominal and $\pm 1 \sigma$ values. Two examples are shown: the blue line shows a ${ }_{-1 \%}^{+2 \%}$ effect, while the red line shows a much smaller $\underset{+0.5 \%}{-0.2 \%}$ effect.

Note that although two different effects are shown in this figure, they could (but don't have to) correspond to the same NP: some given source of uncertainty, $A$, could have two separate effects on two different aspects of the analysis. Furthermore, as 
illustrated in this figure, $\theta=+1$ does not always correspond to a positive systematic effect, or vice-versa with $\theta=-1$. An example where both these aspects come into play is in the electron resolution: $\mathrm{a}+1 \sigma$ variation of the electron resolution systematic, corresponding to $\theta_{\text {el.res. }}=+1$, will increase the detector resolution, and simultaneously decrease the expected yield in the $4 e$ category, because poor resolution means fewer events pass the selection and mass window cut. Effects of this nature will be seen later in this chapter.

\subsection{Sources of systematic uncertainty}

This section is divided according to the source of systematic uncertainty. Section 8.2.1 deals with the theory systematics, Section 8.2.2 deals with the experimental systematics associated to detector performance, and Section 8.2.3 deals with analysis systematics associated to the measurement strategy. In each section several sources of systematic uncertainty are presented according to their NP names. More detail is given to describing the systematic uncertainties which have larger impacts.

\subsubsection{Theory systematics}

The theory uncertainties in this thesis primarily pertain to the rate of processes, and thus their respective yields. In reality they may also affect the shape of distributions, but these effects are considered to be negligible in the context of the other uncertainties entering the analysis.

The theoretical calculation of the rate of any process, via its cross section, inherently has some theory uncertainty. Because cross sections are calculated perturbatively, if the MC generator includes some process calculated only at LO, then an uncertainty exists because of the missing NLO components; if it is calculated at 
NLO, then uncertainty still exists from the NNLO component, and so on. Furthermore, these cross section calculations require knowledge of the parton-density function within the colliding protons - it is evidently important to know the energies of colliding gluons in ggF-Higgs production, or of quarks in $q \bar{q} \rightarrow Z Z^{(*)}$ production. On top of these, there are uncertainties associated both to the energy scale of the process from running couplings in QCD and QED, and from the scale at which partons shower. These uncertainties will appear both for the signal [21], and the backgrounds [96].

The full list of theory uncertainties included in the analysis is as follows.

pdf_ggH Parton-density function uncertainties for ggH signal events from [21]. As ggH events are expected to dominate, theory systematics on other production modes are neglected.

br_H4l Uncertainty on calculation of $\operatorname{BR}\left(H \rightarrow Z Z^{(*)} \rightarrow 4 \ell\right)$.

Signal_comp Uncertainty on signal composition between different production modes as it pertains to varying detector acceptance for $H \rightarrow 4 \ell$ events.

QCDscale_VV QCD scale uncertainties for $q \bar{q} \rightarrow Z Z^{(*)}$.

pdf_qq Parton-density function uncertainties for $q \bar{q} \rightarrow Z Z^{(*)}$.

HOEW_QCD_syst High-order electroweak and QCD corrections for $q \bar{q} \rightarrow Z Z^{(*)}$.

HOEW_syst High-order electroweak corrections for $q \bar{q} \rightarrow Z Z^{(*)}$.

QCDscale_ggVV QCD scale uncertainties for $g g \rightarrow Z Z^{(*)}$.

pdf_gg Parton-density function uncertainties for $g g \rightarrow Z Z^{(*)}$.

scale_VVV Parton-density function and scale uncertainties for $V V V, t \bar{t}+Z$. 


\subsubsection{Experimental systematics}

In additional to the theoretical systematics, several experimental systematics enter into the analysis. Most of these systematics come from aspects of the muon and electron reconstruction and performance - their effects on each lepton are provided by the ATLAS detector performance groups (for muons and electrons, see [4] and [79] respectively), and propagated to the $H \rightarrow 4 \ell$ system by the method described above. Many of the systematics are described in groups, labelled here using a wildcard (e.g. basename_*), as the details of each are beyond the scope of this section.

EG_SCALE_* Electron energy scale uncertainty. The calibration of the EM clusters used to measure electron energy is derived using $Z \rightarrow e^{ \pm} e^{\mp}$ events, and precision knowledge of the $Z$ pole from the LEP experiments. This uncertainty, under the name EG_SCALE_ZEESYST, dominates the electron energy scale uncertainties. The other sources come from material in the ATLAS detector, individual component gain and calibration, detector pedestal correction, and various other minute details.

EG_RESOLUTION_* Electron energy resolution uncertainty. The energy resolution in MC simulation is smeared to match the detector resolution measured in data around the $\mathrm{Z}$ pole, and so the dominant component to this uncertainty is called EG_RESOLUTION_ZSMEARING. Other contributions come from material and calibration effects.

MUON_SCALE Muon momentum scale; the uncertainty on the momentum scale for all muon types. This scale is calibrated using $Z \rightarrow \mu^{ \pm} \mu^{\mp}$ data events, and applied to the MC simulation.

MUON_ID Muon momentum resolution as measured in the ID. The muon reconstruction in the ID has an associated resolution in data. The MC simulation 
often misses the small geometric distortions of the detector, and must be corrected by scale factors to match the data. This is the systematic uncertainty associated to these scale factors.

MUON_MS Muon momentum resolution as measured in the MS. All the same concepts as for MUONS_ID, but in the MS.

MUON_SAGITTA_* A weak rotational distortion in the ATLAS ID led to a correction of the measured muon momentum in data. Two NPs account for the uncertainty on this correction.

$\mathbf{E L} \_\mathbf{E F F} \_$Several systematics associated with the electron efficiency. These arise from isolation $(* I S O *)$ and reconstruction $(* \mathrm{RECO} *)$ efficiencies, which can in general vary by detector region.

MUON_EFF_* Muon efficiency-related uncertainties.

MUON_ISO_* Muon isolation-related uncertainties.

MUON_TTVA_* Muon track-to-vertex-association related uncertainties. This is used in matching muon tracks to vertices for the combined $4 \ell$ vertex fit.

PRW_DATASF Pile-up re-weighting uncertainty. The MC simulation is generated with a certain pileup distribution, often before the data are collected. The simulated events are then reweighted from their generated pileup values to match the data distribution. This is the uncertainty in that procedure.

JET_* Systematics related to jet reconstruction, which can have tangential effects on the $H \rightarrow 4 \ell$ anlaysis through particle overlap and isolation. 


\subsubsection{Analysis Systematics}

Several analysis systematics impact the measurements in this thesis. The luminosity uncertainty is central to ATLAS, while the others are all specific to the analysis in this thesis.

lumi Uncertainty only integrated luminosity recorded by ATLAS, $36.1 \mathrm{fb}^{-1}$. The uncertainty was estimated as 3.2\%, and applied to all MC-based yield estimates.

reducible_llee_* ${ }_{-}$Uncertainty associated to data-driven $Z+e e$ background estimate.

reducible_lluu_* Uncertainty associated to data-driven $Z+\mu \mu$ background estimate.

H4lMCStat Uncertainty associated to the available statistics in the MC simulated signal samples. This uncertainty incorporates the systematic uncertainty due to the choice and implementation of the signal model.

\subsection{Systematic affecting event yields}

This section presents the observed effects of each systematic affecting the signal or background yields. The effects are calculated by the number of weighted events in the corresponding MC simulated sample passing the full selection with the systematic set to its $\pm 1 \sigma$ value, as compared to when it is set at the nominal value,

$$
f(\theta= \pm 1)=\frac{N_{\text {sys at } \pm 1 \sigma}^{\text {pass selection }}}{N_{\text {sys at nominal }}^{\text {pass selection }}} .
$$

Systematics which affect the event yields are incorporated as FIVs multiplying the expected yield of each source in each category. For example, the $q \bar{q} \rightarrow Z Z^{(*)}$ 
normalization in the $2 \mu 2 e$ category with systematics incorporated is

$$
N_{\mathrm{qqZZ}, 2 \mu 2 \mathrm{e}}^{\text {with sys }}=N_{\mathrm{qqZZ}, 2 \mu 2 \mathrm{e}}^{\text {no sys }} \times f_{\mathrm{qqZZ}, 2 \mu 2 \mathrm{e}}^{\text {norm. }}(\boldsymbol{\theta}),
$$

where the FIV, $f_{\mathrm{qqZZ}, 2 \mu 2 \mathrm{e}}^{\text {norm. }}$, is labelled as such because a separate function is needed for every parameter affected by systematic uncertainties.

Experimental and analysis systematics have separate effects in each category, while theory systematics have the same effect in each category. The effect of the theory systematics on the normalizations are shown in Figure 91. The effects of experimental and analysis systematics on the $H \rightarrow 4 \ell$ signal at $m_{H}=125 \mathrm{GeV}$ are shown in Figure 92, and for the qqZZ background in Figure 93. The ZJets systematics are shown in Figure 94; only analysis systematics are included because they dominate so significantly. For ggZZ and VVV, experimental systematics are negligible compared the theory systematics so they are omitted. The dominant systematics affecting the signal yield in each category are summarized in Table 15.

An additional consideration is that the signal uncertainties may in general depend on the Higgs mass. To account for this, the systematic uncertainties are estimated as described above separately for each MC simulated mass point. At each mass point, a FIV is created as described, and then these FIV are interpolated to intermediate values of $m_{H}$, using the BSpline-based interpolation scheme described in Section 7.2.2, where it was used for morphing Template distributions. Two examples of this morphing are shown in Figure 95. 


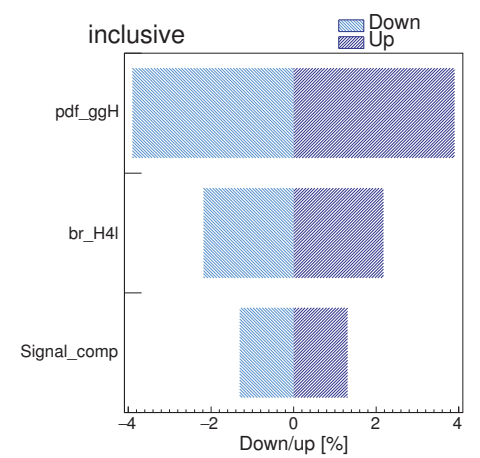

(a) $H \rightarrow 4 \ell$ Signal

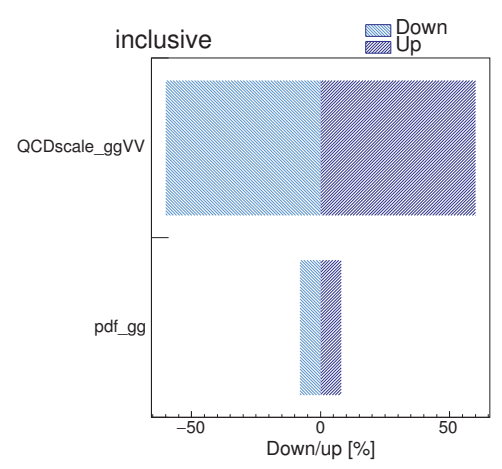

(c) $g g \rightarrow Z Z^{(*)}$

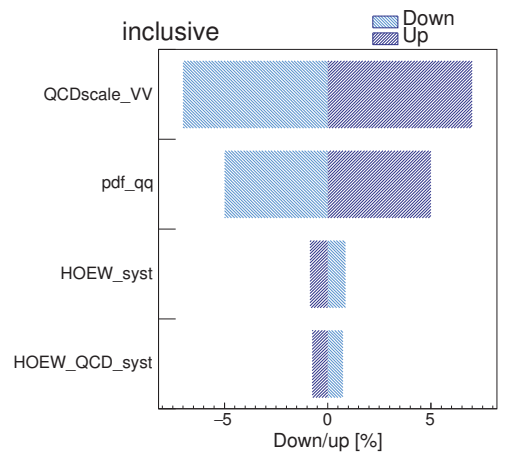

(b) $q \bar{q} \rightarrow Z Z^{(*)}$

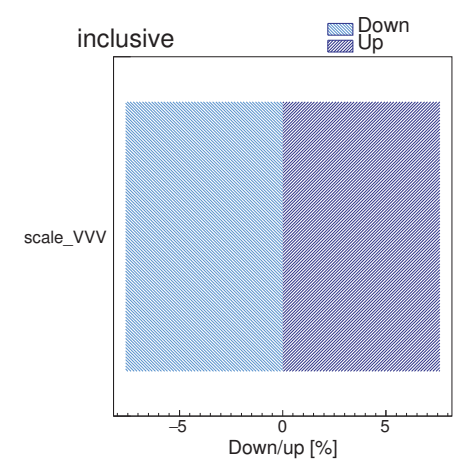

(d) $V V V, t \bar{t}+Z$

Figure 91: The effect of theory systematics on the signal and backgrounds entering the analysis. 

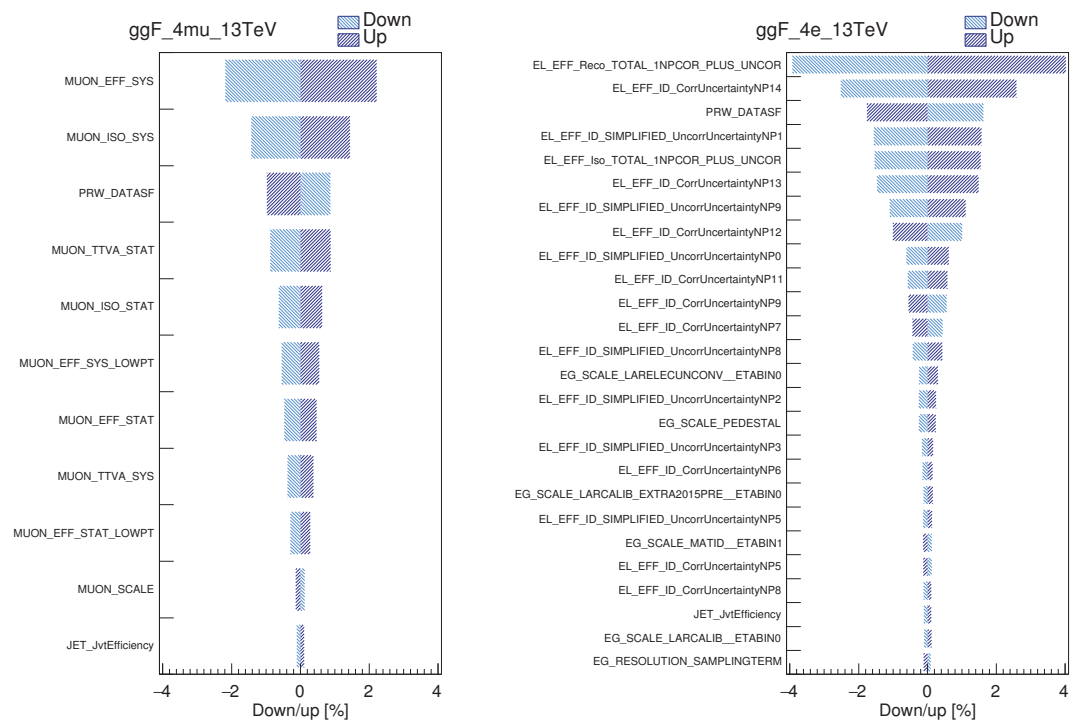

(a) $4 \mu$

(b) $4 e$
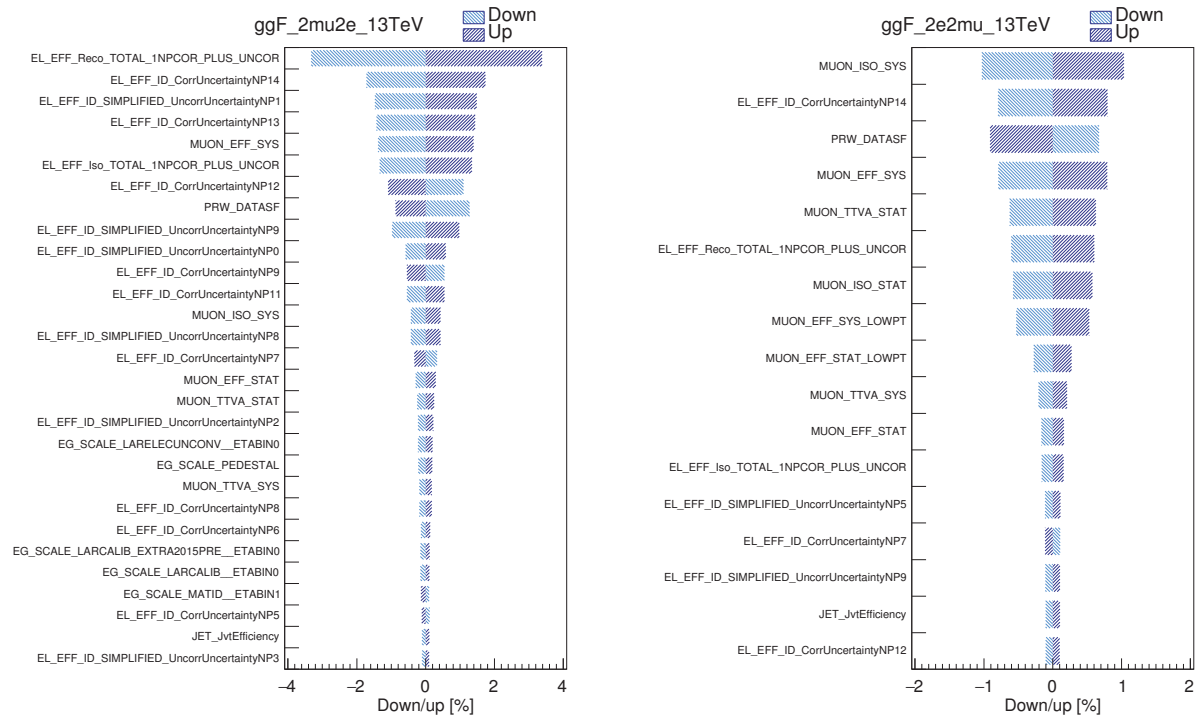

(c) $2 \mu 2 e$

(d) $2 e 2 \mu$

Figure 92: Experimental and analysis systematics affecting the $H \rightarrow 4 \ell$ signal yield for $m_{H}=125 \mathrm{GeV}$. Systematics with effects smaller than $0.2 \%$ are omitted. 


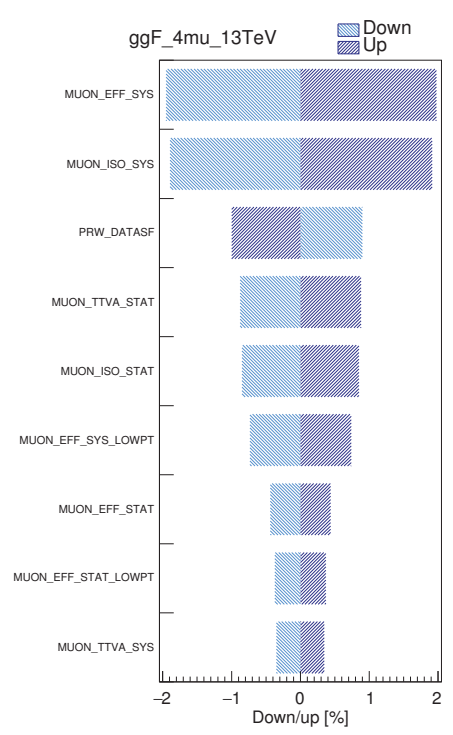

(a) $4 \mu$

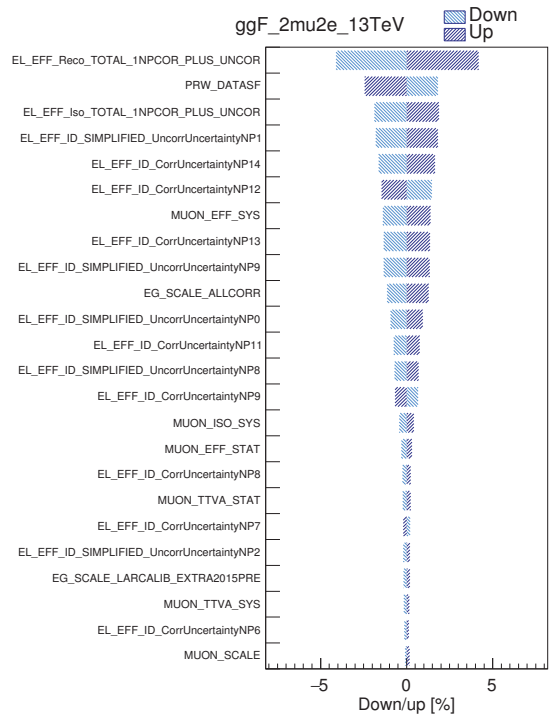

(c) $2 \mu 2 e$

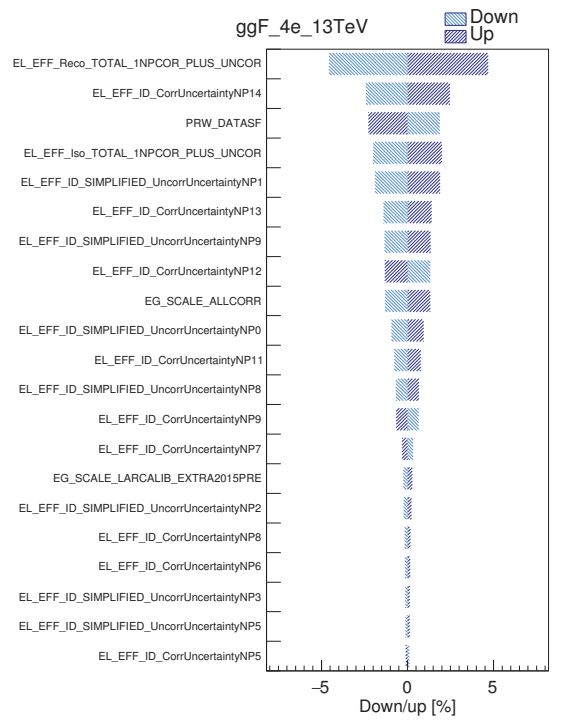

(b) $4 e$

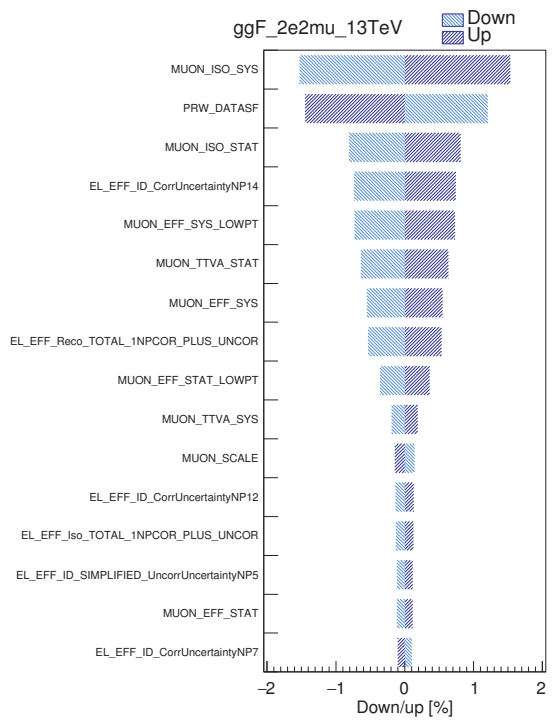

(d) $2 e 2 \mu$

Figure 93: Experimental and analysis systematics affecting the $q \bar{q} \rightarrow Z Z^{(*)}$ background yield. Systematics with effects smaller than $0.2 \%$ are omitted. 


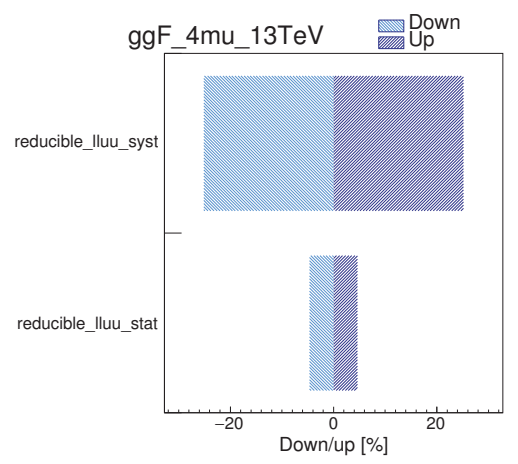

(a) $4 \mu$

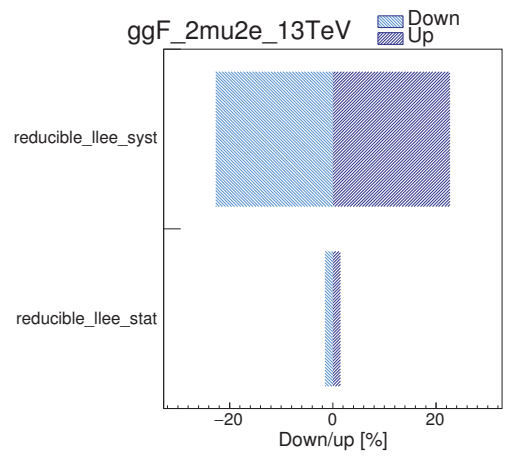

(c) $2 \mu 2 e$

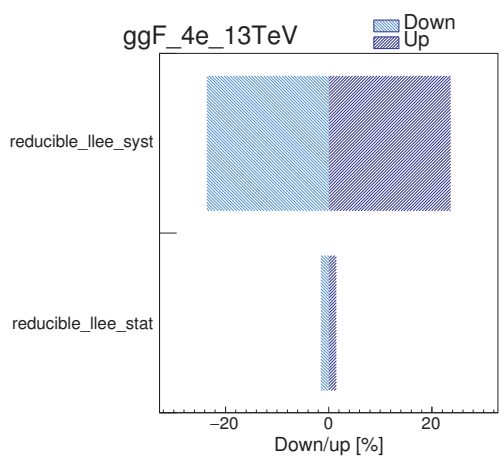

(b) $4 e$

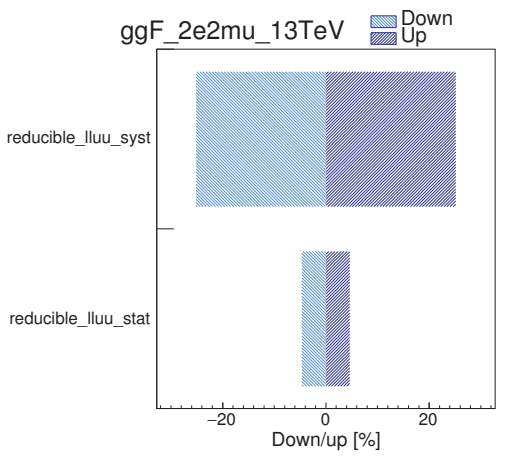

(d) $2 e 2 \mu$

Figure 94: Analysis systematics affecting the $Z+$ Jets, t $\bar{t}, \mathrm{WZ}$ background yield. Only analysis systematics, arising from data-driven background estimates of Section 6.3, are shown as experimental systematic have relatively negligible effects.

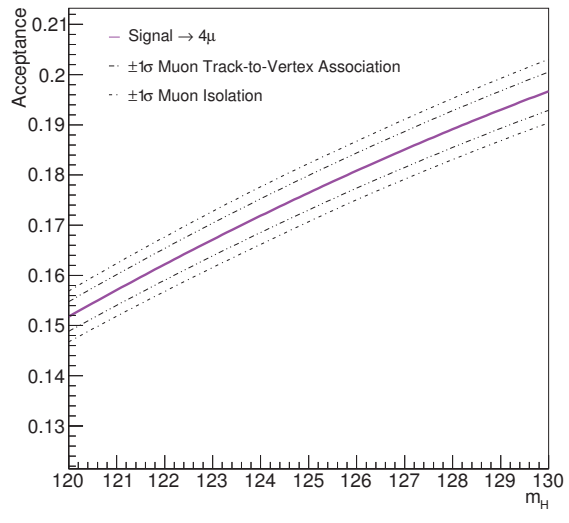

(a) Example in $4 \mu$ category

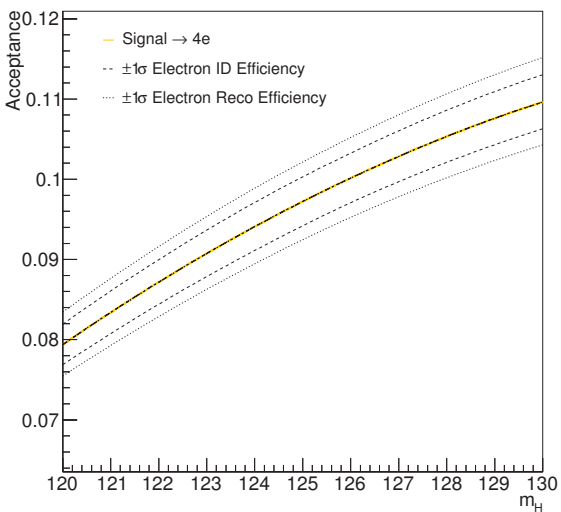

(b) Example in $4 e$ category

Figure 95: Example of morphing of systematic uncertainties on the signal yield. The effects are calculated at discrete mass points, and their effect is interpolated to intermediate values of $m_{H}$. Shown here are a few of the dominant experimental systematics for muons and electrons in the $4 \mu$ and $4 e$ categories, respectively. Their effect is demonstrated on the signal acceptance functions in each category. 
Table 15: Summary of leading systematics affecting signal yield. Systematics are labelled according to the scheme in Section 8.2. Where multiple systematics contribute under one name, the values from Figure 92 were added in quadrature.

\begin{tabular}{lcccc}
\hline \multicolumn{5}{c}{$H \rightarrow 4 \ell$ yield systematic [\%] } \\
Systematic & $4 \mu$ & $4 e$ & $2 \mu 2 e$ & $2 e 2 \mu$ \\
\hline EL_EFF_* & 0.0 & 5.8 & 4.9 & 1.0 \\
pdf_ggH & 3.9 & 3.9 & 3.9 & 3.9 \\
lumi & 3.2 & 3.2 & 3.2 & 3.2 \\
MUON_EFF_* & 2.3 & 0.0 & 1.4 & 1.0 \\
br_H41 & 2.2 & 2.2 & 2.2 & 2.2 \\
PRW_DATASF & 0.9 & 1.7 & 1.1 & 0.8 \\
MUON_ISO_* & 1.6 & 0.0 & 0.4 & 1.2 \\
Signal_comp & 1.3 & 1.3 & 1.3 & 1.3 \\
MUON_TTVA_* & 1.0 & 0.0 & 0.3 & 0.7 \\
EG_SCALE_* & 0.0 & 0.5 & 0.4 & 0.1 \\
EG_RESOLUTION_* & 0.0 & 0.1 & 0.1 & 0.1 \\
MUON_SCALE & 0.1 & 0.0 & 0.1 & 0.1 \\
\hline
\end{tabular}




\subsection{Systematics affecting scale and resolution}

Systematics which affect the shape of the $m_{4 \ell}$ distribution, beyond just its normalization, must be treated differently. Systematics such as these are also handled in a different way for signal and background processes.

For the signal, the effect of each source of systematic uncertainty is decomposed into an effect on the invariant mass scale, and an effect on the invariant mass resolution. To evaluate the effect on the scale, the systematically varied MC samples, after the full event selection, are used. The scale effect is quantified as the change in mean $m_{4 \ell}$ value from all events passing the selection in the systematically varied sample, as compared to the nominal sample,

$$
f_{\text {scale }}(\theta= \pm 1)=\frac{\left\langle m_{4 \ell}^{\text {sys at } \pm 1 \sigma}\right\rangle}{\left\langle m_{4 \ell}^{\text {sys at nominal }}\right\rangle} .
$$

The same strategy is used to evaluate the effect of each systematic uncertainty on the mass resolution, except the standard deviation (denoted here as SD) of the distribution is used to measure the effect,

$$
f_{\text {resolution }}(\theta= \pm 1)=\frac{\mathrm{SD}\left[\mathrm{m}_{4 \ell}^{\text {sys at } \pm 1 \sigma}\right]}{\mathrm{SD}\left[\mathrm{m}_{4 \ell}^{\text {sys at nominal }}\right]}
$$

One exception to this strategy comes from the H4I_MCStat uncertainty. This uncertainty is only considered to affect the mass scale, where it is estimated by the standard deviation of the mean $m_{4 \ell}$ value,

$$
\begin{aligned}
\sigma_{\bar{x}} & =\sigma_{x} / \sqrt{N} \\
\sigma_{M C \text { stat }} & =\mathrm{SD}\left[\mathrm{m}_{4 \ell}\right] / \sqrt{\mathrm{N}_{\mathrm{MC}}}
\end{aligned}
$$

for all events passing the event selection in the $m_{H}=125 \mathrm{GeV}$ MC simulated signal samples, with all production modes and all channels combined. By this calculation, 
the actual value is $\pm 8.1 \mathrm{MeV}$, which ends up being about a factor of five less than the dominant experimental uncertainties. This point is elaborated on later in Section 9.2.2.

Another exception applies only to the Template method. As brought up in Section 7.4, the construction of the smoothed templates by definition assumes the kinematic distribution of the MC simulated samples is correct. Theoretical uncertainties in general give rise to different kinematic distributions, and therefore different $m_{4 \ell}$ lineshapes. This change in the actual $m_{4 \ell}$ distribution with respect to the original template introduces a systematic uncertainty. This uncertainty was estimated by varying the relative fraction in $\mathrm{MC}$ simulation of $\mathrm{ggF}, \mathrm{VBF}, \mathrm{VH}$, ttH within experimental uncertainties, and observing how the mean of the $m_{4 \ell}$ distribution shifted. By this technique, the Template method is assigned an additional $6 \mathrm{MeV}$ scale systematic not present in the PER method (and so not included in the plots that follow).

The sources of systematic uncertainty which impact the scale are shown in Figure 96 for a $m_{H}=125 \mathrm{GeV}$ signal. Those which impact the resolution are shown in Figure 97. Similarly to the signal normalization, this evaluation is done at all simulated mass points, and interpolated to intermediate $m_{H}$ values.

The leading systematics affecting energy scale are summarized in Table 16, where they are expressed in $\mathrm{MeV}$ assuming $m_{4 \ell} \approx 125 \mathrm{GeV}$. The leading resolution systematics are summarized in Table 17.

These results exhibit some of the expected features. The dominant mass scale systematics are those coming from the muon (electron) momentum (energy) scale uncertainty. These effects should translate nearly directly to the $m_{H}$ measurement, which is, in a simplistic sense, just a measurement of the mean $m_{4 \ell}$ of signal events. The systematics which dominate the resolution effects are exactly those designed to describe resolution uncertainties, and also the pile-up re-weighting uncertainty. 


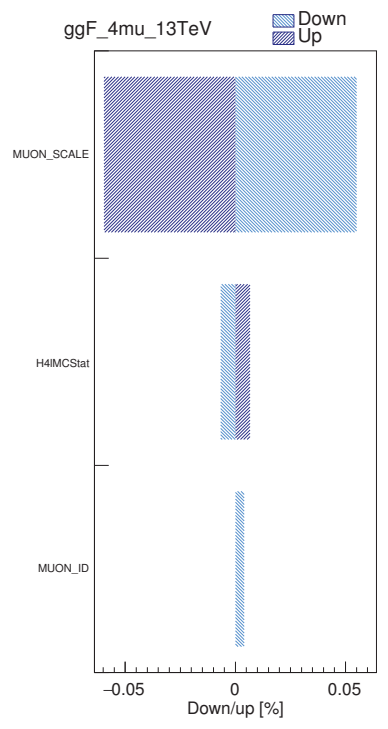

(a) $4 \mu$

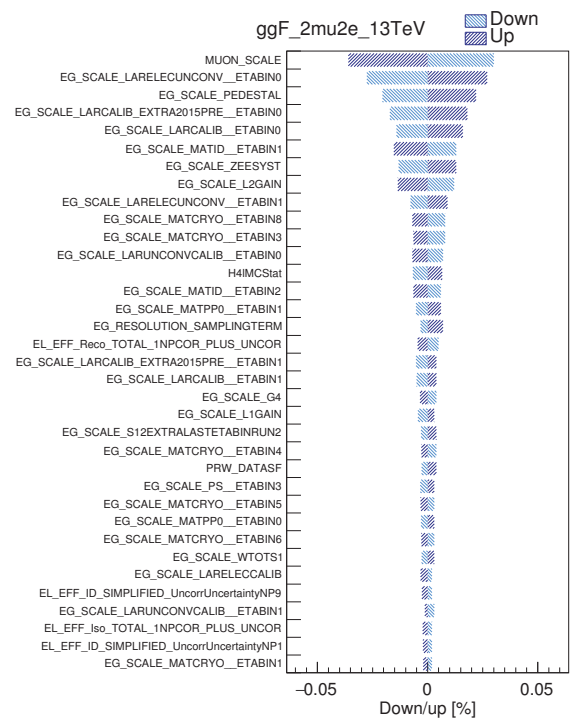

(c) $2 \mu 2 e$

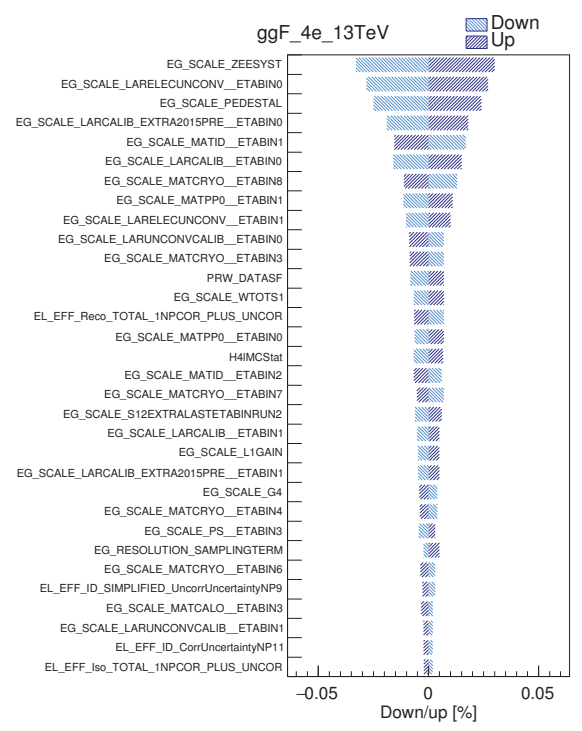

(b) $4 e$

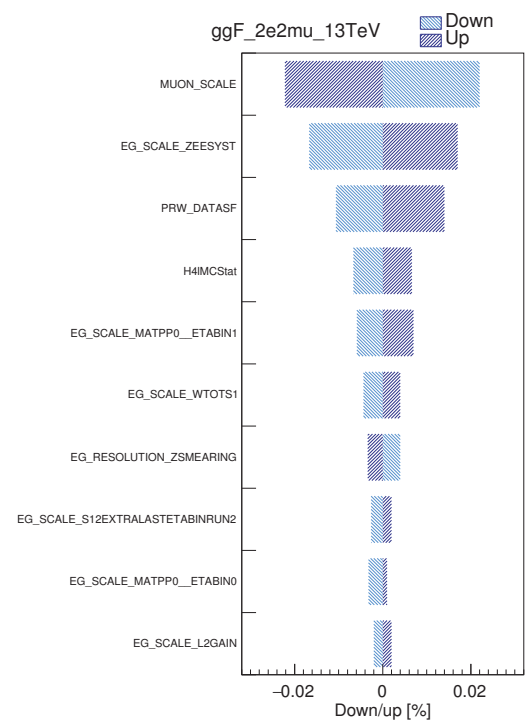

(d) $2 e 2 \mu$

Figure 96: Systematics affecting the $H \rightarrow 4 \ell$ signal mass scale, shown for $m_{H}=125 \mathrm{GeV}$. Systematics with effects smaller than $0.002 \%$ (about $\pm 2.5 \mathrm{MeV}$ ) are omitted. 


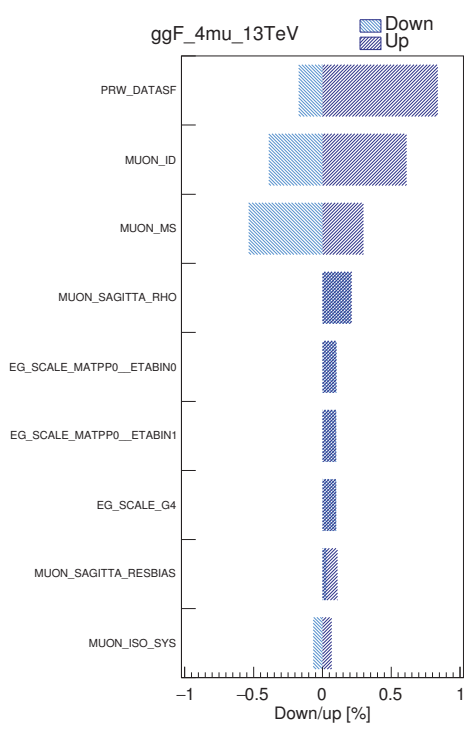

(a) $4 \mu$

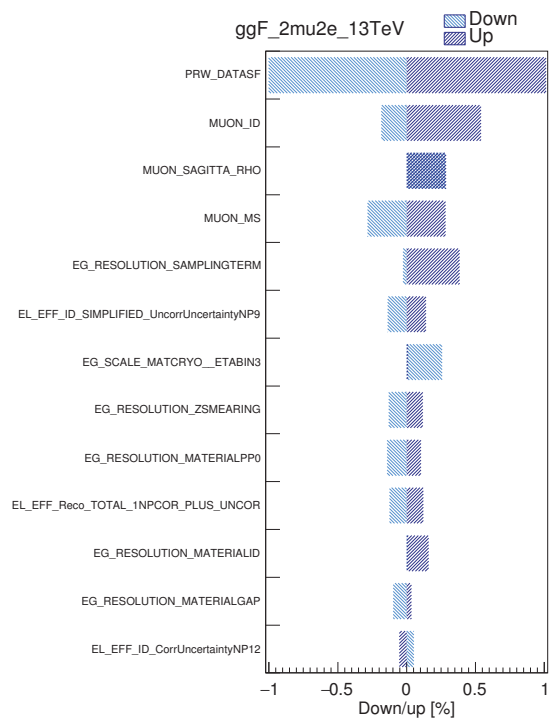

(c) $2 \mu 2 e$

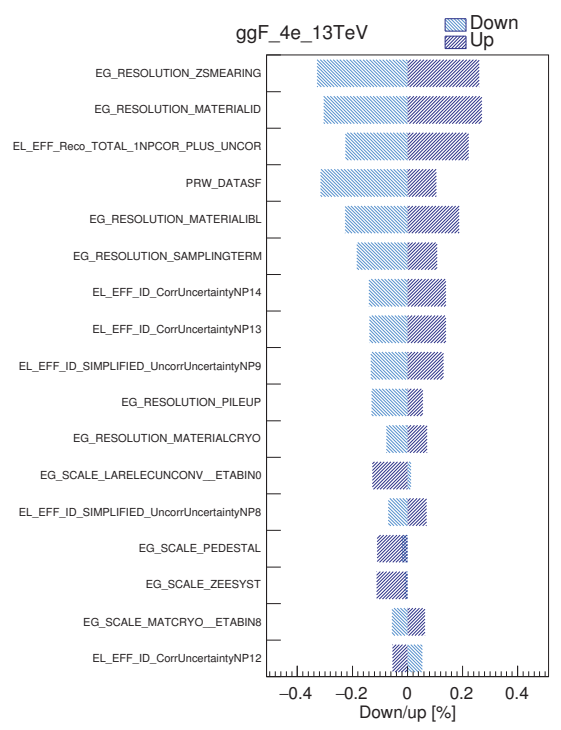

(b) $4 e$

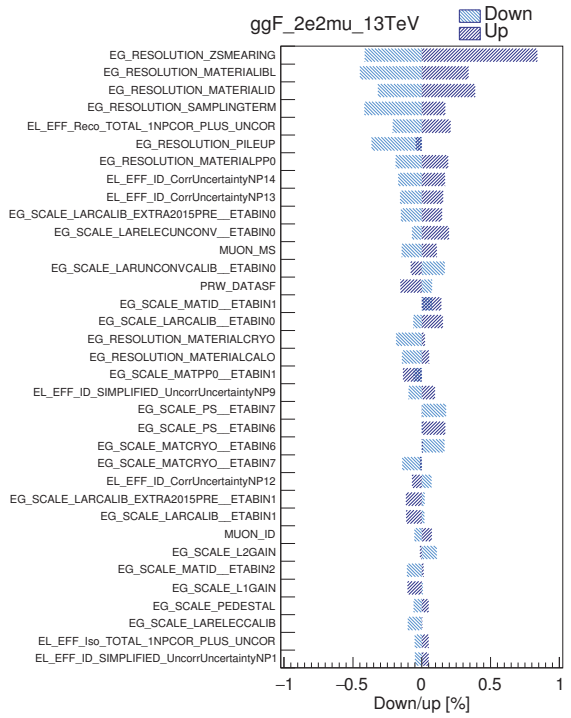

(d) $2 e 2 \mu$

Figure 97: Systematics affecting the $H \rightarrow 4 \ell$ signal mass resolution, shown for $m_{H}=125 \mathrm{GeV}$. Systematics with effects smaller than $0.05 \%$ are omitted. 
In both cases, the systematics from electrons are larger than for muons, which has always been the case for $H \rightarrow 4 \ell$ mass measurement in ATLAS [7].

Table 16: Summary of leading systematics affecting signal mass scale. Systematics are labelled according to the scheme in Section 8.2. Where multiple systematics contribute under one name, the values from Figure 96 were added in quadrature.

\begin{tabular}{lcccc}
\hline \multicolumn{5}{c}{$H \rightarrow$ 4 $\ell$ scale systematic $[\mathrm{MeV}]$} \\
Systematic & $4 \mu$ & $4 e$ & $2 \mu 2 e$ & $2 e 2 \mu$ \\
\hline EG_SCALE_* & 0.9 & 81.4 & 66.1 & 24.0 \\
MUON_SCALE & 71.7 & 0.0 & 41.3 & 27.6 \\
EL_EFF_* & 0.0 & 11.1 & 8.8 & 3.4 \\
PRW_DATASF & 1.0 & 9.5 & 4.2 & 15.4 \\
H4lMCStat & 8.1 & 8.1 & 8.1 & 8.1 \\
EG_RESOLUTION_* & 0.9 & 6.3 & 7.3 & 5.3 \\
\hline
\end{tabular}

Table 17: Summary of leading systematics affecting signal mass resolution. Systematics are labelled according to the scheme in Section 8.2. Where multiple systematics contribute under one name, the values from Figure 97 were added in quadrature.

\begin{tabular}{lcccc}
\hline \multicolumn{5}{c}{$H \rightarrow 4 \ell$ resolution systematic [\%] } \\
Systematic & $4 \mu$ & $4 e$ & $2 \mu 2 e$ & $2 e 2 \mu$ \\
\hline EG_RESOLUTION_* & 0.02 & 0.50 & 0.31 & 0.93 \\
PRW_DATASF & 0.51 & 0.21 & 1.01 & 0.12 \\
MUON_ID & 0.50 & 0.00 & 0.36 & 0.06 \\
EG_SCALE_* & 0.27 & 0.20 & 0.19 & 0.42 \\
MUON_MS & 0.42 & 0.00 & 0.28 & 0.13 \\
EL_EFF_* & 0.00 & 0.35 & 0.21 & 0.35 \\
MUON_SAGITTA & 0.22 & 0.00 & 0.29 & 0.02 \\
\hline
\end{tabular}

The scale systematics are incorporated into the signal model in a somewhat nonintuitive way. These systematics are properties of the detector performance and analysis strategy, not fundamental physics. Accordingly, they belong in the signal 
model as part of the response distribution, and not the physics distribution. However, for technical reasons, they are implemented in exactly the opposite way: the scale systematics are made to transform the $m_{H}$ parameter inside the BW truth distribution of $(91)$,

$$
m_{H}^{2 e 2 \mu}=m_{H} \times f_{\text {scale }}^{2 e 2 \mu}(\boldsymbol{\theta}) .
$$

After this transformation, the parameter to determine is still $m_{H}$, but the mean of the BW distribution in each category is a function thereof. This is just a choice of convenience; the final signal PDF is unaffected by whether the scale systematic was applied on the response or truth component of the distribution. The resolution systematics are incorporated in a more natural way, directly on the response functions where they belong. The Gaussian parameters of the final mass response in (91) are scaled by the relevant systematic. For example,

$$
\sigma_{i, \text { with sys. }}^{2 e 2 \mu}=\sigma_{i, \text { no sys. }}^{2 e 2 \mu} \times f_{\text {resolution }}^{2 e 2 \mu}(\boldsymbol{\theta}) \text {. }
$$

Each Gaussian sigma (indexed by $i$ ) in the sum describing the per-event response is multiplied by this same factor, and all responses in a given category are assumed to have the same systematic uncertainty. Resolution uncertainties are not included in the Template method, as there is no analytical resolution parameter that could be modified, so the technical implementation is much more difficult.

When using the PER signal model, the implementation of signal systematics presents an opportunity for future study. For all the same reasons that each event has a unique mass response, it will also have unique experimental systematics. This fact is neglected in this thesis, where the effects on $m_{4 \ell}$ were calculated as the average effect of individual lepton systematics, much like the Template signal model uses the average response of many individual events. This averaging of systematic uncertainties comes with the same caveats as averaging detector resolution: events with 
below-average systematic uncertainty are assigned unrealistically large uncertainties, and those with above-average systematic uncertainties are given undue importance in the fit. Developing a strategy for implementing per-event systematics into the PER method has potential to yield a reduction in the overall systematic uncertainty on $m_{H}$ measurement, though the magnitude of this difference is expected to be small, and the uncertainty in the $H \rightarrow 4 \ell$ channel will remain by far statistically dominated until at least Run 3 at the LHC.

For the background processes, a different strategy is used. In this case, a histogram of the $m_{4 \ell}$ distribution is built from events passing the full event selection with and without the systematic variations applied at $\pm 1 \sigma$. These histograms use a very coarse $5 \mathrm{GeV}$ binning to minimize effects of statistical fluctuations. The systematically varied histograms are then linearly interpolated from bin-to-bin, and this function is used as the $m_{4 \ell}$-dependent systematic effect on the signal. The class RooStarMomentMorph is used to interpolate (extrapolate) the effect on the PDF to variations smaller (larger) than $\pm 1 \sigma$ at which the systematic was evaluated. One exception to this prescription applies to the Z+Jets background, for which the systematically varied distributions are produced as described in Section 6.3.3.

Two examples of the shape variations created in this way are shown in Figure 98 for the muon momentum scale and the dominant electron resolution systematic. The variations are shown at $\pm 5 \sigma$ to emphasize the effect; when shown at $\pm 1 \sigma$ the difference is invisible. This highlights that the experimental shape uncertainties for the background are less important than those of the signal. This is a result of the nonresonant background shape (as compared to the resonant signal shape) - an energy scale systematic which in general could displace events upward in $m_{4 \ell}$ simultaneously "replaces" them with events moving upwards from still lower values of $m_{4 \ell}$, leaving the shape only marginally affected.

How to assign import to the different systematic uncertainties presented is this 


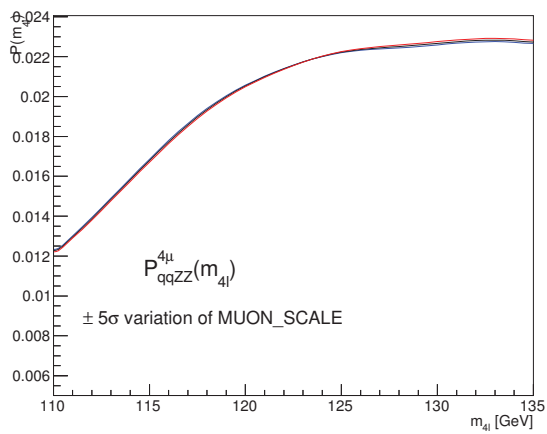

(a) Effect on the $4 \mu$ category from muon momentum scale uncertainty

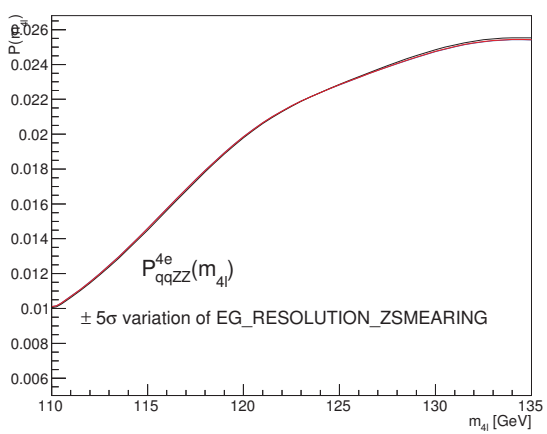

(b) Effect on the $4 e$ category from dominant electron resolution uncertainty

Figure 98: Demonstration of systematic uncertainties affecting the $q \bar{q} \rightarrow Z Z^{(*)} \mathrm{PDF}$.

The effects are shown for the muon momentum scale (MUON_SCALE) and the primary electron resolution systematic (EG_RESOLUTION_ZSMEARING) on the $4 \mu$ and $4 e$ categories, respectively. The nominal PDF of $m_{4 \ell}^{\text {cons }}$ is shown in black, while the $+5 \sigma(-5 \sigma)$ systematic variation is shown in red (blue).

chapter is not always evident. The ultimate question, of course, is what will be their impact on the $m_{H}, \sigma_{H \rightarrow 4 \ell}$, and $\Gamma_{H}$ measurements? Is a $0.01 \%$ scale systematic more or less important than a $5 \%$ normalization systematic? And where does a $1 \%$ resolution systematic fit in that comparison? The following chapter will present the statistical methods used for the property measurement in this thesis, and various validation steps and cross-checks which lead up to the final measurement. Among these checks, Section 9.3 addresses the preceding questions, and estimates the impact of each systematic uncertainty on the final measurements. 


\section{Chapter 9}

\section{Statistics and Property Measurement}

This chapter presents the final statistical methods used for the property measurement in this thesis. All the components of the preceding chapters, especially Chapter 6 and onwards, come together to perform the measurement. The studies and results of all three methods are shown in this chapter: the constrained PER method as the primary measurement strategy, as well as the template method, and unconstrained PER method which serve as cross-checks. The extra checks are shown both as a point of comparison, and to inspire confidence in the constrained PER method as a measurement strategy. The constrained PER method is the exclusive source of the final results of this thesis, summarized in Chapter 10.

This chapter is laid out as follows: Section 9.1 presents the statistical formalism and background needed to understand the results in the following sections. Section 9.2 presents checks of the measurement using an effective dataset built from simulation. Section 9.3 presents a break-down of the systematic uncertainties by their effect on the final measurements. Section 9.4 presents checks using pseudo-datasets built with realistic statistics from what is expected in the data. Section 9.5 presents a validation of the PER method using the $Z \rightarrow 4 \ell$ resonance in simulation and data. Finally Section 9.6 presents the results of the $H \rightarrow 4 \ell$ property measurements in the data. 


\subsection{The method of maximum likelihood}

The main statistical tool used to make inferences in this thesis is the method of maximum likelihood [83]. In this method, a likelihood function is constructed to convert the complex dataset (many numbers, $\boldsymbol{x}$ ) into a likelihood value (a single number $L(\boldsymbol{x}))$, so as to make inferences from that value. Generally, the likelihood function will depend on some parameters of interest (POIs, denoted $\boldsymbol{\mu}$ ), so we can find the best fit value of these parameters by finding where the likelihood is at its maximum value, i.e. the most likely values of the parameters. Confounding this strategy are the NPs associated to systematic uncertainties, $\boldsymbol{\theta}$, which enter the likelihood as additional free parameters.

The observables, $\boldsymbol{x}$, depend on the model being used:

$$
\begin{aligned}
\text { constrained PER: } & \boldsymbol{x}=\left\{m_{4 \ell}^{\mathrm{cons}}, \vec{g}\right\}, \\
\text { Template method: } & \boldsymbol{x}=\left\{m_{4 \ell}^{\mathrm{cons}}\right\}, \\
\text { unconstrained PER: } & \boldsymbol{x}=\left\{m_{4 \ell}^{\mathrm{fsr}}, \vec{g}\right\},
\end{aligned}
$$

where the curly braces, e.g. $\{m\}$, denote "the set of all $m$ in data", and the $\vec{g}$ are the Gaussian-sum parameters representing the mass response, defined in (91) and the text that follows. Note that $\vec{g}$ are conditional observables - their distribution is not specified by the model; it is determined by the dataset.

The POIs, $\boldsymbol{\mu}$, are some subset of the Higgs mass, $m_{H}$, cross section times branching ratio to four leptons, $\sigma_{H \rightarrow 4 \ell}$, and the natural width, $\Gamma_{H}$, depending on the goal of a given fit. For any given fit, the POI is just one of these parameters, while the others are treated as NPs, except for $\Gamma_{H}$ which is fixed at $4.4 \mathrm{MeV}$ (unless it is the POI). As only one parameter is considered the POI at a time, we can simply denote $\boldsymbol{\mu}=\mu$. Note that the POIs and NPs are both free in the fit; the distinction between the two is that we are interested in the value of the POIs, but not that of the NPs. Applying 
this rule gives the following:

$$
\begin{array}{rll}
\text { fitting mass: } & \mu=m_{H}, & \boldsymbol{\theta}=\left(\sigma_{H \rightarrow 4 \ell}, \theta_{i}, \theta_{j}, \ldots\right), \\
\text { fitting cross section: } & \mu=\sigma_{H \rightarrow 4 \ell}, & \boldsymbol{\theta}=\left(m_{H}, \theta_{i}, \theta_{j}, \ldots\right), \\
\text { fitting width: } & \mu=\Gamma_{H}, & \boldsymbol{\theta}=\left(m_{H}, \sigma_{H \rightarrow 4 \ell}, \theta_{i}, \theta_{j}, \ldots\right) .
\end{array}
$$

The above may seem an unnecessarily complex way to re-label the observables and parameters, but it allows the formulation which follows to be more universal to all signal models and types of fit. With these parameters defined, we are prepared to construct the likelihood.

For a given set of parameters $\mu, \boldsymbol{\theta}$, the likelihood of each event $x_{i}$ can be expressed as

$$
P\left(x_{i} \mid \mu, \boldsymbol{\theta}\right)=\sum_{s}^{\text {sources }} N_{s, c}^{\exp }(\mu, \boldsymbol{\theta}) P_{s, c}\left(x_{i}, \mu, \boldsymbol{\theta}\right) .
$$

Recall that when the PER model is used, the signal component of this function differs on an event-by-event basis, whereas for the Template method it is fixed within a given category. Each event can be given a weight, $w_{i}$, which appears as an exponent on this value. Data events always have $w_{i}=1$, while $\mathrm{MC}$ simulated events have non-unit weights in general, as discussed in Section 4.1. If all events are treated as independent observations, then their combined likelihood can be expressed as

$$
\prod_{i}^{\text {events }}\left[\sum_{s}^{\text {sources }} N_{s, c}^{\text {exp. }}(\mu, \boldsymbol{\theta}) P_{s, c}\left(x_{i}, \mu, \boldsymbol{\theta}\right)\right]^{w_{i}}
$$

To add some further complication, these events must be divided into categories, and within each category we must include an extended term accounting for the Poisson probability of observing $N^{o b s}$ events when $N^{e x p}$ are expected,

$$
\prod_{c}^{\text {categories }} \operatorname{Pois}\left(N_{c}^{\text {obs. }} ; N_{c}^{\text {exp. }}(\mu, \boldsymbol{\theta})\right)
$$


where $c$ is an index over each category. This term effectively introduces a penalty in the likelihood when the overall number of events differs from the expected number (for a given set of POIs and NPs), which adds statistical power in constraining the parameters which affect normalization. The overall expected number of events per category is defined by

$$
N_{c}^{\text {exp. }}(\mu, \boldsymbol{\theta})=\sum_{s}^{\text {sources }} N_{s, c}^{\text {exp. }}(\mu, \boldsymbol{\theta}) .
$$

Also included in the likelihood are constraint terms for each of the systematic uncertainties entering the model,

$$
\prod_{\alpha}^{\text {systematics }} G\left(\theta_{\alpha} ; 0,1\right) .
$$

When all these pieces are put together, the extended likelihood can written down, according to the predicted distribution of the dataset $\boldsymbol{x}$, with parameter set $\mu, \boldsymbol{\theta}$, as

$$
\begin{aligned}
L(\boldsymbol{x} \mid \mu, \boldsymbol{\theta})=\prod_{c}^{\text {categories }} & {\left[\operatorname{Pois}\left(N_{c}^{\text {obs. }} ; N_{c}^{\text {exp. }}(\mu, \boldsymbol{\theta})\right) \times \prod_{i}^{\text {events }}\left[\sum_{s}^{\text {sources }} N_{s, c}^{\exp .}(\mu, \boldsymbol{\theta}) P_{s, c}\left(x_{i}, \mu, \boldsymbol{\theta}\right)\right]^{w_{i}}\right] } \\
& \times \prod_{\alpha}^{\text {systematics }} G\left(\theta_{\alpha} ; 0,1\right) .
\end{aligned}
$$

The parameters $\mu, \boldsymbol{\theta}$ which maximize this value are called maximum likelihood estimators, and are denoted as $\hat{\mu}, \hat{\boldsymbol{\theta}}$. More colloquially, they are just the best fit values.

To get the most use of this likelihood function, consider the profile likelihood ratio [97], defined by

$$
\lambda(\mu)=\frac{L(\boldsymbol{x}, \mu, \hat{\hat{\boldsymbol{\theta}}}(\mu))}{L(\boldsymbol{x}, \hat{\mu}, \hat{\boldsymbol{\theta}})},
$$

where $\hat{\hat{\boldsymbol{\theta}}}(\mu)$ indicates the best fit values of $\boldsymbol{\theta}$ for the given POI $\mu$. Using the profile likelihood ratio, three test statistics can be defined that support all measurements in this thesis.

The first and most important test statistic used for measurement of $m_{H}$ and $\sigma_{H \rightarrow 4 \ell}$ 
is defined as

$$
t_{\mu}=-2 \ln \lambda(\mu),
$$

The use of a natural logarithm is computationally convenient for a few reasons. The most basic is that $t_{\mu}=0$ at the best fit value, and is larger everywhere else. Additionally, the quotient in (108) becomes a simple subtraction of a constant (only the numerator inside the $\log$ needs to be re-evaluated with each $\mu$ ), the repeated multiplications of (103) and (104) become repeated additions, and, instead of multiplying very small numbers repeatedly, relatively modest numbers are added, so that floating-point arithmetic is no longer an important concern.

To perform a measurement using $t_{\mu}$, we utilize the property that it is asymptotically distributed as a chi-square distribution with one degree of freedom [97],

$$
p\left(t_{\mu} \mid \mu\right)=\frac{1}{\sqrt{2 \pi}} \frac{1}{\sqrt{t_{\mu}}} e^{-t_{\mu} / 2} .
$$

Using this property, a two-sided confidence region in parameter $\mu$ can be set where $t_{\mu}^{\text {data }}<t_{\mu}^{\text {crit }}$. The critical value is defined where

$$
\int_{t_{\mu}^{\text {crit }}}^{\infty} p\left(t_{\mu} \mid \mu\right) d t_{\mu}=1-\mathrm{CL}
$$

where CL is the desired confidence level of parameter $\mu$. Using this expression, we find that

$$
\begin{aligned}
& \int_{1.00}^{\infty} p\left(t_{\mu} \mid \mu\right) d t_{\mu}=1-68.3 \%, \\
& \int_{3.84}^{\infty} p\left(t_{\mu} \mid \mu\right) d t_{\mu}=1-95.0 \%,
\end{aligned}
$$

which indicates the $\pm 1 \sigma$ ( $68.3 \%$ CL) confidence region is set within the bounds where $t_{\mu}<1.00$. The corresponding $\pm 2 \sigma(95 \% \mathrm{CL})$ confidence region is set within $t_{\mu}<3.84$. With this information, doing a fit is as simple as scanning $t_{\mu}$ across values of $\mu$, finding where it is zero to determine the best fit value, and where it crosses these critical 
values $^{1}$ to find the uncertainty - this procedure is called a likelihood scan.

The other two test statistics are used only for $\Gamma_{H}$. These new test statistics must be used, instead of $t_{\mu}$, because the existing dataset does not have sufficient statistics to perform a two-sided direct measurement of $\Gamma_{H}$. In the following, the POI $\mu=\Gamma_{H}$ in every equation, even though it is written as $\mu$ for generality.

First, the consistency of the observed data with the hypothesis $\Gamma_{H}=\Gamma_{H}^{\mathrm{SM}}$ is checked using the test statistic defined by

$$
q_{0}= \begin{cases}-2 \ln \lambda(0), & \text { when } \hat{\mu}>\mu^{\text {null }}, \\ 0, & \text { when } \hat{\mu}<\mu^{\text {null }}\end{cases}
$$

where $\mu^{\text {null }}=\Gamma_{H}^{\mathrm{SM}}=4.1 \mathrm{MeV}$. The conditional equation is designed such that downwards fluctuations in the observed width are not interpreted as being inconsistent with the null hypothesis of $\Gamma_{H}=\Gamma_{H}^{\mathrm{SM}}$. The asymptotic PDF of $q_{0}$ under the null hypothesis is given by

$$
p\left(q_{0}\right)=\frac{1}{2} \delta\left(q_{0}\right)+\frac{1}{2 \sqrt{2 \pi}} \frac{1}{\sqrt{q_{0}}} e^{-q_{0} / 2},
$$

where $\delta\left(q_{0}\right)$ is the Dirac delta function. This distribution arises under the mathematical assumption that half of datasets will have $\hat{\mu}>\mu^{\text {null }}$, and the other half $\hat{\mu}<\mu^{\text {null }}$ (i.e. the maximum likelihood estimator is symmetric about the true value). The former will be distributed as in (110), while the latter will be assigned a value of zero, resulting in the $\delta\left(q_{0}\right)$ function. Consistency with the SM hypothesis is typically quoted as a pvalue, defined by

$$
p_{0}=\int_{q_{0}^{\mathrm{data}}}^{\infty} p\left(q_{0}\right) d q_{0}
$$

\footnotetext{
${ }^{1}$ To work out any other CL or critical value, the integral in (110) is equal to erfc $\left(\sqrt{\mathrm{t}_{\mu}^{\text {crit }} / 2}\right)$, where erfc is the complementary error function.
} 
which specifies the probability to have observed a deviation in data as large (or larger) than that observed, if the SM hypothesis is correct. In particle physics, evidence for a deviation from the SM is usually claimed at $p_{0}<1.35 \times 10^{-3}$, while a discovery (such as that of the Higgs boson) is claimed when $p_{0}<2.86 \times 10^{-7}$, corresponding to $3 \sigma$ and $5 \sigma$ Gaussian significance, respectively.

If no significant deviation from the SM is observed, then we turn to the final test statistic, which can be used to set an upper limit (UL) on $\Gamma_{H}$. It is defined by

$$
q_{\mu}= \begin{cases}-2 \ln \lambda(\mu), & \text { when } \hat{\mu} \leq \mu \\ 0, & \text { when } \hat{\mu}>\mu\end{cases}
$$

The effect of this conditional expression is to remove the low- $\Gamma_{H}$ rejection region caused by upwards fluctuations of $\Gamma_{H}$ (labelled in (116) as $\mu$ ), and therefore set an upper limit on the width. Similar to the other test statistics, $q_{\mu}$ is asymptotically distributed as

$$
p\left(q_{\mu}\right)=\frac{1}{2} \delta\left(q_{\mu}\right)+\frac{1}{2 \sqrt{2 \pi}} \frac{1}{\sqrt{q_{\mu}}} e^{-q_{\mu} / 2},
$$

where the two conditions in (116) lead to the two terms in this expression. By computing the integral in the right-hand tail, we find

$$
\begin{aligned}
& \int_{0.22}^{\infty} p\left(q_{\mu} \mid \mu\right) d q_{\mu}=1-68.3 \% \\
& \int_{2.71}^{\infty} p\left(q_{\mu} \mid \mu\right) d q_{\mu}=1-95.0 \%
\end{aligned}
$$

so that a one-sided $68.3 \%$ (95\%) frequentist confidence level UL can be set where $q_{\mu}$ rises to $0.22(2.71)$.

The differences in the definitions of $t_{\mu}$ and $q_{\mu}$ are mostly immaterial for this thesis, but are presented in the standard way for consistency with the literature. For analyses performing a search (for a new resonance for example), the prospect of a downward 
or upward fluctuation causing misinterpretation of the results is a much more real effect. In this analysis, the two test statistics are only differentiated by where on the likelihood scan the critical values (and thus the exclusion regions) are drawn. For measurements of $m_{H}$ and $\sigma_{H \rightarrow 4 \ell}, \pm 1 \sigma( \pm 2 \sigma)$ exclusion regions are set where $t_{\mu}>1.00(3.84)$. For measurements of $\Gamma_{H}$, a one-sided $68.3 \%(95 \%)$ exclusion region is set where $q_{\mu}>0.22(2.71)$.

\subsection{Tests using an effective dataset}

The first set of tests used to validate the measurement strategy are based on an effective dataset. This effective dataset is similar to an Asimov dataset [97], where a dataset is created matching exactly the expected distributions and yields with no statistical fluctuation. The purpose of such a dataset is to test the statistical power (significance, discovery potential, expected precision) of a measurement without requiring huge numbers of pseudo-experiments. When actual statistics are sufficiently high, the precision from fitting this Asimov dataset will reflect the median precision that would have been found from pseudo-experiments.

The effective dataset used in this section serves both this same purpose, and another purpose inaccessible to an Asimov dataset created as described above. The effective dataset is constructed by combining all the MC simulated samples for the different processes which enter the analysis: the signal (all production modes), qqZZ, ggZZ, VVV, Z+Jets. All samples are combined according to their SM cross sections, except for Z+Jets which uses the data-driven estimates to renormalize the samples. By this process, the sum of weights, $\sum_{i} w_{i}$, of each sample exactly matches the expected number of events in the likelihood, $N_{s, c}^{\exp }$. For the background samples only, the $m_{4 \ell}^{\text {cons }}$ and $m_{4 \ell}^{\mathrm{fsr}}$ values in the simulated samples are replaced by evaluating the PDF for each, $P_{s, c}\left(x_{i}, \boldsymbol{\theta}=\boldsymbol{\theta}_{\text {nominal }}\right)$, in steps of $0.25 \mathrm{GeV}$, such that background exhibits 
no statistical fluctuation (similar to an Asimov dataset). For the signal samples, the events are used directly from the MC simulation without alteration, and when the PER method is being used, the response parameter $\vec{g}$ are also used without alteration. This permits an important check: while Chapter 7 validated the signal model(s) on signal samples alone, using this strategy permits the validation of the signal model(s) in the presence of backgrounds. This check is not possible using the Asimov dataset, which assumes the full signal and background model is perfect, and generates events from this combined PDF. Conversely, this could be seen as a disadvantage of the PER method: because the $\vec{g}$ are not specified by the model, the PER model is not capable of generating events directly from the PDF, unless a proto-dataset with values of $\vec{g}$ is provided (which would itself have statistical fluctuations), so generating a true Asimov dataset is not possible using the PER method.

\subsubsection{Tests of expected resolution}

For the first round of tests using the effective dataset, $m_{H}=125 \mathrm{GeV}$ MC simulated samples are used for the signal. The NLL scans of $m_{H}$ are shown for the unconstrained PER, the Template method, and the constrained PER in Figure 99. Each plot includes the likelihood scans from each category individually, and that from the combined model, with and without systematics. The systematic uncertainties affect the mass scale are extremely small, and are barely visible on these plots.

The same set of scans for $\sigma_{H \rightarrow 4 \ell}$ are shown in Figure 100, and for $\Gamma_{H}$ in Figure 101. Note that for scans on $\Gamma_{H}$, only a one sided 95\% CL upper limit is set. Also be reminded that the Template method does not include the $\Gamma_{H}$ parameter, so is not able to serve as a cross check of this measurement.

These scans are used to extract the expected resolution of each measurement, 


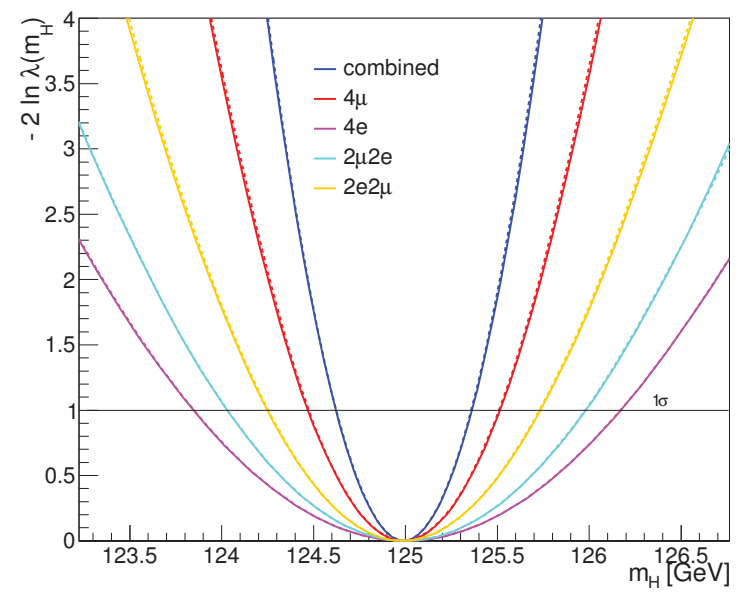

(a) constrained PER

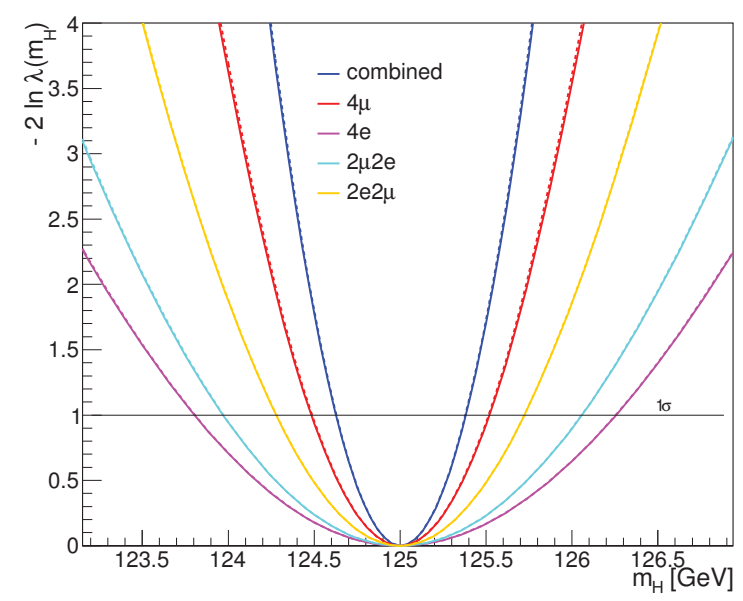

(b) Template

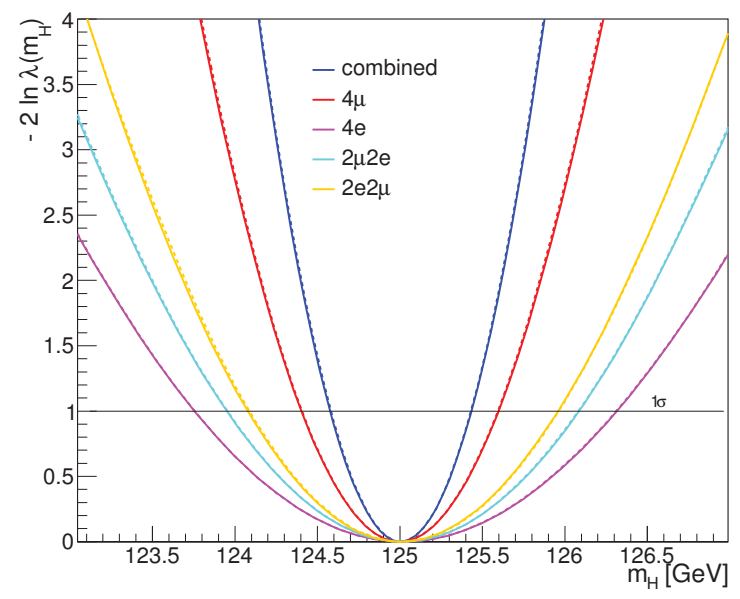

(c) unconstrained PER

Figure 99: Scans of the NLL on parameter $m_{H}$ using an effective dataset with $m_{H}=125 \mathrm{GeV}$. Subfigures show the scans using the different signal models as labelled. The NLL scans including (not including) systematics are shown with solid (dashed - barely visible) lines.

assuming a SM $m_{H}=125 \mathrm{GeV}$ Higgs boson. Since the SM production cross sections and the detector resolution vary as a function of $m_{H}$, the expected measurement resolutions will vary as a function of $m_{H}$, but by a relatively small amount. The results of these scans are recorded in Table 18, including all statistical and systematic uncertainties. These results indicate the constrained PER provides an improvement of approximately $2.6 \%$ on the $m_{H}$ resolution, and $1.7 \%$ on the $\sigma_{H \rightarrow 4 \ell}$ resolution, compared to the template method. The unconstrained PER method, while a satisfactory 


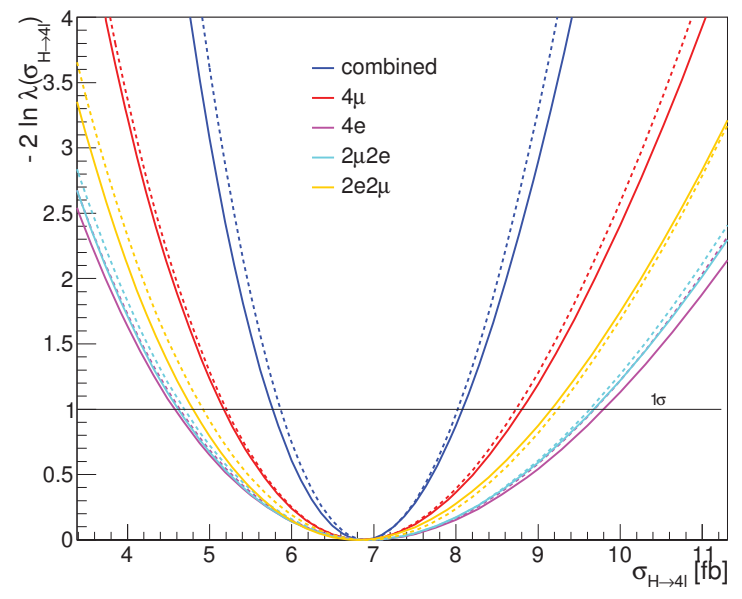

(a) constrained PER

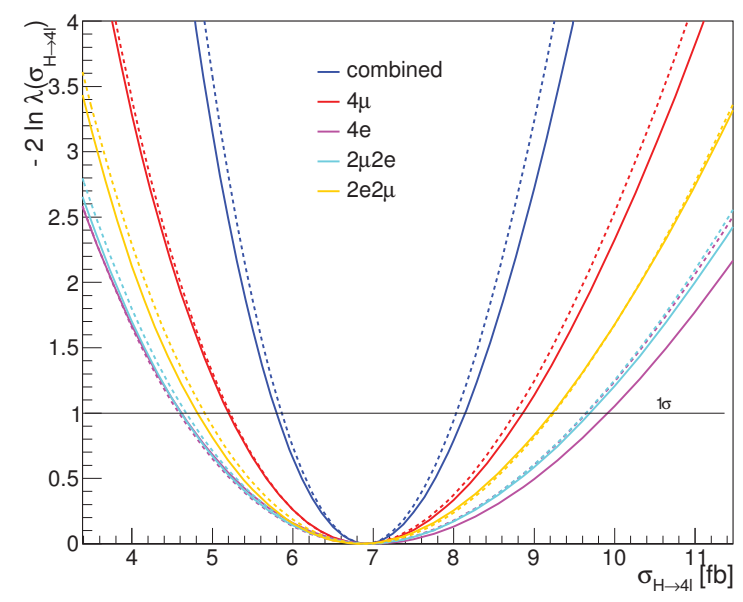

(b) Template

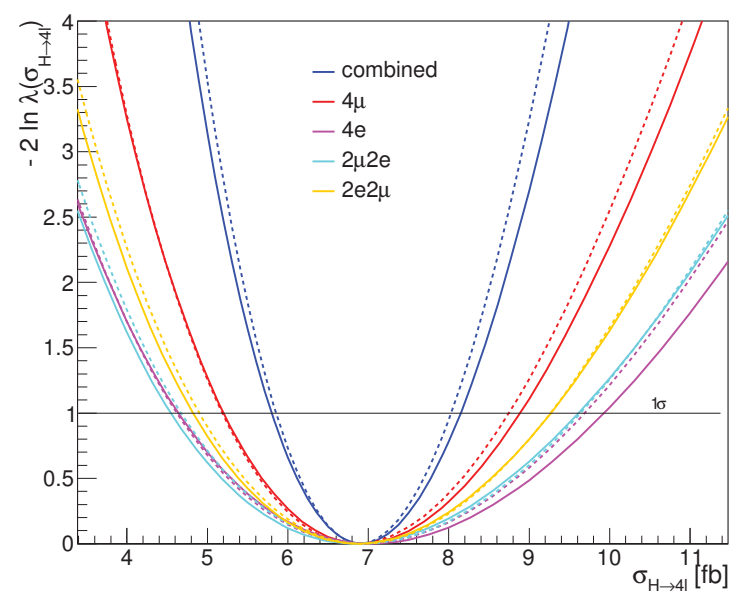

(c) unconstrained PER

Figure 100: Scans of the NLL on parameter $\sigma_{H \rightarrow 4 \ell}$ using an effective dataset with $m_{H}=125 \mathrm{GeV}$. Subfigures show the scans using the different signal models as labelled. The NLL scans including (not including) systematics are shown with solid (dashed) lines.

cross-check, does not attain the expected resolution of the other two methods on $m_{H}$ measurement, but is competitive with the template method in the $\sigma_{H \rightarrow 4 \ell}$ measurement. 


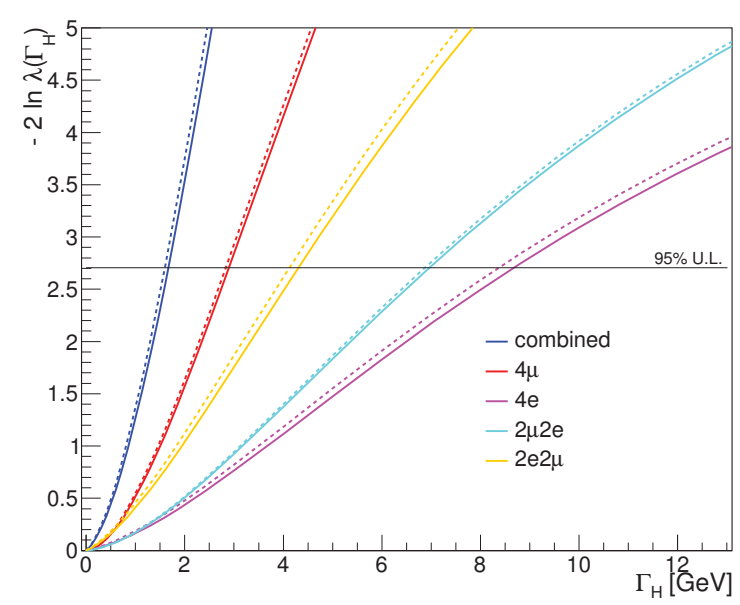

(a) constrained PER

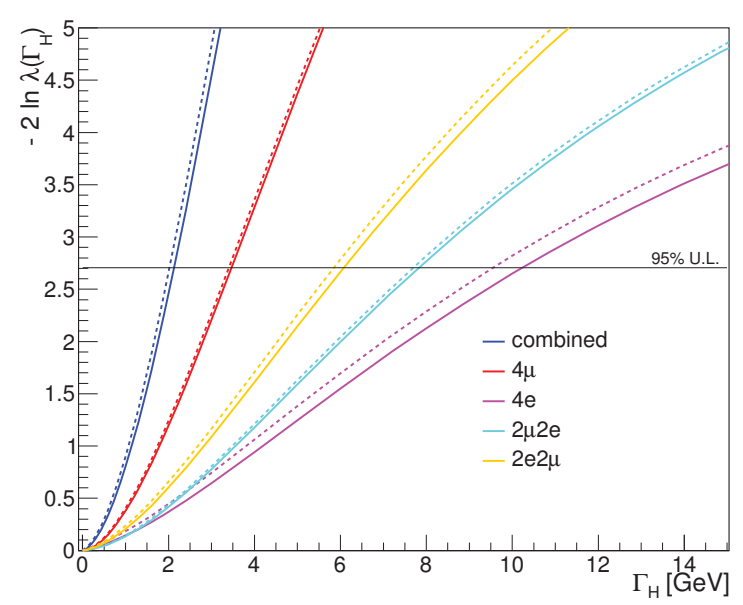

(b) unconstrained PER

Figure 101: Scans of the NLL on parameter $\Gamma_{H}$ using an effective dataset with $m_{H}=125 \mathrm{GeV}$. Subfigures show the scans using the different signal models as labelled. The NLL scans including (not including) systematics are shown with solid (dashed) lines. 
Table 18: Summary table of effective dataset results on $m_{H}, \sigma_{H \rightarrow 4 \ell}, \Gamma_{H}$ for the three signal models. The effective dataset is the same in all cases, with the true values from the input dataset as shown. Values inside the table use the same units as the reference values. Upper limits for $\Gamma_{H}$ are given at $95 \% \mathrm{CL}$.

\begin{tabular}{cccc}
\hline & constrained PER & Template & unconstrained PER \\
\hline$m_{H}$ & \multicolumn{2}{c}{ Input dataset value $m_{H}=125.00 \mathrm{GeV}$} \\
\hline $4 \mu$ & $124.99_{-0.52}^{+0.53}$ & $124.98_{-0.52}^{+0.52}$ & $125.00_{-0.60}^{+0.61}$ \\
$4 e$ & $125.00_{-1.15}^{+1.17}$ & $125.00_{-1.20}^{+1.25}$ & $125.02_{-1.27}^{+1.30}$ \\
$2 \mu 2 e$ & $125.00_{-0.97}^{+0.98}$ & $124.99_{-1.03}^{+1.06}$ & $125.01_{-1.06}^{+1.07}$ \\
$2 e 2 \mu$ & $124.98_{-0.73}^{+0.75}$ & $124.98_{-0.72}^{+0.72}$ & $125.00_{-0.93}^{+0.96}$ \\
combined & $124.99_{-0.37}^{+0.37}$ & $124.99_{-0.38}^{+0.38}$ & $125.00_{-0.43}^{+0.43}$ \\
\hline$\sigma_{H \rightarrow 4 \ell}$ & \multicolumn{1}{c}{ Input dataset value $\sigma_{H \rightarrow 4 \ell}=6.90 \mathrm{fb}$} \\
\hline $4 \mu$ & $6.85_{-1.69}^{+1.95}$ & $6.87_{-1.70}^{+1.96}$ & $6.85_{-1.71}^{+1.97}$ \\
$4 e$ & $6.94_{-2.37}^{+2.86}$ & $6.90_{-2.40}^{+2.90}$ & $6.94_{-2.40}^{+2.89}$ \\
$2 \mu 2 e$ & $6.94_{-2.29}^{+2.77}$ & $6.93_{-2.30}^{+2.78}$ & $6.94_{-2.30}^{+2.77}$ \\
$2 e 2 \mu$ & $6.91_{-2.01}^{+2.36}$ & $6.90_{-2.03}^{+2.38}$ & $6.92_{-2.02}^{+2.40}$ \\
combined & $6.90_{-1.10}^{+1.21}$ & $6.90_{-1.12}^{+1.23}$ & $6.90_{-1.12}^{+1.23}$ \\
\hline$\Gamma_{H}$ & \multicolumn{1}{c}{ Input dataset value $\Gamma_{H}=0.0041 \mathrm{GeV}$} \\
\hline $4 \mu$ & $<2.90$ & n.a. & $<3.47$ \\
$4 e$ & $<8.68$ & n.a. & $<10.23$ \\
$2 \mu 2 e$ & $<6.97$ & n.a. & $<7.83$ \\
$2 e 2 \mu$ & $<4.31$ & n.a. & $<6.08$ \\
combined & $<1.67$ & & $<2.13$ \\
\hline
\end{tabular}




\subsubsection{Tests of model closure}

The second set of tests using the effective dataset are a validation of the signal model(s) in the presence of background. They seek to better understand why the best fit values of $m_{H}$ appearing in Table 18 often show a small deviation from the input mass $m_{H}=125 \mathrm{GeV}$, where they appear as $124.99 \mathrm{GeV}$ or $125.01 \mathrm{GeV}$, and so forth. The test is only particularly meaningful for $m_{H}$ measurement, and not the $\sigma_{H \rightarrow 4 \ell}$ or $\Gamma_{H}$ measurements.

As alluded to in Section 8.4, the best fit value in a given signal dataset is expected to fluctuate in accordance with MC statistics by an amount given in (97) as

$$
\sigma_{M C \text { stat }}=\mathrm{SD}\left[\mathrm{m}_{4 \ell}\right] / \sqrt{\mathrm{N}_{\mathrm{MC}}}
$$

While it is the overall fluctuation that gets assigned as a systematic uncertainty, it is the per-category fluctuations that matter when interpreting the values in Table 18. To this point, Table 19 compiles the statistical variation in the mean $m_{4 \ell}$, and thus best fit $m_{H}$, expected from each fit.

Table 19: Standard deviation of the mean $m_{4 \ell}$ value, translating to the variations in the best fit $m_{H}$ value, for independent signal datasets with the same actual statistics as the MC simulated signal samples used in this thesis. The unconstrained combined value was used as a systematic uncertainty in Section 8.4.

\begin{tabular}{ccc}
\hline & \multicolumn{2}{c}{$H \rightarrow 4 \ell$ scale systematic $[\mathrm{MeV}]$} \\
category & unconstrained $\sigma_{M C \text { stat }}[\mathrm{MeV}]$ & constrained $\sigma_{M C \text { stat }}[\mathrm{MeV}]$ \\
\hline $4 \mu$ & 13.8 & 13.4 \\
$4 e$ & 20.2 & 19.6 \\
$2 \mu 2 e$ & 18.7 & 18.3 \\
$2 e 2 \mu$ & 15.7 & 15.0 \\
\hline combined & 8.1 & 7.8 \\
\hline
\end{tabular}

To get a better indication of whether the small shifts observed in Table 18 arise 
purely from MC statistical fluctuation, three independent effective datasets are created with signals at different MC simulated mass points $m_{H}=124,125,126 \mathrm{GeV}$. For each dataset, NLL scans were performed, and the best fit mass, $\hat{m}_{H}$, was compared with the simulated mass, $m_{H}^{\text {simulated }}$, to calculate the residual bias,

$$
\Delta_{m_{H}} \equiv \hat{m}_{H}-m_{H}^{\text {simulated }}
$$

If the signal and background model(s) are flawless, then in a mathematical sense these values were randomly sampled from a Gaussian distribution with zero mean, and widths as specified in Table $19, G\left(\Delta_{m_{H}} ; 0, \sigma_{M C}\right.$ stat $)$. Using the three independent datasets gives an indication of the truth of this postulate. The residual biases for the different datasets, models, and categories are collected in Table 20.

At first glance, the observed residual biases are of comparable magnitude to that expected from statistical fluctuation alone, suggesting that the signal models introduce no additional uncertainty. The number of samples available is too limited to do a formal test on a per-category basis, so instead all categories are combined. Each of the twelve values per-method (the combined values are derivatives of the others, so can't be used here) are standardized into twelve values of $z=\Delta_{m_{H}} / \sigma_{M C \text { stat }}$. The sample mean, standard deviation, and their respective errors are calculated [98] for each set of values to be

$$
\begin{aligned}
\bar{z}^{\text {constrained PER }} & =-0.49 \pm 0.34, & s_{z}^{\text {constrained PER }} & =1.14 \pm 0.55 \\
\bar{z}^{\text {Template }} & =-0.51 \pm 0.21, & s_{z}^{\text {Template }} & =0.71 \pm 0.22 \\
\bar{z}^{\text {unconstrained PER }} & =-0.08 \pm 0.32, & s_{z}^{\text {unconstrained PER }} & =1.07 \pm 0.49
\end{aligned}
$$

which are consistent with the proposal of $\bar{z}=0.00, s_{z}=1.00$, i.e. that the observed residual biases come from MC statistical fluctuations only. 
Table 20: Residual bias $\Delta_{m_{H}}$ calculated using NLL scans on effective datasets at different mass values for the three signal models.

\begin{tabular}{|c|c|c|c|}
\hline & $m_{H}=124 \mathrm{GeV}$ & $m_{H}=125 \mathrm{GeV}$ & $m_{H}=126 \mathrm{GeV}$ \\
\hline & \multicolumn{3}{|c|}{ constrained PER, $\Delta_{m_{H}}[\mathrm{MeV}]$} \\
\hline $4 \mu$ & +8.7 & -7.0 & -11.0 \\
\hline $4 e$ & -27.9 & +0.7 & -45.1 \\
\hline $2 \mu 2 e$ & +34.9 & +6.6 & -21.6 \\
\hline $2 e 2 \mu$ & -0.9 & -13.9 & -23.2 \\
\hline \multirow[t]{2}{*}{ combined } & +6.2 & -5.8 & -19.1 \\
\hline & \multicolumn{3}{|c|}{ Template, $\Delta_{m_{H}}[\mathrm{MeV}]$} \\
\hline $4 \mu$ & -9.0 & -10.6 & -13.0 \\
\hline $4 e$ & -29.4 & +1.6 & -13.7 \\
\hline $2 \mu 2 e$ & +3.4 & +22.4 & -13.5 \\
\hline $2 e 2 \mu$ & -6.1 & -16.0 & -11.6 \\
\hline \multirow[t]{2}{*}{ combined } & -8.5 & -13.3 & -12.5 \\
\hline & \multicolumn{3}{|c|}{ unconstrained PER, $\Delta_{m_{H}}[\mathrm{MeV}]$} \\
\hline $4 \mu$ & +2.8 & +1.7 & -10.4 \\
\hline $4 e$ & -20.3 & +22.5 & -24.2 \\
\hline $2 \mu 2 e$ & +26.2 & +11.5 & -21.3 \\
\hline $2 e 2 \mu$ & -29.5 & +12.8 & +12.4 \\
\hline combined & +9.9 & +8.7 & -8.7 \\
\hline
\end{tabular}


The tests in this section leave the encouraging conclusion that the different methods under study - primarily the constrained PER method, but also the two crosschecks methods - are robust across a range of possible Higgs masses, and will serve as effective tools for measurement of $m_{H}$.

\subsection{Effects of systematics}

The likelihood scans in the preceding section are sufficient to see the combined effect of all systematics on the measurement; this can be read directly from the difference between the likelihood scans with systematics and those without. It is still very useful to decompose the effect of the systematics on each measurement, which allows the systematics to be ranked according to their impact on the measurement. To this end, the following quantities are calculated, for each source of systematic uncertainty $\alpha$ :

$\hat{\theta}_{\alpha} \quad$ The best fit value and uncertainty of NP $\theta_{\alpha}$ when all other parameters are fixed at their best fit values, called the pull of $\alpha$.

$\hat{\hat{\mu}}\left(\theta_{\alpha}= \pm 1\right.$ ) The best fit value of POI $\mu$ (or upper limit of $\Gamma_{H}$ ) when all other parameters are fixed at their best fit values, except $\theta_{\alpha}$ which is set to \pm 1 . These values are compared against the global best fit (or upper limit) of $\mu$ (when all other parameters, including $\theta_{\alpha}$, are at their best fit values) to see the effect of $\alpha$.

Since the implementation of systematics is common to all methods, this study was performed using only the constrained PER method. The effects were studied on the effective dataset of Section 9.2 with $m_{H}=125 \mathrm{GeV}$, and in the data. The decomposition of systematic uncertainties for the $m_{H}$ measurement is shown in Figure 102, for the $\sigma_{H \rightarrow 4 \ell}$ measurement in Figure 103, and for the $\Gamma_{H}$ measurement in Figure 104. 


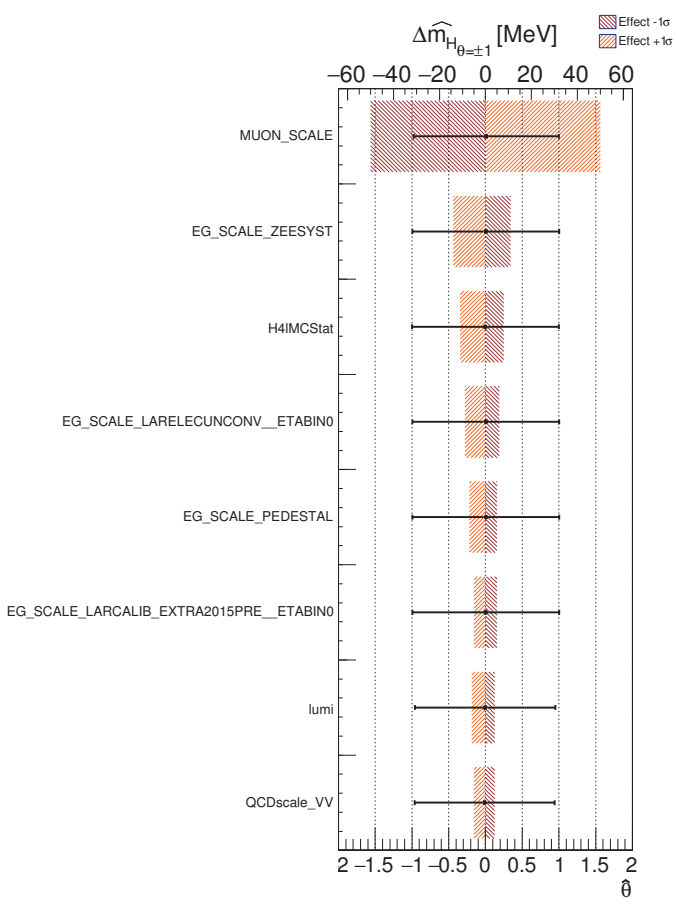

(a) Effective dataset

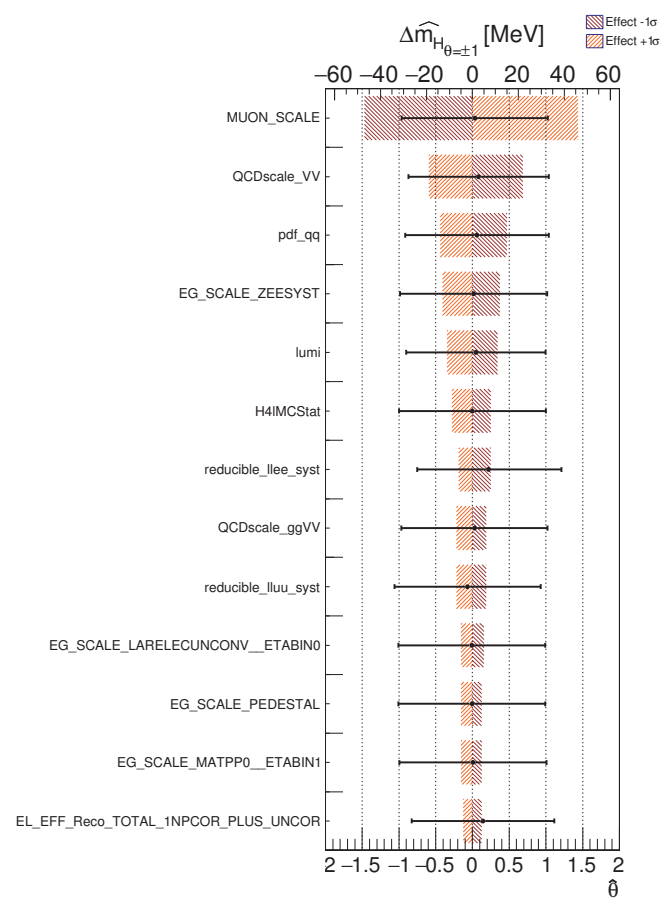

(b) Data

Figure 102: Effects of different sources of systematic uncertainty on the $m_{H}$ measurement, as seen in the effective dataset from simulation with $m_{H}=125 \mathrm{GeV}$ described in Section 9.2, and in the data. Shown are the pulls (black points and error bars) of each NP, and its effect on $m_{H}$ when set to \pm 1 . Systematics with effects less than $\pm 4 \mathrm{MeV}$ are omitted.

In the effective dataset, the pulls should indicate $\theta_{\alpha}=0.00 \pm 1.00$ since all systematics are implicitly at their nominal values. In the data, some statistical fluctuation is expected (and observed), since the global best-fit value of the NPs is a-priori unknown.

In interpreting the effects in these plots, note that the systematics are ordered from top to bottom in decreasing order of their effect ${ }^{2}$. These plots can therefore be used to determine the most important systematic uncertainties for each measurement, something not possible from examining the fit inputs in Chapter 8 alone. Table 21 summarizes the dominant systematics observed for each measurement. Note that

\footnotetext{
${ }^{2}$ Specifically, they are sorted by $\left|\hat{\hat{\mu}}\left(\theta_{\alpha}=+1\right)-\hat{\hat{\mu}}\left(\theta_{\alpha}=-1\right)\right|$
} 


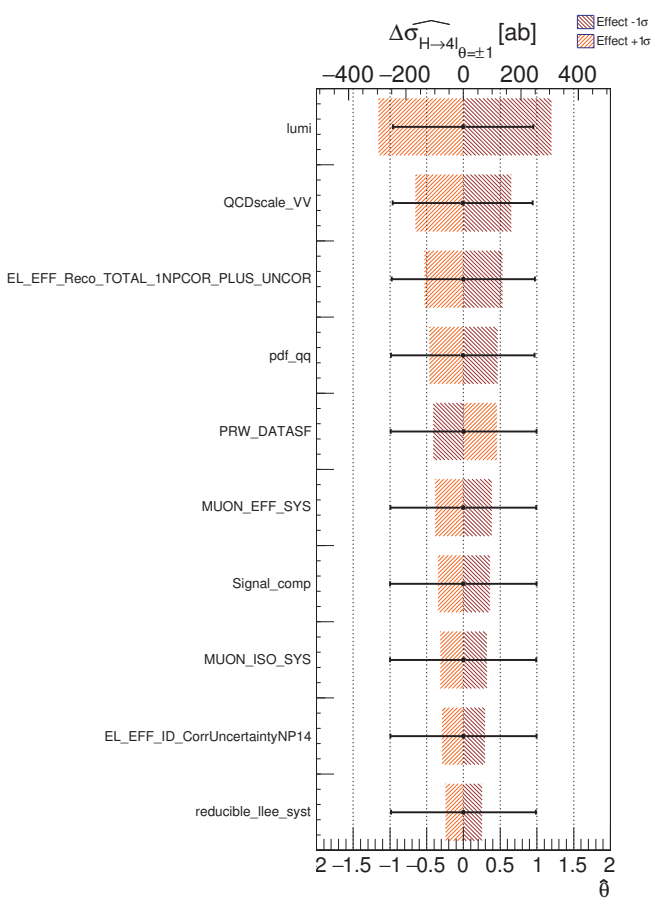

(a) Effective dataset

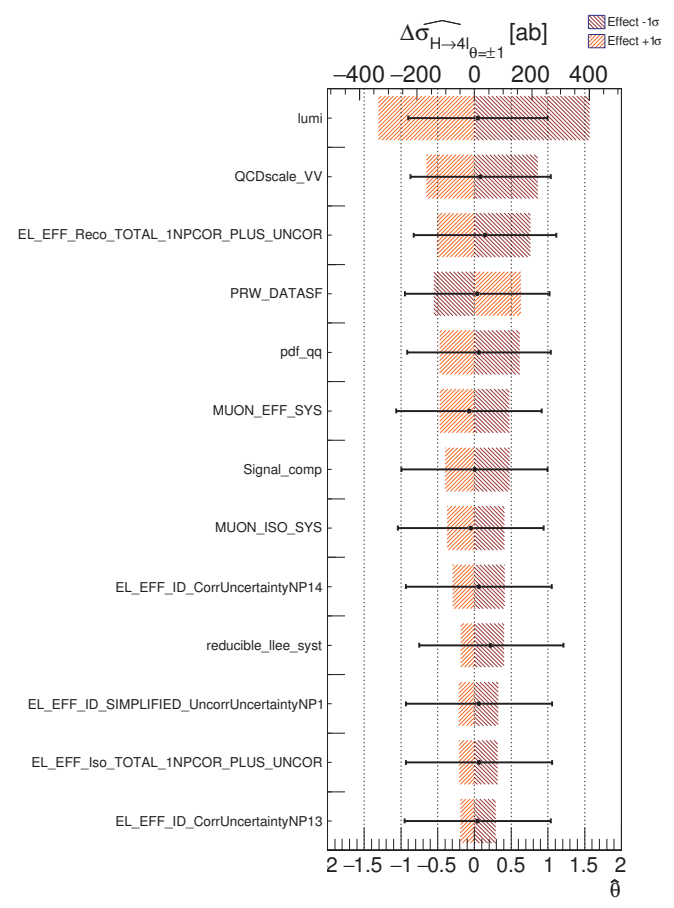

(b) Data

Figure 103: Effects of different sources of systematic uncertainty on the $\sigma_{H \rightarrow 4 \ell}$ measurement, as seen in the effective dataset from simulation with $m_{H}=125 \mathrm{GeV}$ described in Section 9.2, and in the data. Shown are the pulls (black points and error bars) of each NP, and its effect on $\sigma_{H \rightarrow 4 \ell}$ when set to \pm 1 . Systematics with effects less than \pm 60 ab are omitted.

the values do not exactly match the plots, as the table adds (in quadrature) some systematics which where omitted from the plots.

One noteworthy feature can be seen by looking at systematics on the $m_{H}$ measurement. While the effect of the electron energy scale uncertainties on the $4 e$ category are about $12 \%$ larger than the effect of the muon momentum scale on the $4 \mu$ category (refer back to Section 8.4), in the combined fit the effect of the muon momentum scale is about $130 \%$ larger. This occurs because the $4 \mu$ category dominates the measurement statistically, so systematics in this category have a larger impact on the combination. 


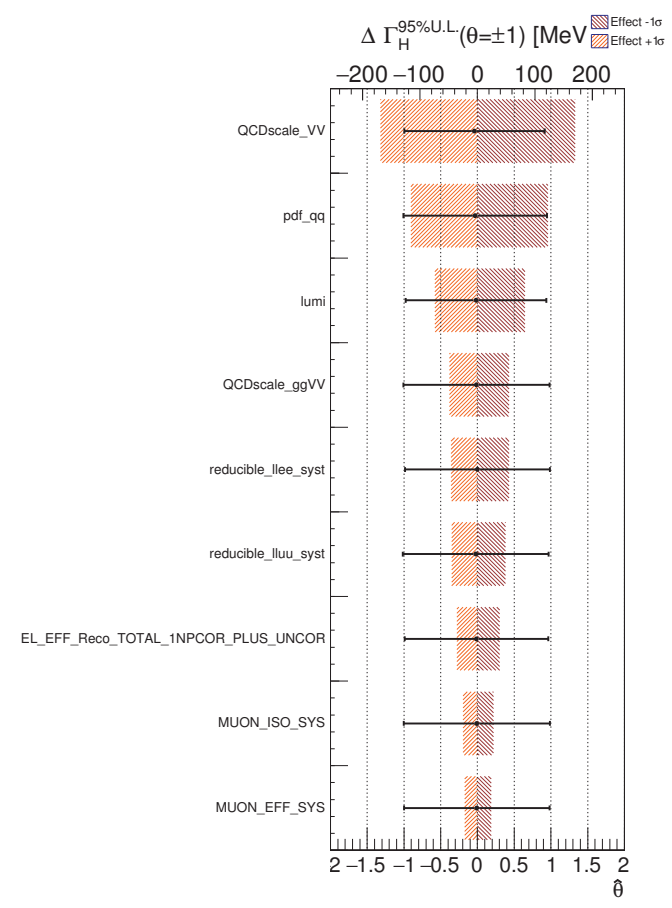

(a) Effective dataset

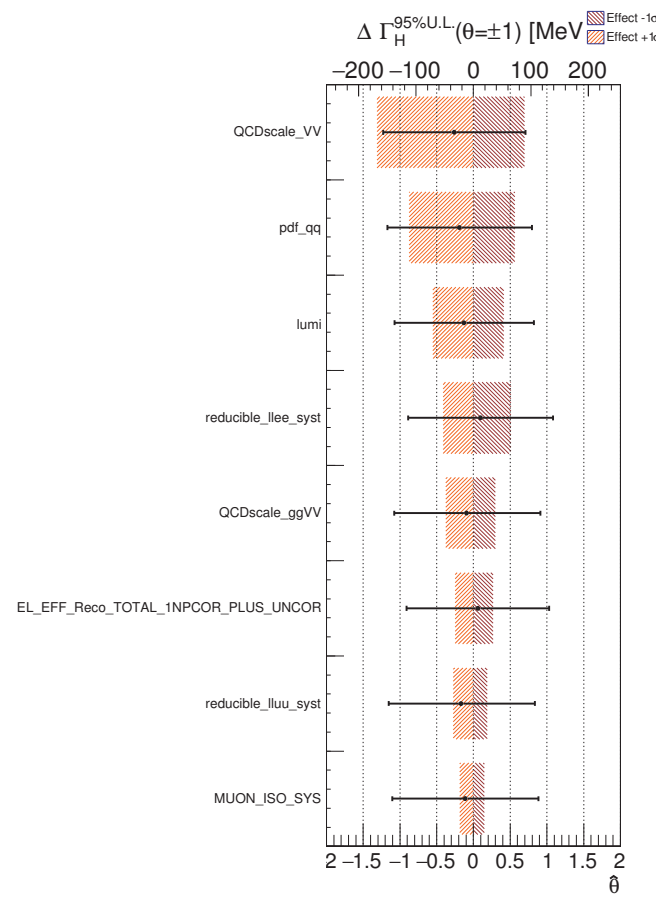

(b) Data

Figure 104: Effects of different sources of systematic uncertainty on the $\Gamma_{H}$ measurement, as seen in the effective dataset from simulation with $m_{H}=125 \mathrm{GeV}$ described in Section 9.2, and in the data. Shown are the pulls (black points and error bars) of each NP, and its effect o the upper limit of $\Gamma_{H}$ when set to \pm 1 . Systematics with effects less than $\pm 20 \mathrm{MeV}$ are omitted.

The ranking of the sources of systematic uncertainty in this section is useful in directing future measurement strategy. Although statistical uncertainties are at this stage by far the dominant source of uncertainty, that will not aways be the case. The systematic uncertainties which dominate the measurements now are those on which the greatest focus should be to improve in future. For all measurements, the theoretical calculation of the dominant $q \bar{q} \rightarrow Z Z^{(*)}$ background is one of the leading effects. For the $m_{H}$ measurement specifically, the electron energy and muon momentum scales are both very important. For the $\sigma_{H \rightarrow 4 \ell}$ measurement, the electron efficiency systematics also play a very important role, followed closely by pile-up uncertainties, and muon efficiency and isolation. For the $\Gamma_{H}$ measurement, the dominant sources of systematic uncertainty are all related to the event yields, and (somewhat surprisingly) 
Table 21: Summary table of dominant systematics affecting measurement of $m_{H}, \sigma_{H \rightarrow 4 \ell}$, and upper limits of $\Gamma_{H}$. The value presented are the observed effect of each systematic, coming from Figure 102b, Figure 103b, Figure 104b. The naming scheme introduced in Section 8.2 is used to group systematics where necessary.

\begin{tabular}{lclc}
\hline \multicolumn{4}{c}{ Dominant systematics for $m_{H}[\mathrm{MeV}]$} \\
\hline MUON_SCALE & 46 & QCDscale_VV & 20 \\
EG_SCALE_* & 17 & pdf_qq & 14 \\
lumi & 11 & H4lMCStat & 8 \\
reducible_llee_* & 7 & EL_EFF_* & 7 \\
total & 58 & & \\
\hline \multicolumn{4}{c}{ Dominant systematics $\sigma_{H \rightarrow 4 \ell}[\mathrm{ab}]$} \\
\hline lumi & 370 & EL_EFF_* & 230 \\
QCDscale_VV & 190 & PRW_DATASF & 150 \\
pdf_qq & 140 & MUON_EFF_* & 120 \\
Signal_comp & 110 & MUON_ISO_* & 100 \\
total & 570 & & 92 \\
\hline \multicolumn{1}{c}{ Dominant systematic for } & $\Gamma_{H}$ upper limit $[\mathrm{MeV}]$ \\
\hline QCDscale_VV & 130 & pdf_qq & 59 \\
lumi & 62 & reducible_llee_* & 53 \\
QCDscale_ggVV & 43 & EL_EFF_* & MUON_ISO_* \\
reducible_lluu_* & 30 & & 23 \\
total & 190 & & \\
\hline
\end{tabular}

not the resolution. This arises because as the natural width increases, the contribution to the overall lineshape width from resolution systematics falls off. Also as the natural width increases, the signal looks more and more background-like, and so the background normalizations play a large role in where the $\Gamma_{H}$ upper limit can be set. Focusing time and effort in reducing these sources of systematic uncertainties will pay the most benefit in the resolution of future measurements. 


\subsection{Tests using pseudo-experiments}

The effective dataset approach used in Section 9.2 is very powerful for determining properties of the fit. In particular, it is very useful for determining the median expected fit result [97]. However, the median behaviour is not always a satisfactory metric, especially in the low-statistics regime of the current measurement. One reason is that by using the median behaviour of a huge number of events, the highly variable mass responses around which the PER method is built are averaged out and appear more like the Template method. So the effective dataset in practice hides the aspects of the PER which distinguish it from the Template method. This section will demonstrate that in the low-statistics regime expected in the data, the constrained PER method outperforms the Template method, specifically because of its use of perevent resolution information. As the methods all use a common implementation and values for systematic uncertainties (with the exception of uncertainties on the signal resolution being omitted for the Template method), the inclusion of systematics is not useful in differentiating these methods ${ }^{3}$. For this reason, no systematic uncertainties are included in these studies.

To test the measurement at low statistics, a large number of pseudo-experiments were performed, also called toys. The datasets in each toy, called pseudo-datasets, were built using events drawn from $\mathrm{MC}$ simulated samples with the event selection and analysis mass window applied. By using directly $\mathrm{MC}$ simulation, no assumption is placed on the validity of the signal or background PDFs. The events were drawn in random order with probabilities proportional to their respective event weights. This procedure forces the toys to have the same composition on average as the effective dataset of Section 9.2. The number of events drawn from each source in each category

\footnotetext{
${ }^{3}$ Systematics would be useful in differentiating these measurements from ATLAS' $m_{H}$ measurement in Run 1 [7], which used different analysis method with larger systematic uncertainties.
} 
was a Poisson-distributed random number based on the expected number of events,

$$
N_{q q Z Z, 2 e 2 \mu}^{\text {toy } i}=\text { random.Poisson }\left(N_{q q Z Z, 2 e 2 \mu}^{\text {exp. }}\right) .
$$

For the signal, $m_{H}=125 \mathrm{GeV}$ samples were used for each production mode, and the total number of signal events to be sampled was split up among production modes according to their relative contributions inside the mass range using SM cross sections and the simulated samples ${ }^{4}$.

$$
\begin{aligned}
N_{g g F, 2 e 2 \mu}^{\text {exp. }} & =88.2 \% N_{\text {signal }, 2 e 2 \mu}^{\text {exp. }}, \\
N_{V B F, 2 e 2 \mu}^{\text {exp. }} & =7.6 \% N_{\text {signal }, 2 e 2 \mu}^{\text {exp. }}, \\
N_{V H, 2 e 2 \mu}^{\text {exp. }} & =3.5 \% N_{\text {signal }, 2 e 2 \mu}^{\text {exp. }}, \\
N_{t t H+b b H, 2 e 2 \mu}^{\text {exp. }} & =0.7 \% N_{\text {signal }, 2 e 2 \mu}^{\text {exp. }} .
\end{aligned}
$$

Using these fractions, a random number of events, following the same prescription as for the backgrounds, was drawn to be used in each toy. This procedure was used to construct a large number of toys (15,000 or more, depending on which measurement), each representing what could be seen in the data, if $m_{H}=125 \mathrm{GeV}$. These toys can be used to validate and compare the fit methods in a complementary fashion to the effective dataset approach.

\subsubsection{Fit results in pseudo-experiments}

The first set of tests using the toy experiments comes from fitting each model (constrained PER, Template, unconstrained PER) on the toy datasets; the results of the fits are used to make comparisons between the methods. The three metrics by which

\footnotetext{
${ }^{4}$ Note that these are different than the SM production fractions in Table 3 because of the mass window applied - fewer WH,ZH,ttH events survive when the mass window is applied, because the four-lepton candidate can often contain a lepton that did not originate from the Higgs decay. As a result, the relative fractions of $\mathrm{ggF}$ and $\mathrm{VBF}$ are increased.
} 
the toys are compared are:

$\hat{\boldsymbol{\mu}} \quad$ The best fit value of the parameter of interest $\mu$ (e.g. $\left.m_{H}\right)$. The distribution of best fit values is expected to be Gaussian, centered on the true value, with width equal to the resolution of the measurement. For $\Gamma_{H}$, it is expected to be zero, with a right-hand tail (negative $\Gamma_{H}$ is not possible, by definition).

$\boldsymbol{p}_{\boldsymbol{\mu}} \quad$ The pull of $\mu$, defined by the residual of the best fit value, divided by the estimated error. The pull is, by definition, expected to be distribution as a dimensionless Gaussian with zero mean, and unit width. Deviations of the mean indicate a measurement bias, while deviations of the width indicate mis-estimation of uncertainties in the fit. The pull is ill-defined by $\Gamma_{H}$, and is not shown.

95\% UL The 95\% CL upper limit, applicable to $\Gamma_{H}$ toys only.

The pull can be calculated in the case of asymmetric errors[99] by

$$
p_{\mu}= \begin{cases}\frac{\hat{\mu}-\mu^{\text {true }}}{\left|\sigma_{\mu}^{+}\right|} & \text {when } \hat{\mu}<\mu^{\text {true }}, \\ \frac{\hat{\mu}-\mu^{\text {true }}}{\left|\sigma_{\mu}^{-}\right|} & \text {when } \hat{\mu}>\mu^{\text {true }}\end{cases}
$$

where $\sigma_{\mu}^{ \pm}$are the positive and negative errors of the fit. This definition is constructed such that $68.3 \%$ of pulls will fall with \pm 1 , if the errors are accurately estimated in the fit.

The distributions of the best fit values and pulls from the three methods for $m_{H}$ are found in Figure 105, and for $\sigma_{H \rightarrow 4 \ell}$ in Figure 106. In each case, the distribution is fit with a Gaussian. The mean and standard deviation of the histogram itself are recorded on the plot as mean ${ }_{\text {hist }}$ and $\mathrm{RMS}_{\text {hist }}$, respectively. The mean and width of the Gaussian fit are recorded as $\mu_{\text {fit }}$ and $\sigma_{\text {fit }}$, respectively. The equivalent plots, separated 
by analysis category, are shown in Appendix C.3. The values of these Gaussian fits are recorded in Table 22 for $m_{H}$, and Table 23 for $\sigma_{H \rightarrow 4 \ell}$. Recall that the uncertainty on $\mu_{\text {fit }}$ is approximately $\sigma_{\text {fit }} / \sqrt{N_{\text {toys }}}$, falling with the number of toys ${ }^{5}$.

The distributions of the best fit values and $95 \%$ CL upper limits on $\Gamma_{H}$ from the PER method with and without ZMC are found in Figure 107. The mean, median, and standard deviation of the histogram is recorded on both plots. The equivalent plots, separated by analysis category, are shown in Appendix C.3. The median fit value is exactly zero ${ }^{6}$ for both methods (and in all individual categories). The median and mean 95\% CL upper limits from the toy experiments are recorded in Table 24.

Table 22: Summary table of toy experiment fits of $m_{H}$. The parameters of the Gaussian fits in Figure 105 are recorded here. Units in the table match the header.

\begin{tabular}{ccccccc}
\hline & \multicolumn{2}{c}{ constrained PER } & \multicolumn{3}{c}{ Template } & \multicolumn{3}{c}{ unconstrained PER } \\
& $\mu_{\text {fit }}$ & $\sigma_{\text {fit }}$ & $\mu_{\text {fit }}$ & $\sigma_{\text {fit }}$ & $\mu_{\text {fit }}$ & $\sigma_{\text {fit }}$ \\
\hline \multicolumn{6}{c}{$\hat{m}_{H}}$, & $m_{H}^{\text {true }}=125 \mathrm{GeV}$ \\
\hline $4 \mu$ & 125.00 & 0.52 & 124.99 & 0.52 & 125.00 & 0.61 \\
$4 e$ & 125.01 & 1.08 & 124.97 & 1.12 & 125.04 & 1.20 \\
$2 \mu 2 e$ & 125.02 & 0.93 & 124.99 & 0.98 & 125.02 & 1.01 \\
$2 e 2 \mu$ & 124.94 & 0.72 & 124.95 & 0.72 & 124.95 & 0.88 \\
combined & 124.99 & 0.36 & 124.98 & 0.37 & 125.00 & 0.43 \\
\hline \multicolumn{6}{c}{$p_{m_{H}}$} \\
\hline $4 \mu$ & -0.01 & 1.00 & -0.02 & 1.01 & -0.01 & 1.01 \\
$4 e$ & 0.01 & 1.02 & -0.03 & 1.05 & 0.02 & 1.03 \\
$2 \mu 2 e$ & 0.01 & 1.01 & -0.02 & 1.04 & 0.01 & 1.01 \\
$2 e 2 \mu$ & -0.09 & 1.01 & -0.07 & 1.03 & -0.06 & 1.03 \\
combined & -0.03 & 0.98 & -0.05 & 1.00 & -0.01 & 0.99 \\
\hline
\end{tabular}

\footnotetext{
${ }^{5}$ The specific number of toys entering each number is printed on the corresponding plot

${ }^{6}$ Technically, it is the lower limit of the fit parameter, which isn't allowed to go below $10^{-5}$ so that the BW remains finite.
} 

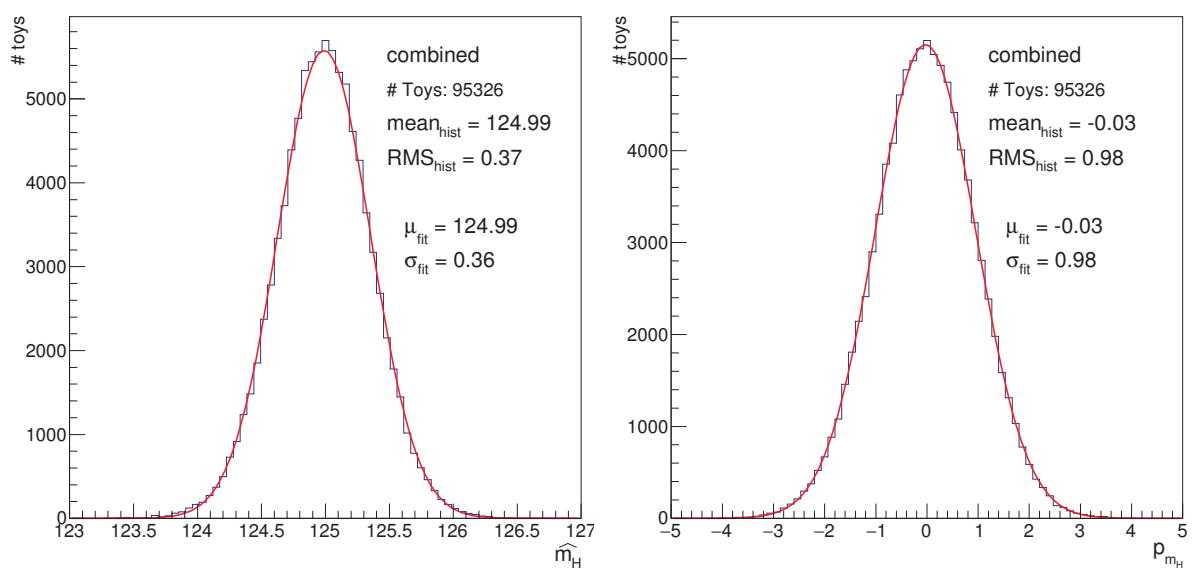

(a) constrained PER
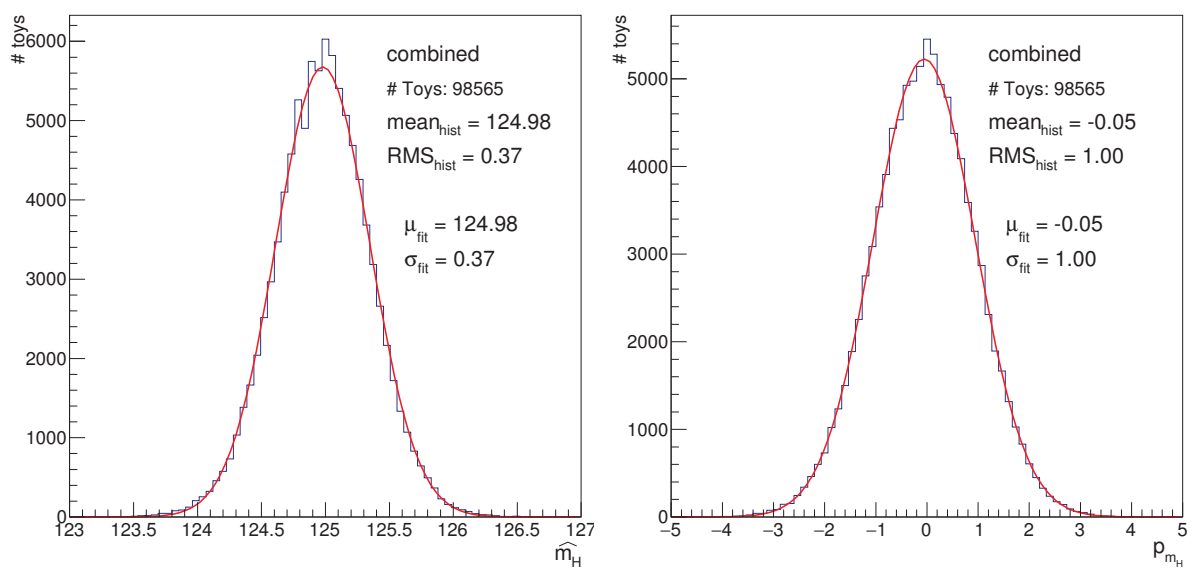

(b) Template
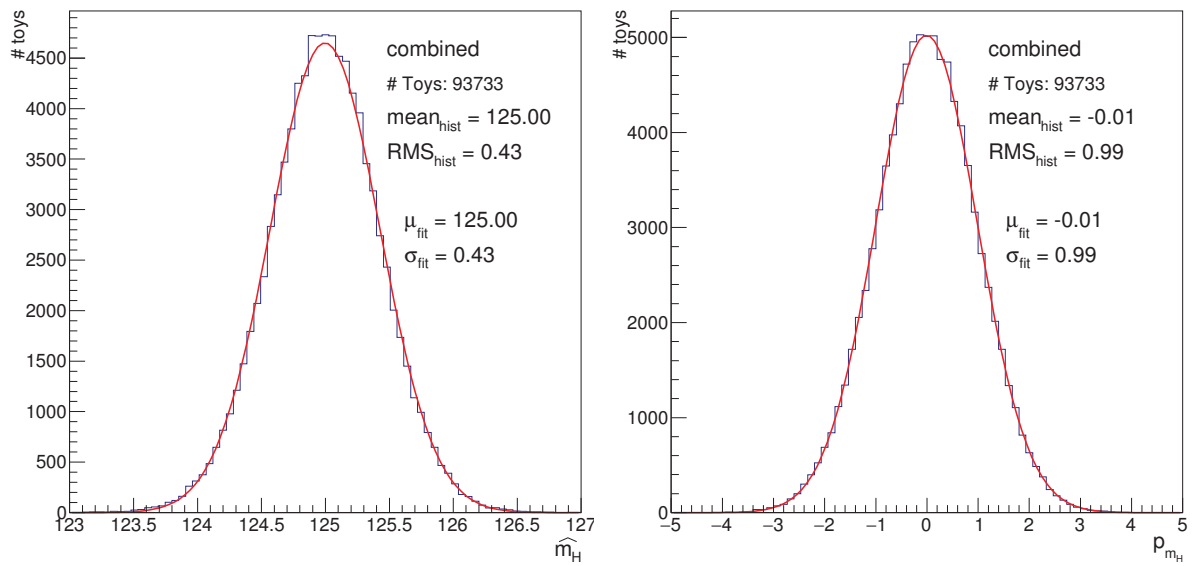

(c) unconstrained PER

Figure 105: Results of $m_{H}$ fits from three methods on toy datasets. Shown are the best fit values $\hat{m_{H}}$ (left), and the pulls $p_{m_{H}}$ (right) from each method. The raw distribution of results from the toys is shown in blue, and a Gaussian fit is shown with a red line. 

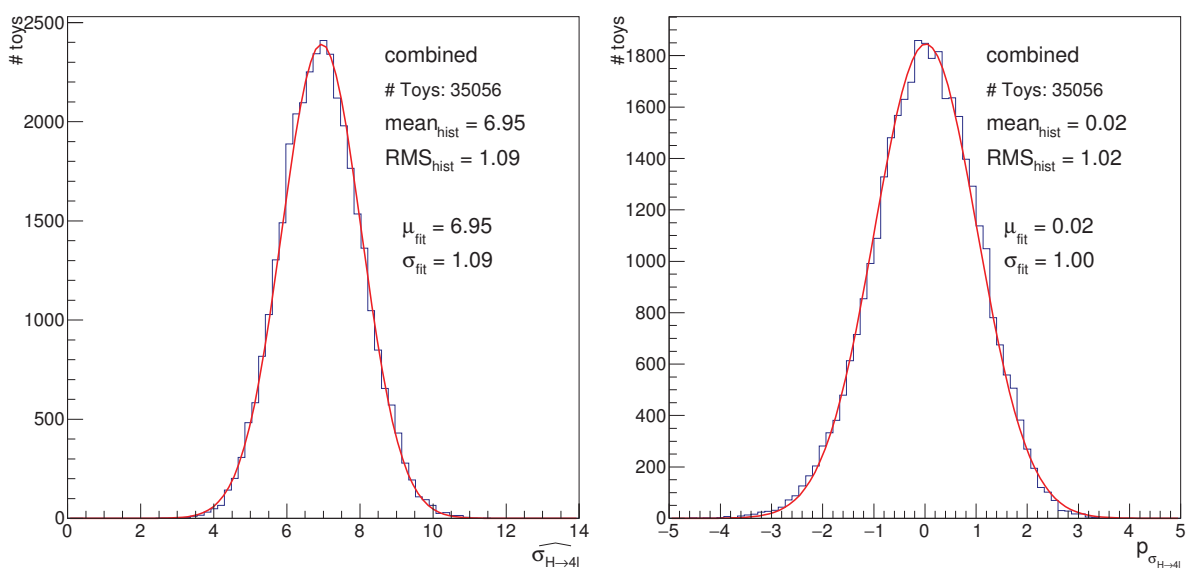

(a) constrained PER
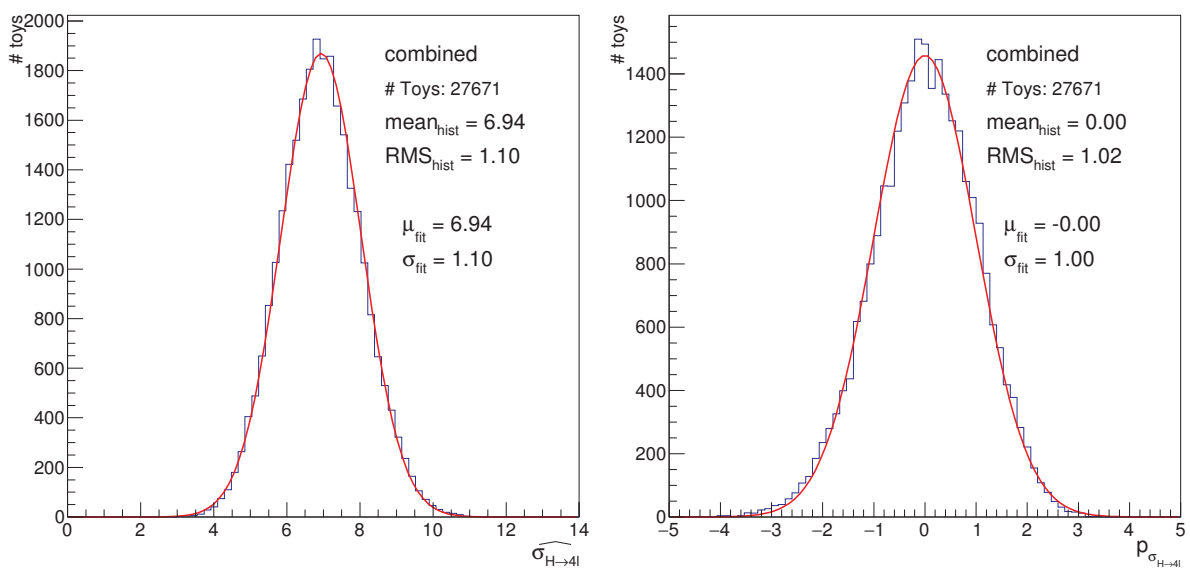

(b) Template
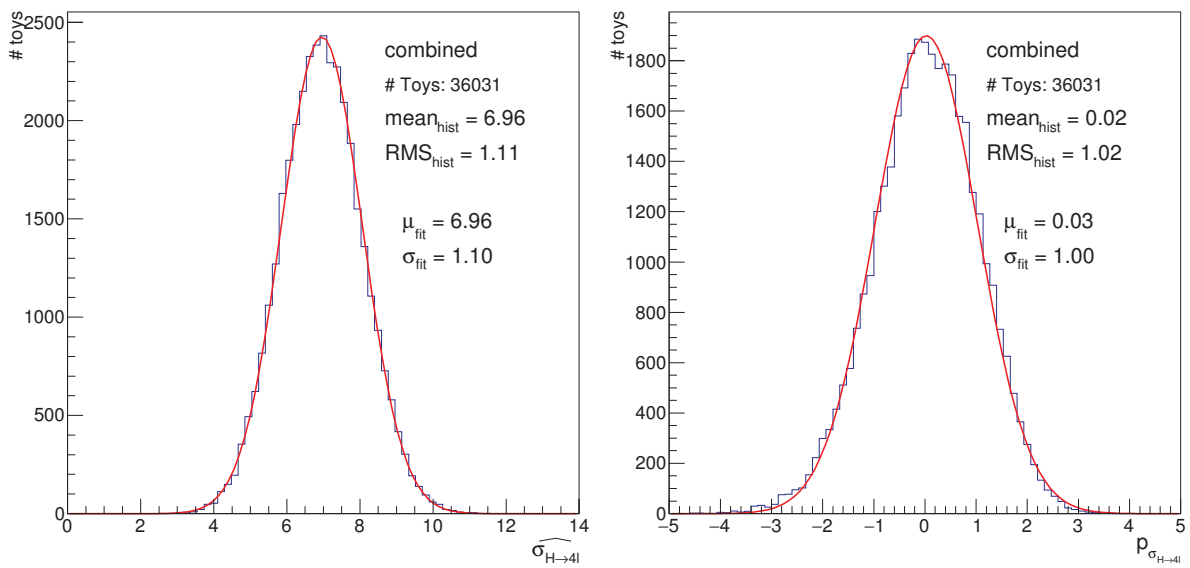

(c) unconstrained PER

Figure 106: Results of $\sigma_{H \rightarrow 4 \ell}$ fits from three methods on toy datasets. Shown are the best fit values $\sigma_{H \rightarrow 4 \ell}$ (left), and the pulls $p_{\sigma_{H \rightarrow 4 \ell}}$ (right) from each method. The raw distribution of results from the toys is shown in blue, and a Gaussian fit is shown with a red line. 

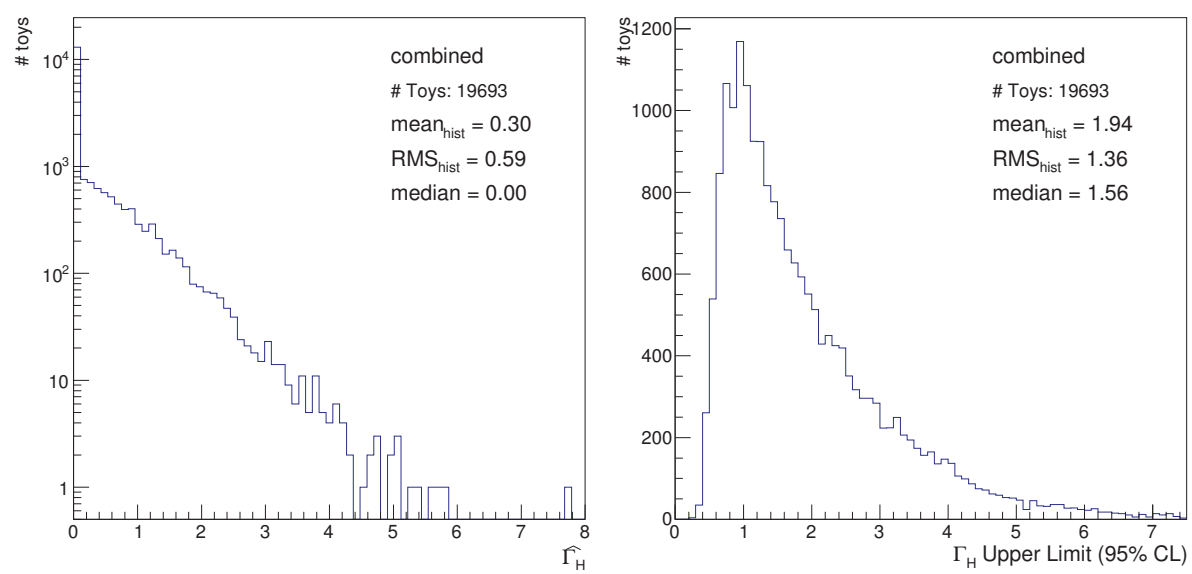

(a) constrained PER
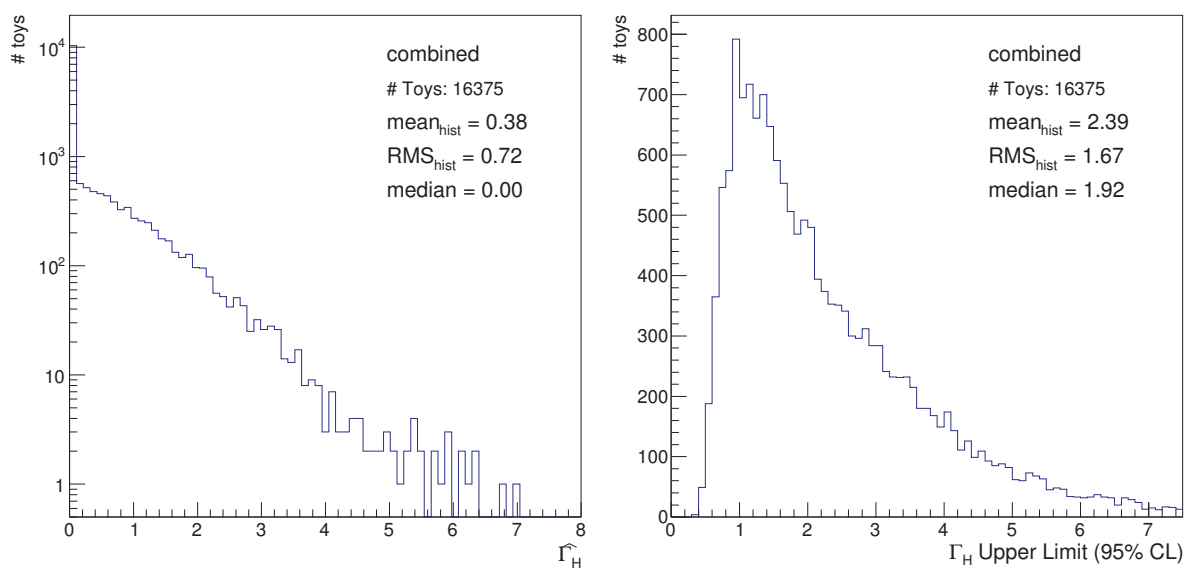

(b) unconstrained PER

Figure 107: Results of $\Gamma_{H}$ fits from Per-event response method, with and without

$\mathrm{Z}$ Mass Constraint, on toy datasets. Shown are the best fit values $\hat{\Gamma_{H}}$ (left of each subfigure), and the 95\% CL upper limit (right) from each method. The raw distribution of results from the toys is shown in blue. Note the log axis and large first bin in the left-hand plots.

The upper section of Table 22 can be used to compare the constrained PER model with the Template method in a context closely related to the actual data. The widths of the $\hat{m}_{H}$ distribution indicate the expected resolution of the measurement: the constrained PER method shows no difference in the $4 \mu$ category, a $3.6 \%$ improvement in the $4 e$ category, a $5.1 \%$ improvement in the $2 \mu 2 e$ category, no change in the the $2 e 2 \mu$ category, and a $2.6 \%$ improvement overall, demonstrating that it is more precise. These improvements are consistent with what was found using the effective dataset 
in Section 9.2.1. While the effective dataset can test the bias of the models at high statistics, where all models were found to be unbiased in Section 9.2.2, it does not test the bias expected in data, where limited statistics are available. The means of the $\hat{m}_{H}$ distribution provide a measure of the bias of the methods in this low statistics regime. In the combined fit toys, the constrained PER method shows less bias compared to the Template method (mean of $\hat{m}_{H}$ is 124.99 versus 124.98), indicating it is also more accurate. These are two of the key reasons why the PER method is such a powerful tool for measurement of resonance mass.

Table 23: Summary table of toy experiment fits of $\sigma_{H \rightarrow 4 \ell}$. The parameters of the Gaussian fits in Figure 106 are recorded here. Units in the table match the header.

\begin{tabular}{|c|c|c|c|c|c|c|}
\hline & \multicolumn{2}{|c|}{ constrained PER } & \multicolumn{2}{|c|}{ Template } & \multicolumn{2}{|c|}{ unconstrained PER } \\
\hline & $\mu_{\text {fit }}$ & $\sigma_{\text {fit }}$ & $\mu_{\mathrm{fit}}$ & $\sigma_{\text {fit }}$ & $\mu_{\mathrm{fit}}$ & $\sigma_{\text {fit }}$ \\
\hline & \multicolumn{6}{|c|}{$\hat{\sigma}_{H \rightarrow 4 \ell}, \quad \sigma_{H \rightarrow 4 \ell}^{\text {true }}=6.90 \mathrm{fb}$} \\
\hline $4 \mu$ & 6.99 & 1.71 & 6.98 & 1.72 & 7.01 & 1.74 \\
\hline $4 e$ & 7.14 & 2.38 & 7.07 & 2.37 & 7.09 & 2.41 \\
\hline $2 \mu 2 e$ & 7.11 & 2.33 & 7.12 & 2.31 & 7.07 & 2.35 \\
\hline $2 e 2 \mu$ & 6.95 & 2.03 & 6.94 & 2.06 & 6.96 & 2.04 \\
\hline \multirow[t]{2}{*}{ combined } & 6.95 & 1.09 & 6.94 & 1.10 & 6.96 & 1.10 \\
\hline & \multicolumn{6}{|c|}{$p_{\sigma_{H \rightarrow 4 \ell}}$} \\
\hline $4 \mu$ & 0.01 & 0.97 & 0.01 & 0.97 & 0.02 & 0.97 \\
\hline $4 e$ & 0.05 & 0.95 & 0.02 & 0.95 & 0.04 & 0.95 \\
\hline $2 \mu 2 e$ & 0.05 & 0.95 & 0.04 & 0.95 & 0.03 & 0.94 \\
\hline $2 e 2 \mu$ & -0.02 & 0.96 & -0.03 & 0.96 & -0.02 & 0.95 \\
\hline combined & 0.02 & 1.01 & 0.00 & 1.00 & 0.03 & 1.00 \\
\hline
\end{tabular}

Examining Table 23 reveals an interesting feature. All methods in (nearly) all categories on average fit a $\sigma_{H \rightarrow 4 \ell}$ value higher than the input to the toys. Curiously, the average fit value in the individual categories is (almost) always higher than the combined average fit value. How can this be? The cause is the Greedy Bump Bias 
[100], wherein the floating $m_{H}$ in the toy fits allows the signal shape to "chase after" upward statistical fluctuations of the background. With more statistics in the combined fit, $m_{H}$ is more restricted from chasing statistics, and the fit is "less greedy". The lower section of Table 23 demonstrates that this bias overall is $\mathcal{O}(2 \%)$ of the statistical uncertainty, so is negligible next to this and to the other systematic uncertainties in the fit result. The overall resolution from the constrained PER method has a slight advantage, approximately $1 \%$ improvement, over the Template method. This is also consistent with the improvement found from the effective dataset.

Table 24: Summary table of toy experiment fits of $\Gamma_{H}$. The median and mean $95 \%$ CL upper limits from toy experiments are recorded here, as found in Figure 107. Units in the table match the header.

\begin{tabular}{ccccc}
\hline & \multicolumn{2}{c}{ constrained PER } & \multicolumn{2}{c}{ unconstrained PER } \\
& median $95 \%$ UL & mean $95 \%$ UL & median 95\% UL & mean 95\% UL \\
\hline \multicolumn{4}{c}{$\Gamma_{H}^{\text {true }}=0.0041 \mathrm{GeV}$} \\
\hline$\mu$ & 2.51 & 3.74 & 3.00 & 4.61 \\
$4 e$ & 7.58 & 15.10 & 8.90 & 17.01 \\
$2 \mu 2 e$ & 5.81 & 11.64 & 6.68 & 13.23 \\
$2 e 2 \mu$ & 4.25 & 8.75 & 5.75 & 11.16 \\
combined & 1.56 & 1.94 & 1.92 & 2.39 \\
\hline
\end{tabular}

Comparing the median upper limits from toys in Table 24 with the upper limits in the effective dataset in Table 18 suggests that the effective dataset strategy overestimates the upper limits that can be set by about $10 \%$, depending on the category. This difference arises because the upper-limit setting on the width is extremely sensitive to statistical fluctuation, far more than the $m_{H}$ or $\sigma_{H \rightarrow 4 \ell}$ measurements, so the effective dataset is less able to replicate the median expected result that a true Asimov dataset would have done.

A few other interesting points arise from comparing the fit results with toys (Table 22, 23) to those with the effective dataset (Table 18). Both methods are intended 
to provide an estimate of the expected resolution of the fit on data, but under different assumptions. By focusing on the constrained PER toys fitting $m_{H}$ as an example, the results indicate that the $4 \mu$ uncertainty is well-estimated by the effective dataset approach (both methods suggest $0.52 \mathrm{GeV}$ ), as well as the $2 e 2 \mu$ (about $0.72 \mathrm{GeV}$ from both methods). Contrarily, in the $4 e$ category the effective dataset overestimates the resolution (toys show $1.08 \mathrm{GeV}$ versus $1.16 \mathrm{GeV}$ from the effective dataset), as it also does in the $2 \mu 2 e$ category $(0.93 \mathrm{GeV}$ versus $0.97 \mathrm{GeV})$. The combined fit uncertainty agrees from both methods, indicating $0.36 \mathrm{GeV}$ expected resolution on $m_{H}$ (without systematics). The same comparison is more difficult for $\sigma_{H \rightarrow 4 \ell}$, where the uncertainties from the effective dataset are significantly asymmetric for the individual categories. For the combined fit, the effective dataset and toys are in agreement on the expected resolution on $\sigma_{H \rightarrow 4 \ell}$.

Complementary to this feature are the widths of the pull distributions, which are equal to 1.00 when the uncertainties are perfectly estimated. Deviations from this value would indicate uncertainties are not being accurately estimated, and so the asymptotic approximation, used in Section 9.1 to define rejection regions of the test statistics may not be satisfied. However, for all three methods, the width of the combined fit pulls of $m_{H}$ and $\sigma_{H \rightarrow 4 \ell}$ are within 0.98-1.01, indicating the uncertainties are being accurately estimated even at low statistics. In the individual categories, the deviations are slightly larger, but all pull widths are within 0.94-1.05, suggesting even the individual categories have mostly sufficient statistics to satisfy the asymptotic approximation and set confidence regions as described.

\subsubsection{Toy-by-toy comparison}

Comparing the properties of an ensemble of toy experiments yields important, yet not complete, information. Some properties become more illustrative when they can be compared on a toy-by-toy basis. This is more akin to the situation in data, where 
there is only a single dataset, and the results between the methods will be compared only on that dataset. Fortunately, the process by which toys are created allows them to be uniquely "barcoded", so that the result of fitting one particular dataset with the constrained PER method can be compared with the fit results when fitting the Template method on the exact same set of events (but without using the per-event response information). This barcoding strategy does not allow the unconstrained PER method to be compared on a toy-by-toy basis, because the application of the ZMC will move events in or out of the mass window, and so the population of MC simulated events from which the toys are drawn is not equivalent between the unconstrained and constrained toy generation. This is not a great loss, as the preceding checks have demonstrated the unconstrained PER is a valid, but lower-resolution method. This section will therefore make toy-by-toy comparisons only of the constrained PER and Template methods.

Figure 108 contains the two-dimensional correlations of $\hat{m}_{H}$ and $\hat{\sigma}_{H \rightarrow 4 \ell}$, respectively, from the constrained PER method and the Template method. These plots indicate that the fit results are highly correlated. The correlation coefficient $\rho$ is printed on each plot, and is found to be ordered by the expected number of events per-category (higher statistics yields higher correlation, not surprisingly). The same plots separated by category can be found in Appendix C.3.

While the correlations are visually interesting, other distributions can be more useful in quantifying the models. Two interesting things to explore are how much the models agree with each other, and how much the models agree with the truth. The former, which contains all the same information as Figure 108 but in one dimension, is shown in Figure 109. This quantifies how much the two models are expected to agree (or disagree) when fit on the data. The latter can be understood, in a somewhat less intuitive fashion, by comparing the absolute difference between the best fit values and the truth value from the input to the toys $\left(m_{H}=125 \mathrm{GeV}\right.$, and $\left.\sigma_{H \rightarrow 4 \ell}=6.90 \mathrm{fb}\right)$. 


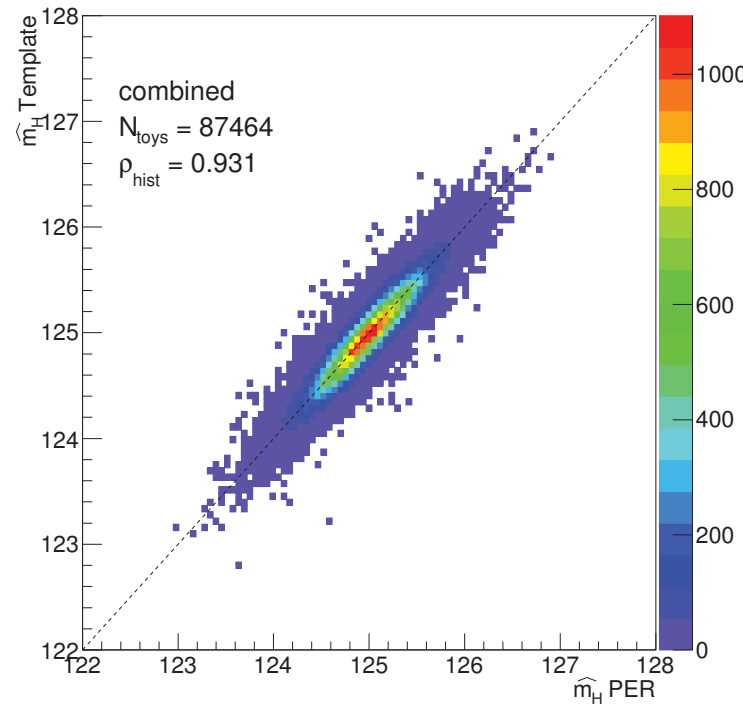

(a) $\hat{m}_{H}$

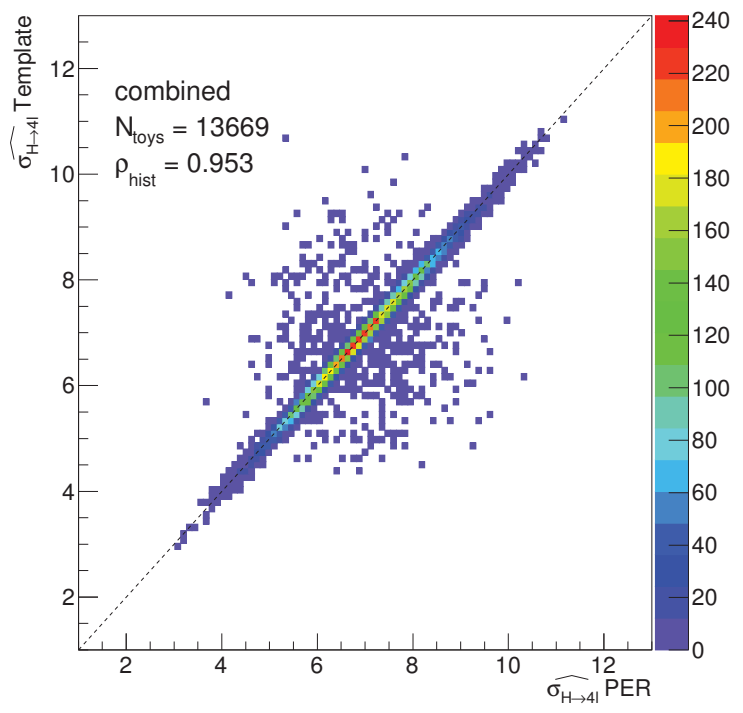

(b) $\hat{\sigma}_{H \rightarrow 4 \ell}$

Figure 108: Correlation plots of $\hat{m}_{H}$ and $\hat{\sigma}_{H \rightarrow 4 \ell}$, respectively, between the constrained PER model, and the Template model, from pseudo-experiments. The diagonal line indicates where both methods agree exactly.

This comparison is shown in Figure 110. Entries to the left of zero are cases where the constrained PER model fit a more accurate value (closer to the truth) than the Template model, and entries to the right of zero are cases where the Template model returned a more accurate result. Consequently, the mean value shows how much closer to the true value the constrained PER method is, on average. The area under the distribution from negative infinity to zero gives the fraction of toy datasets in which the constrained PER model was more accurate. The most impressive difference comes from $m_{H}$ measurement in the $4 e$ category, where the constrained PER method is on average $72 \mathrm{MeV}$ closer to the true $m_{H}$, and more accurate in $54.5 \%$ of datasets. The differences in the other categories are more subdued, so that the combined result is that the PER method fits more accurately the true $m_{H}\left(\sigma_{H \rightarrow 4 \ell}\right)$ value in $51.0 \%$ $(50.2 \%)$ of datasets, being on average $4 \mathrm{MeV}(<1 \mathrm{ab})$ closer to the true value. These quantities are summarized in Table 25, and the corresponding plots for each category 


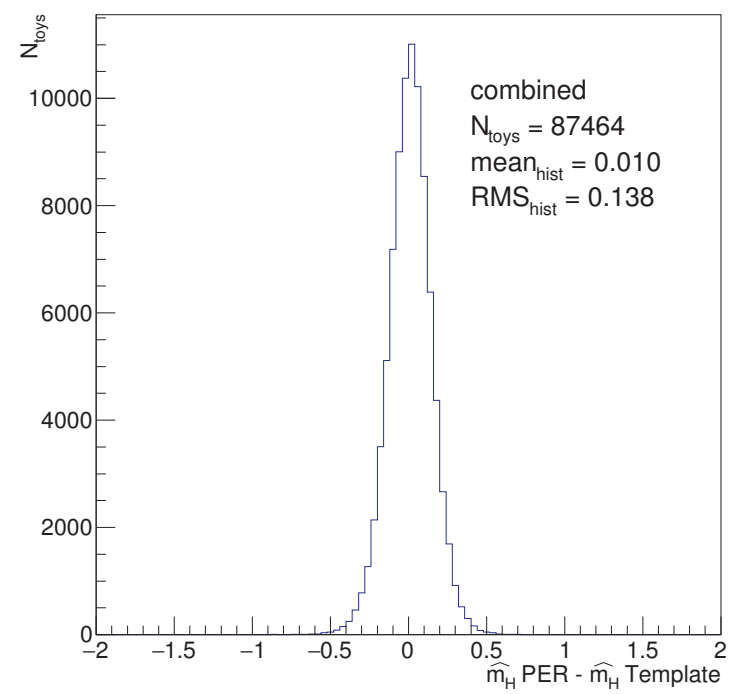

(a) $m_{H}$

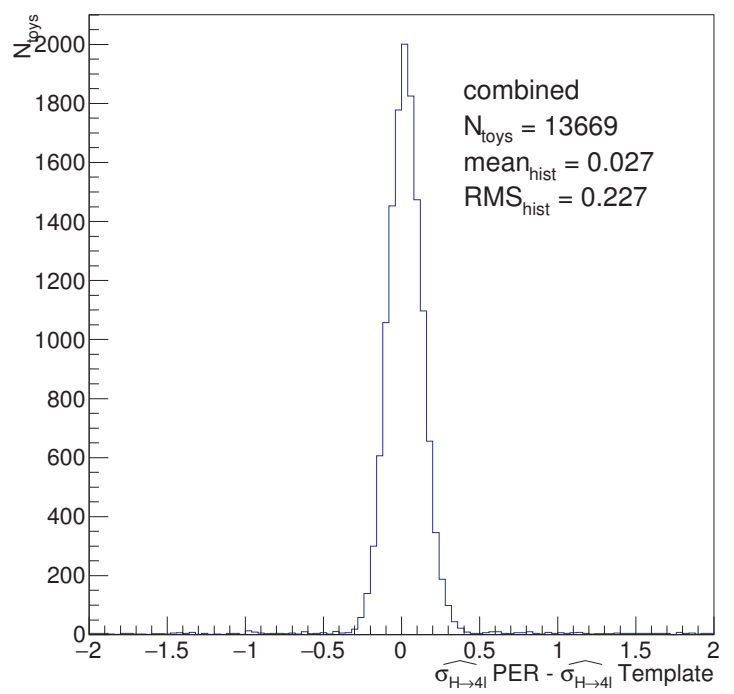

(b) $\sigma_{H \rightarrow 4 \ell}$

Figure 109: Difference in best fit values of $m_{H}$ and $\sigma_{H \rightarrow 4 \ell}$, respectively, between the constrained PER model, and the Template model, from pseudo-experiments. The standard deviation, recorded on the plots as $R M S_{\text {hist }}$, gives an indication of how much the two models may agree or disagree when fit on the data.

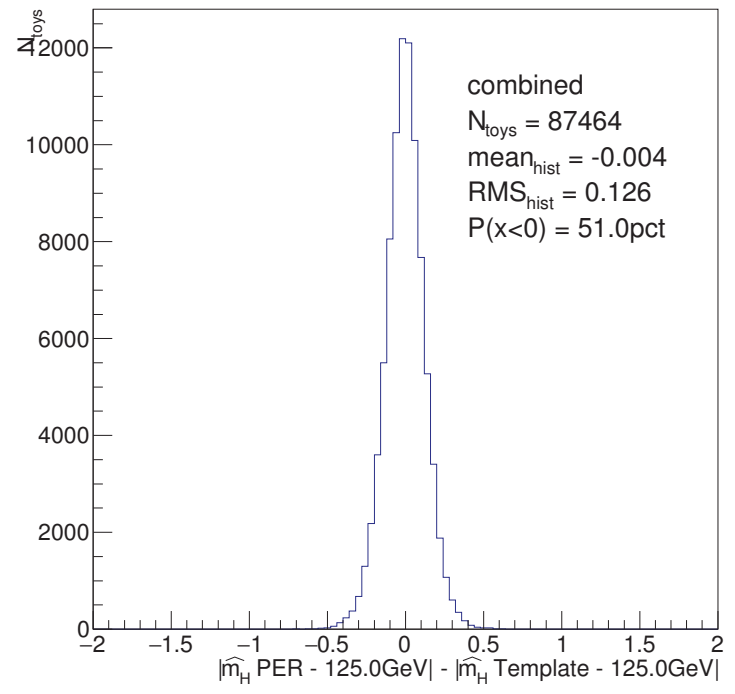

(a) $m_{H}$

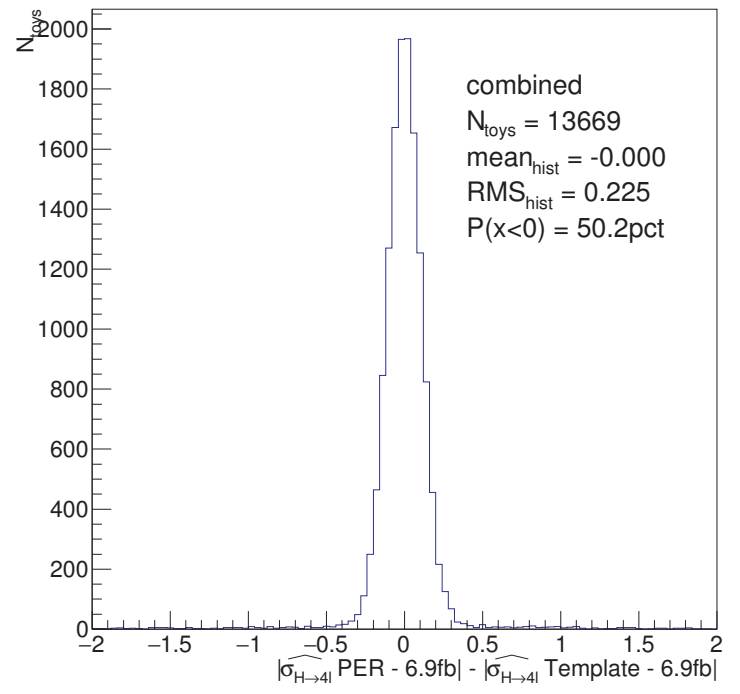

(b) $\sigma_{H \rightarrow 4 \ell}$

Figure 110: Comparison of distance from the best fit value to the true value, of $m_{H}$ and $\sigma_{H \rightarrow 4 \ell}$, one a toy-by-toy basis. In toys to the left of zero, the constrained PER model produces a more accurate fit result than the Template model. In toys to the right of zero, the Template method was more accurate. 
Table 25: Expected improvement in fit results in constrained PER method compared to Template method. The second column shows the RMS deviation between the best fit values of $m_{H}\left(\sigma_{H \rightarrow 4 \ell}\right)$ from the two methods. The third column shows the average improvement; that is, on average, how much closer to the true value of $m_{H}\left(\sigma_{H \rightarrow 4 \ell}\right)$ is the constrained PER method compared to the Template method. The fourth column shows in what percentage of datasets the constrained PER method is expected to produce a fit result closer to the true value of $m_{H}\left(\sigma_{H \rightarrow 4 \ell}\right)$ than the Template method.

\begin{tabular}{cccc}
\hline category & RMS deviation & Average improvement & More accurate results \\
\hline \multicolumn{4}{c}{$m_{H}$} \\
$4 \mu$ & $182 \mathrm{MeV}$ & $1 \mathrm{MeV}$ & $50.0 \%$ \\
$4 e$ & $530 \mathrm{MeV}$ & $72 \mathrm{MeV}$ & $54.5 \%$ \\
$2 \mu 2 e$ & $381 \mathrm{MeV}$ & $54 \mathrm{MeV}$ & $55.1 \%$ \\
$2 e 2 \mu$ & $373 \mathrm{MeV}$ & $4 \mathrm{MeV}$ & $50.0 \%$ \\
combined & $138 \mathrm{MeV}$ & $4 \mathrm{MeV}$ & $51.0 \%$ \\
\hline \multicolumn{4}{c}{$\sigma_{H \rightarrow 4 \ell}$} \\
\hline $4 \mu$ & $222 \mathrm{ab}$ & $11 \mathrm{ab}$ & $51.2 \%$ \\
$4 e$ & $350 \mathrm{ab}$ & $-11 \mathrm{ab}$ & $49.2 \%$ \\
$2 \mu 2 e$ & $290 \mathrm{ab}$ & $4 \mathrm{ab}$ & $51.4 \%$ \\
$2 e 2 \mu$ & $315 \mathrm{ab}$ & $5 \mathrm{ab}$ & $50.9 \%$ \\
combined & $227 \mathrm{ab}$ & $<1 \mathrm{ab}$ & $50.2 \%$ \\
\hline
\end{tabular}

are found in Appendix C.3.

\subsection{Validation using the $Z \rightarrow 4 \ell$ resonance}

As a final check before looking at the data in the signal region, the PER methods can be tested on another resonance with well-known properties: the $Z \rightarrow 4 \ell$ resonance described in Section 6.1. Measuring these known quantities in data gives an additional handle on the performance of the PER technique. Section 9.5.1 describes some of the additional considerations necessary to describe the lineshape of the $Z$, and Section 9.5.2 reports measurements of the resonance properties. As this section is 
intended as a validation of the PER method, rather than a standalone result, the tests in this section are all reported with statistical uncertainties only, while no systematics are included. The result could otherwise stand on its own as a valid measurement, but because the statistics are so limited compared to a $Z \rightarrow \ell \ell$ measurement, the resolution is several orders of magnitude worse than existing measurements. Another contributing factor is that the LHC is a $p-p$ collider, and so the collisions are far messier than at LEP, the $e^{+}-e^{-}$collider where the best existing measurements of the $Z$ boson properties were performed.

The category definitions used for the $Z \rightarrow 4 \ell$ analysis are the same as in the primary analysis, except a mass window of $78 \mathrm{GeV} \leq m_{4 \ell} \leq 104 \mathrm{GeV}$ is used.

\subsubsection{Per-event response and the $Z \rightarrow 4 \ell$ lineshape}

The lepton response model developed to describe the $m_{4 \ell}$ response of the Higgs resonance, described in Section 7.3.2, can be used for the $Z$ resonance. One caveat to this arises because $m_{Z}$ is significantly lower than $m_{H}$. The lower resonance mass means that a lepton with a given energy has a different response distribution when originating from a $Z$, than if it had come from a Higgs ${ }^{7}$. For this reason a separate set of tuning parameters, originally described in Section 7.3.4, is required when fitting the $Z \rightarrow 4 \ell$ peak.

An additional consideration is the interpretation of $\epsilon\left(m_{4 \ell}^{\text {true }}\right)$, first defined in Section 7.3.1. This function is necessary to properly describe the true distribution of events before reconstruction,

$$
\mathrm{BW}_{\epsilon}\left(\mathrm{m}_{4 \ell}^{\text {true }} ; \mathrm{m}_{\mathrm{Z}}, \Gamma_{\mathrm{Z}}\right)=\epsilon\left(\mathrm{m}_{4 \ell}^{\text {true }}\right) \mathrm{BW}\left(\mathrm{m}_{4 \ell}^{\text {true }} ; \mathrm{m}_{\mathrm{Z}}, \Gamma_{\mathrm{Z}}\right)
$$

\footnotetext{
${ }^{7}$ If this seems bizarre, consider a specific example: a lepton reconstructed with $E_{i}^{\text {reco }}=20 \mathrm{GeV}$. If asked the estimate the PDF of its true energy $P\left(E_{i}^{\text {true }}\right)$, would the answer be the same with the knowledge this lepton came from a $Z$, as it would knowing that it came from a $H$ ? Surely it would not be the case. While this effect is present within the $m_{H}$ fit range itself, it is considered to be absorbed by the range of samples used in building the lepton model in Section 7.3.2.
} 
The efficiency function $\epsilon\left(m_{4 \ell}^{\text {true }}\right)$ was initially defined to account for non-uniform detector efficiency as a function of $m_{4 \ell}^{\text {true }}$. Now it must be re-interpreted to absorb two other effects: Many of the selected Z1 di-lepton pairs are produced off-shell creating a significant low-side tail, and, even more important, on the high side there is a continuous transition between $q \bar{q} \rightarrow Z \rightarrow 4 \ell$ into $q \bar{q} \rightarrow Z Z^{*} \rightarrow 4 \ell$ (described in Section 6.1), creating a high-side tail. The function $\epsilon\left(m_{4 \ell}^{\text {true }}\right)$ as it is used in this section incorporates both these effects. It is described by a third-order polynomial, fit on the ratio of the $m_{4 \ell}^{\text {true }}$ distribution from $\mathrm{MC}$ simulation and a pure $B W\left(m_{4 \ell}^{\text {true }} ; m_{Z}, \Gamma_{Z}\right)$ distribution. These fits are shown in Figure 111. Note that the efficiency functions are fit on a wider range than the category definitions to encourage smooth behaviour. The discrepancies at the extreme ends are therefore unimportant for the purposes of this cross-check.

Another point of note is that the likelihood of (107) is more simple for this measurement because the vast majority of events are from the $Z \rightarrow 4 \ell$ signal itself; the measurement is nearly background-free. The only background contribution is from Z+Jets, which is nearly two orders of magnitude smaller than the signal inside the mass range used. One could have also considered the right-hand $q \bar{q} \rightarrow Z Z^{(*)} \rightarrow 4 \ell$ tail as a separate background, but this is more difficult to disentangle in MC simulation, with no particular benefit. The signal normalization in the likelihood is parameterized with respect to the SM as,

$$
N_{Z \rightarrow 4 \ell, \mathrm{c}}^{\mathrm{exp}}=\mu_{Z} N_{Z \rightarrow 4 \ell, \mathrm{c}}^{\mathrm{SM}}
$$

where $\mu_{Z}$ is a free parameter called the signal strength, equal to 1.00 in the SM.

Also, because $\Gamma_{Z}$ is orders of magnitude larger than $\Gamma_{H}$, ATLAS has sufficient experimental resolution to make a two-sided width measurement of the $\mathrm{Z}$, and so the $t_{\mu}$ test statistic is used to perform the measurement in the same way as for $m_{Z}$ and $\mu_{Z}$. 


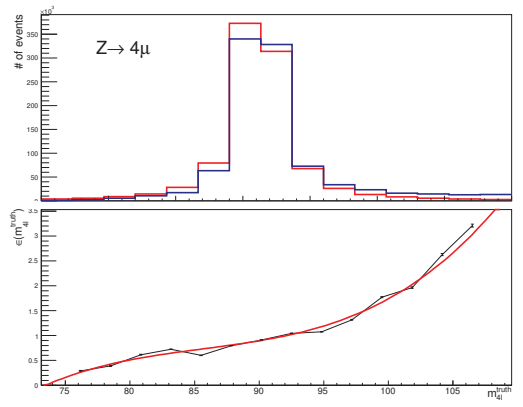

(a) $4 \mu$

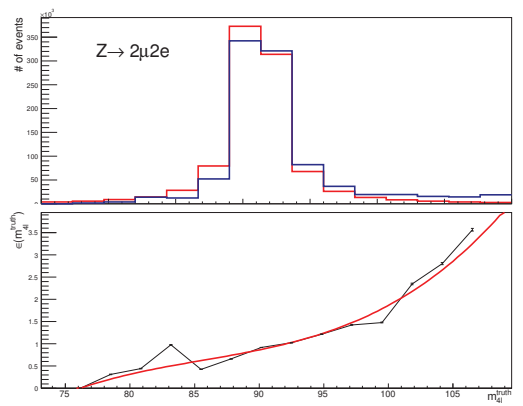

(c) $2 \mu 2 e$

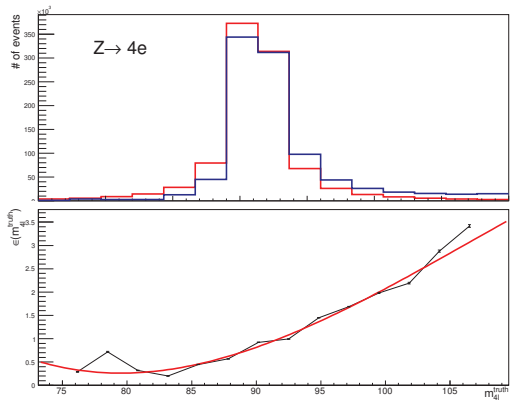

(b) $4 e$

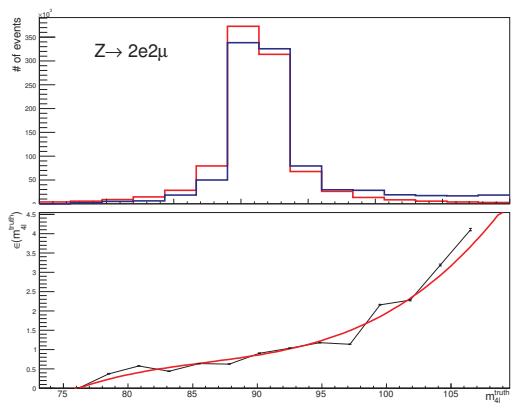

(d) $2 e 2 \mu$

Figure 111: $Z \rightarrow 4 \ell$ efficiency function, $\epsilon\left(m_{4 \ell}^{\text {true }}\right)$, modelling the difference between the MC simulated distribution of $m_{4 \ell}^{\text {true }}$ and a pure BW distribution, in each category. The upper plot shows the MC simulated distribution (blue line), and pure BW distribution (red line). The lower plots shows their ratio (black points and line), and the 3rd order polynomial used for $\epsilon\left(m_{4 \ell}^{\text {true }}\right)$ (red curve).

The final important difference between fitting the $Z \rightarrow 4 \ell$ peak, as compared to the $H \rightarrow 4 \ell$ peak is that the unconstrained PER and the constrained PER are expected to give nearly identical results. The reason is that the ZMC is designed to constrain the Z1 di-lepton pair to have an invariant mass near $m_{Z}$. But this is of course not possible if the four-lepton invariant mass is already near $m_{Z}$ itself. So in practice, the $m_{4 \ell}$ values of events in the mass window used here shift upwards by a small amount $(\approx 0.1 \mathrm{GeV})$, but the resolution of the $Z$ peak is not improved, so neither is the expected resolution from the constrained PER as compared to the unconstrained (in data, subject to statistical fluctuations, they may differ). For this reason, only the constrained PER is used in what follows, while the unconstrained results (expected and observed), are kept in Appendix C.4. 
For this study, the transformation parameters of the constrained PER model were derived on simulated $q \bar{q} \rightarrow Z \rightarrow 4 \ell$ and $q \bar{q} \rightarrow Z Z^{(*)} \rightarrow 4 \ell$ events. These fits are shown in Figure 112, demonstrating that the model can work very effectively at describing the $Z \rightarrow 4 \ell$ resonance.

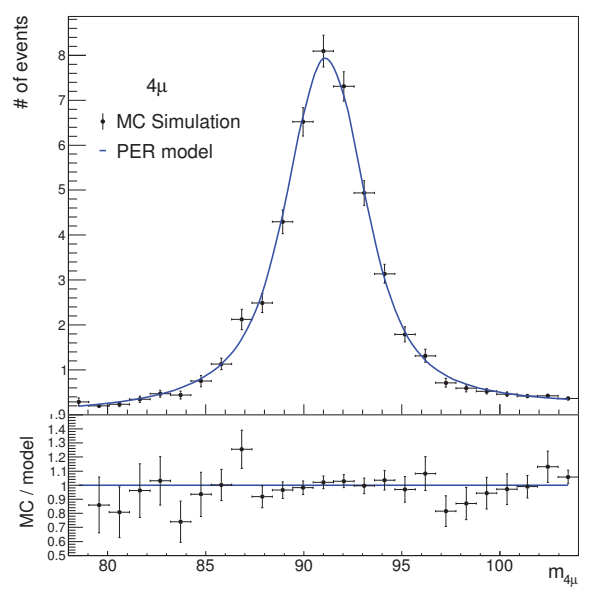

(a) $4 \mu$

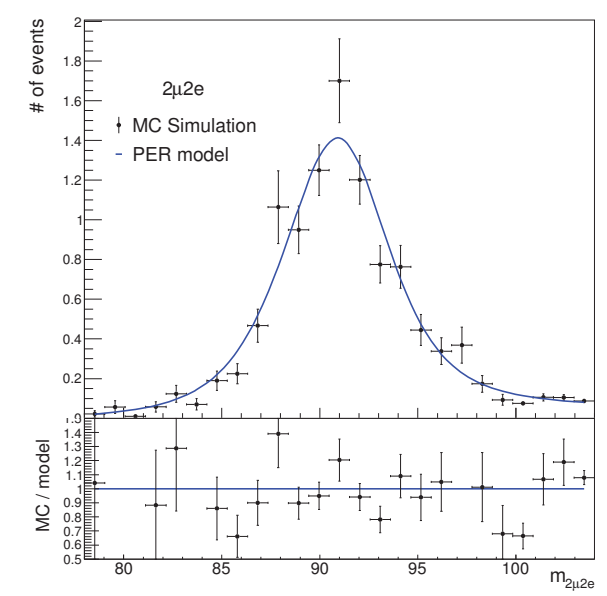

(c) $2 \mu 2 e$

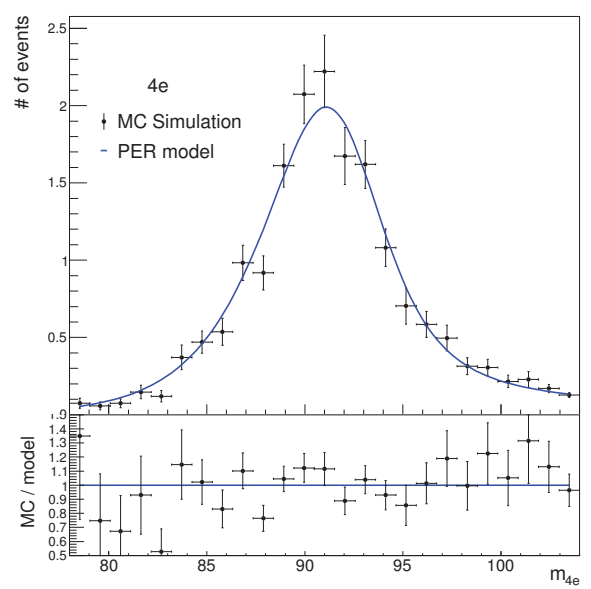

(b) $4 e$

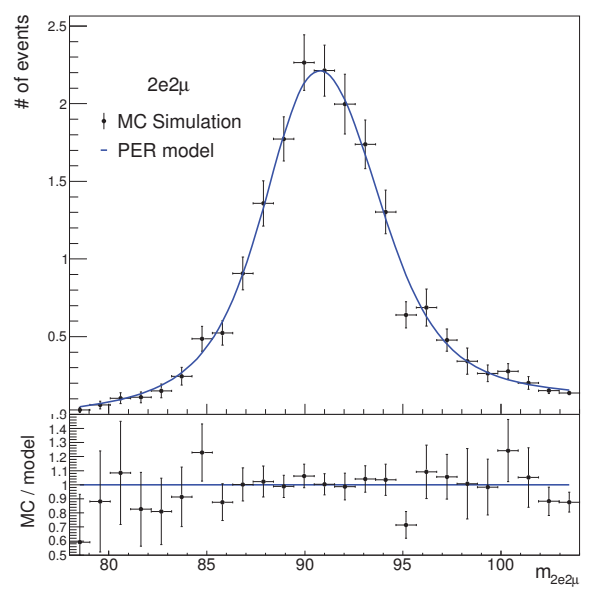

(d) $2 e 2 \mu$

Figure 112: Agreement between MC simulation and constrained PER model on $m_{4 \ell}^{\text {cons }}$ distribution of the $Z \rightarrow 4 \ell$ resonance, in each of the analysis categories. The resonance parameters $m_{Z}, \Gamma_{Z}$ are set at their best measured values from [16]. 


\subsubsection{Property measurement of the $Z \rightarrow 4 \ell$ resonance}

To validate the method, maximum likelihood fits were performed on an effective dataset containing the same events shown in Figure 112, with a small additional component from $Z+$ Jets. The $Z \rightarrow 4 \ell$ component of the dataset is weighted to the SM cross section, while the $\mathrm{Z}+$ Jets component is weighted to its data-driven estimate. One drawback to this dataset is that it has limited statistics compared to the $H \rightarrow 4 \ell$ simulation used in Section 9.2, as far fewer events were simulated for this resonance.

The configurations of parameters were handled in an analogous way to the $H \rightarrow 4 \ell$ resonance: for fits on $m_{Z}, \mu_{Z}$ was left to float; for fits on $\mu_{Z}, m_{Z}$ was left to float; for fits on $\Gamma_{Z}$, both $\mu_{Z}$ and $m_{Z}$ were left to float. As no systematics were applied for the $Z \rightarrow 4 \ell$ study, there are no nuisance parameters included in the fit. Likelihood scans of $m_{Z}, \mu_{Z}$, and $\Gamma_{Z}$ on this dataset are found in Figure 113.

Scans of $m_{Z}$ exhibit residual biases smaller than $5 \mathrm{MeV}$. This value is smaller than that of the $H \rightarrow 4 \ell$ analysis, both because this measurement is nearly backgroundfree, and because the tuning parameters are derived on the very same sample as used for this test, so the closure arises more automatically. For the same reason, $\mu_{Z}$ is guaranteed to show no residual bias. The width, $\Gamma_{Z}$, is the most difficult of the three, as it is highly sensitive to the dataset statistics. The limited $Z \rightarrow 4 \ell$ $\mathrm{MC}$ statistics cause some fluctuation in this case, as large as $130 \mathrm{MeV}$ in the $2 \mu 2 e$ category, which has the fewest simulated events. The combined measurement of $\Gamma_{Z}$ on the simulated data converges within $15 \mathrm{MeV}$ to the reference value, approximately $2.5 \%$ the statistical resolution of the measurement.

The checks on the MC simulation demonstrate the PER model is an effective fit strategy, and is prepared to be used on $Z \rightarrow 4 \ell$ data. Table 26 summarizes the numbers of events observed in each category, compared to the SM expectation. Although no systematic uncertainties were evaluated or used in the $Z \rightarrow 4 \ell$ measurements, the 


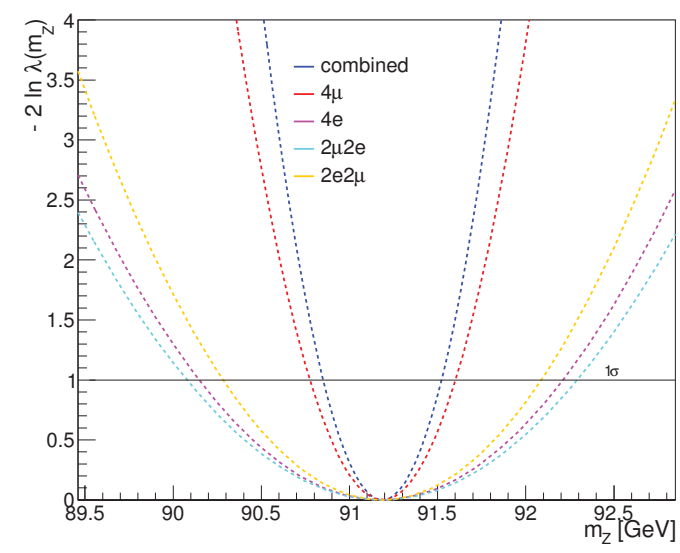

(a) $m_{Z}$

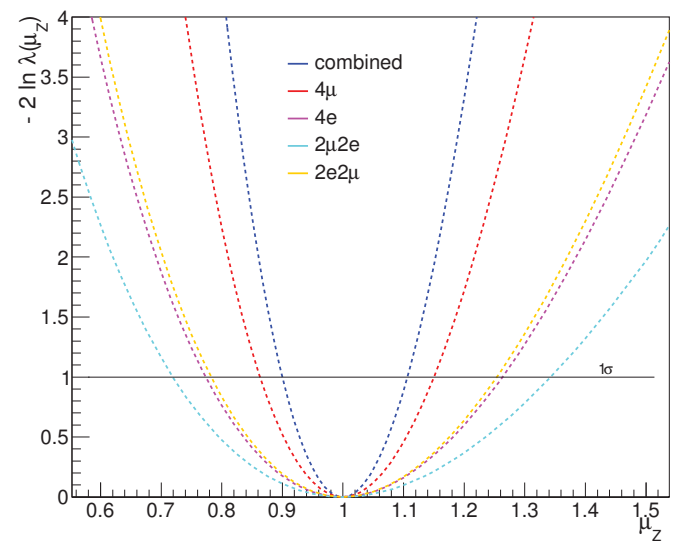

(b) $\mu_{Z}$

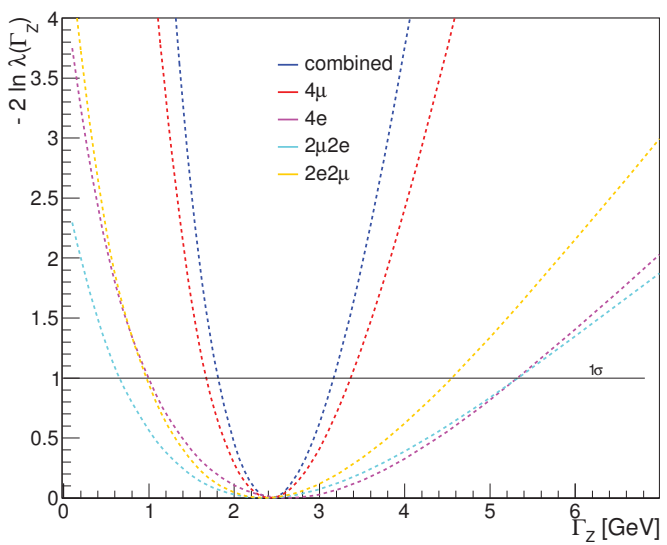

(c) $\Gamma_{Z}$

Figure 113: Likelihood scans of $m_{Z}, \mu_{Z}, \Gamma_{Z}$ on the effective dataset using the constrained PER model. Dashed lines indicate the scans include only statistical uncertainty; no systematics are included.

table includes a conservative $10 \%$ uncertainty on the expected number of $Z \rightarrow 4 \ell$ events. This is roughly consistent with the total uncertainty on the $q \bar{q} \rightarrow Z Z^{(*)}$ background yield in the $H \rightarrow 4 \ell$ analysis, though in the case presented here the uncertainty is not decomposed into all its sources. The uncertainty on the (small) Z+Jets contribution comes from the data-driven estimate. The likelihood scans of $m_{Z}, \mu_{Z}$, and $\Gamma_{Z}$ on the data are found in Figure 114.

The distribution of data events is shown in Figure 115 together with the constrained PER model drawn at the best fit values of $m_{Z}, \mu_{Z}$, and with $\Gamma_{Z}$ set to its 
Table 26: Expected and observed yields in the mass window $78 \mathrm{GeV} \leq m_{4 \ell}^{\text {cons }} \leq 104 \mathrm{GeV}$ for the $Z \rightarrow 4 \ell$ validation study.

\begin{tabular}{cccc}
\hline category & $Z \rightarrow 4 \ell$ & Z+Jets & observed \\
\hline $4 \mu$ & $48.8 \pm 4.8$ & $0.20 \pm 0.05$ & 45 \\
$4 e$ & $17.0 \pm 1.7$ & $0.28 \pm 0.07$ & 18 \\
$2 \mu 2 e$ & $10.6 \pm 1.0$ & $0.29 \pm 0.07$ & 8 \\
$2 e 2 \mu$ & $18.2 \pm 1.9$ & $0.20 \pm 0.05$ & 23 \\
combined & $94.4 \pm 9.4$ & $0.97 \pm 0.25$ & 94 \\
\hline
\end{tabular}

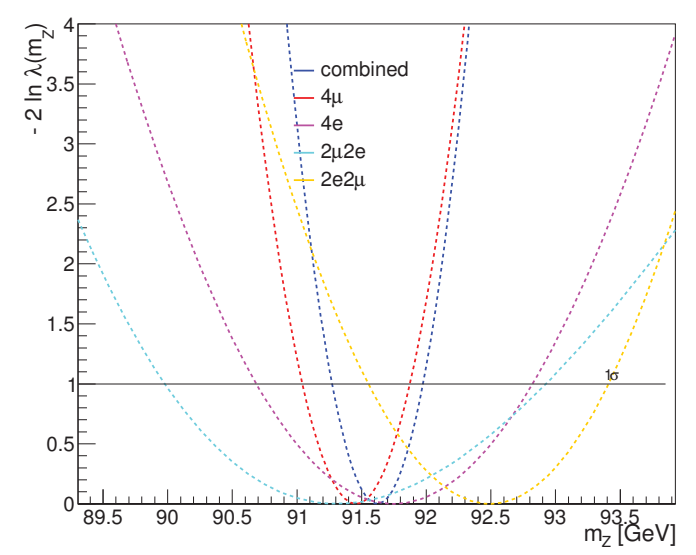

(a) $m_{Z}$

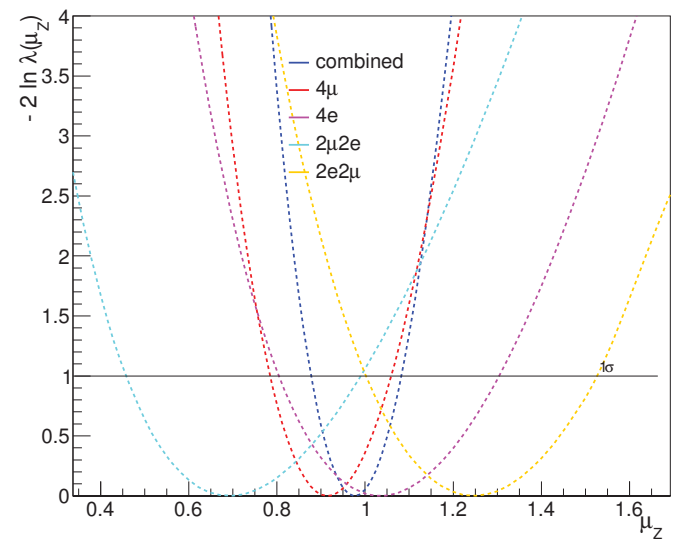

(b) $\mu_{Z}$

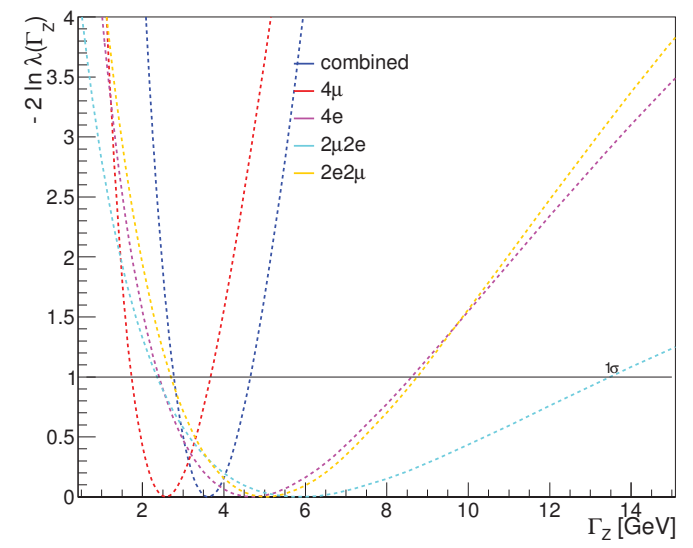

(c) $\Gamma_{Z}$

Figure 114: Likelihood scans of $m_{Z}, \mu_{Z}, \Gamma_{Z}$ on the data using the constrained PER model. Dashed lines indicate the scans include only statistical uncertainty; no systematics are included.

SM value. 


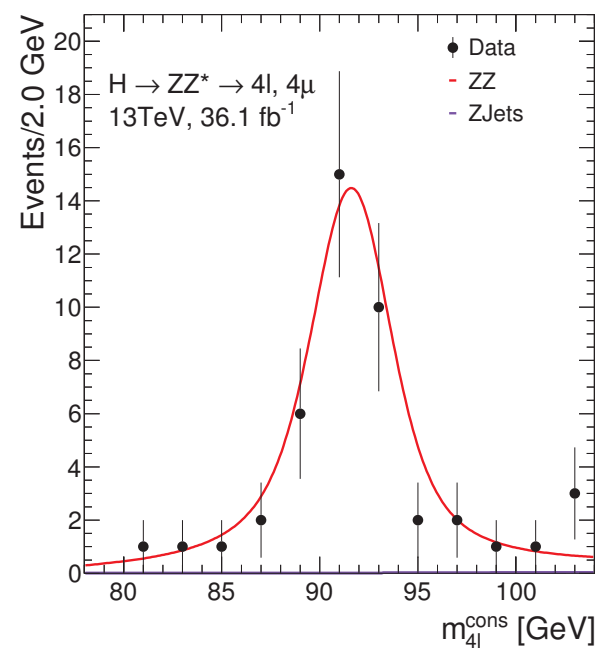

(a) $4 \mu$

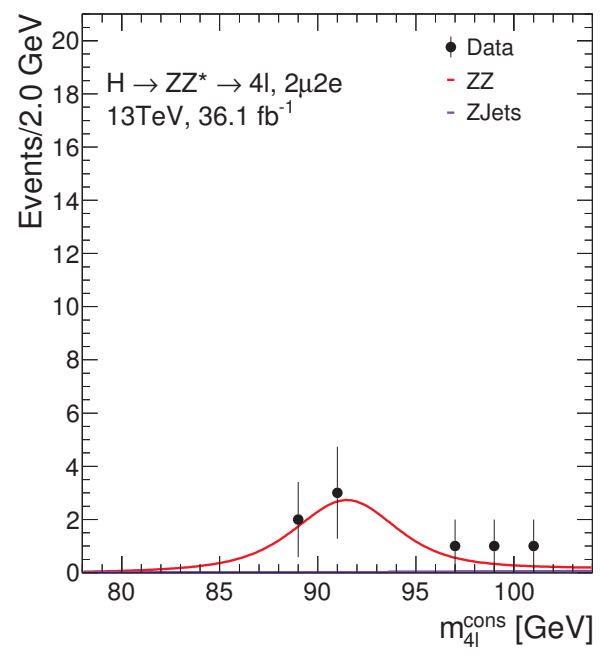

(c) $2 \mu 2 e$

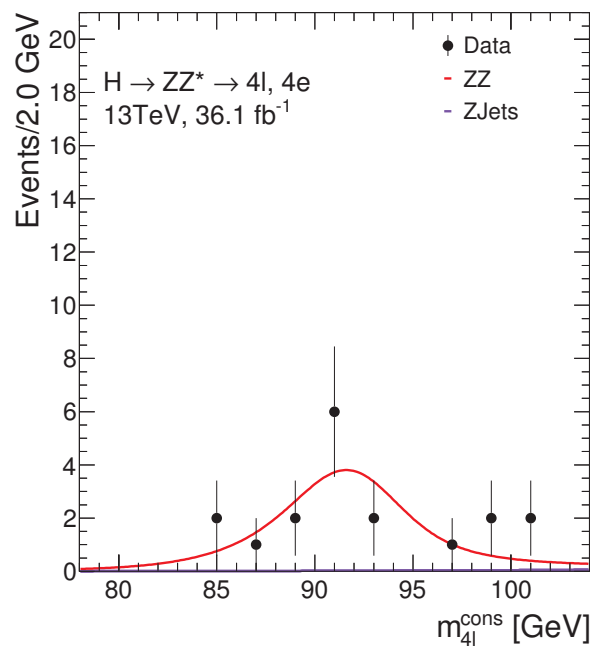

(b) $4 e$

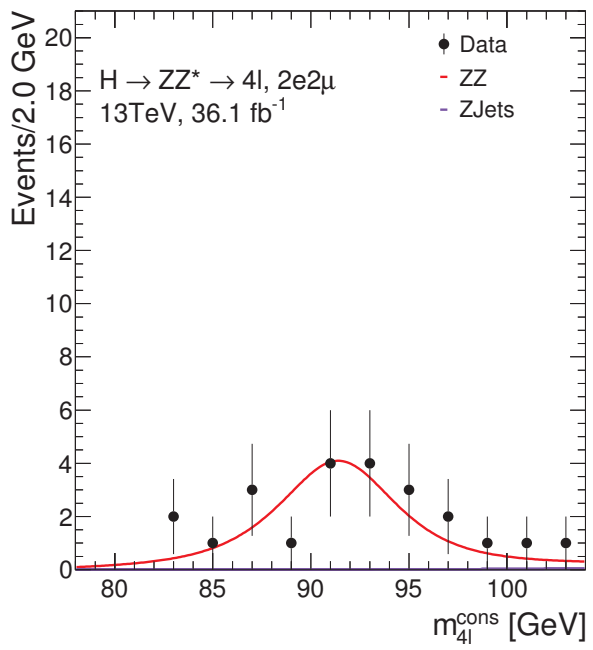

(d) $2 e 2 \mu$

Figure 115: Plot of the $m_{4 \ell}^{\text {cons }}$ distribution of the $Z \rightarrow 4 \ell$ resonance in data, in each of the analysis categories. The constrained PER model is plotted after the fit, with parameters $m_{Z}, \mu_{Z}$ set at their best-fit values.

The results of all $Z \rightarrow 4 \ell$ likelihood scans are summarized in Table 27. All measurements are within $2 \sigma$ of the reference values, while most are within $1 \sigma$. In particular, the measured $m_{Z}$ shows a $+1.2 \sigma$ deviation, $\mu_{Z}$ shows a $-0.2 \sigma$ deviation, and $\Gamma_{Z}$ shows a $+1.3 \sigma$ deviation from their respective reference values. Recall that the quoted uncertainties are conservative as they include only statistical uncertainty, and so these quoted deviations would be reduced in a more complete analysis. The measurements of the $Z \rightarrow 4 \ell$ resonance in this section serve to verify the readiness of 
the constrained PER model for measuring the $H \rightarrow 4 \ell$ resonance in data.

Table 27: Summary table of results from constrained PER $Z \rightarrow 4 \ell$ likelihood scans of $m_{Z}, \mu_{Z}$, and $\Gamma_{Z}$, in MC simulated data, and in the real data. The "SM value" of $m_{Z}, \Gamma_{Z}$ indicates the current best-measured value, used as input in other SM calculations, not a fundamental prediction of the SM. Units in the table match those of the reference values.

\begin{tabular}{ccc}
\hline Category & MC simulated dataset & observed data \\
\hline$m_{Z}$ & SM value $m_{Z}=91.1876 \mathrm{GeV}$ \\
\hline $4 \mu$ & $91.19_{-0.41}^{+0.41}$ & $91.46_{-0.41}^{+0.42}$ \\
$4 e$ & $91.18_{-1.03}^{+1.33}$ & $91.75_{-1.06}^{+1.08}$ \\
$2 \mu 2 e$ & $91.18_{-1.11}^{+1.11}$ & $91.31_{-1.33}^{+1.62}$ \\
$2 e 2 \mu$ & $91.18_{-0.90}^{+0.90}$ & $92.49_{-0.94}^{+0.91}$ \\
combined & $91.19_{-0.34}^{+0.34}$ & $91.62_{-0.35}^{+0.35}$ \\
\hline$\mu_{Z}$ & \multicolumn{1}{c}{ SM value $\mu_{Z}=1.00$} \\
\hline $4 \mu$ & $1.00_{-0.14}^{+0.15}$ & $0.92_{-0.13}^{+0.14}$ \\
$4 e$ & $1.00_{-0.22}^{+0.26}$ & $1.03_{-0.23}^{+0.27}$ \\
$2 \mu 2 e$ & $1.00_{-0.28}^{+0.34}$ & $0.69_{-0.23}^{+0.30}$ \\
$2 e 2 \mu$ & $1.00_{-0.22}^{+0.25}$ & $1.25_{-0.24}^{+0.28}$ \\
combined & $1.00_{-0.10}^{+0.11}$ & $0.98_{-0.10}^{+0.11}$ \\
\hline$\Gamma_{Z}$ & $\mathrm{SM}$ value $\Gamma_{Z}=2.4952 \mathrm{GeV}$ \\
\hline $4 \mu$ & $2.42_{-0.75}^{+0.95}$ & $2.57_{-0.84}^{+1.10}$ \\
$4 e$ & $2.63_{-1.63}^{+2.70}$ & $4.69_{-2.28}^{+3.92}$ \\
$2 \mu 2 e$ & $2.35_{-1.70}^{+2.97}$ & $5.83_{-3.47}^{+7.65}$ \\
$2 e 2 \mu$ & $2.35_{-1.39}^{+2.20}$ & $5.05_{-2.35}^{+3.68}$ \\
combined & $2.43_{-0.62}^{+0.74}$ & $3.61_{-0.84}^{+1.04}$ \\
\hline
\end{tabular}




\subsection{Results with data}

The preceding sections are all intended to engender confidence in the measurement strategy. Section 9.2 demonstrated that the constrained PER model introduces no apparent bias within the available MC simulated statistics, and provided expected resolutions, assuming the SM, for each measurement to be performed. Section 9.3 broke down all sources of systematic uncertainty entering the analysis, ranking them according to their importance for each measurement. Section 9.4 tested the measurement strategy with realistic statistics like those expected in the data, demonstrating that the method is robust in the existing regime of low statistics. As a final validation step, Section 9.5 showed a measurement of $m_{Z}$, and $\Gamma_{Z}$ using the constrained PER model on the $Z \rightarrow 4 \ell$ resonance in $\mathrm{MC}$ simulation and data, demonstrating the high degree of flexibility of the method. In each case where appropriate, the Template method and unconstrained PER method were validated alongside the constrained PER method both as cross-checks of the final measurement, and as benchmarks of performance. With this confidence in hand, we are prepared to do the measurement on the data.

The expected and observed yields in each category are recorded in Table 28. The expected signal yields are given for a $m_{H}=125 \mathrm{GeV}$ SM Higgs boson.

The distributions in data of $m_{4 \ell}^{\text {cons }}$ are shown in Figure 116, overlaid on top of the expected distributions from the difference sources of signal and background. For the signal, the constrained PER PDF is drawn with $m_{H}$ and $\sigma_{H \rightarrow 4 \ell}$ is set to their best fit values (to be presented shortly), and $\Gamma_{H}$ to its SM value. The corresponding distributions of $m_{4 \ell}^{\mathrm{fsr}}$ can be found in Appendix C.1. These plots deliberately bin the model to allow comparison bin-by-bin; the unbinned post-fit signal+background PDFs plotted on top of the same data can be found in Appendix C.2, for both the constrained and unconstrained PER model. 
Table 28: Table of expected and observed event yields. For the $H \rightarrow 4 \ell$ signal yield, the values are given for a $m_{H}=125 \mathrm{GeV}$ Higgs.

\begin{tabular}{|c|c|c|c|c|c|}
\hline category & qqZZ & $\operatorname{ggZZ}$ & VVV & Z+Jets & $H \rightarrow 4 \ell$ \\
\hline $4 \mu$ & $15.18 \pm 1.49$ & $0.65 \pm 0.39$ & $0.18 \pm 0.01$ & $1.56 \pm 0.40$ & $20.86 \pm 1.35$ \\
\hline $4 e$ & $6.39 \pm 0.74$ & $0.29 \pm 0.18$ & $0.13 \pm 0.01$ & $1.97 \pm 0.47$ & $11.12 \pm 0.92$ \\
\hline $2 \mu 2 e$ & $6.83 \pm 0.77$ & $0.20 \pm 0.12$ & $0.11 \pm 0.01$ & $2.05 \pm 0.47$ & $11.31 \pm 0.87$ \\
\hline $2 e 2 \mu$ & $10.59 \pm 1.02$ & $0.31 \pm 0.19$ & $0.18 \pm 0.01$ & $1.56 \pm 0.40$ & $14.79 \pm 0.89$ \\
\hline \multirow[t]{2}{*}{ combined } & $39.00 \pm 3.84$ & $1.45 \pm 0.88$ & $0.60 \pm 0.05$ & $7.14 \pm 1.23$ & $58.08 \pm 3.73$ \\
\hline & & total expected & observed & & \\
\hline $4 \mu$ & & $38.43 \pm 2.40$ & 38 & & \\
\hline $4 e$ & & $19.91 \pm 1.56$ & 24 & & \\
\hline $2 \mu 2 e$ & & $20.49 \pm 1.51$ & 26 & & \\
\hline $2 e 2 \mu$ & & $27.43 \pm 1.60$ & 34 & & \\
\hline combined & & $106.27 \pm 6.39$ & 122 & & \\
\hline
\end{tabular}

Perhaps more interesting for the PER model is the 2D distribution of $m_{4 \ell}^{\text {cons }}$ vs. $\sigma_{\text {eff }}$ (defined in Section 7.3.6). This distribution, colour-coded by category, is shown in Figure 117. Note that a narrower window of $m_{4 \ell}^{\text {cons }}$ is used here than in the fit, so that the plot puts more emphasis on the signal events. To interpret this plot, consider that each event will effectively "pull" $m_{H}$ toward its $m_{4 \ell}^{\text {cons }}$ value (actually, slightly above its $m_{4 \ell}^{\text {cons }}$ value, because the detector response is not symmetric). The strength of this pull is dictated by the resolution, so that events with smaller values of $\sigma_{\text {eff }}$ have stronger pulls, and events with larger values have weaker pulls. Marked on the plot are the average $\sigma_{e f f}$ from MC simulation, and its standard deviation. In this case, the observed $4 e$ and $2 \mu 2 e$ events are worse than the average expected from simulation, while the other two categories are approximately equal. Using this plot is very useful in interpreting the difference between the PER method, which uses this information, and the Template method, which assumes every event in a given category has the same resolution. Of course, the effective resolution is not a complete 
picture when describing the detector response: each event has a unique, generally complex and asymmetric response distribution. The full response distributions of each event in the data, as modelled using the constrained PER method, are collected in Appendix C.5. These distributions provide everything needed to interpret the results in data.

The likelihood scans for $m_{H}$ in the full $36.1 \mathrm{fb}^{-1}$ dataset are found in Figure 118, and the results are summarized in Table 29. The combined best fit value of $m_{H}$ of the two cross-checks agree with the constrained PER method to within $140 \mathrm{MeV}$, a value consistent with the level of disagreement that could be expected from the same dataset based on the toy experiments (second column of Table 25). Some of differences between the constrained PER and Template method in individual channels are worth highlighting, as they serve well to differentiate the two methods.

- In the $4 \mu$ category, the events observed have quite average resolutions, according to Figure 117a, and so the two methods agree very closely. However, as seen in Figure 116a, the events are spread more broadly in $m_{4 \ell}^{\text {cons }}$ than expected from MC simulation, which results in a worse-than-expected $m_{H}$ resolution in this category.

- In the $4 e$ category, the constrained PER returns a higher $m_{H}$ value than the Template method. This is a result of the distribution of events in Figure 117b - both methods are drawn by the clump of events near $122 \mathrm{GeV}$, but the constrained PER method gives extra importance to the well-measured event near $124 \mathrm{GeV}$, which pulls the best fit value upwards.

- Similarly, in the $2 \mu 2 e$ category, the constrained PER is pulled to a lower fit value, and given a larger negative uncertainty, by a cluster of well-measured events between $123-124 \mathrm{GeV}$, seen in Figure 117c. The Template method cannot 


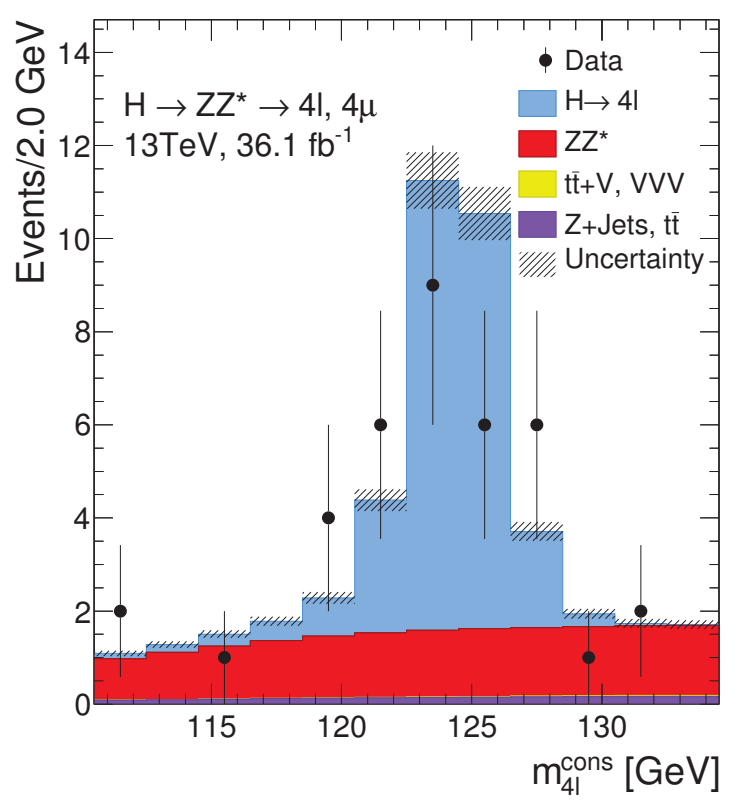

(a) $4 \mu$

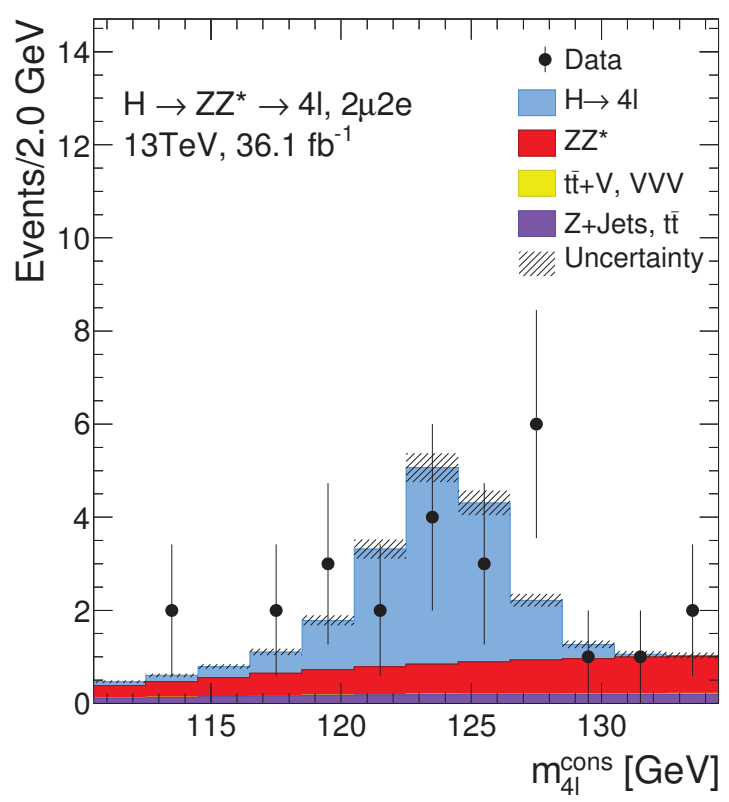

(c) $2 \mu 2 e$

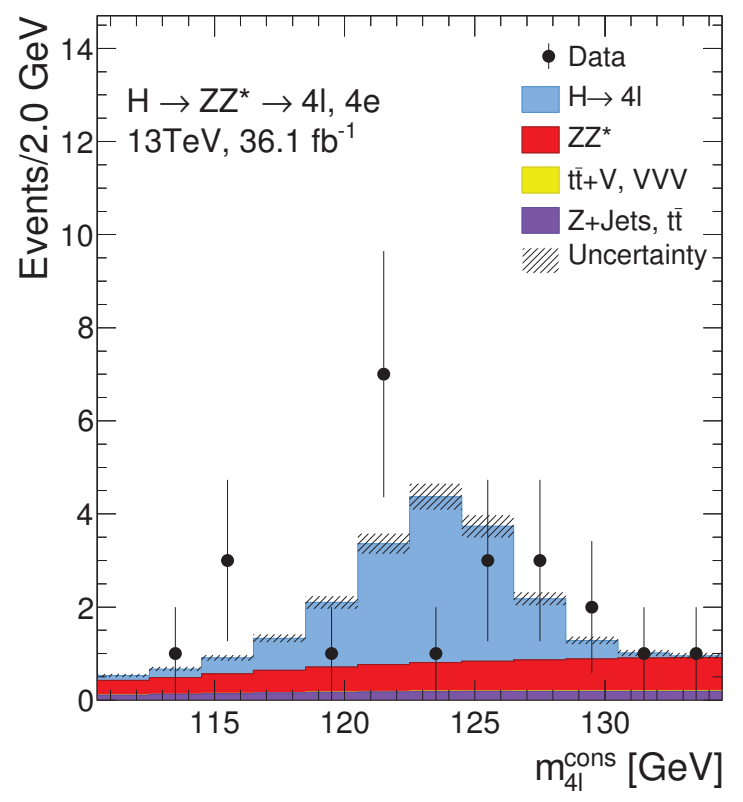

(b) $4 e$

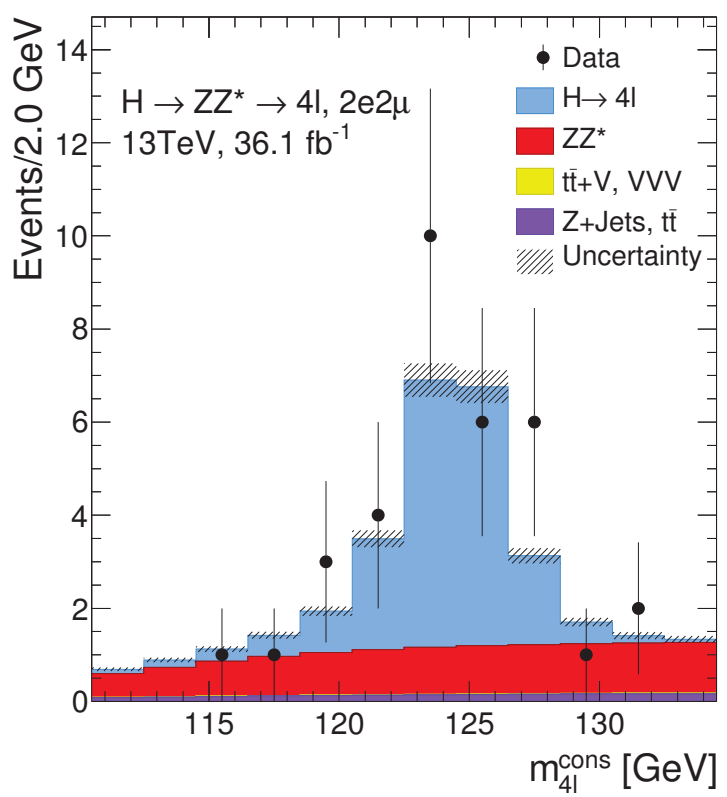

(d) $2 e 2 \mu$

Figure 116: Distribution in data of $m_{4 \ell}^{\text {cons }}$ in the four analysis categories drawn with the distribution predicted by the signalbackground model, using the constrained PER model with $m_{H}$ and $\sigma_{H \rightarrow 4 \ell}$ set to their best fit values. The plotted axis range is reduced from the fit range by $0.5 \mathrm{GeV}$ on either side to allow tidier binning. 


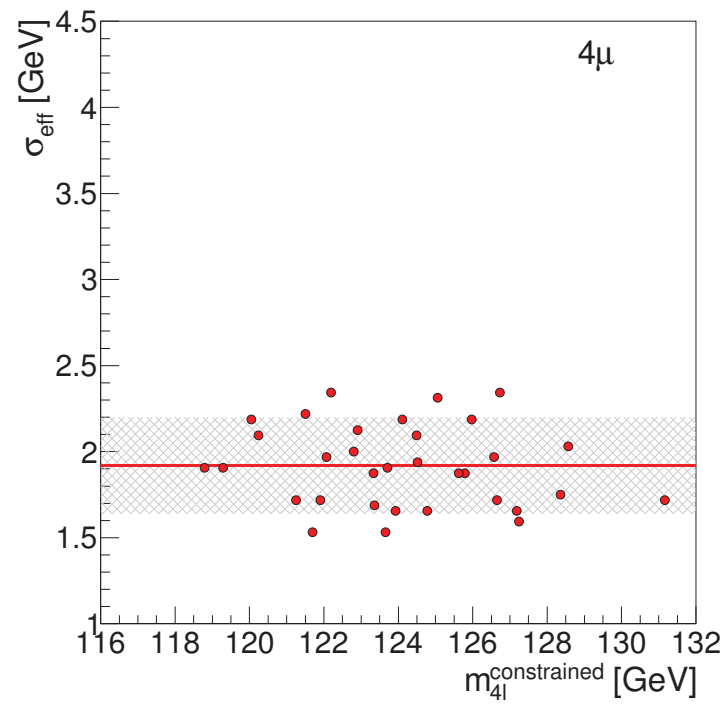

(a) $4 \mu$

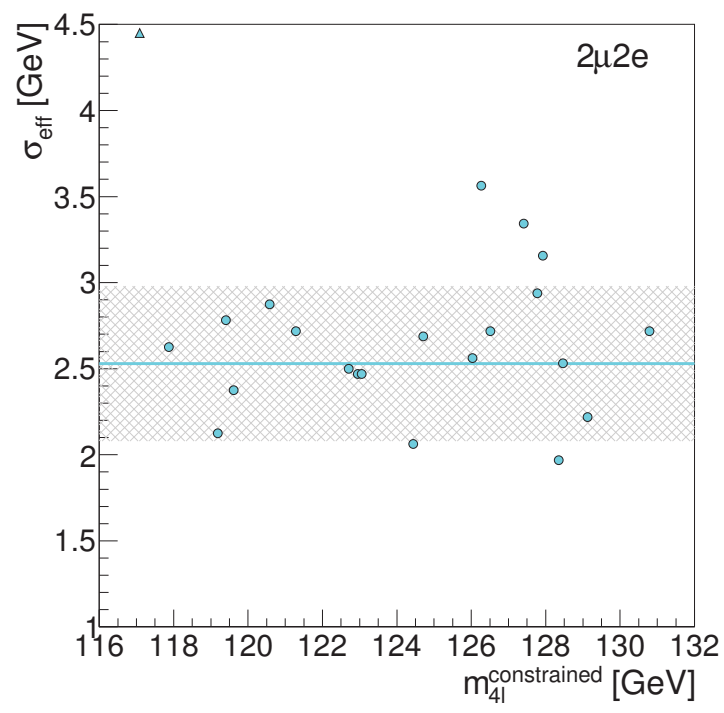

(c) $2 \mu 2 e$

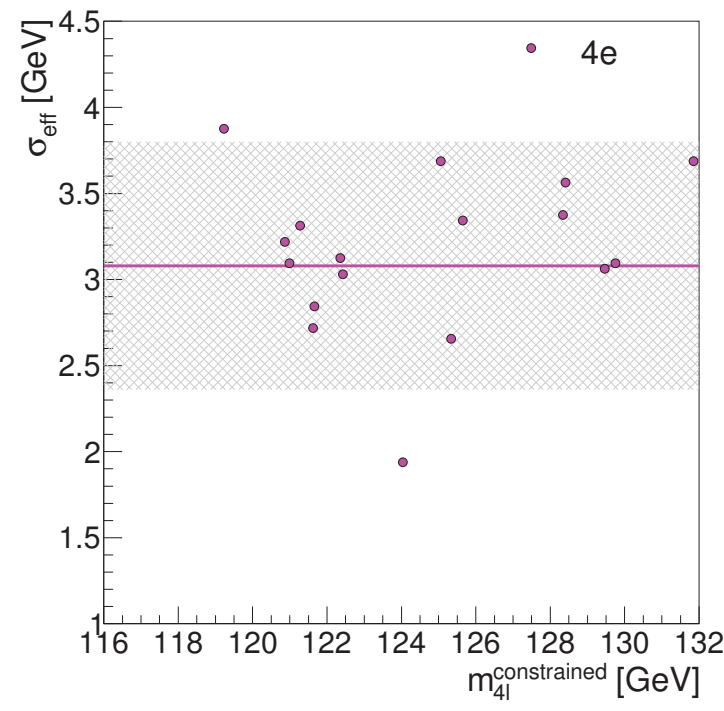

(b) $4 e$

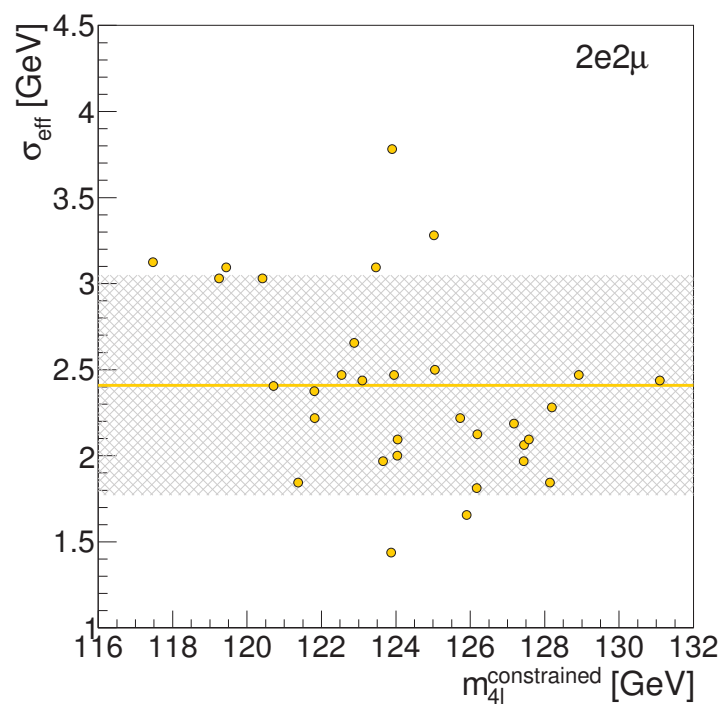

(d) $2 e 2 \mu$

Figure 117: Distribution of $m_{4 \ell}^{\text {cons }}$ vs $\sigma_{\text {eff }}$ in data. Each datum is marked by a round point. Triangles instead of points indicate the actual value is off the plotted range. The horizontal line represents the average $\sigma_{\text {eff }}$ of the events in simulation, and the hashed area represents the standard deviation of those values. Events below the line have a stronger-than-average pull on the $m_{H}$ value, while events above the line have a weaker-than-average pull on $m_{H}$.

"see" the resolution of these events, so is only pulled by the $m_{4 \ell}^{\text {cons }}$ values, of which the majority fall in a slightly higher range.

- In the $2 e 2 \mu$ category, a cluster of well-measured events around $126-128 \mathrm{GeV}$ 
in Figure 117d pull the $m_{H}$ value upwards in the constrained PER method, compared to the Template method.

These results demonstrate one of the key design principles of the PER method, that better-measured events are given more importance in the fit result than poorly measured events. Whether this results in a more accurate measurement is often up to good luck in the events that are observed, but as Table 25 demonstrated, the odds are better than even.

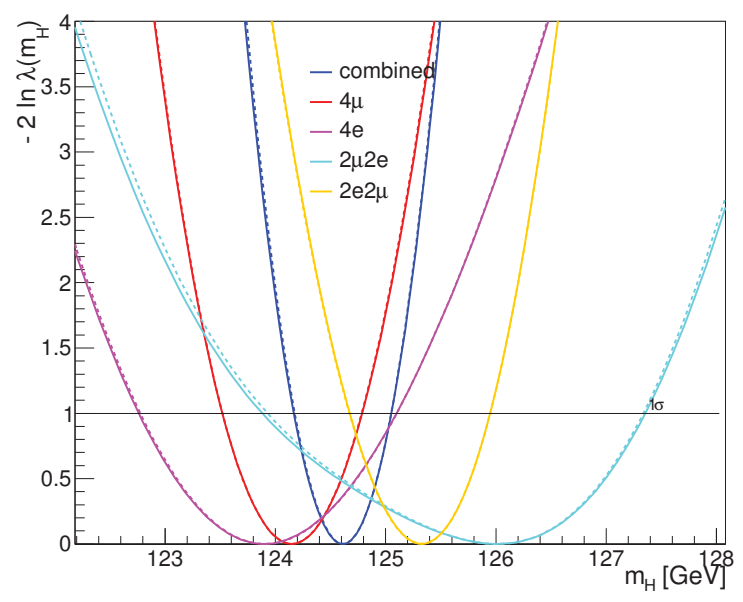

(a) constrained PER

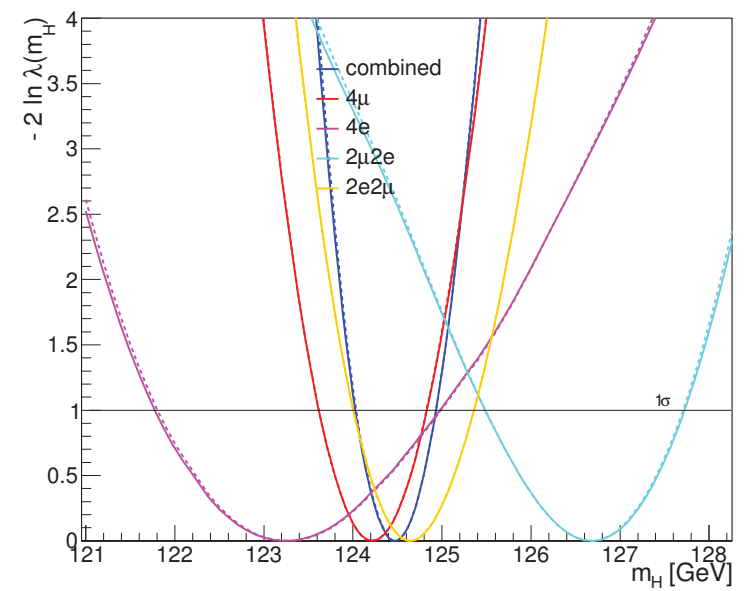

(b) Template

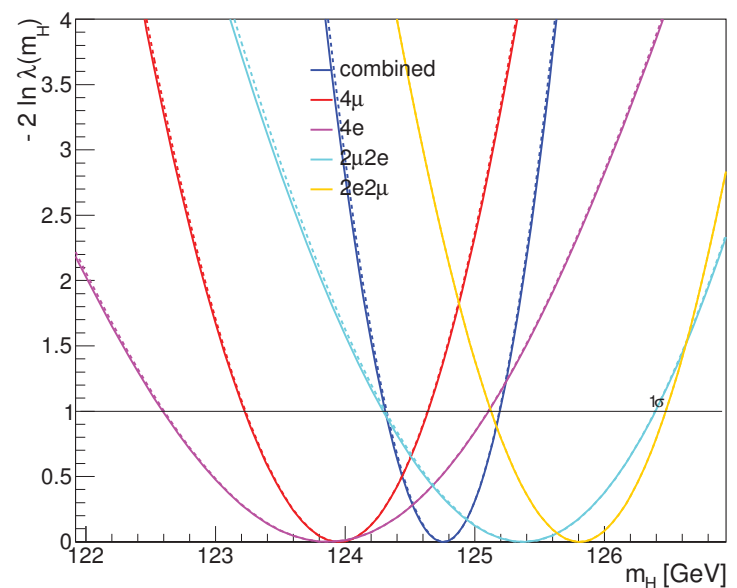

(c) unconstrained PER

Figure 118: Scans of the NLL on parameter $m_{H}$ in data. Subfigures show the scans using the different signal models as labelled. The NLL scans including (not including) systematics are shown with solid (dashed) lines. 
The likelihood scans for $\sigma_{H \rightarrow 4 \ell}$ are found in Figure 119, with the results summarized in Table 29. The constrained PER and Template methods agree exactly on the final best fit value, with slight differences in the uncertainty, and unconstrained PER method returns only a slightly higher value. The fit results in each category also show excellent agreement between all methods.

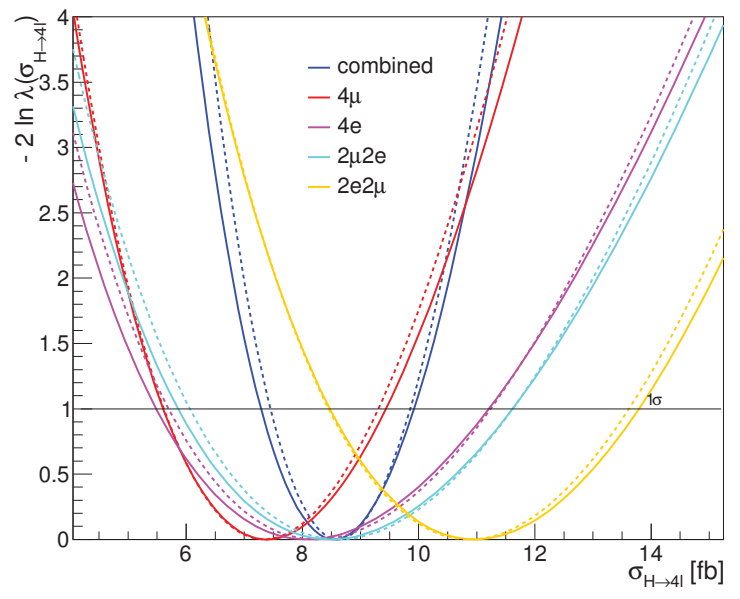

(a) constrained PER

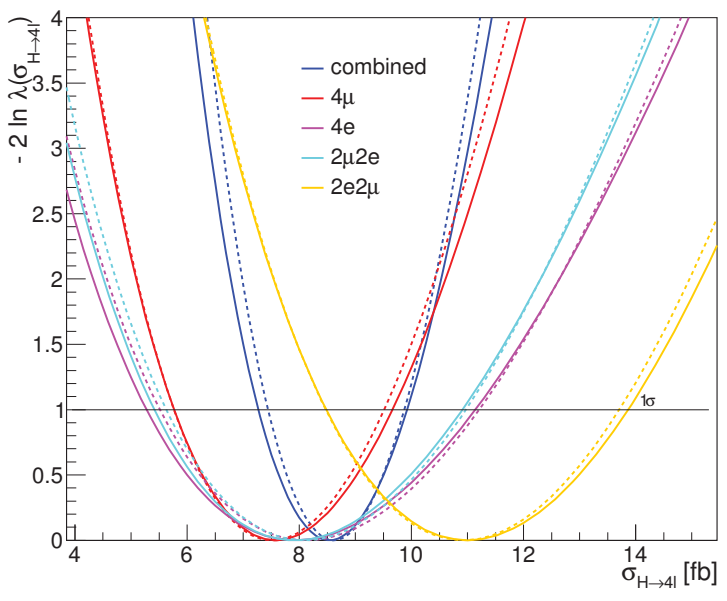

(b) Template

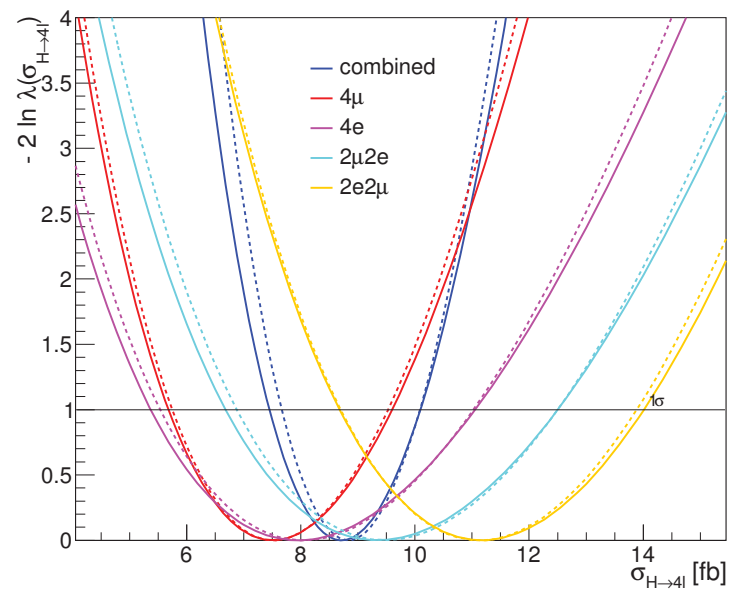

(c) unconstrained PER

Figure 119: Scans of the NLL on parameter $\sigma_{H \rightarrow 4 \ell}$ in data. Subfigures show the scans using the different signal models as labelled. The NLL scans including (not including) systematics are shown with solid (dashed) lines.

The likelihood scans for $\Gamma_{H}$ are found in Figure 120. The value of the test statistic $q_{0}$, defined in (113), can be read as the left-most value of the combined (blue) curve, at 
$\Gamma_{H}=4.1 \mathrm{MeV}$. For the constrained (unconstrained) PER model, it is $q_{0}=4.14(2.09)$. Using (115), the probability of SM fluctuations producing as large or larger an observed width is $p_{0}=0.0089(0.041)$. These values can be checked by considering the distribution of $\hat{\Gamma}_{H}$ from toys, in Figure 107 which suggest a similar probability for such an excess. As these $p_{0}$ values do not meet the criteria for identifying an excess, an upper limit is set on $\Gamma_{H}$, as prescribed in Section 9.1. The $q_{\mu}$ test statistic is equal to the plotted likelihood curve everywhere to the right of $\hat{\Gamma}_{H}$. Most the observed upper limit-setting power comes from the $4 \mu$ and $2 e 2 \mu$ categories, whereas in the $4 e$ and $2 \mu 2 e$ categories, only extremely loose limits are able to be set.

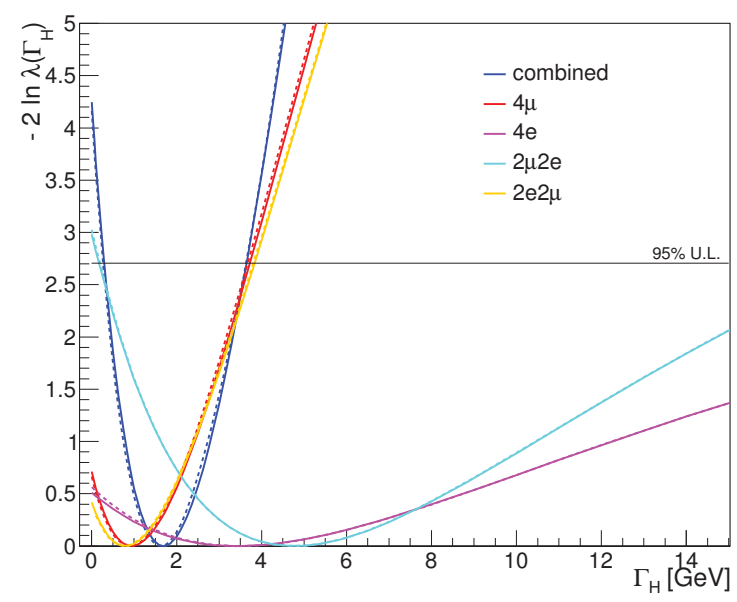

(a) constrained PER

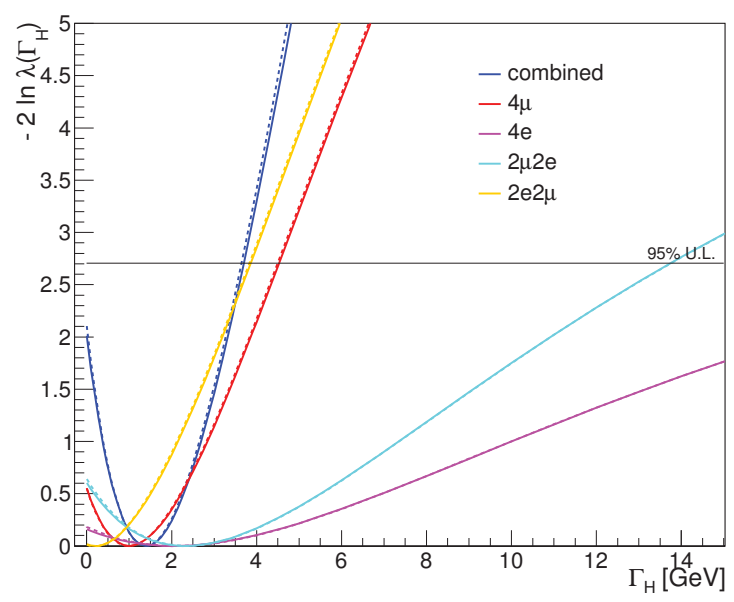

(b) unconstrained PER

Figure 120: Scans of the NLL on parameter $\Gamma_{H}$ in data. Subfigures show the scans using the different signal models as labelled. The NLL scans including (not including) systematics are shown with solid (dashed) lines.

The fit results from the constrained PER model in Table 29 represent the ultimate product of this thesis. The uncertainties in the table can be decomposed into their statistic and systematic components trivially using Table 21, and $\sigma_{\text {stat }+ \text { sys }}^{2}=\sigma_{\text {stat }}^{2}+\sigma_{\text {sys }}^{2}$, which is how the final results of Chapter 10 are presented.

The results can be compared with existing measurement and the SM predictions 
Table 29: Summary table of the final data fit results on $m_{H}, \sigma_{H \rightarrow 4 \ell}, \Gamma_{H}$ for the three signal models. Compare these numbers with expected resolutions from simulation in Table 18. Upper limits for $\Gamma_{H}$ are given at 95\% CL.

\begin{tabular}{cccc}
\hline & constrained PER & Template & unconstrained PER \\
\hline \multicolumn{3}{c}{$m_{H}[\mathrm{GeV}]$} \\
\hline$\mu$ & $124.15_{-0.63}^{+0.64}$ & $124.22_{-0.60}^{+0.61}$ & $123.95_{-0.73}^{+0.69}$ \\
$2 \mu 2 e$ & $123.90_{-1.13}^{+1.20}$ & $123.24_{-1.47}^{+1.74}$ & $123.86_{-1.27}^{+1.24}$ \\
$2 e 2 \mu$ & $126.01_{-2.13}^{+1.34}$ & $126.68_{-1.20}^{+1.05}$ & $125.37_{-1.07}^{+1.02}$ \\
combined & $125.33_{-0.65}^{+0.62}$ & $124.65_{-0.64}^{+0.72}$ & $125.81_{-0.68}^{+0.67}$ \\
\hline & $124.61_{-0.44}^{+0.44}$ & $124.47_{-0.44}^{+0.46}$ & $124.76_{-0.45}^{+0.44}$ \\
\hline $4 \mu$ & & $\sigma_{H \rightarrow 4 \ell}[\mathrm{fb}]$ \\
$4 e$ & $7.39_{-1.78}^{+2.05}$ & $7.58_{-1.82}^{+2.08}$ \\
$2 \mu 2 e$ & $8.08_{-2.60}^{+3.13}$ & $7.93_{-2.65}^{+3.21}$ & $7.51_{-1.83}^{+2.09}$ \\
$2 e 2 \mu$ & $8.49_{-2.63}^{+3.13}$ & $7.92_{-2.50}^{+2.99}$ & $7.95_{-2.58}^{+3.11}$ \\
combined & $10.96_{-2.48}^{+2.83}$ & $11.00_{-2.51}^{+2.87}$ & $9.35_{-2.67}^{+3.15}$ \\
\hline & $8.55_{-1.26}^{+1.38}$ & $8.54_{-1.27}^{+1.38}$ & $8.78_{-2.49}^{+2.84}$ \\
\hline $4 \mu$ & & $\Gamma_{H}[\mathrm{GeV}]$ & \\
$4 e$ & $<3.73$ & n.a. & $<4.53$ \\
$2 \mu 2 e$ & $<30.58$ & n.a. & $<23.55$ \\
$2 e 2 \mu$ & $<18.23$ & n.a. & $<13.75$ \\
combined & $<3.83$ & n.a. & $<3.87$ \\
\hline & $<3.65$ & n.a. & $<3.71$ \\
\hline
\end{tabular}

using

$$
z[\sigma]=\frac{x_{1}-x_{2}}{\sqrt{\sigma_{x_{1}}^{2}+\sigma_{x_{2}}^{2}}},
$$

where for asymmetric uncertainties, the $\sigma_{x_{i}}$ is taken in the direction of the other measurement. The comparisons are signed to express the direction of compatibility, and expressed in units of Gaussian $\sigma$. These comparisons are shown only for the constrained PER results, although the results of the cross checks have similar compatibility. Some of the measurements being compared have correlated systematic 
uncertainties, although this is not taken into account in the simple consistency check as the measurements in this thesis are highly statistically dominated.

The results of the constrained PER measurements are highly compatible with existing measurements. The Higgs boson mass measured in this thesis, $m_{H}=124.61 \pm 0.44 \mathrm{GeV}$, is compatible with:

- The ATLAS and CMS Run 1 combined $m_{H}$ measurement [8], $m_{H}=125.09 \pm 0.24 \mathrm{GeV}$, with compatibility $-0.96 \sigma$.

- The ATLAS Run $1 H \rightarrow Z Z^{(*)} \rightarrow 4 \ell m_{H}$ measurement [7], $m_{H}=124.51 \pm 0.52 \mathrm{GeV}$, with compatibility $+0.15 \sigma$.

- A more recent CMS $H \rightarrow Z Z^{(*)} \rightarrow 4 \ell m_{H}$ measurement [101], $m_{H}=124.50_{-0.46}^{+0.48} \mathrm{GeV}$, with compatibility $+0.17 \sigma$.

The mass measurement presented in this thesis exceeds the precision of the two $H \rightarrow 4 \ell$ measurements entering into [8], and that in [101], making it the most precise single-experiment Higgs boson mass measurement in the $H \rightarrow 4 \ell$ channel to date.

The measurement of $\sigma_{H \rightarrow 4 \ell}$ requires slightly more care in its comparison with existing measurements, as the existing measurements can be framed in different ways and must first be converted to a common reference, in this case $\sigma_{H \rightarrow 4 \ell}$ as it is defined in this thesis. The measurement can also only be compared with Run 2 measurements taken at $\sqrt{s}=13 \mathrm{TeV}$ collisions, as the cross section depends on the collision energy. The measurement of this thesis, $\sigma_{H \rightarrow 4 \ell}=8.55_{-1.26}^{+1.38} \mathrm{fb}$, is compatible with:

- The SM prediction for $\sigma_{H \rightarrow 4 \ell}$ at the observed Higgs boson mass [21], $\sigma_{H \rightarrow 4 \ell}^{S M}=6.63 \pm 0.30 \mathrm{fb}$, to within $+1.57 \sigma$.

- The most recent ATLAS $H \rightarrow Z Z^{(*)} \rightarrow 4 \ell$ measurement [5], which measured $\sigma_{H, t o t}=81_{-16}^{+18} \mathrm{pb}$. Using the branching ratio $\mathrm{BR}(H \rightarrow 4 \ell)$ as in this conference 
note to translate this to a $\sigma_{H \rightarrow 4 \ell}$ value, the approximate corresponding measurement is $\sigma_{H \rightarrow 4 \ell}=10 \pm 2 \mathrm{fb}$, with which the measurement in this thesis is compatible within $-0.61 \sigma$. Note that these two measurements use an overlapping dataset, so this is not a wholly independent comparison.

- The most recent CMS measurement [101], which measured the quantity $\mu_{H \rightarrow 4 \ell}=N_{H \rightarrow 4 \ell}^{o b s} / N_{H \rightarrow 4 \ell}^{S M}=0.99_{-0.26}^{+0.33}$. This can be translated, using the SM predictions at $m_{H}=125.09 \mathrm{GeV}$ to $\sigma_{H \rightarrow 4 \ell}=6.8_{-1.8}^{+2.3} \mathrm{fb}$, with which the measurement in this thesis is compatible within $+0.68 \sigma$.

The measurement of $\sigma_{H \rightarrow 4 \ell}$ at $\sqrt{s}=13 \mathrm{TeV}$ is also the most precise measurement of this quantity to date. The above-mentioned measurements are the first performed at $\sqrt{s}=13 \mathrm{TeV}$, with a smaller dataset than the one used in this thesis. Measurement strategies focused on the cross section, rather than designed around measuring $m_{H}$, may be able to exceed to precision of this thesis using the same dataset.

The observed $\Gamma_{H}$ upper limit, $\Gamma_{H}<3.65 \mathrm{GeV}$ at $95 \% \mathrm{CL}$, is not the most stringent set so far. As demonstrated using pseudo-experiments in Section 9.4, the PER technique has the potential to set a very stringent limit (as far as direct limits go) on $\Gamma_{H}$, potentially even below $1 \mathrm{GeV}$. However, by the same demonstration, in some datasets only very weak limits can be set. The observed data happen to the latter, not the former, and so only a weak limit is set on $\Gamma_{H}$. For comparison, other direct $\Gamma_{H}$ measurements are:

- By ATLAS using the Run $1 H \rightarrow Z Z^{(*)} \rightarrow 4 \ell$ data and the PER technique in its early stages [7], an upper limit of $\Gamma_{H}<2.6 \mathrm{GeV}$ was set.

- A CMS combination of $H \rightarrow Z Z^{(*)} \rightarrow 4 \ell$ and $H \rightarrow \gamma \gamma$ using the Run 1 data [89] set an upper limit $\Gamma_{H}<2.6 \mathrm{GeV}$ (in $H \rightarrow Z Z^{(*)} \rightarrow 4 \ell$ and $H \rightarrow \gamma \gamma$ alone it was $2.4 \mathrm{GeV}$ and $3.4 \mathrm{GeV}$, respectively). 
All these limits pale in comparison to the strength of indirect limits on $\Gamma_{H}$, of which the strongest [102] is $\Gamma_{H}<13 \mathrm{MeV}$. These limits are generally accompanied by significantly more model assumptions than the direct limit set by this thesis, and so the two can still be complementary.

The measurements in this chapter serve to refine our knowledge of one of nature's unknown parameters, $m_{H}$, and put the SM to the test with a precision measurement of $\sigma_{H \rightarrow 4 \ell}$, and upper limit set on $\Gamma_{H}$. No significant deviations from the SM, or from existing measurements, were observed. 


\section{Chapter 10}

\section{Conclusion}

In July of 2012, the ATLAS and CMS collaborations jointly announced the discovery of a new a particle consistent with the Standard Model Higgs boson. This elusive particle holds a critical role in the Standard Model, where it is responsible for giving fundamental particles their mass. To understand whether the newly discovered particle was indeed the predicted Higgs boson, a wide range of property measurements have been conducted by ATLAS and CMS using the full Run 1 LHC dataset, all of which are so far consistent with Standard Model predictions within their uncertainties.

In the higher energy collisions of Run 2 at the LHC, Higgs bosons are being produced at more than twice the rate of Run 1, offering a significant opportunity to measure the Higgs more precisely than ever before. One of the most powerful tools for Higgs property measurement is the $H \rightarrow Z Z^{(*)} \rightarrow 4 \ell$ decay channel because all the final state particles from the Higgs decay can be fully and precisely reconstructed. For this reason it was one of the discovery channels for the Higgs, and continues to be one of the most powerful for Higgs boson property measurements. Precision measurement of the properties of the Higgs boson continue to be an effective tool for testing the Standard Model to its limits, and garnering clues for what may lay beyond it.

This thesis presented a measurement of the Higgs boson mass, $m_{H}$, its cross section

times branching ratio to four electrons or muons, $\sigma_{H} \times \mathrm{BR}(H \rightarrow 4 \ell=e, \mu)$, and an 
upper limit on its natural width, $\Gamma_{H}$. While the mass is an unknown free parameter of the Standard Model, the other two are predicted once the mass is known. The mass measurement has ramifications in a huge range of Higgs analyses from ATLAS and CMS, as all the Higgs coupling and branching ratio predictions depend on it.

The measurements were conducted using an unbinned maximum likelihood fit on the four-lepton invariant mass, $m_{4 \ell}$, providing statistical models for the signal and background distributions. The signal model used was the per event response method, which decouples the detector resolution component of the $m_{4 \ell}$ distribution from the physics component. It works by building a model of lepton resolutions dependent on their kinematics, and propagates this analytically as a Gaussian mixture to the final detector $m_{4 \ell}$ response. As the response is predicted on an event-by-event basis, wellmeasured events are given more importance in the fit than poorly-measured events. The measurements in this thesis were all conducted using the full Run 2 dataset collected by ATLAS so far, $36.1 \mathrm{fb}^{-1}$ of $p-p$ collisions at $\sqrt{s}=13 \mathrm{TeV}$. Using this technique and dataset, the Higgs boson properties were measured to be

$$
\begin{aligned}
m_{H} & =124.61_{-0.44}^{+0.44}(\text { stat. })_{-0.06}^{+0.06} \text { (syst.) } \mathrm{GeV} \\
\sigma_{H} \times \operatorname{BR}(H \rightarrow 4 \ell) & =8.55_{-1.15}^{+1.23} \text { (stat.) }{ }_{-0.52}^{+0.63} \text { (syst.) fb } \\
\Gamma_{H} & <3.65 \text { (stat.) } \oplus 0.19 \text { (syst.) } \mathrm{GeV} \text { at } 95 \% \mathrm{CL} .
\end{aligned}
$$

The measured values are consistent within their uncertainties with all existing measurements and the Standard Model predictions. The $m_{H}$ measurement exceeds the precision of any single-experiment $m_{H}$ measurement in the $H \rightarrow 4 \ell$ channel by ATLAS or CMS conducted to this point. The measurement of $\sigma_{H \rightarrow 4 \ell}$ also exceeds the precision of other such measurements at $\sqrt{s}=13 \mathrm{TeV}$. The $\Gamma_{H}$ upper limit is not the most stringent set, but complements existing limits. All three are the first measurements of their kind using the full 2015 and $2016 \sqrt{s}=13 \mathrm{TeV}$ LHC collision datasets. 
The results in this thesis are just a small piece of the Higgs boson property measurements being performed by ATLAS and CMS. Despite this, they represent a valuable contribution to the field as they, in particular the $m_{H}$ measurement, help refine the SM predictions against which other Higgs property measurements are compared. Tests such as these could eventually uncover whether the Higgs boson near $125 \mathrm{GeV}$ is indeed the Higgs boson of the Standard Model, or is a manifestation of an underlying theory. 


\section{Appendix A}

\section{Backgrounds supplementary plots}

This appendix supplements Chapter 6, providing plots of the smoothing used to create PDFs of the unconstrained mass, $m_{4 \ell}^{\mathrm{fsr}}$. The unconstrained smoothing results for qqZZ, ggZZ, VVV , and are found in Figure 121, Figure 122, Figure 123, respectively. Due to the large uncertainty on the Z+Jets background yield and shape, the $m_{4 \ell}^{\text {cons }}$ and $m_{4 \ell}^{\mathrm{fsr}}$ distributions are considered to be interchangeable. 


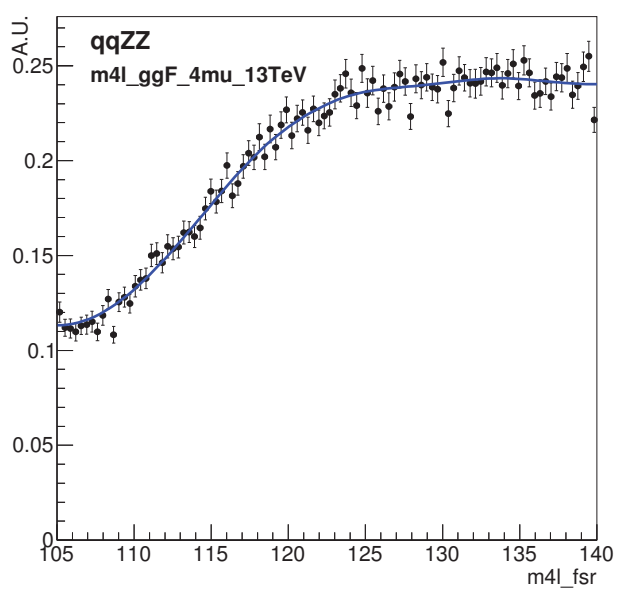

(a) $4 \mu$

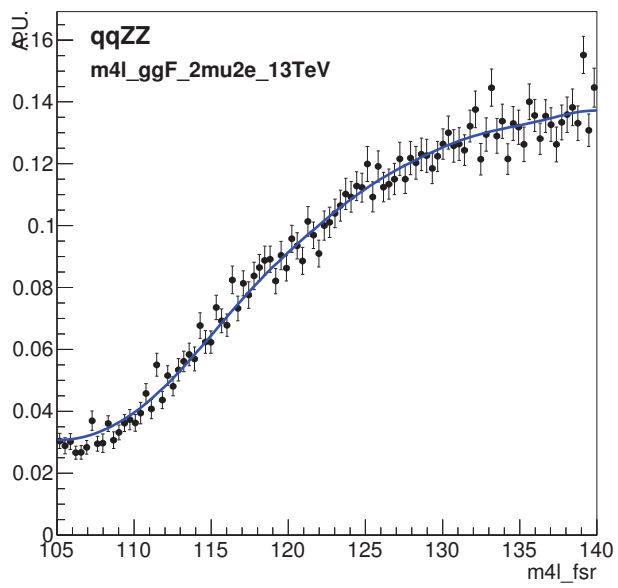

(c) $2 m u 2 e$

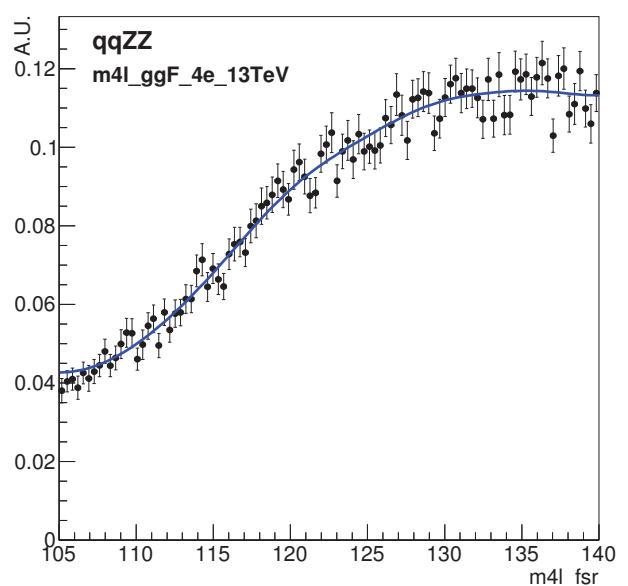

(b) $4 e$

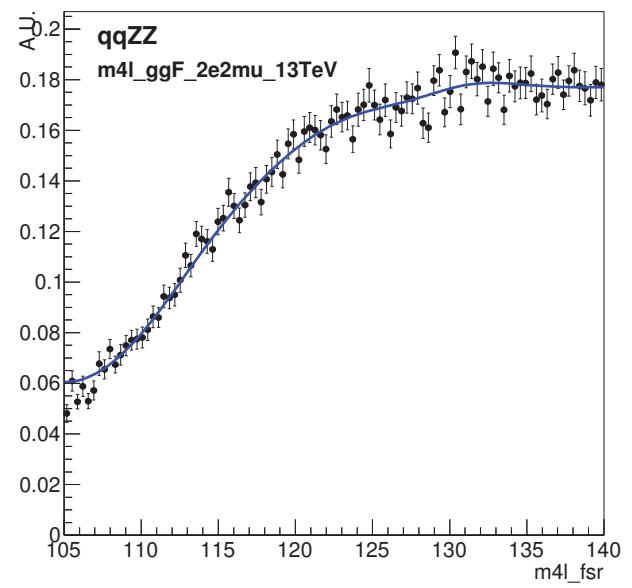

(d) $2 e 2 m u$

Figure 121: Smoothed distribution of $m_{4 \ell}^{\mathrm{fsr}}$ from simulated $q \bar{q} \rightarrow Z Z^{(*)}$ events. The black points represent the simulation, the solid blue line is the kernel smoothing result used as the PDF. 


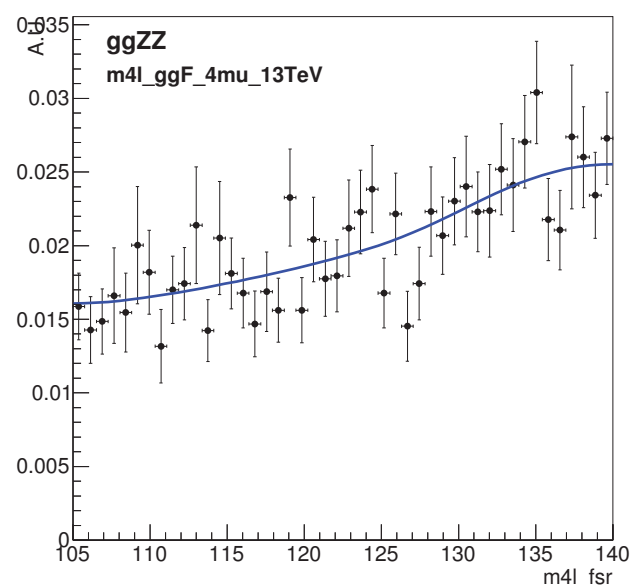

(a) $4 \mu$

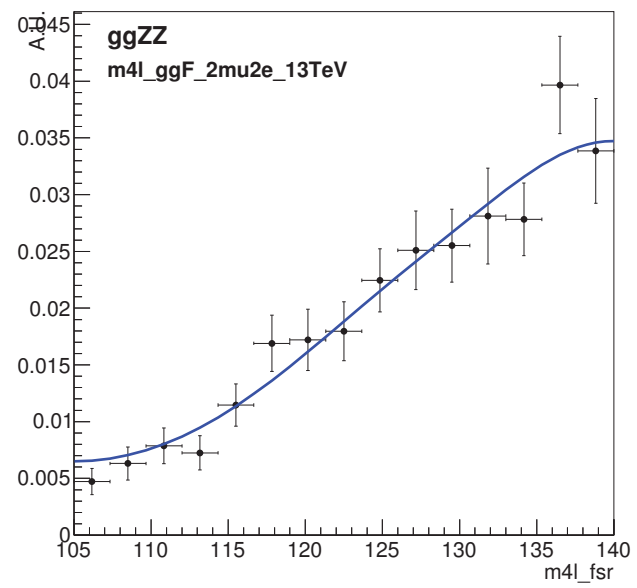

(c) $2 m u 2 e$

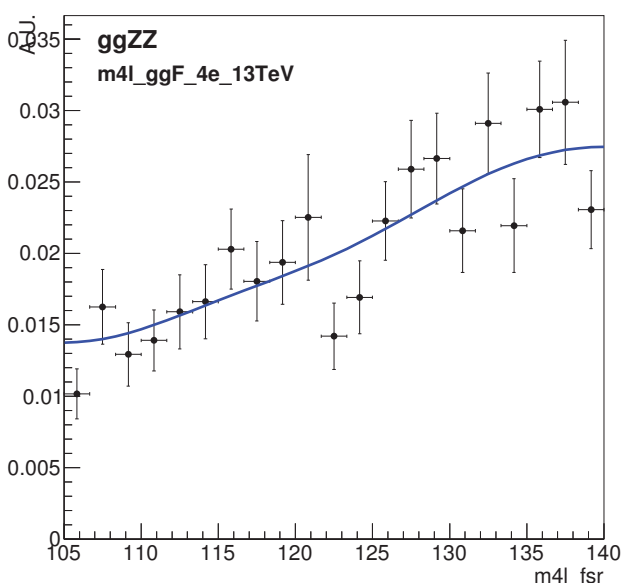

(b) $4 e$

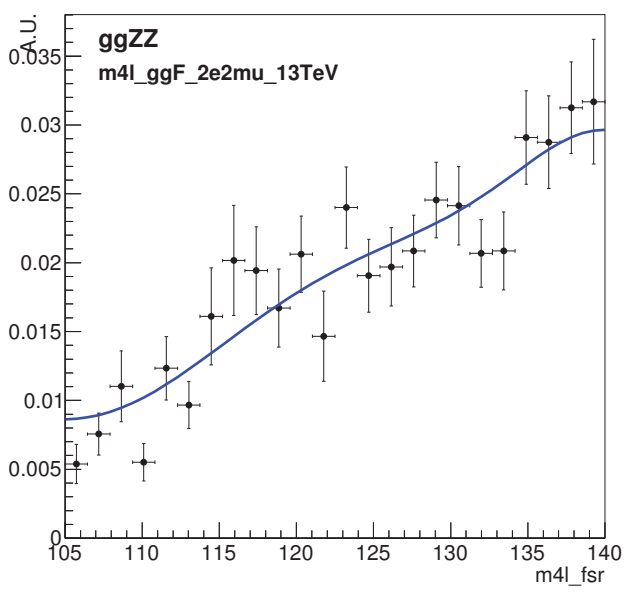

(d) $2 e 2 m u$

Figure 122: Smoothed distribution of $m_{4 \ell}^{\mathrm{fsr}}$ from simulated $g g \rightarrow Z Z^{(*)}$ events. The black points represent the simulation, the solid blue line is the kernel smoothing result used as the PDF. 


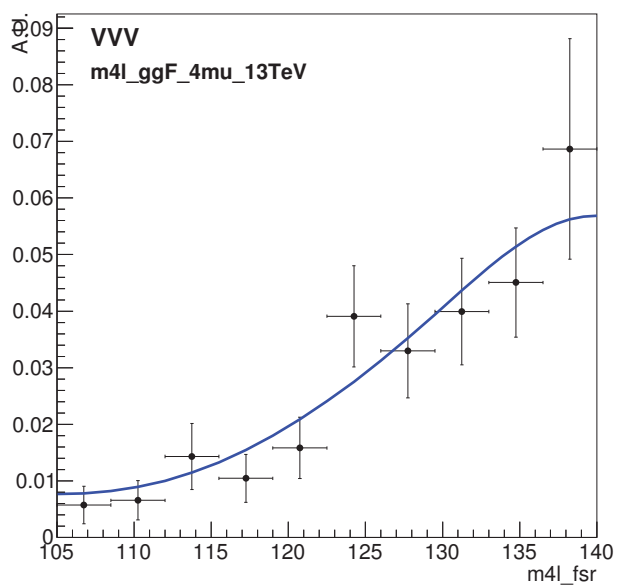

(a) $4 \mu$

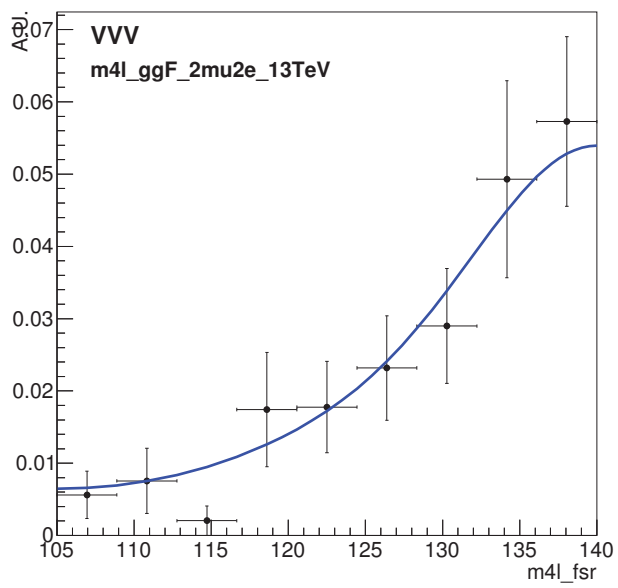

(c) $2 m u 2 e$

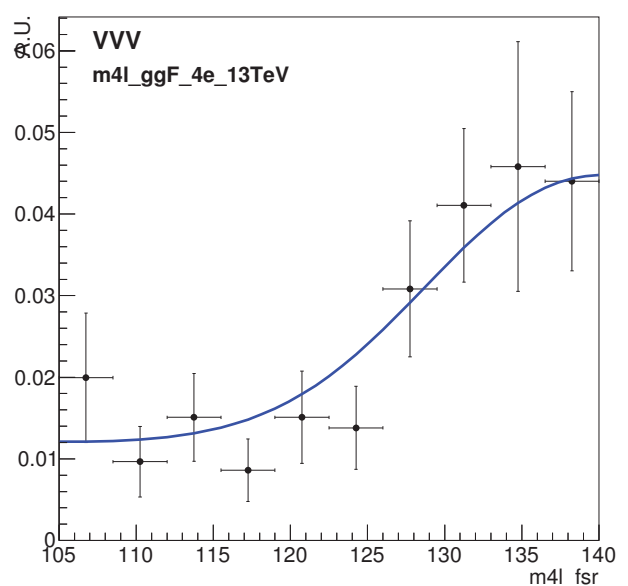

(b) $4 e$

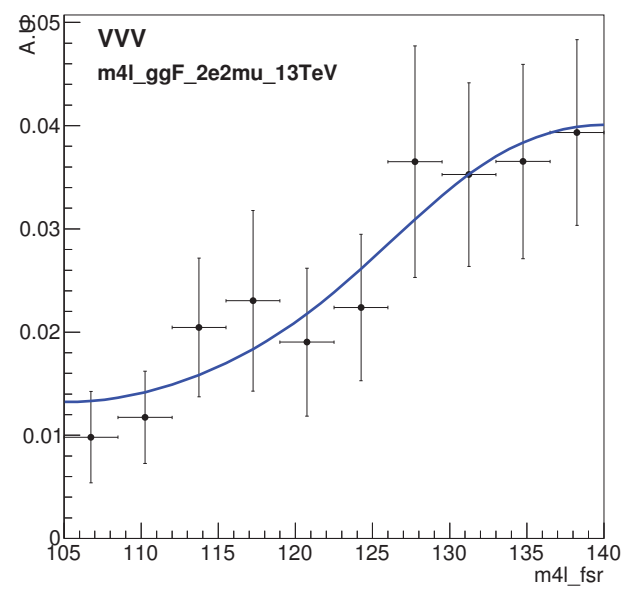

(d) $2 e 2 m u$

Figure 123: Smoothed distribution of $m_{4 \ell}^{\mathrm{fsr}}$ from simulated $V V V, t \bar{t}+Z$ events. The black points represent the simulation, the solid blue line is the kernel smoothing result used as the PDF. 


\section{Appendix B}

\section{Extra Discussion of the Signal Model}

This appendix contains supplementary information concerning the signal models. Appendix B.1 verifies the stability of the Template method's morphing function. Appendix B.2 provides an explanation of the non-conventional definition of the lepton response. Appendix B.3 presents supplementary information on the individual lepton response fits. Appendix B.4 presents the overall behaviour of the fit parameters in the response model. Appendix B.5 presents a test of the kinematic model indpendence of the PER model.

\section{B.1 Stability of Template interpolation}

One potential drawback to the Template method is that the model could become over-tuned to the particular input samples used to build it. To demonstrate that this is not the case, individual signal mass points were removed from the morphing, and the prediction $P\left(m_{4 \ell}^{\text {cons }} \mid m_{H}\right)$ with and without those mass points were compared. This comparison is shown in Figure 124, where the Template drawn is in each category at $m_{H}=124.5 \mathrm{GeV}$ for three distinct cases: when all simulated mass points are used to build the morphed shape, when all except the $m_{H}=124 \mathrm{GeV}$ sample are used to build the morphed shape, and when all except the $m_{H}=125 \mathrm{GeV}$ sample are used to build the morphed shape. From this test, we can see that the interpolation is highly 
stable, even when individual mass points are removed. The actual uncertainty on the interpolation procedure is smaller than the variations from these plots, as they by definition use less input information.

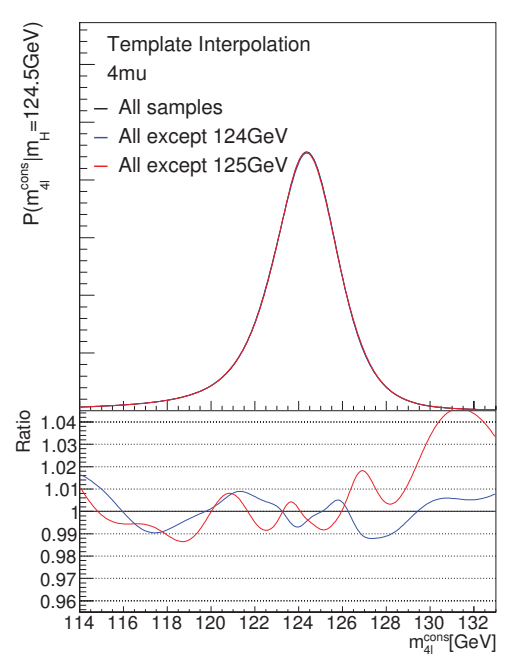

(a) $4 \mu$

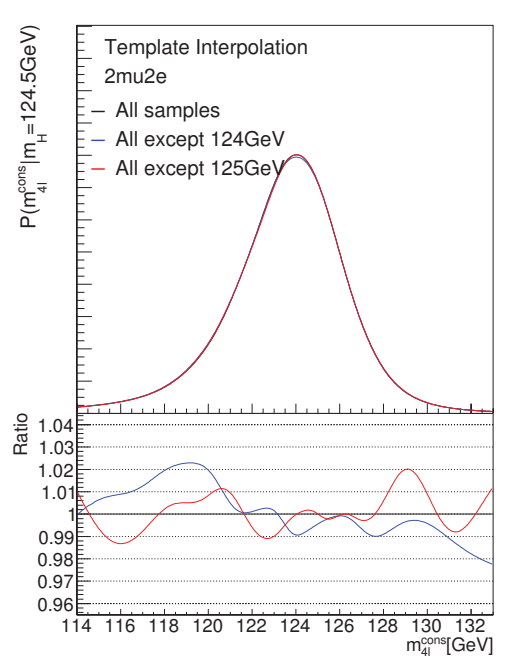

(c) $2 \mu 2 e$

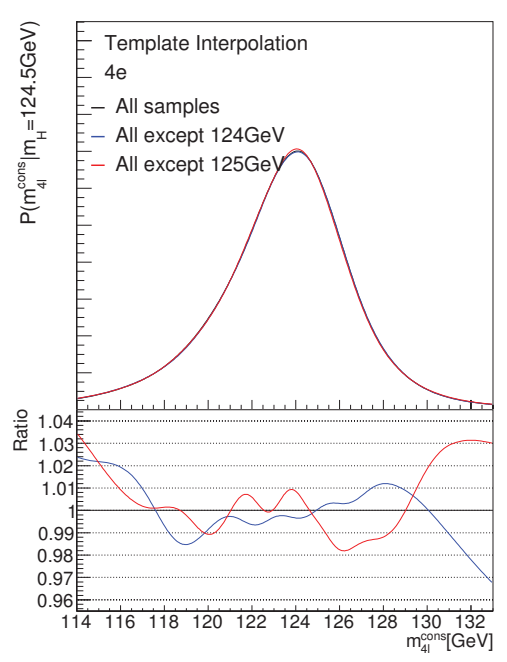

(b) $4 e$

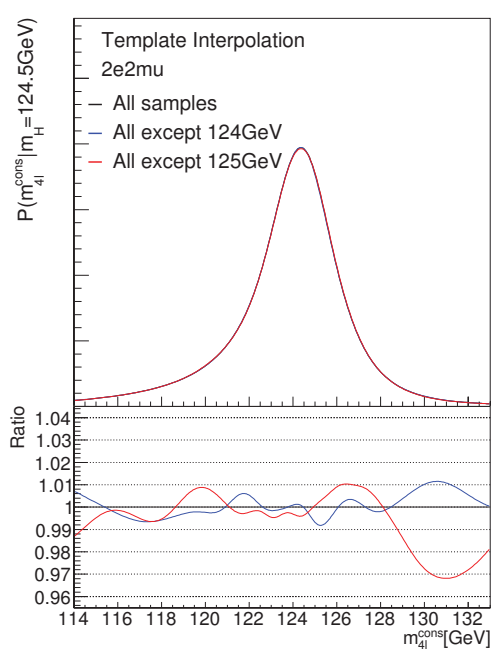

(d) $2 e 2 \mu$

Figure 124: Demonstration of the stability of the Template interpolation (morphing). Each plot shows the Template PDF drawn at $m_{H}=124.5 \mathrm{GeV}$, when the input to the morphing is altered along the following lines: all samples used (black curve), all except the $124 \mathrm{GeV}$ sample (blue curve), and all except the $125 \mathrm{GeV}$ sample (red curve). A the bottom, the ratio of each with respect to the black curve is shown. 


\section{B.2 On the definition of $x_{i}$}

In the derivation of Section 7.3.1, the reader may notice that the definition of the lepton response, replicated from (75) as

$$
x_{i}=\frac{E_{i}^{\mathrm{fsr}}-E_{i}^{\mathrm{true}}}{E_{i}^{\mathrm{fsr}}},
$$

does not match the typical definition, of

$$
x_{i}^{\mathrm{typ}}=\frac{E_{i}^{\mathrm{fsr}}-E_{i}^{\mathrm{true}}}{E_{i}^{\mathrm{true}}}
$$

or similar. It may even appear that the choice was arbitrary. However, the definition made in (75) is quite deliberate. If the same derivation is continued using $x_{i}^{\text {typ }}$ instead of $x_{i}$, then (79), which is replicated here

$$
m_{4 \ell}^{\mathrm{fsr}}-m_{4 \ell}^{\mathrm{true}}=\frac{1}{2 m_{4 \ell}^{\mathrm{fsr}}} \sum_{i}\left(\sum_{j \neq i}\left(m_{i j}^{\mathrm{fsr}}\right)^{2}\right) x_{i},
$$

becomes instead

$$
m_{4 \ell}^{\mathrm{fsr}}-m_{4 \ell}^{\mathrm{true}}=\frac{1}{2 m_{4 \ell}^{\mathrm{fsr}}} \sum_{i}\left(\sum_{j \neq i}\left(m_{i j}^{\mathrm{true}}\right)^{2}\right) x_{i}^{\mathrm{typ}} .
$$

While this new expression is mathematically valid, it is unfortunately of no use in predicting the response. To calculate the response of a reconstructed event using the right hand side of this expression, we would need to know the true lepton kinematics to calculate $m_{i j}^{\text {true }}$. While this could serve as a validation tool in simulation, where the true kinematics are known, it would be useless on data, where only reconstructed kinematics are available. It is the atypical definition of lepton energy response in (75) which allows the predicted mass response to be calculated. 


\section{B.3 Response model fits}

This section contains supplementary plots of the lepton response model fits in various kinematic bins. The plots shown here represent only a small subset of the greaterthan 8000 fits used to build the models. Table 30 summarizes where to find the plots for the per-event response model with and without Z Mass Constraint. Also find Figure 131 which shows the $\chi^{2} / n$ distributions for the constrained lepton response model.

Table 30: Reference table for response model example fits

\begin{tabular}{cc}
\hline muon response & electron response \\
\hline unconstrained & (more in Section 7.3.2) \\
Figure 125 & Figure 126 \\
constrained & \\
Figure 127 & Figure 129 \\
Figure 128 & Figure 130 \\
\hline
\end{tabular}



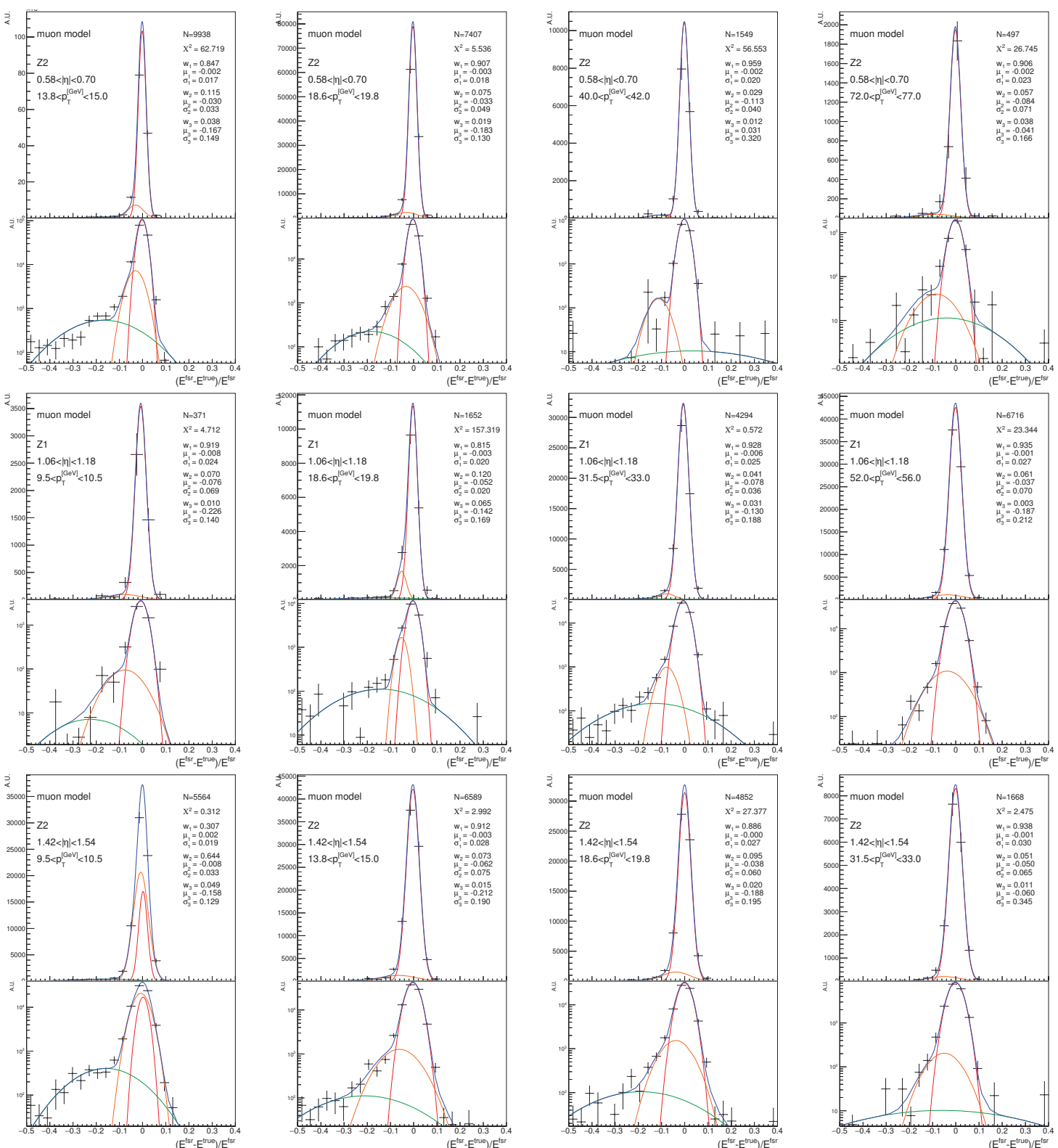

Figure 125: Example fits of unconstrained muon energy response (2/2). The distributions are built from MC simulation (black points) fit using a sum of three Gaussians (blue curve). Each plot shows the same fit in linear and log scale. The kinematic bins and the fit parameters are printed on each plot. See Table 30 for more plots. 

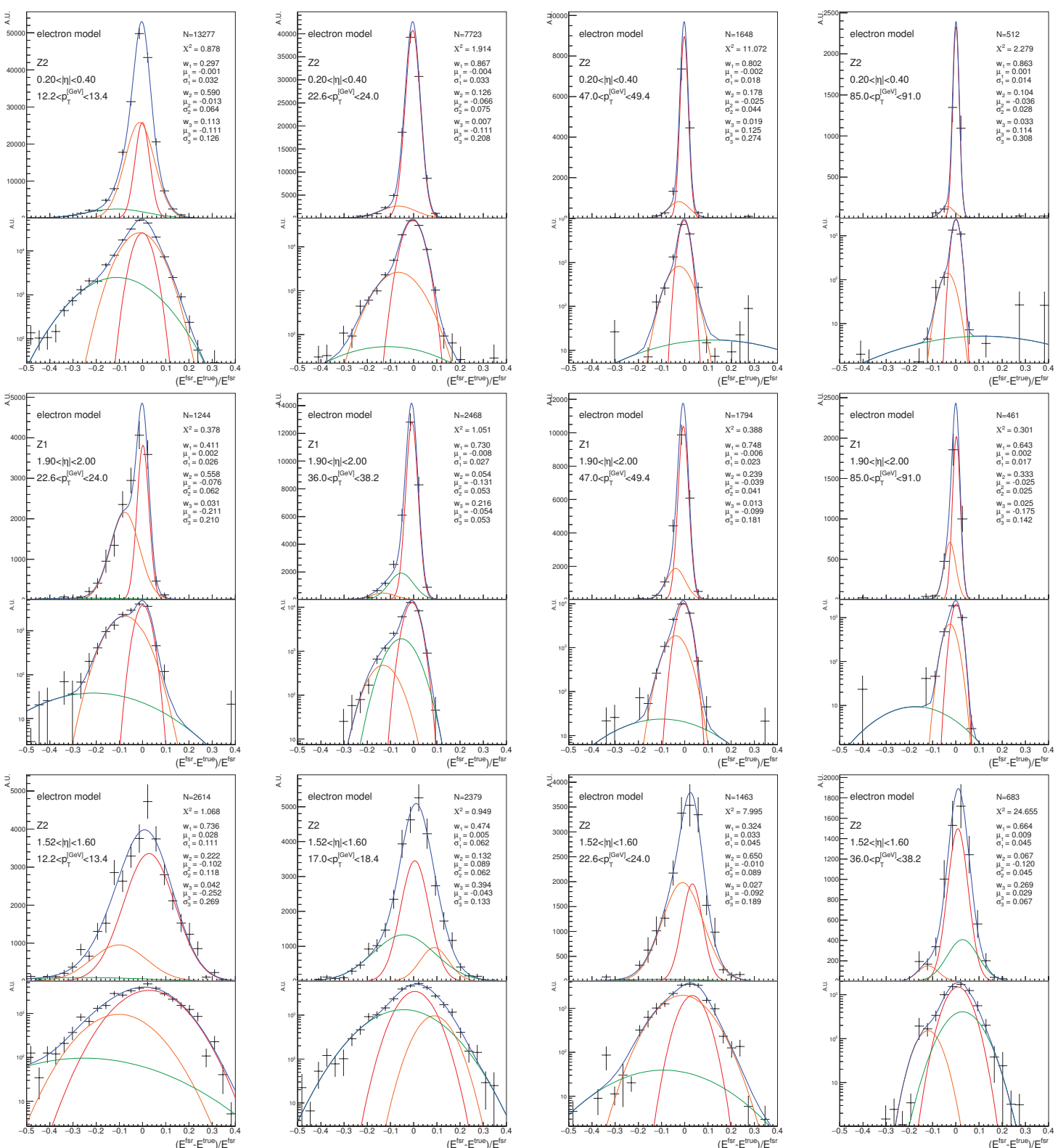

Figure 126: Example fits of unconstrained electron energy response (2/2). The distributions are built from MC simulation (black points) fit using a sum of three Gaussians (blue curve). Each plot shows the same fit in linear and log scale. The kinematic bins and the fit parameters are printed on each plot. See Table 30 for more plots. 

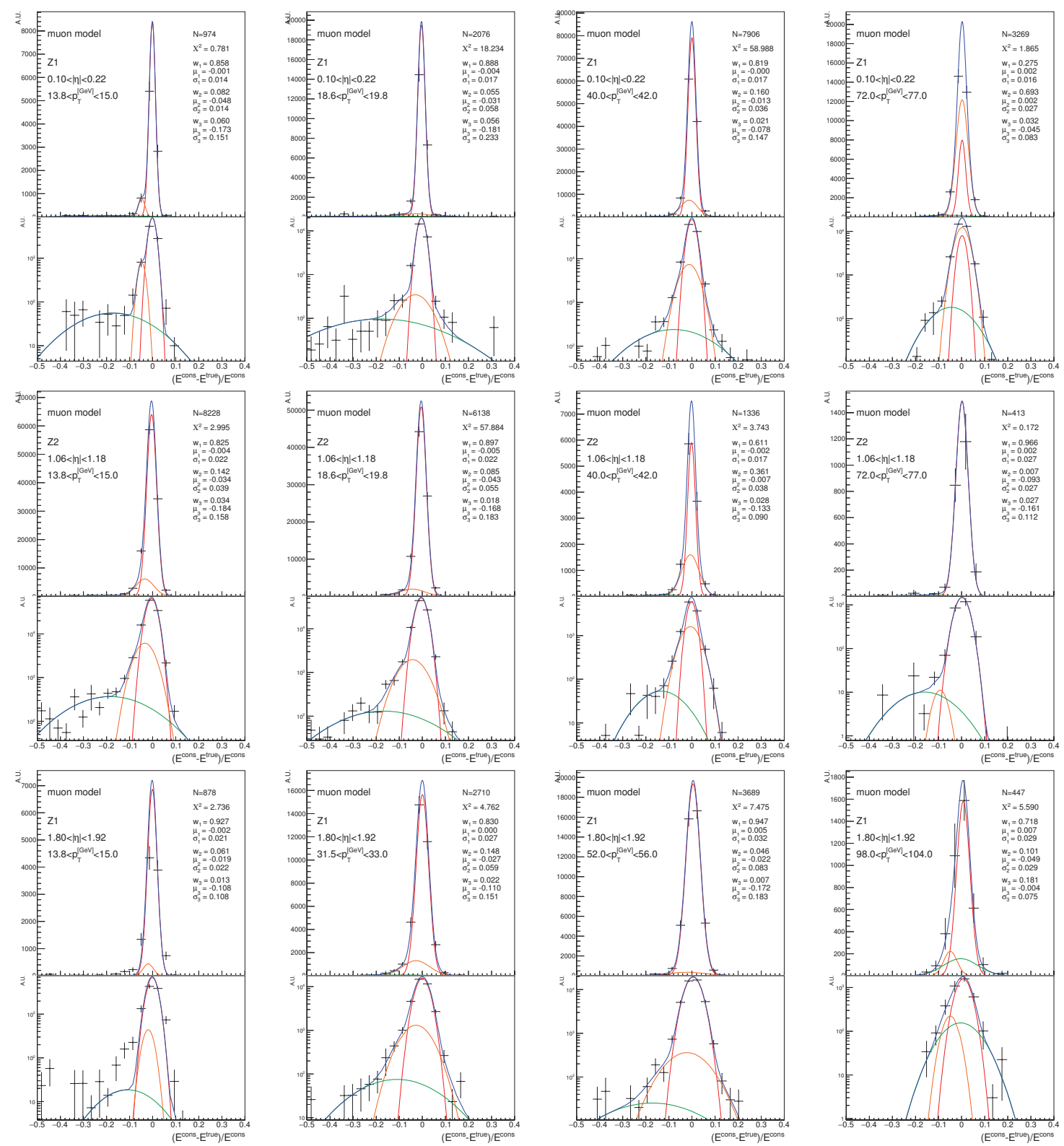

Figure 127: Example fits of constrained muon energy response (1/2). The distributions are built from MC simulation (black points) fit using a sum of three Gaussians (blue curve). Each plot shows the same fit in linear and log scale. The kinematic bins and the fit parameters are printed on each plot. See Table 30 for more plots. 

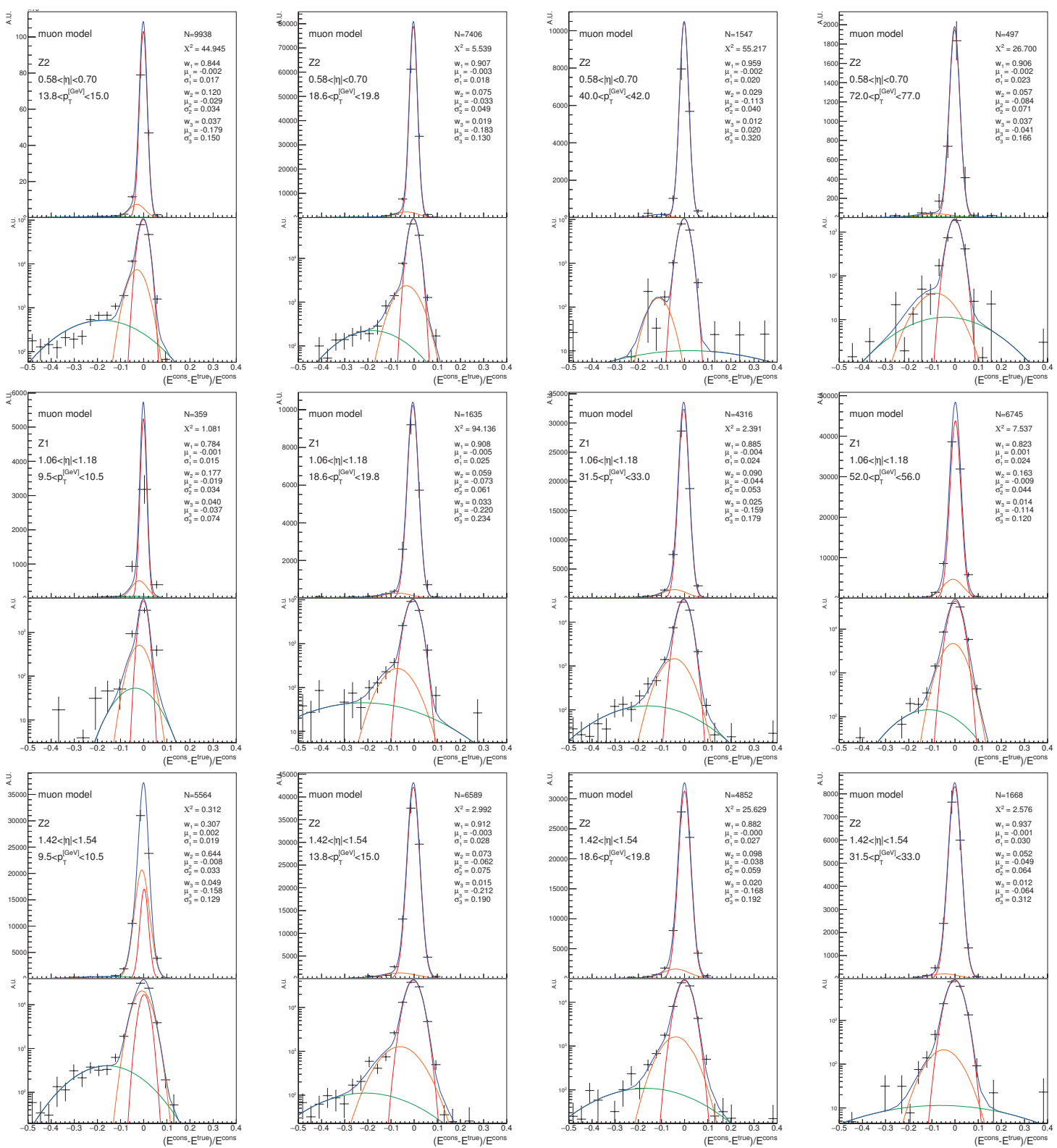

Figure 128: Example fits of constrained muon energy response (2/2). The distributions are built from MC simulation (black points) fit using a sum of three Gaussians (blue curve). Each plot shows the same fit in linear and log scale. The kinematic bins and the fit parameters are printed on each plot. See Table 30 for more plots. 

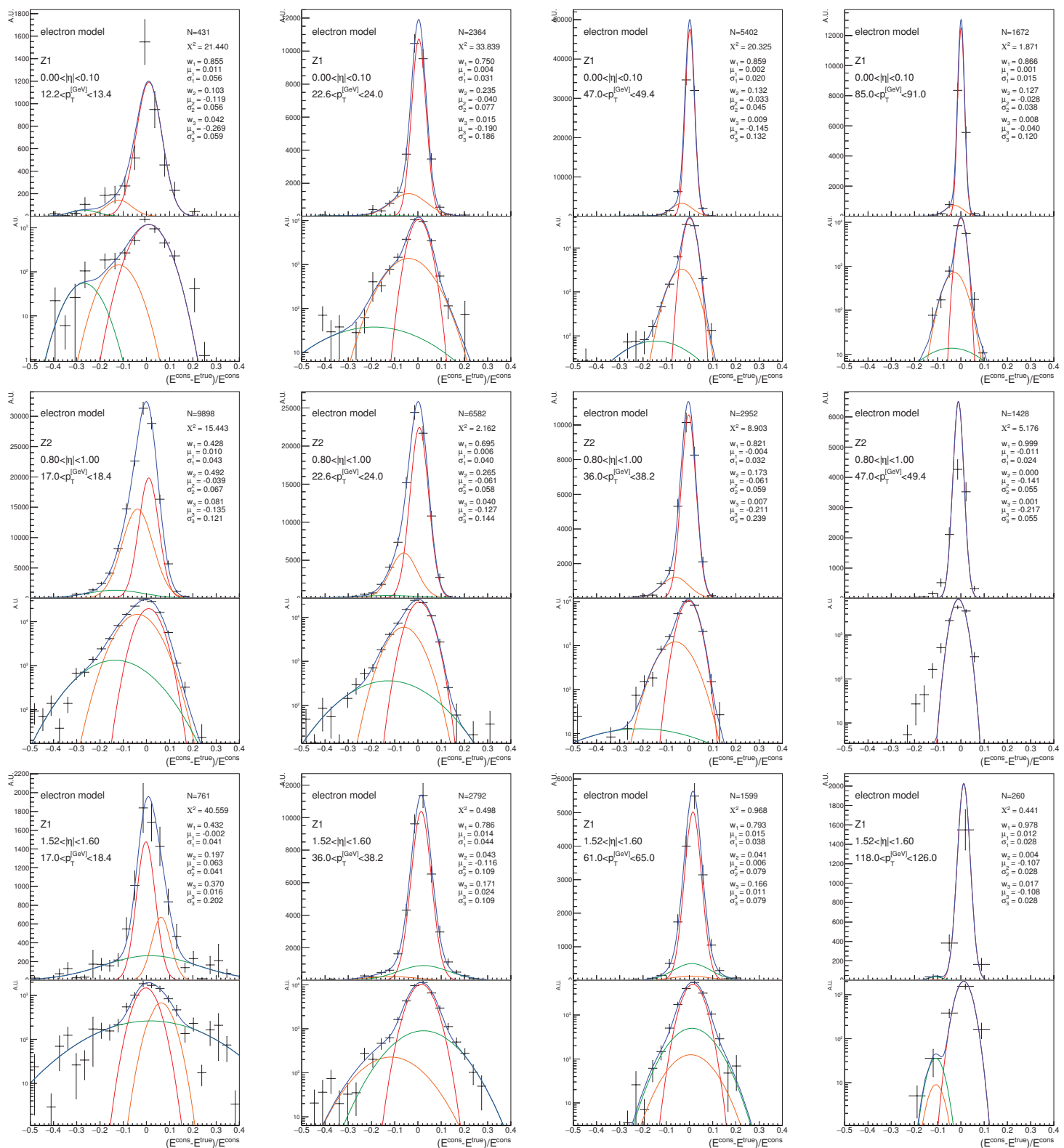

Figure 129: Example fits of constrained electron energy response (1/2). The distributions are built from MC simulation (black points) fit using a sum of three Gaussians (blue curve). Each plot shows the same fit in linear and log scale. The kinematic bins and the fit parameters are printed on each plot. See Table 30 for more plots. 

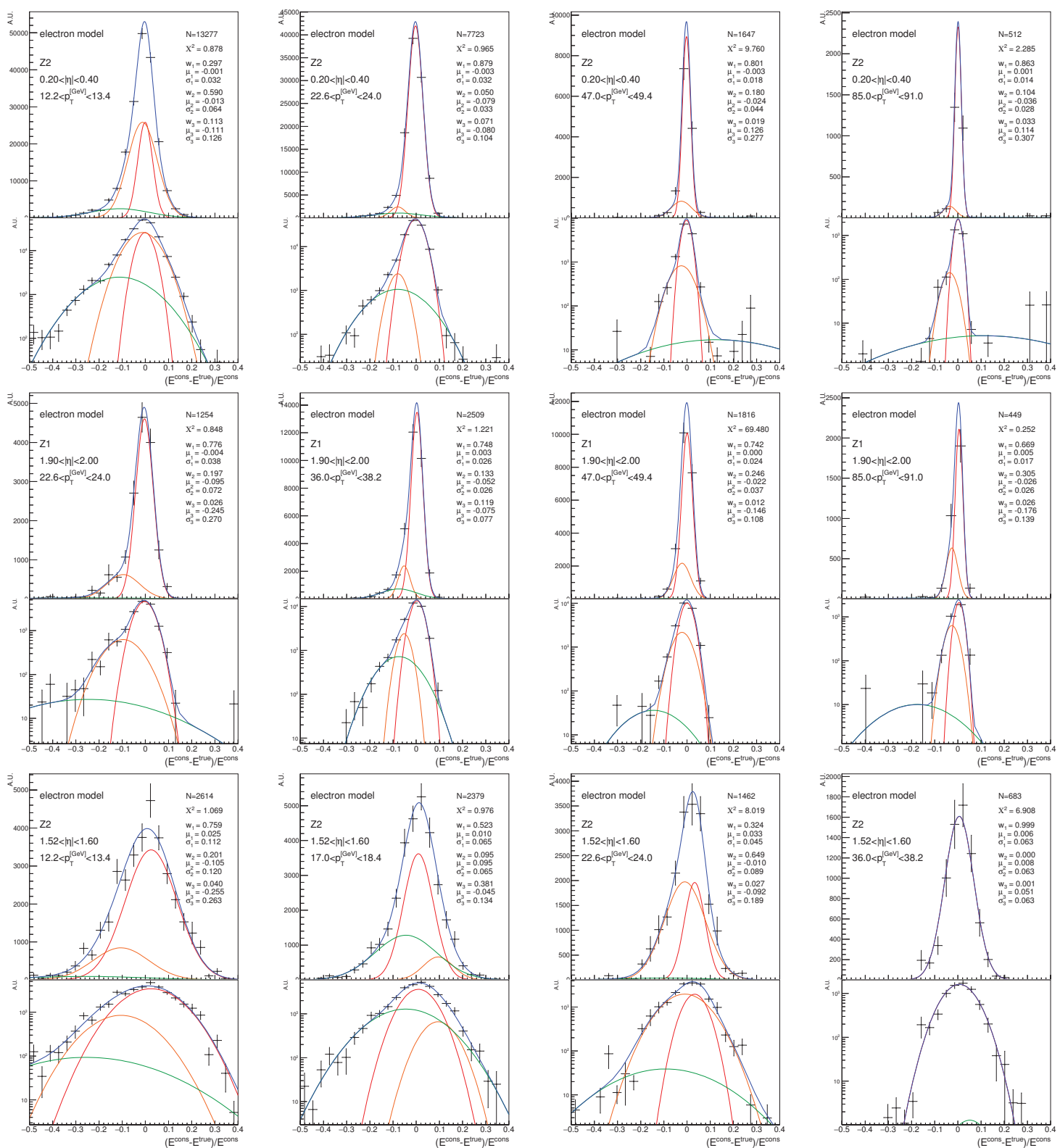

Figure 130: Example fits of constrained electron energy response (2/2). The distributions are built from MC simulation (black points) fit using a sum of three Gaussians (blue curve). Each plot shows the same fit in linear and log scale. The kinematic bins and the fit parameters are printed on each plot. See Table 30 for more plots. 


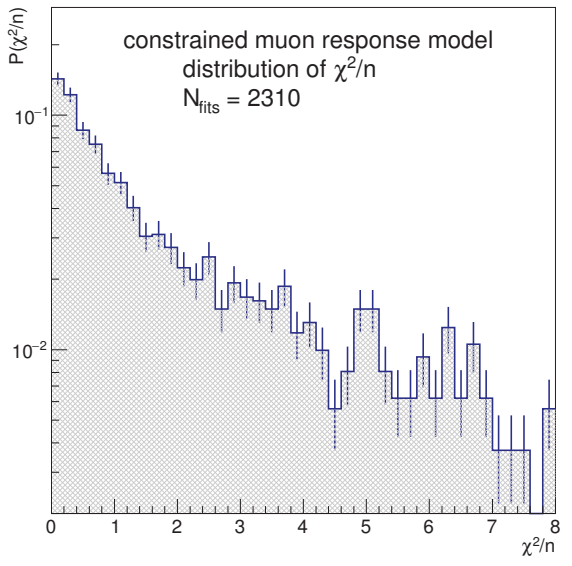

(a) muons

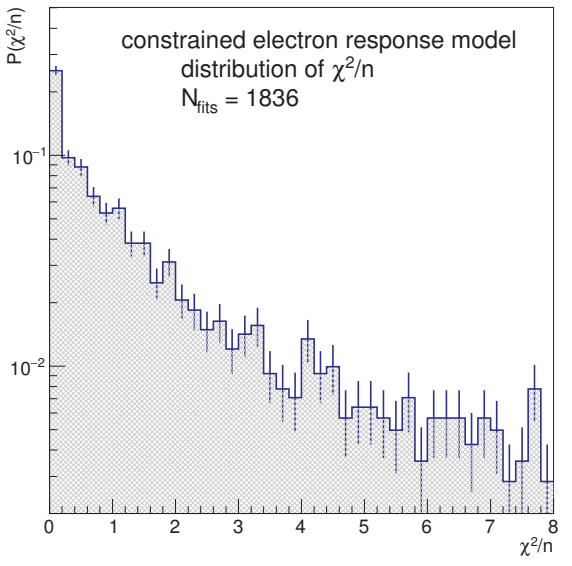

(b) electrons

Figure 131: Distribution of $\chi^{2} / n$ from constrained lepton response model fits. 


\section{B.4 Response model parameters}

This section presents how the parameters of $P\left(x_{i}\right)$ (defined in (83)) describing the lepton response PDF depend on selected Z, $|\eta|$, and $p_{T}$. Parameter $\sigma_{1}$ is the most easily recognized as the lepton energy resolution, while the others characterize additional properties of the response distribution and can't be so easily interpreted in isolation. Table 31 summarizes where to find each of the parameters for two models - with Z Mass Constraint ("constrained") and without ("unconstrained").

Note that the parameter $w_{2}$ contained in these plots is a recursive weight, while in (83) it is absolute (meaning $\left.w_{2}^{\text {plotted }}=w_{2}^{(83)} /\left(1-w_{1}\right)\right)$ ). The parameter $w_{1}$ is unaffected by this definition. The $p_{T}$ range used in these plots is reduced as compared to the full model coverage to emphasize the region where most $H \rightarrow 4 \ell$ leptons are reconstructed.

Table 31: Reference table for response model plots for each of the parameters of (83) for model with and without Z Mass Constraint.

\begin{tabular}{ccc}
\hline parameter & unconstrained model & constrained model \\
\hline$\sigma_{1}$ & Figure 132 & Figure 140 \\
$\sigma_{2}$ & Figure 133 & Figure 141 \\
$\sigma_{3}$ & Figure 134 & Figure 142 \\
$\mu_{1}$ & Figure 135 & Figure 143 \\
$\mu_{2}$ & Figure 136 & Figure 144 \\
$\mu_{3}$ & Figure 137 & Figure 145 \\
$w_{1}$ & Figure 138 & Figure 146 \\
$w_{2}$ & Figure 139 & Figure 147 \\
\hline
\end{tabular}




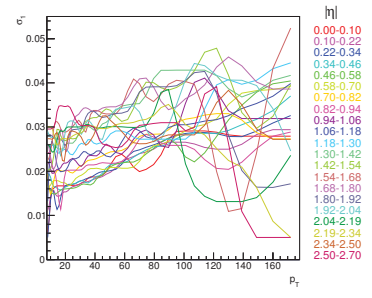

(a) muon Z1

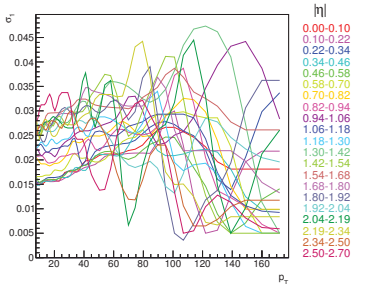

(b) muon Z2

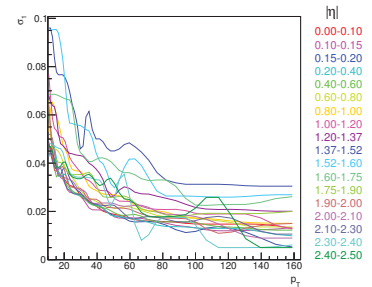

(c) electron Z1

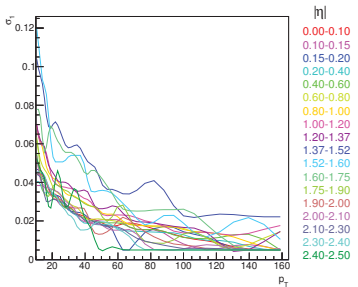

(d) electron Z2

Figure 132: Behaviour of parameter $\sigma_{1}$ in unconstrained lepton response models. See

Table 31 for other parameters.

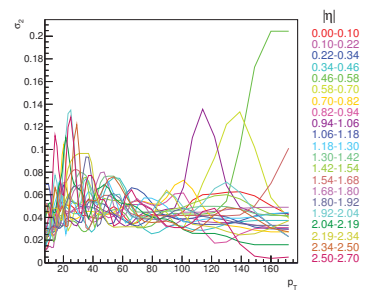

(a) muon Z1

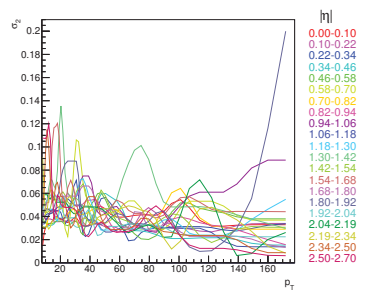

(b) muon Z2

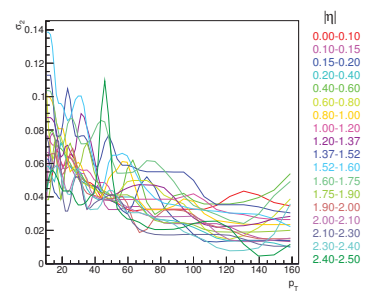

(c) electron Z1

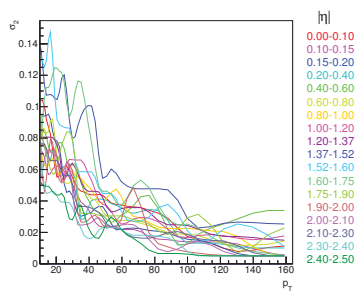

(d) electron Z2

Figure 133: Behaviour of parameter $\sigma_{2}$ in unconstrained lepton response models. See Table 31 for other parameters.

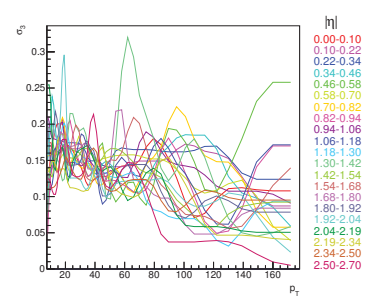

(a) muon Z1

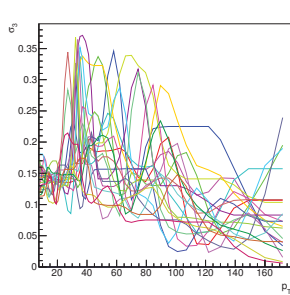

(b) muon Z2

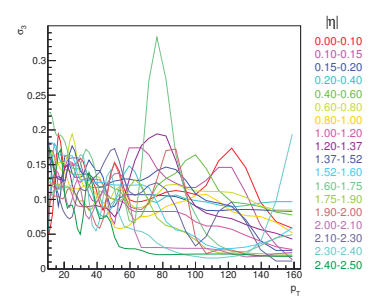

(c) electron Z1

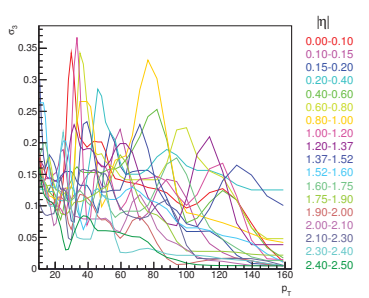

(d) electron Z2

Figure 134: Behaviour of parameter $\sigma_{3}$ in unconstrained lepton response models. See Table 31 for other parameters.

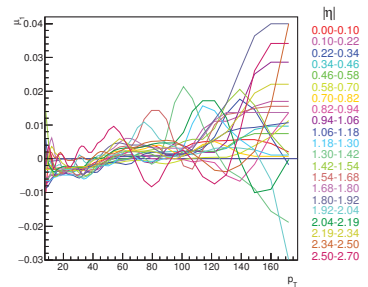

(a) muon Z1

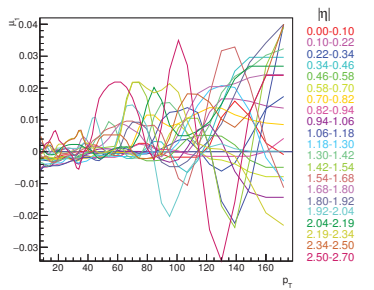

(b) muon Z2

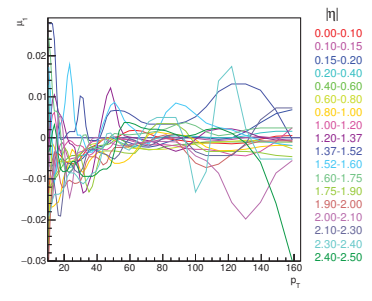

(c) electron Z1

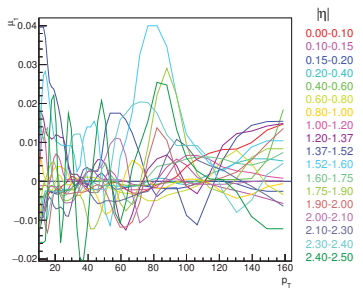

(d) electron Z2

Figure 135: Behaviour of parameter $\mu_{1}$ in unconstrained lepton response models. See

Table 31 for other parameters. 


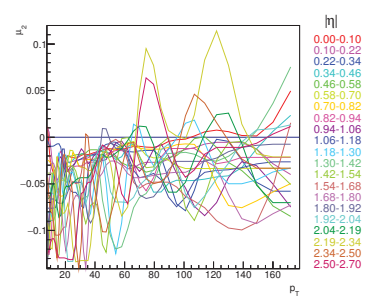

(a) muon Z1

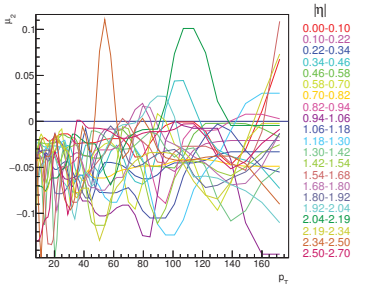

(b) muon Z2

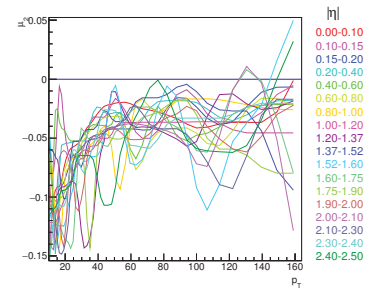

(c) electron Z1

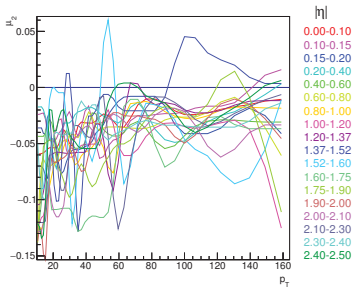

(d) electron Z2

Figure 136: Behaviour of parameter $\mu_{2}$ in unconstrained lepton response models. See

Table 31 for other parameters.

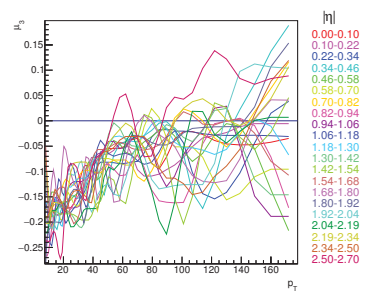

(a) muon Z1

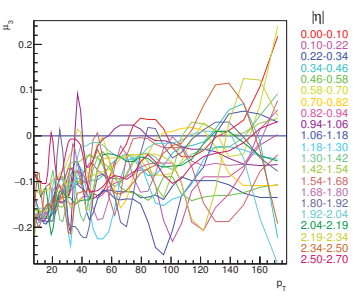

(b) muon Z2

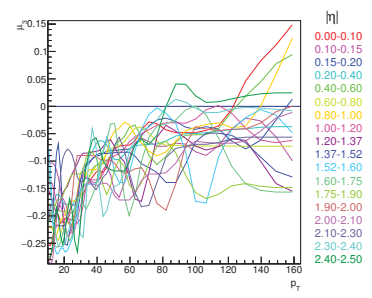

(c) electron Z1

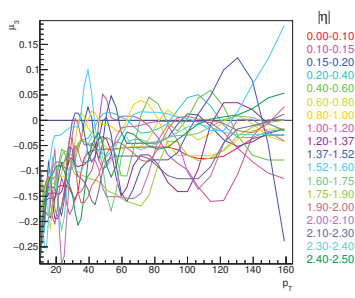

(d) electron Z2

Figure 137: Behaviour of parameter $\mu_{3}$ in unconstrained lepton response models. See Table 31 for other parameters.

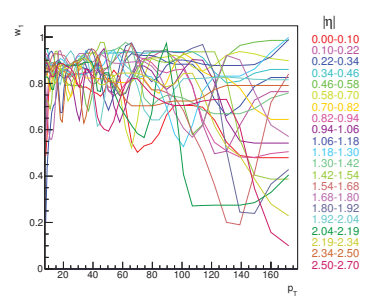

(a) muon Z1

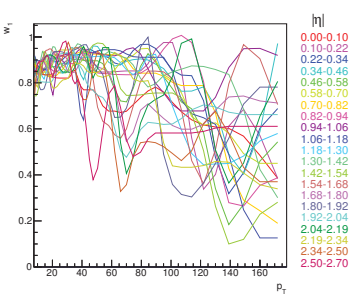

(b) muon Z2

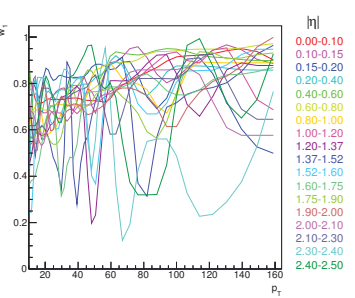

(c) electron Z1

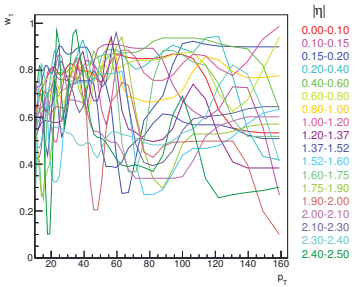

(d) electron Z2

Figure 138: Behaviour of parameter $w_{1}$ in unconstrained lepton response models. See Table 31 for other parameters.

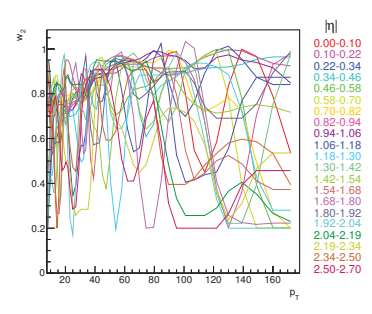

(a) muon Z1

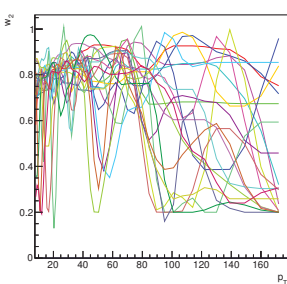

(b) muon Z2
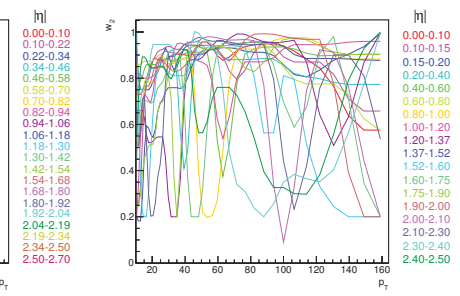

(c) electron Z1

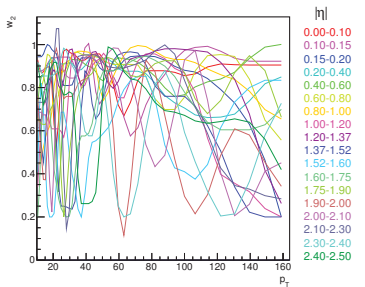

(d) electron Z2

Figure 139: Behaviour of parameter $w_{2}$ in unconstrained lepton response models. See

Table 31 for other parameters. 


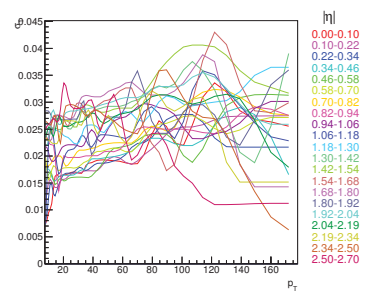

(a) muon Z1

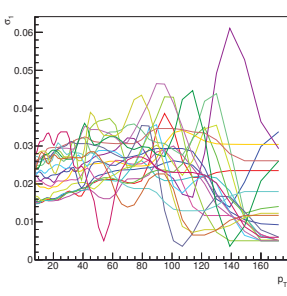

(b) muon Z2

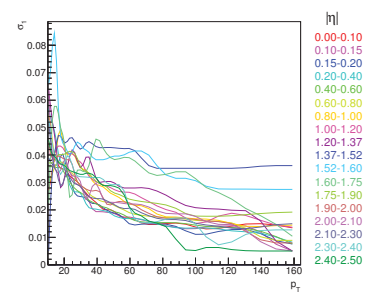

(c) electron Z1

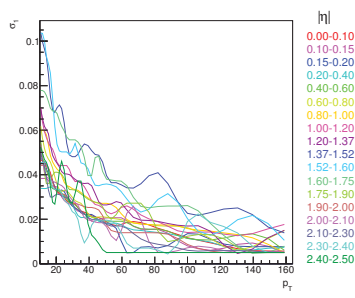

(d) electron Z2

Figure 140: Behaviour of parameter $\sigma_{1}$ in constrained lepton response models. See Table 31 for other parameters.

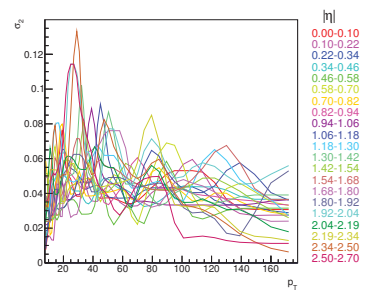

(a) muon Z1

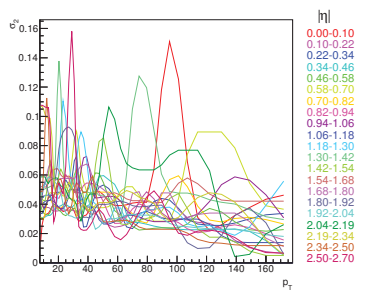

(b) muon Z2

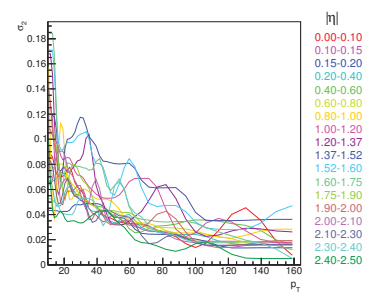

(c) electron Z1

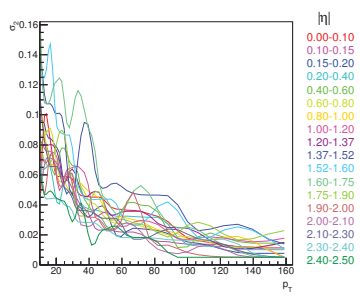

(d) electron Z2

Figure 141: Behaviour of parameter $\sigma_{2}$ in constrained lepton response models. See Table 31 for other parameters.

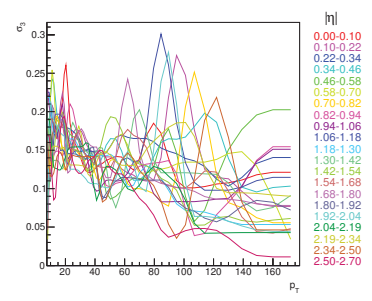

(a) muon Z1

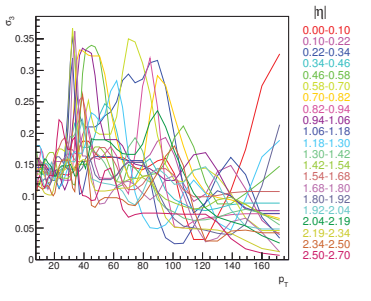

(b) muon Z2

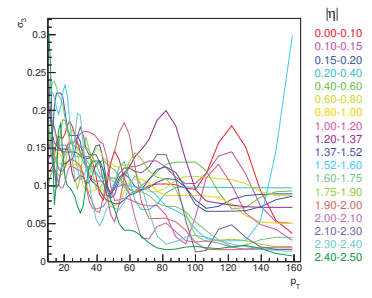

(c) electron Z1

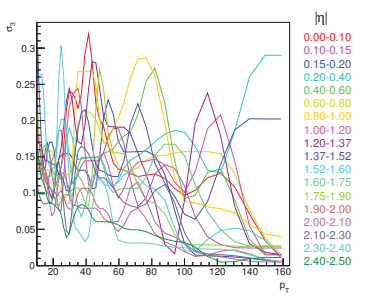

(d) electron Z2

Figure 142: Behaviour of parameter $\sigma_{3}$ in constrained lepton response models. See Table 31 for other parameters. 


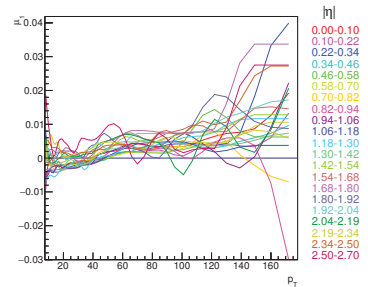

(a) muon Z1

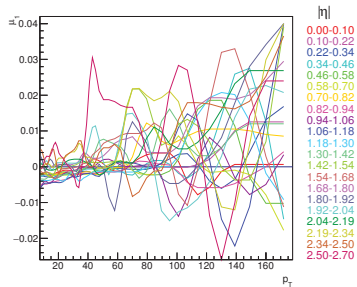

(b) muon Z2

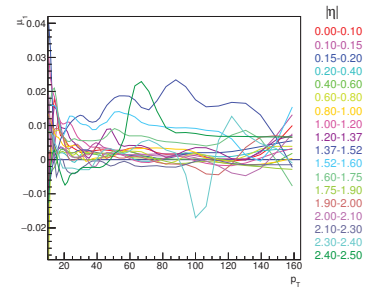

(c) electron Z1

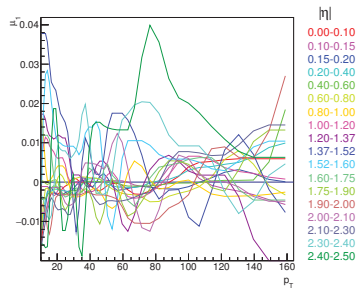

(d) electron Z2

Figure 143: Behaviour of parameter $\mu_{1}$ in constrained lepton response models. See

Table 31 for other parameters.

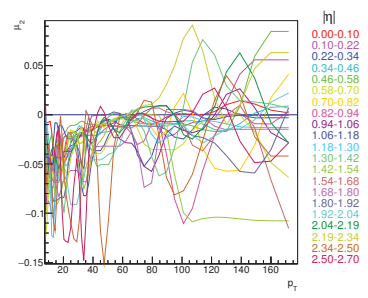

(a) muon Z1

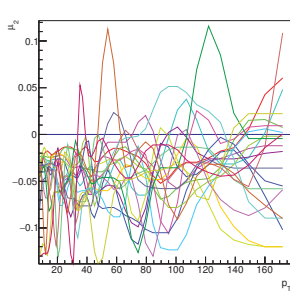

(b) muon Z2

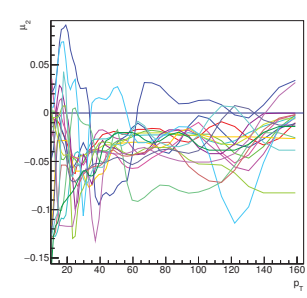

(c) electron Z1

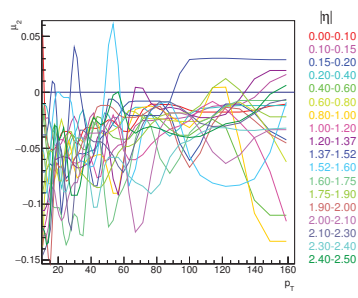

(d) electron Z2

Figure 144: Behaviour of parameter $\mu_{2}$ in constrained lepton response models. See Table 31 for other parameters.

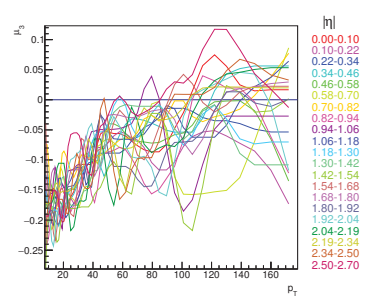

(a) muon Z1

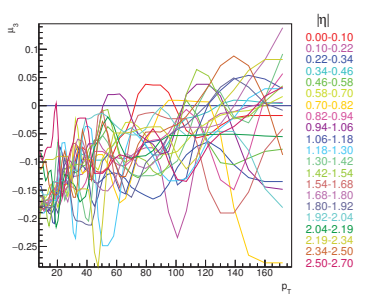

(b) muon Z2

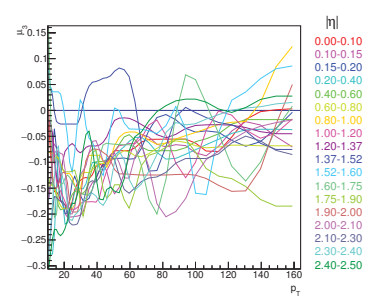

(c) electron Z1

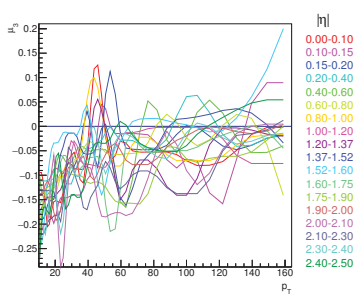

(d) electron Z2

Figure 145: Behaviour of parameter $\mu_{3}$ in constrained lepton response models. See Table 31 for other parameters.

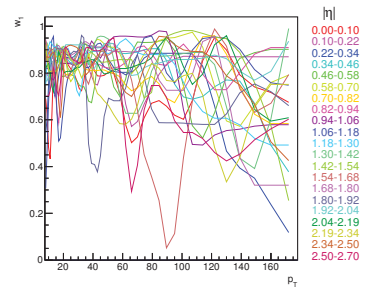

(a) muon Z1

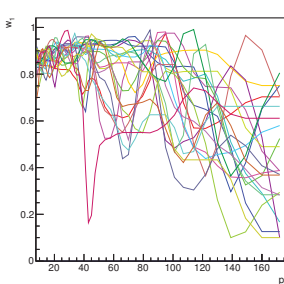

(b) muon Z2
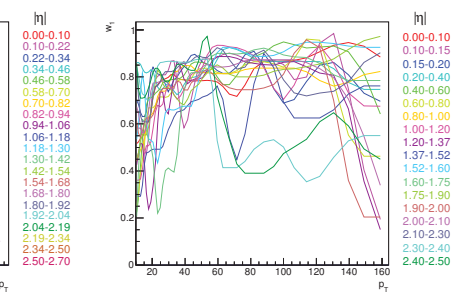

(c) electron Z1

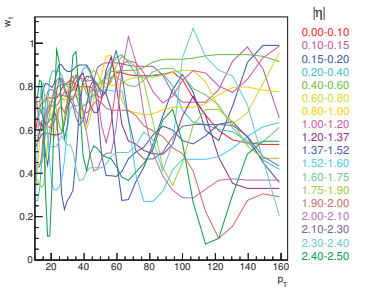

(d) electron Z2

Figure 146: Behaviour of parameter $w_{1}$ in constrained lepton response models. See

Table 31 for other parameters. 


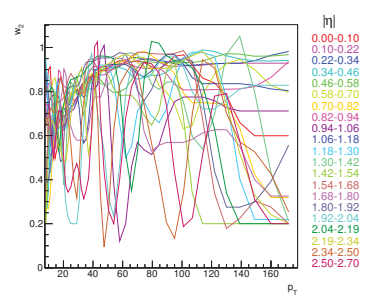

(a) muon Z1

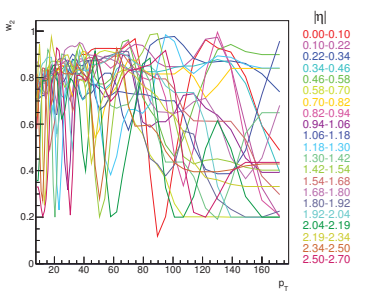

(b) muon Z2

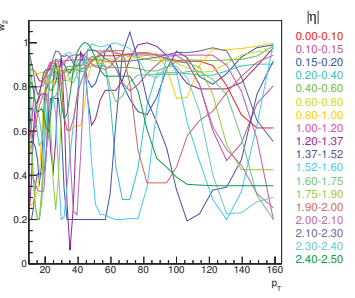

(c) electron Z1

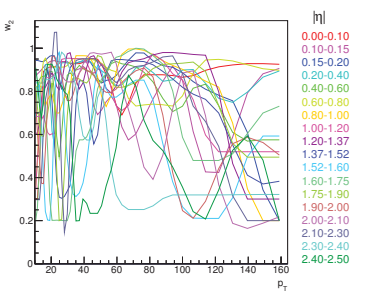

(d) electron Z2

Figure 147: Behaviour of parameter $w_{2}$ in constrained lepton response models. See Table 31 for other parameters.

\section{B.5 Model Independence of the PER signal model}

Theoretical uncertainties in general give rise to kinematic differences of the $H \rightarrow 4 \ell$ events, which will result in an altered invariant mass distribution. These can come from parton-density-function uncertainties, or more directly from calculation of the Higgs boson cross section. If the signal model does not account for these differences, a bias can be introduced. This appendix is meant to demonstrate that the Per-Event Response model is independent of any kinematic hypothesis for the signal model, and contrast it with the Template method which by definition assumes that the kinematic distributions of the events in the MC simulated samples used to built the templates are correct, and is therefore sensitive to the aforementioned theoretical uncertainties. To do so, simulated ggF samples are used in three configurations:

1. The nominal with all weights according to the SM,

2. a very hard Higgs, which is the nominal sample re-weighted by $w^{\prime}=w \times 0.03\left(p_{T}^{4 \ell} / G e V\right)$

3. a very forward Higgs, which is the nominal re-weighted by $w^{\prime}=w \times\left(y^{4 \ell}\right)$

The overall weight is arbitrary for this test, and so all samples are re-weighted to the same total cross section. The PER model, and Template model are drawn on top of these three MC configurations in Figure 148, using the $4 e$ channel as an example. 
Note that in all cases, the models are drawn "as nominal", and no fit is done. As can be seen from the plots, the Template PDF is identical in all cases; for a given $m_{H}$, its shape is fixed. However, the PER method adapts dynamically to the kinematics in each event, and succeeds at describing the shape. So any alteration of event kinematics from a new theory, or higher-order calculation, etc., will not affect the best fit mass value produced by the PER method. For this reason, it is called "model independent", depending only on accurate description of detector effects.

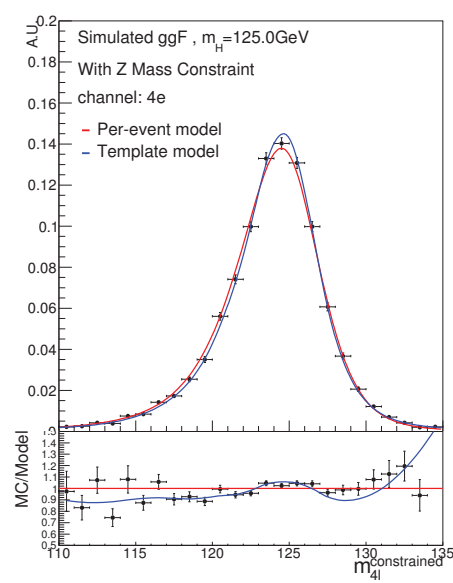

(a) nominal

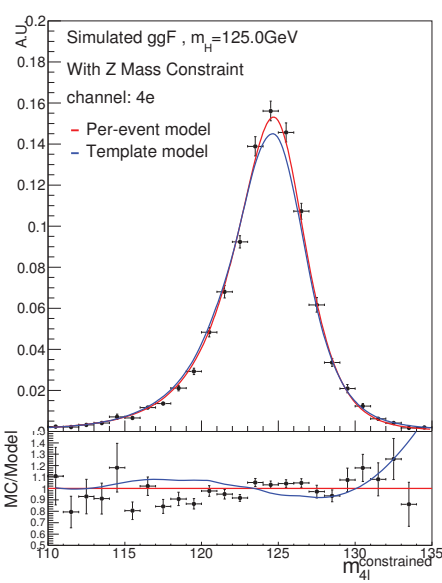

(b) very hard Higgs

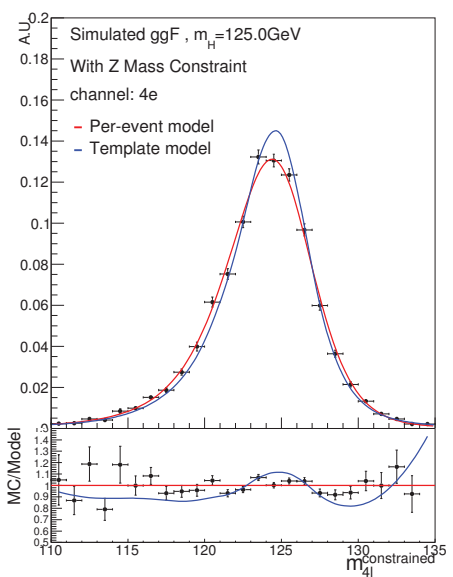

(c) very forward Higgs

Figure 148: Per-event Response model (red line) and Template model (blue line) drawn on top of $\mathrm{ggF} m_{H}=125 \mathrm{GeV}$ Higgs MC (black points) in the $4 e$ category. The bottom of each plot shows the ratio of the MC and Template model to the PER model. The three plots show the MC simulation as compared to each model, in each of the different variations described in the text. The PER model adapts dynamically to the changing kinematic distributions, while the Template model is static and fails to describe the altered distributions.

The variations presented in this appendix are not meant to be physically reasonable variations. They are extreme examples to demonstrate (i) how the reconstructed distribution depends on the kinematics of the $H \rightarrow 4 \ell$ events, and (ii) how the PER signal model automatically adapts to these kinematics to be able to describe arbitrarily altered kinematic models. This demonstration shows that the PER technique is as model-independent as possible. 


\section{Appendix $\mathrm{C}$}

\section{Statistics and Measurement Supplement}

This appendix supplements several aspects of Chapter 9. Appendix C.1 shows data distributions using $m_{4 \ell}^{\mathrm{fsr}}$. Appendix C.2 shows the data distributions with the fitted S+B model on top. Appendix C.3 shows supplementary plots of the toy experiments, presenting many of the same plots from the main body of the thesis separated by analysis category. Appendix C.4 shows the $Z \rightarrow 4 \ell$ analysis conducted using $m_{4 \ell}^{\mathrm{fsr}}$ instead of $m_{4 \ell}^{\text {cons }}$. Appendix C.5 shows the response distribution of $m_{4 \ell}^{\text {cons }}$ for every recorded data event in the $m_{H}$ fit range.

\section{C.1 Data distributions of $m_{4 \ell}^{\mathrm{fsr}}$}

The distributions in data of $m_{4 \ell}^{\mathrm{fsr}}$ are found in Figure 149, together with expectations from each source. For the signal, the unconstrained PER model is drawn with $m_{H}$ and $\sigma_{H \rightarrow 4 \ell}$ set to their best fit values. The $2 \mathrm{D}$ distributions of $m_{4 \ell}^{\mathrm{fsr}}$ and $\sigma_{\text {eff }}$ are found in Figure 150. 


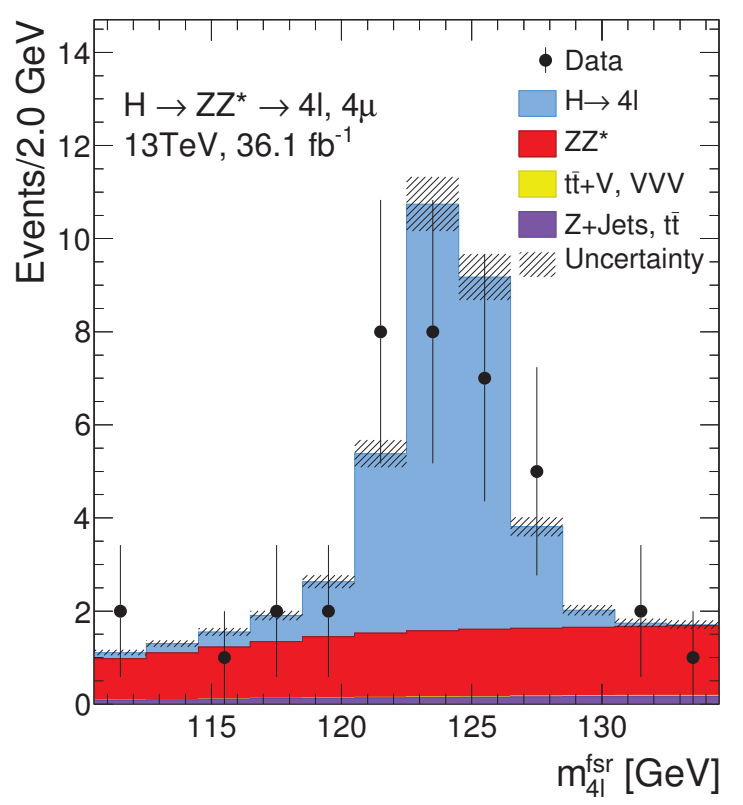

(a) $4 \mu$

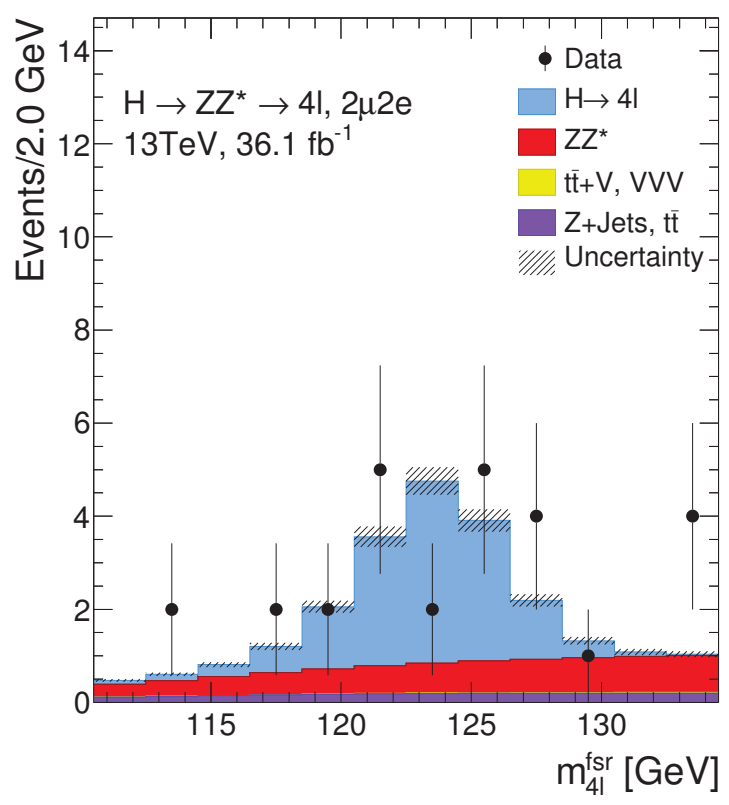

(c) $2 \mu 2 e$

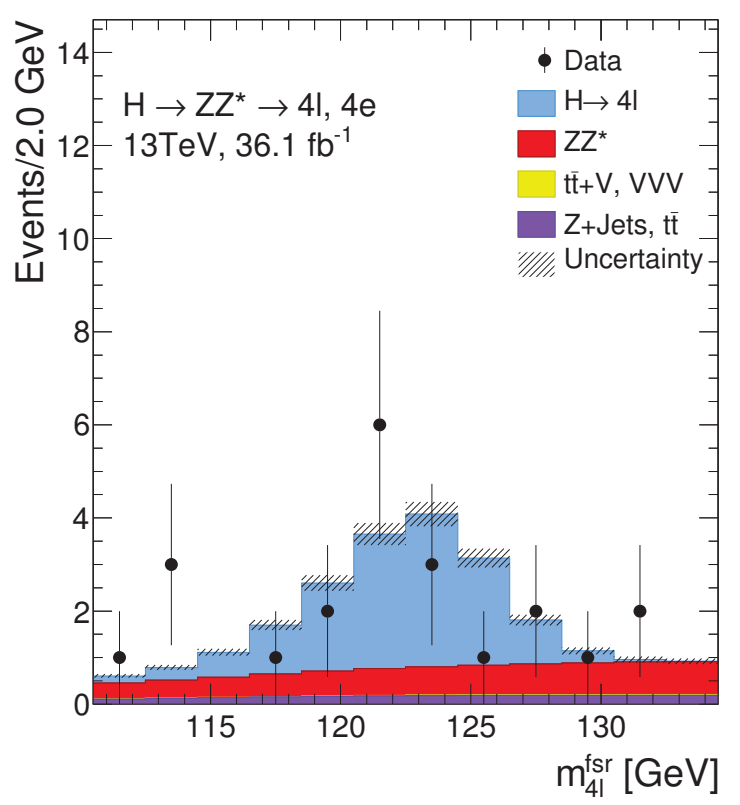

(b) $4 e$

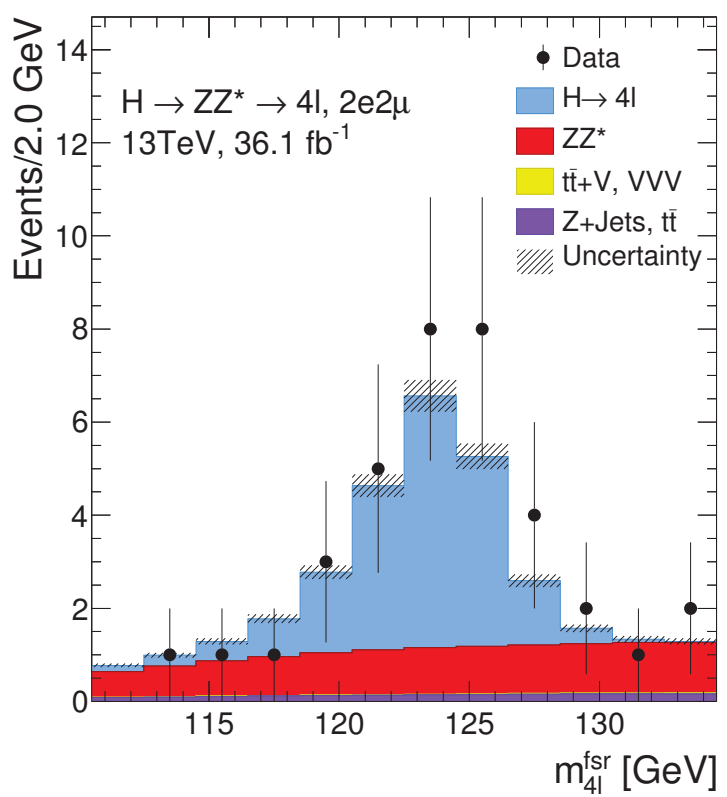

(d) $2 e 2 \mu$

Figure 149: Distribution in data of $m_{4 \ell}^{\mathrm{fsr}}$ in the four analysis categories drawn with the distribution predicted by the signalbackground model, using the constrained PER model with $m_{H}$ and $\sigma_{H \rightarrow 4 \ell}$ set to their best fit values. The plotted axis range is reduced from the fit range by $0.5 \mathrm{GeV}$ on either side to allow tidier binning. 


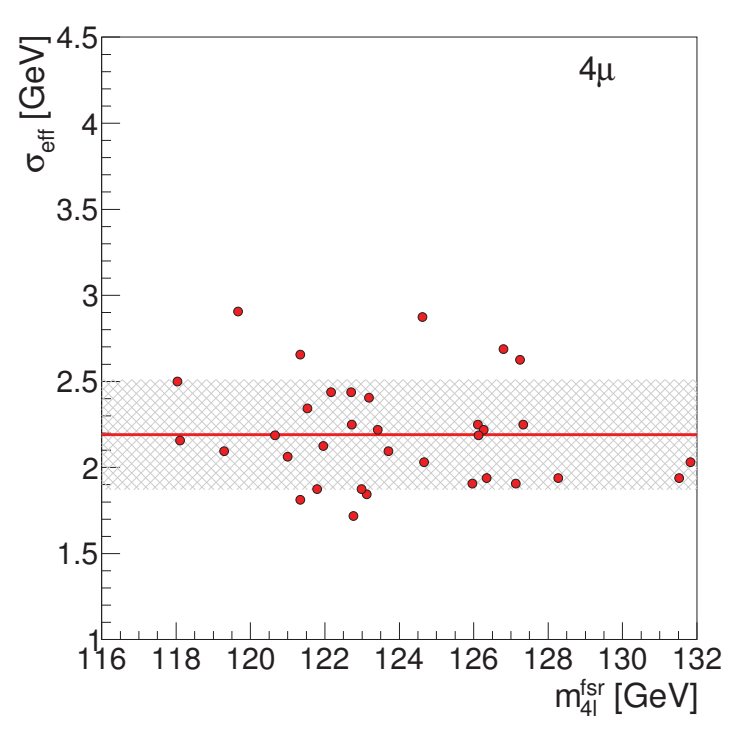

(a) $4 \mu$

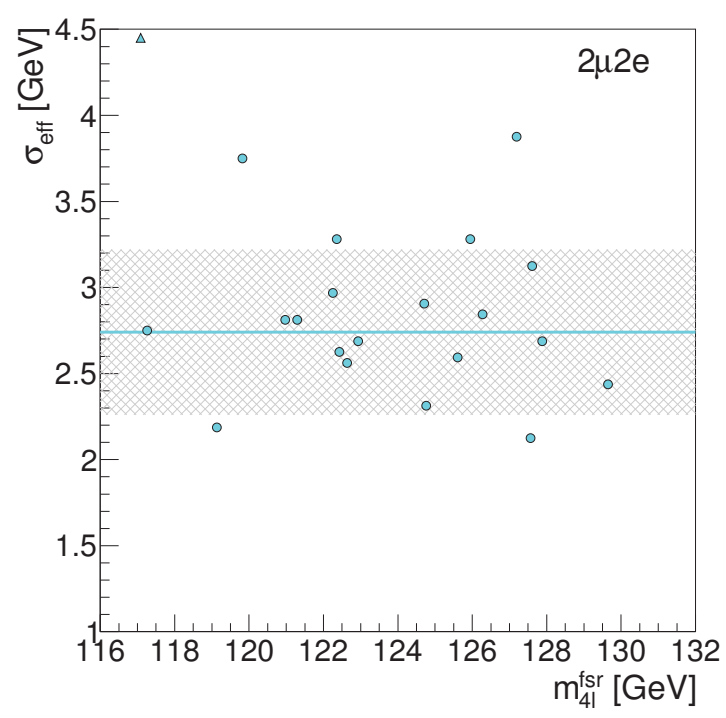

(c) $2 \mu 2 e$

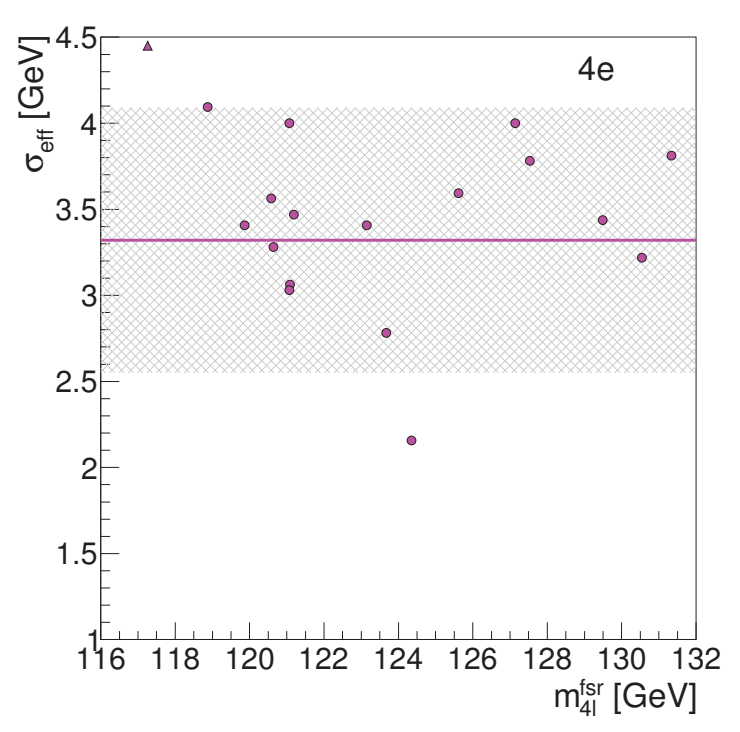

(b) $4 e$

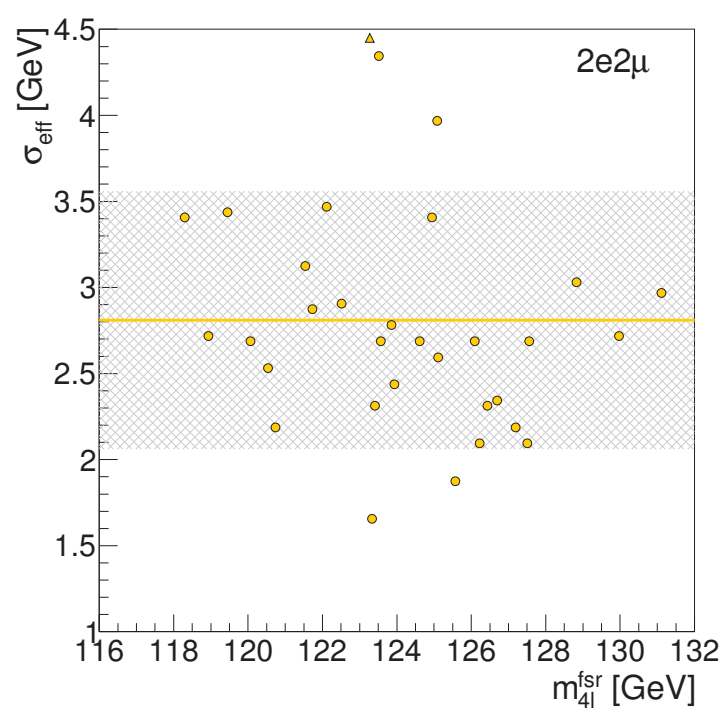

(d) $2 e 2 \mu$

Figure 150: Distribution of $m_{4 \ell}^{\mathrm{fsr}}$ vs $\sigma_{\text {eff }}$ in data. Each datum is marked by a round point. Triangles instead of points indicate the actual value is off the plotted range. The horizontal line represents the average $\sigma_{\text {eff }}$ of the events in simulation, and the hashed area represents the standard deviation of those values. Events below the line have a stronger-than-average pull on the $m_{H}$ value, while events above the line have a weaker-than-average pull on $m_{H}$. 


\section{C.2 Post-fit PDF}

The data distributions of $m_{4 \ell}^{\text {cons }}$ with the combined signal+background PDF of the constrained PER model, with $m_{H}, \sigma_{H \rightarrow 4 \ell}$ set to their final best fit values, and $\Gamma_{H}$ to its SM value, are shown in Figure 151. The corresponding plots using $m_{4 \ell}^{\mathrm{fsr}}$ and the unconstrained PER model are shown in Figure 152. 


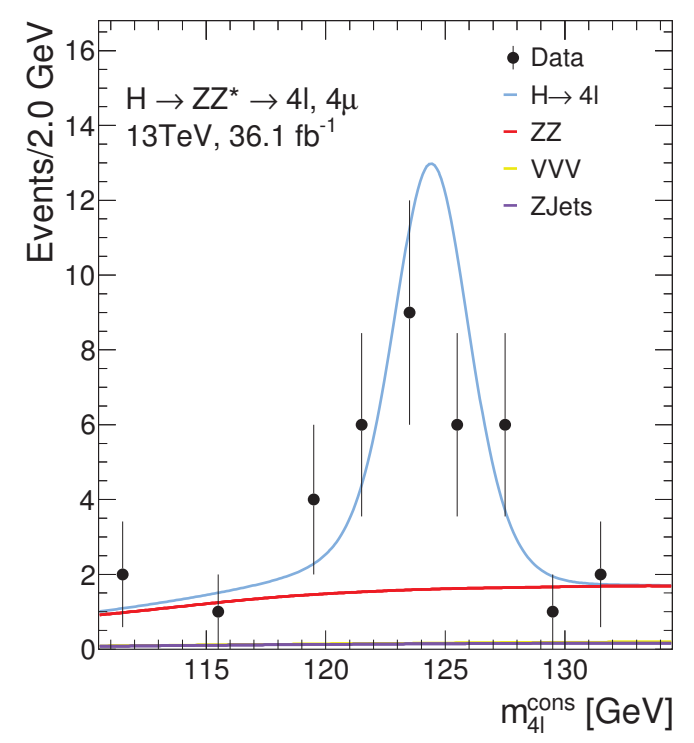

(a) $4 \mu$

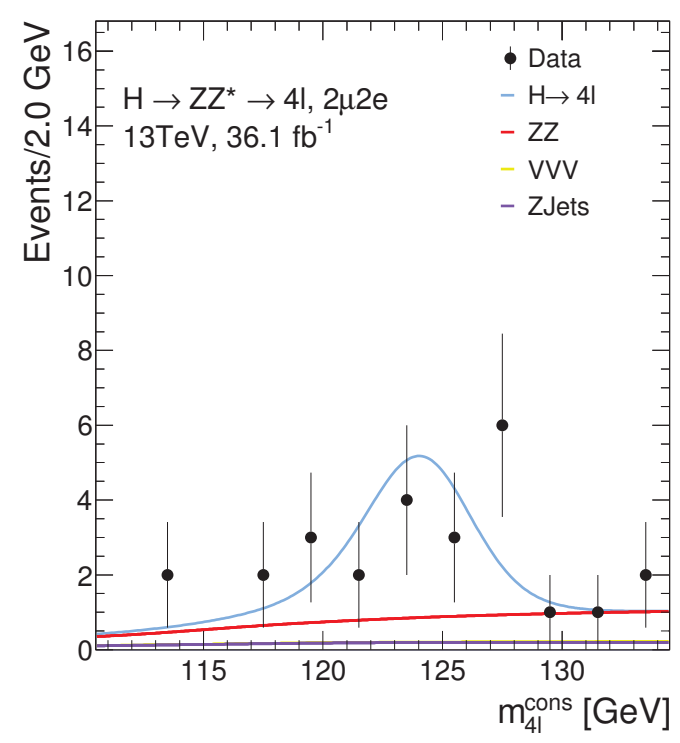

(c) $2 \mu 2 e$

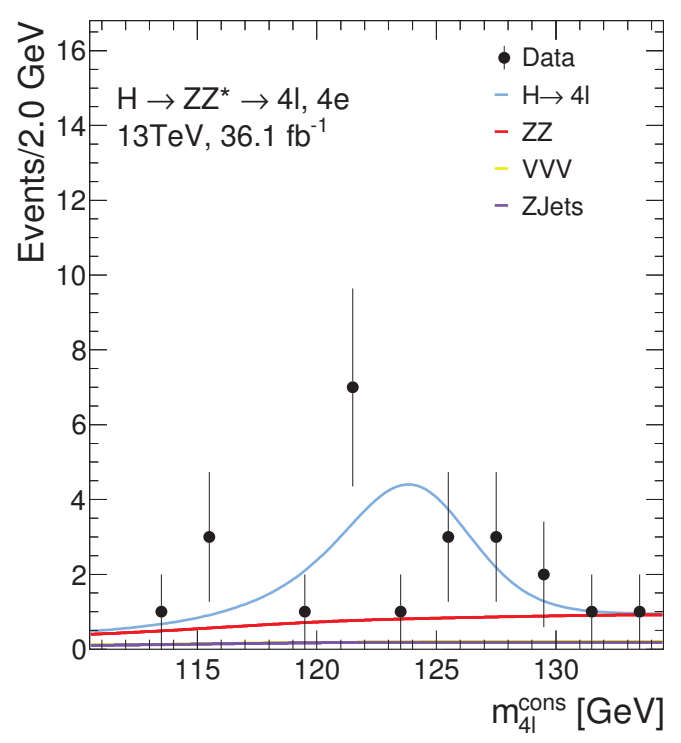

(b) $4 e$

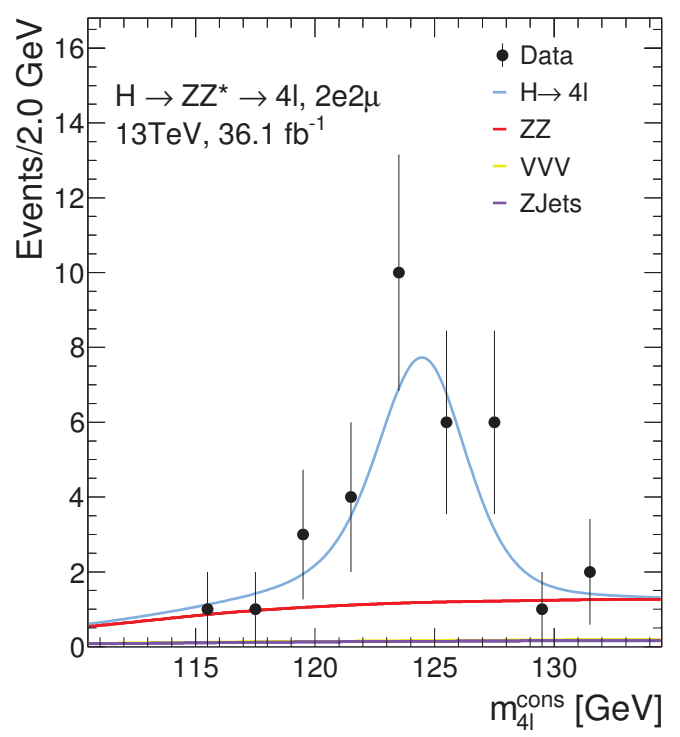

(d) $2 e 2 \mu$

Figure 151: Distribution in data of $m_{4 \ell}^{\text {cons }}$ in the four analysis categories drawn with the full signal+background model, using the constrained PER model with $m_{H}$ and $\sigma_{H \rightarrow 4 \ell}$ set to their best fit values. The plotted axis range is reduced from the fit range by $0.5 \mathrm{GeV}$ on either side to allow tidier binning. 


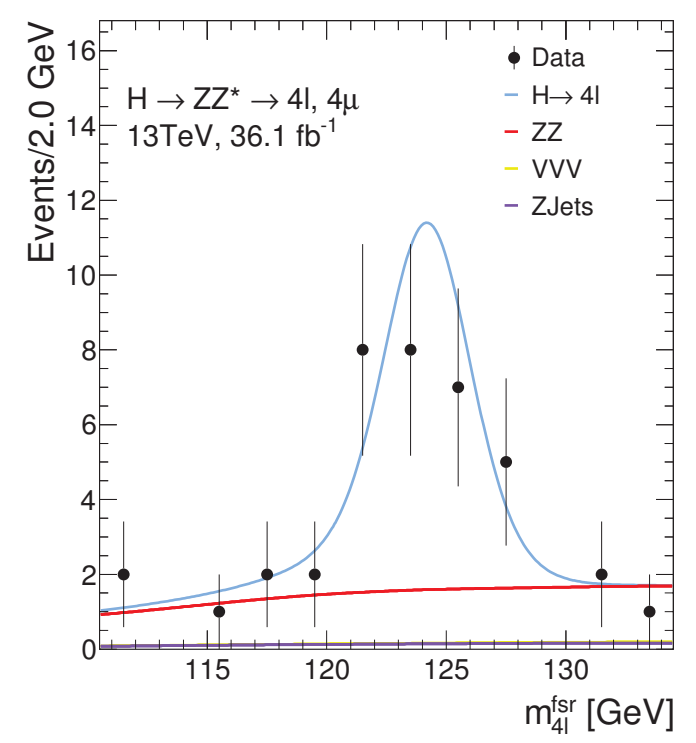

(a) $4 \mu$

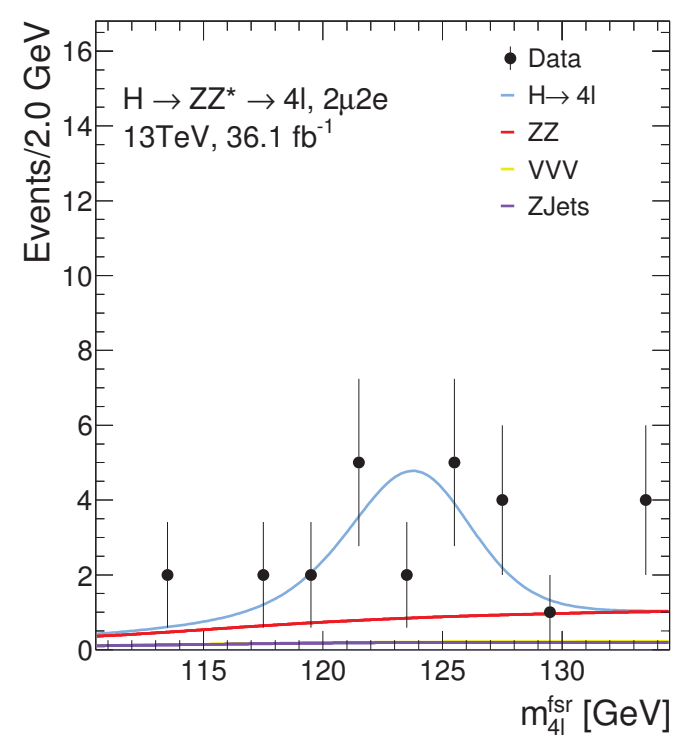

(c) $2 \mu 2 e$

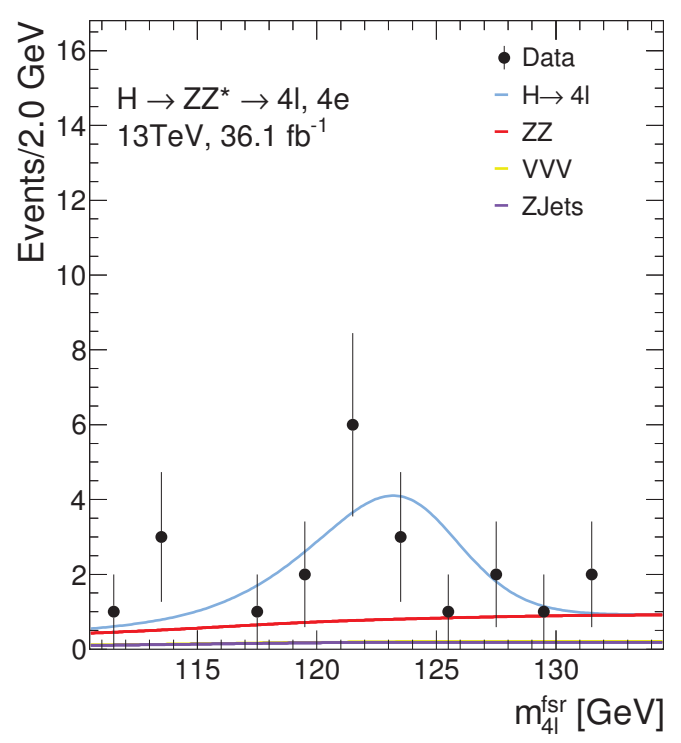

(b) $4 e$

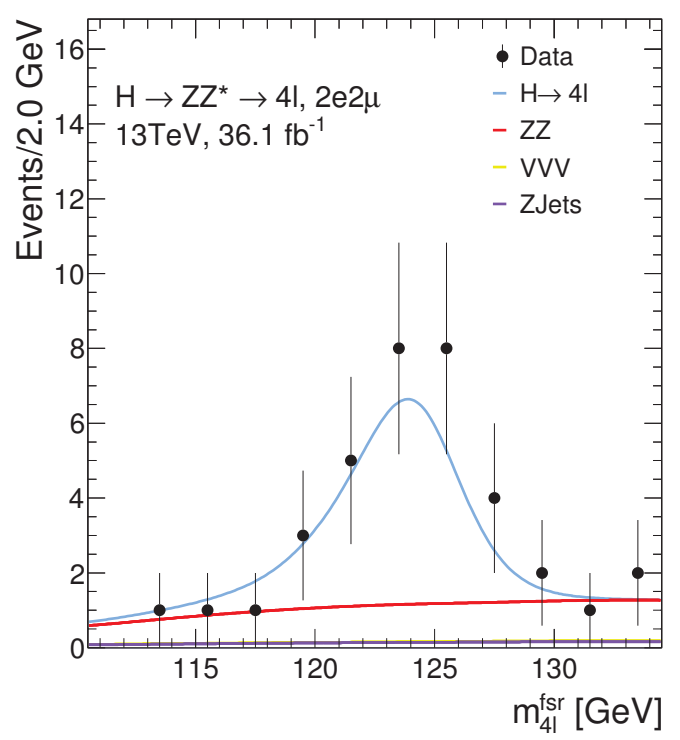

(d) $2 e 2 \mu$

Figure 152: Distribution in data of $m_{4 \ell}^{\mathrm{fsr}}$ in the four analysis categories drawn with the full signal+background model, using the unconstrained PER model with $m_{H}$ and $\sigma_{H \rightarrow 4 \ell}$ set to their best fit values. The plotted axis range is reduced from the fit range by $0.5 \mathrm{GeV}$ on either side to allow tidier binning. 


\section{C.3 More results from pseudo-experiments}

This section contains supplementary plots to Section 9.4, where tests of the three prepared methods were performed using pseudo-experiments.

To complement Figure 105, the fit results and pulls of $m_{H}$ in the toy datasets separated by category are shown for the constrained PER, Template, and unconstrained PER models in Figure 153, Figure 154, and Figure 155, respectively. Correspondingly, the per-category results of toy fits of $\sigma_{H \rightarrow 4 \ell}$, shown for all category combined in Figure 106, are shown for the same three methods in Figure 156, Figure 157, and Figure 158, respectively. The per-category results for $\Gamma_{H}$, which can only be fit using the PER technique, are shown for the constrained and unconstrained PER models in Figure 159 and Figure 160, respectively.

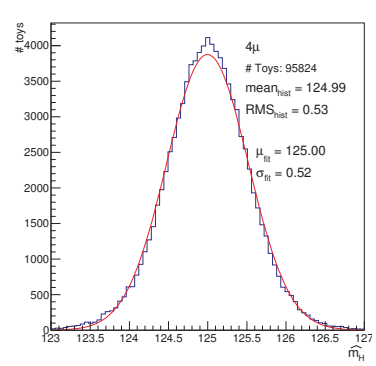

(a) $4 \mu$
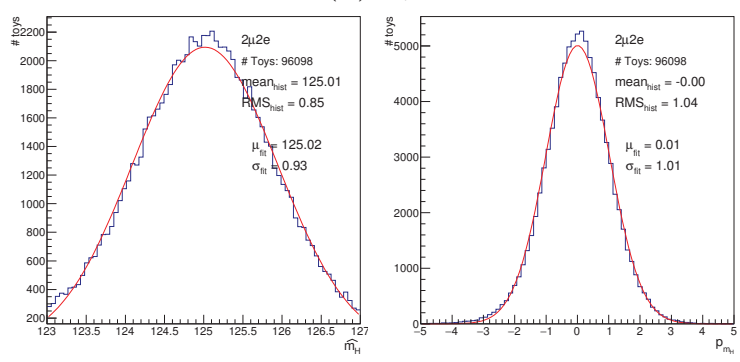

(c) $2 \mu 2 e$

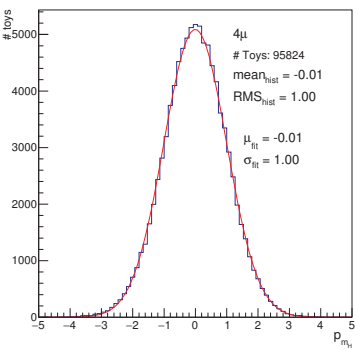

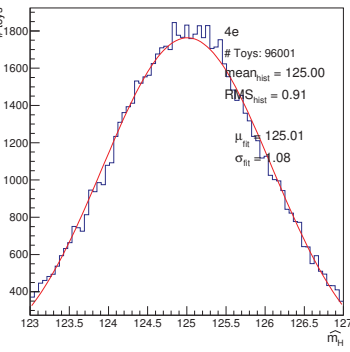

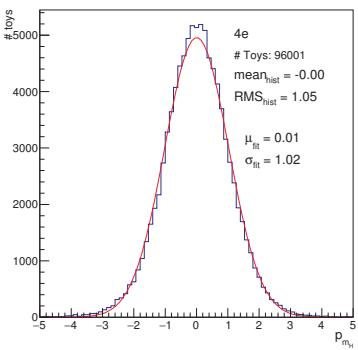

(b) $4 e$
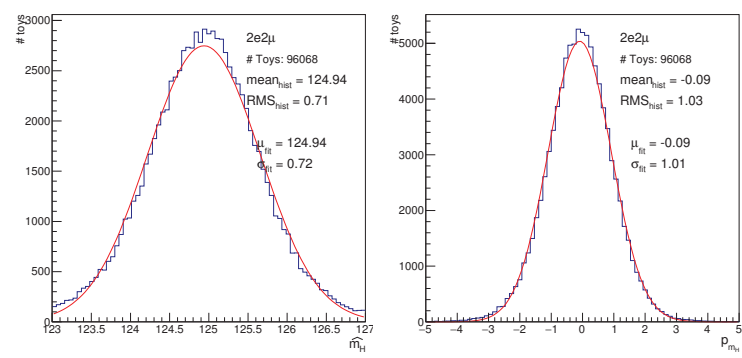

(d) $2 e 2 \mu$

Figure 153: Results of $m_{H}$ fits using the constrained PER model, per category. Shown are the best fit values $\hat{m_{H}}$ (left of each subfigure), and the pulls $p_{m_{H}}$ (right) in each category. The raw distribution of results from the toys is shown in blue, and a Gaussian fit is shown with a red line. 

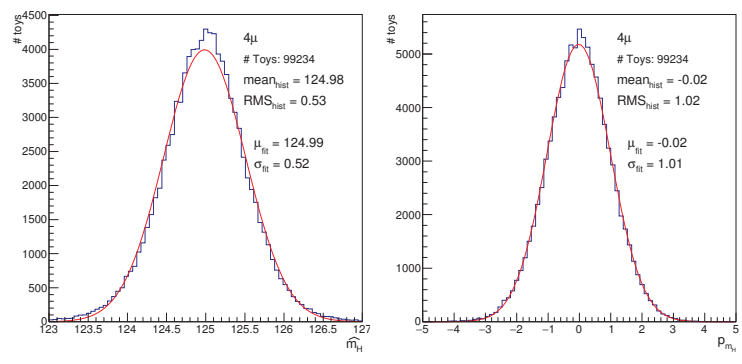

(a) $4 \mu$
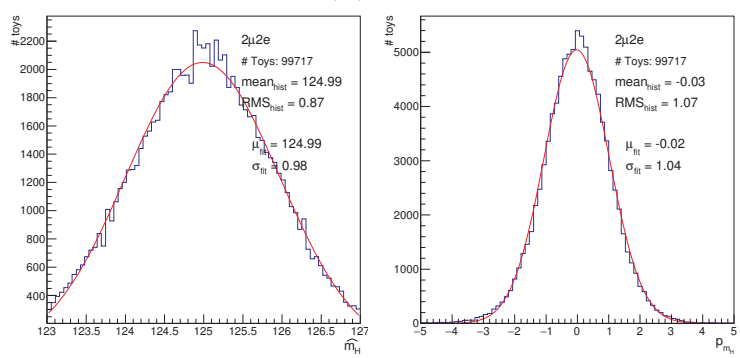

(c) $2 \mu 2 e$
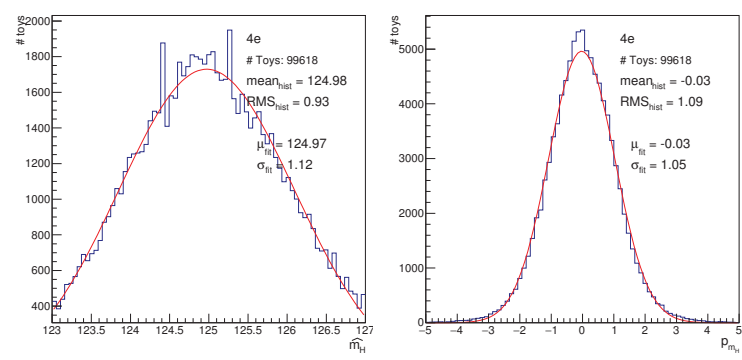

(b) $4 e$
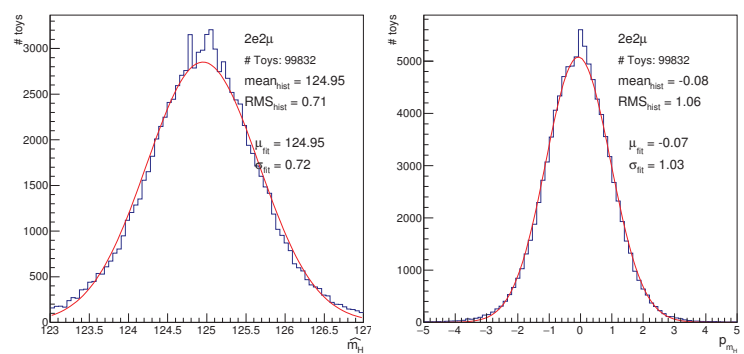

(d) $2 e 2 \mu$

Figure 154: Results of $m_{H}$ fits using the Template model, per category. Shown are the best fit values $\hat{m}_{H}$ (left of each subfigure), and the pulls $p_{m_{H}}$ (right) in each category. The raw distribution of results from the toys is shown in blue, and a Gaussian fit is shown with a red line.
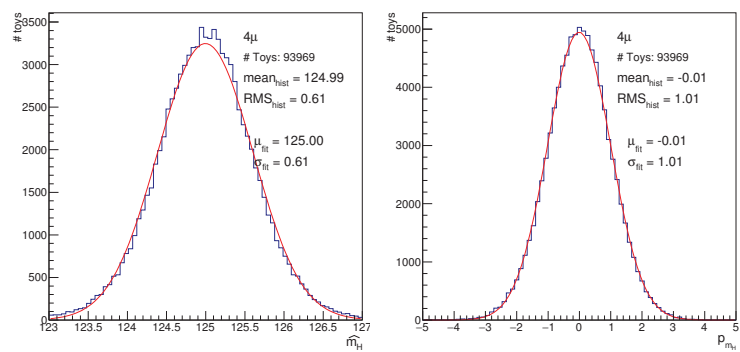

(a) $4 \mu$
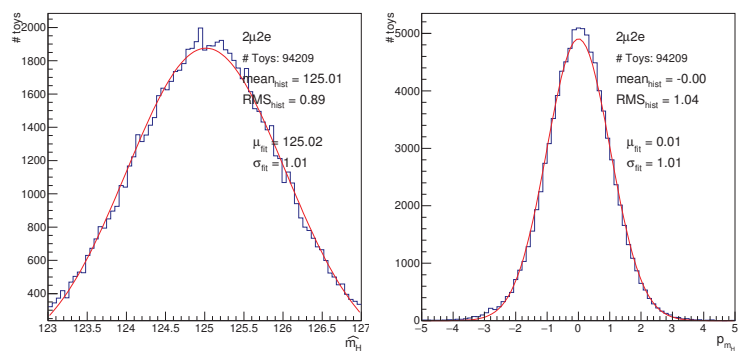

(c) $2 \mu 2 e$
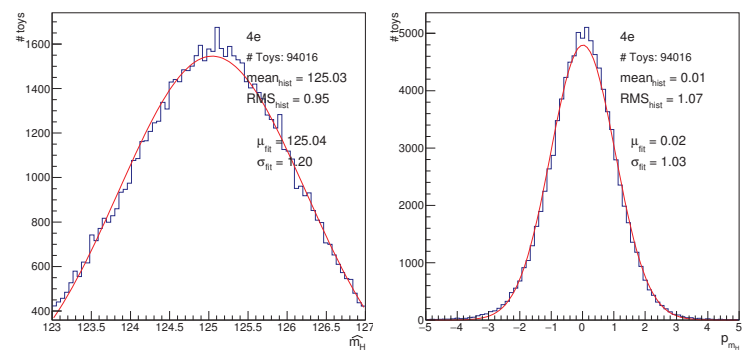

(b) $4 e$
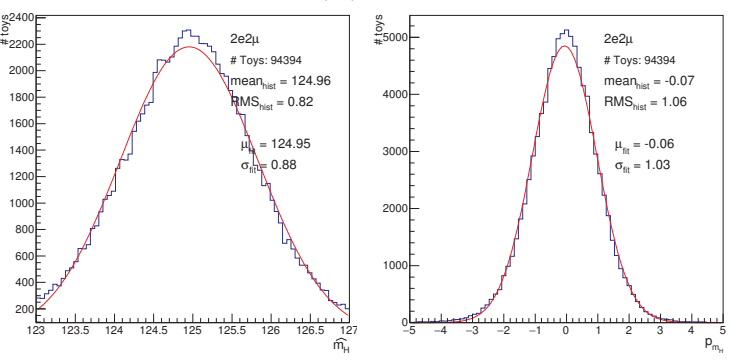

(d) $2 e 2 \mu$

Figure 155: Results of $m_{H}$ fits using the unconstrained PER model, per category. Shown are the best fit values $\hat{m}_{H}$ (left of each subfigure), and the pulls $p_{m_{H}}$ (right) in each category. The raw distribution of results from the toys is shown in blue, and a Gaussian fit is shown with a red line. 

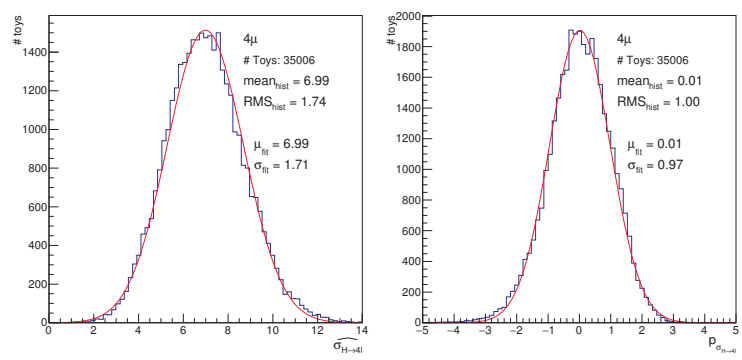

(a) $4 \mu$
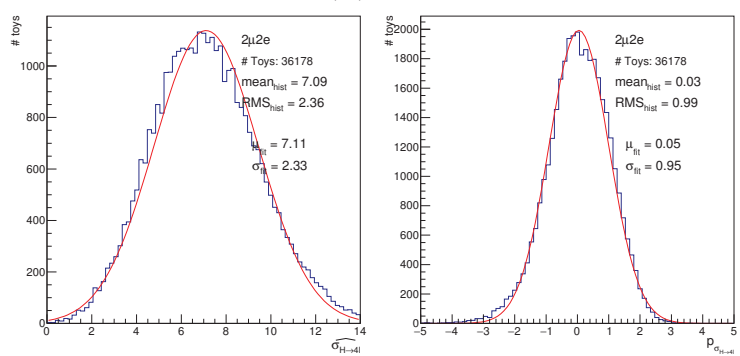

(c) $2 \mu 2 e$
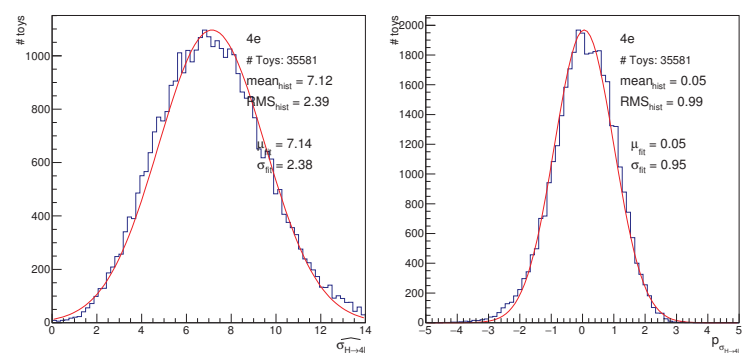

(b) $4 e$
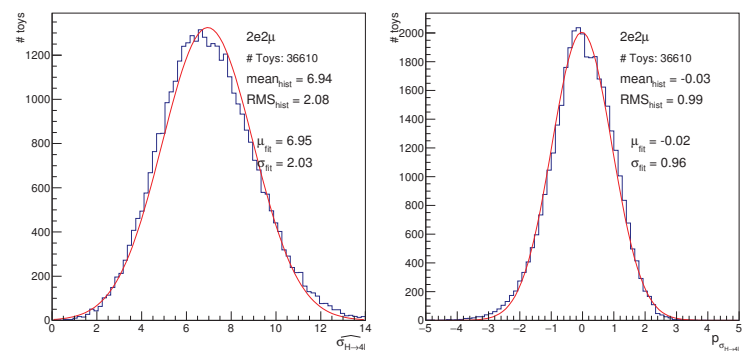

(d) $2 e 2 \mu$

Figure 156: Results of $\sigma_{H \rightarrow 4 \ell}$ fits using the constrained PER model, per category. Shown are the best fit values $\sigma_{H \rightarrow 4 \ell}$ (left of each subfigure), and the pulls $p_{\sigma_{H \rightarrow 4 \ell}}$ (right) in each category. The raw distribution of results from the toys is shown in blue, and a Gaussian fit is shown with a red line.
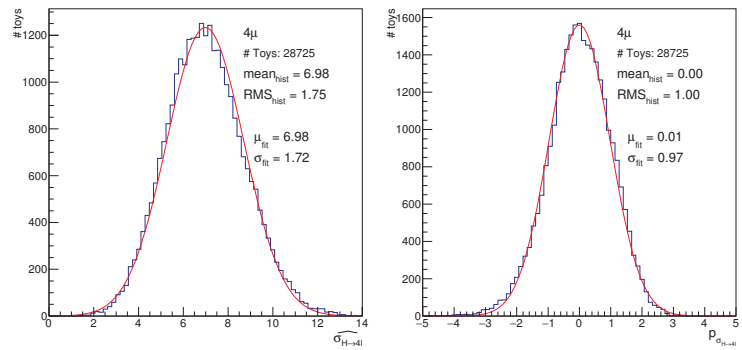

(a) $4 \mu$
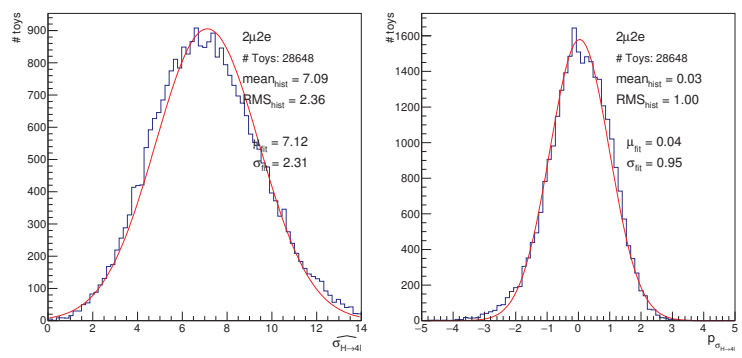

(c) $2 \mu 2 e$
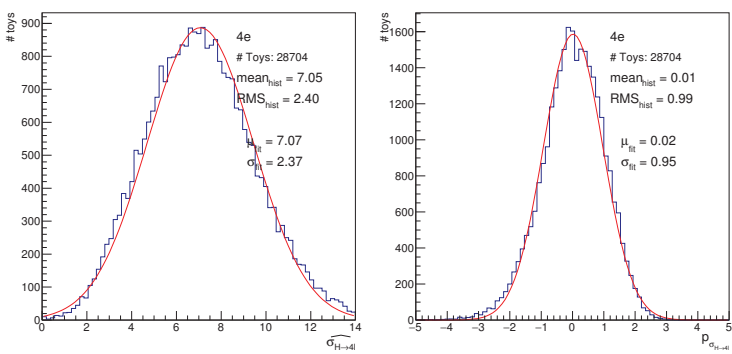

(b) $4 e$
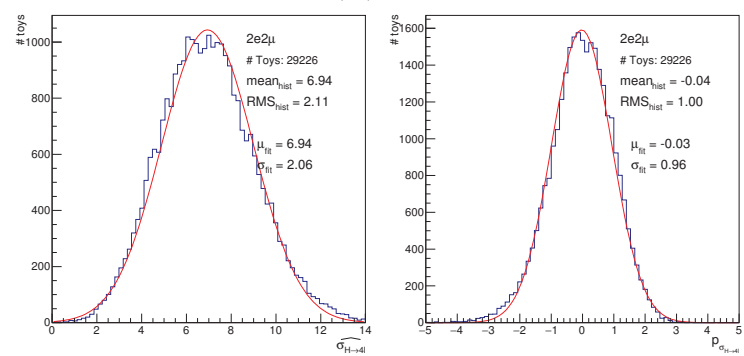

(d) $2 e 2 \mu$

Figure 157: Results of $\sigma_{H \rightarrow 4 \ell}$ fits using the Template model, per category. Shown are the best fit values $\sigma_{H \rightarrow 4 \ell}$ (left of each subfigure), and the pulls $p_{\sigma_{H \rightarrow 4 \ell}}$ (right) in each category. The raw distribution of results from the toys is shown in blue, and a Gaussian fit is shown with a red line. 

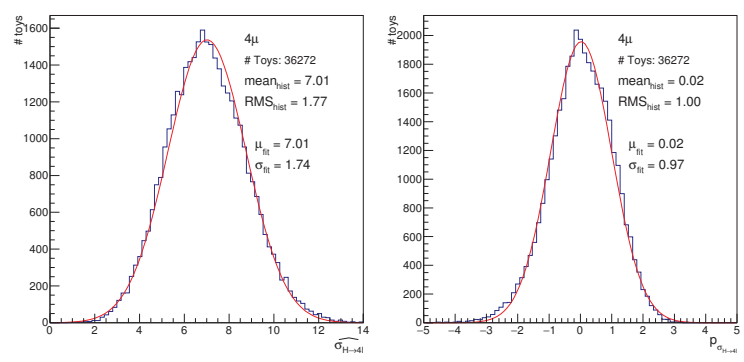

(a) $4 \mu$
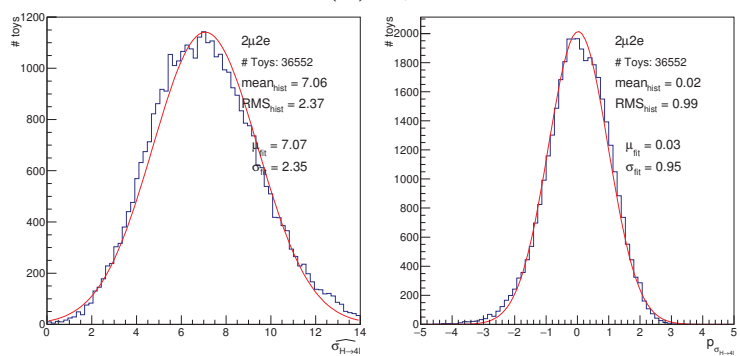

(c) $2 \mu 2 e$
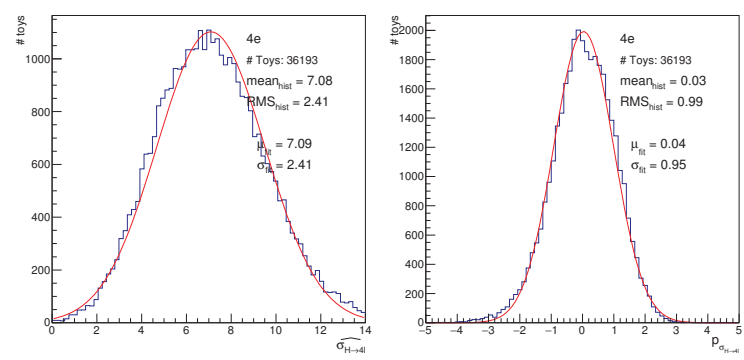

(b) $4 e$
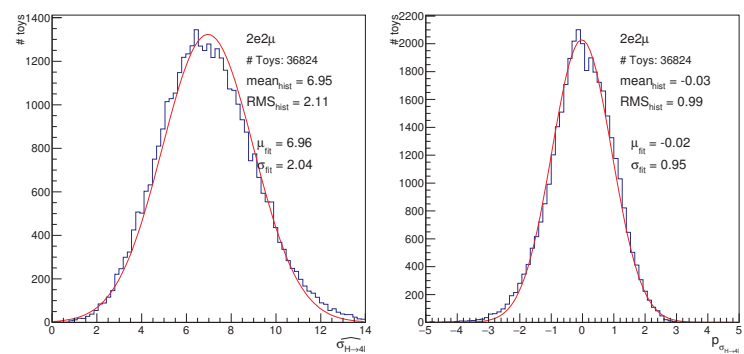

(d) $2 e 2 \mu$

Figure 158: Results of $\sigma_{H \rightarrow 4 \ell}$ fits using the unconstrained PER model, per category. Shown are the best fit values $\sigma_{H \rightarrow 4 \ell}$ (left of each subfigure), and the pulls $p_{\sigma_{H \rightarrow 4 \ell}}$ (right) in each category. The raw distribution of results from the toys is shown in blue, and a Gaussian fit is shown with a red line.
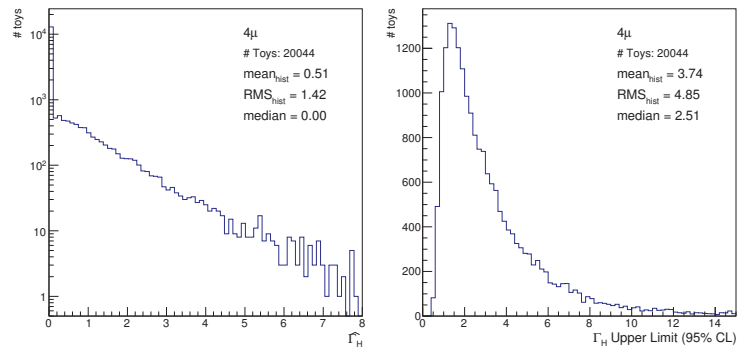

(a) $4 \mu$
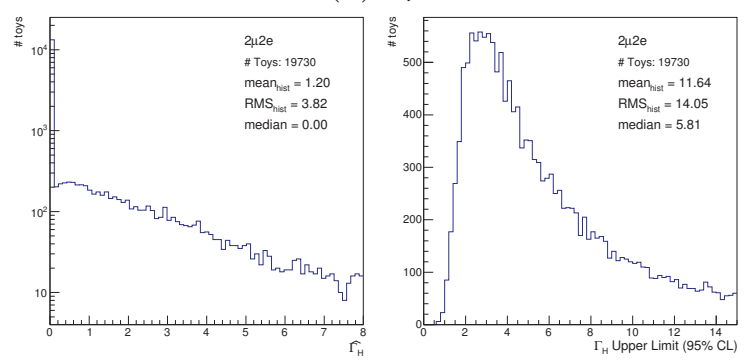

(c) $2 \mu 2 e$
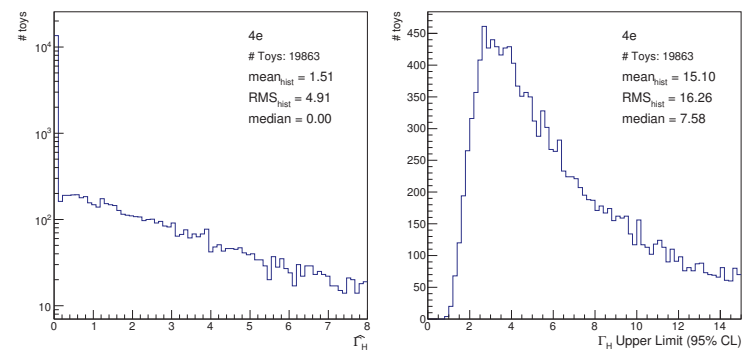

(b) $4 e$
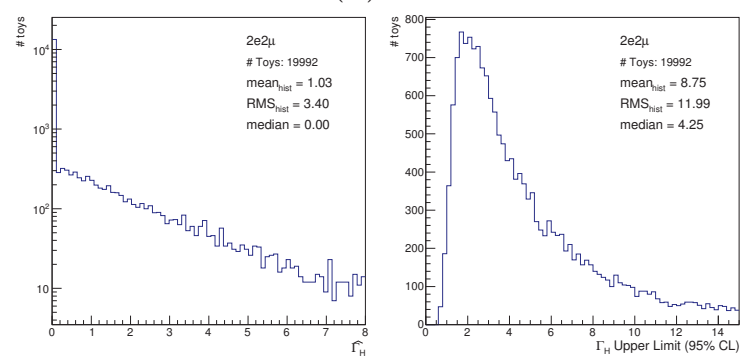

(d) $2 e 2 \mu$

Figure 159: Results of $\Gamma_{H}$ fits using the constrained PER model, per category. Shown are the best fit values $\hat{\Gamma_{H}}$ (left of each subfigure), and the 95\% CL upper limit (right) in each category. The raw distribution of results from the toys is shown in blue. Note the log axis and large first bin in the left-hand plots. 

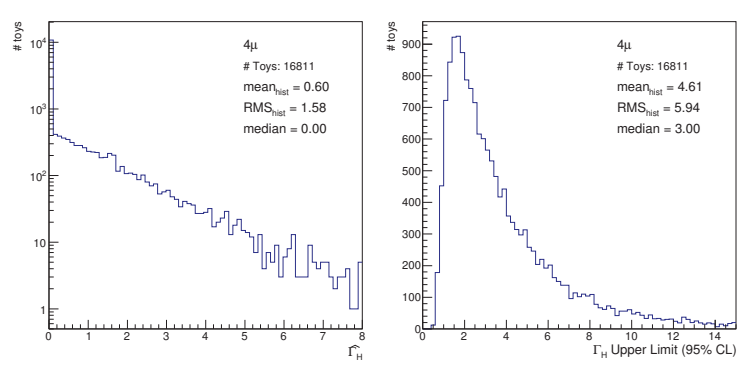

(a) $4 \mu$
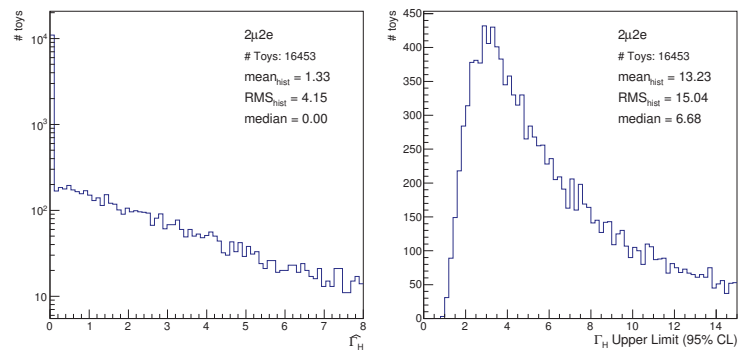

(c) $2 \mu 2 e$
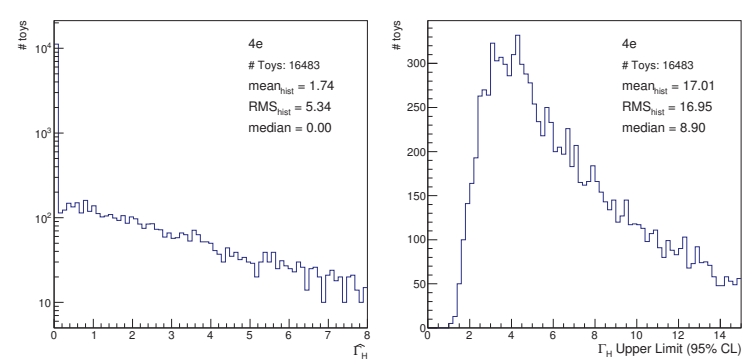

(b) $4 e$
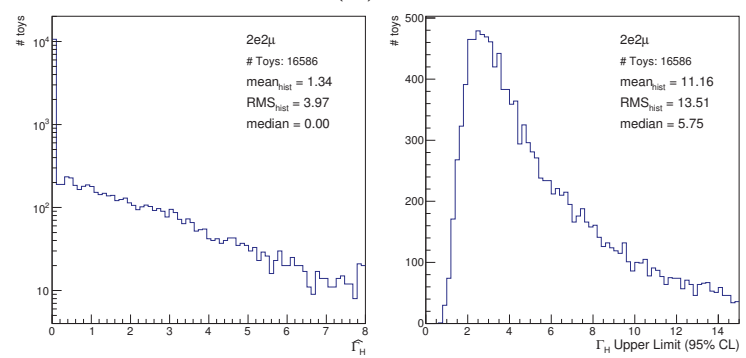

(d) $2 e 2 \mu$

Figure 160: Results of $\Gamma_{H}$ fits using the unconstrained PER model, per category. Shown are the best fit values $\hat{\Gamma_{H}}$ (left of each subfigure), and the $95 \%$ CL upper limit (right) in each category. The raw distribution of results from the toys is shown in blue. Note the log axis and large first bin in the left-hand plots. 
To complement Figure 108, the 2D correlation of $\hat{m_{H}}$ between the constrained PER and Template models, separated by category, are shown in Figure 161. Those for $\sigma_{\hat{H \rightarrow 4 \ell}}$ are shown in Figure 162.

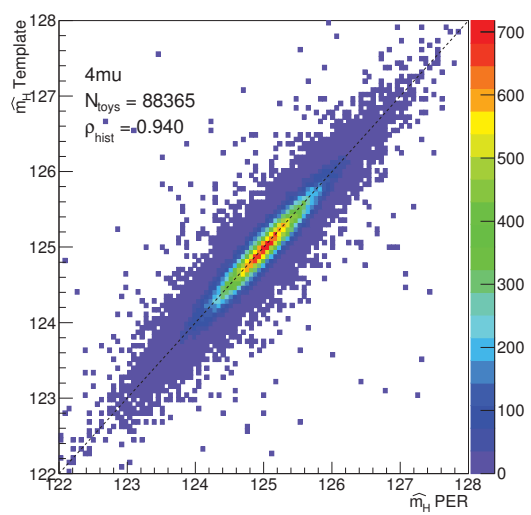

(a) $4 \mu$

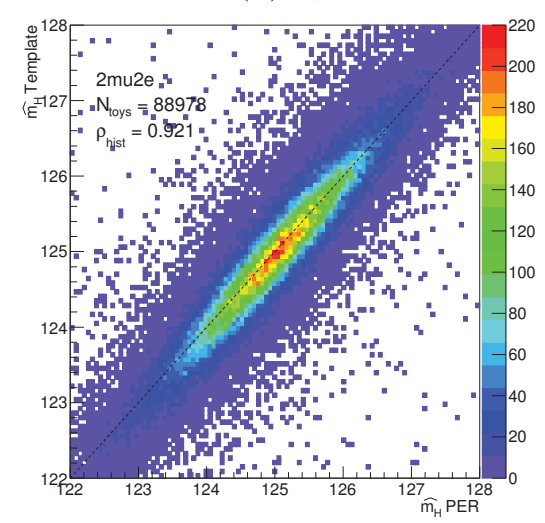

(c) $2 \mu 2 e$

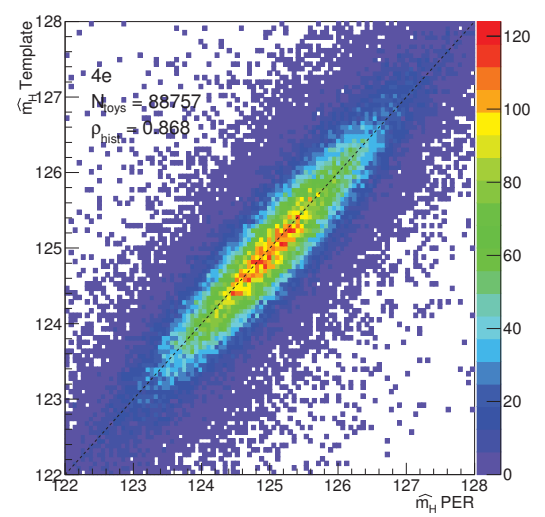

(b) $4 e$

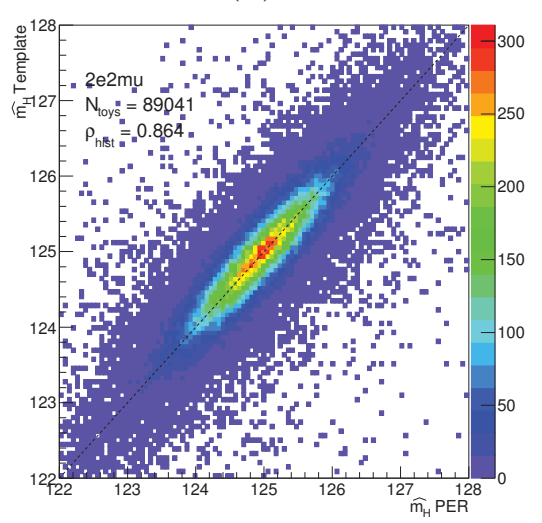

(d) $2 e 2 \mu$

Figure 161: Correlation plots of $\hat{m}_{H}$ between the constrained PER model, and the Template model, from pseudo-experiments in each analysis category. The diagonal line indicates where both methods agree exactly.

To complement Figure 109, the difference in best fit $m_{H}$ between the constrained PER and Template models, separated by category, is shown in Figure 163. Those for $\sigma_{H \rightarrow 4 \ell}$ are shown in Figure 164.

To complement Figure 110, the actual fit accuracy (based on the distance to the true value) of the constrained PER and Template models are compared for fits of $m_{H}$ 


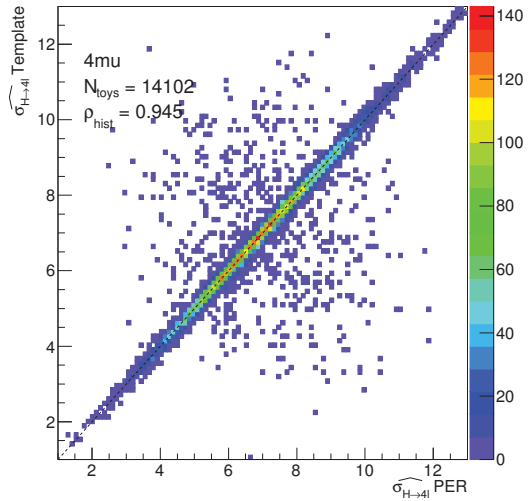

(a) $4 \mu$

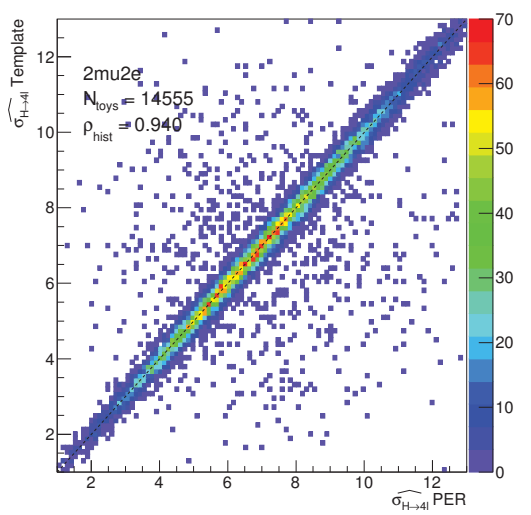

(c) $2 \mu 2 e$

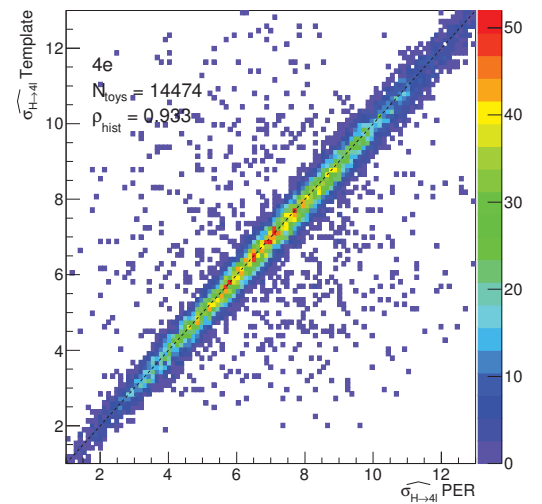

(b) $4 e$

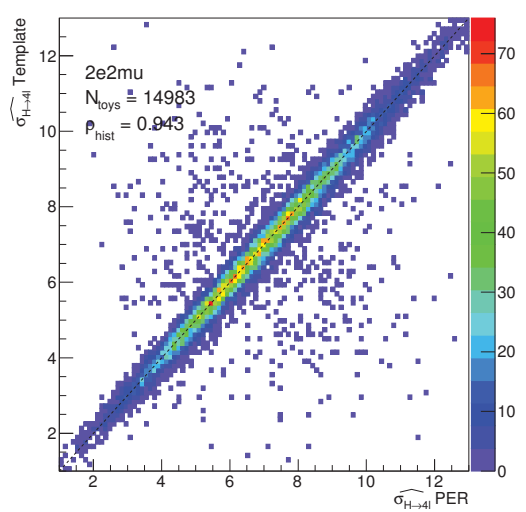

(d) $2 e 2 \mu$

Figure 162: Correlation plots of $\hat{\sigma}_{H \rightarrow 4 \ell}$ between the constrained PER model, and the Template model, from pseudo-experiments in each analysis category. The diagonal line indicates where both methods agree exactly.

in Figure 165, and of $\sigma_{H \rightarrow 4 \ell}$ Figure 166. 


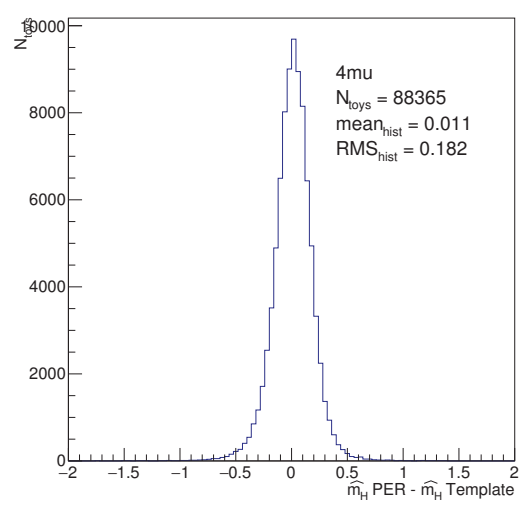

(a) $4 \mu$

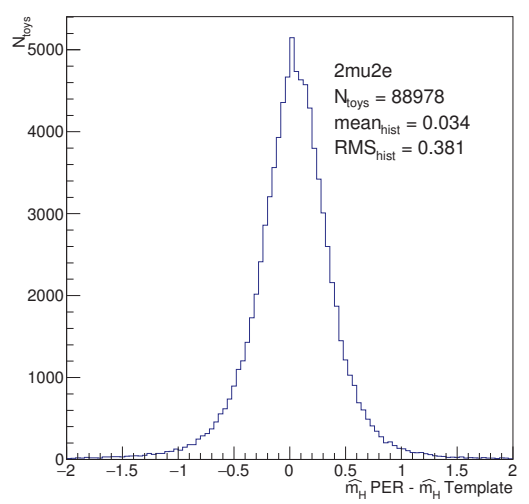

(c) $2 \mu 2 e$

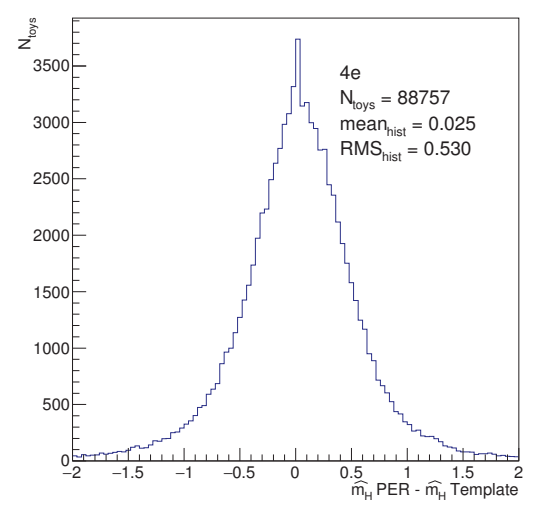

(b) $4 e$

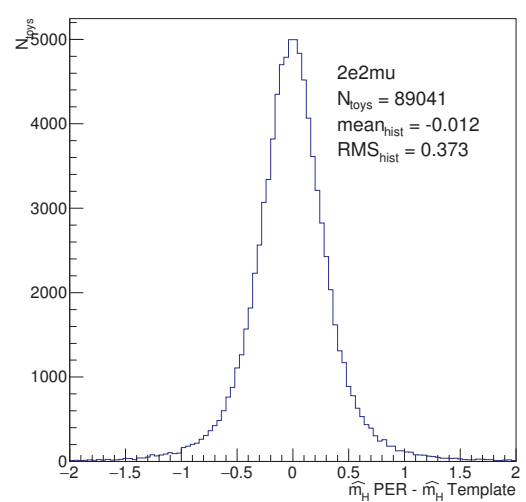

(d) $2 e 2 \mu$

Figure 163: Difference in best fit values of $m_{H}$ between the constrained PER model, and the Template model, from pseudo-experiments in each analysis category. The standard deviation, recorded on the plots as $R M S_{\text {hist }}$, gives an indication of how much the two models may agree or disagree when fit on the data. 


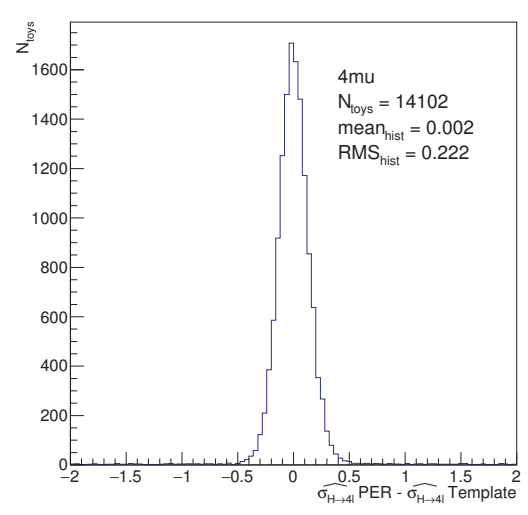

(a) $4 \mu$

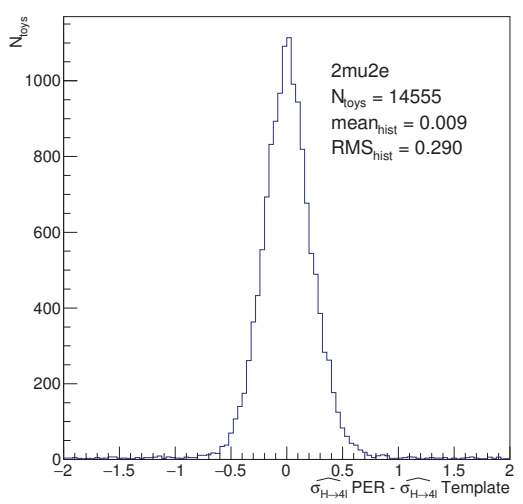

(c) $2 \mu 2 e$

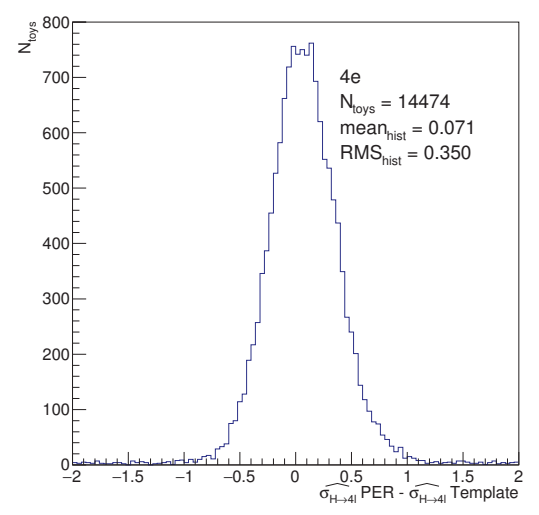

(b) $4 e$

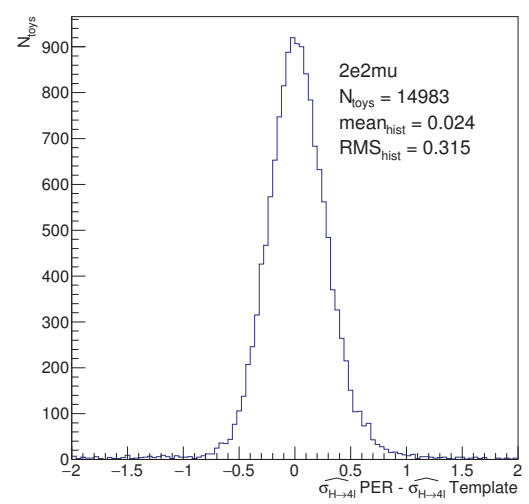

(d) $2 e 2 \mu$

Figure 164: Difference in best fit values of $\sigma_{H \rightarrow 4 \ell}$ between the constrained PER model, and the Template model, from pseudo-experiments in each analysis category. The standard deviation, recorded on the plots as $R M S_{\text {hist }}$, gives an indication of how much the two models may agree or disagree when fit on the data. 


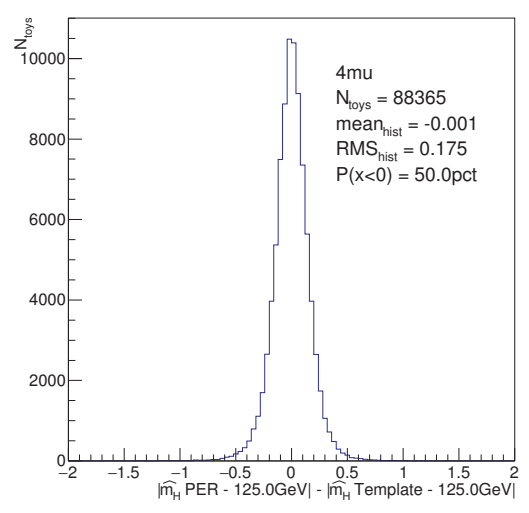

(a) $4 \mu$

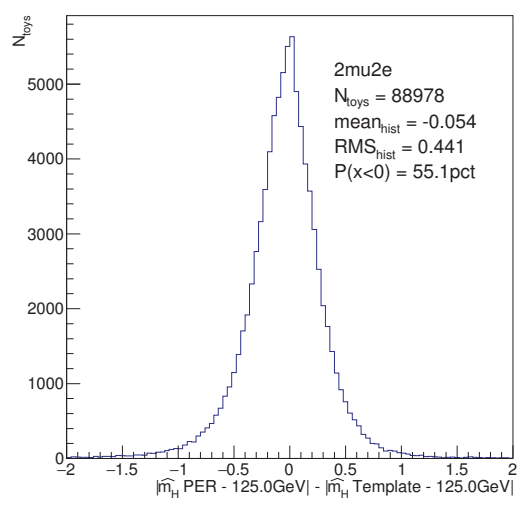

(c) $2 \mu 2 e$

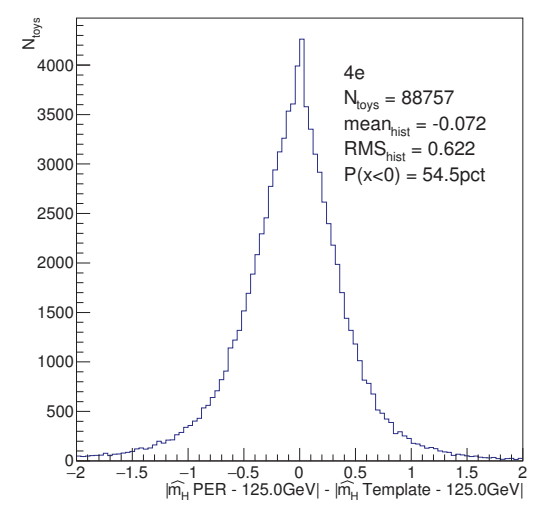

(b) $4 e$

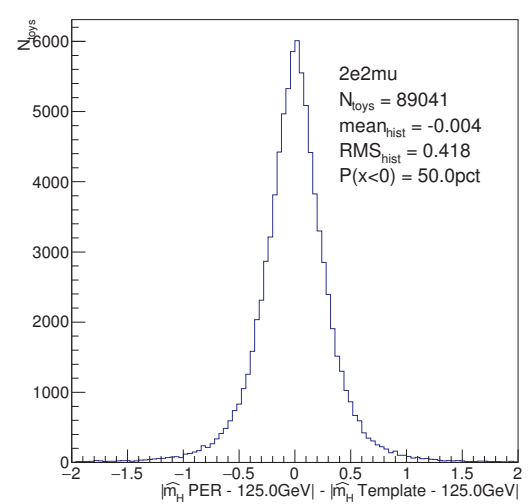

(d) $2 e 2 \mu$

Figure 165: Comparison of distance from the best fit value to the true value of $m_{H}$ one a toy-by-toy basis. In toys to the left of zero, the constrained PER model produces a more accurate fit result than the Template model. In toys to the right of zero, the Template method was more accurate. 


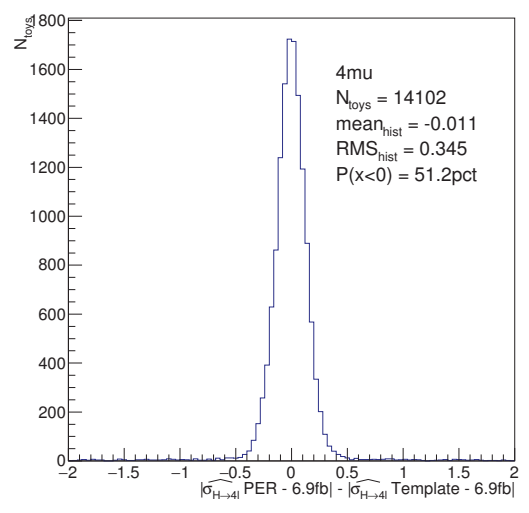

(a) $4 \mu$

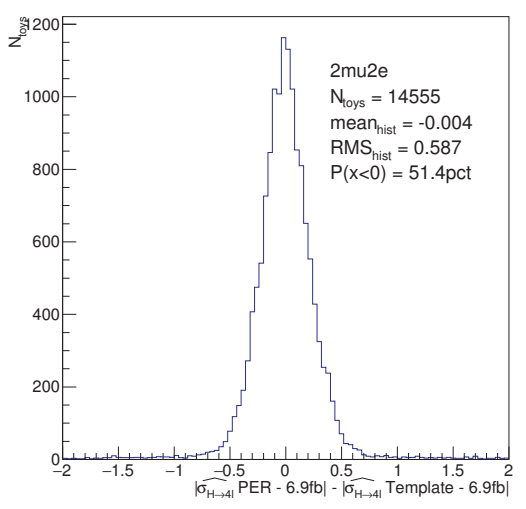

(c) $2 \mu 2 e$

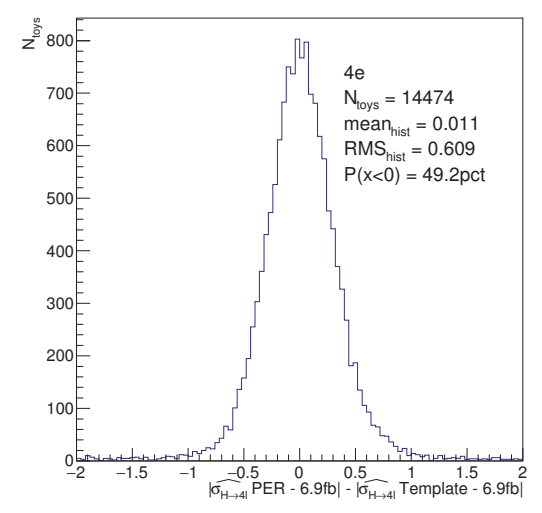

(b) $4 e$

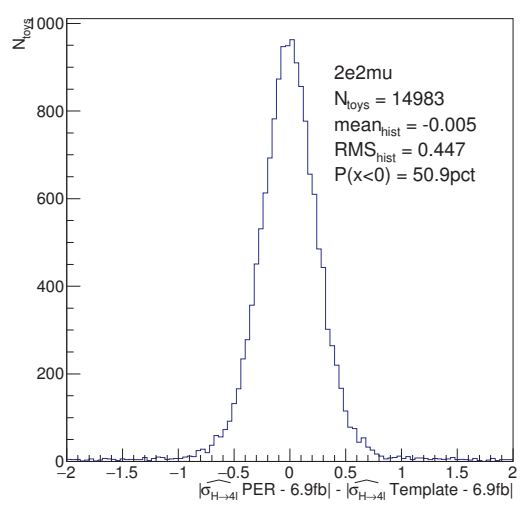

(d) $2 e 2 \mu$

Figure 166: Comparison of distance from the best fit value to the true value of $\sigma_{H \rightarrow 4 \ell}$ one a toy-by-toy basis. In toys to the left of zero, the constrained PER model produces a more accurate fit result than the Template model. In toys to the right of zero, the Template method was more accurate. 


\section{C.4 $Z \rightarrow 4 \ell$ analysis using $m_{4 \ell}^{\mathrm{fsr}}$}

The $Z \rightarrow 4 \ell$ analysis using $m_{4 \ell}^{\mathrm{fsr}}$ proceeds exactly as for $m_{4 \ell}^{\text {cons }}$ detailed in Section 9.5, using the same $m_{4 \ell}^{\mathrm{fsr}}$ window, using all the same samples for the tuning fit (separate tuning parameters) and for the effective dataset, and the same configuration of free and fixed variables during the fits. With these points considered, the likelihood scans of $m_{Z}, \mu_{Z}$, and $\Gamma_{Z}$ on this dataset are found in Figure 167. The expected and observed event yields for $m_{4 \ell}^{\mathrm{fsr}}$ are found in Table 32. The likelihood scans on data are found in Figure 168. The data distribution of $m_{4 \ell}^{\mathrm{fsr}}$ is plotted in Figure 169 with the unconstrained PER model plotted at the best fit $m_{Z}, \mu_{Z}$, with $\Gamma_{Z}$ set to its SM value. A summary of the expected and observed results are found in Table 33, which confirms that the ZMC has a relatively minor effect on the $Z \rightarrow 4 \ell$ analysis, and does not improve the resolution of this measurement.

Table 32: Expected and observed yields in the mass window $78 \mathrm{GeV} \leq m_{4 \ell}^{\mathrm{fsr}} \leq 104 \mathrm{GeV}$ for the $Z \rightarrow 4 \ell$ unconstrained validation study.

\begin{tabular}{cccc}
\hline category & $Z \rightarrow 4 \ell$ & Z+Jets & observed \\
\hline $4 \mu$ & $48.8 \pm 4.8$ & $0.20 \pm 0.05$ & 45 \\
$4 e$ & $17.1 \pm 1.7$ & $0.28 \pm 0.07$ & 18 \\
$2 \mu 2 e$ & $10.6 \pm 1.0$ & $0.29 \pm 0.07$ & 8 \\
$2 e 2 \mu$ & $18.4 \pm 1.9$ & $0.20 \pm 0.05$ & 23 \\
combined & $94.4 \pm 9.4$ & $0.97 \pm 0.25$ & 94 \\
\hline
\end{tabular}




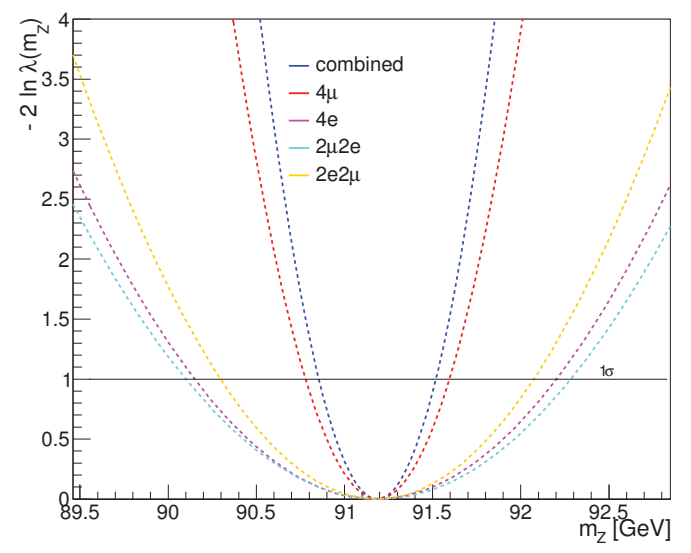

(a) $m_{Z}$

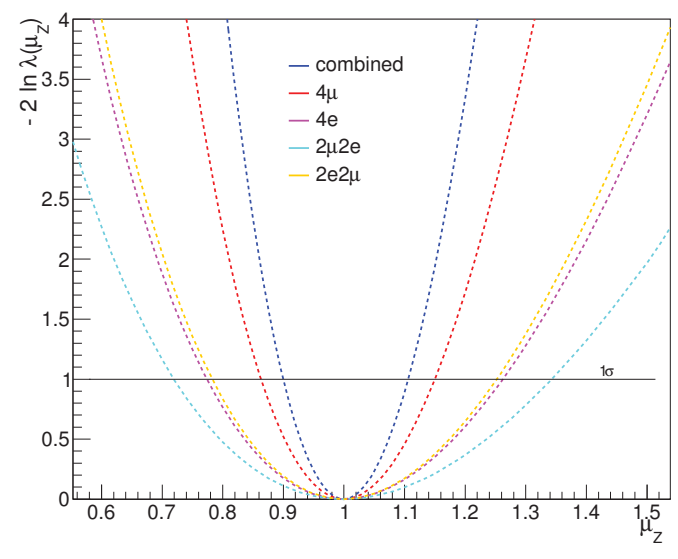

(b) $\mu_{Z}$

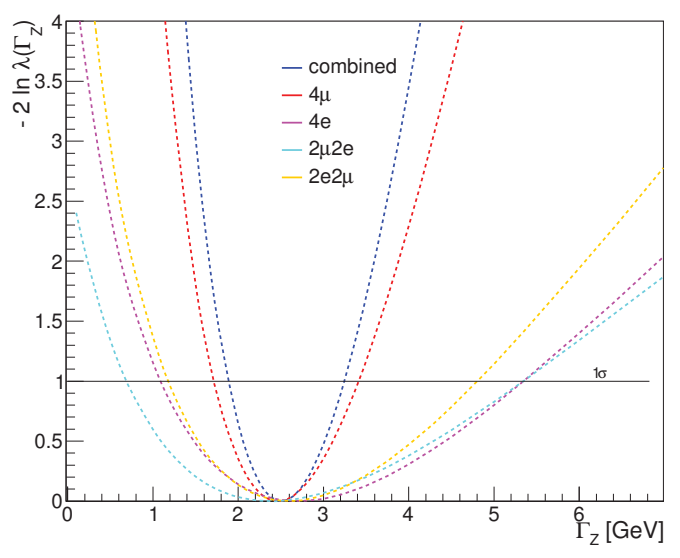

(c) $\Gamma_{Z}$

Figure 167: Likelihood scans of $m_{Z}, \mu_{Z}, \Gamma_{Z}$ on the effective dataset using the unconstrained PER model. Dashed lines indicate the scans include only statistical uncertainty; no systematics are included. 


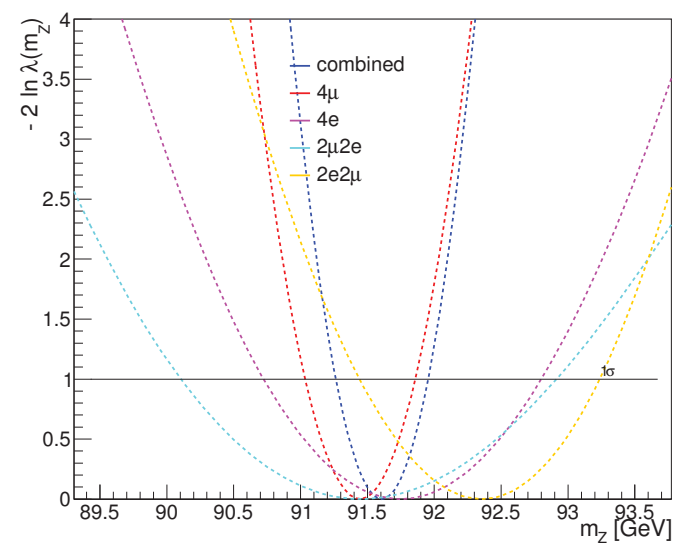

(a) $m_{Z}$

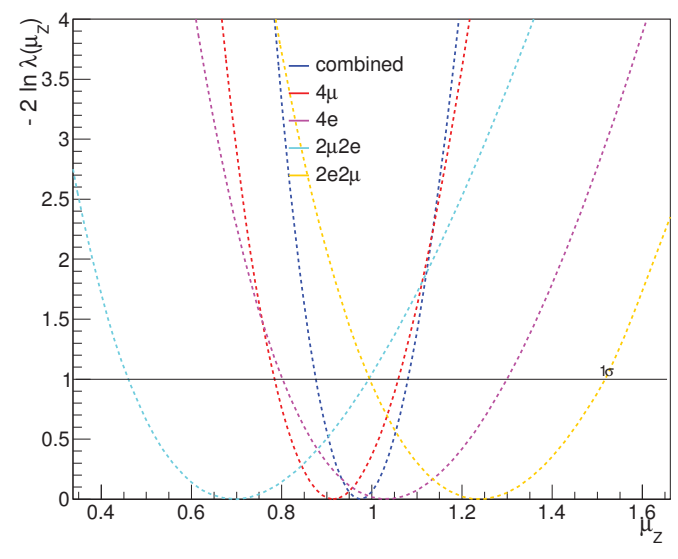

(b) $\mu_{Z}$

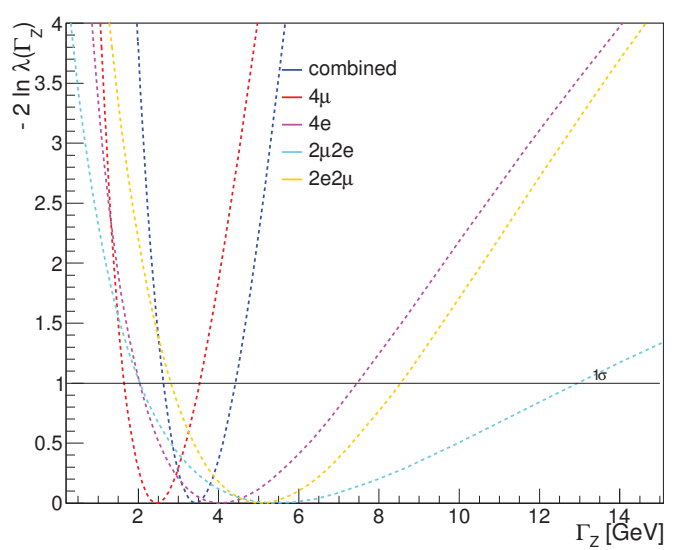

(c) $\Gamma_{Z}$

Figure 168: Likelihood scans of $m_{Z}, \mu_{Z}, \Gamma_{Z}$ on the data using the unconstrained PER model. Dashed lines indicate the scans include only statistical uncertainty; no systematics are included. 


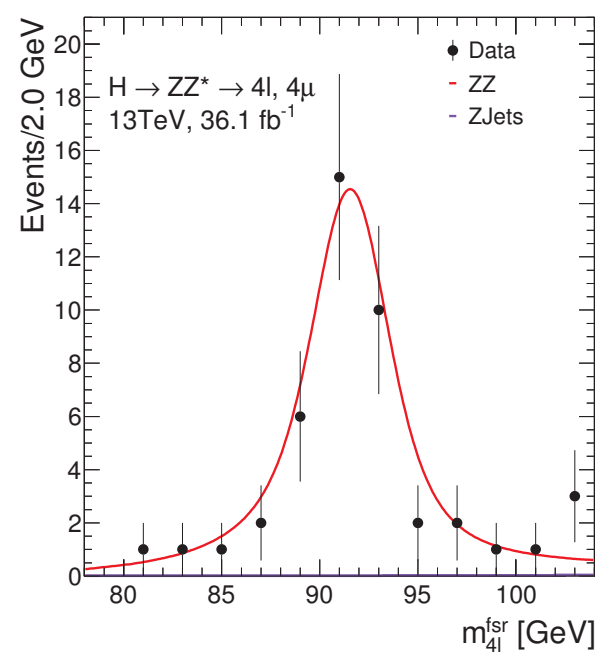

(a) $4 \mu$

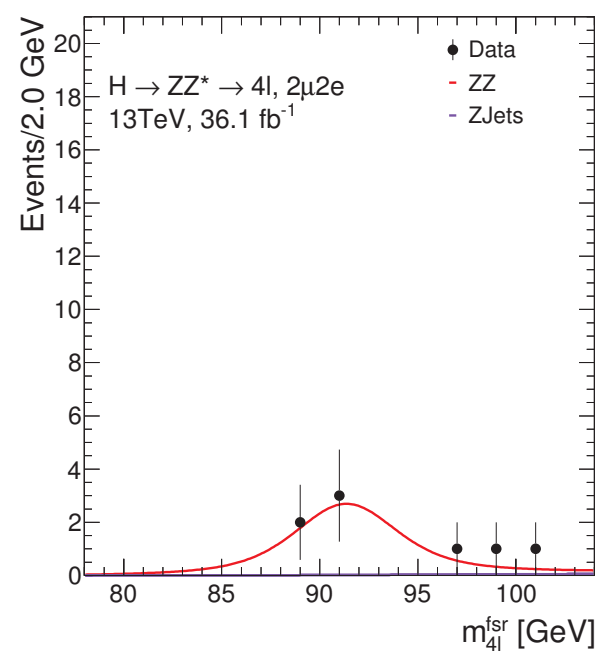

(c) $2 \mu 2 e$

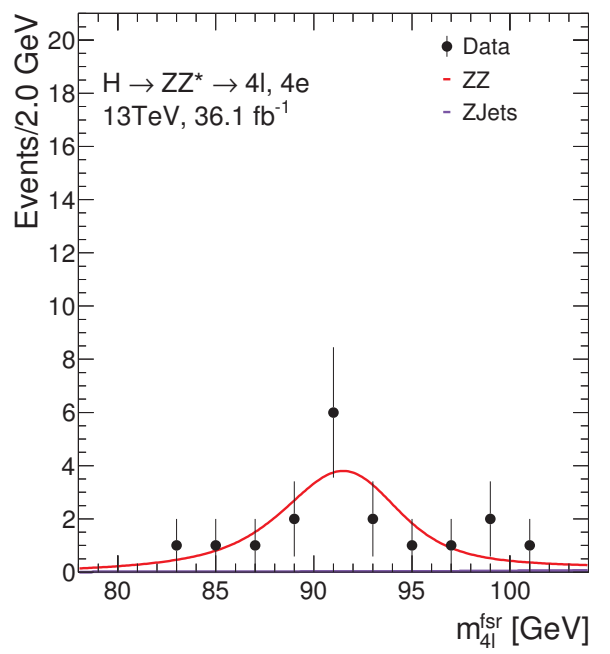

(b) $4 e$

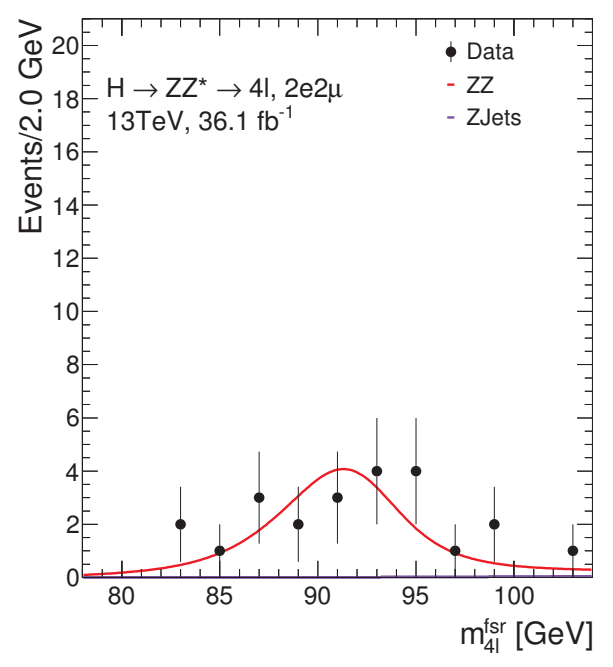

(d) $2 e 2 \mu$

Figure 169: Plot of the $m_{4 \ell}^{\mathrm{fsr}}$ distribution of the $Z \rightarrow 4 \ell$ resonance in data, in each of the analysis categories. The unconstrained PER model is plotted after the fit, with parameters $m_{Z}, \mu_{Z}$ set at their best-fit values. 
Table 33: Summary table of results from unconstrained PER $Z \rightarrow 4 \ell$ likelihood scans of $m_{Z}, \mu_{Z}$, and $\Gamma_{Z}$, in MC simulated data, and in the real data. The "SM value" of $m_{Z}, \Gamma_{Z}$ indicates the current best-measured value, used as input in other SM calculations, not a fundamental prediction of the SM. Units in the table match those of the reference values.

\begin{tabular}{ccc}
\hline Category & MC simulated dataset & observed data \\
\hline$m_{Z}$ & SM value $m_{Z}=91.1876 \mathrm{GeV}$ \\
\hline $4 \mu$ & $91.19_{-0.41}^{+0.41}$ & $91.44_{-0.41}^{+0.41}$ \\
$4 e$ & $91.18_{-1.03}^{+1.03}$ & $91.75_{-1.03}^{+1.05}$ \\
$2 \mu 2 e$ & $91.20_{-1.10}^{+1.09}$ & $91.45_{-1.35}^{+1.46}$ \\
$2 e 2 \mu$ & $91.18_{-0.89}^{+0.89}$ & $92.36_{-0.91}^{+0.88}$ \\
combined & $91.19_{-0.33}^{+0.33}$ & $91.61_{-0.34}^{+0.35}$ \\
\hline$\mu_{Z}$ & \multicolumn{1}{c}{$\mathrm{SM}$ value $\mu_{Z}=1.00$} \\
\hline $4 \mu$ & $1.00_{-0.14}^{+0.15}$ & $0.91_{-0.13}^{+0.14}$ \\
$4 e$ & $1.00_{-0.22}^{+0.26}$ & $1.03_{-0.23}^{+0.27}$ \\
$2 \mu 2 e$ & $1.00_{-0.28}^{+0.34}$ & $0.69_{-0.23}^{+0.30}$ \\
$2 e 2 \mu$ & $1.00_{-0.22}^{+0.25}$ & $1.24_{-0.24}^{+0.28}$ \\
combined & $1.00_{-0.10}^{+0.11}$ & $0.97_{-0.10}^{+0.11}$ \\
\hline$\Gamma_{Z}$ & $\mathrm{SM} \mathrm{value} \Gamma_{Z}=2.4952 \mathrm{GeV}$ \\
\hline $4 \mu$ & $2.46_{-0.75}^{+0.95}$ & $2.46_{-0.81}^{+1.07}$ \\
$4 e$ & $2.70_{-1.61}^{+2.64}$ & $4.04_{-2.01}^{+3.42}$ \\
$2 \mu 2 e$ & $2.38_{-1.69}^{+2.96}$ & $5.45_{-3.41}^{+7.50}$ \\
$2 e 2 \mu$ & $2.60_{-1.41}^{+2.21}$ & $5.07_{-2.25}^{+3.46}$ \\
combined & $2.50_{-0.62}^{+0.74}$ & $3.43_{-0.80}^{+0.99}$ \\
\hline
\end{tabular}




\section{C.5 Response distribution of data events}

The rarity of $H \rightarrow 4 \ell$ events may limit the statistical precision of the measurement, but at least one consolation to this fact is that the number of events in data is small enough to examine one at a time. This allows one to see the dramatic effect of the Per-event response technique: each event has a uniquely predicted signal distribution, for a given mass hypothesis. This appendix contains the per-event signal PDF, drawn for each event drawn with $m_{H}$ set to its combined best fit value. Table 34 indicates where to find the PDFs, which are organized by category of the events. On each plot the actual value of $m_{4 \ell}^{\text {cons }}$ is marked by a red line. The height of the red line (the value of the PDF at that $m_{4 \ell}$ value) indicates roughly how likely such an event is, if it came from a signal decay.

Table 34: Summary of figures containing Per-event signal PDFs for the data events. The constrained PER signal model is drawn with $m_{H}$ set to the best fit value, $124.61 \mathrm{GeV}$.

\begin{tabular}{cc}
\hline category & \multicolumn{1}{c}{ figures references } \\
\hline $4 \mu$ & Figure 170 and Figure 171 \\
$4 e$ & Figure 172 and Figure 173 \\
$2 \mu 2 e$ & Figure 174 and Figure 175 \\
$2 \mu 2 e$ & Figure 176 and Figure 177 \\
\hline
\end{tabular}



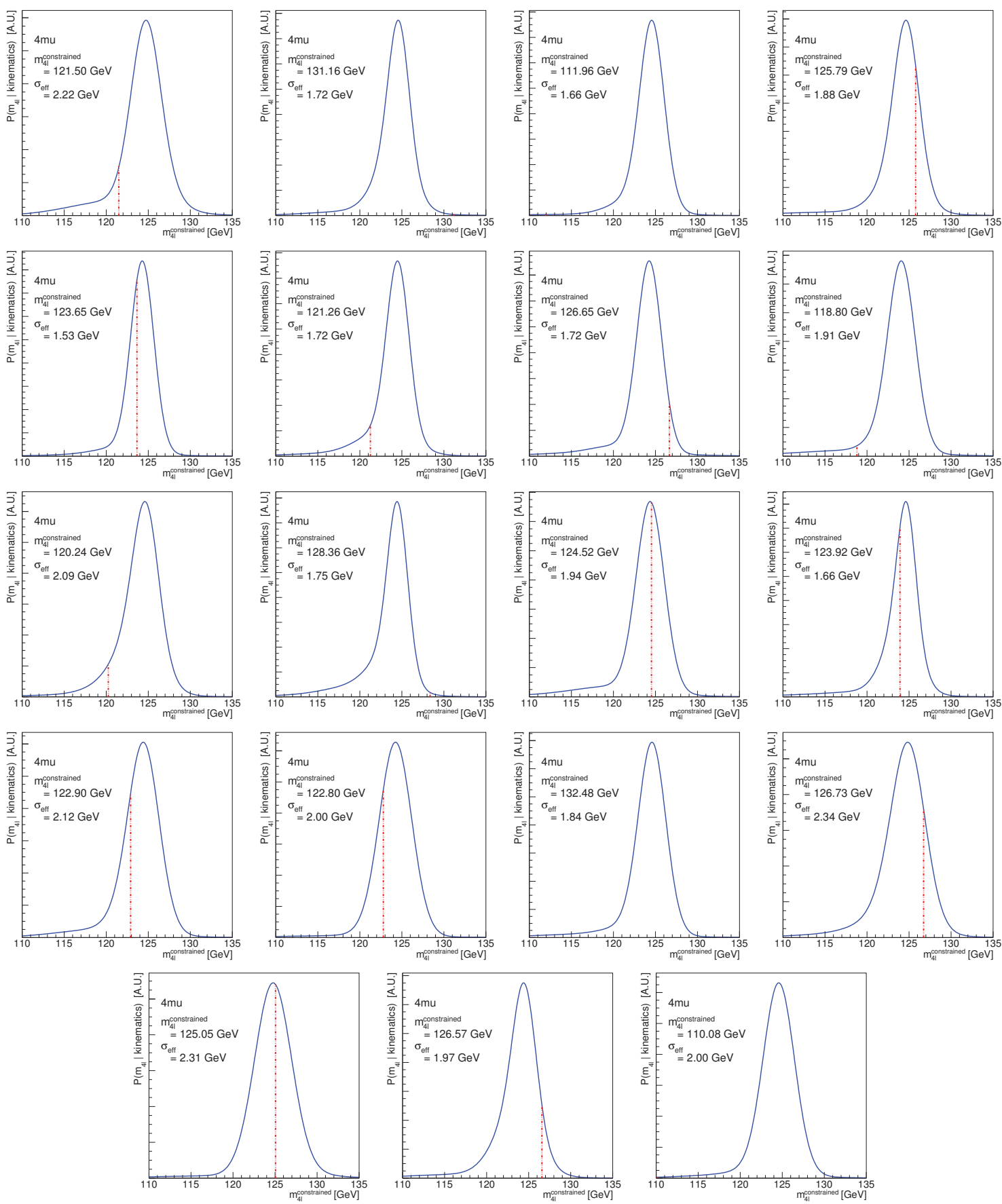

Figure 170: Per-event signal PDF of $4 \mu$ events in data according to the constrained PER model (1/2). Printed on each plot is the reconstructed mass of the event after ZMC and FSR recovery, and the effective resolution $\sigma_{\text {eff }}$ as it is defined in Section 7.3.6. The dashed red line is drawn at the observed value of $m_{4 \ell}^{\text {cons }}$ for this event. 

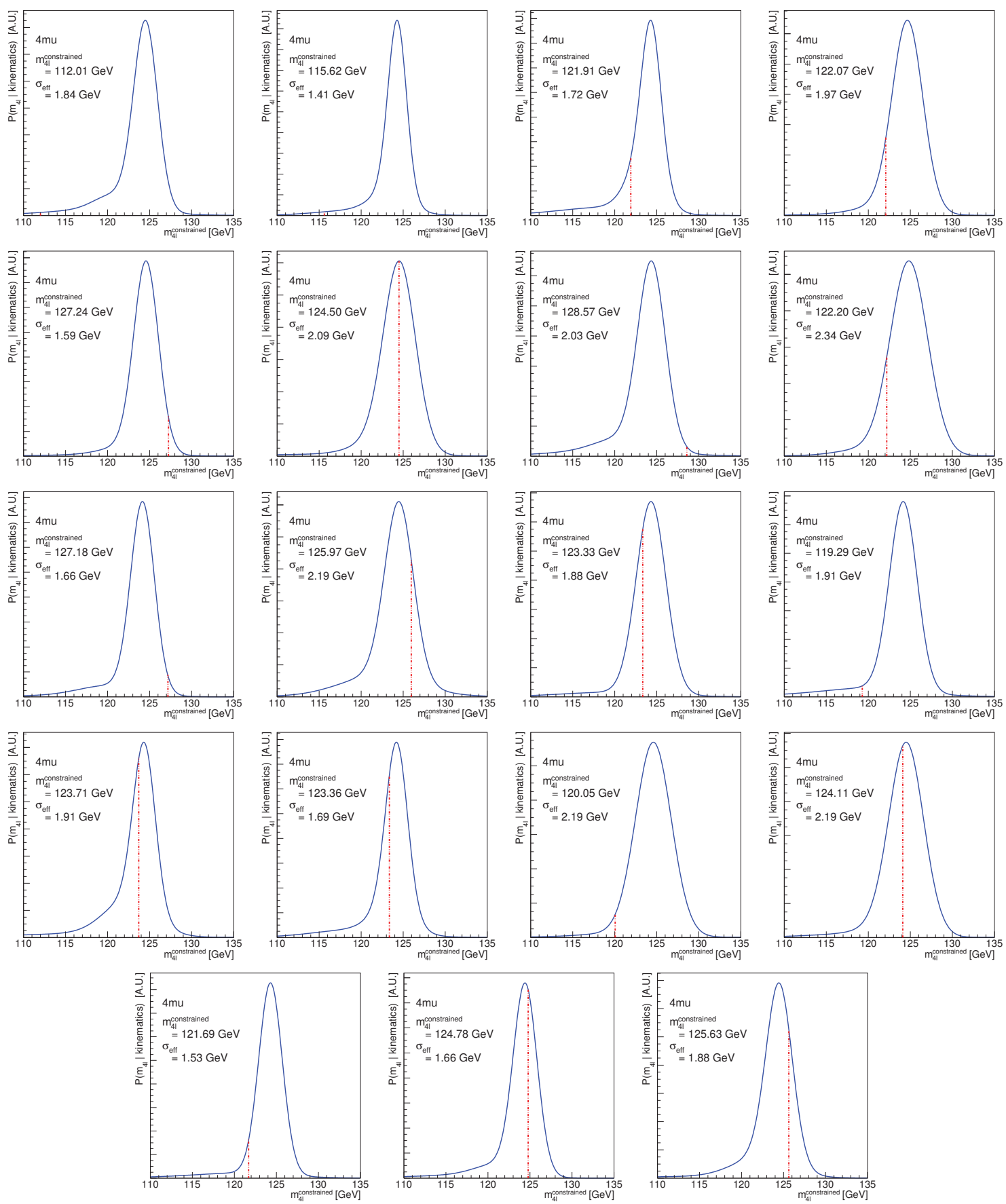

Figure 171: Per-event signal PDF of $4 \mu$ events in data according to the constrained PER model (2/2). Printed on each plot is the reconstructed mass of the event after ZMC and FSR recovery, and the effective resolution $\sigma_{\text {eff }}$ as it is defined in Section 7.3.6. The dashed red line is drawn at the observed value of $m_{4 \ell}^{\text {cons }}$ for this event. 

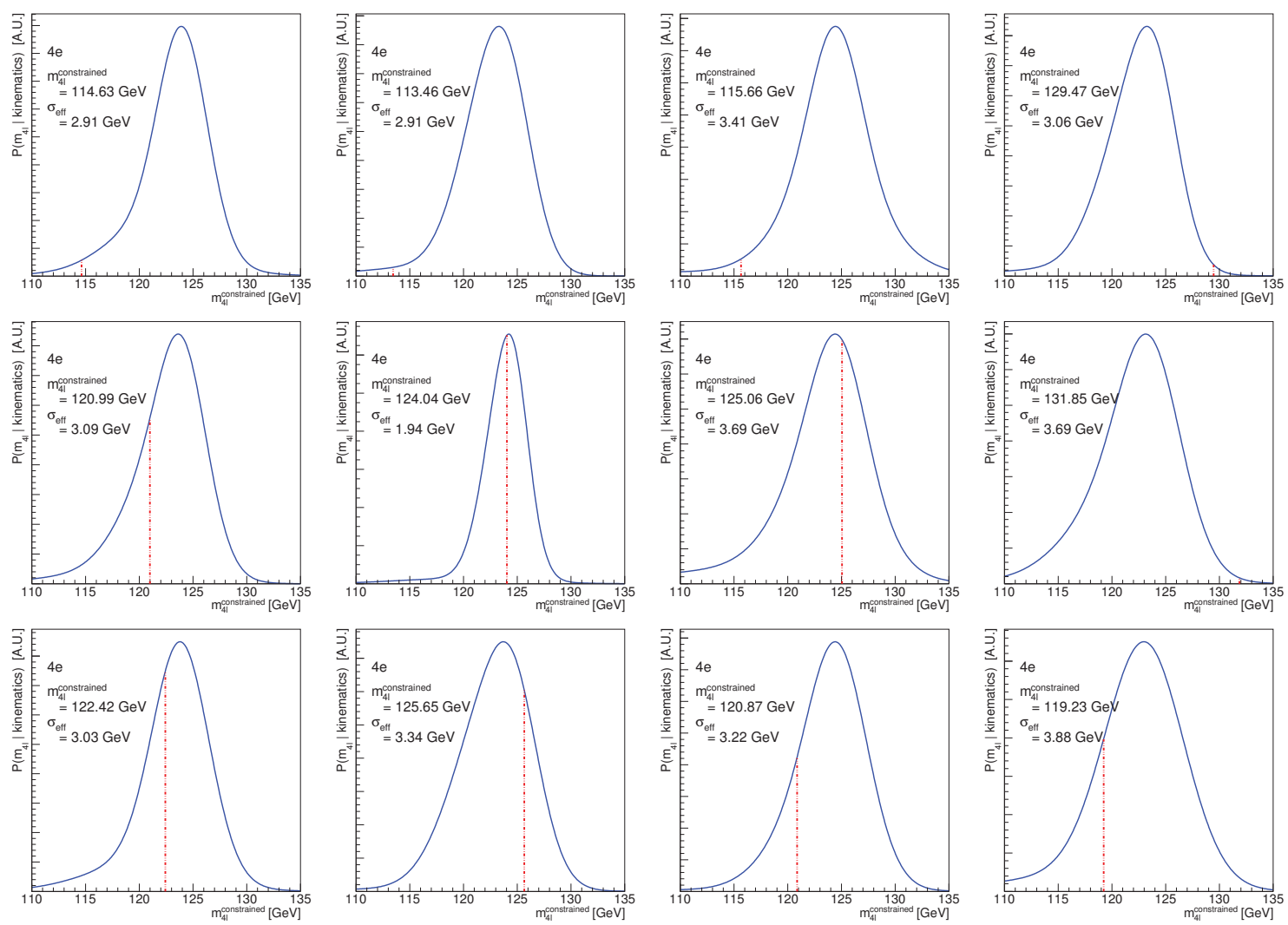

Figure 172: Per-event signal PDF of $4 e$ events in data according to the constrained PER model (1/2). Printed on each plot is the reconstructed mass of the event after ZMC and FSR recovery, and the effective resolution $\sigma_{\text {eff }}$ as it is defined in Section 7.3.6. The dashed red line is drawn at the observed value of $m_{4 \ell}^{\text {cons }}$ for this event. 

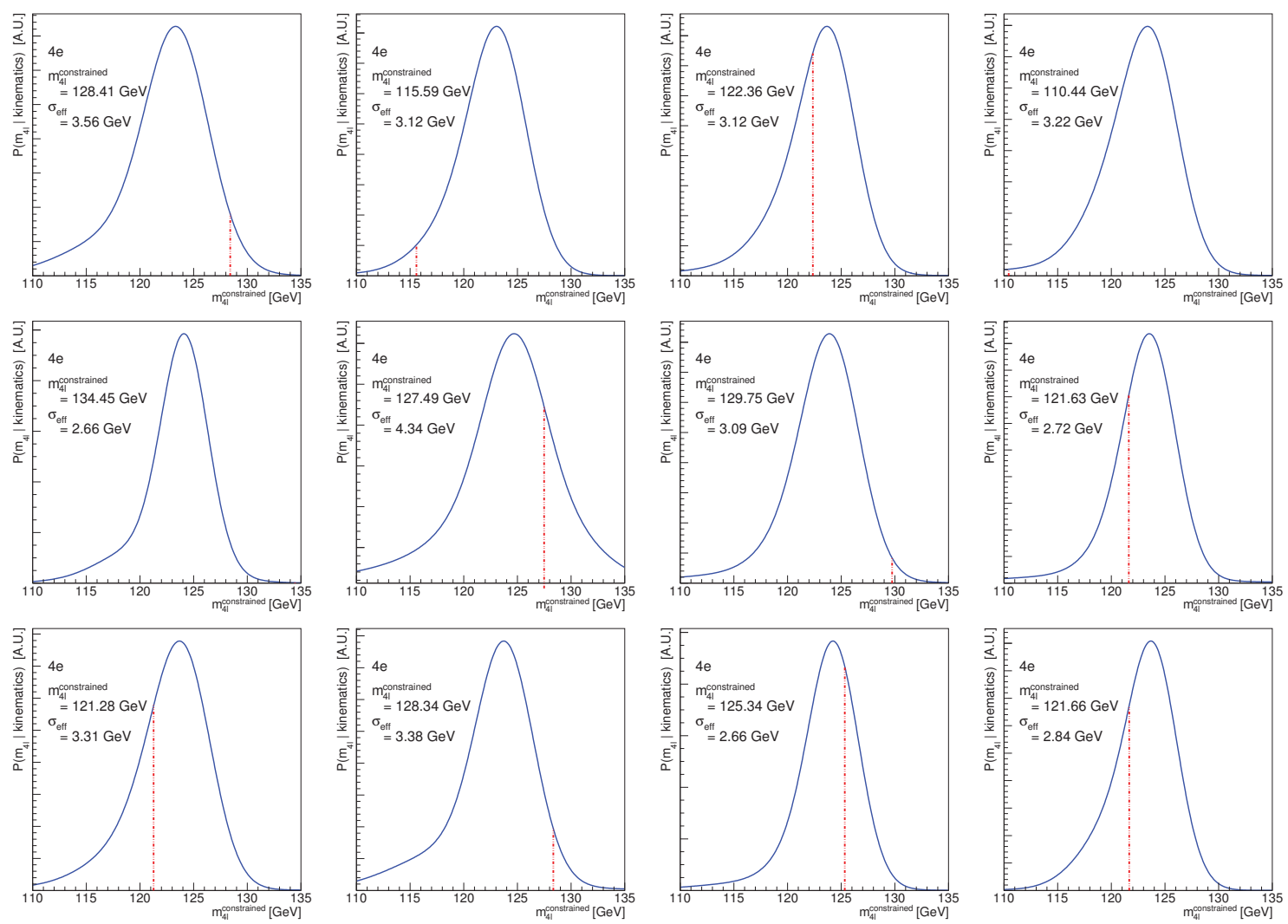

Figure 173: Per-event signal PDF of $4 e$ events in data according to the constrained PER model (2/2). Printed on each plot is the reconstructed mass of the event after ZMC and FSR recovery, and the effective resolution $\sigma_{\text {eff }}$ as it is defined in Section 7.3.6. The dashed red line is drawn at the observed value of $m_{4 \ell}^{\text {cons }}$ for this event. 

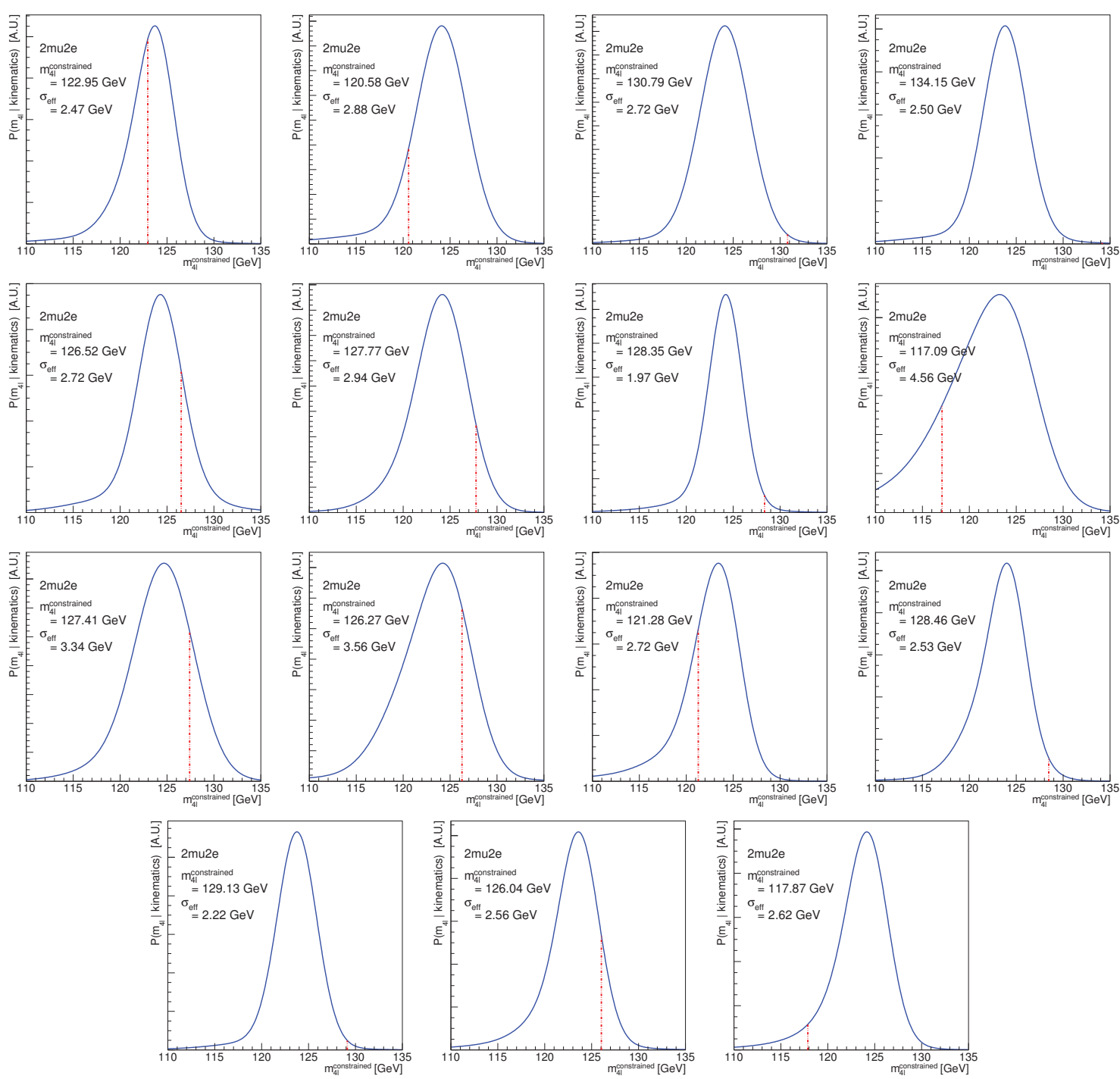

Figure 174: Per-event signal PDF of $2 \mu 2 e$ events in data according to the constrained PER model $(1 / 2)$. Printed on each plot is the reconstructed mass of the event after ZMC and FSR recovery, and the effective resolution $\sigma_{\text {eff }}$ as it is defined in Section 7.3.6. The dashed red line is drawn at the observed value of $m_{4 \ell}^{\text {cons }}$ for this event. 

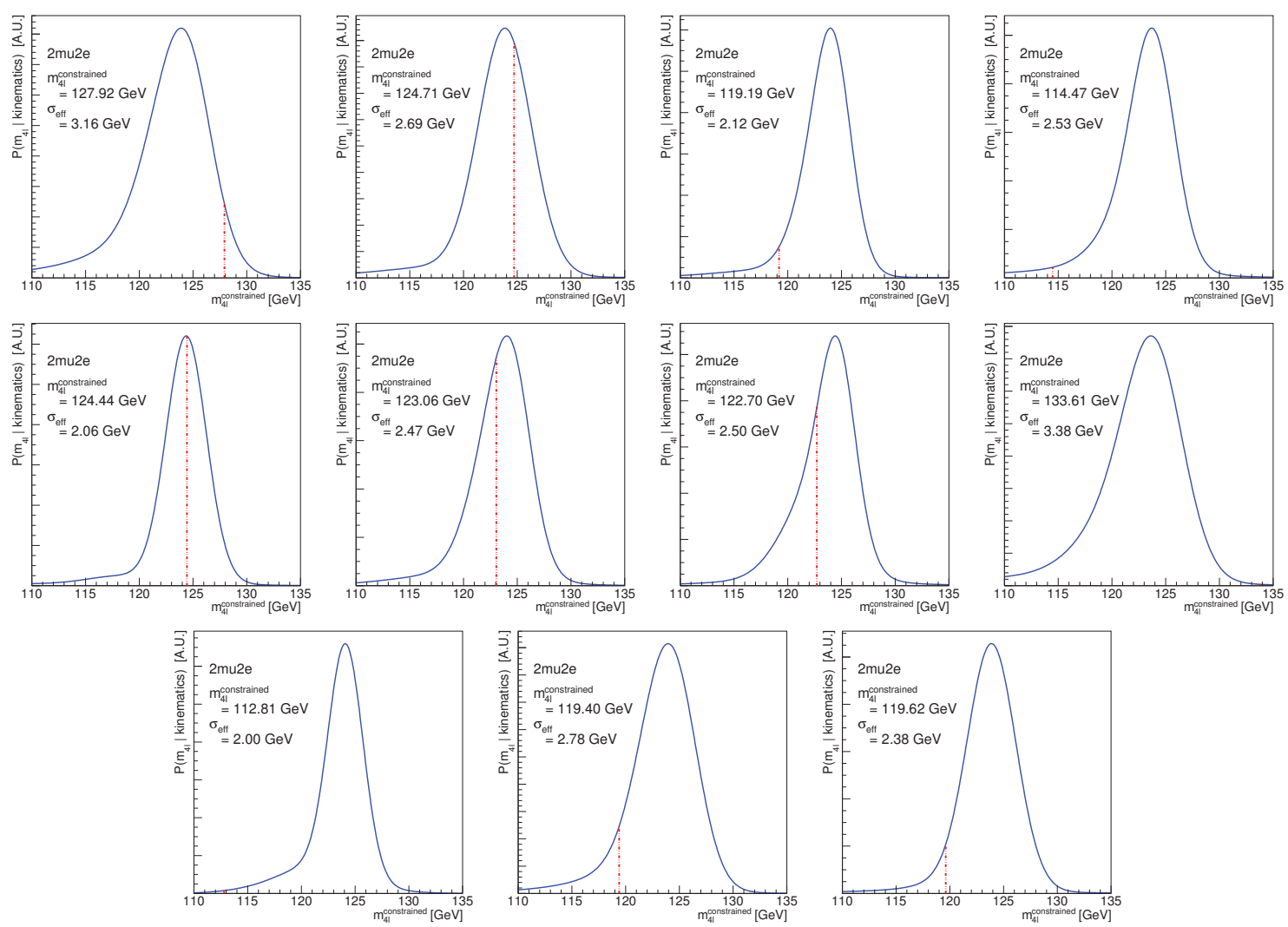

Figure 175: Per-event signal PDF of $2 \mu 2 e$ events in data according to the constrained PER model (2/2). Printed on each plot is the reconstructed mass of the event after ZMC and FSR recovery, and the effective resolution $\sigma_{\text {eff }}$ as it is defined in Section 7.3.6. The dashed red line is drawn at the observed value of $m_{4 \ell}^{\text {cons }}$ for this event. 

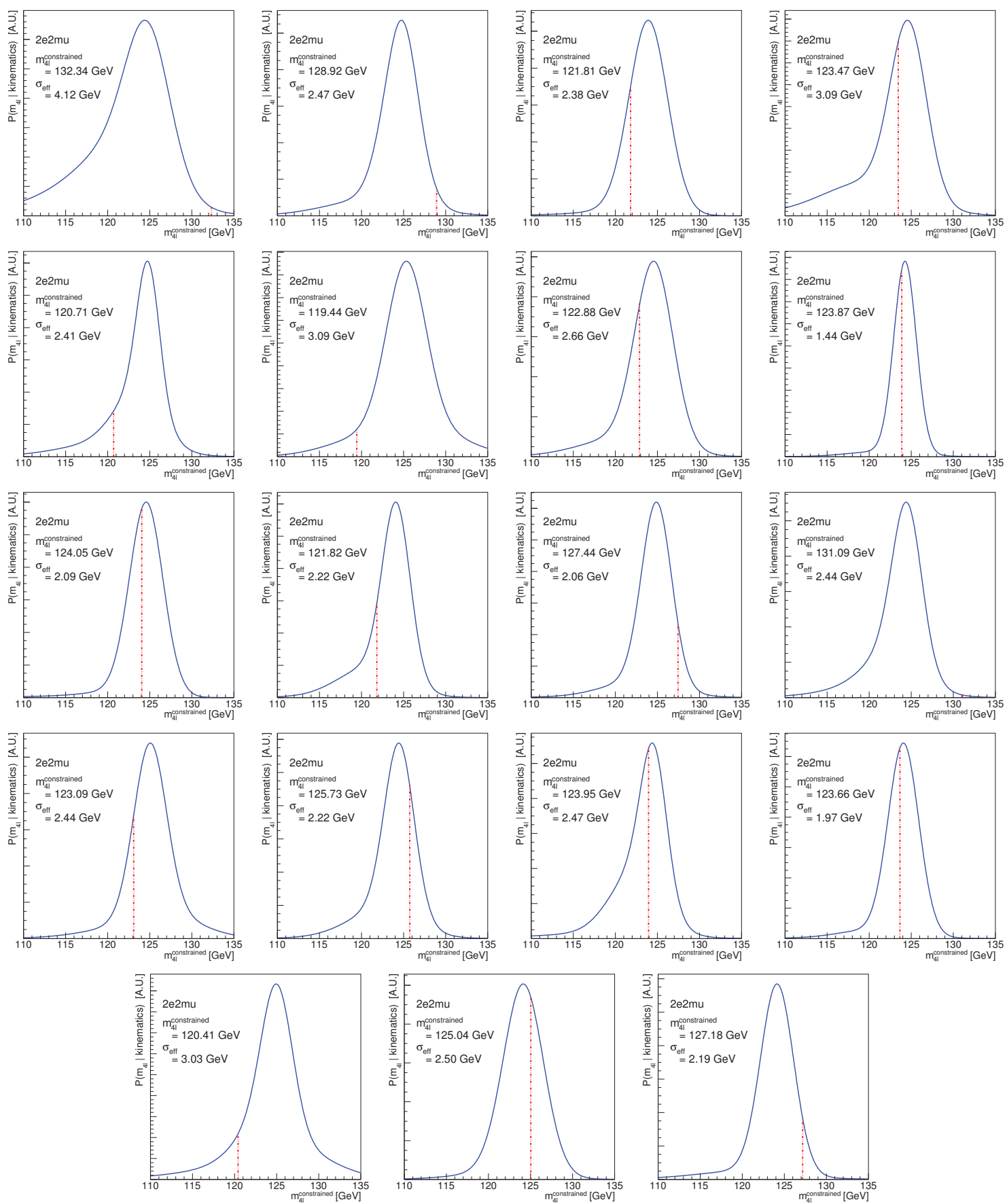

Figure 176: Per-event signal PDF of $2 e 2 \mu$ events in data according to the constrained PER model (1/2). Printed on each plot is the reconstructed mass of the event after ZMC and FSR recovery, and the effective resolution $\sigma_{\text {eff }}$ as it is defined in Section 7.3.6. The dashed red line is drawn at the observed value of $m_{4 \ell}^{\text {cons }}$ for this event. 

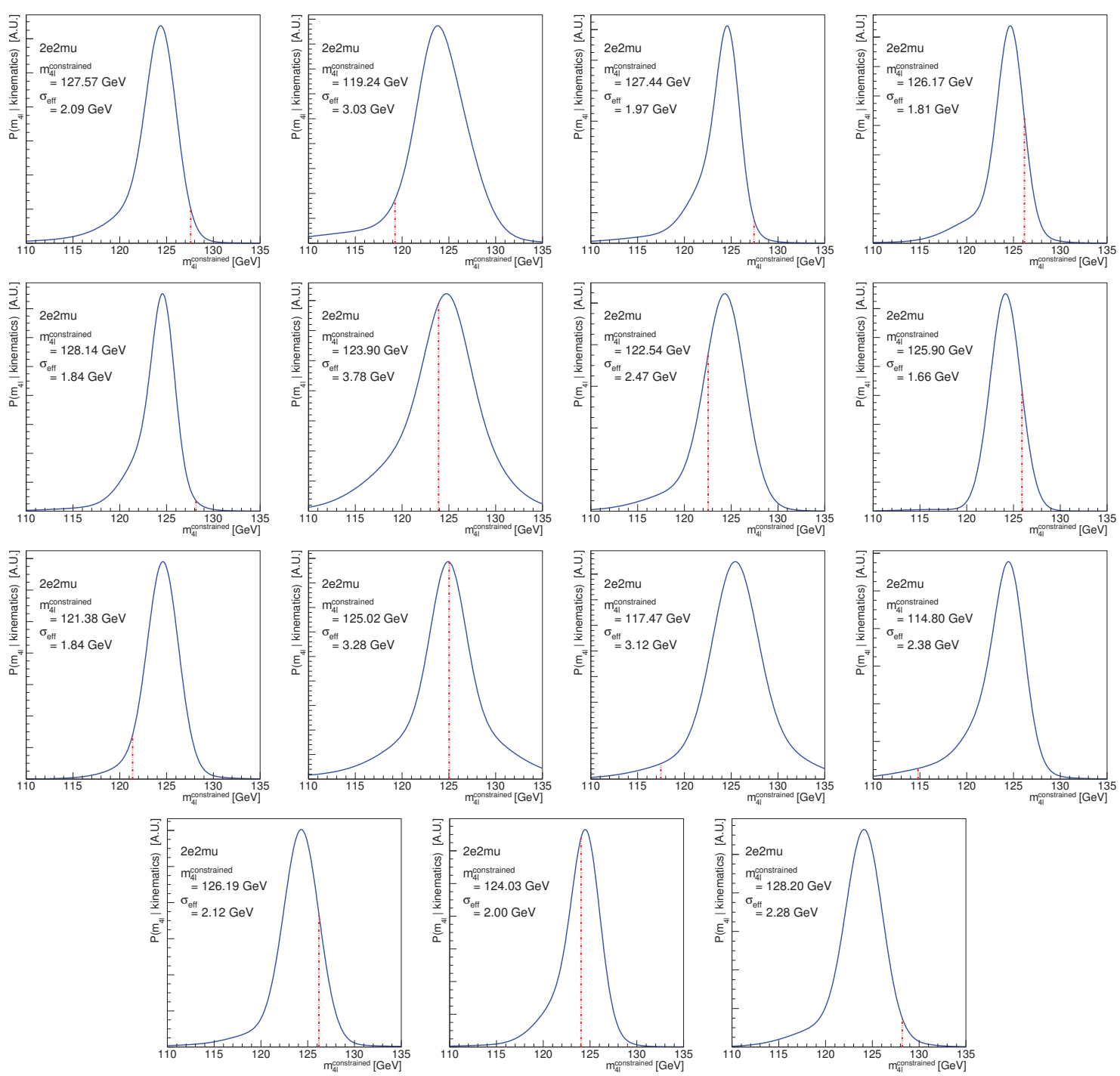

Figure 177: Per-event signal PDF of $2 e 2 \mu$ events in data according to the constrained PER model $(2 / 2)$. Printed on each plot is the reconstructed mass of the event after ZMC and FSR recovery, and the effective resolution $\sigma_{\text {eff }}$ as it is defined in Section 7.3.6. The dashed red line is drawn at the observed value of $m_{4 \ell}^{\text {cons }}$ for this event. 


\section{List of References}

[1] P. W. Higgs, "Broken symmetries and the masses of gauge bosons," Phys. Rev. Lett., vol. 13, pp. 508-509, 161964.

[2] ATLAS Collaboration, "Observation of a new particle in the search for the Standard Model Higgs boson with the ATLAS detector at the LHC," Physics Letters B, vol. 716, no. 1, 2012.

[3] CMS Collaboration, "Observation of a new boson at a mass of $125 \mathrm{GeV}$ with the CMS experiment at the LHC," Phys. Lett. B, vol. 716, no. 1, 2012.

[4] ATLAS Collaboration, "Muon reconstruction performance of the ATLAS detector in protonproton collision data at $\sqrt{s}=13 \mathrm{TeV}$," Eur. Phys. J., vol. C76, no. 5, p. 292, 2016. arXiv: 1603.05598 [hep-ex].

[5] ATLAS Collaboration, "Study of the Higgs boson properties and search for high-mass scalar resonances in the $H \rightarrow Z Z^{*} \rightarrow 4 \ell$ decay channel at $\sqrt{s}=13$ TeV with the ATLAS detector," CERN, Geneva, Tech. Rep. ATLAS-CONF2016-079, 2016.

[6] "Measurements of the Higgs boson production cross section at 7, 8 and 13 $\mathrm{TeV}$ centre-of-mass energies and search for new physics at $13 \mathrm{TeV}$ in the $H \rightarrow$ $Z Z^{*} \rightarrow \ell^{+} \ell \ell^{\prime+} \ell^{\prime}$ final state with the ATLAS detector," CERN, Geneva, Tech. Rep. ATLAS-CONF-2015-059, 2015. 
[7] ATLAS Collaboration, "Measurement of the Higgs boson mass from the $H \rightarrow$ $\gamma \gamma$ and $H \rightarrow Z Z^{*} \rightarrow 4 \ell$ channels with the ATLAS detector using $25 \mathrm{fb}^{-1}$ of $p p$ collision data," Phys. Rev., vol. D90, no. 5, p. 052 004, 2014. arXiv: 1406.3827 $[$ hep-ex].

[8] ATLAS Collaboration and CMS Collaboration, "Combined Measurement of the Higgs Boson Mass in $p p$ Collisions at $\sqrt{s}=7$ and $8 \mathrm{TeV}$ with the ATLAS and CMS Experiments," Phys. Rev. Lett., vol. 114, p. 191803, 192015.

[9] G. Cree, "Search for a high mass neutral Higgs boson using the ATLAS detector," 2015.

[10] D. Griffiths, Introduction to Elementary Particles. Wiley-VCH, 2008.

[11] CMS Collaboration, "Measurement of the top quark mass using proton-proton data at $\sqrt{s}=7$ and $8 \mathrm{TeV}, " 2015$. arXiv: 1509.04044 [hep-ex].

[12] SNO Collaboration, "Measurement of the Rate of $\nu_{e}+d \rightarrow p+p+e^{-}$Interactions Produced by ${ }^{8} B$ Solar Neutrinos at the Sudbury Neutrino Observatory," Phys. Rev. Lett., vol. 87, p. 071301, 72001.

[13] Super-Kamiokande Collaboration, "Evidence for oscillation of atmospheric neutrinos," Phys. Rev. Lett., vol. 81, pp. 1562-1567, 81998.

[14] B. N. Laboratory. (). Facility upgrades: rhic-ii and erhic, [Online]. Available: https://www. bnl.gov/rhic/RHIC2.asp.

[15] M. E. Peskin and D. V. Schroeder, An Introduction to Quantum Field Theory. Westview Press, 1995.

[16] Particle Data Group, "Review of Particle Physics," Chin. Phys., vol. C38, p. $090001,2014$.

[17] F. Englert and R. Brout, "Broken Symmetry and the Mass of Gauge Vector Mesons," Phys. Rev. Lett., vol. 13, pp. 321-323, 1964. 
[18] G. S. Guralnik, C. R. Hagen, and T. W. B. Kibble, "Global Conservation Laws and Massless Particles," Phys. Rev. Lett., vol. 13, pp. 585-587, 1964.

[19] L. Alvarez-Gaume and J. Ellis, "Eyes on a prize particle," Nature Physics, pp. 2-3, 2011.

[20] C. ATLAS, "Studies of the ATLAS potential for Higgs self-coupling measurements at a High Luminosity LHC," CERN, Geneva, Tech. Rep. ATL-PHYSPUB-2013-001, 2013.

[21] D. de Florian et al., "Handbook of LHC Higgs Cross Sections: 4. Deciphering the Nature of the Higgs Sector," 2016. arXiv: 1610.07922 [hep-ph].

[22] J. D. Lykken, "Beyond the Standard Model," in CERN Yellow Report CERN2010-002, 101-109, 2010. arXiv: 1005.1676 [hep-ph].

[23] M. Roos, "Dark Matter: The evidence from astronomy, astrophysics and cosmology," 2010. arXiv: 1001.0316 [astro-ph.CO].

[24] ATLAS Collaboration, "Search for invisible decays of a Higgs boson using vector-boson fusion in $p p$ collisions at $\sqrt{s}=8 \mathrm{TeV}$ with the ATLAS detector," JHEP, vol. 01, p. 172, 2016. arXiv: 1508.07869 [hep-ex].

[25] J. F. Gunion, "Extended Higgs sectors," in Supersymmetry and unification of fundamental interactions. Proceedings, 10th International Conference, SUSY'02, Hamburg, Germany, June 17-23, 2002, 2002, pp. 80-103. arXiv: hep-ph/0212150 [hep-ph].

[26] ATLAS Collaboration, "Study of the Higgs boson properties and search for high-mass scalar resonances in the $H \rightarrow Z Z^{*} \rightarrow 4 \ell$ decay channel at $\sqrt{s}=13$ TeV with the ATLAS detector," CERN, Geneva, Tech. Rep. ATLAS-CONF2016-079, 2016. 
[27] J. Unger, M. Kobel, and D. Stoeckinger, "Higgs Boson Production in the Atmosphere of the Earth," Presented 20 Nov 2014, PhD thesis, Dresden, Tech. U., 2014.

[28] O. S. Brning, P. Collier, P Lebrun, S. Myers, R. Ostojic, J. Poole, and P. Proudlock, LHC Design Report. Geneva: CERN, 2004.

[29] ATLAS Collaboration, "The ATLAS Experiment at the CERN Large Hadron Collider," J. Instrum., vol. 3, S08003. 437 p, 2008, Also published by CERN Geneva in 2010.

[30] CMS Collaboration, "The CMS Experiment at the CERN LHC," JINST, vol. 3, S08004, 2008.

[31] ALICE Collaboration, "The ALICE experiment at the CERN LHC," JINST, vol. 3, S08002, 2008.

[32] LHCb Collaboration, "The LHCb Detector at the LHC," JINST, vol. 3, S08005, 2008.

[33] L. Evans and P. Bryant, "Lhc machine," Journal of Instrumentation, vol. 3, no. 08, S08001, 2008.

[34] Nobel Media, The Nobel Prize in Physics 1984.

[35] UA1 Collaboration, "Further evidence for charged intermediate vector bosons at the $\{$ SPS $\}$ collider," Physics Letters B, vol. 129, no. 34, pp. $273-282,1983$.

[36] UA1 Collaboration, "Observation of muonic $Z^{0}$-decay at the pp collider," Physics Letters B, vol. 147, no. 1, pp. 241 -248, 1984.

[37] ATLAS Collaboration, "Improved luminosity determination in pp collisions at $\sqrt{s}=7 \mathrm{TeV}$ using the ATLAS detector at the LHC," Eur. Phys. J. C, vol. 73, no. arXiv:1302.4393. CERN-PH-EP-2013-026, 2518. 40 p, 2013. 
[38] CMS Collaboration, "Search for quark compositeness in dijet angular distributions from $p p$ collisions at $\sqrt{s}=7 \mathrm{TeV}$," JHEP, vol. 05, p. 055, 2012. arXiv: 1202.5535 [hep-ex].

[39] S. Chatrchyan et al., "Measurement of the Underlying Event Activity at the LHC with $\sqrt{s}=7 \mathrm{TeV}$ and Comparison with $\sqrt{s}=0.9 \mathrm{TeV}$," JHEP, vol. 09, p. 109, 2011. arXiv: 1107.0330 [hep-ex].

[40] J. M. Butterworth, G. Dissertori, and G. P. Salam, "Hard Processes in ProtonProton Collisions at the Large Hadron Collider," Ann. Rev. Nucl. Part. Sci., vol. 62, pp. 387-405, 2012. arXiv: 1202.0583 [hep-ex].

[41] J. Pequenao, "Computer generated image of the whole ATLAS detector," 2008.

[42] E. Daw, Lecture 7 - rapidity and pseudorapidity, 2012.

[43] ATLAS Collaboration, "Track Reconstruction Performance of the ATLAS Inner Detector at $\sqrt{s}=13 \mathrm{TeV}, "$ CERN, Geneva, Tech. Rep. ATL-PHYS-PUB2015-018, 2015.

[44] ATLAS Collaboration, "A study of the material in the ATLAS inner detector using secondary hadronic interactions," JINST, vol. 7, P01013, 2012. arXiv: 1110.6191 [hep-ex].

[45] M Capeans, G Darbo, K Einsweiller, M Elsing, T Flick, M Garcia-Sciveres, C Gemme, H Pernegger, O Rohne, and R Vuillermet, "ATLAS Insertable BLayer Technical Design Report," CERN, Geneva, Tech. Rep. CERN-LHCC2010-013. ATLAS-TDR-19, 2010.

[46] J. Pequenao, "Computer Generated image of the ATLAS calorimeter," 2008.

[47] S. Palestini, "The muon spectrometer of the atlas experiment," Nuclear Physics B-Proceedings Supplements, vol. 125, pp. 337-345, 2003. 
[48] J. Pequenao, "Computer generated image of the ATLAS Muons subsystem," 2008 .

[49] ATLAS Collaboration, "Standalone vertex finding in the ATLAS muon spectrometer," JINST, vol. 9, P02001, 2014. arXiv: 1311 . 7070 [physics.ins-det].

[50] T Kawamoto, S Vlachos, L Pontecorvo, J Dubbert, G Mikenberg, P Iengo, C Dallapiccola, C Amelung, L Levinson, R Richter, and D Lellouch, "New Small Wheel Technical Design Report," CERN, Geneva, Tech. Rep. CERN-LHCC2013-006. ATLAS-TDR-020, 2013, ATLAS New Small Wheel Technical Design Report.

[51] ATLAS Collaboration, "Expected Performance of the ATLAS Experiment Detector, Trigger and Physics," 2009. arXiv: 0901.0512 [hep-ex].

[52] S. Weinzierl, "Introduction to Monte Carlo methods," 2000. arXiv: hep-ph/ 0006269 [hep-ph].

[53] S. A. et al., "Geant4a simulation toolkit," Nuclear Instruments and Methods in Physics Research Section A: Accelerators, Spectrometers, Detectors and Associated Equipment, vol. 506, no. 3, pp. 250 -303, 2003.

[54] S. Alioli, P. Nason, C. Oleari, and E. Re, "NLO Higgs boson production via gluon fusion matched with shower in POWHEG," JHEP, vol. 04, p. 002, 2009. arXiv: 0812.0578 [hep-ph].

[55] P. Nason and C. Oleari, "NLO Higgs boson production via vector-boson fusion matched with shower in POWHEG," JHEP, vol. 02, p. 037, 2010. arXiv: 0911. 5299 [hep-ph].

[56] D. de Florian, G. Ferrera, M. Grazzini, and D. Tommasini, "Transversemomentum resummation: Higgs boson production at the Tevatron and the LHC," JHEP, vol. 11, p. 064, 2011. arXiv: 1109.2109 [hep-ph]. 
[57] T. Sjostrand, S. Mrenna, and P. Z. Skands, "A Brief Introduction to PYTHIA 8.1," Comput. Phys. Commun., vol. 178, pp. 852-867, 2008. arXiv: 0710.3820 $[$ hep-ph].

[58] D. J. Lange, "The EvtGen particle decay simulation package," Nucl. Instrum. Meth., vol. A462, pp. 152-155, 2001.

[59] J. Alwall, R. Frederix, S. Frixione, V. Hirschi, F. Maltoni, O. Mattelaer, H. S. Shao, T. Stelzer, P. Torrielli, and M. Zaro, "The automated computation of tree-level and next-to-leading order differential cross sections, and their matching to parton shower simulations," JHEP, vol. 07, p. 079, 2014. arXiv: 1405.0301 [hep-ph].

[60] M. Bahr et al., "Herwig++ Physics and Manual," Eur. Phys. J., vol. C58, pp. 639-707, 2008. arXiv: 0803.0883 [hep-ph].

[61] T. Gleisberg, S. Hoeche, F. Krauss, M. Schonherr, S. Schumann, F. Siegert, and J. Winter, "Event generation with SHERPA 1.1," JHEP, vol. 02, p. 007, 2009. arXiv: 0811.4622 [hep-ph].

[62] T. Sjostrand, S. Mrenna, and P. Z. Skands, "PYTHIA 6.4 Physics and Manual," JHEP, vol. 05, p. 026, 2006. arXiv: hep-ph/0603175 [hep-ph].

[63] P. Golonka and Z. Was, "PHOTOS Monte Carlo: A Precision tool for QED corrections in $Z$ and $W$ decays," Eur. Phys. J., vol. C45, pp. 97-107, 2006. arXiv: hep-ph/0506026 [hep-ph].

[64] S. Jadach, Z. Was, R. Decker, and J. H. Kuhn, "The tau decay library TAUOLA: Version 2.4," Comput. Phys. Commun., vol. 76, pp. 361-380, 1993.

[65] ATLAS Collaboration, "Measurements of the Total and Differential Higgs Boson Production Cross Sections Combining the $H \rightarrow \gamma \gamma$ and $H \rightarrow Z Z^{(*)} \rightarrow 4 \ell$ Decay Channels at $\sqrt{s}=8 T e V$ with the ATLAS Detector," Phys. Rev. Lett., vol. 115, p. $091801,92015$. 
[66] I Bird, P Buncic, F Carminati, M Cattaneo, P Clarke, I Fisk, M Girone, J Harvey, B Kersevan, P Mato, R Mount, and B Panzer-Steindel, "Update of the Computing Models of the WLCG and the LHC Experiments," Tech. Rep. CERN-LHCC-2014-014. LCG-TDR-002, 2014.

[67] Y. Nakahama, "The atlas trigger system: ready for run-2," Journal of Physics: Conference Series, vol. 664, no. 8, p. 082 037, 2015.

[68] ATLAS Collaboration, "Charged-particle distributions in $\sqrt{s}=13 \mathrm{TeV} p p$ interactions measured with the ATLAS detector at the LHC," Phys. Lett., vol. B758, pp. 67-88, 2016. arXiv: 1602.01633 [hep-ex].

[69] ATLAS Collaboration, "Electron and photon energy calibration with the ATLAS detector using LHC Run 1 data," Eur. Phys. J., vol. C74, no. 10, p. 3071, 2014. arXiv: 1407.5063 [hep-ex].

[70] ATLAS Collaboration, "Electron and photon energy calibration with the ATLAS detector using data collected in 2015 at $\sqrt{s}=13 \mathrm{TeV}$," CERN, Geneva, Tech. Rep. ATL-PHYS-PUB-2016-015, 2016.

[71] A. Keith Morley, "Electron Bremsstrahlung Studies and Track Based Alignment of the ATLAS Detector," PhD thesis, Melbourne U., 2010.

[72] ATLAS Collaboration, "Improved electron reconstruction in ATLAS using the Gaussian Sum Filter-based model for bremsstrahlung," CERN, Geneva, Tech. Rep. ATLAS-CONF-2012-047, 2012.

[73] T. M. Atkinson, "Electron reconstruction with the ATLAS inner detector," Presented 2006, PhD thesis, Melbourne U., 2006.

[74] S. Kullback and R. A. Leibler, "On Information and Sufficiency," Ann. Math. Statist., vol. 22, no. 1, pp. 79-86, Mar. 1951.

[75] "auto-parallelization and auto-vectorization", https : / / msdn . microsoft . com/en-us/library/hh872235. aspx, Accessed: 2016-10-17. 
[76] J. Illingworth and J. Kittler, "A survey of the hough transform," Computer Vision, Graphics, and Image Processing, vol. 44, no. 1, pp. 87 -116, 1988.

[77] ATLAS Collaboration, "Jet energy measurement and its systematic uncertainty in proton-proton collisions at $\sqrt{s}=7 \mathrm{TeV}$ with the ATLAS detector," The European Physical Journal C, vol. 75, no. 1, pp. 1-101, 2015.

[78] A. Roodman, "Blind analysis in particle physics," eConf, vol. C030908, TUIT001, 2003, [,166(2003)]. arXiv: physics/0312102 [physics.data-an].

[79] ATLAS Collaboration, "Electron efficiency measurements with the ATLAS detector using 2012 LHC proton-proton collision data," 2016. arXiv: 1612. 01456 [hep-ex].

[80] ATLAS Collaboration, "Selection of jets produced in $13 \mathrm{TeV}$ proton-proton collisions with the ATLAS detector," CERN, Geneva, Tech. Rep. ATLASCONF-2015-029, 2015.

[81] S. Schael et al., "Precision electroweak measurements on the $Z$ resonance," Phys. Rept., vol. 427, pp. 257-454, 2006. arXiv: hep-ex/0509008 [hep-ex].

[82] V. Ippolito, "Measurement of the properties of the new particle observed within the search for the Higgs boson in the $H \rightarrow Z Z^{(*)} \rightarrow 4 \ell$ channel at ATLAS.," PhD thesis, Rome U., 2013-12-06.

[83] G. Cowan, Statistical Data Analysis. Oxford University Press, Oxford, 1998.

[84] K. S. Cranmer, "Kernel estimation in high-energy physics," Comput. Phys. Commun., vol. 136, pp. 198-207, 2001. arXiv: hep-ex/0011057 [hep-ex].

[85] M. Pivk, "sPLOT: A Quick introduction," in Statistical Problems in Particle Physics, Astrophysics and Cosmology (PHYSTAT 05): Proceedings, Oxford, UK, September 12-15, 2005, 2006, pp. 173-177. arXiv: physics/0602023 [physics]. 
[86] UA1 Collaboration, "Intermediate-vector-boson properties at the CERN Super Proton Synchrotron collider," Europhys. Lett., vol. 1, no. CERN-EP-85-185, 327-345. 23 p, 1986.

[87] CMS Collaboration, "W-like measurement of the $\mathrm{Z}$ boson mass using dimuon events collected in pp collisions at $\sqrt{s}=7 \mathrm{TeV}$," CERN, Geneva, Tech. Rep. CMS-PAS-SMP-14-007, 2016.

[88] K. C. Sven Kreiss, $H \rightarrow Z Z^{*} \rightarrow 4 \ell$ Likelihood in ATLAS, Likelihoods for the LHC Searches, CERN Workshop, 2012.

[89] CMS Collaboration, "Precise determination of the mass of the Higgs boson and tests of compatibility of its couplings with the standard model predictions using proton collisions at 7 and 8 TeV," Eur. Phys. J., vol. C75, no. 5, p. 212, 2015. arXiv: 1412.8662 [hep-ex].

[90] M. Oreglia, "A Study of the Reactions $\psi^{\prime} \rightarrow \gamma \gamma \psi$," PhD thesis, SLAC, 1980.

[91] W. Spearman, "Measurement of the mass and natural width of the Higgs boson in the $H$ to $Z Z$ to $4 \ell$ decay channel with the ATLAS detector," PhD thesis, Harvard U. (main), 2014-09-11.

[92] "Proposal for truth particle observable definitions in physics measurements," CERN, Geneva, Tech. Rep. ATL-PHYS-PUB-2015-013, 2015.

[93] N. D. Gagunashvili, "Comparison of weighted and unweighted histograms," ArXiv Physics e-prints, May 2006. eprint: physics/0605123.

[94] R. Fernow, Introduction to experimental particle physics. Cambridge University Press, 1986.

[95] CMS Collaboration, "Measurement of the properties of a Higgs boson in the four-lepton final state," Phys. Rev., vol. D89, no. 9, p. 092007,2014 . arXiv: 1312.5353 [hep-ex]. 
[96] M. Grazzini, S. Kallweit, and D. Rathlev, "ZZ production at the LHC: fiducial cross sections and distributions in NNLO QCD," Phys. Lett., vol. B750, pp. 407-410, 2015. arXiv: 1507.06257 [hep-ph].

[97] G. Cowan, K. Cranmer, E. Gross, and O. Vitells, "Asymptotic formulae for likelihood-based tests of new physics," Eur. Phys. J., vol. C71, p. 1554, 2011, [Erratum: Eur. Phys. J.C73,2501(2013)]. arXiv: 1007 . 1727 [physics.data-an].

[98] S. Ahn and J. A. Fessler, Standard Errors of Mean, Variance, and Standard Deviation Estimators, 2003.

[99] L. Demortier and L. Lyons, "Everything you always wanted to know about pulls," The Rockefeller University, University of Oxford, Tech. Rep., 2002.

[100] Thomas Dorigo and Michael Schmitt, "On the Significance of the Dimuon Mass Bump and the Greedy Bump Bias," Tech. Rep., 2000.

[101] "Measurements of properties of the Higgs boson and search for an additional resonance in the four-lepton final state at $\sqrt{s}=13 \mathrm{TeV}$," CERN, Geneva, Tech. Rep. CMS-PAS-HIG-16-033, 2016.

[102] CMS Collaboration, "Search for Higgs boson off-shell production in protonproton collisions at 7 and $8 \mathrm{TeV}$ and derivation of constraints on its total decay width," JHEP, vol. 09, p. 051, 2016. arXiv: 1605.02329 [hep-ex]. 